\title{
aerospace
}

Advances in

Hybrid Rocket

Technology and

Related Analysis

Methodologies

Edited by

Carmine Carmicino

Printed Edition of the Special Issue Published in Aerospace 


\section{Advances in Hybrid Rocket Technology and Related Analysis Methodologies}





\section{Advances in Hybrid Rocket Technology and Related Analysis Methodologies}

Editor

Carmine Carmicino 
Editor

Carmine Carmicino

Università di Napoli "Federico II"

Italy

\section{Editorial Office}

MDPI

St. Alban-Anlage 66

4052 Basel, Switzerland

This is a reprint of articles from the Special Issue published online in the open access journal Aerospace (ISSN 2226-4310) (available at: https://www.mdpi.com/journal/aerospace/special_issues/hybrid_ rocket).

For citation purposes, cite each article independently as indicated on the article page online and as indicated below:

LastName, A.A.; LastName, B.B.; LastName, C.C. Article Title. Journal Name Year, Article Number, Page Range.

ISBN 978-3-03943-390-2 (Hbk)

ISBN 978-3-03943-391-9 (PDF)

(C) 2020 by the authors. Articles in this book are Open Access and distributed under the Creative Commons Attribution (CC BY) license, which allows users to download, copy and build upon published articles, as long as the author and publisher are properly credited, which ensures maximum dissemination and a wider impact of our publications.

The book as a whole is distributed by MDPI under the terms and conditions of the Creative Commons license CC BY-NC-ND. 


\section{Contents}

About the Editor $\ldots \ldots \ldots \ldots \ldots \ldots \ldots \ldots \ldots \ldots \ldots$

\section{Carmine Carmicino}

Special Issue "Advances in Hybrid Rocket Technology and Related Analysis Methodologies"

Reprinted from: Aerospace 2019, 6, 128, doi:10.3390/aerospace6120128 . . . . . . . . . . . . 1

\section{Carmine Carmicino}

Postscript for Special Issue "Advances in Hybrid Rocket Technology and Related

Analysis Methodologies"

Reprinted from: Aerospace 2020, 7, 117, doi:10.3390/aerospace7080117 . . . . . . . . . . . 5

Heejang Moon, Seongjoo Han, Youngjun You and Minchan Kwon

Hybrid Rocket Underwater Propulsion: A Preliminary Assessment

Reprinted from: Aerospace 2019, 6, 28, doi:10.3390/aerospace6030028 . . . . . . . . . . . 7

\section{Lorenzo Casalino, Filippo Masseni and Dario Pastrone}

Viability of an Electrically Driven Pump-Fed Hybrid Rocket for Small Launcher Upper Stages

Reprinted from: Aerospace 2019, 6, 36, doi:10.3390/aerospace6030036 . . . . . . . . . . . . 27

Landon Kamps, Kazuhito Sakurai, Yuji Saito and Harunori Nagata

Comprehensive Data Reduction for $\mathrm{N}_{2} \mathrm{O}$ /HDPE Hybrid Rocket Motor Performance Evaluation

Reprinted from: Aerospace 2019, 6, 45, doi:10.3390/aerospace6040045 . . . . . . . . . . . 43

Suhang Chen, Yue Tang, Wei Zhang, Ruiqi Shen, Hongsheng Yu, Yinghua Ye and

Luigi T. DeLuca

Innovative Methods to Enhance the Combustion Properties of Solid Fuels for Hybrid

Rocket Propulsion

Reprinted from: Aerospace 2019, 6, 47, doi:10.3390/aerospace6040047 . . . . . . . . . . . 65

Giuseppe Daniele Di Martino, Carmine Carmicino, Stefano Mungiguerra and

Raffaele Savino

The Application of Computational Thermo-Fluid-Dynamics to the Simulation of Hybrid Rocket Internal Ballistics with Classical or Liquefying Fuels: A Review

Reprinted from: Aerospace 2019, 6, 56, doi:10.3390/aerospace6050056 . . . . . . . . . . . 87

Jérôme Messineo and Toru Shimada

Theoretical Investigation on Feedback Control of Hybrid Rocket Engines

Reprinted from: Aerospace 2019, 6, 65, doi:10.3390/aerospace6060065

Timothy Marquardt and Joseph Majdalani

Review of Classical Diffusion-Limited Regression Rate Models in Hybrid Rockets

Reprinted from: Aerospace 2019, 6, 75, doi:10.3390/aerospace6060075 . . . . . . . . . . . . 169

\section{Mitchell McFarland and Elsa Antunes}

Small-Scale Static Fire Tests of 3D Printing Hybrid Rocket Fuel Grains Produced from Different Materials

Reprinted from: Aerospace 2019, 6, 81, doi:10.3390/aerospace6070081 . . . . . . . . . . . . 187 
Daniele Bianchi, Giuseppe Leccese, Francesco Nasuti, Marcello Onofri and

Carmine Carmicino

Modeling of High Density Polyethylene Regression Rate in the Simulation of Hybrid Rocket

Flowfields

Reprinted from: Aerospace 2019, 6, 88, doi:10.3390/aerospace6080088 _ . . . . . . . . . . . 199

Francesco Battista, Daniele Cardillo, Manrico Fragiacomo, Giuseppe Daniele Di Martino,

Stefano Mungiguerra and Raffaele Savino

Design and Testing of a Paraffin-Based 1000 N HRE Breadboard

Reprinted from: Aerospace 2019, 6, 89, doi:10.3390/aerospace6080089 . . . . . . . . . . . . . 225

\section{Christian Paravan}

Nano-Sized and Mechanically Activated Composites: Perspectives for Enhanced Mass Burning

Rate in Aluminized Solid Fuels for Hybrid Rocket Propulsion

Reprinted from: Aerospace 2019, 6, 127, doi:10.3390/aerospace6120127 . . . . . . . . . . . . . 241

\section{Stephen A. Whitmore}

Nytrox as "Drop-in" Replacement for Gaseous Oxygen in SmallSat Hybrid Propulsion Systems

Reprinted from: Aerospace 2020, 7, 43, doi:10.3390/aerospace7040043 . . . . . . . . . . . . . . 275

\section{Francesca Heeg, Lukas Kilzer, Robin Seitz and Enrico Stoll}

Design and Test of a Student Hybrid Rocket Engine with an External Carbon Fiber

Composite Structure

Reprinted from: Aerospace 2020, 7, 57, doi:10.3390/aerospace7050057 . . . . . . . . . . . . 313

\section{Olexiy Shynkarenko and Domenico Simone}

Oxygen-Methane Torch Ignition System for Aerospace Applications

Reprinted from: Aerospace 2020, 7, 114, doi:10.3390/aerospace7080114 . . . . . . . . . . . . 333 


\section{About the Editor}

Carmine Carmicino is currently a new-product-introduction test project engineer at Baker Hughes; in the same company (previously GE Oil \& Gas), he held the position of senior engineer in the centrifugal compressor design department. In 2019, he was a visiting associate professor at ISAS-JAXA (Japan).

From 2006 to 2007, he was a system engineer at CIRA, the Italian Aerospace Research Center.

From 2003 to 2006, he was a postdoctoral appointee of the Department of Space and Engineering Science "L. G. Napolitano" of Università di Napoli “Federico II" (Italy).

He is currently a research consultant for the Department of Industrial Engineering at the Università di Napoli "Federico II". He has also worked in collaboration with the Università di Roma "La Sapienza" and Politecnico di Torino, on several aspects of hybrid rocket propulsion.

He has been a member of the AIAA Hybrid Rockets TC since 2006.

He received his Ph.D. in Aerospace Engineering from the Università di Napoli “Federico II" in 2003, and has the Italian National Qualification to the rank of Associate Professor in Aerospace Engineering.

His main research topics encompass all the hybrid rocket system aspects, including engine performance, internal ballistics, thermo-acoustics, oxidizer injector design, fuel regression rate modeling, measurement and improvement, applied computational fluid dynamics, lower order numerical modeling, firing tests design and conduction. 
To Roo, who has given me the key to access the unknowns into the depths of my soul.

"Humpty Dumpty sat on a wall.

Humpty Dumpty had a great fall.

All the Kings Horses and all the Kings Men,

couldn't put Humpty together again." 


\title{
Editorial \\ Special Issue "Advances in Hybrid Rocket Technology and Related Analysis Methodologies"
}

\author{
Carmine Carmicino \\ Department of Industrial Engineering-Aerospace Division, University of Naples "Federico II", \\ Piazzale Tecchio, 80-80125 Naples, Italy; carmicin@unina.it
}

Received: 21 November 2019; Accepted: 21 November 2019; Published: 26 November 2019

Hybrid rockets are chemical propulsion systems that, in the most common configuration, employ a liquid oxidizer (or gaseous in much rarer cases) and a solid fuel; the oxidizer, stored in tanks, is properly injected in the combustion chamber where the solid fuel grain is bonded. In the classical arrangement, one or more ports are present in the fuel grain whereby the oxidizer flows and burns with the fuel vapors. When speaking about hybrid rockets, one cannot avoid stating that, although they may seem to lie somewhere between a liquid and a solid propellant system, this propulsion technology, thanks to the phase separation of the two propellants and the vast choice of available inert fuels, features unique advantages, well known in the propulsion community [1,2], which are not enjoyed by either liquids or solids. In fact, because they essentially preserve performance comparable to the high level of the most complicated liquid rocket engines, their several benefits, spanning lower development cost, higher safety and less environmental impact, can lead to extensive employment as game changing technology in the current space arena characterized by a dramatic upsurge of worldwide activities and the parallel emergence of different types of space actors [3,4].

The hybrid rocket concept dates back to the early twentieth century [5], but till about a decade ago, hybrids have been perceived as a niche technology; nowadays, however, they are attracting renewed interest from both the propulsion technical community and industry. The number of researchers involved in this subject has increased more and more all over the globe along with the launch of student sounding rockets $[6,7]$.

Hybrid propellant engines can be used in practically all applications where a rocket is needed, but there are certain cases where they present a superior fit, such as the above-mentioned sounding rockets, tactical missile systems, launch boosters and the emerging field of commercial space transportation. The novel space tourism business will definitely benefit from their safety and lower recurrent development costs. The famous Virgin Galactic SpaceShipTwo is, indeed, a spacecraft propelled by a hybrid rocket engine that aims to take tourists on brief trips to suborbital space at an unusually large flight frequency [8]. Manufactured by The Spaceship Company, the vehicle is currently in an advanced testing stage. Furthermore, it was last October 8 that Boeing announced a strategic investment in Virgin Galactic, which could eventually support high-speed future passenger transportation systems [9]. Those are all clear signs of growing, genuine attention toward the hybrid rockets alternative, and probably the real challenge facing researchers is inseminating the hybrid culture to enable the widespread adoption of this technology, which is still hindered not for technical reasons, but due to societal factors like the stereotype represented by the mature solid and liquid propellant rockets. Within this framework and with the latter purpose, the Special Issue of Aerospace "Advances in Hybrid Rocket Technology and Related Analysis Methodologies" was born.

The current key research areas include systems to improve the slow fuel regression rate, such as the selection of paraffin-wax-based fuel casting; the enhancement of wall heat transfer with nonstandard oxidizer injection methods and/or fuel grain configurations; the effects of the addition of energetic ingredients into the fuel, the suppression of combustion instability; and the optimization of engine components. 
Scientists from Japan, USA, China, France, Australia, South Korea and Italy have contributed to make this Special Issue an amazing collection of papers drawing a picture of the state of the art. I am honored to present twelve excellent articles from some of the most accredited scholars active in the sector as well as from emerging research organizations covering a wide range of topics, which encompass nearly all the subjects just listed above, from fundamental research to real-world applications.

Three review papers appear in the Special Issue; Marquardt and Majdalani [10] revise the Marxman's classical diffusion-limited regression rate model with the purpose of complementing the existing literature, providing a unique combination of detail and brevity that will be appreciated by newcomers entering the field. The development of accurate simulations of reacting flows incorporating the capability of modeling the fuel surface regression poses significant challenges for computational methods, and the article from Di Martino et al. [11] is concerned with the application of computational thermo-fluid-dynamics to the simulation of the internal ballistics of rockets burning either standard polymeric or liquefying fuels; finally, Chen et al. [12] conducted an excellent survey of several innovative methods under testing to improve the solid fuels' combustion properties, presenting a number of experimental results.

Three research papers address particularly innovative themes in the hybrid rocket literature: the article from Messineo and Shimada [13] is centered around a non-conventional engine configuration where the oxidizer injection in the fuel port is split in two separated streams: one of which is axial and the other is tangential with the idea of controlling, independently, both the injected flow rates to optimize the mixture ratio with a given thrust profile. A theoretical investigation into the feedback control of hybrid rocket engines is, thus, addressed; Casalino et al. [14] examined the viability of an electrically driven pump-fed hybrid rocket for small launcher upper stages of the Vega class, demonstrating that it can be a suitable option for the replacement of the conventional pressurized gas feed system. The third paper from Moon et al. [15] reports on an assessment of a hybrid rocket for underwater propulsion, suggesting that the throttleable hybrid engine could be an effective candidate for a short-duration, high-speed marine boosting device as an alternative to the solid propulsion system.

The remaining research articles deal with more standard topics, yet are well deserving of attention. They focus on the scaling up problem, the CFD modeling of the regression rate, the application of ballistic reconstruction methods to the engine performance evaluation and the effect of energetic ingredients to improve the regression rate. Experimental results from a number of firings of a paraffin-based, $1 \mathrm{kN}$ thrust rocket are discussed by Battista et al. [16]; the results of numerical simulations carried out with an ad-hoc CFD code are reported by Bianchi et al. [17] for the high-density polyethylene regression rate calculation, highlighting the influence of the gas-phase radiation contribution to the total heat flux to the surface; some preliminary data from small-scale static firings of 3D printed fuel grains made by several materials are shown in [18], by Mc Farland and Antunes; the experimental performance data acquired from a hybrid rocket fed by nitrous oxide and high density polyethylene for the application to an apogee kick motor were treated by Kamps et al. [19]. Regression rate and mass burning rate obtained with the addition of nano- or micron-sized aluminum powders and oxidizer-containing fuel-rich composites to HTPB (Hydroxyl-terminated Polybutadiene) are investigated in comparison to the baseline pure fuel in the paper of Paravan [20].

Finally, the subject analyzed by Whitmore [21] in the twelfth paper, which at the moment of this foreword is still on the publication path, is the investigation into a fluid blend of nitrous oxide and gaseous oxygen as a significantly safer and higher volumetrically-efficient alternative for the current generation of environmentally-unsustainable spacecraft propellants.

The first article appearing in this issue was published on 6 March 2019, whereas the last was just a few days ago; since then, from the MDPI articles' access metrics service, one can see a continuous increase of interest in the papers, with peak numbers of about 1300 downloads and 2000 views per article. The latter data are extremely encouraging and, considering the relatively small competent community compared to the solid and liquid propulsion groups, make us hope one day soon we will see large-scale development of hybrid rocket engines. 
Acknowledgments: I am grateful to all the authors who accepted to publish a piece of their work in this Special Issue, making it a success; I would like to thank the numerous referees who briskly and accurately worked on the multiple reviews of the papers: they all did an outstanding job, which guaranteed the high quality of this Special Issue. Additionally, special thanks go to the Aerospace editorial office; in particular, to Peter Liu for his relentless support.

Conflicts of Interest: The author declares no conflict of interest.

\section{References}

1. Altman, D.; Humble, R. Hybrid Rocket Propulsion Systems. In Space Propulsion Analysis and Design; Humble, R.W., Henry, G.N., Larson, W.J., Eds.; The McGraw-Hill Companies, Inc., Primis Custom Publishing: New York, NY, USA, 1995; pp. 365-370.

2. Altman, D.; Holzman, A. Overview and History of Hybrid Rocket Propulsion. In Fundamentals of Hybrid Rocket Combustion and Propulsion; Kuo, K., Chiaverini, M., Eds.; Progress in Astronautics and Aeronautics; AIAA: Reston, VA, USA, 2007; pp. 1-36.

3. Schmierer, C.; Kobald, M.; Tomilin, K.; Fischer, U.; Schlechtriem, S. Low cost small-satellite access to space using hybrid rocket propulsion. Acta Astronautica 2019, 158, 578-583. [CrossRef]

4. Jens, E.T.; Cantwell, B.J.; Hubbard, G.S. Hybrid rocket propulsion systems for outer planet exploration missions. Acta Astronautica 2016, 128, 119-130. [CrossRef]

5. Altman, D. Hybrid Rocket Development History. In Proceedings of the 27th AIAA/ASME/SAE/ASEE Joint Propulsion Conference and Exhibit, Sacramento, CA, USA, 24-26 June 1991.

6. Okninski, A. On use of hybrid rocket propulsion for suborbital vehicles. Acta Astronautica 2018, 145, 1-10. [CrossRef]

7. Kobald, M.; Fischer, U.; Tomilin, K.; Schmierer, C.; Petrarolo, A. Hybrid Sounding Rocket HEROS: TRL 9. In Proceedings of the 7th European Conference for Aeronautics and Aerospace Sciences (EUCASS), Milano, Italy, 3-7 July 2017.

8. Grossman, D. Richard Branson's Plans for Space Tourism Sure Are Aggressive. Popular Mechanics. 11 September 2019. Available online: https://www.popularmechanics.com/space/rockets/a28987245/virgingalactic-space-flight-plans/ (accessed on 26 November 2019).

9. Foustm, J. Boeing to invest in Virgin Galactic. Spacenews. 8 October 2019. Available online: https: //spacenews.com/boeing-to-invest-in-virgin-galactic/ (accessed on 26 November 2019).

10. Marquardt, T.; Majdalani, J. Review of Classical Diffusion-Limited Regression Rate Models in Hybrid Rockets. Aerospace 2019, 6, 75. [CrossRef]

11. Di Martino, G.D.; Carmicino, C.; Mungiguerra, S.; Savino, R. The Application of Computational Thermo-Fluid-Dynamics to the Simulation of Hybrid Rocket Internal Ballistics with Classical or Liquefying Fuels: A Review. Aerospace 2019, 6, 56. [CrossRef]

12. Chen, S.; Tang, Y.; Zhang, W.; Shen, R.; Yu, H.; Ye, Y.; DeLuca, L.T. Innovative Methods to Enhance the Combustion Properties of Solid Fuels for Hybrid Rocket Propulsion. Aerospace 2019, 6, 47. [CrossRef]

13. Messineo, J.; Shimada, T. Theoretical Investigation on Feedback Control of Hybrid Rocket Engines. Aerospace 2019, 6, 65. [CrossRef]

14. Casalino, L.; Masseni, F.; Pastrone, D. Viability of an Electrically Driven Pump-Fed Hybrid Rocket for Small Launcher Upper Stages. Aerospace 2019, 6, 36. [CrossRef]

15. Moon, H.; Han, S.; You, Y.; Kwon, M. Hybrid Rocket Underwater Propulsion: A Preliminary Assessment. Aerospace 2019, 6, 28. [CrossRef]

16. Battista, F.; Cardillo, D.; Fragiacomo, M.; Di Martino, G.D.; Mungiguerra, S.; Savino, R. Design and Testing of a Paraffin-Based 1000 N HRE Breadboard. Aerospace 2019, 6, 89. [CrossRef]

17. Bianchi, D.; Leccese, G.; Nasuti, F.; Onofri, M.; Carmicino, C. Modeling of High Density Polyethylene Regression Rate in the Simulation of Hybrid Rocket Flowfields. Aerospace 2019, 6, 88. [CrossRef]

18. McFarland, M.; Antunes, E. Small-Scale Static Fire Tests of 3D Printing Hybrid Rocket Fuel Grains Produced from Different Materials. Aerospace 2019, 6, 81. [CrossRef]

19. Kamps, L.; Sakurai, K.; Saito, Y.; Nagata, H. Comprehensive Data Reduction for $\mathrm{N}_{2} \mathrm{O} / \mathrm{HDPE}$ Hybrid Rocket Motor Performance Evaluation. Aerospace 2019, 6, 45. [CrossRef]

20. Paravan, C. Nano-Sized and Mechanically Activated Composites: Perspectives for Enhanced Mass Burning Rate in Aluminized Solid Fuels for Hybrid Rocket Propulsion. Aerospace 2019, 6, 127. [CrossRef] 
21. Whitmore, $\mathrm{S} . \mathrm{N}_{2} \mathrm{O} / \mathrm{O}_{2}$ Blend as an Inherently Safe and Volumetrically Efficient Oxidizer for Small Spacecraft Hybrid Propulsion Systems. Aerospace 2019, in press.

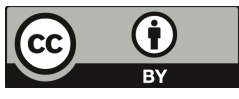

(C) 2019 by the author. Licensee MDPI, Basel, Switzerland. This article is an open access article distributed under the terms and conditions of the Creative Commons Attribution (CC BY) license (http://creativecommons.org/licenses/by/4.0/). 


\title{
Editorial \\ Postscript for Special Issue "Advances in Hybrid Rocket Technology and Related Analysis Methodologies"
}

\author{
Carmine Carmicino \\ Department of Industrial Engineering-Aerospace Division, University of Naples "Federico II", \\ Piazzale Tecchio, 80-80125 Naples, Italy; carmicin@unina.it \\ Received: 10 August 2020; Accepted: 13 August 2020; Published: 14 August 2020
}

Since the Editorial [1] of this Special Issue was published last 26 November 2019, I have had the pleasure to accept the submission of a couple of new articles which are now included in the Issue; moreover, for the sake of clarification, I want to mention that, over the revision process, the title of the paper [2] — which at that time was thought of as the last to be published-has been changed to more fairly comply with the revised topics.

The last two papers now appearing in the Special Issue deal, in the temporal order of publication, with the design and testing of a student hybrid rocket engine featuring an external carbon fiber composite structure [3], and with the development of an oxygen-methane torch ignition system designed for a hybrid rocket and later improved to be used in the testing of solid and liquid ramjet engines [4].

The former was developed in the framework of the German educational program Studentische Experimental-Raketen (STERN), by students of the Technische Universität Braunschweig, whereas the latter reports on a part of a research funded by the Foundation for the Scientific Research Support of the Brazilian Federal District at the University of Brasilia. I have particularly welcomed these two articles, in that, both being born in institutions recently involved in hybrids, they further prove the nice spread of the research activities in this subject across the world.

The aim of this short addendum is to give the final picture of the contents of the Special Issue, which collects 14 papers and 1 Editorial, 3 of which are review papers, 10 are original research papers, and 1 is a technical note.

The success of this experience has laid the groundwork for the "Hybrid Rocket (Volume II)", edited in collaboration with Toru Shimada and Arif Karabeyoglu [5], which, alongside articles addressing the advances in hybrid rocket technology and related analysis methodologies, will welcome papers dealing with novel space transportation systems, new flight systems, and mission concepts and optimization using hybrid rockets.

Funding: This research received no external funding.

Conflicts of Interest: The author declares no conflict of interest.

\section{References}

1. Carmicino, C. Special Issue "Advances in Hybrid Rocket Technology and Related Analysis Methodologies". Aerospace 2019, 6, 128. [CrossRef]

2. Whitmore, S.A. Nytrox as "Drop-in" Replacement for Gaseous Oxygen in SmallSat Hybrid Propulsion Systems. Aerospace 2020, 7, 43. [CrossRef]

3. Heeg, F.; Kilzer, L.; Seitz, R.; Stoll, E. Design and Test of a Student Hybrid Rocket Engine with an External Carbon Fiber Composite Structure. Aerospace 2020, 7, 57. [CrossRef]

4. Shynkarenko, O.; Simone, D. Oxygen-Methane Torch Ignition System for Aerospace Applications. Aerospace 2020, 7, 114. [CrossRef] 
5. Special Issue "Hybrid Rocket (Volume II)". Available online: https://www.mdpi.com/journal/aerospace/ special_issues/Hybrid_Rocket_II (accessed on 8 July 2020).

(c)

(C) 2020 by the author. Licensee MDPI, Basel, Switzerland. This article is an open access article distributed under the terms and conditions of the Creative Commons Attribution (CC BY) license (http://creativecommons.org/licenses/by/4.0/). 


\title{
Hybrid Rocket Underwater Propulsion: A Preliminary Assessment
}

\author{
Heejang Moon ${ }^{1, *}$, Seongjoo Han ${ }^{1}$, Youngjun You ${ }^{2}$ and Minchan Kwon ${ }^{2}$ \\ 1 Aerospace and Mechanical Engineering, Korea Aerospace University, Goyang 10540, Korea; \\ freemanswill@gmail.com \\ 2 Agency for Defense Development, Daejeon 34186, Korea; yjyou@add.re.kr (Y.Y.); mckwon@add.re.kr (M.K.) \\ * Correspondence: hjmoon@kau.ac.kr; Tel.: +82-2-300-0118
}

Received: 28 January 2019; Accepted: 22 February 2019; Published: 6 March 2019

\begin{abstract}
This paper presents an attempt to use the hybrid rocket for marine applications with a $500 \mathrm{~N}$ class hybrid motor. A 5-port high density polyethylene (HDPE) fuel grain was used as a test-bed for the preliminary assessment of the underwater boosting device. A rupture disc preset to burst at a given pressure was attached to the nozzle exit to prevent water intrusion where a careful hot-firing sequence was unconditionally required to avoid the wet environment within the chamber. The average thrust level around $450 \mathrm{~N}$ was delivered by both a ground test and an underwater test using a water-proof load cell. However, it was found that instantaneous underwater thrusts were prone to vibration, which was due in part to the wake structure downstream of the nozzle exit. Distinctive ignition curves depending on the rupture disc bursting pressure and oxidizer mass flow rate were also investigated. To assess the soft-start capability of the hybrid motor, the minimum power thrust, viewed as the idle test case, was evaluated by modulating the flow controlling valve. It was found that an optimum valve angle, delivering $16.3 \%$ of the full throttle test case, sustained the minimum thrust level. This preliminary study suggests that the throttable hybrid propulsion system can be a justifiable candidate for a short-duration, high-speed marine boosting system as an alternative to the solid underwater propulsion system.
\end{abstract}

Keywords: hybrid rocket; marine propulsion; rupture disc; idling operation; underwater environment

\section{Introduction}

Underwater propulsion systems using conventional chemical rockets are uncommon and quite rare because of their short operating time, high noise, non-stealthiness, and shallow water depth operability with respect to the screw propeller driven system. These result from the limitations of a hot gas jet propulsive mechanism, which differs from the mechanical driver device where two-phase flow and high water-to-gas density ratios are present at the exit of the convergent/divergent nozzle, complicating the flow structure [1,2]. Mechanical driver devices, i.e., screw propellers, are usually driven by diesel engines, gas turbines, or even nuclear reactors for marine vessels, while, with the exception of rare trials using rocket propulsion, compressed heated air, electric motors, monopropellants, and gas turbines are used mostly for torpedoes. Modern marine vehicle development, either for watercrafts or torpedoes, focuses on speed augmentation, longer range and noise reduction, and additional depth increase for the underwater apparatus [3]. None of these factors are favorable for rocket application for marine propulsion, except for the speed augmentation, since the objective of a high-speed exhaust gas jet is a means of propulsion rather than noise reduction [4]. If a short-range and high-speed vehicle is enough for tactical purpose without taking any countermeasure for the noise, the underwater rocket propulsion could be an answer [4-6]. 
From the 20th century until today, marine propulsion designers and engineers have worked to increase torpedo speeds such that the modern high performing torpedo generally operates at around 50 knots. It is known that the increase of necessary power is proportional to the cubic of the rotational shaft speed, and therefore $240 \%$ increase in power output is required for a speed increase from 45 to 60 knots with a mechanically driven screw propeller [5,6]. Thus, rocket propulsion has been sporadically considered even up to the present time. From the first US Navy trial of the Ericsson rocket [4] proposed in the early 1880s to the recent "supercavitating rocket" [7], the underwater rocket has its own heritage with respect to the conventional torpedo in terms of cost, lack of moving parts, simplicity, minimal preparation, low maintenance, and low weight, depending on the rocket type. Research interest in high-speed torpedoes relying on solid propellant rockets increased in the past mid-twentieth century following World War II due to their speed, low cost, reliability, and negligible pre-launch preparation requirements [4]. To the authors' knowledge, projects such as HEYDAY [4], CAMROSE [4,5] for anti-torpedo mission shown in Figure 1a, and BOOTLEG [4,5] for anti-ship missions were the earliest rocket-propelled torpedo studies conceived by the British since World War II. However, these projects were all abandoned due to the lack of financial support, which instead prioritized noise reduction studies. The focus of this study is not on supercavitating rockets nor on torpedo applications. Rather, the work proposed in this paper is focused on conventional chemical rocket propulsion systems for marine vessels.

In addition to a pure underwater main propulsion device, a rocket can also be envisaged as an auxiliary propulsion device for boosting the already running watercraft for some duration when used together with the conventional screw propeller driven system [8-12]. If a further rocket thrust variation is achievable, it would increase the mission flexibility of a marine vessel. It is also important to mention that thrust control for a soft-start is essential for marine vessels because the sudden acceleration in the start-up stage is dangerous, since it can disrupt the balance of the marine vehicle. Hence, the throttable rocket propulsion system can not only be applied to conventional vessels but also to high-speed vessels, which can be faster than the speed of "Ghost" (50 knots), built by Juliet Marine System shown in Figure 1b, which uses a gas turbine based engine [13].

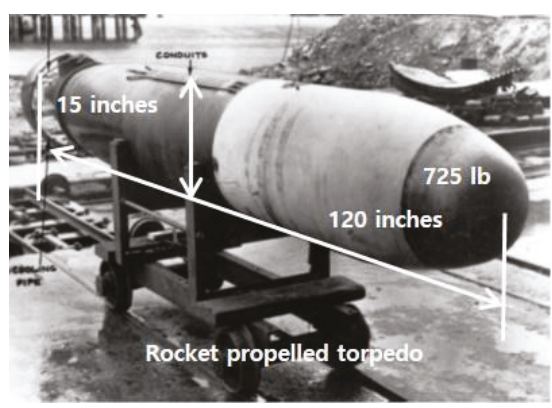

(a)

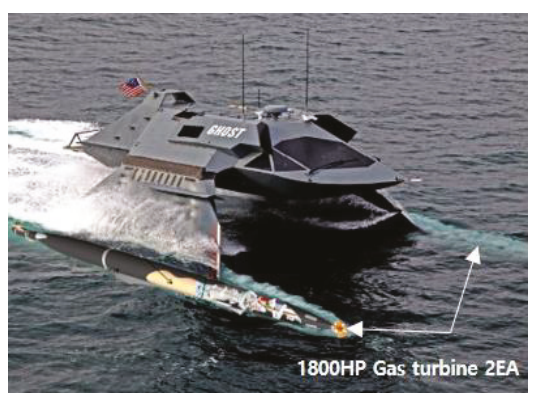

(b)

Figure 1. (a) CAMROSE dynamic test vehicle c.1954 [5]; (b) Ghost (Juliet Marine system) [13].

Until now, there has been no systematic classification of solid, liquid, and hybrid rockets for underwater environments. Marine propulsion using a solid propellant rocket has two main drawbacks: (1) the lack of thrust modulation capability with respect to hybrid and liquid counterparts; and (2) the low thrust level required for somewhat longer underwater operations. For the former, a lack of thrust modulation capability by means of propellant feed rate is a key classical drawback of solid propellant rockets since the thrust controllability is not frequently demanded for solid propellant systems. For the latter, solid propellant underwater propulsion systems have no choice but to have low thrust levels because these solid propellant rockets are forced to have "end-burning" grain configurations for 
prolonged operation time. The enlargement of propellant grain diameter required for a tubular port solid propellant grain is not a good solution for increasing the thrust level or for increasing the operating time because the drag force is increased as the square of the diameter. Concerning the liquid propellant rocket, it is obvious that its relatively heavy weight, large dimension, and complicated plumbing system are not very effective for underwater application.

Meanwhile, with its thrust controllability, the hybrid rocket used to be known as a cost-saving rocket with many advantages in terms of insensitivity of fuel, multiple shut on/off capability, easy handling, and environmental friendliness compared to solid and liquid propellant rockets. In addition, a typical hybrid propellant system can achieve a higher specific impulse than a solid propellant system, even though the belief that the hybrid propulsion system has a lower thrust/weight ratio than the solid propulsion system due to the addition of an oxidizer tank is still controversial in the field. Nonetheless, the throttleability of a propulsion system using a solidified fuel by means of a single oxidizer flow is very attractive since it reduces tremendous plumbing unlike conventional liquid bi-propellant systems. Owing to this, the underwater hybrid rocket can be an attractive alternative to solid or liquid counterparts, not to mention a safer alternative. Furthermore, common solid fuels used in the hybrid rocket propulsion system are suitable in humid environments due to the moisture-resistance of these fuels. From this point of view, a novel application of a hybrid rocket propulsion system for underwater application is introduced in this paper.

This study aimed to demonstrate the feasibility of the hybrid rocket application in an underwater environment rather than underlying interests on motor performance, e.g., use of high performing cryogenic oxidizers like liquid oxygen (LOX) [14-16] and use of high regression rate fuels with energetic particle addition [16-18] or swirl assisted injectors $[19,20]$. For this reason, nitrous oxide $\left(\mathrm{N}_{2} \mathrm{O}\right)$ was selected as the oxidizer since it does not require an additional pressurization system, while high density polyethylene (HDPE) was selected as the fuel since HDPE is known to be easily accessible for academic purposes in laboratory scale experiments. This is one of the simplest and most compact hybrid propulsion systems that can minimize the vehicle weight and the number of components. A series of hot firing tests was conducted to demonstrate the feasibility of the hybrid rocket application in an underwater environment where the $500 \mathrm{~N}$ class lab-scale hybrid rocket motors were used together for the underwater experimental set-up. A rupture disc was attached to the nozzle exit during each test to prevent the intrusion of water. Special emphasis was also placed on investigating the oxidizer supply timing and ignition characteristics. We also investigated the feasibility of an underwater hybrid rocket system in terms of full power thrust and factors to be assessed for minimum power thrust, i.e., the idle case.

\section{Underwater Setup}

\subsection{Underwater Experimental System}

Figure 2 shows the schematic of the experimental setup for the $500 \mathrm{~N}$ class lab-scale hybrid rocket motor used for the static-tests. The experimental setup of each unit was composed of an oxidizer feed system, ignition system, data acquisition (DAQ) system, and the hybrid rocket motor, where the unit number 8 represented the water filled tank. For the ground test, the hybrid motor was out of the water tank, while for the underwater test, a supplemental cap plugged in the rocket nozzle exit and the water filled tank accommodating the hybrid motor were additionally used.

The cross-sectional view of the lab-scale hybrid motor used both for ground and underwater combustion experiments is shown in Figure 3a. From the head-end, a shower head injector, pre-chamber, fuel grain, post-combustion chamber and a water-cooled copper nozzle used to prevent over-heating of the environment were all attached in-line making a total motor length of $457 \mathrm{~mm}$. Pressure transducers were each mounted in the pre-chamber and post-chamber for static pressure measurement with a $\mathrm{K}$ type thermocouple to monitor the temperature level in the nozzle section. Figure $3 \mathrm{~b}$ is an outer tank view showing the vertical transfer line, nozzle cooling line, and a cap 
plugged into the nozzle exit with the motor unit. The supplemental cap for the underwater firing test comprised a rupture disc provided by FDC Co. Ltd. (shown in Figure 4a,b) that not only prevented the intrusion of water during the ignition stage, but also blasts at a given preset chamber pressure.

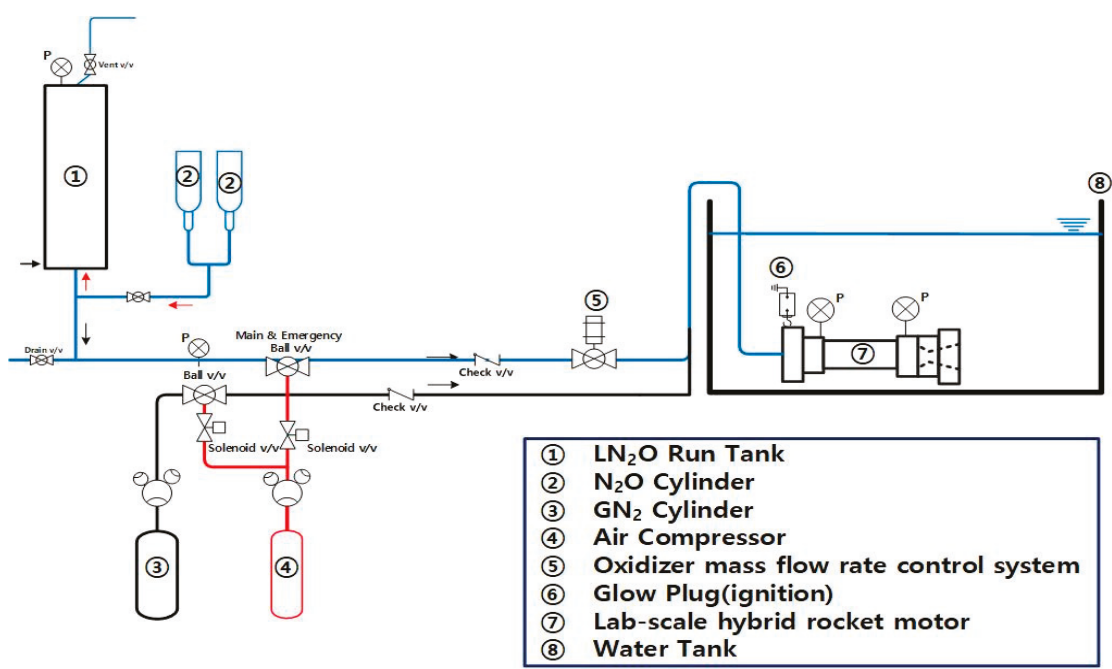

Figure 2. Schematic of experimental setup for underwater hot-firing test.

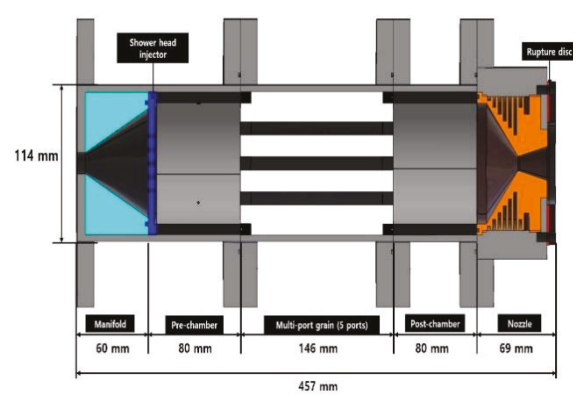

(a)

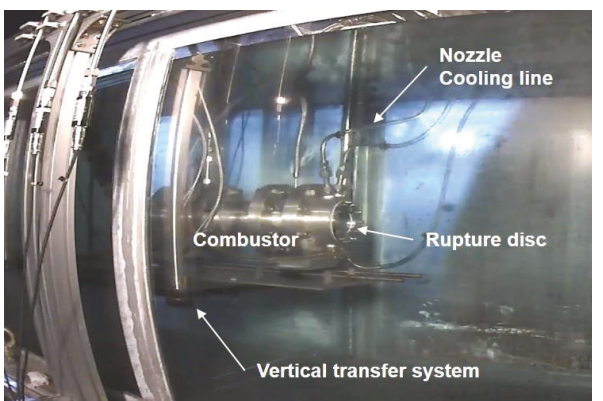

(b)

Figure 3. (a) Cross-sectional view of lab-scale motor; (b) lab-scale hybrid rocket motor mounted in the water tank.

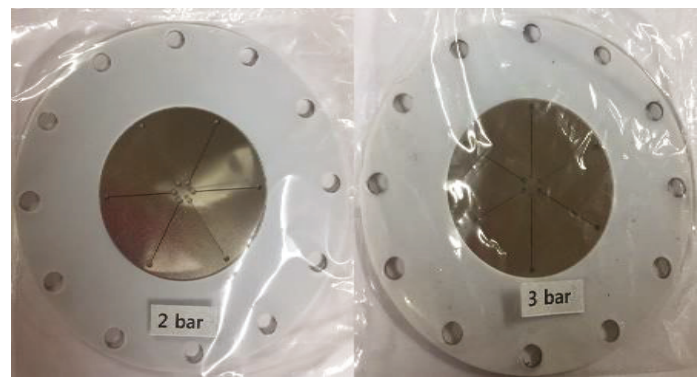

(a)

(b)

Figure 4. Rupture disc (provided by FDC Co. Ltd. Gimhae, Korea), (a) bursting pressure set to 2 bar gauge; (b) bursting pressure set to 3 bar gauge. 
Figure 5 shows the oxidizer supply system for the ground and underwater experiments. All combustion experiments were conducted in blow down mode using liquid $\mathrm{N}_{2} \mathrm{O}$ by assuming no spontaneous mixing of the liquid and vapor during the evacuation of $\mathrm{N}_{2} \mathrm{O}$ from the tank. The oxidizer run tank was charged from two $\mathrm{N}_{2} \mathrm{O}$ tanks, and the oxidizer mass flow rate was measured by a load cell capable of measuring the weight change in the run tank. Additionally, plumbing was installed at the bottom of the oxidizer run tank so that the liquid $\mathrm{N}_{2} \mathrm{O}$ could be discharged in advance of gas phase $\mathrm{N}_{2} \mathrm{O}$.

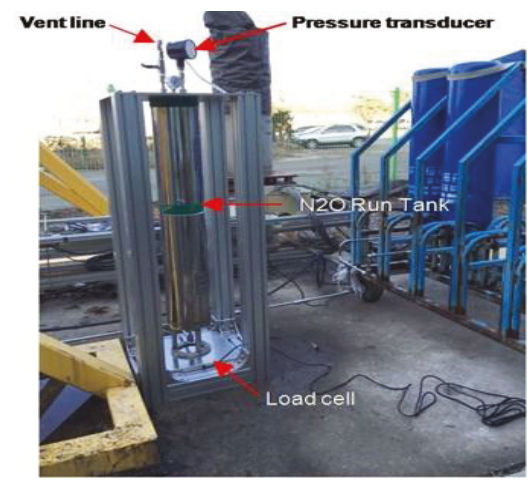

Figure 5. Oxidizer run tank and mass flow measurement.

\subsection{Internal Ballistics}

Table 1 lists some specifications of the basic experimental conditions and fuel geometry, where liquid nitrous oxide and HDPE were used for the oxidizer and solid fuel, respectively. A 5-port fuel grain was chosen to shorten the motor length instead of a single port grain to avoid an unrealistically long motor unit. Figure $6 \mathrm{a}, \mathrm{b}$ shows the front and side view of the 5-port grain, respectively, with an initial port diameter of $10 \mathrm{~mm}$ and distance between port's centers of $25.5 \mathrm{~mm}$ before the test. On the other hand, Figure 7a,b each shows the front and rear view of the grain after combustion. One can notice that with a burning time of $10 \mathrm{~s}$, there was no merging event between ports during the hot firing tests. An in-depth analysis on port merging can be found in reference [21].

Table 1. Specifications of the experimental conditions for underwater firing test.

\begin{tabular}{cc}
\hline Heading & Heading \\
\hline Design thrust $(\mathrm{kgf})$ & 50 \\
Oxidizer & Liquid nitrous oxide \\
Solid fuel & High density polyethylene (HDPE) \\
Igniter & Potassium nitrate/sorbitol (KNSB) propellant \\
Fuel density $\left(\mathrm{kg} / \mathrm{m}^{3}\right)$ & 950 \\
Burning time $(\mathrm{s})$ & 10 \\
Oxidizer mass flow rate range $(\mathrm{g} / \mathrm{s})$ & $15-134$ \\
Initial port diameter $(\mathrm{mm})$ & 10 \\
Grain outer diameter $(\mathrm{mm})$ & 104.5 \\
Port number & 5 \\
Grain length $(\mathrm{mm})$ & 146 \\
\hline
\end{tabular}

In a typical hybrid rocket internal ballistic design, knowledge of the fuel regression rate is of primary importance and crucial for the right performance prediction. Therefore, for the design of the $500 \mathrm{~N}$ class motor, the empirical regression rate of our previous works [21-24] for multi-port grains 
were used. The empirical relation used in this study for the overall regression rate, $\overline{\dot{r}}$, was a time-space (burning time-fuel length) averaged value:

$$
\overline{\dot{r}}=0.01 \bar{G}_{o}^{0.83}
$$

The overall regression rate can be evaluated with the measured fuel mass difference $\Delta m$, initial and final port radius $\left(R_{p i}\right.$ and $\left.R_{p f}\right)$ from the cross-section areas $\left(A_{p i}\right.$ and $\left.A_{p f}\right)$, the port number $N$, the fuel density $\rho$, and the fuel length $L$ by following equations:

$$
\begin{gathered}
\frac{\Delta m}{N \rho}=\left(A_{p f}-A_{p i}\right) L \\
\overline{\dot{r}} \cong \frac{R_{p f}-R_{p i}}{t_{b}}
\end{gathered}
$$

$\bar{G}_{o}$ is the averaged oxidizer mass flux defined as:

$$
\bar{G}_{o} \cong \frac{4 \overline{\dot{m}}_{o}}{\pi\left(R_{p i}+R_{p f}\right)^{2} N}
$$

where $\overline{\dot{m}}_{o}$ is the averaged oxidizer mass flow rate. The complete details of the procedure can be found in references [21-24]. Figure 8 shows the $\mathrm{N}_{2} \mathrm{O} / \mathrm{HDPE}$ multi-port regression rate correlations [22] where Equation (1) is the empirical relation corresponding to "three to seven" ports shown by the red dotted line. It was found that three to seven-port HDPE laboratory scale grain can be fitted by a single empirical regression rate. Results of ground and underwater hot-firing experiments using the 5-port grain motor are shown in Section 3.1 and idle cases in Section 3.4.

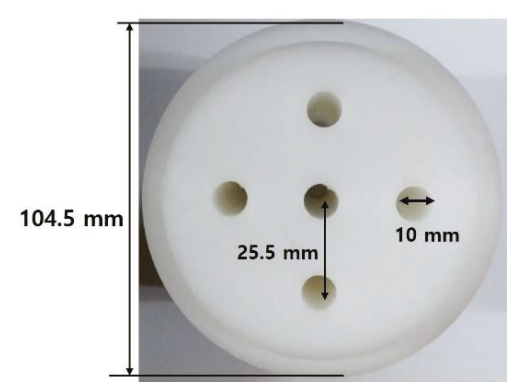

(a)

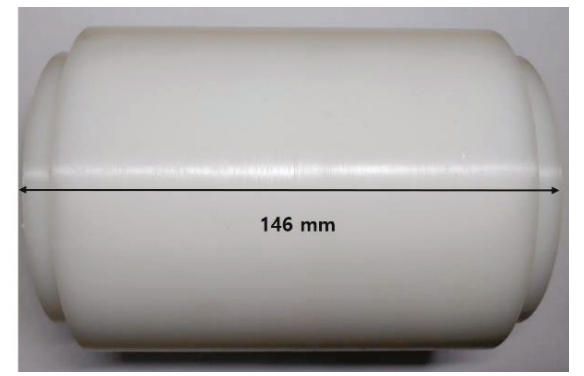

(b)

Figure 6. Fuel grain configuration, (a) front view; (b) side view.

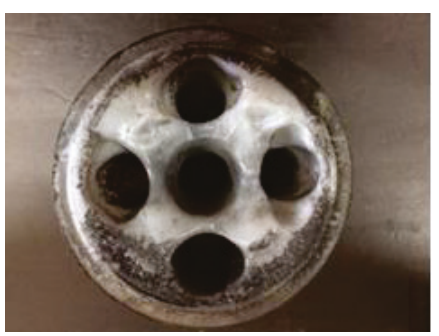

(a)

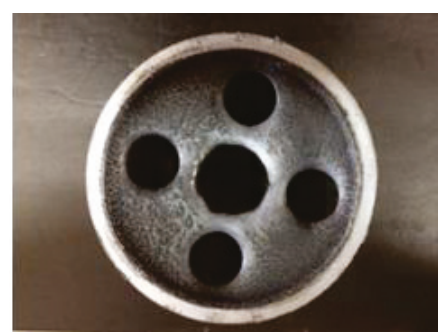

(b)

Figure 7. Fuel grain after combustion, (a) front view; (b) rear view. 


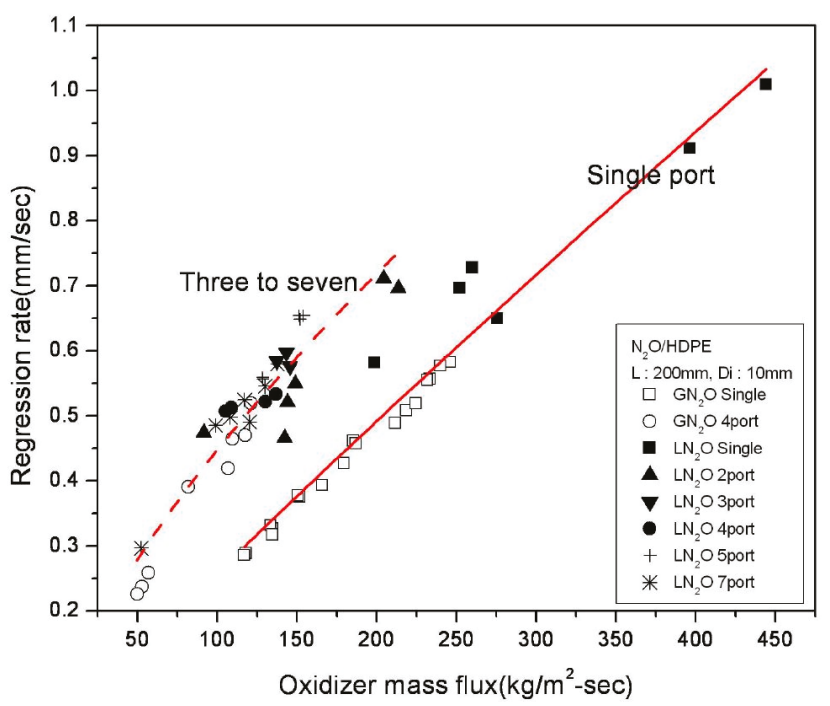

Figure 8. $\mathrm{N}_{2} \mathrm{O} / \mathrm{HDPE}$ multi-port regression rates correlations [22].

\subsection{Underwater Subsystem (Ignition, Flow Control, Thrust Measurement)}

Concerning the ignition system, Potassium Nitrate/Sorbitol (KNSB), widely used in semi-professional rocketry, was used as a pyrotechnic ignition for simplicity. The KNSB igniter was mounted upstream of the fuel port entrance inside the pre-combustion chamber, as shown in Figure 9. The amount of igniter chosen was either $30 \mathrm{~g}$ or $60 \mathrm{~g}$ depending on the preset rupture disc bursting pressure. The oxidizer mass flow control system was made using a commercially available throttle valve with a stepping motor in which they are connected by a coupling for accurate alignment. Among numerous valve types, pintle valves are known to be best fitted for hybrid and liquid propellant rockets. However, they are expensive and custom-made. For this study, being a preliminary assessment of an underwater hybrid rocket application, with the exception of the water-proof load cell, which directly contacted the water, all underwater systems were assembled using conventional systems that were widely available and cost effective, such as the ball valve, stepping motor, and KNSB ignition system. Thus, the ball valve was selected for the oxidizer flow control considering the temperature range of liquid nitrous oxide $\left(-40\right.$ to $\left.24^{\circ} \mathrm{C}\right)$. The specification of the oxidizer control valve and stepping motor are presented in Tables 2 and 3, respectively.

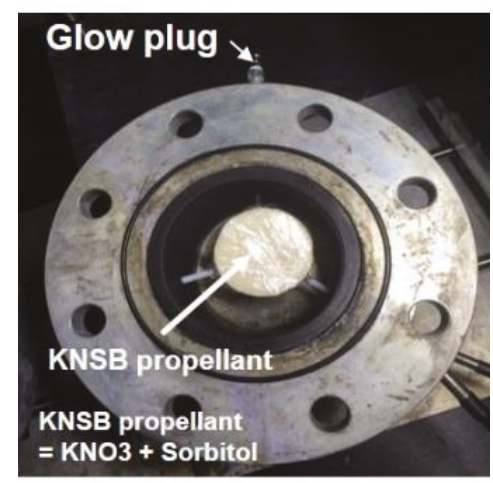

Figure 9. KNSB igniter mounted in pre-chamber. 
Table 2. Specification of oxidizer mass flow control valve.

\begin{tabular}{ccccc}
\hline Model & $\begin{array}{c}\text { Supplier } \\
\text { (Country) }\end{array}$ & $\begin{array}{c}\text { Orifice Diameter } \\
(\mathbf{m m})\end{array}$ & $\begin{array}{c}\text { Temperature Rating } \\
\left({ }^{\circ} \mathbf{C}\right)\end{array}$ & $\begin{array}{c}\text { Pressure Rating } \\
\text { (bar) }\end{array}$ \\
\hline VL82A-D-4T-S & DK-lok (KOR) & 3.2 & -54 to 65 & 172 \\
\hline
\end{tabular}

Table 3. Specification of oxidizer mass flow control motor.

\begin{tabular}{cccccc}
\hline Model & $\begin{array}{c}\text { Supplier } \\
\text { (Country) }\end{array}$ & Motor Type & Motion & $\begin{array}{c}\text { Max. Resolution } \\
\text { (deg/pulse) }\end{array}$ & $\begin{array}{c}\text { Min. Resolution } \\
\text { (deg/pulse) }\end{array}$ \\
\hline MDrive 23 Plus & $\begin{array}{c}\text { Schneider } \\
\text { Electric (USA) }\end{array}$ & Stepping Motor & CW/CCW & 1.8 & 0.007 \\
\hline
\end{tabular}

\subsection{Water Filled Tank Containing the Motor}

Figure 10 shows the accommodated laboratory scale motor within the filled water tank prior to the underwater test. Three pressure transducers each measuring the static pressure of the injector manifold, pre-chamber and post-chamber can be seen with a water-proof load cell (CAS SB-100L) situated at the head end of the motor. The water tank was designed to have a minimum length and width so that the wake reflected back from the far end of the tank wall would not disturb the jet flame coming out of the nozzle. For the time being, we assumed that the reflected wake would merely disturb the nozzle exit area. The width and depth were set as $1 \mathrm{~m} \times 1 \mathrm{~m}$ with a total water capacity of 3 tons. The side wall of the water tank was made of tempered glass to aid in visualizing and observing the wake structure. Figure 10 also shows the vertical transfer system that submerged the hybrid rocket motor into the water where the motor base was set to be located at $70 \mathrm{~cm}$ beneath the water surface.

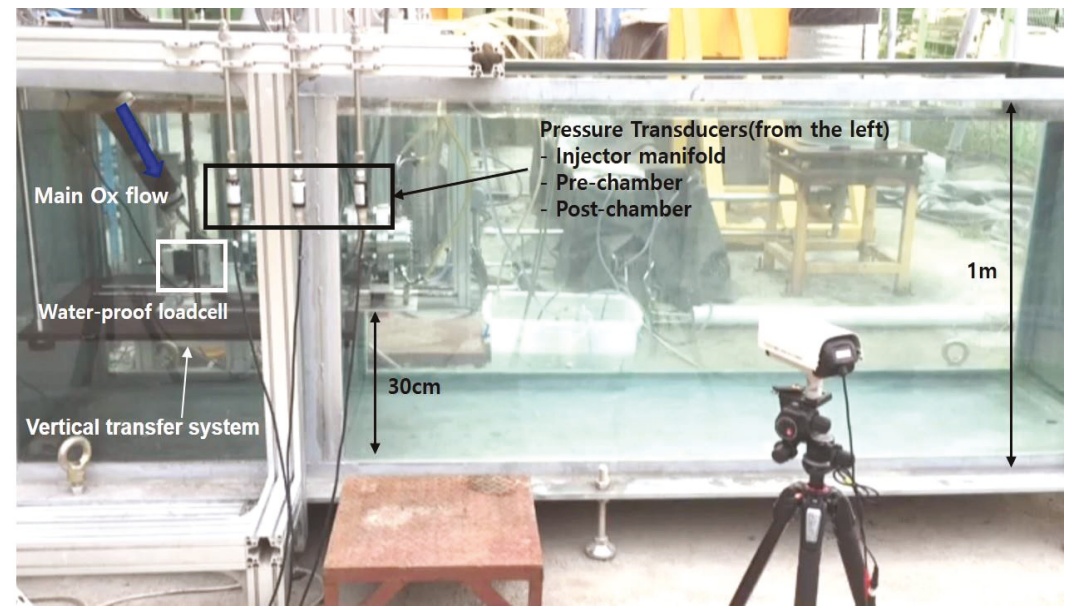

Figure 10. Water tank and vertical transfer system of hybrid motor.

\subsection{Signal Command and Sequence}

In order to guarantee a smooth and uniform ignition curve, the "pressure signal triggering loop" was tested following the flow chart shown in Figure 11. During the ignition sequence, at the time when the pre-chamber pressure attained the "preset Ox. v/v opening pressure", the main oxidizer valve was opened, and the igniter power was turned off simultaneously. The "preset Ox. v/v opening pressure" was preset (before the combustion test) to 1.2 bar due to a delay time $(0.5-0.7 \mathrm{~s})$ existing between power signal "on" and the real valve opening moment. As the rupture disc bursting pressure was set to 2 bar (or 3 bar), the valve opening command was signaled earlier than expected to compensate 
this delay time. Figure $4 \mathrm{a}, \mathrm{b}$ shows two rupture discs set to burst at a gauge pressure of 2 bar and $3 \mathrm{bar}$, respectively. If the hot firing test of $10 \mathrm{~s}$ duration is successful, nitrogen is purged for $8 \mathrm{~s}$ and the sequence is terminated. On the contrary, if ignition does not occur within $15 \mathrm{~s}$ and thus, the pre-chamber pressure does not rise, the test is stopped immediately. Detailed experimental cases are shown in Table 4.

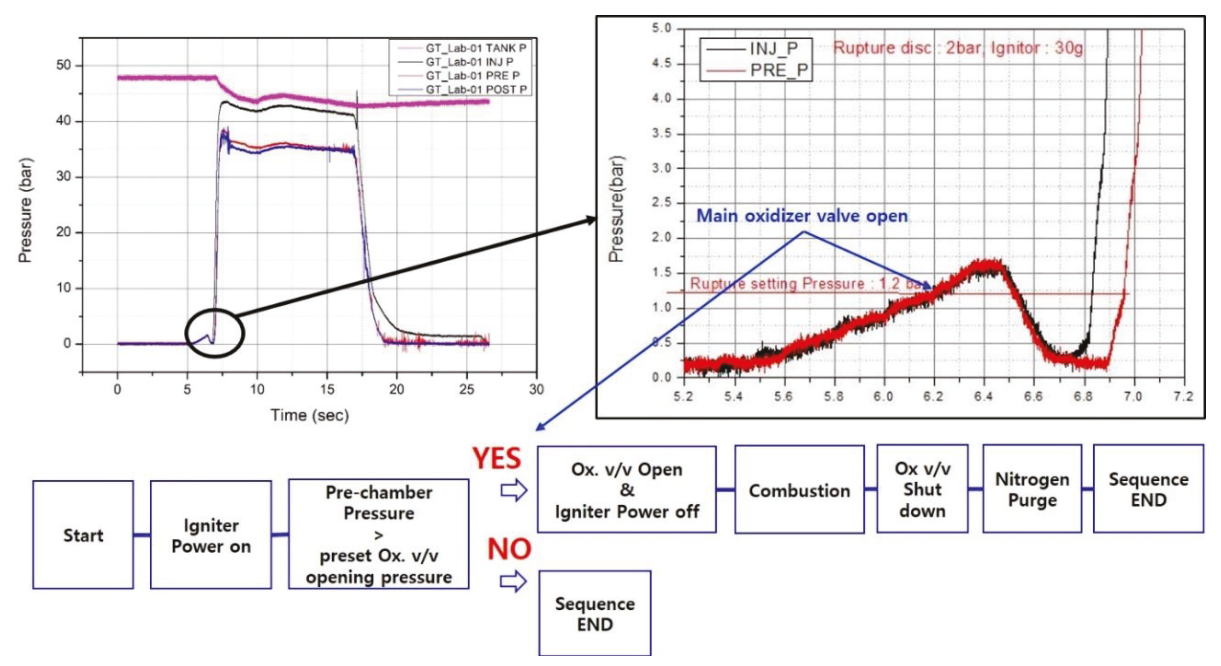

Figure 11. Pressure signal triggering loop.

Table 4. Ground and underwater hot-firing test cases.

\begin{tabular}{cccccc}
\hline Identifier & GT_Lab-01 & UT_Lab-01 & UT_Lab-02 & UT_Lab-03 & UT_Lab-04 \\
\hline Test environment & Ground & \multicolumn{4}{c}{ Underwater } \\
\hline Oxidizer control valve angle $(\mathrm{deg})$ & $\mathrm{w} / \mathrm{o}$ v/v & $\mathrm{w} / \mathrm{o}$ v/v & 52 & 54 & 56 \\
\hline Average oxidizer mass flow rate $(\mathrm{g} / \mathrm{s})$ & 121.1 & 134.6 & 15.0 & 22.0 & 32.0 \\
\hline Average fuel mass flow rate $(\mathrm{g} / \mathrm{s})$ & 21.2 & 21.8 & - & 8.5 & 10.5 \\
\hline Average oxidizer-to-fuel $(\mathrm{O} / \mathrm{F})$ ratio & 5.7 & 6.2 & - & 2.6 & 3.0 \\
\hline Rupture disc bursting pressure (bar) & 2 & 2 & 3 & 3 & 3 \\
\hline Preset Ox. v/v opening pressure (bar) & 1.2 & 1.2 & 1.2 & 1.2 & 1.2 \\
\hline Amount of pyrotechnic igniter $(\mathrm{g})$ & 30 & 30 & 60 & 60 & 60 \\
\hline
\end{tabular}

\section{Results and Discussion}

\subsection{Ground Tests versus Underwater Tests}

In order to assess the technical feasibility of the hybrid rocket application in the underwater environment, comparisons between underwater test results with ground test results were performed. Firing test comparisons on these two different mediums were necessary to check any difference that may exist on the chamber pressure and thrust level, or that may be revealed during the ignition time and combustion period. Therefore, two identical hybrid motors, one for the ground test and one another for the underwater test, were made and designated for their own medium. The pressure and thrust curves of the ground tests are shown in Figure 12a,b, respectively, while those of underwater tests are shown in Figure 12c,d. The unstable wavy pressure curve (from 7.5 to $11 \mathrm{~s}$ ) in the early stage of the ground test (GT_Lab-01) was due to the lengthy oxidizer feeding line, which required a settling time until a sufficient amount of oxidizer was stably supplied. Due to the inherent characteristics of the self-pressurized liquid nitrous oxide, whose tank pressure depends highly on the ambient 
temperature, the injected oxidizer supply pressure of ground and underwater cases could show a difference. However, as seen in Figure 12a,c, the difference was minor. Additionally, the average thrust level around $45 \mathrm{kgf}$, which corresponded to $90 \%$ of the design thrust, was observed for both cases. Comparing the ground test data of Figure 12a,b with the underwater test data of Figure 12c,d, it was found that there were no significant qualitative differences in chamber pressure curves and mean thrust levels. The snapshots of hot-firing tests in atmospheric and underwater media are shown in Figures 13 and 14, respectively.

It should be pointed out that as far as the nozzle throat is choked, the built-up pressure of the combustion chamber for both the ground and underwater tests would behave similarly for the two mediums. Conversely, it is expected that the combustion characteristics within the chamber of the underwater rocket motor would differentiate with respect to the ground test case for the non-choked nozzle, since the underwater back pressure, downstream of the nozzle exit, is subject to a violent change during jet evacuation. However, in terms of thrust behavior in the underwater environment and under choked nozzle conditions, it is questionable whether the underwater environment gives a notable effect on thrust variation with respect to the ground case. Therefore, the following section assesses the outer motor underwater environment.

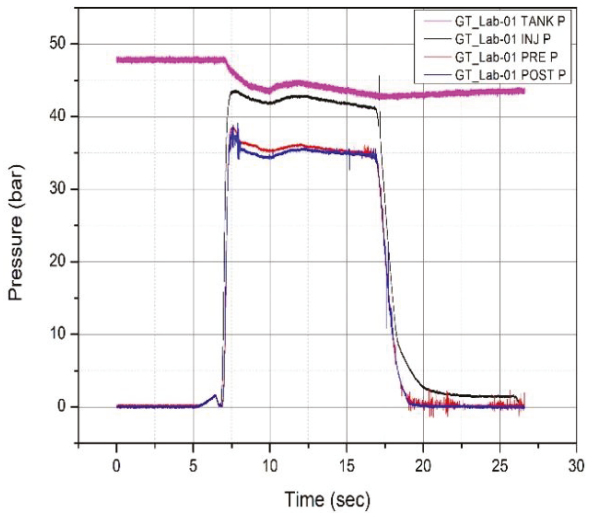

(a)

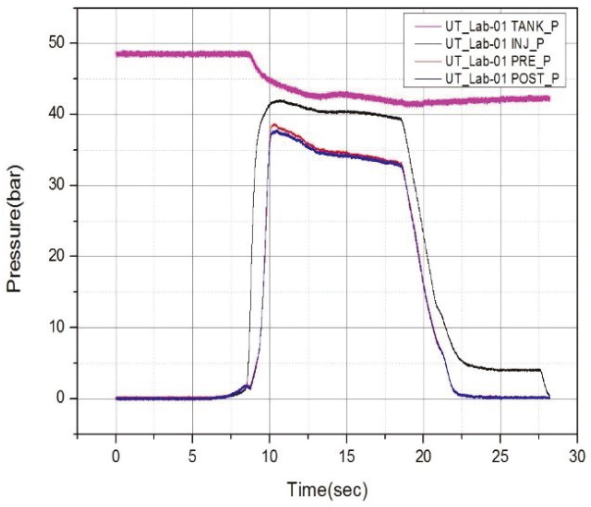

(c)

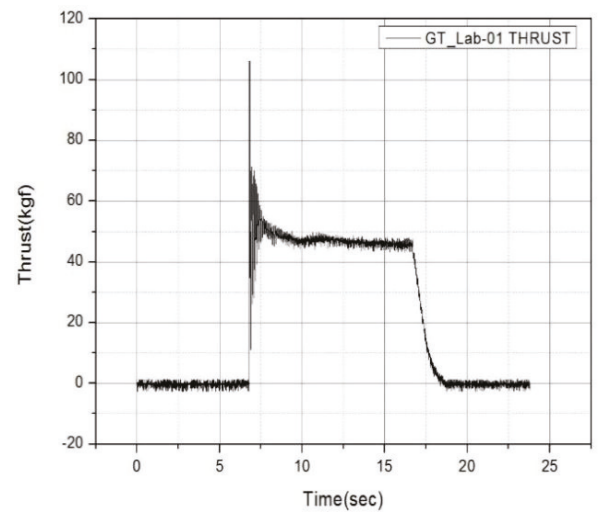

(b)

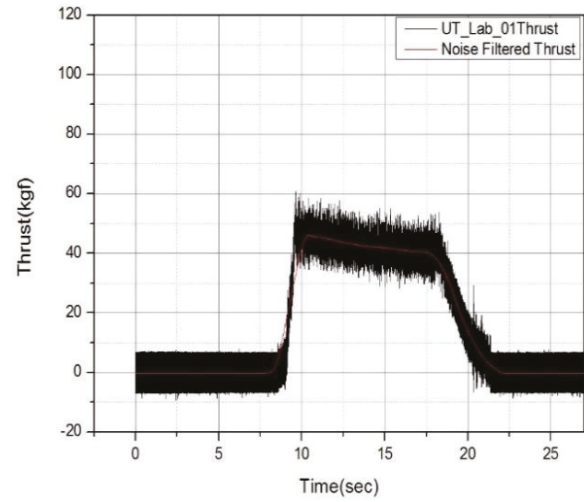

(d)

Figure 12. (a) Pressure curve of ground test (GT_Lab-01); (b) thrust curve of ground test (GT_Lab-01); (c) pressure curve of underwater test (UT_Lab-01); (d) thrust curve of underwater test (UT_Lab-01). 


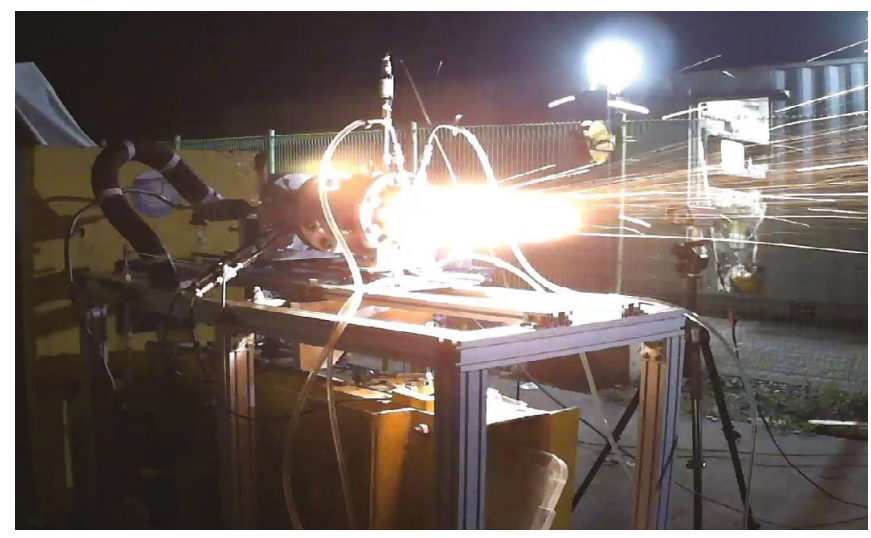

Figure 13. Snapshot of ground hot-firing test.

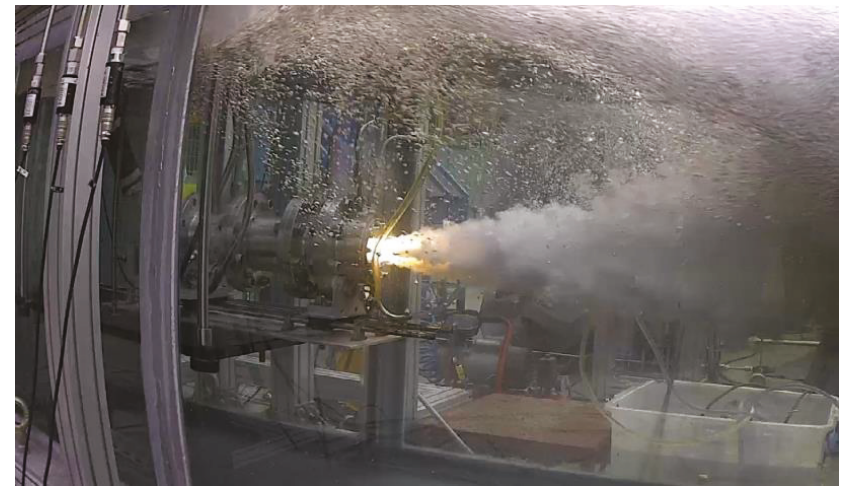

Figure 14. Snapshot of underwater hot-firing test.

\subsection{Thrust Oscillation in Underwater Environment}

Extensive works $[1,2,25-31]$ have reported that the gaseous jet injected into water results in complicated flow structures due mainly to the high water-to-gas density ratio leading to an extremely unsteady and turbulent multiphasic environment. In addition, the intermittent pulses of back pressure and nozzle exit pressure make the prediction and measurement of the flow structures extremely challenging either by computational fluid dynamics or experiments. From the literature [1,2], the nozzle exit environment can be categorized as expansion, bulge, necking/breaking, and back-attack depending on the formation and dissipation of the gas bag at the rear of the nozzle. In order to check and validate the gas bag formation encountered in this study, snapshots of exiting gaseous jets as well as Fast Fourier Transform (FFT) analysis of chamber pressure and of thrust were performed. Figure 15a,b shows the snapshots of the flow structure at the onset of jet exhaust into the water where the aforementioned four categorized phenomena can be depicted. The three snapshots of Figure 15b show the presence of necking/breaking and back-attack phenomena in which a high pressurized gas bag situated just downstream of the necking region is clearly seen. Following Tang et al. [1,2], it is believed that the oscillating back pressure can result in oscillating thrust. Accordingly, this fact may have triggered the thrust vibration observed in Figure 12d. It should be pointed out that the back-pressure variation of an underwater medium does not affect the chamber pressure for the case of the maximum supply oxidizer flow rate, i.e., with a choked nozzle throat. At the contrary, the chamber pressure can interact with the gas bag (filled with hot gas) downstream of the nozzle for the case of 
low oxidizer flow rate, i.e., with non-choking condition. To clarify this, FFT analyses were performed with the data from post-chamber pressure (Figure 16) and from thrust data (Figure 17). From the comparison shown in Figure 16, one can observe that the chamber pressure FFTs of the ground and underwater firing tests were nearly the same for both environments. Thus, one can confirm that the underwater test was conducted well within the choked condition. The observed frequency band of 18 $\mathrm{Hz}$ and $33 \mathrm{~Hz}$ was the signal noise frequency. Meanwhile, the thrust FFTs analyses of GT_lab-01 and UT_lab-01, shown in Figure 17, behaved differently as a consequence of gas bag presence in the water. Comparing the UT_Lab-01 spectrum shown in Figures 17 and 18 of Tang et al. [1,2], one can remark that the amplitude observed in the frequency bandwidth of $0-100 \mathrm{~Hz}$ of this study nearly matched the one observed by Tang et al.

(a)

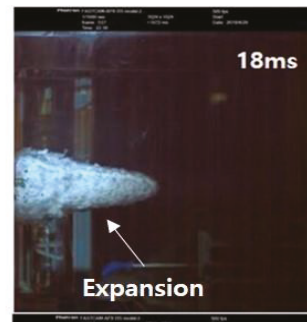

(b)

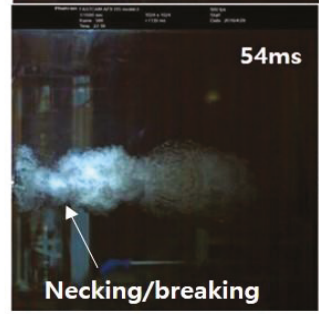

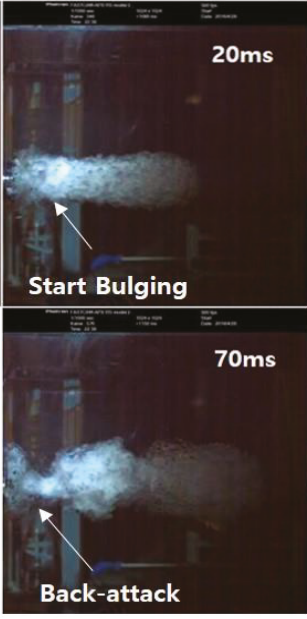

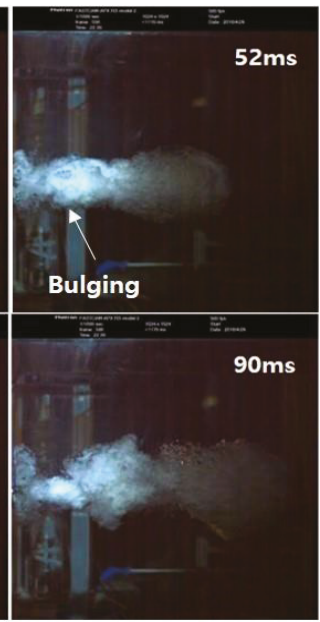

Figure 15. Snapshots of flow structures during underwater hot-firing test, (a) expansion and bulging process; (b) necking/breaking and back-attack process.

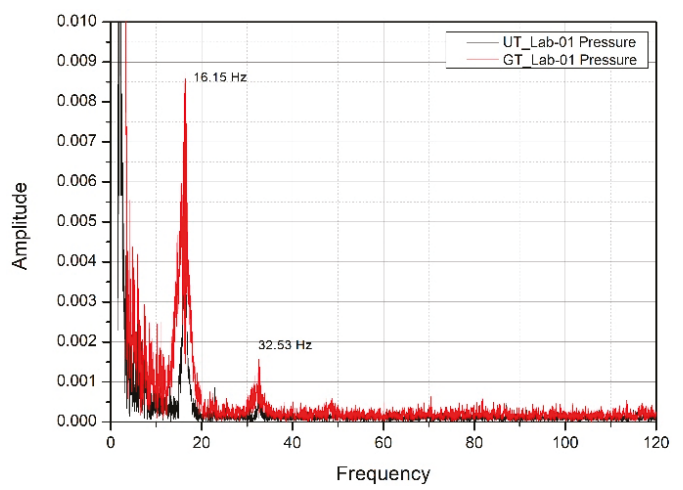

Figure 16. Fast Fourier transform (FFT) results of post-chamber pressure for ground and underwater test. 


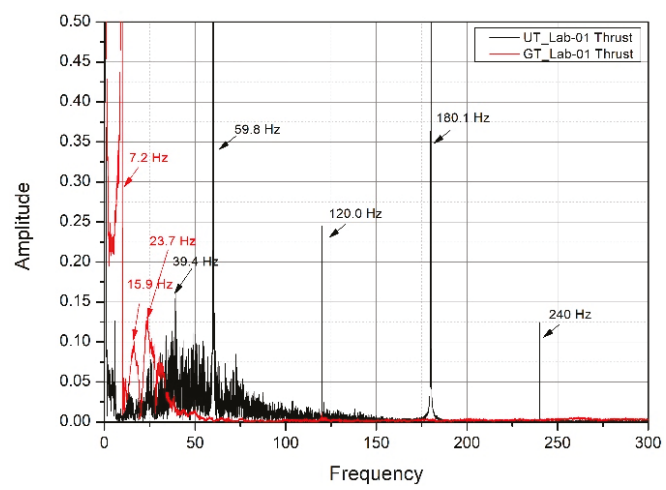

Figure 17. FFT results of thrust data for ground and underwater test.

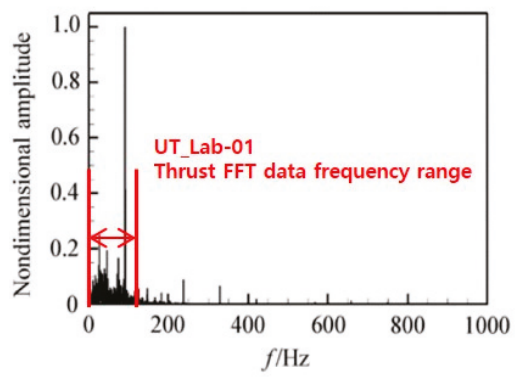

Figure 18. FFT results of back pressure oscillation from Tang et al. [1,2].

\subsection{Ignition Characteristics}

For a rocket motor submerged in the water, a dry environment of the combustion chamber is necessary especially for pyrotechnic ignition. The rupture disc is used to prevent the eventual intrusion of water and to keep the environment in the chamber dry, at least during the initial stage of ignition. The pyrotechnic ignition characteristics, with a rupture disc bursting at 2 bar for ground and underwater environments, are shown in Figure 19a,b, respectively. These two tests were conducted under identical experimental conditions (i.e., with the same rupture disc bursting pressure, the same "preset Ox. v/v opening pressure", and the same amount of igniter).

As cited in Section 2.5, when the growing pre-chamber pressure attains the "preset Ox. v/v opening pressure", the main oxidizer valve opening signal is triggered (signal on), and the igniter power is turned off simultaneously. The "preset Ox. v/v opening pressure" was preset to $1.2 \mathrm{bar}$ due to a delay time (0.5- $0.7 \mathrm{~s})$ existing between the power signal "on" and the real valve opening moment. The rupture disc bursting pressure being set to 2 bar (gauge), the valve opening command was signaled on earlier to compensate this delay time. The optimal condition would be the exact moment when the oxidizer valve opens at the bursting event of the rupture disc. In order to find out the valve opening delay time after the command signal, numerous ignition tests were performed. It was found that a preset gauge pressure of 1.2 bar for the "preset Ox. v/v opening pressure" was the best preset value to resolve this delay. Since then, the "Identifier" number was given, e.g., UT_Lab-01. 


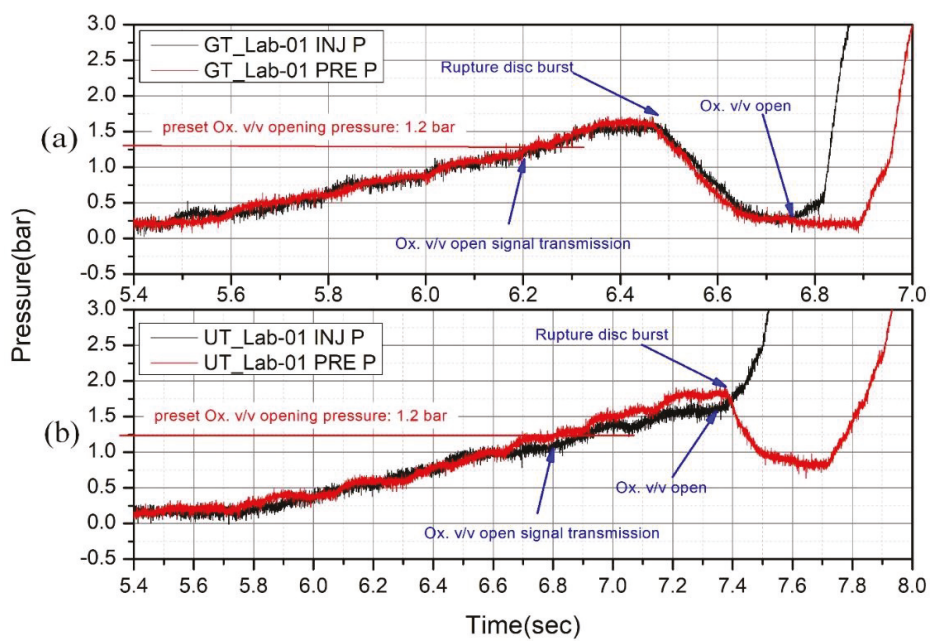

Figure 19. Ignition curve, (a) GT_Lab-01 w/rupture disc 2 bar; (b) UT_Lab-01 w/rupture disc 2 bar.

Figures 19 and 20 show the moment of signal transmission time, rupture disc bursting time, and oxidizer valve opening time for a clearer observation of the ignition sequence. It can be seen that after the rupture disc burst, the chamber pressure started to decrease toward atmospheric pressure followed later by the opening of the oxidizer valve that triggered the combustion leading to an abrupt pressure rise. It was observed that water intrusion was prevented during the ignition stage of the underwater tests. Figure 19a,b also shows early bursting events (below 2 bar) prior to the expected preset rupture disc bursting pressure. It seems that this was a consequence of the direct exposure of high temperature gases to the plate of the rupture disc, which resided in the small internal volume of the lab-scale motor.

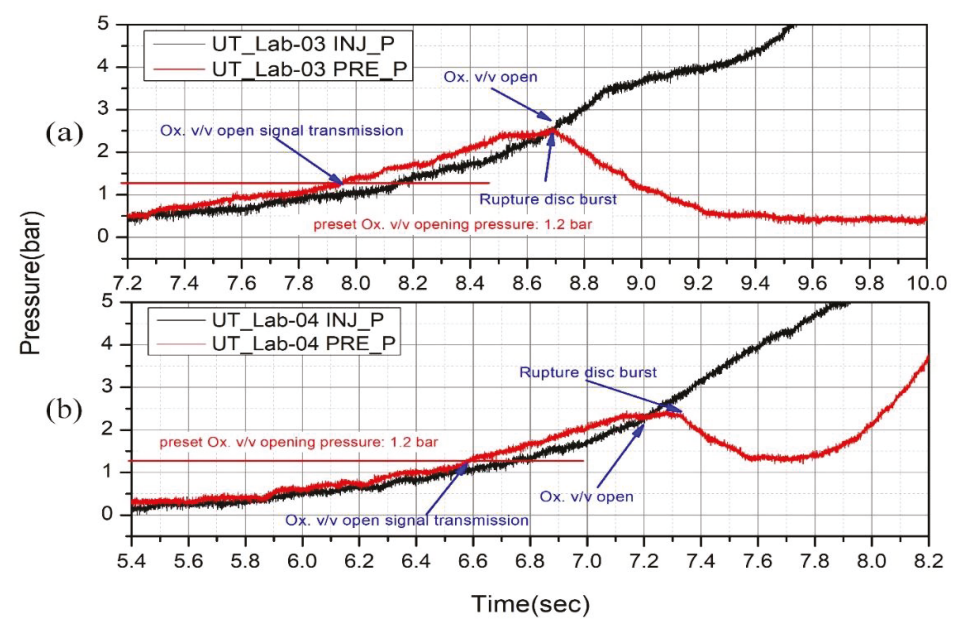

Figure 20. Ignition curve, (a) UT_Lab-03 w/rupture disc 3 bar; (b) UT_Lab-04 w/rupture disc 3 bar.

When the minimum ignitable oxidizer mass flow rate was supplied to the combustion chamber, the pressure rise occurred very slowly with respect to the full throttle test case and there may have been a possibility of extinguishment due to water intrusion. In order to find out the safe operating condition for the minimum achievable and ignitable oxidizer mass flow rate, the amount of KNSB 
propellant as well as the rupture disc bursting pressure were varied and investigated (cf. Table 4). Figure 20a,b shows the case of the oxidizer valve angle of 54 degrees (UT_lab-03) and of 56 degrees (UT_lab-04), respectively. These correspond to $16.3 \%$ and $23.7 \%$ of the maximum (UT_Lab-01) oxidizer mass flow rate, respectively, where the minimum chamber pressure could be maintained at a gauge pressure of 0.5 bar and 1.2 bar, respectively. These conditions were achieved by increasing the amount of KNSB to $60 \mathrm{~g}$.

\subsection{Motor Idling Condition in Underwater Environment}

The sudden acceleration in the start-up stage is dangerous since it can disrupt the balance of the marine vehicles. Thus, finding idling conditions for the soft-start is crucial for stable operation. Experiments were carried out to find the optimum valve angle that can sustain the minimum thrust level. Figure 21a-c shows the results of experiments performed in the water where the valve angles were set to 52 degrees, 54 degrees, and 56 degrees, representing around $11.1 \%, 16.3 \%$ and $23.7 \%$ of the full throttle oxidizer supply, respectively. For all tests, the oxidizer tank pressure was initially set to 48 bar intentionally to retain the exact initial conditions of Figure 12c, i.e., the full throttle case. For all three Figures, one can clearly see that a bump in pre-chamber pressure trace was observed following the "rupture disc burst" event. This bump represents the pressure build-up section where the main oxidizer started to deliver fresh oxidizer to the combustion chamber mixed with KNSB propellant, which was still continuously burning. Looking to the case of valve angle 52 degrees (Figure 21a), it was confirmed that $11.1 \%$ of maximum flow rate was not enough to trigger any pressure rise, since the oxidizer mass flux was too low to sustain the main combustion in the chamber. Thus, the flame extinguished due to the extremely low oxidizer-to-fuel $(\mathrm{O} / \mathrm{F})$ ratio. However, three pressure build-up sections are depicted in Figure $21 \mathrm{~b}, \mathrm{c}(16.3 \%$ and $23.7 \%$ of the maximum flow rate, respectively) where the combustion was sustained, representing the motor idle case. The first pressure build-up section was due to KNSB propellant burning before the "rupture disc burst" event, while the second pressure build-up section that lies on the bump was due to the mixture of supplied fresh main oxidizer with the still burning KNSB as cited earlier. Finally, the third pressure build-up section was purely caused by the main combustion of $\mathrm{N}_{2} \mathrm{O} / \mathrm{HDPE}$ in which the low slopes shown in Figure 21b,c resulted from the low oxidizer supply of 54 degrees and 56 degrees valve angles, respectively. It is worthwhile to note that the ignition delay after the second pressure build-up was decreased for augmented oxidizer mass flow. The ignition delays for the 54 degrees and 56 degrees cases were approximately $5 \mathrm{~s}$ and $2.3 \mathrm{~s}$, respectively, while for the final combustion chamber pressure, it attained up to 3 bar and 7.5 bar, respectively. Meanwhile, Figure 22 shows the thrust and normalized thrust curve for underwater idling conditions. Due to the existing "bump" in the early operating stage and the self-pressurizing $\mathrm{N}_{2} \mathrm{O}$ blow down nature, fluctuations of instantaneous thrust were inevitable. For a clearer quantitative observation, we displayed the normalized thrust in percent with a double Y-axis. The thrust delivered from the main combustion of $\mathrm{N}_{2} \mathrm{O} / \mathrm{HDPE}$ starting at $14.5 \mathrm{~s}$ (UT_Lab-03) and the one starting at $10 \mathrm{~s}$ (UT_Lab-04) each reached $6 \%$ and $12 \%$ of the maximum thrust, respectively. 


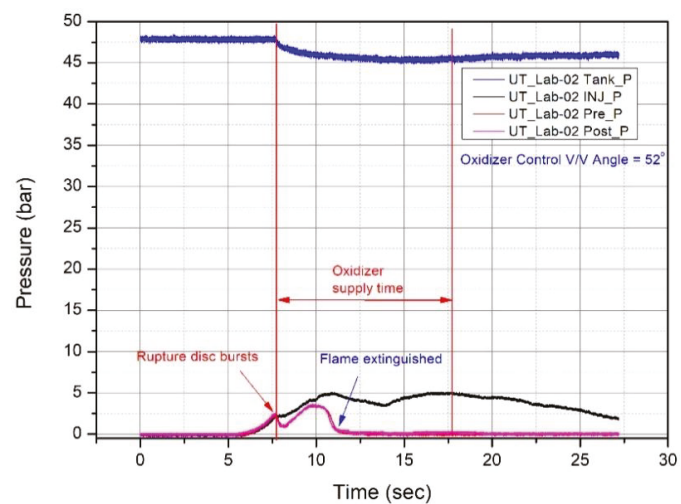

(a)

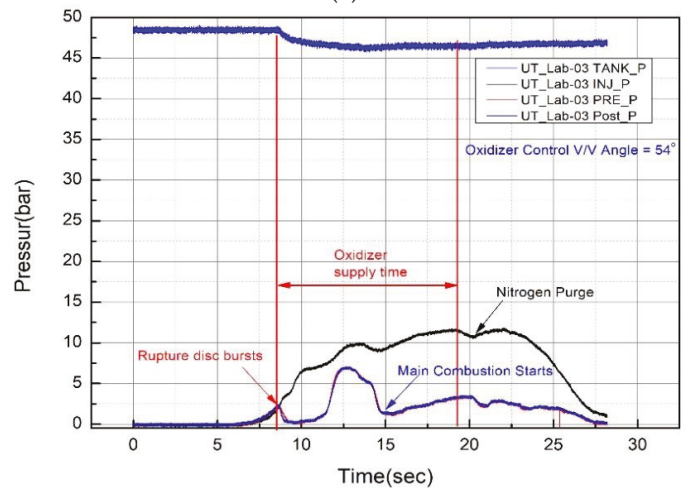

(b)

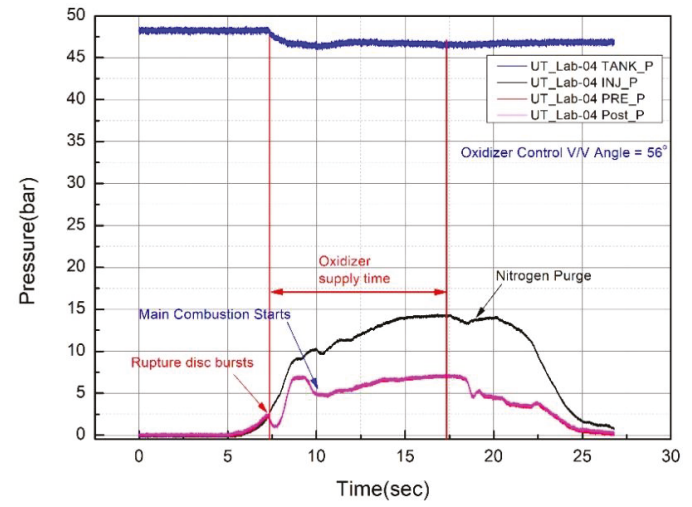

(c)

Figure 21. Pressure curves at idling condition, (a) UT_Lab-02 (52 degrees oxidizer control v/v angle); (b) UT_Lab-03 (54 degrees oxidizer control v/v angle); (c) UT_Lab-04 (56 degrees oxidizer control v/v angle). 


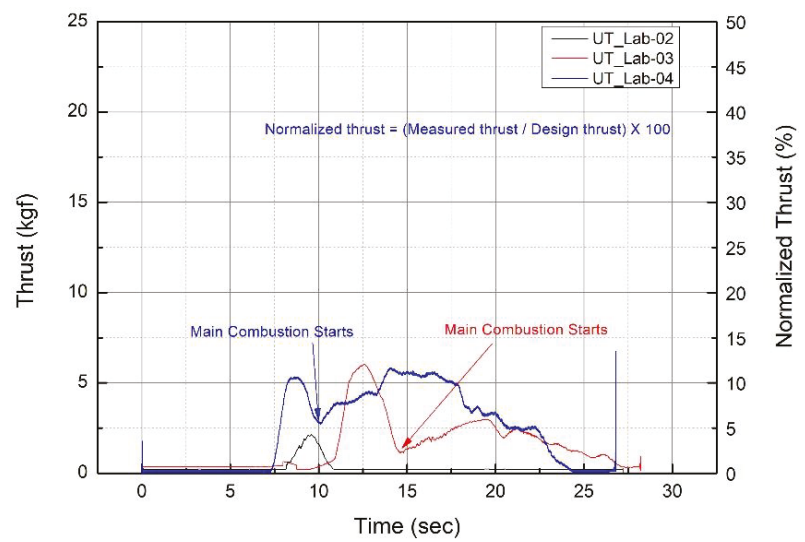

Figure 22. Thrust and normalized thrust curves for underwater idling conditions.

\section{Conclusions}

In this study, attempts at hybrid rocket motor hot-firing tests were conducted in an underwater environment for marine application. A laboratory scale hybrid motor of $500 \mathrm{~N}$ class using liquid nitrous oxide and 5-port high density polyethylene (HDPE) fuel grain was used as a test-bed for the preliminary assessment of the underwater main propulsion or auxiliary boosting device. The use of a rupture disc for preventing the water intrusion, and the rupture disc blast timing both required a special precaution of the ignition signal command and sequence. From the FFT analysis of chamber pressure for the ground and underwater tests, no critical and notable difference was observed for the combustion chamber environment, while FFT analysis of underwater motor thrust revealed a vibration-prone environment, which was due in part to the wake structure downstream of the nozzle exit, a common fact encountered for a jet propulsive system compared to a screw propeller system.

Compared to the solid rocket counterpart, the hybrid rocket, with its throttleability, can offer a soft-start capability, which is essential for a marine vessel's early stage of operation. The minimum power thrust, viewed as the idle test case with stable operation, was evaluated depending on the flow controlling optimum valve angle. It was found that $16.3 \%$ of the full throttle case sustained the minimum thrust level. Under full power, severe thrust oscillation was not observed in the underwater environment. These factors imply that the throttable hybrid rocket propulsion system can be a justifiable candidate for a short duration, high speed, marine boosting system or applied as a tactical system for naval application as an alternative to the solid propellant underwater rocket.

Author Contributions: Supervision, H.M.; conceptualization, H.M. and S.H.; investigation, S.H.; writing-review and editing, H.M. and S.H.; project management, Y.Y. and M.K.

Funding: This research was funded by the Agency for Defense Development (No. UE161021GD) and Advanced Research Center Program (NRF-2013R1A5A1073861).

Acknowledgments: This work was supported by the Defense Acquisition Program Administration and Agency for Defense Development (UE161021GD) Korea and supported by the Advanced Research Center Program (NRF-2013R1A5A1073861) through the National Research Foundation of Korea (NRF) grant funded by the Korea government (MSIP), contracted through the Advanced Space Propulsion Research Center at Seoul National University.

Conflicts of Interest: The authors declare no conflict of interest 


\section{Nomenclature}

$\begin{array}{lll}A_{p i} & \text { Initial cross-section area } & \left(\mathrm{m}^{2}\right) \\ A_{p f} & \text { Final cross-section area } & \left(\mathrm{m}^{2}\right) \\ \bar{G}_{o} & \text { Averaged oxidizer mass flux } & \left(\mathrm{kg} \cdot \mathrm{m}^{-2} \cdot \mathrm{s}^{-1}\right) \\ L & \text { Fuel grain length } & (\mathrm{m}) \\ \Delta m & \text { Fuel mass difference } & (\mathrm{kg}) \\ \overline{\dot{m}}_{o} & \text { Averaged oxidizer mass flow rate } & \left(\mathrm{kg} \cdot \mathrm{s}^{-1}\right) \\ N & \text { Port number } & (-) \\ \overline{\dot{r}} & \text { Overall regression rate } & \left(\mathrm{m} \cdot \mathrm{s}^{-1}\right) \\ R_{p i} & \text { Initial port radius } & (\mathrm{m}) \\ R_{p f} & \text { Final port radius } & (\mathrm{m}) \\ t_{b} & \text { Burning time } & (\mathrm{s}) \\ \rho & \text { Fuel density } & \left(\mathrm{kg} \cdot \mathrm{m}^{-3}\right)\end{array}$

\section{References}

1. Tang, J.N.; Wang, N.F.; Shyy, W. Flow structures of gaseous jets injected into water for underwater propulsion. Acta Mech. Sin. 2011, 27, 461-472. [CrossRef]

2. Tang, J.; Tseng, C.C.; Wang, N.; Shyy, W. Flow structures of gaseous gets injected into water for underwater propulsion. In Proceedings of the 49th AIAA Aerospace Sciences Meeting including the New Horizons Forum and Aerospace Exposition, Orlando, FL, USA, 4-7 January 2011.

3. Burke, A.E. Torpedoes and Their Impact on Naval Warfare; 0704-0188; Naval Undersea Warfare Center Division: Newport, RI, USA, 2017.

4. Kirby, G. A history of the torpedo. J. R. Nav. Sci. Serv. 1972, 27, 30-105.

5. Kirby, G. The Development of Rocket-Propelled Torpedoes. Available online: http://www.geoffkirby.co.uk/ rocket-torpedoes.pdf (accessed on 26 January 2019).

6. Garanin, I. The hydro-reacting marine solid fuel rocket engine. In Thermal to Mechanical Energy Conversion: Engines and Requirements-Volume II; United Nations Educational Scientific and Cultural Organization: Paris, France, 2009; Volume 8, pp. 201-240.

7. $\mathrm{Ng}, \mathrm{K}$. Overview and future research directions of undersea weapon design \& optimization. In Proceedings of the 9th AIAA/ISSMO Symposium on Multidisciplinary Analysis and Optimization, Atlanta, GA, USA, 4-6 September 2002.

8. Han, S.J.; Moon, K.H.; Ko, S.H.; Kim, J.K.; Moon, H.J.; You, Y.J.; Kwon, M.C. Feasibility study and demonstration of an underwater lab-scale hybrid rocket propulsion. In Proceedings of the 53rd AIAA/SAE/ASEE Joint Propulsion Conference, Atlanta, GA, USA, 10-12 July 2017.

9. Hwang, H.S.; Kim, H.S.; You, Y.J. Analysis on initial stability test results of underwater vehicle using the HR propulsion system. In Proceedings of the Korean Society of Propulsion Engineers, Jeju, Korea, 31 May-2 June 2017.

10. Woo, K.J.; Min, M.K.; Lee, J.H.; Choo, B.K.; Lee, S.H.; Kim, G.M.; Kim, H.J.; Kim, J.M.; Hwang, H.S.; You, Y.J. A Study of hybrid rocket for underwater operation. In Proceedings of the Korean Society of Propulsion Engineers, Jeju, Korea, 31 May-2 June 2017.

11. Han, S.J.; Moon, K.H.; Ko, S.H.; Kim, J.K.; You, Y.J.; Kwon, M.C.; Moon, H.J. Ignition characteristics of hybrid underwater propulsion system with rupture disc. In Proceedings of the Korean Society of Propulsion Engineers, Pusan, Korea, 22-24 November 2016.

12. Kim, S.J.; Kim, H.C.; Kim, K.H.; Park, Y.H.; Park, S.J.; Lee, D.G.; Kim, J.K.; Moon, H.J.; You, Y.J.; Kwon, M.C. Underwater combustion test using lab-scale hybrid rocket motor. In Proceedings of the Korean Society of Propulsion Engineers, Jeju, Korea, 25-27 May 2016.

13. Juliet-Marine-Brochure-1. Available online: http://www.julietmarine.com/pdfs/Juliet-Marine-Brochure1.pdf (accessed on 26 January 2019).

14. Sakurai, T.; Tomizawa, T. Applicability of a LOx vaporization preburner for swirling-flow hybrid rocket engines. In Proceedings of the 51st AIAA/SAE/ASEE Joint Propulsion Conference, Orlando, FL, USA, 27-29 July 2015. 
15. Calabro, M. LOx/HTPB/AlH3 hybrid propulsion for launch vehicle boosters. In Proceedings of the 40th AIAA/ASME/SAE/ASEE Joint Propulsion Conference and Exhibit, Fort Lauderdale, FL, USA, 11-14 July 2004.

16. Rice, E.; Gramer, D. Methane and methane/aluminum cryogenic hybrid rocket engines for Mars ISRU propulsion. In Proceedings of the 38th Aerospace Sciences Meeting and Exhibit, Reno, NV, USA, 10-13 January 2000.

17. Risha, G.; Boyer, E.; Wehrman, R.; Evans, B.; Kuo, K. Nano-sized aluminum and boron-based solid fuel characterization in a hybrid rocket engine. In Proceedings of the 39th AIAA/ASME/SAE/ASEE Joint Propulsion Conference and Exhibit, Huntsville, AL, USA, 20-23 July 2003.

18. Marothiya, G.; Ramakrishna, P. Utilization of mechanically activated aluminum in hybrid rockets. J. Propuls. Power 2018, 34, 1206-1213. [CrossRef]

19. Paccagnella, E.; Barato, F.; Pavarin, D.; Karabeyoğlu, A. Scaling parameters of swirling oxidizer injection in hybrid rocket motors. J. Propuls. Power 2017, 33, 1378-1394. [CrossRef]

20. Arena, Z.; Athougies, A.; Rodulfo, A.; DeTurris, D. Swirl injection hybrid rocket motor design and testing. In Proceedings of the 47th AIAA/ASME/SAE/ASEE Joint Propulsion Conference \& Exhibit, San Diego, CA, USA, 31 July-3 August 2011.

21. Kim, S.J.; Lee, J.P.; Moon, H.J.; Kim, J.K.; Sung, H.G.; Kwon, O.C. Regression characteristics of the cylindrical multiport grain in hybrid rockets. J. Propuls. Power 2013, 29, 573-581. [CrossRef]

22. Kim, G.H. A Study on Combustion Characteristic of the Cylindrical Multi-Port Grain for Hybrid Rocket Motor Using PE/ $\mathrm{N}_{2} \mathrm{O}$. Master's Thesis, Korea Aerospace University, Goyang, Korea, 20 January 2010.

23. Kim, S.J.; Kim, J.K.; Moon, H.J.; Sung, H.G.; Lee, J.P.; Kim, G.H.; Cho, J.T.; Park, S.H. Combustion characteristics of the cylindrical multi-port grain for hybrid rocket motor. In Proceedings of the 45th AIAA/ASME/SAE/ASEE Joint Propulsion Conference \& Exhibit, Denver, CO, USA, 2-5 August 2009.

24. Kim, G.H.; Kim, S.J.; Lee, J.P.; Cho, J.T.; Moon, H.J.; Sung, H.G.; Kim, J.K. A study on merge effect in the multi-port fuel grain of hybrid rockets. In Proceedings of the 2009 Asia-Pacific International Symposium on Aerospace Technology, Gifu, Japan, 4-6 November 2009.

25. Chen, K.; Richter, H. Instability analysis of the transition from bubbling to jetting in a gas injected into a liquid. Int. J. Multiph. Flow 1997, 23, 699-712. [CrossRef]

26. Dai, Z.; Wang, B.; Qi, L.; Shi, H. Experimental study on hydrodynamic behaviors of high-speed gas jets in still water. Acta Mech. Sin. 2006, 22, 443-448. [CrossRef]

27. Linck, M.B.; Gupta, A.K.; Yu, K.H. Submerged combustion and two-phase exhaust jet instabilities. J. Propuls. Power 2009, 25, 522-532. [CrossRef]

28. Loth, E.; Faeth, G.M. Structure of plane under expanded air jets into water. J. AIChE 1990, 36, 818-826. [CrossRef]

29. Shi, H.; Wang, B.; Dai, Z. Research on the mechanics of underwater supersonic gas jets. Sci. China Phys. Mech. 2010, 53, 527-535. [CrossRef]

30. Shi, H.H.; Guo, Q.; Wang, C.; Dong, R.L.; Zhang, L.T.; Jia, H.X.; Wang, X.G.; Wang, B.Y. Oscillation flow induced by underwater supersonic gas jets. Shock Waves 2010, 20, 347-352. [CrossRef]

31. Weiland, C.; Vlachos, P.P. Round gas jets submerged in water. Int. J. Multiph. Flow 2013, 48, 46-57. [CrossRef]

(c) 2019 by the authors. Licensee MDPI, Basel, Switzerland. This article is an open access article distributed under the terms and conditions of the Creative Commons Attribution (CC BY) license (http:/ / creativecommons.org/licenses/by/4.0/). 



\title{
Viability of an Electrically Driven Pump-Fed Hybrid Rocket for Small Launcher Upper Stages
}

\author{
Lorenzo Casalino ${ }^{\dagger}$, Filippo Masseni $^{\dagger}$ and Dario Pastrone ${ }^{*, \dagger}$ \\ Dipartimento di Ingegneria Meccanica e Aerospaziale, Politecnico di Torino, Corso Duca degli Abruzzi, 24, \\ 10129 Torino, Italy; lorenzo.casalino@polito.it (L.C.); filippo.masseni@polito.it (F.M.) \\ * Correspondence: dario.pastrone@polito.it \\ + These authors contributed equally to this work.
}

Received: 31 January 2019; Accepted: 11 March 2019; Published: 14 March 2019

\begin{abstract}
An electrically driven pump-fed cycle for a hybrid rocket engine is proposed and compared to a simpler gas-pressurized feed system. A liquid-oxygen/paraffin-based fuel hybrid rocket engine which powers the third stage of a Vega-like launcher is considered. Third-stage ignition conditions are assigned, and engine design and payload mass are defined by a proper set of parameters. Uncertainties in the classical regression rate correlation coefficients are taken into account and robust design optimization is carried out with an approach based on an epsilon-constrained evolutionary algorithm. A mission-specific objective function, which takes into account both the payload mass and the ability of the rocket to reach the required final orbit despite uncertainties, is determined by an indirect trajectory optimization approach. The target orbit is a $700 \mathrm{~km}$ altitude polar orbit. Results show that electrically driven pump-fed cycle is a viable option for the replacement of the conventional gas-pressurized feed system. Robustness in the design is granted and a remarkable payload gain is achieved, using both present and advanced technologies for electrical systems.
\end{abstract}

Keywords: hybrid rocket engines; multidisciplinary design optimization; robust optimization; electric feed system

\section{Introduction}

Hybrid rocket engine (HRE) performance is comparable to semi-cryo or storable liquid rocket engines (LREs) and solid rocket motors (SRMs). HREs have higher density-specific impulse $I_{\rho}$ than typical bi-propellant LREs, whereas their mean specific impulse $I_{S P}$ is higher than SRMs. HREs gather many favorable properties from SRMs such as safety, reliability, and low cost. On the other hand, HREs and LREs share shut-off/restart capabilities and can be throttled within a wide thrust range. Moreover, HREs are more environmentally friendly than both LREs and SRMs. Thus, many research programs worldwide are focusing on the development of HREs. Examples of applications are micro-gravity platforms, hypersonic accelerators, small satellites, upper stage for small launchers, launchers from Mars, Moon landers, debris removal, and commercial space flights [1-5].

In previous studies the authors analyzed the effect of the presence of uncertainties in the design parameters, such as in the regression rate, on rocket performance and mission goals [6,7]. Results showed that even small uncertainty in the determination of the regression rate may jeopardize vehicle performance and threaten seriously the mission. A proper robust design multidisciplinary approach [8] has been developed to couple propulsion system design and trajectory optimization, while reducing the sensitivity of the engine performance to uncertainties. The concept of "robustness" can be summarized as "the capability of the system to grant a fixed level of performance" (i.e., to match mission goals), "minimizing the effect of uncertainties in the design parameters without eliminating their causes" [9,10]. A Vega-like upper stage, powered by a gas-pressurized liquid-oxygen (LOX)/paraffin-based fuel HRE, was considered as test case. Results showed that robustness in the 
design was achieved with a small payload reduction with respect to the optimal deterministic design. Gas-pressurized feed systems were adopted aiming to keep cost as low as possible. However, the high tank pressure, required to keep the thrust magnitude and regression rate sufficiently large during operation, results in relevant auxiliary and oxidizer tanks masses. Due to the relevant value of the total impulse of the upper stage, the liquid oxidizer tank is relatively large and the use of a turbopump feed system can reduce the engine dry mass. The tank weight reduction and the elimination of possible gas vessels overcome the turbopump system weight.

In a turbopump feed system, the pump is classically driven by a turbine, which is fed by gas or liquid working fluid. Electrical pump feed systems (EPFS), where a battery-powered electric motor replaces the turbine, have also been proposed for bi-propellant LREs. Different applications have been considered since the 1990s [11,12], and the advances in batteries and electric motor technologies have been making this feed system increasingly competitive [13-16]. In 2016 an EPFS bi-propellant engine was considered, alongside other classical propulsion systems, to power a Mars ascent vehicle (MAV) [17]. Results showed that EPFS, although lower in technology readiness level, was able to outperform a conventional gas-pressurized feed system. The application to small-sat launcher appears to be very appealing and feasible [18], as the use of the Rutherford engine proved (this LOX/RP1 (Rocket Propellant 1) engine, used to power the Rocket Lab's Electron launch vehicle in its recent successful second flight test, has an electrically driven pump feed system). Recently, Ref. [19] assessed the viability of an EPFS LOX/kerosene upper stage for the Korean Space Launch Vehicle-II, which is designed to insert $1500 \mathrm{~kg}$ into a $700 \mathrm{~km}$ Single Stage to Orbit (SSO) orbit.

In an HRE, the presence of just one liquid propellant makes the generation of a proper working fluid for the turbine more challenging. A gas-generator cycle cannot be adopted, unless auxiliary liquid fuel is embarked. Hydrogen peroxide could produce hot gases by means of a catalyzer, but this is not the case of LOX, here considered. LOX heating would not be so effective, while a tap-off cycle would introduce complexity, reduce reliability, and increase costs. Thus, the electric pump solution is even more appealing than in LREs. Nevertheless, no great attention has been devoted to EPFS in HRE. In a previous work [20], the authors proposed the EPFS for a HP (Hydrogen Peroxide)/PE (Polyethylene) upper stage, performing a deterministic optimization. In the present work, a robust-based design and optimization is performed considering EPFS with LOX/paraffin-based fuel. Only uncertainties in the classical regression rate correlation are taken into account. The authors use a combined procedure: an indirect method optimizes the trajectory for each combination of engine parameters $[21,22]$ which, in turn, are selected by a particle swarm optimization algorithm [23]. The robust-based objective function is evaluated as a linear combination of an index that quantifies the effective reaching of the target orbit, based on the average performance under uncertainty, and the payload (that instead is not affected by uncertainties).

In the following sections, we sum up the main features of grain geometry, ballistic model, feed systems, and indirect optimization procedure. Then we compare the performance of gas-pressurized and pump feed systems, making our conclusions.

\section{Numerical Models}

\subsection{Grain Geometry and Ballistic Model}

In the present work, the authors considered LOX/paraffin-based fuel as propellant combination for HREs design. Cryogenic LOX is stored in liquid phase in a tank and injected into the combustion chamber during operation. The combustion chamber stores the wax in solid phase as a cylindrical grain. Paraffin-based fuels, such as wax, present an unstable melt liquid layer that causes the entrainment of droplets into the gas stream [24]. The fuel mass transfer rate into the flame zone is strongly increased by the entrainment of droplets. Combustion takes place in the flame zone through diffusive mixing of oxidizer and fuel coming from the grain. For this reason, regression rate is relatively large, and a single 
circular port can be adopted for the fuel grain, whereas classical fuels would require a multi-port grain design to avoid excessive length to diameter ratio (L/D) [25].

The grain outer radius $R_{g}$, the web thickness $w$, and the grain length $L_{b}$ define the geometry of the circular-port grain as shown in Figure 1. The initial inner radius, i.e., the port radius before ignition, results to be $R_{i}=R_{g}-w$. The burning perimeter $P$ and the port area $A_{p}$, for any given burning distance $y(0 \leq y \leq w)$, can be computed as:

$$
\begin{gathered}
P=2 \pi\left(R_{i}+y\right) \\
A_{p}=\pi\left(R_{i}+y\right)^{2}
\end{gathered}
$$

An approximate relation, between chamber head-end pressure $p_{1}$ and chamber nozzle-stagnation pressure $p_{c}$, is used to take into account pressure losses inside the combustion chamber [26]:

$$
p_{1}=\left[1+0.2\left(\frac{A_{t h}}{A_{p}}\right)^{2}\right] p_{c}
$$

where $A_{t h}$ is the throat area. The authors assumed a uniform regression rate along the port axis while the combustion of the lateral end is neglected. Its value is determined by the oxidizer mass flow rate $\dot{m}_{O}$ and grain geometry:

$$
\dot{y}=a\left(\dot{m}_{O} / A_{p}\right)^{n}
$$

In the present work $a$ and $n$ are assumed to be uncertain parameters. $a=9.1 \times 10^{-5} \mathrm{~m}^{2 n+1} \mathrm{~s}^{n-1} \mathrm{~kg}^{-n}$ and $n=0.69$ are taken into consideration as reference nominal values when International System (SI) of units are used [24].

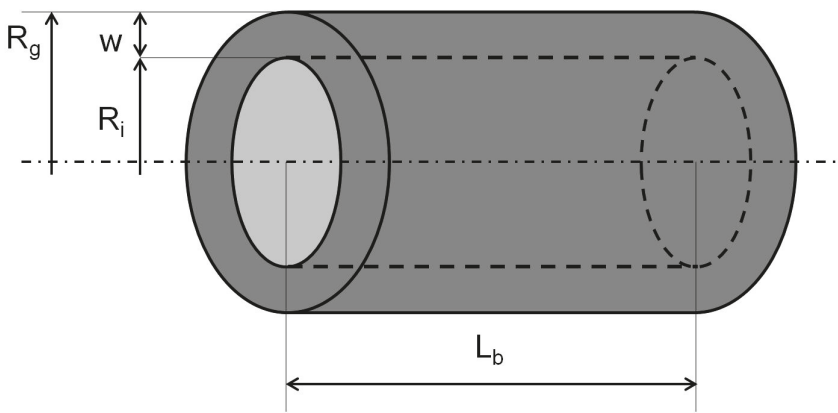

Figure 1. Schematic of the grain geometry.

The oxidizer flow rate is determined by the hydraulic resistance $Z$ in the oxidizer flow path and the pressure provided by the feed system. Under the assumption of incompressible turbulent flow:

$$
\dot{m}_{O}=\sqrt{\left(p_{f s}-p_{1}\right) / Z}
$$

where $p_{f s}$ is the feed system pressure. We assume a constant value of $Z$ during the operation. Fuel mass flow $\dot{m}_{F}$ can be obtained as:

$$
\dot{m}_{F}=\rho_{F} \dot{y} A_{b}=\rho_{F} \dot{y} L_{b} P
$$

where $\rho_{F}$ is the fuel grain density, $A_{b}$ is the burning area. One can compute the mixture ratio $\alpha$ as:

$$
\alpha=\frac{\dot{m}_{O}}{\dot{m}_{F}} \propto \dot{m}_{O}^{1-n} A_{p}^{n} / A_{b}
$$


An isentropic expansion in the nozzle is assumed, and the chamber nozzle-stagnation pressure $p_{\mathcal{C}}$ can be determined by:

$$
p_{c}=\frac{\left(\dot{m}_{O}+\dot{m}_{F}\right) c^{*}}{A_{t h}}
$$

The authors used a 10-bar chamber pressure in performance evaluation of the propellant combination as a function of the mixture ratio $\alpha$. Even though the actual pressure in the combustion chamber can span over a wide range during engine operations, the performance error, due to the constant chamber pressure assumption, is small for pressures and mixture ratios considered in this article. Frozen equilibrium expansion is assumed: exhaust gas composition is fixed throughout the nozzle and equal to combustion chamber one. We adopt the conservative assumption of frozen equilibrium expansion to account for the low combustion efficiency of HREs. Moreover, a 0.96 $c^{*}$-efficiency [27] is introduced. Third-degree polynomial curves, fitting the characteristic velocity and specific heat ratio, are embed in the code, to compute accurately and quickly the proper values as the mixture ratio changes during operation [28]. Thrust coefficient $C_{F}$ can be evaluated as:

$$
C_{F}=0.98\left\{\sqrt{\frac{2 \gamma^{2}}{\gamma-1}\left(\frac{2}{\gamma+1}\right)^{\frac{\gamma+1}{\gamma-1}}\left[1-\left(\frac{p_{e}}{p_{c}}\right)^{\frac{\gamma-1}{\gamma}}\right]}+E \frac{p_{e}}{p_{c}}\right\}-E \frac{p_{0}}{p_{c}}
$$

where a 0.98 correction factor is introduced to modify the vacuum thrust coefficient of a 1-D isentropic expansion to the exit pressure $p_{e}$ with constant heat ratio $\gamma$. Atmospheric pressure-related term $E \cdot p_{0} / p_{c}$ is always small since the third stage always flies at high altitude. One can determine mass flow rate at ignition (i.e., at $t=0$ ) as:

$$
\left(\dot{m}_{p}\right)_{i}=\left(1+\alpha_{i}\right)\left(\dot{m}_{F}\right)_{i}=\frac{1+\alpha_{i}}{\alpha_{i}}\left(\dot{m}_{O}\right)_{i}
$$

Then initial throat area $\left(A_{t h}\right)_{i}$ and initial port area $\left(A_{p}\right)_{i}$ can be obtained:

$$
\left(A_{t h}\right)_{i}=\frac{\left(\dot{m}_{p}\right)_{i}}{\left(p_{c}\right)_{i} c_{i}^{*}} ; \quad\left(A_{p}\right)_{i}=\frac{\left(A_{t h}\right)_{i}}{J}
$$

Nozzle throat erosion is here considered [29,30]. Bartz's method is used to model the dependence of the rate of throat erosion $\dot{s}$ on throat radius $R_{t h}$ and chamber pressure $p_{c}$ :

$$
\dot{s}=\dot{s}_{r e f}\left(\frac{p_{c}}{p_{c, r e f}}\right)^{0.8}\left(\frac{R_{t h, r e f}}{R_{t h}}\right)^{0.2}
$$

$R_{t h}$ and $E$ values are computed by integrating Equation (12). $\dot{s}_{r e f}=0.1 \mathrm{~mm} / \mathrm{s}$, obtained from CFD analysis on the ablation of a carbon/carbon nozzle for LOX/wax HREs [31], is here adopted. Our model does not consider erosion along the nozzle, obtaining a greater reduction of $E$ and a conservative solution. Eroded mass is not taken into account, either for thrust augmentation or for rocket mass reduction.

\subsection{Feed Systems}

In the present work, the authors compare the performance of two different feed systems: a gas-pressurized feed system (GPFS) and an EPFS. During engine operation of a gas-pressurized feed system, the oxidizer mass flow rate is determined by the pressure drop between tank and chamber head-end pressures. The tank pressure is maintained constant by a pressurizing gas, contained in a high-pressure auxiliary tank during an initial regulated phase, which is then followed by a blow-down phase. The pressure at the pump outlet is instead considered in an EPFS. EPFS requires additional 
masses for batteries, electric motor, and pump while GPFS needs a heavier oxidizer tank, due to its higher pressure, and auxiliary gas and vessels. The performance of GPFS here considered, and put into comparison with EPFS, are taken from a previous optimization work by the authors [8]. Two sets of electrical properties for batteries and electric motor and pump are used in EPFS performance evaluation. Set A has already been employed by the authors, back in 2010, for the deterministic optimization of a HP/PE powered HRE [20]. Set B, instead, is taken from the most recent literature available [19].

\subsubsection{GPFS}

The GPFS has two operational modes. Initially, constant tank pressure is maintained by helium flowing from an auxiliary tank, thus $p_{f_{s}}$ is constant too. Later, a subsequent blow-down (BD) phase is performed and $p_{f_{s}}$ decreases during operation. The authors assume an initial ullage volume equal to $3 \%$ of the oxidizer volume to have a stable regulator response when the out flow starts [32]. The GPFS is characterized by two design parameters: the auxiliary gas tank volume $V_{a}$ and the initial pressurizing gas pressure $p_{a}$. The exhausted oxidizer mass at the beginning of the BD phase $\left(m_{O}\right)_{B D}$ is conveniently used instead of $V_{a}$ while the initial pressurizing gas pressure is kept constant at $p_{a}=200$ bar. During the constant pressure mode, $p_{f s}=\left(p_{t}\right)_{i}=25$ bar, whereas, during the BD phase, $p_{f s}$ is calculated assuming an isentropic expansion of the pressurizing gas in the tank:

$$
p_{f s}=\left(p_{t}\right)_{i}\left[\frac{\left(V_{g}\right)_{B D}}{V_{g}}\right]^{\gamma_{g}}
$$

where the gas volume in the tank $V_{g}=\left(V_{g}\right)_{i}+m_{O} / \rho_{O}$ depends on the oxidizer mass $m_{O}$ that has been exhausted, $\left(V_{g}\right)_{B D}=\left(V_{g}\right)_{i}+\left(m_{O}\right)_{B D} / \rho_{O}$ and $\gamma_{g}$ is the specific heat ratio of the pressurizing gas.

\subsubsection{EPFS}

In the EPFS, an electric motor is used to drive the pump which feeds the oxidizer to the combustion chamber. A battery pack supplies the energy required by the electric motor during operation. Pump, electrical systems and batteries masses are evaluated by means of typical power density (power to mass ratio) values provided by existing literature. Thus, the electric motor and pump mass is evaluated as:

$$
m_{e p}=\frac{P_{e, \max }}{\delta_{e p}}
$$

where $P_{e, \max }$ is the maximum electrical power required. In the following, two values for the power density $\delta_{e p}$ has been assumed for the electric drive system plus pump: $\left(\delta_{e p}\right)_{A}=1.25 \mathrm{~kW} / \mathrm{kg}$ represents current technology [13,33] and $\left(\delta_{e p}\right)_{B}=3.92 \mathrm{~kW} / \mathrm{kg}$ for advances designs [19]. The electrical power required by the motor to drive the pump is:

$$
P_{e}=\frac{\dot{m}_{O}\left(p_{f s}-p_{t}\right)}{\rho_{O} \eta_{e p}}
$$

where $p_{f s}$ is equal to the pump discharge pressure $p_{d}$ and $p_{t}$ is the oxidizer tank pressure. The tank pressure is assumed to be constant during operation and equal to 1 bar. The mass used to keep $p_{t}$ constant during operation is small and then can be neglected. The conversion of electrical energy, stored in the batteries, into flow head rise is taken into account by the overall efficiency $\eta_{e p}$. In the present work, we assume two values for the overall efficiency, $\left(\eta_{e p}\right)_{A}=0.64$ and $\left(\eta_{e p}\right)_{B}=0.53$. One can notice that $\left(\eta_{e p}\right)_{B}<\left(\eta_{e p}\right)_{A}$, but $m_{e p} \propto\left(\delta_{e p} \cdot \eta_{e p}\right)^{-1}$ which is actually smaller for the newer Set $\mathrm{B}$ quantities. Battery pack mass is constrained by the most stringent requirement between the maximum electrical power required $P_{e, \max }$ and the total electrical energy $E_{e, \text { tot }}$ needed to drive the 
pump during the whole burning time. In the present case, the discharge time is equal to the overall HRE burning time $t_{b u r n}$. Thus, the needed electrical energy is:

$$
E_{e, t o t}=\int_{0}^{t_{\text {burn }}} P_{e} d t
$$

Batteries mass $m_{b}$ can be evaluated by means of typical values of power density $\delta_{b p}$ and energy density $\delta_{b e}$ (energy to mass ratio) that can be found in literature. A safety factor of 1.2 is assumed and therefore:

$$
m_{b}=1.2 \max \left(\frac{P_{e, \max }}{\delta_{b p}}, \frac{E_{e, t o t}}{\delta_{b e}}\right)
$$

Due to their small size and light weight compared to all other technologies, Lithium batteries, are considered for the present application. The authors considered two couples of densities to evaluate battery pack mass: $\left(\delta_{b p}\right)_{A}=3.0 \mathrm{~kW} / \mathrm{kg}$ and $\left(\delta_{b e}\right)_{A}=90 \mathrm{Wh} / \mathrm{kg}$, based on Ragone plots [34], and $\left(\delta_{b p}\right)_{B}=6.95 \mathrm{~kW} / \mathrm{kg}$ and $\left(\delta_{b e}\right)_{B}=198.5 \mathrm{Wh} / \mathrm{kg}$, based on state of the art Lithium-Polymer batteries [19]. These kinds of batteries are suitable for HRE applications because they require high power levels for a relatively short time, so that high rate capability is required. The pump is operated at constant power $P_{e}=P_{e, \max }$. Thus, Equation (16) can be easily integrated and:

$$
E_{e, t o t}=P_{e, \max } t_{b u r n}
$$

The value of $\left(p_{d}\right)_{i}$ at the beginning of the HRE operation is an additional parameter, used in the direct optimization procedure. It fixes the value of $P_{e, \max }=P_{\mathcal{e}}=$ constant, which is crucial for feed system and engine performance.

One can notice that power-constrained batteries mass can be evaluated before the actual trajectory is optimized, while energy-constrained mass must be computed "a posteriori". The authors define a characteristic burn time $t_{b u r n}^{*}=\delta_{b e} / \delta_{b p}$ that represents the simultaneous fulfillment of both constraints. If $t_{b u r n} \leq t_{\text {burn }}^{*}$, power-constrained mass is larger than energy-constrained one. Hence, battery pack mass does not depend on the actual ascent trajectory. On the other hand, if $t_{\text {burn }} \geq t_{\text {burn }}^{*}$ energy-constrained mass is larger than power-constrained one. In this case, batteries mass must be checked "a posteriori" to take into account the additional energy required by a longer mission. Electric properties values are summarized in Table 1 for the sake of clarity.

Table 1. Electric properties.

\begin{tabular}{cccccc}
\hline Design Set & $\begin{array}{c}\delta_{b p} \\
\mathbf{k W} / \mathbf{k g}\end{array}$ & $\begin{array}{c}\delta_{b e} \\
\mathbf{W h} / \mathbf{k g}\end{array}$ & $\begin{array}{c}\delta_{\text {ep }} \\
\mathbf{k W} / \mathbf{k g}\end{array}$ & $\begin{array}{c}\eta_{e p} \\
-\end{array}$ & $\begin{array}{c}\boldsymbol{t}_{\text {burn }}^{*} \\
\mathbf{s}\end{array}$ \\
\hline $\mathrm{A}$ & 3.00 & 90.00 & 1.25 & 0.68 & 108 \\
$\mathrm{~B}$ & 6.95 & 198.50 & 3.92 & 0.53 & 103 \\
\hline
\end{tabular}

\subsection{Trajectory Optimization}

Once engine design parameters have been defined, an indirect procedure optimizes the orbit insertion trajectory [21]. A point mass rocket is considered for the trajectory optimization. State equations provide the derivative of position $r$ (radius, latitude and longitude), velocity $v$ (radial, eastward and northward components) and rocket mass $M$. In a vectorial form one has:

$$
\frac{\mathrm{d} r}{\mathrm{~d} t}=v \quad \frac{\mathrm{d} v}{\mathrm{~d} t}=g+\frac{\boldsymbol{F}-\boldsymbol{D}}{M} \quad \frac{\mathrm{d} M}{\mathrm{~d} t}=-\frac{|\boldsymbol{F}|}{c^{*} C_{F}}
$$

An inverse-square gravity field is assumed:

$$
g=-\frac{G M_{\oplus}}{\left\|r^{3}\right\|} r
$$


where $G$ is the gravitational constant and $M_{\oplus}$ is Earth mass. An interpolation of the standard atmosphere, as a function of the rocket altitude, provides density and pressure evaluation. Equations of motion are written in a non-dimensional form to improve the accuracy of the numerical integration. Indirect optimization procedure details are here only summarized and can be found in Ref. [35].

An adjoint variable is associated with each equation; the theory of optimal control provides Euler-Lagrange equations, algebraic equations that determine the control variables (i.e., the thrust direction), and the boundary conditions for optimality (which also implicitly define the engine switching times). A Newton's method-based procedure is used to solve the multi-point boundary value problem which arises. Further details about this procedure can be found in Ref. [36]. Tentative values are initially chosen for the problem unknowns and progressively modified to fulfill the boundary conditions. The unknown parameters are the time lengths of each phase and the initial values of five adjoint variables (the variable corresponding to longitude is null, the one corresponding to the mass is fixed at one, as the problem is homogeneous in the adjoint variables, which can therefore be arbitrarily scaled). Moreover, the overall oxidizer mass and the grain radius are additional unknowns. Dynamic pressure, heat flux and acceleration constraints are checked "a posteriori" and are not explicitly imposed during the trajectory optimization. However, the authors add a constraint that forces horizontal flight at the end of the first burn, to prevent the rocket from reentering the lower layers of the atmosphere (where the heat flux would become larger). Analogously, an additional unknown (the adjoint variable corresponding to the horizontal velocity component has a free discontinuity at the end of the first burn) is introduced in the trajectory optimization procedure.

In the present work, an HRE suitable for the replacement of third (solid) and fourth (liquid) stage of the Vega launcher is considered [37]. The performance of the first stage, second stage, and the exhausted masses are given. The conditions at the ignition of the third stage, consistent with a launch from Kourou, are assigned: altitude $h=86.88 \mathrm{~km}$, latitude $\phi=9.11^{\circ}$, velocity components in the radial, eastward and northward directions $u_{r}=0.142 \mathrm{~km} / \mathrm{s}, v_{e}=0.230 \mathrm{~km} / \mathrm{s}, w_{n}=4.146 \mathrm{~km} / \mathrm{s}$, respectively, and mass $m_{i}=14,522 \mathrm{~kg}$ [25]. Altitude, eccentricity and inclination (700 km, zero and $90 \mathrm{deg}$, respectively) define target final orbit. The longitude of the ascending node is left free.

\subsection{Robust Design Model}

Robust optimization problem can be formally cast as [10]:

$$
\begin{array}{ll}
\text { find } & \boldsymbol{b} \in \mathbb{R}^{n} \\
\text { to maximize } & \Phi_{a v g}(\boldsymbol{b}, \boldsymbol{p}) \\
\text { subject to } & g_{j}\left(\boldsymbol{b}, \boldsymbol{p}+\boldsymbol{z}^{p}\right) \leqslant 0, j=1, \ldots, r \\
\text { and to } & \boldsymbol{b}_{L} \leqslant \boldsymbol{b} \leqslant \boldsymbol{b}_{U}
\end{array}
$$

where $\boldsymbol{b}$ is the design variables vector, $\boldsymbol{p}$ is the uncertain variables vector, $z^{p}$ is the noise vector of $\boldsymbol{p}, g_{j}$ is the $j$-th inequality constraint, $\boldsymbol{b}_{L}$ and $\boldsymbol{b}_{U}$ are the lower and upper boundary of the design variables, respectively.

Here the initial mass of the upper stage is given at ignition and the hydraulic resistance $Z$ is evaluated to have $p_{t} / p_{c}=2.5$ at ignition in nominal condition. Six parameters are required to define the propulsion system: the grain outer radius $R_{g}$, the web thickness $w$, the fuel grain length $L_{b}$, the final exhausted oxidizer mass $\left(m_{O}\right)_{f}$ and the initial nozzle area ratio $E_{i}$. The sixth parameter is the exhausted oxidizer mass at the beginning of the BD phase $\left(m_{O}\right)_{B D}$, when GPFS is used, while the pump discharge pressure $p_{d}$ is needed when EPFS is considered. Therefore $\boldsymbol{b}_{G P F S}=\left[R_{g}, w, L_{b},\left(m_{O}\right)_{f},\left(m_{O}\right)_{B D}, E_{i}\right]$ and $\boldsymbol{b}_{E P F S}=\left[R_{g}, w, L_{b},\left(m_{O}\right)_{f}, p_{d}, E_{i}\right]$. Upper and lower boundary of the design variables for GPFS and EPFS are shown in Tables 2 and 3 respectively. 
Table 2. Design parameters ranges: GPFS.

\begin{tabular}{ccccccc}
\hline Boundary & $\begin{array}{c}\boldsymbol{R}_{\boldsymbol{g}} \\
\mathbf{m}\end{array}$ & $\begin{array}{c}w \\
\mathbf{m}\end{array}$ & $\begin{array}{c}\boldsymbol{L}_{\boldsymbol{b}} \\
\mathbf{m}\end{array}$ & $\begin{array}{c}\left(m_{O}\right)_{f} \\
\mathbf{k g}\end{array}$ & $\begin{array}{c}\left(m_{O}\right)_{B D} \\
\mathbf{k g}\end{array}$ & $\begin{array}{c}E_{i} \\
-\end{array}$ \\
\hline $\boldsymbol{b}_{L}$ & 0.55 & 0.25 & 4.3 & 6971 & 3195 & 15 \\
$\boldsymbol{b}_{U}$ & 0.60 & 0.35 & 4.5 & 7697 & 3631 & 20 \\
\hline
\end{tabular}

Table 3. Design parameters ranges: EPFS.

\begin{tabular}{ccccccc}
\hline Boundary & $\begin{array}{c}\boldsymbol{R}_{\boldsymbol{g}} \\
\mathbf{m}\end{array}$ & $\begin{array}{c}w \\
\mathbf{m}\end{array}$ & $\begin{array}{c}\boldsymbol{L}_{\boldsymbol{b}} \\
\mathbf{m}\end{array}$ & $\begin{array}{c}\left(m_{O}\right)_{f} \\
\mathbf{k g}\end{array}$ & $\begin{array}{c}p_{d} \\
\mathbf{b a r}\end{array}$ & $\begin{array}{c}\boldsymbol{E}_{i} \\
-\end{array}$ \\
\hline $\boldsymbol{b}_{L}$ & 0.55 & 0.25 & 4.3 & 6971 & 10 & 15 \\
$\boldsymbol{b}_{U}$ & 0.60 & 0.35 & 4.5 & 7697 & 50 & 20 \\
\hline
\end{tabular}

Uncertain parameters are the regression rate coefficients $a$ and $n$, i.e., $p=[a, n]$. Uncertainties are taken into account assigning three different levels for each uncertain variables: $a_{i} \times 10^{5}=9,9.1,9.2 \mathrm{~m}^{2 n+1} \mathrm{~s}^{n-1} \mathrm{~kg}^{-n}$ for $i=1,2,3$, respectively, and $n_{j}=0.68,0.69,0.7$ for $j=1,2,3$, respectively. The altitude of the attained orbit $h_{i j}$ is evaluated for each of the nine possible couples $a_{i}, n_{j}$. Since two objectives are relevant (i.e., payload $\mu$ and altitude $h$ ), an $\epsilon$-constraint approach is adopted [38] to find the Pareto front of robust solutions. Here only the average performance is analyzed. The average constraint violation $\Delta_{a v g}=\sum_{i j} p_{i} p_{j} \max _{i j}\left(0, h^{*}-h_{i j}\right)$ is considered. We assume a binomial distribution giving $p_{1}=p_{3}=0.25$ and $p_{2}=0.5$. The average altitude is then $h_{\text {avg }}=h^{*}-\Delta_{\text {avg }}$ and the objective function can be computed as:

$$
\Phi_{a v g}=\mu-k \max \left(0, \epsilon-h_{a v g}\right)
$$

$k=20 \mathrm{~kg} / \mathrm{km}$ is selected to force the average altitude at $\epsilon$. Only the case $\epsilon=h^{*}=700 \mathrm{~km}$ (that is the most demanding in terms of robustness) is here considered.

The selection of the optimal robust design is performed by means of a particle swarm optimization (PSO) algorithm. PSO is an optimization procedure belonging to the class of evolutionary algorithms (EAs) that search for an optimal solution in a prescribed search space. It is inspired by the social behavior showed by flock of birds and school of fishes [39,40]. Solutions, here called particles, fly through the problem space by following the optimum particle (i.e., the alpha-member of the flock or school). Cognitive and social acceleration drive the motion of each particle and their position and speed are updated at each iteration. Basic steps of PSO are shown in Figure 2 and PSO settings are presented in Table 4. Additional details about PSO implementation and tuning can be found in Refs. [23,41].

Table 4. PSO settings.

\begin{tabular}{cc}
\hline Parameter & Value \\
\hline Number of generations, $N_{G}$ & 100 \\
Number of particles, $N_{I}$ & 20 \\
Dimension of particles & 6 \\
Ranges of particles & $\boldsymbol{b}_{U}-\boldsymbol{b}_{L}$ \\
PSO method & 1-trelea type 1 \\
Cognitive acceleration, C1 & 2.0 \\
Social acceleration, C2 & 2.0 \\
Check population method & Saturation \\
End velocity weight & 0.4 \\
Linear varying factor & 0.2 \\
Maximum velocity, $\boldsymbol{v}_{\text {max }}$ & $0.25\left(\boldsymbol{b}_{U}-\boldsymbol{b}_{L}\right)$ \\
Mass mutation parameter & $98 \%$ \\
\hline
\end{tabular}




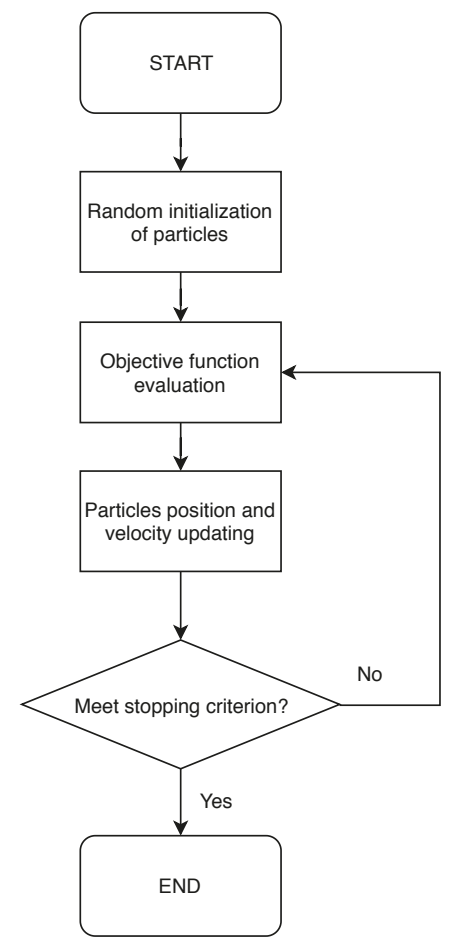

Figure 2. PSO: flow chart.

\section{Numerical Results}

As described in the previous sections, a two-layer optimization is employed for trajectory and engine design: the indirect trajectory optimization maximizes the final mass given engine geometry, which, in turn, is optimized by means of a robust-based design approach. The average height violation $\Delta_{\text {avg }}$ is forced to zero, due to the large value for $k$ in Equation (22), and $\Phi$ coincides with the payload for all the robust designs summarized in Table 5. Please note that the final burn of the HRE has a very short duration and a limited influence on the rocket performance. Hence subsequent figures show only the first burn for the sake of simplicity.

Table 5. Design parameters and performance.

\begin{tabular}{ccccccccccccc}
\hline Case & $\begin{array}{c}\boldsymbol{R}_{\boldsymbol{g}} \\
\mathbf{m}\end{array}$ & $\begin{array}{c}w \\
\mathbf{m}\end{array}$ & $\begin{array}{c}\boldsymbol{L}_{\boldsymbol{b}} \\
\mathbf{m}\end{array}$ & $\begin{array}{c}\left(m_{O}\right)_{f} \\
\mathbf{k g}\end{array}$ & $\begin{array}{c}E_{\boldsymbol{i}} \\
-\end{array}$ & $\begin{array}{c}\left(m_{O}\right)_{B D} \\
\mathbf{k g}\end{array}$ & $\begin{array}{c}p_{d} \\
\mathbf{b a r}\end{array}$ & $\begin{array}{c}\mu \\
\mathbf{k g}\end{array}$ & $\begin{array}{c}\boldsymbol{\Delta}_{\text {avg }} \\
\mathbf{k m}\end{array}$ & $\begin{array}{c}\boldsymbol{\Phi}_{\text {avg }} \\
\mathbf{k g}\end{array}$ & $\begin{array}{c}\boldsymbol{t}_{\text {burn }} \\
\mathbf{s}\end{array}$ & $\begin{array}{c}\boldsymbol{I}_{S P} \\
\mathbf{s}\end{array}$ \\
\hline $\mathrm{GPFS}$ & 0.591 & 0.294 & 4.360 & 7403 & 17.32 & 3195 & - & 2069.8 & 0.0 & 2069.8 & 177 & 296 \\
$\mathrm{EPFS}_{A}$ & 0.579 & 0.303 & 4.438 & 7408 & 17.44 & - & 27.7 & 2321.7 & 0.0 & 2321.7 & 157 & 296 \\
$\mathrm{EPFS}_{B}$ & 0.563 & 0.334 & 4.368 & 7381 & 18.14 & - & 39.8 & 2467.7 & 0.0 & 2467.7 & 158 & 296 \\
\hline
\end{tabular}

When pump feed system is adopted, mixture ratio and regression rate tend to be larger at the end of the engine burn with respect to GPFS values, as shown in Figures 3 and 4 respectively. Figures 5 and 6 display that pump discharge pressure and oxidizer mass flow variations are actually limited (less than $5 \%$ ), due to pump constant power operation during the engine burn. Hence efficiency and electrical density assumption for the electric motor and pump system are valid. Thrust history and longitudinal acceleration exhibit different behavior for GPFS and EPFS as reported in Figures 7 and 8 respectively. 


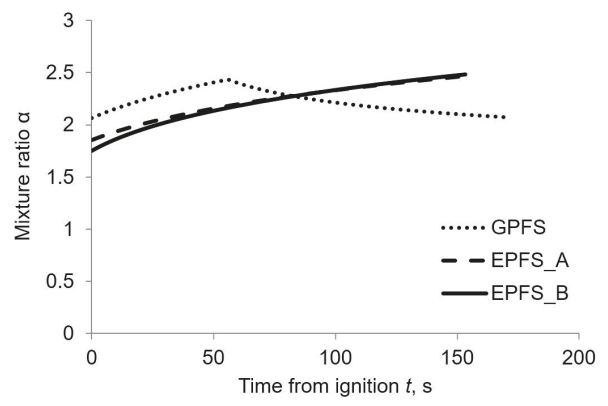

Figure 3. Mixture ratio history.

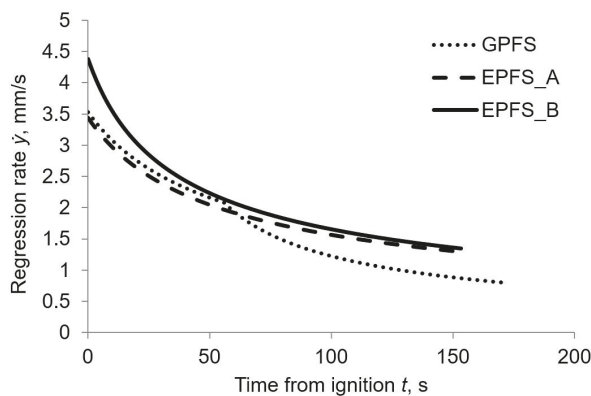

Figure 4. Regression rate history.

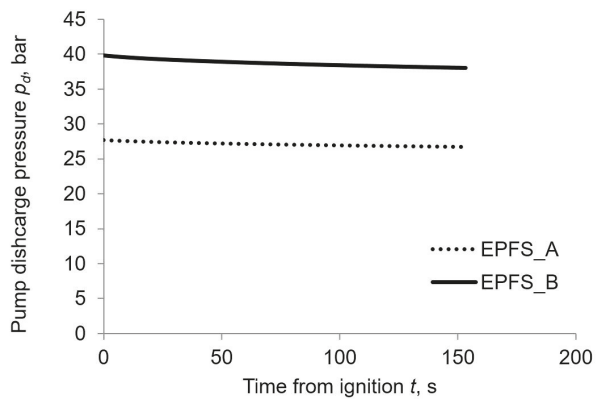

Figure 5. Discharge pressure history.

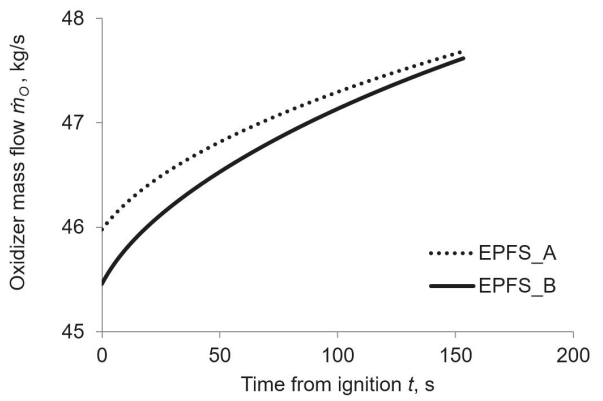

Figure 6. Oxidizer mass flow history. 


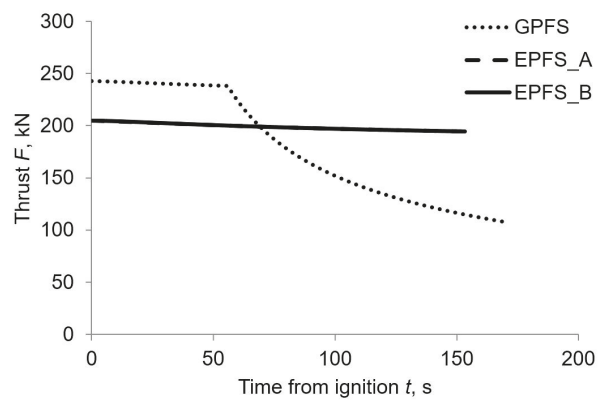

Figure 7. Thrust history.

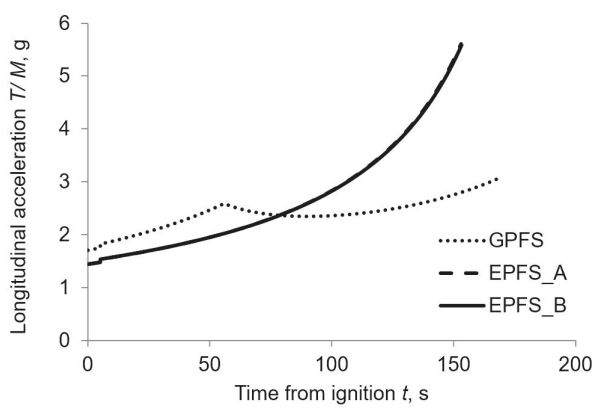

Figure 8. Longitudinal acceleration history.

The thrust in the first half of the burn is larger for the GPFS, with respect to EPFS one, to maintain an acceptable thrust level during the subsequent BD phase. On the other hand, EPFS can maintain an almost constant thrust for all the burn duration and thus its initial thrust level can be lower than GPFS one.

One can notice that $\mathrm{EPFS}_{A}$ and $\mathrm{EPFS}_{B}$ have almost the same thrust history. Nevertheless, the thrust level is obtained in two different ways: $\mathrm{EPFS}_{A}$ optimal design is characterized by lower discharge pressure (i.e., low chamber pressure) and a larger nozzle (i.e., large throat area) than $\mathrm{EPFS}_{B}$ ones. This behavior is forced by the low electric motor and pump power density $\left(\delta_{e p}\right)_{A}$ that penalizes high-pressure designs. On the other hand, $\mathrm{EPFS}_{B}$ optimal design favors a higher discharge, and thus chamber, pressures alongside a smaller and lighter nozzle (i.e., small throat area) than $\mathrm{EPFS}_{A}$ ones. The latter optimization strategy is allowed by the advanced electrical properties considered in set $B$ and grants a remarkable nozzle mass saving without thrust reduction.

Mass budgets for robust solutions are reported in Table 6 whereas normalized mass ratios are summarized in Table 7. The current Vega configuration payload for the mission considered in the present work is equal to $1500 \mathrm{~kg}$ [37]. The main sources of performance improvement, for both GPFS and EPFS designs, are a remarkable saving in the dry masses of the upper stages and reduced $\Delta v$ losses. Furthermore, one can notice that $\mathrm{EPFS}_{A}$ saves roughly $350 \mathrm{~kg}$ due to lighter tank (pumps allow for unpressurized oxidizer tank), smaller nozzle, and far lower pressurizing gas mass than GPFS one. Moreover, EPFS does not require the auxiliary tank. Thus, the total mass saving is close to $500 \mathrm{~kg}$ with respect to GPFS. On the other hand, EPFS requires electric motor and batteries masses that are equal to roughly $230 \mathrm{~kg}$ for $\mathrm{EPFS}_{A}$ and $150 \mathrm{~kg}$ for $\mathrm{EPFS}_{B}$. Hence, the payload gain when EPFS is considered, with respect to GPFS, is equal to $252 \mathrm{~kg}$ and $398 \mathrm{~kg}$ when current or advanced technologies are considered in electric motor and pump masses evaluation. $\mathrm{EPFS}_{B}$ motor, pump and batteries masses are smaller than $\mathrm{EPFS}_{A}$ ones due to better electrical properties despite an actually larger electric power consumption (i.e., higher initial discharge pressure) and electric energy requirement. 
Table 6. Mass budget comparison.

\begin{tabular}{ccccccccccccc}
\hline Case & $\begin{array}{c}\mu \\
\mathbf{k g}\end{array}$ & $\begin{array}{c}m_{O} \\
\mathbf{k g}\end{array}$ & $\begin{array}{c}m_{F} \\
\mathbf{k g}\end{array}$ & $\begin{array}{c}m_{\text {sl }} \\
\mathbf{k g}\end{array}$ & $\begin{array}{c}m_{c c} \\
\mathbf{k g}\end{array}$ & $\begin{array}{c}m_{t} \\
\mathbf{k g}\end{array}$ & $\begin{array}{c}m_{n z} \\
\mathbf{k g}\end{array}$ & $\begin{array}{c}m_{\text {case }} \\
\mathbf{k g}\end{array}$ & $\begin{array}{c}m_{g} \\
\mathbf{k g}\end{array}$ & $\begin{array}{c}m_{\boldsymbol{a}} \\
\mathbf{k g}\end{array}$ & $\begin{array}{c}m_{\boldsymbol{b}} \\
\mathbf{k g}\end{array}$ & $\begin{array}{c}m_{e p} \\
\mathbf{k g}\end{array}$ \\
\hline $\mathrm{GPFS}$ & 2069.8 & 7403.5 & 3364.0 & 0.00 & 160.2 & 299.4 & 351.1 & 165.6 & 23.6 & 149.8 & - & - \\
$\mathrm{EPFS}_{A}$ & 2321.7 & 7407.7 & 3392.4 & 0.00 & 147.4 & 12.0 & 309.7 & 163.7 & 0.0315 & - & 98.0 & 134.4 \\
$\mathrm{EPFS}_{B}$ & 2467.7 & 7380.5 & 3414.6 & 0.00 & 162.3 & 12.0 & 239.7 & 158.4 & 0.0314 & - & 77.3 & 74.4 \\
\hline
\end{tabular}

Table 7. Mass budget comparison: normalized ratios.

\begin{tabular}{cccc}
\hline Case & $\frac{\mu}{m_{i}}$ & $\frac{m_{p}}{m_{i}}$ & $\frac{m_{p}}{m_{p}+m_{\text {dry }}}$ \\
& - & - & - \\
\hline GPFS & 0.143 & 0.741 & 0.904 \\
EPFS $_{A}$ & 0.160 & 0.744 & 0.926 \\
EPFS $_{B}$ & 0.170 & 0.743 & 0.937 \\
\hline
\end{tabular}

\section{Conclusions}

An indirect trajectory optimization procedure coupled with an evolutionary algorithm has been used to assess the performance viability of an electrically driven pump-fed HRE. The performance of EPFS have been compared to GPFS ones. The optimization of an upper stage of a Vega-like launcher has been considered. The optimization is performed for a given insertion orbit and lift-off weight. The authors take into account uncertainties in the regression rate to grant robustness of the optimal solution. A linear combination of average altitude and payload has been used as mission-specific performance index. The solution is forced to nullify the spread of the orbit altitude by the chosen index formulation, thus assuring the required robustness.

Results show that electric pumps are a viable option for the replacement of a GPFS for the present application. Present technology in batteries and electric motor allows for a payload improvement of approximately $250 \mathrm{~kg}$ with respect to GPFS, granting the same level of robustness. A further payload gain is achieved when advanced batteries are considered. Pump power control law can be taken into consideration in future works to improve engine performance and reduce electrical systems masses and maximum longitudinal acceleration.

Author Contributions: Conceptualization, L.C. and D.P.; Data curation, F.M.; Formal analysis, L.C., F.M. and D.P.; Methodology, L.C., F.M. and D.P.; Software, L.C., F.M. and D.P.; Supervision, L.C. and D.P.; Writing-original draft, F.M.; Writing-review \& editing, L.C., F.M. and D.P.

Funding: This research received no external funding.

Conflicts of Interest: The authors declare no conflict of interest.

\section{Abbreviations}

The following abbreviations are used in this manuscript:

BD Blow-Down

CFD Computational Fluid Dynamics

EA(s) Evolutionary Algorithm(s)

EPFS Electric Pump Feed System

GPFS Gas-Pressurized Feed System

HP Hydrogen Peroxide

HRE(s) Hybrid Rocket Engine(s)

LOX Liquid-Oxygen 


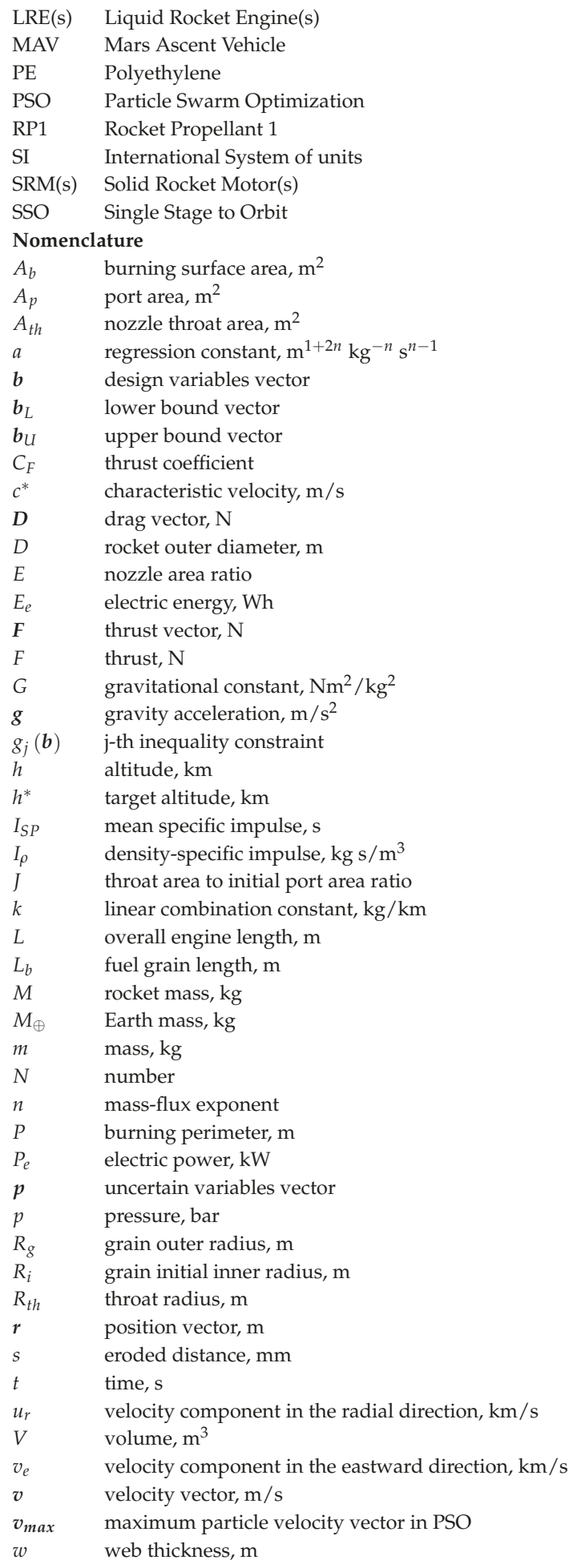


$w_{n} \quad$ velocity component in the northward direction, $\mathrm{km} / \mathrm{s}$

y burning distance, $\mathrm{m}$

$\mathrm{Z} \quad$ hydraulic resistance, $1 /(\mathrm{kg} \mathrm{m})$

$z^{p} \quad$ noise vector of $p$

$\alpha \quad$ mixture ratio

$\gamma \quad$ specific heat ratio

$\delta_{e p} \quad$ electric motor and pump power density, $\mathrm{kW} / \mathrm{kg}$

$\delta_{b e} \quad$ batteries energy density, $\mathrm{Wh} / \mathrm{kg}$

$\delta_{b p} \quad$ batteries power density, $\mathrm{kW} / \mathrm{kg}$

$\Delta \quad$ altitude violation, $\mathrm{m}$

$\epsilon \quad$ multi-objective constraint, $\mathrm{km}$

$\eta_{e p} \quad$ electric motor and pump efficiency

$\mu \quad$ payload, $\mathrm{kg}$

$\rho$ density, $\mathrm{kg} / \mathrm{m}^{3}$

$\Phi \quad$ objective function, $\mathrm{kg}$

$\phi \quad$ latitude, deg

Superscripts

time derivative

characteristic

\section{Subscripts}

0 ambient

1 combustion chamber at head-end

A set A electric properties

a auxiliary gas

avg average

$B \quad$ set $B$ electric properties

$B D$ beginning of blow-down phase

$b \quad$ batteries

burn engine burn

c combustion chamber at nozzle entrance

cc combustion chamber

d discharge

dry dry

$e \quad$ nozzle exit

ep electric motor and pump

$F$ fuel

$f \quad$ final

$f_{S} \quad$ feed system

$G$ generations

$g \quad$ pressurizing gas

I individuals

$i \quad$ initial value

$\max$ maximum

$n z \quad$ nozzle

O oxidizer

$p \quad$ overall propellant (oxidizer + fuel)

ref reference

sl sliver

$t \quad$ oxidizer propellant tank

th throat

tot total 


\section{References}

1. Casalino, L.; Pastrone, D. Optimal design of hybrid rocket motors for microgravity platform. J. Propuls. Power 2008, 24, 491-498. [CrossRef]

2. Jens, E.; Karp, A.C.; Nakazono, B.; Eldred, D.B.; DeVost, M.E.; Vaughan, D. Design of a Hybrid CubeSat Orbit Insertion Motor. In Proceedings of the 52nd AIAA/SAE/ASEE Joint Propulsion Conference, AIAA Propulsion and Energy Forum, Salt Lake City, UT, USA, 25-27 July 2016.

3. Dornheim, M.A. Reaching $100 \mathrm{~km}$. AW \& ST 2004, 161, 45-46.

4. Casalino, L.; Pastrone, D. Optimization of Hybrid Sounding Rockets for Hypersonic Testing. J. Propuls. Power 2012, 28, 405-411. [CrossRef]

5. Karp, A.C.; Nakazono, B.; Manrique, J.B.; Shotwell, R.; Vaughan, D.; Story, G.T. A Hybrid Mars Ascent Vehicle Concept for Low Temperature Storage and Operation. In Proceedings of the 52nd AIAA/SAE/ASEE Joint Propulsion Conference, AIAA Propulsion and Energy Forum, Salt Lake City, UT, USA, 25-27 July 2016.

6. Pastrone, D. Approaches to Low Fuel Regression Rate in Hybrid Rocket Engines. IJAE 2012, 2012. [CrossRef]

7. Casalino, L.; Pastrone, D. A Straightforward Approach for Robust Design of Hybrid Rocket Engine Upper Stage. In Proceedings of the 51st AIAA/SAE/ASEE Joint Propulsion Conference, AIAA Propulsion and Energy Forum, Orlando, FL, USA, 27-29 July 2015. [CrossRef]

8. Casalino, L.; Masseni, F.; Pastrone, D. Comparison of Robust Design Approaches for Hybrid Rocket Engines. In Proceedings of the 53rd AIAA/SAE/ASEE Joint Propulsion Conference, AIAA Propulsion and Energy Forum, Atlanta, GA, USA, 10-12 July 2017. [CrossRef]

9. Taguchi, G.; Chowdhury, S.; Taguchi, S. Robust Engineering; McGraw-Hill: New York, NY, USA, 2000; ISBN 978-0071347822.

10. Park, G.J.; Lee, T.H.; Lee, K.H.; Hwang, K.H. Robust Design: An Overview. AIAA J. 2006, 44, 181-191. [CrossRef]

11. Johnsson, G.; Bigert, M. Development of Small Centrifugal Pumps for an Electric Propellant Pump System. Acta Astronaut. 1990, 21, 429-438. [CrossRef]

12. Schneider, S.J. Low thrust chemical rocket technology. In Proceedings of the 43rd Congress of the International Astronautical Federation, Washington, DC, USA, 28 August-5 September 1992.

13. Abel, T.M.; Velez, T.A. Electrical Drive System for Rocket Engine Propellant Pumps. U.S. Patent 6457306B1, 1 October 2002.

14. Soldà, N.; Lentini, D. Opportunities for a Liquid Rocket Feed System Based on Electric Pumps. J. Propuls. Power 2008, 24, 1340-1346. [CrossRef]

15. Pavlov Rachov, P.A.; Tacca, H.; Lentini, D. Electric Feed Systems for Liquid-Propellant Rockets. J. Propuls. Power 2013, 29, 1171-1180. [CrossRef]

16. Bahn, P.R.E. Rocket Engine Systems. U.S. Patent 9677503B2, 13 June 2017.

17. Vaughan, D.; Nakazono, B.; London, A.; Mehra, A. Technology Development of an Electrically Driven Pump Fed Storable Liquid Bi-Propellant for a Mars Ascent Vehicle. In Proceedings of the 68th International Astronautical Congress, Adelaide, Australia, 25-29 September 2017.

18. Waxenegger-Wilfing, G.; Hahn, R.H.S.; Deeken, J. Studies on Electric Pump-Fed Liquid Rocket Engines for Micro-Launchers. In Proceedings of the Space Propulsion Conference, Seville, Spain, 14-18 May 2018.

19. Kwak, H.D.; Kwon, S.; Choi, C.H. Performance Assessment of Electrically Driven Pump-Fed LOX/Kerosene Cycle Rocket Engine: Comparison with Gas Generator Cycle. Aerosp. Sci. Technol. 2018, 77, 67-82. [CrossRef]

20. Casalino, L.; Pastrone, D. Optimization of a Hybrid Rocket Upper Stage with Electric Pump Feed System. In Proceedings of the 46th AIAA/ASME/SAE/ASEE Joint Propulsion Conference and Exhibit, Nashville, TN, USA, 25-28 July 2010.

21. Casalino, L.; Colasurdo, G.; Pastrone, D. Optimal Low-Thrust Escape Trajectories Using Gravity Assist. J. Guid. Control Dyn. 1999, 22, 637-642. [CrossRef]

22. Casalino, L.; Pastrone, D. Oxidizer Control and Optimal Design of Hybrid Rockets for Small Satellites. J. Propuls. Power 2005, 21, 230-238. [CrossRef]

23. Sentinella, M.R.; Casalino, L. Hybrid Evolutionary Algorithm for the Optimization of Interplanetary Trajectories. J. Spacecr Rocket. 2009, 46, 365-372. [CrossRef]

24. Karabeyoglu, M.A.; Altman, D.; Cantwell, B.J. Combustion of Liquefying Hybrid Propellants: Part 1, General Theory. J. Propuls. Power 2002, 18, 610-620. [CrossRef] 
25. Casalino, L.; Pastrone, D. Optimal Robust Design of Hybrid Rocket Engines. In Springer Optimization and Its Applications; Springer: New York, NY, USA, 2016; Volume 114, pp. 269-285, ISBN 978-3-319-41506-2.

26. Barrere, M.; Jaumotte, A.; De Veubeke, B.F.; Vandenkerckhove, J. Rocket Propulsion; Elsevier Publishing Company: Amsterdam, The Netherlands, 1960; pp. 251-256, ISBN B0000CKFWU.

27. Sutton, G.P.; Biblarz, O. Rocket Propulsion Elements, 7th ed.; Wiley: New York, NY, USA, 2001; ISBN 978-0471326427.

28. Mc Bride, B.J.; Reno, M.A.; Gordon, S. CET93 and CETPC: An Interim Updated Version of the NASA Lewis Computer Program for Calculating Complex Chemical Equilibria with Applications; TM-4557; NASA: Cleveland, OH, USA, 1994.

29. Casalino, L.; Letizia, F.; Pastrone, D. Optimization of Hybrid Upper-Stage Motor with Coupled Evolutionary/Indirect Procedure. J. Propuls. Power 2014, 30, 1390-1398. [CrossRef]

30. Ellis, R.A. Solid Rocket Motor Nozzles—NASA Space Vehicle Design Criteria (Chemical Propulsion); SP-8115; NASA: Cleveland, OH, USA, 1975.

31. Bianchi, D.; Nasuti, F. Numerical Analysis of Nozzle Material Thermochemical Erosion in Hybrid Rocket Engines. J. Propuls. Power 2013, 29, 547-558. [CrossRef]

32. Brown, C.D. Spacecraft Propulsion; AIAA Education Series: Washington, DC, USA, 1992; p. 82, doi:10.2514/4.862441.

33. NASA Lewis Research Center. Turbopump Systems for Liquid Rocket Engines; SP-8107; NASA: Cleveland, OH, USA, 1974.

34. Battery and Energy Technologies. Available online: http://www.mpoweruk.com/performance.htm (accessed on 1 June 2010).

35. Casalino, L.; Pastrone, D. Optimal Design and Control of Hybrid Rockets for Access to Space. In Proceedings of the 41st AIAA/ASME/SAE/ASEE Joint Propulsion Conference \& Exhibit, Joint Propulsion Conferences, Tucson, AZ, USA, 10-13 July 2005. [CrossRef]

36. Colasurdo, G.; Pastrone, D. Indirect Optimization Method for Impulsive Transfer. In Proceedings of the Astrodynamics Conference, Guidance, Navigation, and Control and Co-located Conferences, Scottsdale, AZ, USA, 1-3 August 1994. [CrossRef]

37. Isakowitz, S.J.; Hopkins, J.A.; Hopkins, J.A., Jr. International Reference Guide to Space Launch Systems, 4th ed.; AIAA: Portland, OR, USA, 2004; pp. 517-524. [CrossRef]

38. Haimes, Y.; Lasdon, L.; Wismer, D. On a Bicriterion Formulation of the Problems of Integrated System Identification and System Optimization. IEEE Trans. Syst. Man Cybern. 1971, SMC-1, 296-297. [CrossRef]

39. Eberhart, R.; Kennedy, J. Particle Swarm Optimization. In Proceedings of the IEEE International Conference on Neural Networks, Perth, Australia, 27 November-1 December 1995; pp. 1942-1948. [CrossRef]

40. Eberhart, R.; Kennedy, J. A New Optimizer Using Particle Swarm Theory. In Proceedings of the Sixth International Symposium on Micro Machine and Human Science, Nagoya, Japan, 6 August 2002; pp. $39-43$. [CrossRef]

41. Sentinella, M.R. Development of New Procedures and Hybrid Algorithms for Space Trajectories Optimisation. Ph.D. Thesis, Politecnico di Torino, Turin, Italy, March 2008

(C) 2019 by the authors. Licensee MDPI, Basel, Switzerland. This article is an open access article distributed under the terms and conditions of the Creative Commons Attribution (CC BY) license (http:/ / creativecommons.org/licenses/by/4.0/). 


\title{
Comprehensive Data Reduction for $\mathrm{N}_{2} \mathrm{O} / \mathrm{HDPE}$ Hybrid Rocket Motor Performance Evaluation
}

\author{
Landon Kamps ${ }^{1, *,+}{ }^{\dagger}$, Kazuhito Sakurai ${ }^{1,+}$, Yuji Saito ${ }^{2}$ and Harunori Nagata ${ }^{3}$ \\ 1 Department of Mechanical and Space Engineering, Hokkaido University, Sapporo 060-8628, Japan; \\ sakurai371@gmail.com \\ 2 Department of Aerospace Engineering, Tohoku University, Sendai 980-8579, Japan; \\ saito@aero.mech.tohoku.ac.jp \\ 3 Faculty of Engineering, Hokkaido University, Sapporo 060-8628, Japan; nagata@eng.hokudai.ac.jp \\ * Correspondence: landon_kamps@eis.hokudai.ac.jp; Tel.: +81-11-706-7226 \\ + These authors contributed equally to this work.
}

Received: 31 January 2019; Accepted: 15 April 2019; Published: 17 April 2019

\begin{abstract}
Static firing tests of a hybrid rocket motor using liquid nitrous oxide $\left(\mathrm{N}_{2} \mathrm{O}\right)$ as the oxidizer and high-density polyethylene (HPDE) as the fuel are analyzed using a novel approach to data reduction that allows histories for fuel mass consumption, nozzle throat erosion, characteristic exhaust velocity $\left(c^{*}\right)$ efficiency, and nozzle throat wall temperature to be determined experimentally. This is done by firing a motor under the same conditions six times, varying only the burn time. Results show that fuel mass consumption was nearly perfectly repeatable, whereas the magnitude and timing of nozzle throat erosion was not. Correlations of the fuel regression rate result in oxidizer port mass flux exponents of 0.62 and 0.76 . There is a transient time in the $c^{*}$ efficiency histories of around $2.5 \mathrm{~s}$, after which $c^{*}$ efficiency remains relatively constant, even in the case of excessive nozzle throat erosion. Although nozzle erosion was not repeatable, the erosion onset factors were similar between tests, and greater than values in previous research in which oxygen was used as the oxidizer. Lastly, nozzle erosion rates exceed $0.15 \mathrm{~mm} / \mathrm{s}$ for chamber pressures of 4 to $5 \mathrm{MPa}$.
\end{abstract}

Keywords: ballistic reconstruction technique; fuel regression; nozzle erosion; $c^{*}$ efficiency

\section{Introduction}

Hybrid rockets are currently the focus of countless aerospace propulsion projects worldwide. Possibly the most prominent example is Virgin Galactic Ltd.'s hybrid rocket-powered spaceplane, "SpaceShipTwo", which is projected to begin commercial operations this year (FY2019) [1]. The attention on hybrid rocket development is justified by the logistical cost savings and risk reduction during operations of hybrid rockets compared to their liquid bi-propellant and solid rocket counterparts [2,3]. One potential use for the current state-of-the-art hybrid rockets is as apogee kick motors, which will alleviate satellite operators from relying solely on piggy-backing on larger satellite buses to destinations beyond geostationary transfer orbit (GTO), thus reducing launch wait times, and increasing freedom of movement to desired orbital placements.

Kuo and Chiaverini summarize the advantages of hybrid rockets for upper-stage use as having high specific impulse, throttling capability, safe manufacturing, and low cost [4] (p. 632). These attributes are especially attractive when considering the application of hybrid rockets as apogee kick motors. Jens et al. reported extensively on the concept of a hybrid rocket-powered apogee kick motor for placing CubeSats into deep space [5-7]. A key aspect of their proposed design is the storage of gaseous oxygen at very high pressures-roughly $50 \mathrm{MPa}$ - to keep the storage volume at a minimum. Thus, one major technical limitation to such a vehicle is the maximum size and cost of the pressure vessels necessary to store the oxidizer. In the case where payloads larger than CubeSats are desired, it 
may be advantageous to have a vehicle that uses an oxidizer that can be stored as a liquid to avoid the requirement for large and expensive pressure vessels.

Heister and Wernimont elaborate on the practicality of hybrid rockets for space applications which demand storable oxidizers, highlighting nitrous oxide $\left(\mathrm{N}_{2} \mathrm{O}\right)$ for its low toxicity and self-pressurization ability [8]. To clarify the self-pressurizing ability of $\mathrm{N}_{2} \mathrm{O}$, values for density, $\rho$, in $\mathrm{kg} / \mathrm{m}^{3}$ and vapor pressure, $P_{v}$, in $\mathrm{Pa}$ are plotted as functions of temperature in Figure 1 based on data from the National Institute of Standards and Technology (NIST) online database [9]. The main conclusion that can be drawn from this figure is that $\mathrm{N}_{2} \mathrm{O}$ can be self-pressurized as a liquid with densities ranging from $700 \mathrm{~kg} / \mathrm{m}^{3}$ to $900 \mathrm{~kg} / \mathrm{m}^{3}$ at pressures ranging from $4 \mathrm{MPa}$ to $6 \mathrm{MPa}$ for a range of temperatures typically permissible to satellite operators-roughly $280 \mathrm{~K}$ to $300 \mathrm{~K}$.

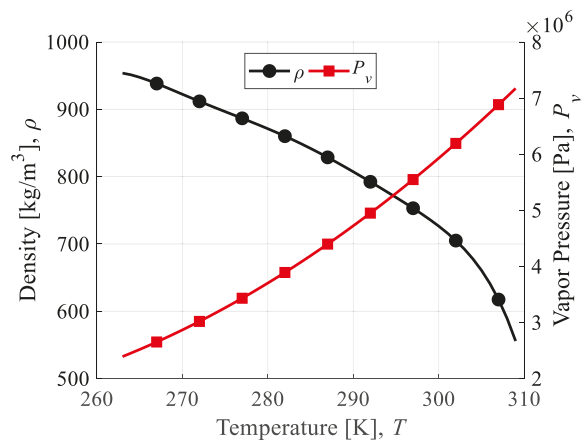

Figure 1. Thermodynamic properties of liquid nitrous oxide $\left(\mathrm{N}_{2} \mathrm{O}\right)$.

Many noteworthy hybrid rocket demonstrations in recent years have been achieved in part by taking advantage of the self-pressurizing ability of $\mathrm{N}_{2} \mathrm{O}$. These include the aforementioned SpaceShipTwo, as well as its predecessor vehicle, SpaceShipOne [10], the highly successful Stuttgart University student-based hybrid sounding rocket, HEROS 3, reported by Kobald et al. [11], the rocket test sled trials of Muroran Institute of Technology reported by Nakata et al. [12], the small launch vehicles of TiSPACE Inc. reported by Chen and Wu [13], as well as the sounding rockets of Space Forest Ltd. reported by Gamal et al. [14]. However, it is also important to point out that $\mathrm{N}_{2} \mathrm{O}$ can be pressurized above its vapor pressure to increase the flow rate capability of, and prevent two-phase flow within, the feed system. For example, Whitmore et al. used helium to pressurize a liquid $\mathrm{N}_{2} \mathrm{O}$ feed system for closed-loop throttling and thrust control [15]. Similarly, the Peregrine sounding rocket project of NASA, Stanford University, and SPG Inc. reported by Zilliac et al. used helium to pressurize an $\mathrm{N}_{2} \mathrm{O}$ reservoir to slightly above the vapor pressure [16]. The current research uses a similar feed system, which will be described in detail in the Materials and Methods section.

There are two distinct fuel design strategies used in the aforementioned $\mathrm{N}_{2} \mathrm{O}$ hybrid rocket projects to achieve high thrust-to-weight ratios. One strategy is to use non-liquifying fuels with multiple ports and/or chambers to increase the burning surface area and/or create a vortex-like flow field. This is true for SpaceShipOne/Two, and the hybrid rockets reported by Chen and $\mathrm{Wu}$, and Whitmore et al. in $[1,13,15]$. The other strategy is to use liquefying fuels with a single port, in which case the entrainment of liquid droplets from the surface of the melting fuel leads to high burning rates. This is true for the hybrid rockets reported by Kobald et al., Gamal et al., and Zilliac et al. in [11,14,16]. In a 5 kN thrust-class sounding rocket launch reported by Nagata et al., the former strategy was employed with great success using a high-density polyethylene (HDPE) fuel of the Cascaded Multistage Impinging-jet (CAMUI) design and liquid oxygen as the oxidizer [17]. With a characteristic exhaust velocity, $c^{*}$, efficiency, $\eta^{*}$, of $99 \%$ and a sustained acceleration during launch of $4 \mathrm{G}$ to $5 \mathrm{G}$, the major concern of this development project shifted to nozzle thermochemical erosion. The tests in follow-on research to investigate nozzle erosion consistently showed values of $\eta^{*}$ greater than $95 \%$. Furthermore, the 
mass "equivalent" regression rates, based on the fuel grain outer dimensions and mass consumption rates, reached values upwards of $3 \mathrm{~mm} / \mathrm{s}$, which is comparable to or exceeding that of liquefying propellants [18]. The main drawback of using a CAMUI-type fuel, or something similar in nature, is the rigor involved in the fuel design itself. It can be said that the main benefit of using liquefying fuels in place of geometrically complex fuels, like CAMUI, is the simplicity of the single port fuel design that can be used. Mazzetti et al. make a strong argument on behalf of hybrid rockets in general, but specifically for liquefying fuel-based hybrid rockets because of the combination of the high fuel regression rate and fuel design simplicity [19].

In the context of designing an apogee kick motor, achieving a high thrust-to-weight ratio becomes less critical than in the context of a planetary launch vehicle. For example, to achieve a transfer from GTO to Mars orbit, a change in velocity of roughly $1200 \mathrm{~m} / \mathrm{s}$ is required. Even at a constant acceleration of only $1 \mathrm{G}$, this apogee kick would require only a two-minute burn time. Thus, it can be said that achieving a high specific impulse, low manufacturing costs, and minimal combustion oscillations is more important in the development of an apogee kick motor than improving the fuel regression rate. In fact, Jens et al. selected the non-liquefying fuel, polymethyl methacrylate (PMMA), over a liquefying wax fuel for their hybrid rocket apogee kick motor development specifically to prevent acceleration from exceeding $3 \mathrm{G}[5]$.

In the fiscal year of 2018, the authors of this paper began the development of a hybrid rocket apogee kick motor in collaboration with the Japanese Institute of Space and Astronautical Science (JAXA/ISAS) that will be capable of transporting small satellites (less than $100 \mathrm{~kg}$ ) from GTO to lunar orbits and beyond. Nitrous oxide $\left(\mathrm{N}_{2} \mathrm{O}\right)$ was selected to be the oxidizer for its long-term storability, non-toxicity, non-corrosiveness, low cost, widespread commercial availability, self-pressurizing ability, and successful role in the numerous projects mentioned in the previous paragraphs. High-density polyethylene (HDPE) was also selected for its non-toxicity, low cost, and widespread commercial availability, as well as for its mechanical strength and heritage in the development of the CAMUI-type hybrid rocket by the authors and their predecessors.

The propellant combination, $\mathrm{N}_{2} \mathrm{O} / \mathrm{HDPE}$, has mostly gone overlooked in previous research due to the heavy focus on the development of launch vehicles with high thrust-to-weight ratios. The latest version of the SpaceShipTwo engine may be an exception to this claim; however, the details of the current propellant combination have not been made public yet. The two previous publications that were closely related to the proposed work were an experimental study conducted by Doran et al. on a laboratory-scale $\mathrm{N}_{2} \mathrm{O} / \mathrm{HDPE}$ hybrid rocket [20], and a numerical study of nozzle thermochemical erosion conducted by Bianchi and Nasuti [21]. Doran et al. reported $\eta^{*}$ values ranging from $90 \%$ to $95 \%$, and a weak correlation between fuel regression rates-ranging from $0.4 \mathrm{~mm} / \mathrm{s}$ to $0.6 \mathrm{~mm} / \mathrm{s}$-and oxidizer port mass flux. Bianchi and Nasuti predicted that the erosion rate of graphite is $0.06 \mathrm{~mm} / \mathrm{s}$ when the equivalence ratio is close to 1 and the pressure is $1 \mathrm{MPa}$. Moreover, they demonstrated that as a general trend among all propellant combinations, the erosion rate decreases with an increasing equivalence ratio and is linearly dependent on pressure.

The results of Doran et al. and Bianchi and Nasuti serve as a valuable starting point for this research, but there are some reasons why this information is inadequate for our purposes. Doran et al.'s experiments had combustion times in the range of $5 \mathrm{~s}$ to $7 \mathrm{~s}$, which is too short of a span to determine if the fuel regression correlations will remain true in time, yet long enough to introduce uncertainty due to the time-averaging of nonlinear terms needed for the correlations. For example, test 42 from Doran et al.'s study shows a duration of $5.9 \mathrm{~s}$ with an initial port diameter of $13.4 \mathrm{~mm}$ and an average regression rate of $0.76 \mathrm{~mm} / \mathrm{s}$. This means the oxidizer port mass flux decreased by roughly $40 \%$ during the test, which for this test would account for a span of almost $100 \mathrm{~kg} / \mathrm{m}^{2}$-s. Also, Doran et al. supplied $\mathrm{N}_{2} \mathrm{O}$ to the motor as a gas, whereas it is crucial for the purposes of this study to supply the oxidizer as a liquid. Bianchi and Nasuti's results will prove to be consistent with this study, but they are only valid when the nozzle throat temperature has reached a steady state. 
Due to the lack of previous work on the propellant combination of $\mathrm{N}_{2} \mathrm{O} / \mathrm{HDPE}$, the authors found it necessary to conduct basic research to form empirical correlations for the fuel regression rate, $c^{*}$, efficiency, $\eta^{*}$, and nozzle erosion for the first time. Of the multitude of measurement techniques introduced in previous research, the latest versions of the data reduction methods referred to as ballistic reconstruction techniques appeared to offer the most effective means of pursuing this research in a cost-effective and expedient way. In general, ballistic reconstruction techniques only require some combination of the following commonly measured experimental values: (1) Oxidizer mass flow rate, $\dot{m}_{o x}$; (2) chamber pressure, $P_{c}$; (3) thrust, $F$; (4) overall fuel mass consumed, $\Delta M_{f u}$; and (5) final nozzle throat diameter, $d_{t, f}$. This means that the same measurement equipment can be used regardless of fuel design, configuration, or scale. This is done by using either the $c^{*}$ equation, thrust equation, or both, in an iterative algorithm to determine instantaneous values of the oxidizer-to-fuel mass ratio, $\xi ; c^{*}$ efficiency, $\eta^{*}$; thrust correction factor, $\lambda$; and, recently, nozzle throat area, $A_{t}$. Wernimont and Heister, and Nagata et al. introduced ballistic reconstruction techniques, which used the $c^{*}$ equation to determine fuel mass consumption under the assumption that $\eta^{*}$ is constant and nozzle throat erosion is negligible [22,23]. Carmicino and Sorge, and Nagata et al. alleviated the need to treat $\eta^{*}$ as a constant by measuring thrust and incorporating the thrust equation [24,25]. Most recently, Kamps et al. combined these works, incorporating both the $c^{*}$ and thrust equations, and treating $\eta^{*}$ as a constant in order to determine the history of the nozzle throat area in an effort to investigate nozzle throat erosion [26]. This ballistic reconstruction technique was titled "Nozzle Throat Reconstruction Technique" or "NTRT," and was later used in a follow-on study by Kamps et al., which demonstrated how to use the results of the NTRT with thermocouple measurements from within the nozzle to determine the wall temperature history at the nozzle throat [27]. This follow-on technique was titled the "Throat Temperature Reconstruction Technique" or "TTRT."

Unfortunately, reconstruction techniques, such as the NTRT, have a region where multiple solutions exist for the results of $\xi$, typically when hybrid rocket tests are conducted in fuel-rich conditions. Nagata et al. [25] and Saito et al. [28] demonstrated that approximations for $c^{*}$ can be used to overcome this problem in the data reduction of hybrid rocket firing tests using oxygen. However, as will be evident in the following section, these approximations are not suitable when using $\mathrm{N}_{2} \mathrm{O}$ as the oxidizer because the region of multiple solutions that exists is too large. The objective of this research is to further develop the experimental methodology introduced by Kamps et al. so that the analysis of tests conducted under the propellant combination of $\mathrm{N}_{2} \mathrm{O} / \mathrm{HDPE}$ yields results for the histories of fuel consumption, nozzle erosion, and characteristic exhaust velocity efficiency regardless of the oxidizer-to-fuel-mass ratio. The purpose of this research is to lay the groundwork for effectively conducting basic combustion research in the development of a hybrid rocket apogee kick motor using $\mathrm{N}_{2} \mathrm{O}$ as the oxidizer and non-liquefying fuels, such as HDPE, as the fuel.

\section{Materials and Methods}

Kamps et al. recently introduced a method, titled the "Nozzle Throat Reconstruction Technique (NTRT)," for determining both $A_{t}$ and $\xi$ histories through the data reduction of commonly measured experimental values with the aim of enabling hybrid rocket researchers to accurately and cost-effectively investigate nozzle erosion experimentally [26]. The two governing equations of this method are the thrust equation shown by (1), and the characteristic exhaust velocity equation shown by (2):

$$
\begin{gathered}
F=\lambda \dot{m} u_{e}+\left(P_{e}-P_{a}\right) A_{e} \\
c_{t h}^{*}=P_{c} A_{t} / \eta^{*} \dot{m}
\end{gathered}
$$

Here, the thrust, $F$, is in $\mathrm{N}$ and the theoretical characteristic exhaust velocity, $c_{t h^{\prime}}^{*}$ is in $\mathrm{m} / \mathrm{s}$. The term, $\lambda$, is the dimensionless thrust correction factor, $\dot{m}$ is the propellant mass flow rate in $\mathrm{kg} / \mathrm{s}, u_{e}$ is the nozzle exit velocity in $\mathrm{m} / \mathrm{s}, P_{e}$ and $P_{a}$ are the nozzle exit pressure and atmospheric pressure in $\mathrm{Pa}$, and $A_{t}$ and $A_{e}$ are the nozzle throat area and exit area in $\mathrm{m}^{2}$. The thrust correction factor, $\lambda$, accounts for 
the momentum losses due to non-one-dimensionality and non-isentropicity of flow, as well as the momentum losses in the axial direction due to the divergence angle of the nozzle exit. The propellant mass flow rate can be shown in terms of the oxidizer-to-fuel-mass ratio according to Equation (3):

$$
\dot{m}=\dot{m}_{o x}(1+1 / \xi)
$$

where the subscript, " $o x$ ", indicates that the mass flow rate term is a property of the "oxidizer" only.

When the propellant combination of N2O/HDPE is used, there exists a region of multiple solutions of $\xi$ where maximum specific impulse can be achieved.

This is shown by breaking down the calculation of the thrust equation. The value of $P_{e}$ is calculated implicitly from Equation (4):

$$
\left(\frac{P_{e}}{P_{c}}\right)^{\frac{1}{\gamma}}\left(\frac{\gamma+1}{2}\right)^{\frac{1}{\gamma-1}} \sqrt{\frac{\gamma+1}{\gamma-1}\left(1-\left(\frac{P_{e}}{P_{c}}\right)^{\frac{\gamma-1}{\gamma}}\right.}=\frac{A_{t}}{A_{e}}
$$

and $u_{e}$ is calculated explicitly from Equation (5):

$$
u_{e}=\eta^{*} c_{t h}^{*} \sqrt{\frac{2 \gamma^{2}}{\gamma-1}\left(\frac{2}{\gamma+1}\right)^{\frac{(\gamma+1)}{(\gamma-1)}}\left[1-\left(\frac{P_{e}}{P_{c}}\right)^{\frac{(\gamma-1)}{\gamma}}\right]}
$$

where $\gamma$, the specific heat ratio of combustion gas, is calculated using the NASA Chemical Equilibrium with Applications (CEA) computer program as a function of $P_{c}$ and $\xi$ [29]. Although it is not a problem in the tests conducted in this study, it is worth noting that Equations (4) and (5) are not valid if shocks occur in the nozzle, which is possible in ground tests where the chamber pressure is relatively low. Figure 2 plots $F$ as a function of $\xi$ for the case where $d_{t}$ is $4 \mathrm{~mm}, P_{c}$ is $4.7 \mathrm{MPa}$, and $\dot{m}_{0 x}$ is $36 \mathrm{~g} / \mathrm{s}$ - which is representative of the tests conducted in this study. Here, the efficiency terms, $\eta^{*}$ and $\lambda$, were assumed to be 1 (i.e., $100 \%$ ). The range of $\xi$ from 4.0 to 7.1 results in roughly the same value for thrust. Specific impulse, $I_{s p}$, has also been plotted in Figure 2 to show that optimal performance is achieved when $\xi$ is roughly equal to 7 . This is important because in a typical hybrid rocket, the value of $\xi$ increases in time. Thus, for the goal of maximizing $I_{s p}$ in long duration firing tests, it may be advantageous to begin operations in the multiple solutions region of $\xi$. Furthermore, from the vantage point of nozzle erosion prevention, it is advantageous to operate with the smallest value of $\xi$ that is possible [21].

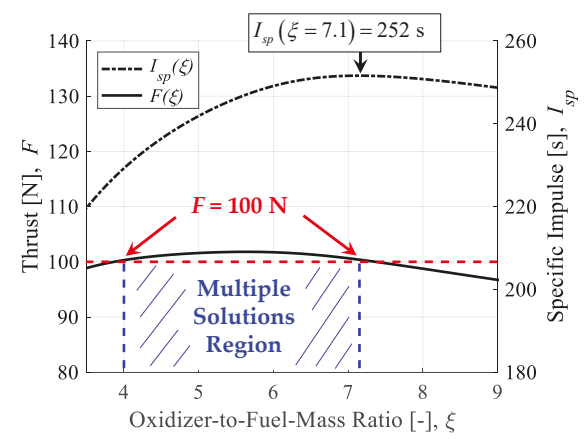

Figure 2. Example of multiple $\xi$ solutions in the Nozzle Throat Reconstruction Technique (NTRT).

In previous data reduction methods, such as the NTRT, data are analyzed separately for each firing test conducted, with no cross-over of data between tests. However, in this research, a different approach is taken. The concept of the method employed in this paper is to include an additional experimental input in the governing set of equations of the NTRT, allowing for an additional output, 
and avoiding the issue of multiple solutions arising from the thrust equation's dependency on $\xi$. More precisely, the value for overall fuel mass consumption, $\Delta M_{f u}$, which is a constant value input to the original NTRT, will be replaced by a fuel mass consumption history, $m_{f u}$. Thus, $\eta^{*}$ can be solved at every time, rather than be treated as a constant. Since the outputs of the data reduction method in this research are in effect the same as the NTRT, the method in this paper will be referred to as "NTRT plus" or "NTRT+."

The operational flow of the comprehensive data reduction procedure used in this research is shown in Figure 3. Data reduction steps are separated into "tiers" based on the information flow between operations. First, a firing test is repeated multiple times, varying only the combustion time between tests. In this way, a trendline can be determined that describes the overall fuel mass consumption progression between tests. This trendline is the only "tier I" operation. It is crucial that there is a clear agreement with the fuel mass consumption history trendline and the overall fuel mass consumption values determined through direct measurement in each test separately. With the fuel mass consumption history determined, it is possible to run all "tier II" operations: The NTRT+, NASA CEA, and fuel regression rate correlation operations. Finally, in this paper, the only "tier III" operation carried out is a simplification of the TTRT, which is used to determine the nozzle throat wall temperature histories.

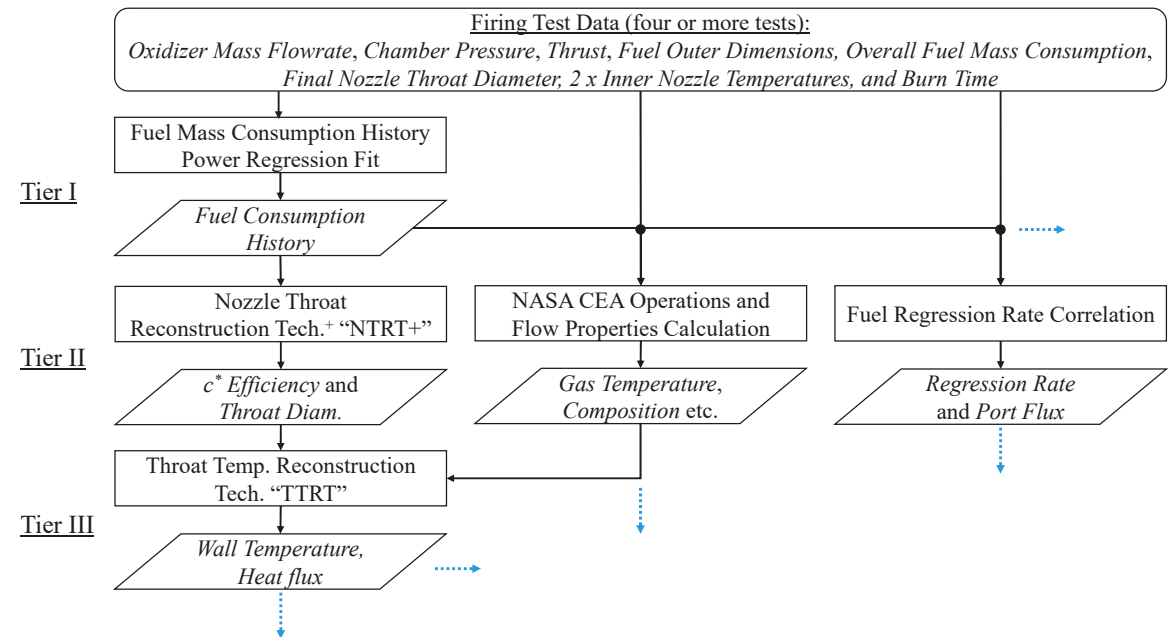

Figure 3. Flowchart of comprehensive data reduction operations in this research.

Although the set of governing equations for the NTRT + is essentially the same as the original NTRT, the algorithm is significantly different. The computational flow of the NTRT+ is shown in Figure 4. There are two iterative loops: Loop A and loop B. Loop A iterations are conducted only once to solve for $\lambda$. Loop B iterations are conducted at every time step to solve for the $\eta^{*}$ history. Convergence of loop A depends on reducing the final throat diameter residual, $\psi_{d}$, to zero, according to Equation (6):

$$
\psi_{d}=\left|1-\frac{d_{t}\left(t_{b}\right)}{d_{t, f}}\right|=f(\lambda)
$$

were $d_{t}\left(t_{b}\right)$ is the calculated value for the nozzle throat diameter at the end of the firing duration. The calculated nozzle throat diameter history is backed out of the value for the nozzle throat area, $A_{t}$, which is determined by rearranging the terms in Equation (2), as shown by Equation (7):

$$
d_{t}=\sqrt{\frac{4}{\pi} A_{t}}=\sqrt{\frac{4}{\pi} \frac{\eta^{*} c_{t h}^{*} \dot{m}}{P_{c}}}
$$


The value of $\lambda$ is iterated until the nozzle throat diameter calculation matches the known value at the end of the firing test. Convergence of loop B depends on reducing the thrust residual, $\psi_{F}$, to zero, according to Equation (8):

$$
\psi_{F}(t)=\left|1-\frac{\lambda \dot{m}(t) u_{e}(t)+\left(P_{e}(t)-P_{a}\right) A_{e}}{F(t)}\right|=f\left(\eta^{*}(t)\right)
$$

The value of $\eta^{*}$ is iterated until the thrust calculation matches the measured value at that time.

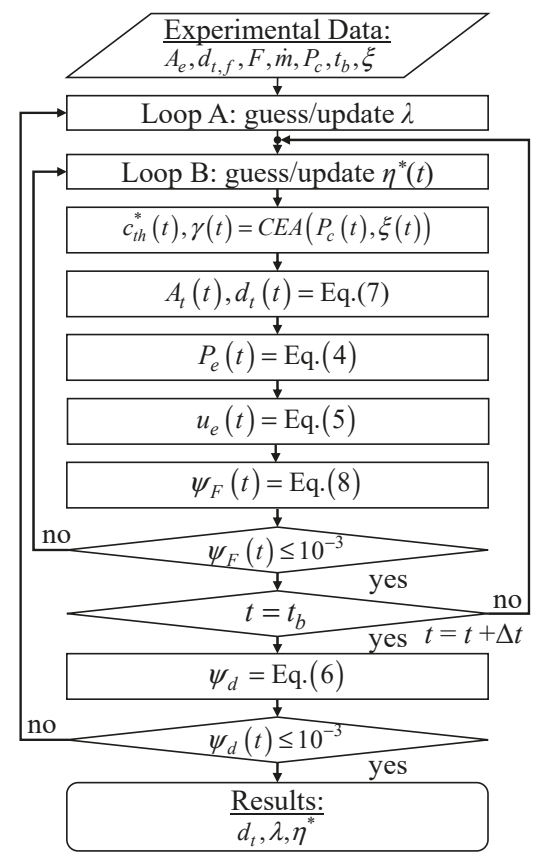

Figure 4. The NTRT+ algorithm computational flowchart.

The most common adaptation of Marxman et al.'s diffusion-limited regression rate model, first introduced in [30], will be used for the analysis in this study. This model is shown by Equation (9):

$$
\dot{r}_{f u}=a G_{o x}^{n}=a\left(\frac{\dot{m}_{o x}}{\pi r_{f u}^{2}}\right)^{n}
$$

where $\dot{r}_{f u}$ is in $\mathrm{m} / \mathrm{s}, a$ and $n$ are empirical constants, $G_{o x}$ is in $\mathrm{kg} / \mathrm{m}-\mathrm{s}^{2}$, and $r_{f u}$ is the fuel port radius in $\mathrm{m}$. Two methods of fuel regression rate correlations are compared in this study. In both cases, the concept behind the correlations is to run tests under the same oxidizer mass flow rate, varying only the combustion time. As the fuel burns, the port diameter will increase and the oxidizer port mass flux will decrease accordingly. This is different than the traditional approach of limiting the combustion time and varying the oxidizer mass flow rate to vary the oxidizer port mass flux. With that in mind, the correlation procedure remains essentially the same. The more conventional correlation procedure is to use only the endpoint data (i.e., changes in value before and after firing) for fuel mass consumption and the measurement of the oxidizer mass flow rate. This method is referred to as the "endpoint" 
method. By separating the variables of the port radius, $r_{f u}$ and time, $t$, Equation (9) can be integrated as follows in Equation (10):

$$
\frac{\pi^{n}\left(r_{f u, f}^{2 n+1}-r_{f u, o}^{2 n+1}\right)}{(2 n+1)}=a \int_{0}^{t_{b}} m_{o x}^{n} d t \cong a \sum m_{o x}^{n} \Delta t
$$

where $\Delta t$ represents the sampling rate of the experimental measurement history in $\mathrm{s}$, and $t_{b}$ is the burn time in s. Burn time is defined as the duration of time from the moment the (gauge) chamber pressure reaches $10 \%$ of the maximum value to the time at the aft-bisector of the pressure drop at the end of burning. This designation is outlined in [31] (p. 459), and was used in [26,27]. The fuel port radius in Equation (9) is calculated based on the fuel mass, $M_{f u}$, fuel density, $\rho_{f u}$, and the outer diameter and length of the fuel, $D_{f u}$ and $L_{f u}$, according to Equation (11):

$$
r_{f u}=\sqrt{\frac{D_{f u}^{2}}{4}-\frac{M_{f u}}{\pi L_{f u} \rho_{f u}}}
$$

where $r_{f u}, D_{f u}$, and $L_{f u}$ are in $\mathrm{m}, M_{f u}$ is in $\mathrm{kg}$, and $\rho_{f u}$ is in $\mathrm{kg} / \mathrm{m}^{3}$. The constant, $a$, is determined by using the least-squares method (for a given value of $n$ ) on Equation (10), and the exponent, $n$, is determined by finding the value that maximizes the correlation of determination (i.e., $R^{2}$ ) of the results of $a$. The second fuel regression correlation method attempted in this paper simply considers the solution to Equation (11) for all times, and performs the least-squares method directly on Equation (9). This is possible because we can integrate the fuel mass consumption history trendline from the tier I operations to determine the history of $M_{f u}$.

The Throat Temperature Reconstruction Technique, or TTRT, employed in this study is a simplification of the method introduced in [27]. A single iterative loop solves for the nozzle throat wall temperature history, $T_{w}$. Convergence of the TTRT depends on reducing the thermocouple temperature residual, $\psi_{T}$, to zero:

$$
\psi_{T}(t)=\left|1-\frac{T_{n 1, \text { calculation }}(t)}{T_{n 1, \text { measured }}(t)}\right|=f\left(T_{w}(t)\right)
$$

Here, $T_{n 1}$ is the temperature at the position of the thermocouple placed closest to the nozzle throat in $\mathrm{K}$. In general, the governing differential equation for 1D conductive heat flux in cylindrical coordinates reduces to Equation (13) by assuming a negligibly small axial temperature gradient and no internal heat generation:

$$
\frac{\partial T}{\partial t}=\frac{\alpha}{r} \frac{\partial}{\partial r}\left(r \frac{\partial T}{\partial r}\right)
$$

Here, $\alpha$ is thermal diffusivity of the nozzle in $\mathrm{m}^{2} / \mathrm{s}, \mathrm{T}$ is the local instantaneous temperature in $\mathrm{K}$, and $r$ is the radial position from the centerline of the nozzle in $\mathrm{m}$. Approximating the partial derivatives in Equation (13) by the first term of the Taylor series expansions near the points of interest, consolidating terms, and rearranging yields the following finite difference equation:

$$
\begin{gathered}
a_{i}^{j} T_{i-1}^{j+1}+b_{i}^{j} T_{i}^{j+1}+c_{i}^{j} T_{i+1}^{j+1}+d_{i}^{j} T_{i}^{j}=0 \\
a_{i}^{j}=2 \alpha^{j} r_{i} \Delta t-\alpha \Delta r \Delta t \\
b_{i}^{i}=-\left(4 \alpha^{j} r_{i} \Delta t+2 r_{i} \Delta r^{2}\right) \\
c_{i}^{j}=\alpha^{j} \Delta r \Delta t+2 \alpha^{j} r_{i} \Delta t \\
d_{i}=2 r_{i} \Delta r^{2}
\end{gathered}
$$

where $\Delta t$ is the time step, subscript $i$ specifies the radial node index, and superscript $j$ represents the time index. Note that the thermal properties of graphite, $k$ and $\alpha$, are treated as functions of temperature, as described in the following paragraph, but this behavior is approximated by using the 
solution of the temperature profile from the previous time step. With one temperature boundary set as the nozzle throat wall temperature, $T_{w}$, and the other set as the nozzle thermocouple measurement temperature, $T_{n 2}$, we can solve for the temperature distribution history within the nozzle according to Equation (15):

$$
\left[\begin{array}{c}
T_{w+\Delta r}^{j+1} \\
T_{w+2 \Delta r}^{j+1} \\
\vdots \\
T_{r_{n 2}-2 \Delta r}^{j+1} \\
T_{r_{n 2}-\Delta r}^{j+1} \\
T_{w} \text { at } j+1
\end{array}\right]=\left[\begin{array}{ccccc}
b_{w+\Delta r}^{j} & c_{w+\Delta r}^{j} & 0 & 0 & 0 \\
a_{w+2 \Delta r}^{j} & b_{w+2 \Delta r}^{j} & c_{w+2 \Delta r}^{j} & 0 & 0 \\
0 & \ddots & \ddots & \ddots & 0 \\
0 & 0 & a_{r_{n 2}-2 \Delta r}^{j} & b_{r_{n 2}-2 \Delta r}^{j} & c_{r_{n 2}-2 \Delta r}^{j} \\
0 & 0 & 0 & a_{r_{n 2}-\Delta r}^{j} & b_{r_{n 2}-\Delta r}^{j}
\end{array}\right]^{-1}\left[\begin{array}{c}
a_{w+\Delta r}^{j} T_{w+\Delta r}^{j} \\
a_{w+2 \Delta r}^{j} T_{w+2 \Delta r}^{j} \\
\vdots \\
a_{r_{n 2}-2 \Delta r}^{j} T_{r_{n 2}-2 \Delta r}^{j} \\
a_{r_{n 2}-\Delta r}^{j} T_{r_{n 2}-\Delta r}^{j} \\
T_{w} \text { at } j
\end{array}\right]-\left[\begin{array}{c}
a_{w+\Delta r}^{j} T_{w w}^{j} \\
0 \\
\vdots \\
0 \\
c_{r_{n 2}-\Delta r}^{j} T_{n 2}^{j} \\
\text { Boundary }
\end{array}\right]
$$

A mesh size of $\Delta r=10^{-5} \mathrm{~m}$ and a time step of $\Delta t=0.5 \mathrm{~s}$ was used for the calculations in this study.

Static firing tests were carried out on a $100 \mathrm{~N}$-thrust class motor. Depictions of the test setup, motor assembly, and fuel and nozzle schematics are shown in Figure 5a-c, respectively. The test setup consisted of three fluid supply lines in parallel: A main oxidizer line with an Ar gas tank and an $\mathrm{N}_{2} \mathrm{O}$ reservoir; a purge gas line with an $\mathrm{N}_{2}$ gas tank; and an ignition assist oxidizer line with an $\mathrm{O}_{2}$ gas tank. The flow in each line was controlled using a Swagelok pneumatic ball valve operated by an SMC solenoid valve. Solenoid valve operations were controlled in LABVIEW7. The $\mathrm{N}_{2} \mathrm{O}$ in the reservoir, which was stored at outdoor atmospheric temperature $(\approx 290 \mathrm{~K})$, was pressurized to $7.6 \mathrm{MPa}$ using Ar gas to ensure that it remained in a liquid phase until injection into the combustion chamber. The oxidizer mass flow rate was determined by measuring the pressure drop across an orifice plate with an orifice diameter of $1 \mathrm{~mm}$ located between the $\mathrm{N}_{2} \mathrm{O}$ reservoir and the motor. The equation used to calculate oxidizer mass flow rate is (16):

$$
\dot{m}_{o x}=C_{o r}\left(\frac{\pi}{4} d_{o r}^{2}\right) \sqrt{\rho_{o x}\left(P_{u p}-P_{d w}\right)}
$$

where the oxidizer mass flow rate is in $\mathrm{kg} / \mathrm{s}, \mathrm{C}_{\text {or }}$ is a dimensionless orifice flow coefficient determined experimentally to be $0.92, d_{\text {or }}$ is the orifice hole diameter in $\mathrm{m}$ (i.e., $0.001 \mathrm{~m}$ ), $\rho_{\mathrm{ox}}$ is the oxidizer density in $\mathrm{kg} / \mathrm{m}^{3}$, and $P_{u p}$ and $P_{d w}$ are the pressure upstream and downstream of the orifice in Pa, respectively. Note that the density of $\mathrm{N}_{2} \mathrm{O}$ is a function of temperature, and was calculated based on the following equation determined from the NIST database [9]:

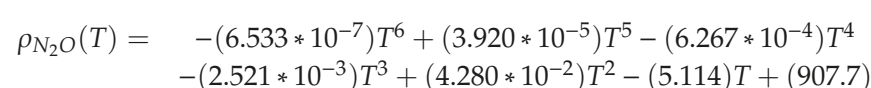

where, in this case, $\mathrm{T}$ is the temperature of $\mathrm{N}_{2} \mathrm{O}$ in ${ }^{\circ} \mathrm{C}$.

All tests were conducted using polyethylene (formula: $\mathrm{C}_{2} \mathrm{H}_{4}$ ) with a density of $955 \mathrm{~kg} / \mathrm{m}^{3}$ as the fuel. Fuel grains were assembled from multiple short cylindrical fuel blocks, which allowed for a pseudo-one-dimensional evaluation of the fuel mass consumption. As is shown in Figure $5 \mathrm{~b}, \mathrm{c}$, the initial fuel port diameter of the first four blocks (blocks (1)-(4)) was $20 \mathrm{~mm}$ and that of the last four blocks (blocks (5)-(8) was $30 \mathrm{~mm}$. The step-increase in port diameter between blocks (4) and (5) was used to force a disturbance in the boundary layer and improve mixing. After assembly, fuel grains were loaded into glass fiber-reinforced plastic (GFRP) insulating tubes and sealed in a steel motor case. An impinging-type injector with $4 \times 0.8 \mathrm{~mm}$ holes at a convergence angle of $45^{\circ}$ was used to promote atomization. The nozzles used in all tests were manufactured using the same grade of isotropic graphite, Tokyo Tokai Carbon Ltd. G347. The density and thermal conductivity at atmospheric conditions are listed by the manufacturer to be $\rho_{n}=1850 \mathrm{~kg} / \mathrm{m}^{3}$ and $k=116 \mathrm{~W} / \mathrm{m}-\mathrm{K}$, respectively [32]. The temperature dependency of these and other properties of G347 graphite is not specified by the 
manufacturer, and so values were referenced from previous research on similar graphite. An empirical correlation of data for thermal conductivity, $k$, based on Figure 1 in [33] yields Equation (18):

$$
k(T)=(3712) T^{-0.602} \text { for } 200<T<2500
$$

and an empirical correlation of data for specific heat, $c_{p}$, based on Figure 1 in [34] yields Equation (19):

$$
c_{p}(T)=(651) \ln (T)-2877 \text { for } 200<T<3000
$$

where $k$ is in $\mathrm{W} / \mathrm{m}-\mathrm{K}, c_{p}$ is in $\mathrm{J} / \mathrm{kg}-\mathrm{K}$, and $T$ is in $\mathrm{K}$. The initial nozzle throat diameter, $d_{t, o}$, was $4 \mathrm{~mm}$ in all tests, and the nozzle exit diameter, $d_{e}$, was $8 \mathrm{~mm}$ in all, but one test. The nozzle exit diameter in a single test was lowered to $6 \mathrm{~mm}$ to examine the effect of the nozzle expansion ratio, $\varepsilon$, on the nozzle throat erosion history and thrust correction factor. Thus, in all tests, with the exception of one, $\varepsilon$ is 4 , and in one test, $\varepsilon$ is 2.25 . There are three thermocouple measurement ports at the nozzle throat plane. The initial radial distances from the thermocouple measurement points to the throat are $5 \mathrm{~mm}, 8 \mathrm{~mm}$, and $10 \mathrm{~mm}$, as shown in Figure 5c.

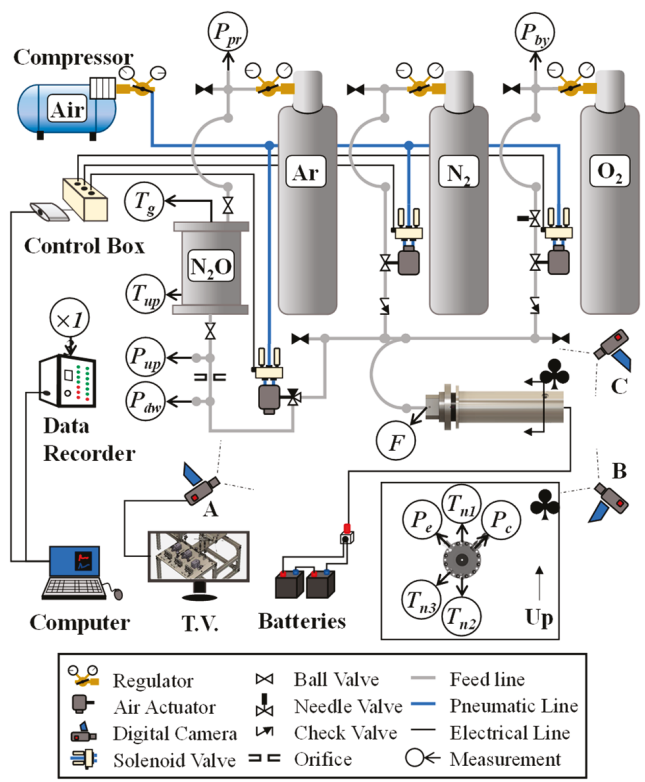

(a)

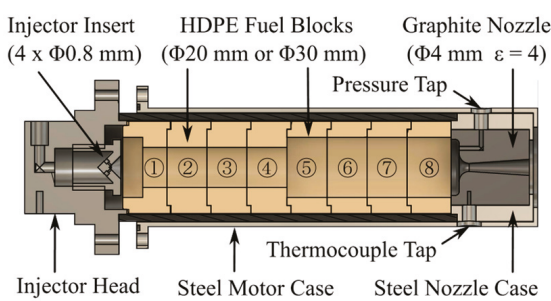

(b)
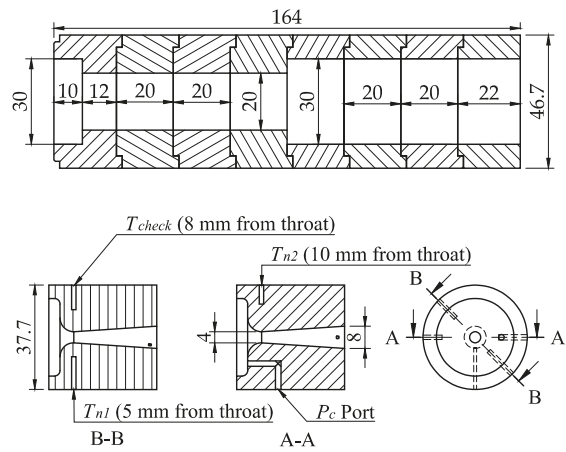

(c)

Figure 5. The experimental apparatus (a) test setup; (b) cut-away view of the motor assembly; (c) detailed schematics of the fuel grain and nozzle (units of $\mathrm{mm}$ ).

The three digital cameras labeled "A", "B", and " $\mathrm{C}$ " in Figure 5 were used to monitor key components of the test apparatus at all times. These cameras were displayed on a T.V. located in a concrete bunker near the test stand. The T.V., computer, control box, and batteries were operated from a desk within the bunker. A depiction of the test sequence using screenshots from camera " $\mathrm{B}$ " (see Figure 5a) is shown in Figure 6. In all tests, ignition was achieved by sending an electrical current through a coil of nichrome wire affixed to the lip of the first fuel block using $1 \mathrm{~g}$ of an epoxy gunpowder 
mixture: $0.8 \mathrm{~g}$ epoxy and $0.2 \mathrm{~g}$ gunpowder. Wire leads were fed through the nozzle exit and attached to a DC voltage source. A test section of wire and nichrome coil was attached in series to the main ignition line to allow for a visual confirmation that the current was large enough to heat the nichrome wire. Nichrome wire was heated for $10 \mathrm{~s}$ before actuating the oxidizer supply valve and initiating the firing test. Ignition was first achieved using $2 \mathrm{~s}$ of a flow of $1 \mathrm{~g} / \mathrm{s}$ of gaseous oxygen $\left(\mathrm{O}_{2}\right)$ to ensure heating and gasification of fuel. After two seconds of $\mathrm{O}_{2}$ ignition, the $\mathrm{O}_{2}$ line was closed, and the main oxidizer $\left(\mathrm{N}_{2} \mathrm{O}\right)$ line was opened. Firing tests were shut down by closing the $\mathrm{N}_{2} \mathrm{O}$ line and opening the $\mathrm{N}_{2}$ purge line. The chamber was purged with $\mathrm{N}_{2}$ for a minimum of $30 \mathrm{~s}$, both to extinguish the combustion of fuel and to cool the nozzle to less than $500 \mathrm{~K}$ for handling.

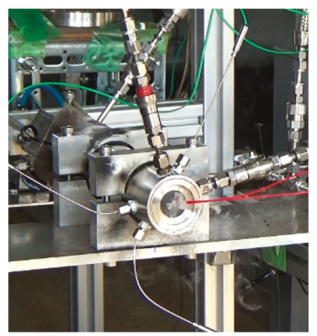

(a)

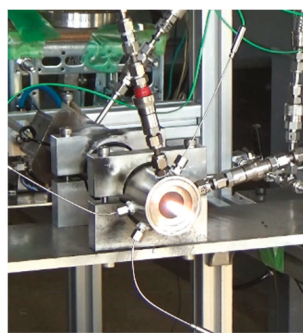

(b)

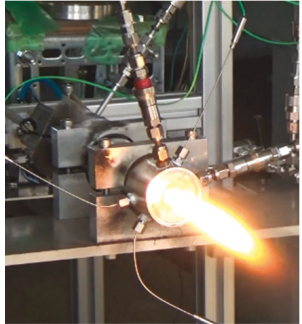

(c)

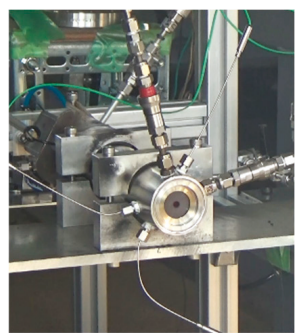

(d)

Figure 6. Screenshots of the firing test sequence (a) nichrome wire heating ( $0 \mathrm{~s}$ to $10 \mathrm{~s})$; (b) $\mathrm{O}_{2}$ ignition assist (10 s to $12 \mathrm{~s})$; (c) main burn (12 $\mathrm{s}$ to $\left.12+t_{b} \mathrm{~s}\right)$; (d) $\mathrm{N}_{2}$ purge/cooling (12 $\left.\mathrm{s}+t_{b} \mathrm{~s}+30 \mathrm{~s}\right)$.

As required for the NTRT+ and TTRT, multiple dynamic and static measurements were taken during the experiments conducted in this study. Pressures were measured using KYOWA DCS-10 MPa and KYOWA DCS-5 MPa pressure sensors with rated accuracies of $\pm 40 \mathrm{kPa}$ and $\pm 28 \mathrm{kPa}$, respectively. Thrust was measured using a KYOWA LMB-A-200N load cell with a rated accuracy of $\pm 3.5 \mathrm{~N}$. These instruments were calibrated by the manufacturer such that the rated accuracies account for uncertainty due to nonlinearity, hysteresis, low-temperature conditions, and external loading. Nozzle temperatures were measured using RC Pro k-type thermocouples rated up to a temperature of $1100{ }^{\circ} \mathrm{C}$, which exhibited a response time of $0.3 \mathrm{~s}$. Dynamic measurements were recorded at $200 \mathrm{~Hz}$ using DCS-100A series software, and later filtered using a 20-point moving average. The reason for applying a moving average was to reduce the presence of oscillations in the reconstructed nozzle throat erosion histories. The uncertainty introduced by applying such moving averages is much smaller than the precision limits of the sensors used, and therefore will not be addressed in detail in this paper (refer to [26]). Initial and final nozzle throat diameter measurements, $d_{t, o}$ and $d_{t, f}$, were taken by the image analysis of digital photographs of the nozzle before and after firing. A length scale was established by placing a plaque of $1 \mathrm{~mm}$-spacing grid paper next to the nozzle being photographed. The nozzle throat diameter was computed based on the area of the lighted region at the throat using ImageJ software [35].

Uncertainty in the experimental measurements of thrust, pressure etc. propagates through the tiers of the comprehensive data reduction operations. The details of this process can be summarized by Equation (20), which expresses the overall uncertainty, $U_{y}$, in some operational output, $y$, as a function of the inputs, $x_{i}$ :

$$
u_{y}=\sqrt{\sum\left(\frac{\partial y}{\partial x_{i}} u_{x_{i}}\right)^{2}}
$$

Here, the $U_{x i}$ terms on the right-hand side represent the uncertainties in the $x_{i}$ measurements. The partial derivative terms in Equation (20) represent the sensitivity of the reconstructed solution to each 
input. Since the algorithms in most of the data reduction operations are coupled non-linear problems, the partial derivative terms in Equation (20) are approximated as (21):

$$
\frac{\partial y}{\partial x_{i}} \cong \frac{y\left(101 \% x_{i}\right)-y\left(100 \% x_{i}\right)}{1 \% x_{i}}
$$

Here, the numerator is the change in value of solution $y$ given that the input parameter, $x_{i}$, has been perturbed by the amount of $1 \%$ of the nominal value.

\section{Results}

Six firing tests were conducted for this study in total. A summary of the experimental results is shown in Table 1. These tests have been labeled according to their burn times for plotting and comparison purposes. The local names of these tests are listed in the second column of Tables 1 and 2 for continuity. Test-3s, 4s, 10s-I and 15s were conducted with the aim of determining the fuel mass consumption trendline necessary for comprehensive data reduction operations. Test-10s-II and Test-10s-III were conducted to assess the repeatability of fuel mass consumption and nozzle throat erosion of Test-10s-I. In Table 1, the change in the nozzle throat diameters due to thermochemical erosion, $\Delta d_{t}$, are listed in $\mathrm{mm}$, the values of overall fuel mass consumption, $\Delta M_{f u}$, are listed in $\mathrm{g}$, the time-averaged thrusts, $\bar{F}$, are listed in $\mathrm{N}$, the time-averaged oxidizer mass flow rates, $\overline{\dot{m}}_{o x}$, are listed in $\mathrm{g} / \mathrm{s}$, the time-averaged chamber pressures, $\bar{P}_{c}$, are listed in MPa, and the time-averaged nozzle temperatures, $\bar{T}_{10 m m}, \bar{T}_{8 m m}$, and $\bar{T}_{5 m m}$, are listed in K. An inspection of the overall fuel mass consumption and final nozzle throat diameters of Tests-10s-I through to III reveals that nozzle throat erosion was not the same even though the initial test conditions were identical, whereas the values of fuel mass consumption were within $1 \%$ of one another. Test- 3 s did not have a measurable amount of nozzle throat erosion, whereas the remaining tests did. The primary reason for this is that the nozzle throat wall temperature did not become high enough to allow for chemical reactions with the combustion gas. This is further discussed in Section 3.4. The greater than symbol " $>$ " means that the thermocouple overheated during the firing, and thus the time-averaged temperature shown is an underestimate of the true value.

Table 1. Summary of direct measurements and their uncertainties.

\begin{tabular}{|c|c|c|c|c|c|c|c|c|c|}
\hline $\begin{array}{c}\text { Test } \\
\text { Label }\end{array}$ & $\begin{array}{l}\text { Local } \\
\text { Name }\end{array}$ & $\begin{array}{l}\Delta d_{t} \\
\mathrm{~mm}\end{array}$ & $\begin{array}{c}\Delta M_{f u} \\
\mathrm{~g}\end{array}$ & $\begin{array}{l}\bar{F} \\
\mathbf{N}\end{array}$ & $\begin{array}{l}\overline{\dot{m}}_{o x} \\
\mathrm{~g} / \mathrm{s}\end{array}$ & $\begin{array}{c}\bar{P}_{c} \\
\mathbf{M P a}\end{array}$ & $\begin{array}{c}\bar{T}_{10 m m} \\
\mathbf{K}\end{array}$ & $\begin{array}{c}\bar{T}_{8 m m} \\
\mathbf{K}\end{array}$ & $\begin{array}{c}\bar{T}_{5 m m} \\
\mathbf{K}\end{array}$ \\
\hline $3 s$ & $(\mathrm{CBX}-5)$ & $0.05 \pm>100 \%$ & $30 \pm<1 \%$ & $74 \pm 4 \%$ & $41 \pm 4 \%$ & $4.1 \pm 1 \%$ & 518 & 551 & 661 \\
\hline $4 s$ & $(\mathrm{CBX}-4)$ & $0.20 \pm 50 \%$ & $35 \pm<1 \%$ & $78 \pm 4 \%$ & $41 \pm 4 \%$ & $4.1 \pm 1 \%$ & 520 & 570 & 717 \\
\hline $10 s-I$ & (CBX-6) & $0.53 \pm 20 \%$ & $74 \pm<1 \%$ & $81 \pm 4 \%$ & $39 \pm 4 \%$ & $4.2 \pm 1 \%$ & 551 & $>582$ & $>745$ \\
\hline $10 s-I I$ & (CBX-8) & $0.75 \pm 10 \%$ & $73 \pm<1 \%$ & $89 \pm 4 \%$ & $38 \pm 4 \%$ & $4.4 \pm 1 \%$ & 710 & 776 & $>874$ \\
\hline $10 \mathrm{~s}-\mathrm{III}$ & (CBX-9) & $1.00 \pm 10 \%$ & $74 \pm<1 \%$ & $83 \pm 4 \%$ & $39 \pm 4 \%$ & $4.1 \pm 1 \%$ & 556 & 600 & $>764$ \\
\hline $15 \mathrm{~s}$ & (CBX-7) & $0.80 \pm 13 \%$ & $110 \pm<1 \%$ & $88 \pm 4 \%$ & $37 \pm 4 \%$ & $4.3 \pm 1 \%$ & 658 & $>723$ & $>859$ \\
\hline
\end{tabular}

Note: Time averages were taken for the entire burning duration, and the greater than symbol, $>$, is used in the case where thermocouples overheated during the test.

The unprocessed pressure cascade and thrust histories are plotted together in Figure 7a,b. The main steps in the test sequence (as shown in Figure 6) have also been labeled in Figure 7a. It is evident that all tests show the same pressure and thrust to within less than $\pm 2.5 \%$ up to $15 \mathrm{~s}$ into the test sequence (i.e., the end of burn in Test-3s), but the pressure and thrust histories beyond $15 \mathrm{~s}$ were not the same. This means that the primary cause of the differing values of pressure and thrust in tests lasting longer than Test- $3 \mathrm{~s}$ were due to the differences in the nozzle throat erosion history. Figure $7 \mathrm{~b}$ shows an expanded view of the first $4 \mathrm{~s}$ of ignition and start-up transients. Each test reached a steady state around $14 \mathrm{~s}$ into the test sequence after a transient lasting $2 \mathrm{~s}$. There is a sharp increase that ends the transient between $13 \mathrm{~s}$ and $14 \mathrm{~s}$ into the firing sequence. The timing of this increase varies between tests, although the rate and trend of the increase is similar. 


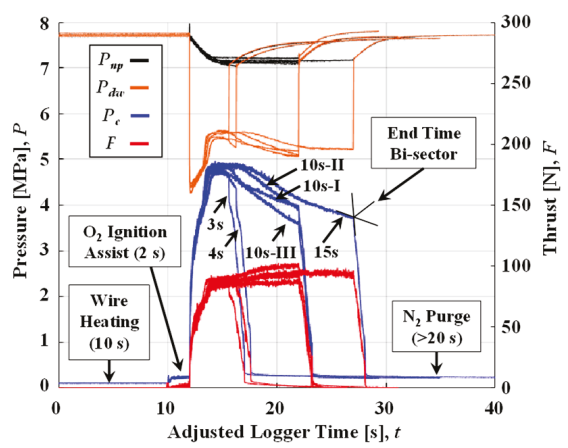

(a)

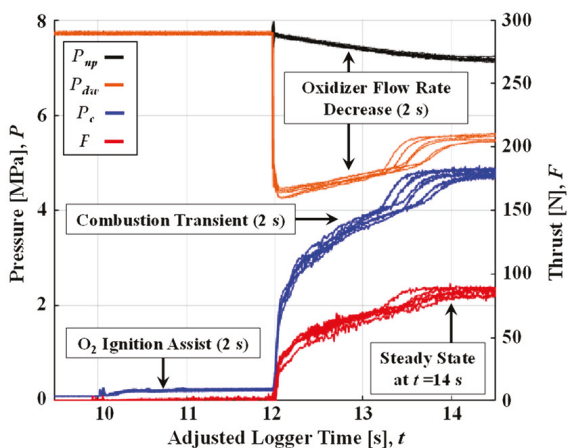

(b)

Figure 7. Unprocessed static firing test data of all tests; (a) the first $40 \mathrm{~s}$ of the firing sequence, (b) expanded graph of the first $4 \mathrm{~s}$ of ignition and start-up transients.

\subsection{Tier I Results: Fuel Mass Consumption History Trendline}

The overall fuel mass consumption and the associated trendline is plotted for all tests in Figure 8a. A power-type trendline yields a near perfect fit for the fuel mass consumption history, in which the fuel consumption of Tests-10s-I to III were within $\pm 1 \%$ of one another. This high degree of repeatability is important for the NTRT+, which operates on the assumption that the trendline in Figure 8a accurately represents the fuel consumption history of any given test. Figure $8 \mathrm{~b}$ shows the overall fuel regression by block in all tests in units of meters. These values were calculated based on the change in mass, according to Equation (11). Blocks (1)-(4) had a greater overall fuel regression than blocks (5)-(8) in all tests. This can be explained by the fact that the first four blocks have a smaller initial port diameter, and thus a larger oxidizer port mass flux. However, it is also interesting to point out that block (1) and block (5), both of which have step changes in port diameter, regressed less than similar blocks downstream.

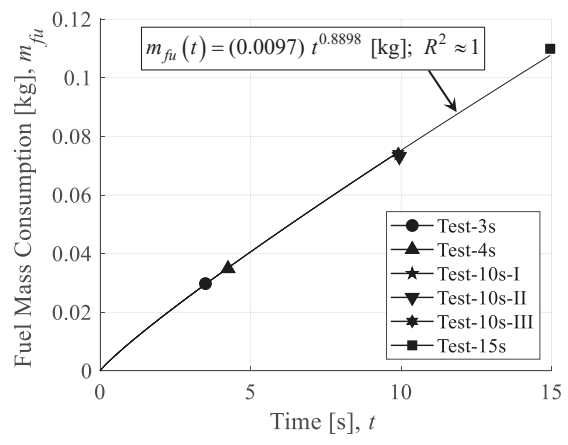

(a)

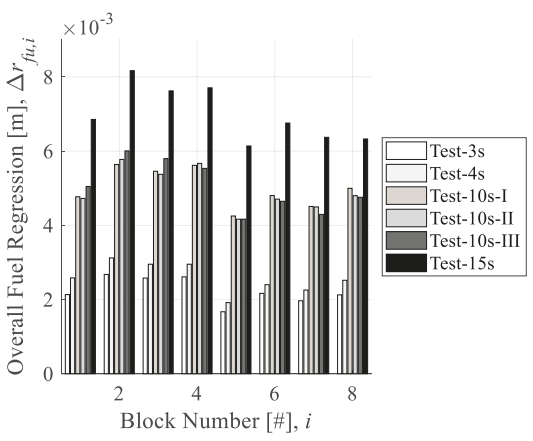

(b)

Figure 8. Fuel consumption analysis (a) fuel mass consumption trendline; (b) fuel regression by block.

Block (1) is immediately downstream of the injector, and thus marks the initiation point of the boundary layer. Reduced fuel regression rates at this location should be expected because the boundary layer is not fully developed. It is implied in the numerical analysis conducted by Kumar and Kumar [36], and by Kumar and Ramakrishna [37] that step increases in fuel port diameter are also likely to create recirculation zones downstream of the disturbance. The results in Figure $8 \mathrm{~b}$ are consistent with this 
analysis. The reduced fuel regression in block (5) can be explained by the presence of a recirculation zone, downstream of which fuel regression increases again. In following, the local peaks in the fuel regression of blocks (2) and (6) are the result of increased turbulent heating downstream of the recirculation zones of blocks (1) and (5), respectively.

\subsection{Tier II Results}

\subsubsection{Fuel Regression Correlations}

In the same way that the fuel mass consumption history trend line had a nearly perfect fit (with a coefficient of determination of $\approx 1$ ), the "endpoint" fuel regression correlation led to a near perfect fit, as shown in Figure 9a. In the "history" method, where the histories of fuel regression rate are plotted directly against the histories of port mass flux, the resulting regression correlation has a coefficient of determination of 0.95 , as shown in Figure 9b. This figure is organized according to the left- and right-hand side of Equation (9), so it is possible to plot and compare all correlations introduced so far in this figure. The waviness that appears in the first three seconds of the fuel regression rate histories is the result of the decrease in the oxidizer mass flow rate in the beginning of each firing due to the chamber pressure transient, as seen in Figure 7. DeLuca et al. [38], Pavaran et al. [39], and Evans et al. [40] examined time-resolved burning rates using direct (optical) measurements and did not observe this degree of waviness. The general trend that fuel regression rate decreases in time with decreasing oxidizer port mass flux is in agreement with [38-40].

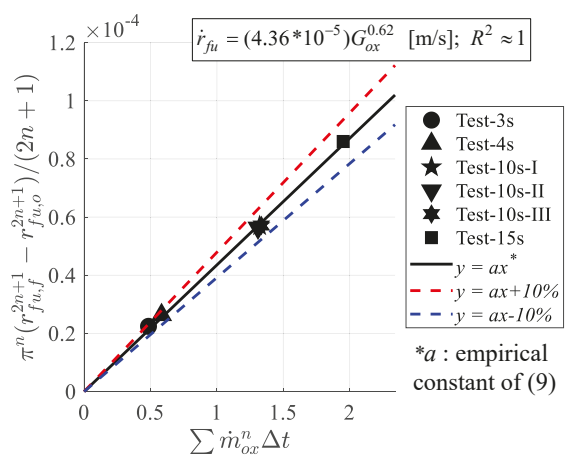

(a)

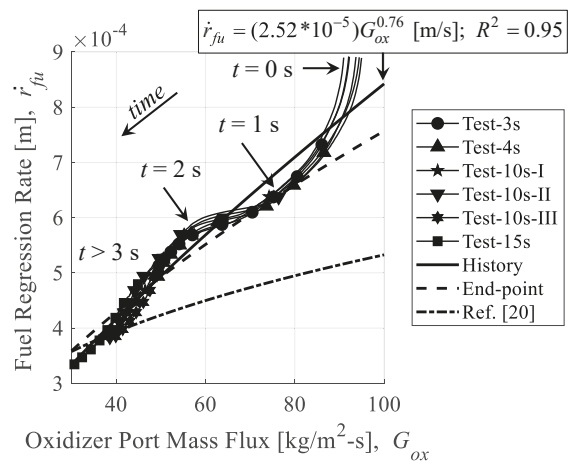

(b)

Figure 9. Fuel regression rate correlations of (a) endpoint correlation; (b) history correlation.

It can be seen from Figure $9 \mathrm{~b}$ that the endpoint correlation slightly underestimates the values of the histories, and the results of Doran et al. grossly underestimate the magnitude and slope of these histories when $G_{0 x}>50 \mathrm{~kg} / \mathrm{m}^{2}$-s. The most important discrepancy is the large difference in the solution of the fuel regression rate exponent, $n$. The history correlation results in an exponent of 0.76 , which is very close to the theoretical value of 0.8 that is expected by Marxman et al.'s diffusion-limited boundary layer combustion theory $[30,41]$, whereas the endpoint method results in an exponent of 0.62 , and Doran et al. reports a value of 0.331 [20]. The departure of the fuel regression correlation exponent, $n$, from a value of 0.331 in Doran et al.'s study [20] to either 0.62 or 0.76 in this study, depending on whether the endpoint method or history method is used, warrants a discussion.

There does not appear to be a very large difference in the test apparatus used in each study. One reason for this discrepancy may simply be that the range of $G_{0 x}$ tested in this study is too narrow. The time-averaged values of $G_{0 x}$ varied from $50 \mathrm{~kg} / \mathrm{m}^{2}$-s to over $300 \mathrm{~kg} / \mathrm{m}^{2}$-s in Doran et al.'s test series, but only from $50 \mathrm{~kg} / \mathrm{m}^{2}$-s to $100 \mathrm{~kg} / \mathrm{m}^{2}$-s in this study. With that stated, it is also clear from this study that 
the largest changes in $G_{0 x}$ take place in the first three seconds of firing, which constitute half of the burn time in Doran et al.'s tests. Given that even the time-averaged endpoint values from this study overestimate the prediction of Doran et al. by nearly $50 \%$, it seems possible that the low exponent observed by Doran et al. may simply be due to the uncertainty introduced by using time-averaged values in the fuel regression rate correlation of Equation (9). If so, the consequences of this are grave, because the increase or decrease in time of the value of $\xi$ depends on whether the exponent, $n$, is greater than or less than 0.5 . In other words, the results of this study will predict an increase in $\xi$ in time, whereas the results of Doran et al. will predict a decrease of $\xi$ in time.

It is important to point out that although the fuel regression is being calculated based on the fuel mass consumption (refer to Equation (11)) and thus does not directly consider the shape and orientation of the burning surface at the end surfaces near the injector in block (1) and at the end face of block (4), the combined surface area of these end faces is a mere $1 \%$ of the main port burning surface area. As the fuel burns and all surfaces blend together with the main port, any possible effects are reduced even further. Thus, the effect of these non-tubular burning surfaces is considered to be negligible for the sake of the analysis presented here.

\subsubsection{Results of the NTRT+}

The results of the NTRT + for thrust correction factor, $\lambda$, and time-averaged $c^{*}$ efficiency, $\bar{\eta}^{*}$, are listed in Table 2. The results of the NTRT+ for oxidizer-to-fuel-mass ratio, $\xi$, history are shown in Figure $10 \mathrm{a}$, while the results for nozzle throat diameter history, $d_{t}$, and $c^{*}$ efficiency history, $\eta^{*}$, are shown in Figure 10b. The histories of the oxidizer mass flow rate, $\dot{m}_{0 x}$, were added to Figure 10a to show that increases in $\dot{m}_{0 x}$ correspond to increases in $\xi$. The close agreement of all throat diameter histories with the known value for $d_{t, o}$ in the beginning of the tests bolsters the reliability of the NTRT + results, since $d_{t, o}$ is a known value that is not used in the NTRT+ algorithm. It can be seen from these results that the timing of the onset of nozzle throat erosion varies by $2 \mathrm{~s}$ between tests, from around $3 \mathrm{~s}$ in Tests-4s and 10s-I to around $5 \mathrm{~s}$ in Tests-10s-II and 15s. Also, the erosion rate of Test-10s-I is noticeably smaller than that of the other tests. Test-10s-I is the one test which employed a smaller nozzle expansion ratio than the other tests-2.25 versus 4.0. Thus, it is likely that the nozzle expansion ratio effected some aspect of the nozzle throat erosion mechanisms. Similar to the chamber pressure and thrust histories, the histories of $\eta^{*}$ were the same during the first three seconds of burn time, during which time they sharply rose to a steady-state value. The time averages of the steady-state values of $\eta^{*}$ are listed in Table 2. The values differ between tests in the range of $0.85<\eta^{*}<0.95$, which is consistent with the results of Doran et al. [20].

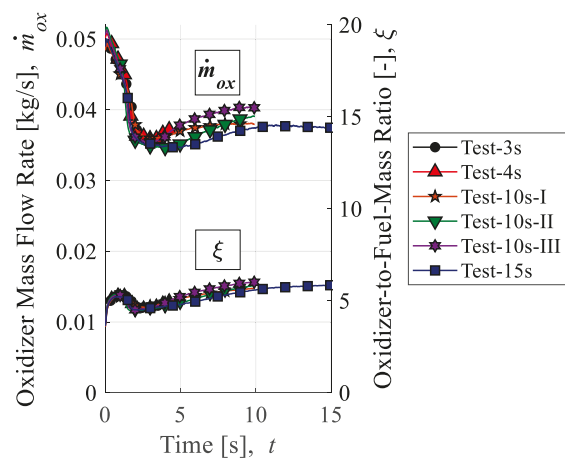

(a)

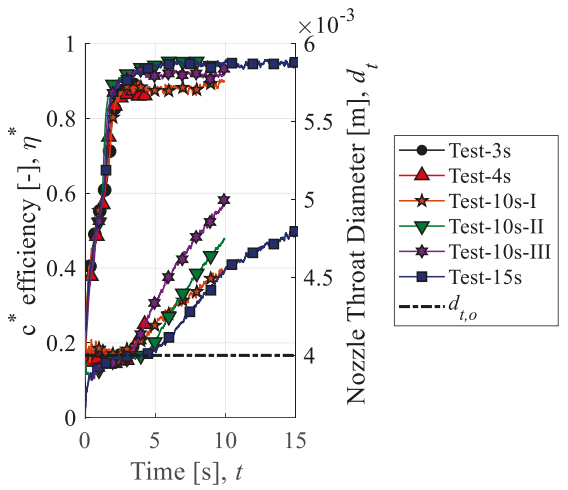

(b)

Figure 10. The NTRT+ analysis (a) propellant flow rates; (b) throat erosion and efficiency histories. 
Table 2. Summary of tier II (NTRT+) and tier III (TTRT) results.

\begin{tabular}{ccccccccc}
\hline $\begin{array}{c}\text { Test } \\
\text { Label }\end{array}$ & $\begin{array}{c}\text { Local } \\
\text { Name }\end{array}$ & $\lambda$ & $\bar{\eta}^{*}$ & $\begin{array}{c}\overline{\boldsymbol{T}}_{w} \\
\mathbf{K}\end{array}$ & $\begin{array}{c}\boldsymbol{t}_{\Pi} \\
\mathbf{s}\end{array}$ & $\mathbf{\Phi}$ & $\begin{array}{c}\boldsymbol{P}_{\Pi} \\
\mathbf{M P a}\end{array}$ & $\begin{array}{c}\boldsymbol{T}_{\Pi} \\
\mathbf{K}\end{array}$ \\
\hline $3 \mathrm{~s}$ & (CBX-5) & $0.97 \pm 10 \%$ & $0.87 \pm 11 \%$ & $1243 \pm 9 \%$ & 3 & $2.03 \pm 4 \%$ & $2.6 \pm 2 \%$ & $1778 \pm 10 \%$ \\
4s & (CBX-4) & $1.00 \pm 10 \%$ & $0.86 \pm 10 \%$ & $1464 \pm 10 \%$ & 3 & $2.02 \pm 4 \%$ & $2.6 \pm 2 \%$ & $2030 \pm 12 \%$ \\
$10 \mathrm{~s}-\mathrm{I}$ & (CBX-6) & $0.97 \pm 10 \%$ & $0.88 \pm 10 \%$ & $1467 \pm 10 \%$ & 4 & $1.95 \pm 4 \%$ & $2.6 \pm 2 \%$ & $2076 \pm 9 \%$ \\
$10 \mathrm{~s}-\mathrm{II}$ & (CBX-8) & $0.98 \pm 9 \%$ & $0.94 \pm 9 \%$ & $1455 \pm 11 \%$ & 5 & $1.96 \pm 4 \%$ & $2.7 \pm 2 \%$ & $1811 \pm 7 \%$ \\
$10 \mathrm{~s}-\mathrm{III}$ & (CBX-9) & $0.90 \pm 8 \%$ & $0.91 \pm 9 \%$ & $1538 \pm 10 \%$ & 3 & $2.05 \pm 4 \%$ & $2.7 \pm 2 \%$ & $1963 \pm 11 \%$ \\
$15 \mathrm{~s}$ & (CBX-7) & $0.94 \pm 8 \%$ & $0.94 \pm 10 \%$ & $1527 \pm 8 \%$ & 5 & $1.95 \pm 4 \%$ & $2.7 \pm 2 \%$ & $1905 \pm 7 \%$ \\
\hline
\end{tabular}

\subsection{Tier III Results: Results of the TTRT}

Kamps et al. demonstrated in [27] that the wall temperature, pressure, and equivalence ratio at the nozzle throat can be used to predict whether or not thermochemical erosion may take place at the nozzle throat. This analysis will be shown in the following section, but first it is necessary to identify the temperature of nozzle throat wall using the TTRT. The results of the TTRT for time-averaged values of the nozzle throat wall temperature are listed in Table 2 as $\bar{T} w$ in K. Note that nozzle throat wall temperature histories could not be calculated for the entire burn time in most tests because the thermocouples nearest to the throat, predominately $T_{5 \mathrm{~mm}}$, but in two occasions $T_{\text {check, }}$, failed due to overheating. This typically happened around $5 \mathrm{~s}$ into the burn time. The results of the TTRT for nozzle throat wall temperature histories, $T_{w}$, in $\mathrm{K}$ are shown in red in Figure 11a. The two thermocouple measurement histories used as input data to the TTRT, at depths of $10 \mathrm{~mm}\left(T_{n 2}\right)$ and $5 \mathrm{~mm}\left(T_{n 1}\right)$ from the nozzle throat, are also shown in in Figure 11a in black and blue, respectively. The procedure for confirming the results of $T_{w}$ is depicted in Figure 11b, where the thermocouple located at a depth of 8 $\mathrm{mm}$ from the nozzle throat $\left(T_{\text {check }}\right)$ was used to verify the calculated value at that position within the nozzle. The solid black lines in Figure 11b show the calculated temperature profiles in the nozzle at $1 \mathrm{~s}$ intervals. The close agreement between calculated and measured values in Test- $3 \mathrm{~s}$ as shown in Figure $11 \mathrm{~b}$ was also observed in all tests for the range of times where the thermocouples did not fail due to overheating.

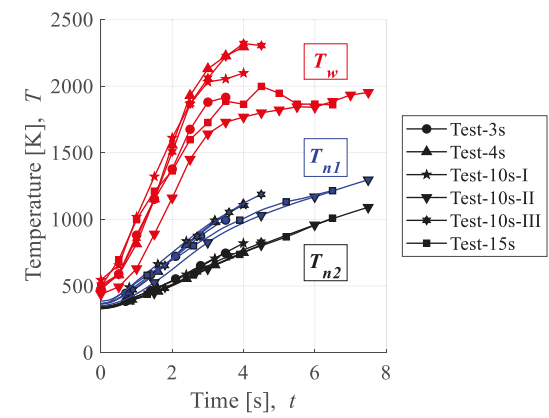

(a)

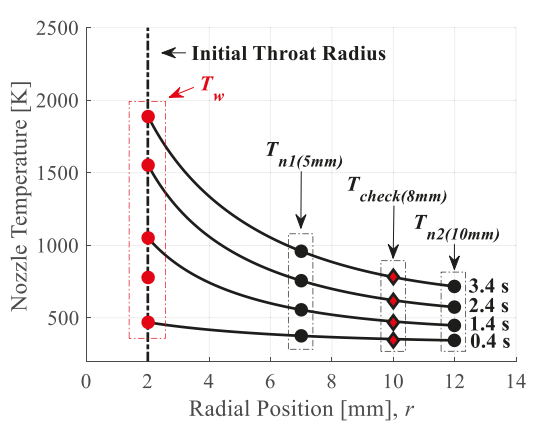

(b)

Figure 11. The TTRT analysis (a) nozzle temperature histories; (b) confirmation of temperature profile histories in Test-3s.

\subsection{Erosion Onset Factor}

The erosion onset factor, $\Pi$, was introduced by Kamps et al. in [27] as a way to model the nozzle temperature and gas pressure that allow for chemical kinetic-limited thermochemical erosion to take place for a given equivalence ratio, $\Phi$ — the ratio of the stoichiometric oxidizer-to-fuel mass ratio, $\xi_{s t o}$ 
$\left(\xi_{\text {sto }}=9.4\right.$ for $\left.\mathrm{N}_{2} \mathrm{O} / \mathrm{HDPE}\right)$, and the measured value of oxidizer-to-fuel mass ratio, $\xi$. Since the timing of the onset of erosion can be identified from Figure 10b, a brief analysis of $\Pi$ will be conducted in this section. The equation for $\Pi$ is (22):

$$
\Pi=P^{n_{\Pi}} \exp \left(-E_{\Pi} / T_{w}\right)
$$

where $P$ is the local combustion gas pressure in $\mathrm{Pa}, n_{\Pi}$ is a dimensionless empirical constant, and $E_{\Pi}$ is an empirical constant with units of $\mathrm{K}$. Based on the results of [27], the following values of empirical constants will be used: $n_{\Pi}=1.03$ and $E_{\Pi}=1408 \mathrm{~K}$. Here, it is necessary to identify the pressure at the nozzle throat and the temperature of the nozzle throat wall position at the onset of nozzle throat erosion-listed as $P_{\Pi}$ in $\mathrm{Pa}$ and $T_{\Pi}$ in $\mathrm{K}$ in Table 2-so that values for the erosion onset factor may be calculated. The subscript, $\Pi$, is used to indicate the data taken at the time of the onset of erosion. Here, the value of $P_{\Pi}$ was taken to be the pressure at the nozzle throat, $P_{t}$, which was approximated by the equation for the isentropic expansion of an ideal gas:

$$
P_{t}=P_{c}\left(\frac{2}{\gamma+1}\right)^{\frac{\gamma}{\gamma-1}}
$$

The times at the onset of erosion, $t_{\Pi}$, were chosen to be the time when the $d_{t}$ histories in Figure 10b departed from the value of $d_{t, o}$ and had an erosion rate of more than $0.02 \mathrm{~mm} / \mathrm{s}$ for longer than $1 \mathrm{~s}$. The values for nozzle throat wall temperature at the onset of erosion were obtained from the $T_{w}$ histories plotted in Figure 11a. The range of equivalence ratios, $\Phi$, was not wide enough to carry out a correlation of $\Pi$, such as that in [27]. Thus, the data from [27] was used as a reference to evaluate the relative erosion characteristics observed in this study. Fortunately, there is a group of data and a trendline from [27] that lines up with the values of $\Phi$ observed in this study. This is shown in Figure 12. We can draw two useful conclusions from this comparison. The first conclusion is that even though nozzle erosion histories were not repeatable between tests in this study, the values of $\Pi$ were. This suggests that the onset of erosion was essentially chemical-controlled. The second conclusion is that the use of $\mathrm{N}_{2} \mathrm{O}$ in place of $\mathrm{O}_{2}$ increased the threshold for $\Pi$. This is expected, since the presence of nitrogen in the combustion gas reduces the concentration of oxidizing species. Similar findings are reported by Bianchi and Nasuti regarding the difference in erosion rates when using $\mathrm{N}_{2} \mathrm{O}$ as an oxidizer versus $\mathrm{O}_{2}$ [21].

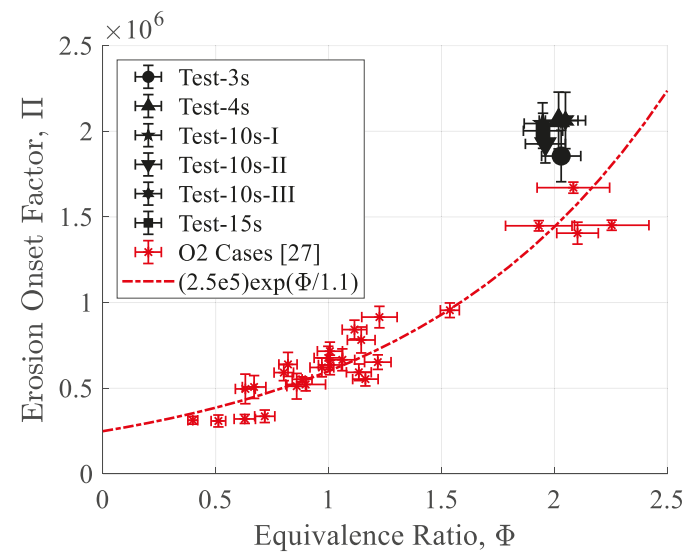

Figure 12. Erosion onset factor is larger when using $\mathrm{N}_{2} \mathrm{O}$ as the oxidizer rather than $\mathrm{O}_{2}$. 


\section{Conclusions}

The propellant combination of nitrous oxide $\left(\mathrm{N}_{2} \mathrm{O}\right)$ and high-density polyethylene (HDPE) shows promise for use in hybrid rocket apogee kick motors for the attributes of storability, non-toxicity, and stable burning with minimal combustion oscillations. However, there has been very little research published on the performance of hybrid rockets employing this propellant combination, and the research that has been made available to the public is limited in scope and inadequate for serious design considerations. This study introduces an improvement to well-tested non-intrusive data collection and analysis methods called ballistic reconstruction techniques in order to cost-effectively collect data on hybrid rockets using this propellant combination. The concept of this improvement is to introduce time-resolved fuel mass consumption into the governing equations of characteristic exhaust velocity and thrust, making it possible to simultaneously collect and evaluate time histories of the fuel regression rate, combustion efficiency, and nozzle throat erosion. The results were a highly cost-effective test series. With just six firing tests, the fuel regression rate could be correlated $\left(a=2.52 \times 10^{-5} ; n=0.76\right.$; such that the regression rate is in units of $\mathrm{m} / \mathrm{s}$ ), the combustion efficiency transients and trends became known ( $2 \mathrm{~s}$ transient; steady state values of $87 \%$ to $94 \%$ ), and the erosion onset factor threshold was shown to be $50 \%$ larger than in the case where $\mathrm{O}_{2}$ is used as the oxidizer. Moreover, now it is clearer how the chamber pressure, equivalence ratio, and nozzle heating transients at the beginning of a burn dictate when nozzle erosion is likely to begin.

Future implantation of the data reduction methodology introduced in this study will greatly expedite the development of hybrid rocket projects using $\mathrm{N}_{2} \mathrm{O} / \mathrm{HDPE}$, including the apogee kick motor being developed by the authors. Essentially, all key aspects of combustion can be examined with a limited number of trials. This method may be especially helpful when studying the behavior of long-duration firing tests, for which neither time-averaged analysis methods nor high-resolution direct measurement techniques, such as $\mathrm{X}$-ray radiography, are practical.

Author Contributions: This research was only possible through the collaboration and cooperation of all four of the authors, however, each author contributed in different ways to achieve the results reported in this paper. H.N. and L.K. held research responsibility for this work and the integration of these results within ongoing projects. This included the management of funds, ordering and receiving experimental equipment and managing experimental facilities. Y.S. and L.K. worked together extensively on the conceptualization and validation of the analysis methodology, and on the development of software for applying these methodologies. K.S. was the lead investigator, and was responsible for preparing and conducting the static firing tests together with L.K. and other graduate students from the University of Hokkaido. L.K. prepared the original draft of this manuscript, and all authors reviewed and edited this draft prior to submission.

Funding: This research is supported by the Grant-in-Aid for Japan Society for the Promotion of Science (JSPS) fellows 18J2087708, as well as by a matching fund program of Centers for Inter-University Collaboration from ISAS (Institute of Space and Astronautical Science), JAXA (Japan Aerospace Exploration Agency).

Acknowledgments: The authors would like to recognize the hard work of the students of the Hokkaido University hybrid rocket systems research team Erika Uchiyama, Taku Inoue, Hanako Ikeda, Seiji Itoh, Lisa Kageyama, and Terutaka Okuda in assisting in the experiments conducted for this study. Furthermore, this research relied heavily on the expert technical support of Kato of the Hokkaido University workshop.

Conflicts of Interest: The authors declare no conflict of interest.

\section{Nomenclature}

$a, n$

$A_{e}$

$A_{t}$

$C_{\text {or }}$

$c_{p}$

$c_{t h}^{*}$

$d_{e}$

$D_{f u}$

$d_{\text {or }}$

$d_{t}$ empirical constants of Equation (9)

nozzle exit area, $\mathrm{m}^{2}$

nozzle throat area, $\mathrm{m}^{2}$

dimensionless orifice flow coefficient

specific heat capacity of the nozzle, J/kg-K

theoretical characteristic exhaust velocity, $\mathrm{m} / \mathrm{s}$

nozzle exit diameter

fuel outer diameter, $\mathrm{m}$

orifice hole diameter, $\mathrm{m}$

nozzle throat diameter, $\mathrm{m}$ 


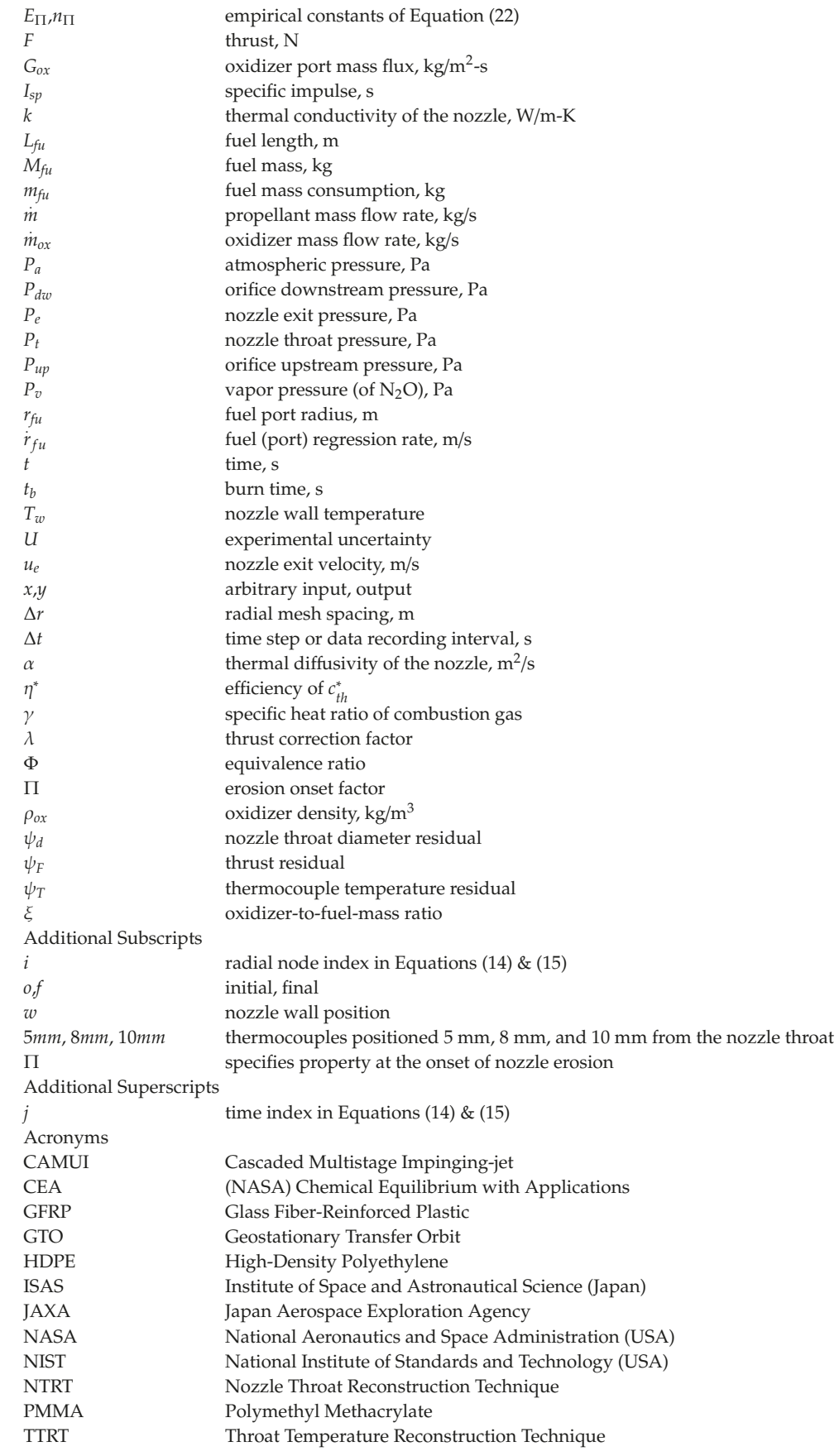

\section{References}

1. The Wall Street Journal. Available online: https://www.wsj.com/articles/richard-bransons-space-tourismcompany-rockets-out-of-atmosphere-for-first-time-11544720558 (accessed on 27 January 2019). 
2. Takahashi, A.; Shimada, T. Essentially Non-Explosive Propulsion Paving a Way for Fail-Safe Space Transportation. Trans. JSASS Aerosp. Tech. Jpn. 2018, 16,1-8. [CrossRef]

3. Altman, D.; Holzman, A. Overview and History of Hybrid Rocket Propulsion. In Fundamentals of Hybrid Rocket Propulsion, 1st ed.; Progress in Astronautics and Aeronautics; Chiaverini, M., Kuo, K., Eds.; AIAA: Washington, DC, USA, 2006; Volume 218, pp. 1-33. [CrossRef]

4. Kuo, K.; Chiaverini, M. Challenges of Hybrid Rocket Propulsion in the 21st Century. In Fundamentals of Hybrid Rocket Propulsion, 1st ed.; Progress in Astronautics and Aeronautics; Chiaverini, M., Kuo, K., Eds.; AIAA: Washington, DC, USA, 2006; Volume 218, pp. 593-638. [CrossRef]

5. Jens, E.T.; Cantwell, B.; Hubbard, G.S. Hybrid Rocket Propulsion Systems for Outer Planet Exploration Missions. Acta Astronaut. 2016, 128, 119-130. [CrossRef]

6. Jens, E.T.; Karp, A.C.; Nakazono, B.; Vaughan, D.; Conte, A.; Contadin, S. Development Testing of Hybrid Rocket Motors Using Classical Fuels for Interplanetary CubeSats. In Proceedings of the 53rd AIAA/SAE/ASEE Joint Propulsion Conference, Atlanta, GA, USA, 10-12 July 2017. [CrossRef]

7. Jens, E.T.; Karp, A.C.; Rabinovich, J.; Nakazono, B.; Conte, A.; Vaughan, D. Design of Interplanetary Hybrid CubeSat and SmallSat Propulsion Systems. In Proceedings of the 54th AIAA/SAE/ASEE Joint Propulsion Conference, Cincinnati, OH, USA, 9-11 July 2018. [CrossRef]

8. Heister, S.; Wernimont, E. Hydrogen Peroxide, Hydroxyl Ammonium Nitrate, and Other Storable Oxidizers. In Fundamentals of Hybrid Rocket Propulsion, 1st ed.; Progress in Astronautics and Aeronautics; Chiaverini, M., Kuo, K., Eds.; AIAA: Washington, DC, USA, 2006; Volume 218, pp. 457-487. [CrossRef]

9. NIST. Thermophysical Properties of Fluid Systems. Available online: https://webbook.nist.gov/chemistry/ fluid (accessed on 1 August 2018).

10. Story, G. Large-Scale Hybrid Motor Testing. In Fundamentals of Hybrid Rocket Propulsion, 1st ed.; Progress in Astronautics and Aeronautics; Chiaverini, M., Kuo, K., Eds.; AIAA: Washington, DC, USA, 2006; Volume 218, pp. 513-591. [CrossRef]

11. Kobald, M.; Fischer, U.; Tomilin, K.; Petrarolo, A.; Schmierer, C. Hybrid Experimental Rocket Stuttgart: A Low-Cost Technology Demonstrator. J. Spacecr. Rocket. 2018, 55, 484-500. [CrossRef]

12. Nakata, D.; Yasuda, K.; Horio, S.; Higashino, K. A Fundamental Study on the Hybrid Rocket Clustering for the Rocket Sled Propulsion System. In Proceedings of the 52nd AIAA/SAE/ASEE Joint Propulsion Conference, Salt Lake City, UT, USA, 25-27 July 2016. [CrossRef]

13. Chen, Y.; Wu, B. Development of a Small Launch Vehicle with Hybrid Rocket Propulsion. In Proceedings of the 54th AIAA/SAE/ASEE Joint Propulsion Conference, Cincinnati, OH, USA, 9-11 July 2018. [CrossRef]

14. Gamal, H.; Matusiewicz, A.; Magiera, R.; Hubert, D.; Karolewski, L.; Zielinski, K. Design, Analysis and Testing of a Hybrid Rocket Engine with a Multi-port Nozzle. In Proceedings of the 54th AIAA/SAE/ASEE Joint Propulsion Conference, Cincinnati, OH, USA, 9-11 July 2018. [CrossRef]

15. Whitmore, S.; Peterson, Z.; Eilers, S. Closed-Loop Precision Throttling of a Hybrid Rocket Motor. J. Propuls. Power 2014, 30, 325-336. [CrossRef]

16. Zilliac, G.; Waxman, B.; Evans, B.; Karabeyoglu, A.; Cantwell, B. Peregrine Hybrid Rocket Motor Development. In Proceedings of the 50th AIAA/SAE/ASEE Joint Propulsion Conference, Cleveland, OH, USA, 28-30 July 2014. [CrossRef]

17. Nagata, H.; Wakita, M.; Totani, T.; Uematsu, T. Development and Flight Demonstration of 5 kN Thrust Class CAMUI Type Hybrid Rocket. Trans. JSASS Aerosp. Tech. 2014, 12, TA_1-TA_4. [CrossRef]

18. Kamps, L.; Nagata, H. Tubular Equivalent Regression Rate in Hybrid Rockets with Complex Geometries. Trans. JSASS 2019. [CrossRef]

19. Mazzetti, A.; Merotto, L.; Pinarello, G. Paraffin-Based Hybrid Rocket Engines Applications: A Review and Market Perspective. Acta Astronaut. 2016, 126, 286-297. [CrossRef]

20. Doran, E.; Dyer, J.; Lohner, K.; Dunn, Z.; Cantwell, B.; Zilliac, G. Nitrous Oxide Hybrid Rocket Motor Fuel Regression Rate Characterization. In Proceedings of the 43rd AIAA/SAE/ASEE Joint Propulsion Conference, Cincinnati, OH, USA, 8-11 July 2007. [CrossRef]

21. Bianchi, D.; Nasuti, F. Numerical Analysis of Nozzle Material Thermochemical Erosion in Hybrid Rocket Engines. J. Propuls. Power 2013, 29, 547-558. [CrossRef]

22. Wernimont, E.; Heister, D. Reconstruction Technique for Reducing Hybrid-Rocket Combustion Data. J. Propuls. Power 1999, 15, 128-136. [CrossRef] 
23. Nagata, H.; Nakayama, H.; Watanabe, M.; Wakita, M.; Totani, T. Accuracy and Applicable Range of a Range of a Reconstruction Technique for Hybrid Rockets. Adv. Aircr. Spacecr. Sci. 2014, 1, 273-289. [CrossRef]

24. Carmicino, C.; Sorge, A. Influence of a Conical Axial Injector on Hybrid Rocket Performance. J. Propuls. Power 2006, 22, 984-995. [CrossRef]

25. Nagata, H.; Saito, Y.; Ishiyama, T.; Inaba, Y.; Wakita, M.; Totani, T. Evaluations of Data Reduction Methods for Hybrid Rockets. In Proceedings of the 65th International Astronautical Congress, Toronto, ON, Canada, 29 September-3 October 2014.

26. Kamps, L.T.; Saito, Y.; Kawabata, R.; Wakita, M.; Totani, T.; Nagata, H. Method for Determining Nozzle-Throat-Erosion-History in Hybrid Rockets. J. Propuls. Power 2017, 33, 1369-1377. [CrossRef]

27. Kamps, L.; Hirai, S.; Sakurai, K.; Viscor, T.; Saito, Y.; Guan, R.; Isochi, H.; Adachi, N.; Itoh, M.; Nagata, H. Investigation of Graphite Nozzle Erosion in Hybrid Rockets Using O2/C2H4. In Proceedings of the 54th AIAA/SAE/ASEE Joint Propulsion Conference, Cincinnati, OH, USA, 9-11 July 2018. [CrossRef]

28. Saito, Y.; Uematsu, T.; Isochi, H.; Wakita, M.; Totani, T.; Nagata, H. Estimation of Hybrid Rocket Nozzle Throat Erosion History. Trans. JSASS 2016, 14, 145-151. [CrossRef]

29. Gordon, S.; McBride, B. Computer Program for Calculation of Complex Chemical Equilibrium Compositions and Applications; NASA RP-1311; NASA: Washington, DC, USA, 1994.

30. Marxman, G.A.; Wooldridge, C.E.; Muzzy, R.J. Fundamentals of Hybrid Boundary-Layer Combustion. In Heterogeneous Combustion, 1st ed.; Progress in Astronautics and Aeronautics; Wolfhard, H.G., Glassman, I., Green, L., Eds.; AIAA: New York, NY, USA, 1964; Volume 15, pp. 485-522. [CrossRef]

31. Sutton, G.; Biblarz, O. Solid Propellant Rocket Fundamentals. In Rocket Propulsion Elements, 8th ed.; Wiley: Hoboken, NJ, USA, 2010; pp. 435-489.

32. G347 Graphite. Available online: https://www.tokaicarbon.co.jp/en/products/fine_carbon/pdf/Isotropic_ graphite.pdf (accessed on 28 January 2019).

33. Lutcov, A.I.; Volga, V.I.; Dymov, B.K. Thermal Conductivity, Electric Resistivity and Specific Heat of Dense Graphite. Carbon 1970, 8, 753-760. [CrossRef]

34. Butland, A.T.D.; Maddison, R.J. The Specific Heat of Graphite: An Evaluation of Measurements. J. Nucl. Mater. 1973, 49, 49-56. [CrossRef]

35. Rasband, W.S. Imagej; U.S. National Institutes of Health: Bethesda, MD, USA, 1997-2018. Available online: https://imagej.nih.gov/ij/ (accessed on 3 August 2018).

36. Kumar, C.P.; Kumar, A. Effect of Diaphragms on Regression Rate in Hybrid Rocket Motors. J. Propuls. Power 2013, 29, 559-572. [CrossRef]

37. Kumar, R.; Ramakrishna, P.A. Enhancement of Hybrid Fuel Regression Rate Using a Bluff Body. J. Propuls. Power 2014, 30, 909-916. [CrossRef]

38. DeLuca, L.T.; Galfetti, L.; Colombo, G.; Maggi, F.; Bandera, A.; Boiocchi, M.; Gariani, G.; Merotto, L.; Paravan, C.; Reina, A. Time-Resolved Burning of Solid Fuels for Hybrid Rocket Propulsion. Prog. Propuls. Phys. 2011, 2, 405-426. [CrossRef]

39. Pavaran, C.; Reina, A.; Sossi, A.; Manzoni, M.; Massini, G.; Rambaldi, G.; Duranti, E.; Adami, A.; Seletti, E.; DeLuca, L.T. Time-Resolved Burning of Solid Fuels for Hybrid Rocket Propulsion. Prog. Propuls. Phys. 2011, 2, 405-426. [CrossRef]

40. Evans, B.; Favorito, N.; Kuo, K. Oxidizer-Type and Aluminum-Particle Addition Effects on Solid-Fuel Burning Behavior. In Proceedings of the 42nd AIAA/SAE/ASEE Joint Propulsion Conference, Sacramento, CA, USA, 9-12 July 2006. [CrossRef]

41. Chiaverini, M. Review of Solid-Fuel Regression Rate Behavior in Classical and Nonclassical Hybrid Rocket Motors. In Fundamentals of Hybrid Rocket Propulsion, 1st ed.; Progress in Astronautics and Aeronautics; Chiaverini, M., Kuo, K., Eds.; AIAA: Washington, DC, USA, 2006; Volume 218, pp. 37-116. [CrossRef] 



\title{
Innovative Methods to Enhance the Combustion Properties of Solid Fuels for Hybrid Rocket Propulsion
}

\author{
Suhang Chen ${ }^{1}$, Yue Tang ${ }^{2}$, Wei Zhang ${ }^{1, *}$, Ruiqi Shen ${ }^{1, *}$, Hongsheng Yu ${ }^{1}$, Yinghua $\mathrm{Ye}^{1}$ and \\ Luigi T. DeLuca ${ }^{1,3}$ \\ 1 Institute of Space Propulsion (ISP), School of Chemical Engineering, Nanjing University of Science and \\ Technology, Nanjing 210094, China; chensuhang_nust@126.com (S.C.); hongshengyu2017@foxmail.com (H.Y.); \\ yyinghua@mail.njust.edu.cn (Y.Y.); luigi.t.deluca@gmail.com (L.T.D.) \\ 2 Department of Forensic Science and Technology, Jiangsu Police Institute, Nanjing, 210031, China; \\ tangyue090@163.com \\ 3 Space Propulsion Laboratory (SPLab), Department of Aerospace Science and Technology, \\ Politecnico di Milano (RET), I-20156 Milan, Italy \\ * Correspondence: wzhang@njust.edu.cn (W.Z.); rqshen@njust.edu.cn (R.S.); \\ Tel.: +86-025-8431-5855 (W.Z.); +86-025-8431-5076 (R.S.)
}

Received: 8 March 2019; Accepted: 17 April 2019; Published: 22 April 2019

\begin{abstract}
The low regression rates for hydroxyl-terminated polybutadiene (HTPB)-based solid fuels and poor mechanical properties for the alternative paraffin-based liquefying fuels make today hybrid rocket engines far from the outstanding accomplishments of solid motors and liquid engines. In this paper, a survey is conducted of several innovative methods under test to improve solid fuel properties, which include self-disintegration fuel structure (SDFS)/paraffin fuels, paraffin fuels with better mechanical properties, high thermal conductivity fuels and porous layer combustion fuels. In particular, concerning $\mathrm{HTPB}$, new results about diverse insert and low-energy polymer particles enhancing the combustion properties of HTPB are presented. Compared to pure HTPB, regression rate can be increased up to $21 \%$ by adding particles of polymers such as $5 \%$ polyethylene or $10 \%$ oleamide. Concerning paraffin, new results about self-disintegrating composite fuels incorporating Magnesium particles (MgP) point out that $15 \% 1 \mu \mathrm{m}$ - or $100 \mu \mathrm{m}-\mathrm{MgP}$ formulations increase regression rates by $163.2 \%$ or $82.1 \%$ respectively, at $335 \mathrm{~kg} / \mathrm{m}^{2}$.s oxygen flux, compared to pure paraffin. Overall, composite solid fuels featuring self-disintegration structure appear the most promising innovative technique, since they allow separating the matrix regression from the combustion of the filler grains. Yet, the investigated methods are at their initial stage. Substantial work of refinement in this paper is for producing solid fuels to fulfill the needs of hybrid rocket propulsion.
\end{abstract}

Keywords: hybrid; regression rate; self-disintegration; HTPB; paraffin; low-energy polymer; magnesium

\section{Introduction}

Hybrid engines testing dates back to the very beginning of rocket propulsion development, both in USA and Russia. The famous American pioneer Robert H. Goddard started his experiments by injecting little quantities of graphite into a small combustion chamber, aiming at controlling burning rate, achieving stop/restart capability, and avoiding the hazards of large amounts of fuel stored in the combustion chamber. Because of the complexity of feeding powder into the combustor, Goddard eventually turned to liquid propulsion and in 1926 was able to launch the first liquid rocket engine reaching $14 \mathrm{~m}$ altitude, followed by 33 more launches up to 1941 [1]. The famous Russian designer Sergei Pavlovich Korolev [2], in 1933, was able to reach $1500 \mathrm{~m}$ altitude with the maiden flight of the GIRD-09 hybrid rocket engine (HRE) burning gelled gasoline and LOx. 
In spite of these very precocious activities, still today hybrid rocket propulsion is far from the huge success later obtained by the companion liquid and solid rocket propulsion. In most cases, it happened that only the solid and liquid rocket systems were developed to operational status. In fact, HRE found applications for small-size engines, but still today no large-size engine exists able to compete with the relative simplicity of solid propulsion or the superior performance of liquid propulsion. Yet, several attempts were made. A first example is the study of high-energy space engines promoted by NASA in the mid-1960s. United Technology Center-Chemical Systems Division (UTC-CSD) considered [1] a hypergolic and very energetic reaction between $\mathrm{Li}$ and $\mathrm{F}_{2}$ by incorporating a mixture of $\mathrm{Li}+\mathrm{LiH}$ in a matrix of HTPB, while $\mathrm{F}_{2}$ was mixed with $\mathrm{O}_{2}$ to create the mixture $\mathrm{F}_{2}+\mathrm{O}_{2}$ (FLOx). The versatility of hybrid propulsion easily allowed to optimize the performance of an otherwise traditional hydrocarbon propellant system. The hybrid engine featured an 11-port wagon wheel solid grain. This system was throttleable, burned smoothly, and exhibited impressive high performance with a specific impulse efficiency of $93 \%$, achieving a delivered vacuum specific impulse of about $380 \mathrm{~s}$ with a nozzle area ratio of 40. A picture of the fire test was reproduced on the cover page of Aviation Week of 26 January 70.

A second example is the high-energy boosters for space launchers. Following the tragic accident of the Challenger Space Shuttle solid boosters in 1986, NASA considered replacing them with hybrid units aiming at increasing safety while decreasing the cost of space access. Two experimental programs, Hybrid Technology Options Project (HyTOP) and Hybrid Propulsion Demonstration Program (HPDM), were carried out for years at Marshall Space Flight Center (MSFC) in cooperation with Defense Advanced Research Projects Agency (DARPA). But after intensive static fire testing of large hybrid engines burning LOx/HTPB (8- or 15-port wagon wheel solid grain) capable of $1.1 \mathrm{MN}$ thrust in vacuum up to $80 \mathrm{~s}$ burning time, NASA gave up despite the potential advantages for safety, cost, and versatility of propellant selection.

The difficulties that hindered the full-scale development of HRE essentially regard the solid-phase fuel: in the classical hybrid formulations, this consists of HTPB (borrowed from composite solid propellants), which has good mechanical properties but too low regression rates; the opposite is true for the alternative paraffin-based liquefying fuels. Moreover, the intrinsic architecture of HRE forces a macroscopic diffusion flame conducive to poor combustion efficiency compared to solid and liquid rockets.

\section{Literature Survey and Objective}

After so much history, the "hybrid option" remains very attractive. The inherent safety in both manufacture and operation (zero TNT equivalent, insensitivity to cracks and defects), low-cost and thrust throttleability, and terminate/restart capability [3] make hybrid rocket engines an economical alternative to suborbital flight, space tourism, small satellite orbital injection [4], booster and upper stage [5], Lunar [6] and Mars lander spacecraft [7]. Unfortunately, the low regression rates of classical hybrid fuels limit the motor thrust, resulting in complicated fuel grain design and scaling, because of the low heat feedback (radial blocking effect and low thermal conduction of gasified products for non-metallized or non-radiating fuel) from the thin diffusion flame to the fuel surface.

Methods for increasing the fuel regression rate mainly consist of improving the heat feedback (nano-metallized fuels), fostering condensed phase reactions (using high-energy binder such as Guanidinium Azo-Tetrazolate (GAT) [8] and Glycidyl Azide Polymer (GAP) to decrease heat of degradation or adding ammonium perchlorate (AP) [9] to get oxygen-poor fuels), resorting to low enthalpy of vaporization fuels like paraffin liquefying fuels [10-14] to promote a mass-transfer mechanism, enhancing oxidation turbulence (swirling injector $[9,15,16]$ or helicoidally shaped spikes port injector [17]), designing multi-port grain (star and wagon wheel type or porous hybrid grains to increase the burning area [18]) and applying more reactive oxidants $\left(\mathrm{LO}_{2}-\mathrm{LF}_{2}\right.$ [19] and $\mathrm{LO}_{2}-\mathrm{N}_{2} \mathrm{O}$ [20,21]). Kuo et al. [22,23] reported $105 \%$ and $123 \%$ increase in regression rate, at the average oxygen mass flux $\overline{\mathrm{G}}_{0 x}=112 \mathrm{~kg} / \mathrm{m}^{2} \cdot \mathrm{s}$, by adding respectively $13 \%$ in mass of Alex (100 to $150 \mathrm{~nm}$ aluminum) and Viton-A coated Alex to a standard HTPB matrix. DeLuca et al. [24] found that introducing $11.2 \%$ in mass 
of $\mathrm{AlH}_{3}$ into an HTPB matrix promotes the regression rate of this fuels by $150 \%$ at $\bar{G}_{0 x}=11 \mathrm{~kg} / \mathrm{m}^{2} \cdot \mathrm{s}$. Carmicino and Russo-Sorge [25] tested a laboratory-scale hybrid rocket burning gaseous oxygen in a center-perforated cylindrical solid grain and, with respect to pure HTPB, verified for several high-energy fuel additives limited effects in terms of regression rate increase (say, 15-20\% based on oxygen mass flux) but also a considerably high characteristic-velocity and impulse efficiencies (around 95\% and 92\%). Karabeyoglu et al. $[13,14]$ demonstrated 3 to 4 times higher regression rates than those of conventional polymeric fuels for a class of paraffin-based fuels, suggesting its great potential for high power HREs after solving the mechanical properties of paraffin.

In this framework, a series of innovative techniques is currently under investigation at Nanjing University of Science and Technology (NUST) in order to overcome the solid fuel deficiencies so far experienced. Shen et al. [26-32] verified new concepts such as:

1. Insert and low-energy polymer particles enhancing HTPB combustion.

2. Self-disintegration fuel structure (SDFS) [26-28].

3. Porous layer combustion fuels [29,30].

4. High thermal conductivity fuels [31].

5. Paraffin fuels with better mechanical properties [32].

In this paper, each of the above new concepts is discussed separately and representative results are given for everyone. In addition, new experimental results are reported about the combustion performance of HTPB loaded with low-energy polymer particles (item 1) and paraffin-based SDFS fuels (item 2).

\section{Experimental}

The combustion characteristics of all tested fuels were examined in a gaseous oxygen flow by a 2D-radial hybrid combustion burner. All experiments were conducted at $1 \mathrm{MPa}$. Only the implemented radial burner is quickly described in this section. Preparation, composition, and properties of fuels are reported under the appropriate section headings that follow.

\section{Combustion Characterization}

The 2D-radial hybrid combustion burner (Figure 1) was previously described [26-32] and is based on the original design by SPLab [33,34]. Gaseous nitrogen is used to stabilize the chamber pressure during combustion and terminate the $\mathrm{HTPB}-\mathrm{O}_{2}$ reaction after turning off oxygen, while compressed air is used to save nitrogen before combustion and cool off the chamber after combustion. Gaseous oxygen is axially injected into the fuel grain port (a center perforated cylinder) at a mass flow rate of $5 \mathrm{~g} / \mathrm{s}$, controlled by a Bronkhorst F202 flowmeter. An automatic control system, which is equipped with a logical circuit, a pressure transducer, four solenoid valves, and four electromagnetic relays, governs the solenoid valves opening or closing every $30 \mathrm{~ms}$ to keep the combustion chamber in a quasi-steady state. A charge (4.5 mm OD $\times 0.8-1.2 \mathrm{~mm}$ thickness) of $\mathrm{B} / \mathrm{KNO}_{3}(40 / 60)$ which is fixed upstream of the fuel grain port is activated by an Nd:YAG laser, followed by the ignition of the inner surface of the cylindrical fuel grain under oxygen flow. A high-speed camera is used to record the regression process of the inner burning surface upstream cross-section at $1500 \mathrm{fps}$.

The high-speed photographs are processed as $\Delta D(t)$ vs. $t, G_{O x}(t)$ vs. $t, \dot{r}(t)$ vs. $t$, and $\dot{r}(t)$ vs. $G_{o x}(t)$, as discussed in detail in References [31,33,34]. $\Delta D(t)$ evolution is the diameter variation of the inner burning surface of the regressing fuel grain at time $t, G_{o x}(t)$ is the oxygen mass flux (mass flow rate per unit area, $\left.\mathrm{kg} /\left(\mathrm{m}^{2} \cdot \mathrm{s}\right)\right)$ at time $t$, and $\dot{r}(t)$ is the regression rate of the solid fuel at time $t$. The resulting function $\dot{r}(t)$ vs. $G_{O x}(t)$ is used to evaluate the combustion characteristics of the fuel under study. Finally, the mass burning rate from the central port fuel grain is simply expressed as $\dot{m}_{f}(t)=A_{b} \dot{r}(t) \rho_{f}$, where 
$A_{b}$ is the combustion surface area and $\rho_{f}$ the density of the fuel. For the regression rate, the classical power law empirical formula of hybrid combustion, based on oxidizer mass flux, is typically used.

$$
r_{f}(t)=a_{r} G_{o x}^{n_{r}}(t)\left(t \geq t_{i g n}>t_{0}\right)
$$

where the reference starting time $t_{0}=0 \mathrm{~s}$ is set, for each test, as the instant at which the external ignition stimulus (laser on) is first applied to the primer charge while the ignition time $t_{\text {ign }}=t_{0}+\Delta t_{\text {ign }}$ is conventionally taken as the first instant at which the sample cross section appears fully inflamed. Thus, the observed ignition delay $\Delta t_{i g n}$ includes laser activation, primer charge ignition, and fuel sample inflammation. Assuming a quasi-steady burning regime has established, all plotted regression rate curves start at $t_{i g n}$ and the investigated measurement range cover the whole combustion process until extinction. In a log plot, $n_{r}$ is the slope of the regression rate straight line evaluated according to Equation (1).

The 2D-radial burner is a lab scale tool and one of its advantages is the possibility of visual and direct observation of the hybrid combustion process. Other 2D burners were designed and are in use at SPLab implementing a simpler planar geometry consisting of a single or double solid fuel slab $[33,34]$. Due to the different boundary conditions, the experimental regression rates differ for each burner. For the standard HTPB fuel, under the same operating conditions (about $0.15 \mathrm{MPa}$ for the planar burners), the measured regression rates in the 2D-radial burner is $80 \%$ larger than that in the companion 2D-double slab, in turn 26\% larger than the corresponding 2D-single slab, the slowest of the crop [33]. With reference to Figure 6 of Reference [25], results from the 2D-radial burner are reasonably close to the line denoted as "HTPB Literature". Other fitting expressions of the solid fuel regression rate, for example, based on total mass flux as recommended in Reference [25], can be implemented as well. Since a large number of results was obtained as above described at SPLab and NUST, for consistency data processing is carried out in this work using Equation (1) based on the oxidizer mass flux, as done in the vast majority of the literature. Moreover, keep in mind that the 2D-radial burner provides instantaneous values of regression rate, while data from engines are in most cases space- and time-averaged.

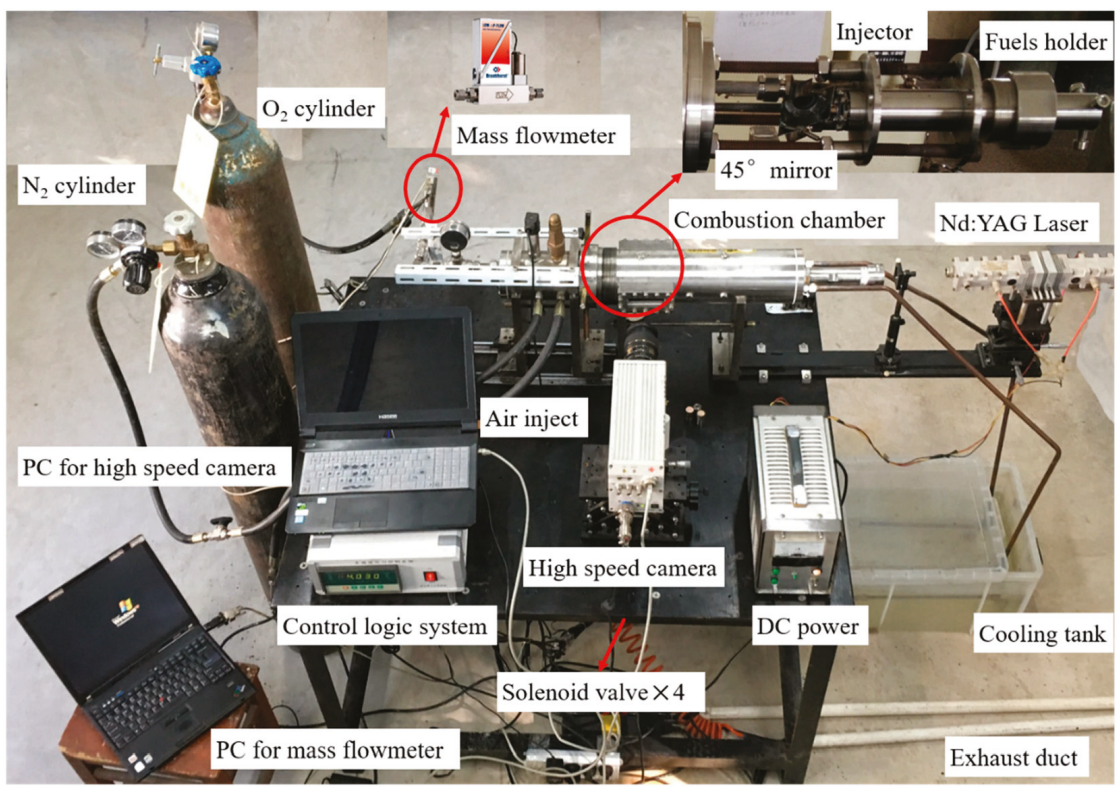

Figure 1. Optical image of the 2D-radial hybrid propulsion burner system. 


\section{Low-Energy Polymer Particles/HTPB}

Although energetic ingredients are the preferred additives in conventional studies, insert and low-energy polymer particles offer an additional method to enhance HTPB combustion. In this work, previously unreported PE paraffin (low molecular mass polyethylene), oleamide, polydextrose, and polyethylene glycol (PEG) particles are all additives with poorer combustion performance and lower decomposition temperature than HTPB. PE paraffin is a commercial polymer which has a lower regression rate than HTPB definitely [35]. Oleamide (- $\mathrm{NH}_{2}$ groups) particles reacting with -OCN groups and reducing the mechanical properties of HTPB, potentially reduce the degree of polymerization and decomposition heat. Polydextrose or PEG particles provide a certain amount of oxygen and their surface contains very few - $\mathrm{OH}$ groups reducing mechanical properties only very slightly. These four kinds of polymers are extremely low-cost (less than $1 \$ / \mathrm{kg}$ ), while HTPB prepolymer needs $15 \$ / \mathrm{kg}$ without considering the more expensive ingredients dioctyl adipate (DOA) and isophorone diisocyanate (IPDI). The cost can further be reduced by introducing a certain amount of polymer particles without lowering the regression rate.

\subsection{Low-Energy Polymer Particles/HTPB Fuel Preparation}

HTPB, IPDI, DOA, dibutyltin diacetate (TIN), and four kinds of polymer particles (PE paraffin, oleamide, polydextrose, and PEG) are the raw materials used for sample preparation. HTPB was purchased from Liming Research and Design Institute of Chemical Industry Co. (Luoyang, China). with a number-average molecular mass of 2940 and $-\mathrm{OH}$ content of $0.787 \mathrm{mmol} / \mathrm{g}$. IPDI, DOA and TIN were obtained from Aladdin. The physical properties of PE paraffin, oleamide, polydextrose, and polyethylene glycol particles are listed in Table 1.

Table 1. Physical properties of the tested polymer particles.

\begin{tabular}{|c|c|c|c|c|c|}
\hline $\begin{array}{l}\text { Polymer } \\
\text { Particles }\end{array}$ & Source & $\begin{array}{l}\text { Molecular } \\
\text { Mass }\end{array}$ & $\begin{array}{l}\text { Melting Point } \\
\left({ }^{\circ} \mathrm{C}, 1 \text { atm }\right)\end{array}$ & $\begin{array}{l}\text { Boiling Point } \\
\left({ }^{\circ} \mathrm{C}, 1 \mathrm{~atm}\right)\end{array}$ & $\begin{array}{l}\text { Density } \\
\left(\mathrm{g} / \mathrm{cm}^{3}\right)\end{array}$ \\
\hline НТРВ & {$[31]$} & - & 413 & 453 & 0.922 \\
\hline PE paraffin & \multirow{2}{*}{$\begin{array}{l}\text { Dinghai Plastic Chemical } \\
\text { Co., Ltd. (Dongguan, China) }\end{array}$} & $2000-5000$ & 96 & 270 & 0.94 \\
\hline Oleamide & & 281.5 & $72-77$ & 433.3 & 0.879 \\
\hline Polydextrose & $\begin{array}{l}\text { Weiduome Food Co., Ltd. } \\
\text { (Shen Zhen, China) }\end{array}$ & Average-3200 & $153-158$ & 410.8 & 1.587 \\
\hline PEG6000 & $\begin{array}{c}\text { Ipsen S.A. } \\
\text { (Boulogne-Billancourt, } \\
\text { France) }\end{array}$ & $5500-7500$ & $64-66$ & $>250$ & 1.27 \\
\hline
\end{tabular}

The compositions of the tested polymer particles/HTPB composite fuels are tabulated in Table 2. The preparation process of HTPB-based fuels was described in our previous studies [31] and is shown in Figure 2. The cured HTPB was prepared at a ratio NCO: OH of 1.05. Briefly, the corresponding content of HTPB was dispersed under vacuum $(<5 \mathrm{kPa})$ with a blade mixer rate of $200 \mathrm{rpm}$ for $10 \mathrm{~min}$, followed by DOA and TIN for $5 \mathrm{~min}$. The resulting mixture was mixed with polymer particles for $20 \mathrm{~min}$, followed by IPDI stirring for another $10 \mathrm{~min}$. The uncured polymer particles/HTPB fuels were cast into several molds to get 4-6 samples (16 mm OD $\times 4 \mathrm{~mm}$ ID $\times 30 \mathrm{~mm}$ length). The samples were solidified at $36^{\circ} \mathrm{C}$ in an oven for $23 \mathrm{~h}$ and a $2 \mathrm{~h}$ post-cure bake at $60{ }^{\circ} \mathrm{C}$.

Table 2. Composition of the tested $0,5 \%, 10 \%$, and 20\% polymer particles/HTPB composite fuels

\begin{tabular}{cccccc}
\hline Fuel Name & HTPB & DOA & IPDI & TIN & Polymer Particles \\
\hline Pure HTPB & 78.86 & 13.04 & 7.67 & 0.43 & $0 \%$ \\
5\% Polymer/HTPB & 74.91 & 12.39 & 7.29 & 0.41 & $5 \%$ \\
10\% Polymer/HTPB & 70.97 & 11.74 & 6.90 & 0.39 & $10 \%$ \\
20\% Polymer/HTPB & 63.09 & 10.43 & 6.14 & 0.34 & $20 \%$ \\
\hline
\end{tabular}




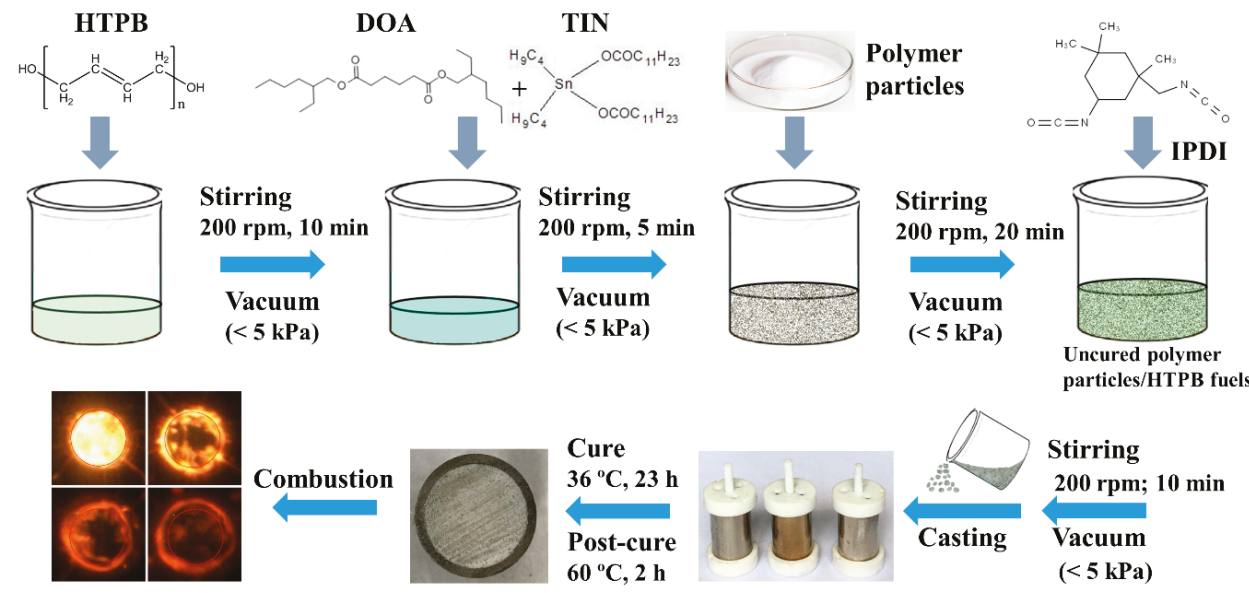

Figure 2. Procedure for the preparation of polymer particles/HTPB (hydroxyl-terminated polybutadiene) composite fuels.

\subsection{Low-Energy Polymer Particles Morphology}

The morphological features and diameter distribution of raw polymer particles were detailed by Confocal Laser Scanning Microscope (CLSM, LEXT OLS3100, Olympus, Tokyo, Japan) on glass substrates; see Figure 3. PE paraffin, oleamide, polydextrose, and PEG respectively have an average diameter of $49.9 \mu \mathrm{m}, 425.4 \mu \mathrm{m}, 44.0 \mu \mathrm{m}$, and $182.0 \mu \mathrm{m}$.
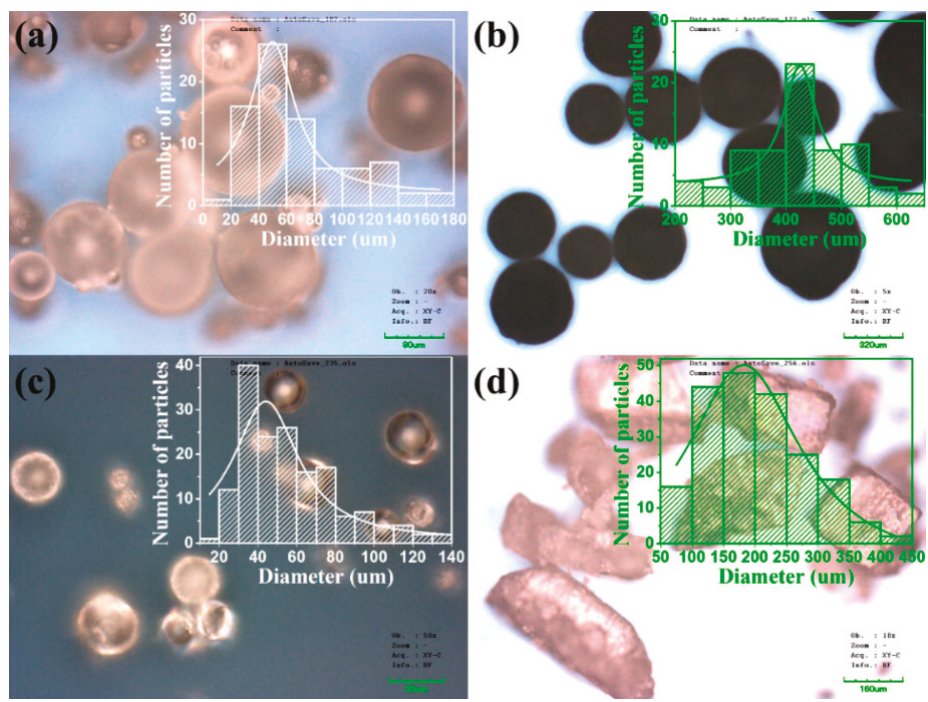

Figure 3. CLSM (Confocal Laser Scanning Microscope) images of (a) PE paraffin, (b) oleamide, (c) polydextrose, (d) PEG6000 particles. 


\subsection{Low-Energy Polymer Particles/HTPB Composite Fuel Combustion}

Figure 4 shows the regression state of $5 \%$ PE particles/HTPB composites during burning at $t=0$ $\mathrm{s}\left(t_{\text {ign }}\right), 1 \mathrm{~s}, 2 \mathrm{~s}, 3 \mathrm{~s}$ and $5 \mathrm{~s}$, respectively. The red circle represents the diameter of the initial internal burning surface diameter $(4 \mathrm{~mm})$. The brightness of the $5 \%$ PE particle formulation image is darker than HTPB, suggesting less energy release compared to HTPB and making difficult regression data collection. From the viewpoint of cross-section diameters which were processed as $\dot{r}(t)$ vs. $t$, the $5 \%$ PE particle formulation shows a slightly enhanced regression rate compared to HTPB.
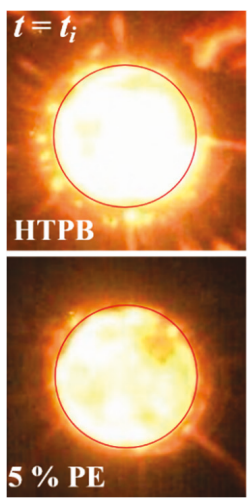
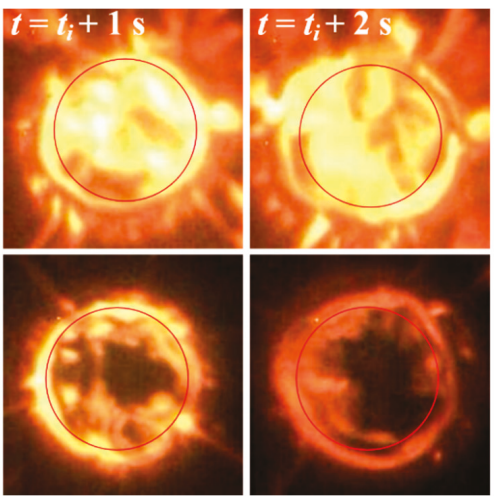
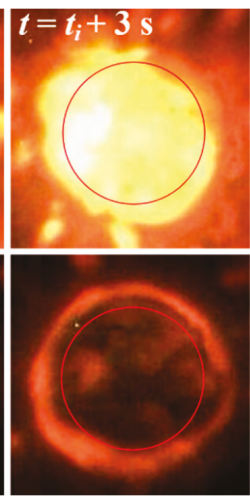
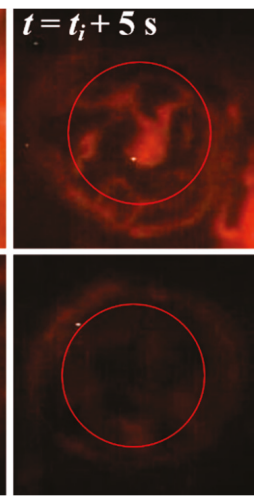

Figure 4. Photograph of regression process of pure HTPB (top) and polymer particles/HTPB (bottom) fuels. All pictures were obtained at the same exposure and aperture.

The fuel density was calculated from the sample mass and dimensions. HTPB containing PE paraffin (5\%,10\%, and 20\%), oleamide particles (5\%,10\%, and $20 \%$ ), $10 \%$ polydextrose, and $10 \%$ PEG6000 particles have a density of 922 (pure HTPB), 925, 927, 931, 918, 917, 914, 980 and 958 kg/m³. Compared to the HTPB matrix alone, the tested fuels reinforce the regression rate at $G_{0 x}=350 \mathrm{~kg} / \mathrm{m}^{2} \cdot \mathrm{s}$ and $G_{0 x}=150 \mathrm{~kg} / \mathrm{m}^{2} \cdot \mathrm{s}$, respectively, as shown in Figure 5 and Table 3. This indicates that the optimal addition of polymers is $5 \%$ PE particles (up to $\approx 21 \%$ increase) and $10 \%$ oleamide particles (up to $\approx 17 \%$ increase), despite the insert polymer particles depress combustion performance in terms of released energy.

Table 3. $r_{f}$ increase and fitting results of $r_{f} / G_{0 x}$ curves for polymer particles/HTPB composite fuels.

\begin{tabular}{|c|c|c|c|c|}
\hline Fuel Name & $\begin{array}{l}r_{f} \text { Increase at } \\
350 \mathrm{~kg} /\left(\mathrm{m}^{2} \cdot \mathrm{s}\right)\end{array}$ & $\begin{array}{l}r_{f} \text { Increase at } \\
150 \mathrm{~kg} /\left(\mathrm{m}^{2} \cdot \mathrm{s}\right)\end{array}$ & $\begin{array}{c}G_{o x} \text { Range from } 150 \text { to } \\
310 \mathrm{~kg} / \mathrm{m}^{2} \cdot \mathrm{s}\end{array}$ & $R^{2}$ \\
\hline Pure HTPB & - & - & $\dot{r}_{f}=\left(6.59 \times 10^{-4}\right) G_{o x} 1.043$ & 0.970 \\
\hline $5 \%$ PE paraffin & $20.7 \%$ & $4.2 \%$ & $\dot{r}_{f}=\left(5.05 \times 10^{-4}\right) G_{o x}{ }^{1.112}$ & 0.970 \\
\hline $10 \%$ PE paraffin & $3.2 \%$ & $-21.1 \%$ & $\dot{r}_{f}=\left(1.58 \times 10^{-4}\right) G_{o x}{ }^{1.255}$ & 0.971 \\
\hline $20 \%$ PE paraffin & $-0.7 \%$ & $-11.3 \%$ & $\dot{r}_{f}=\left(1.91 \times 10^{-4}\right) G_{o x} 1.267$ & 0.970 \\
\hline $5 \%$ oleamide & $3.0 \%$ & $-15.5 \%$ & $\dot{r}_{f}=\left(1.78 \times 10^{-4}\right) G_{o x}{ }^{1.257}$ & 0.970 \\
\hline $10 \%$ oleamide & $7.8 \%$ & $2.9 \%$ & $\dot{r}_{f}=\left(4.56 \times 10^{-4}\right) G_{o x} 1.116$ & 0.970 \\
\hline $20 \%$ oleamide & $18.6 \%$ & $-25.4 \%$ & $\dot{r}_{f}=\left(6.49 \times 10^{-5}\right) G_{o x}{ }^{1.441}$ & 0.971 \\
\hline $10 \%$ polydextrose & $10.6 \%$ & $-14.1 \%$ & $\dot{r}_{f}=\left(1.13 \times 10^{-4}\right) G_{o x} 1.350$ & 0.976 \\
\hline 10\% PEG6000 & 21. $4 \%$ & $-19.0 \%$ & $\dot{r}_{f}=\left(1.66 \times 10^{-4}\right) G_{0 x} 1.277$ & 0.970 \\
\hline
\end{tabular}


$(\mathbf{a})_{0.6}$

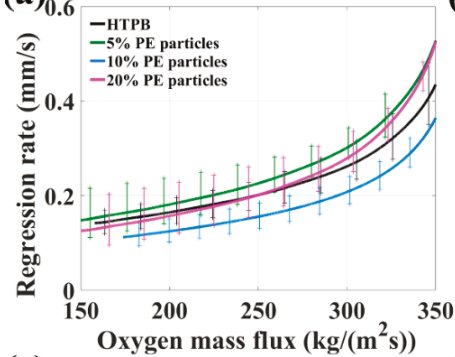

(c)

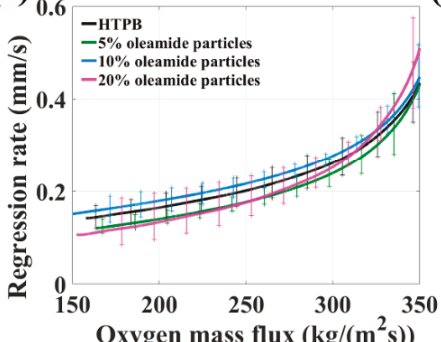

(e) 0.6

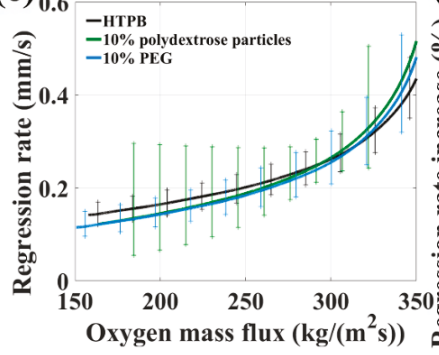

(b)

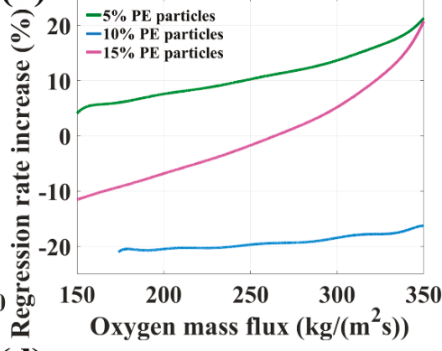

(d)

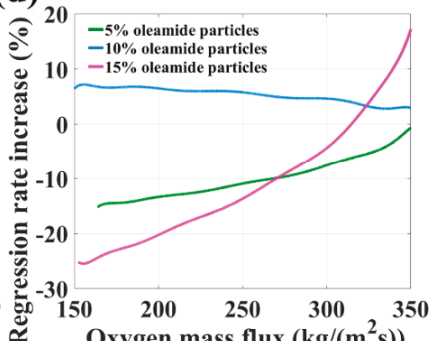

(f)

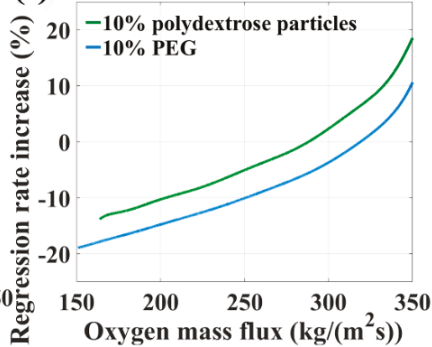

Figure 5. (a,c,e) Regression rate and (b,d,f) percent increase in regression rate vs. oxygen mass flux for low molecular mass polyethylene (PE paraffin) $(5 \%, 10 \%$, and $20 \%) / \mathrm{HTPB}$, oleamide $(5 \%, 10 \%$, and $20 \%$ )/HTPB, $10 \%$ polydextrose/HTPB, and 10\% PEG6000/HTPB composite fuels, respectively.

From the viewpoint of burning surface (Figure 6), 10\% PEG formulation appears more uniform and smoother with several holes which were left by PEG particles melting and flowing, while the melted and partly pyrolytic HTPB reformed porous edge with gas and some black carbon products attaching on the surface. Excess PEG particles are more susceptible to melting, pyrolysis and evaporation, resulting in reduced heat feedback to the HTPB surface, blocking the regression rate of HTPB slightly and exposing the poor combustion properties of polymer particles. $10 \%$ oleamide features a relatively large $a_{r}$ as well as low $n_{r}$ and shows a better regression rate than $5 \%$ and $20 \%$, suggesting a convenient compromise of poor oleamide combustion performance and a relatively lower degree of polymerization. The oxygen element in polydextrose and PEG particles yield no enhancement on HTPB regression rate due to the pyrolysis and evaporation process into the flame zone, involving no condensed phase reaction. 


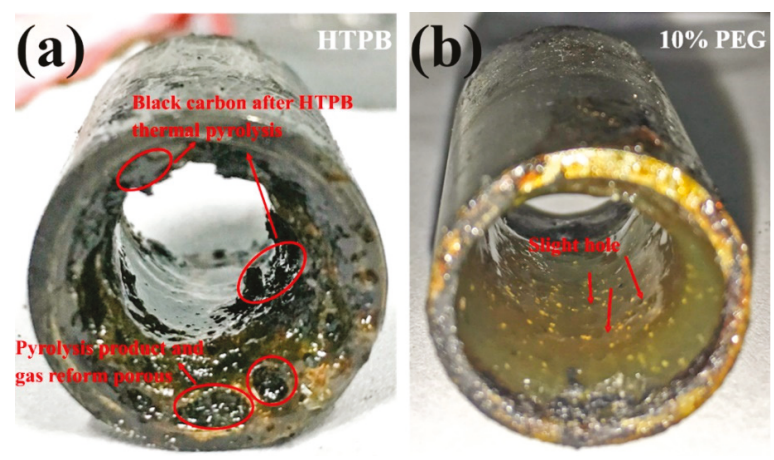

Figure 6. Images of (a) pure HTPB and (b) 10\% PEG particles/HTPB composite fuel grains after terminating combustion.

\section{Self-Disintegration Fuel Structure (SDFS)/Paraffin}

SDFS is a concept borrowed from composite modified double-base propellant [36] and new for hybrid propellant burning, whereby the binder will release fuel particles from a block of composite solid fuel over some critical threshold condition (for example, a melting temperature). Combustion visualization shows a lot of burning granules of the implemented filler escaping from the burning surface into the gas flow; see Figure 7. During combustion, the solid fuels disintegrate into many small parts due to melting and decomposition of binder, and flow out with the sweep of oxygen flow, realizing the structural self-disintegration phenomenon. The self-disintegration process needs less energy and reaction time than the evaporation and thermal decomposition processes of conventional fuels so that the mass transportation per unit time can greatly improve, and the regression rate and mass burning rate will be enhanced. Thus, the scattering of particles associated with the self-disintegrating matrix structure can promote propulsion performance.

Being subject to a variety of chemical and physical effects, the effect of the particle size is not obvious: in general, large filler particles carry away more mass and volume from the regressing surface when they escape into the gas flow, while small filler particles offer a better diffuse particle dispersion and a more abrupt particle scattering. So far four cases were implemented; each is recalled in the following subsections. Paraffin was used as a binder: Paraffin \#90 in Reference [26] and Paraffin \#58 in References $[27,28,32]$ and in this work; their main properties are shown in Table 4.

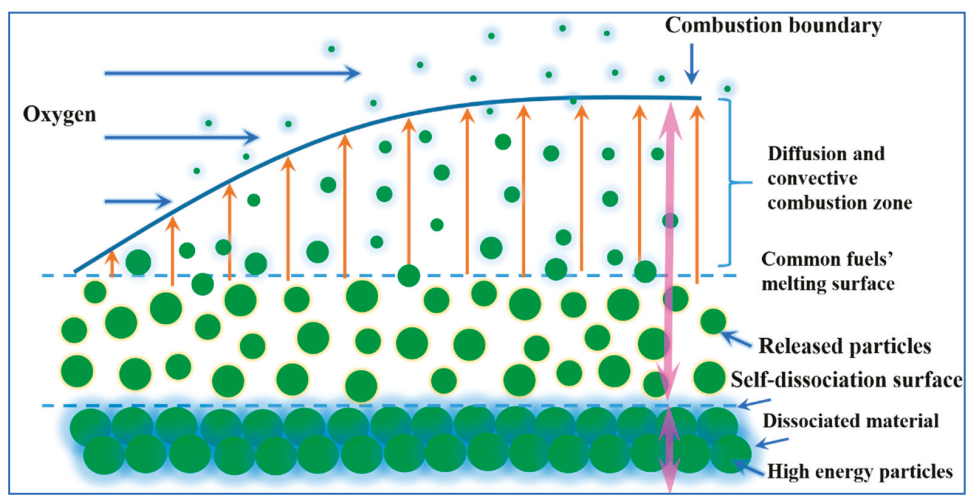

Figure 7. Self-disintegration mechanism of particle/paraffin composite fuels [28]. Copyright Begell House (2017). Reproduced with permission. 
Table 4. Physical properties of the tested kinds of paraffin.

\begin{tabular}{cccccc}
\hline Fuel Name & $\begin{array}{c}\text { Melting Point } \\
\left({ }^{\circ} \mathbf{C}\right)\end{array}$ & $\begin{array}{c}\text { Combustion } \\
\text { Heat } \mathbf{( k J / g )}\end{array}$ & $\begin{array}{c}\text { Density } \\
\left(\mathbf{g} / \mathbf{c m}^{3}\right)\end{array}$ & $\begin{array}{c}\text { Compressive } \\
\text { Strength } \mathbf{( M P a})\end{array}$ & $\begin{array}{c}\text { Tensile Strength } \\
\mathbf{( M P a})\end{array}$ \\
\hline Paraffin \#58 & 58.3 & 47.36 & 0.917 & 2.84 & 2.76 \\
Paraffin \#90 & 72.2 & 46.19 & 0.927 & 1.40 & 2.78 \\
\hline
\end{tabular}

\subsection{Polystyrene Particles/Paraffin Fuel Combustion}

In the first attempt to realize an SDFS structure, inert low-energy polystyrene (PS) particles (D301) were blended with paraffin \#90 [26]. Two sizes of PS grains were tested: small $(0.60-0.71 \mathrm{~mm})$ and large $(0.85-1.00 \mathrm{~mm})$ ones. The experimental trend of the associated SDFS fuel regression rate is the same as that of pure paraffin, but its value is higher up to $25 \%$ and increasing for growing PS particles load in the range $5 \%$ to $15 \%$. For the tested fuel formulations, the power $n_{r}$ decreases with increasing particle size and thus it affects differently the regression rate values trends under high and low oxygen mass flux. In general, for large $G_{o x}$ values, the small size PS grains revealed more efficient than large size, and vice versa for low $G_{0 x}$ values. Only for $15 \%$ PS load, the formulation with large particles appears just slightly faster over the entire test range.

\subsection{Double-Base Particles/Paraffin Fuel Combustion}

In the second example of SDFS structure, active particles of double-base (DB) propellant were blended with paraffin \#58 [27]. Although the presence of solid propellant is not accepted in hybrids for space exploration missions, this formulation is useful to check the validity of the SDFS concept. The loaded DB particles were small cylinders with $0.28 \mathrm{~mm}$ average diameter and $0.65 \mathrm{~mm}$ average length (ranging from 0.47 to $0.81 \mathrm{~mm}$ ). During combustion, the settled particles are disaggregated from the molten paraffin matrix and detached from the burning surface due to the oxidant flow blowing. Over the range $G_{o x}=50-350 \mathrm{~kg} /\left(\mathrm{m}^{2} \cdot \mathrm{s}\right)$, combustion runs show that the regression rates and the power $n_{r}$ of all tested formulations raised monotonically with the loaded mass \% of DB particles; see Table 5 . Moreover, the more content of particles the more splatter. The average regression rates of formulations blended with $5 \%, 10 \%, 15 \%$, and $20 \%$ particles in mass were increased by $30 \%, 38 \%, 47 \%$ and $59 \%$ with respect to pure paraffin, respectively.

Table 5. $r_{f}$ increase and fitting results of $r_{f}$ vs. $G_{o x}$ for double-base (DB) particles/paraffin composite fuels with $0,5 \%, 10 \%, 15 \%, 20 \% \mathrm{DB}$ showing increasing regression rate and slope $n_{r}$ for increasing DB load [27]. Copyright Begell House (2017). Reproduced with permission.

\begin{tabular}{ccccc}
\hline Fuel Name & Average $r_{f}$ Increase & $a_{r}$ & $n_{r}$ & $R^{2}$ \\
\hline Paraffin \#58 & - & $0.048 \pm 0.001$ & $0.566 \pm 0.004$ & 0.917 \\
$5 \%$ DB & $30 \%$ & $0.045 \pm 0.001$ & $0.624 \pm 0.005$ & 0.895 \\
$10 \%$ DB & $38 \%$ & $0.040 \pm 0.001$ & $0.656 \pm 0.006$ & 0.875 \\
$15 \%$ DB & $47 \%$ & $0.039 \pm 0.001$ & $0.669 \pm 0.006$ & 0.889 \\
$20 \%$ DB & $59 \%$ & $0.033 \pm 0.001$ & $0.707 \pm 0.006$ & 0.884 \\
\hline
\end{tabular}

\subsection{Low-Density Polyethylene Particles/Paraffin Fuel Combustion}

In the third example of SDFS structure, inert low-energy particles of low-density polyethylene (LDPE) were blended with paraffin \#58 [28]. Compared with pure paraffin, the average regression rates of formulations blended with $5 \%$ and $10 \%$ LDPE particles increased by $9.0 \%$ and $22.2 \%$, respectively. However, for LDPE addition of $15 \%$ and $20 \%$, the average regression rates were reduced by $5.5 \%$ and $13.2 \%$, which was due to high melting point and high decomposition temperature of LDPE. Thus, the regression rates first increased and then decreased with an increasing load of LDPE particles.

As to the effects of the particle size, see Table 6 , the power $n_{r}$ of the regression fitting law decreased with increasing particle size implying that small particles favor regression rates under high oxygen 
mass flux while large particles favor regression rates under low oxygen mass flux. For example, the regression rates of fuels loaded with $15 \%$ LDPE particles, at $G_{0 x}=367 \mathrm{~kg} /\left(\mathrm{m}^{2} \cdot \mathrm{s}\right)$, increased by $21.0 \%$, $11.2 \%$, and $-1.6 \%$, respectively, moving from small $(0.18-0.45 \mathrm{~mm})$ to medium $(0.45-0.75 \mathrm{~mm})$ and to large $(0.75-1.00 \mathrm{~mm})$ particles.

Table 6. $r_{f}$ increase and fitting results of $r_{f}$ vs. $G_{o x}$ for low-density polyethylene (LDPE) particles/paraffin composite fuels, $15 \%$ LDPE showing decreasing slope $n_{r}$ for increasing particle size [28]. Copyright Begell House (2017). Reproduced with permission.

\begin{tabular}{ccccc}
\hline Fuel Name & $\begin{array}{r}\boldsymbol{r}_{f} \text { Increase at } \\
\left.\mathbf{3 6 7} \mathbf{~ k g} / \mathbf{m}^{2} \cdot \mathbf{s}\right)\end{array}$ & $\boldsymbol{a}_{\boldsymbol{r}}$ & $\boldsymbol{n}_{\boldsymbol{r}}$ & $\boldsymbol{R}^{\mathbf{2}}$ \\
\hline Paraffin \#58 & - & $0.048 \pm 0.001$ & $0.566 \pm 0.004$ & 0.917 \\
15\% LDPE small & $21.0 \%$ & $0.002 \pm 0.0001$ & $1.140 \pm 0.013$ & 0.817 \\
15\% LDPE medium & $11.2 \%$ & $0.006 \pm 0.0004$ & $0.938 \pm 0.012$ & 0.848 \\
15\% LDPE large & $-1.6 \%$ & $0.019 \pm 0.001$ & $0.732 \pm 0.007$ & 0.827 \\
\hline
\end{tabular}

A significant finding is that comparing the combustion response of the same LDPE/paraffin formulation, manufactured as an SDFS composite fuel rather than just a simple blend, points out quite a different behavior in terms of regression rate. For example, for the kinds of paraffin with 5\% and 10\% LDPE previously mentioned, the SDFS samples exhibit an increase of the average regression rate by $9.0 \%$ and $22.2 \%$ respectively, in marked contrast with the corresponding reduction by $39.0 \%$ and $58.9 \%$ featured by the blend formulations; for details, see Section 8. Being the ingredients and formulations the same, only the internal fuel structures are different. This discrepancy is direct proof that the SDFS composite fuels can play an important role in promoting regression rates.

\subsection{Magnesium Particles/Paraffin Fuel Preparation and Combustion}

Two sizes of magnesium particles ( 1 and $100 \mu \mathrm{m})$ were chosen to analyze the effects on combustion of composite fuels based on paraffin \#58. Their main properties are listed in Table 7 while their SEM images are shown in Figure 8. The tested MgP have high purity, and the specific surface area of small particles is much bigger than that of large particles, indicating that the small magnesium powders have higher reactivity and are easier to burn. Figure 9 shows optical microscope images of the two $\mathrm{MgP} /$ paraffin composite fuels and Figure 10 shows two samples ready for combustion test. The composition of the tested $\mathrm{MgP} /$ paraffin fuels is indicated in Table 8.

Figure 11 illustrates the regression process of magnesium particles/paraffin composite fuels and the arrows point out the burning $\mathrm{Mg}$ particles which disintegrate from the paraffin matrix. As the figure suggests, the structural self-disintegration of $\mathrm{Mg}$ particles/paraffin composite fuels was more obvious under high oxidizer mass flow rate, and the flame of magnesium particles was bright which shows high energy.

Table 7. Main properties of the tested magnesium particles.

\begin{tabular}{cccccc}
\hline $\begin{array}{c}\text { Particle Size } \\
(\mu \mathrm{m})\end{array}$ & $\begin{array}{c}\text { Active Content } \\
(\%)\end{array}$ & $\begin{array}{c}\text { Specific Surface } \\
\text { Area }\left(\mathbf{m}^{2} / \mathbf{g}\right)\end{array}$ & $\begin{array}{c}\text { Volume Density } \\
\left(\mathrm{g} / \mathbf{c m}^{3}\right)\end{array}$ & $\begin{array}{c}\text { Density } \\
\left(\mathrm{g} / \mathbf{c m}^{3}\right)\end{array}$ & $\begin{array}{c}\text { Crystal } \\
\text { Shape }\end{array}$ \\
\hline 1 & 99.9 & 18.75 & 1.17 & 1.70 & sphere \\
100 & 99.9 & 3.64 & 3.95 & 1.70 & sphere \\
\hline
\end{tabular}

Table 8. Composition of the tested magnesium particles/paraffin composite fuels.

\begin{tabular}{ccc}
\hline Fuel Name & Formulation (Mass Fraction/\%) & Density $\left(\mathrm{g} / \mathrm{cm}^{\mathbf{3}}\right)$ \\
\hline $15 \% 1 \mu \mathrm{m}-\mathrm{MgP}$ & $15 \% 1 \mu \mathrm{m} \mathrm{Mg}$ particles $+85 \%$ paraffin & 0.957 \\
$15 \% 100 \mu \mathrm{m}-\mathrm{MgP}$ & $15 \% 100 \mu \mathrm{m} \mathrm{Mg}$ particles $+85 \%$ paraffin & 0.928 \\
\hline
\end{tabular}




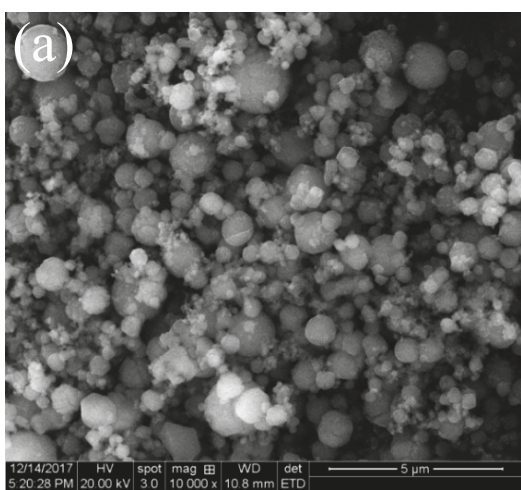

$1 \mu \mathrm{m}$

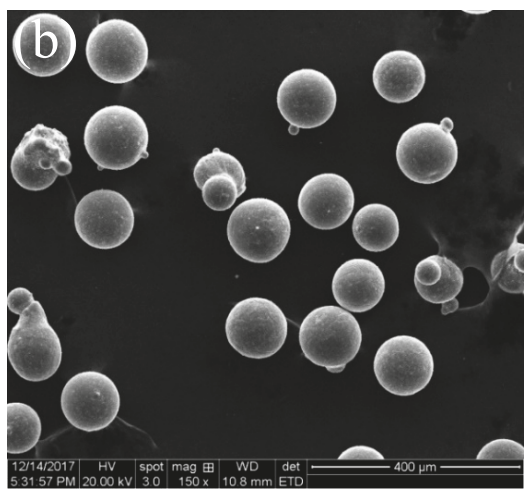

$100 \mu \mathrm{m}$

Figure 8. Scanning Electron Microscopy (SEM) images of small (a) and large (b) magnesium particles.

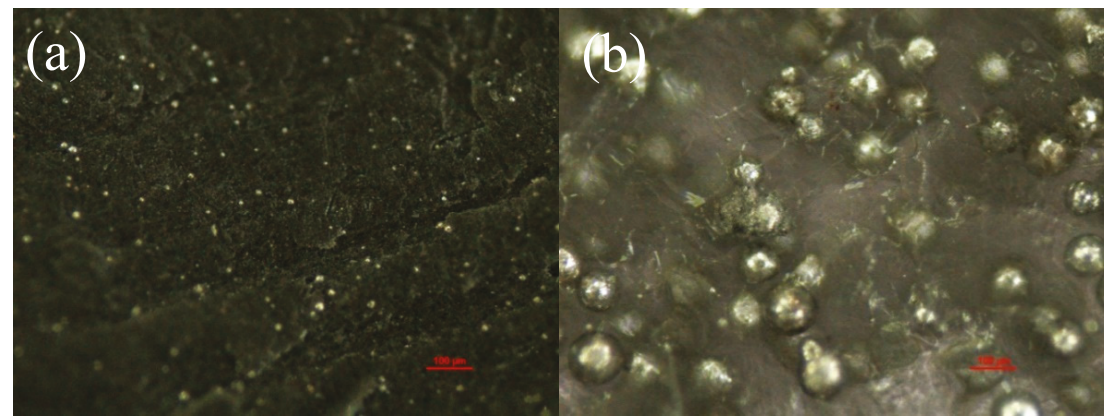

$15 \% 1 \mu \mathrm{m}-\mathrm{MgP}$

15\% $100 \mu \mathrm{m}-\mathrm{MgP}$

Figure 9. Optical microscope images of small (a) and large (b) magnesium particles/paraffin composite fuels.

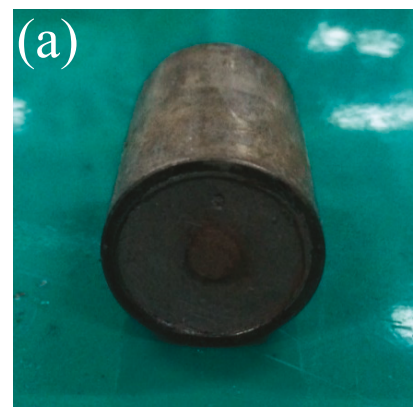

$15 \% 1 \mu \mathrm{m}-\mathrm{MgP}$

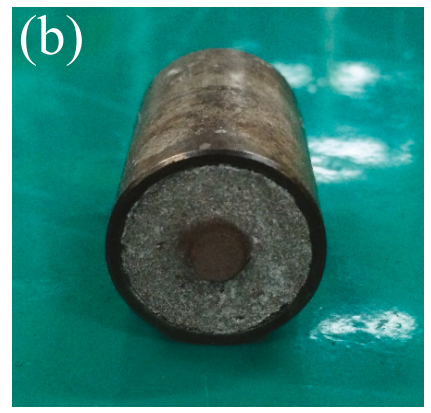

$15 \% 100 \mu \mathrm{m}-\mathrm{MgP}$

Figure 10. Combustion test samples of small (a) and large (b) magnesium particles/paraffin composite fuels. 

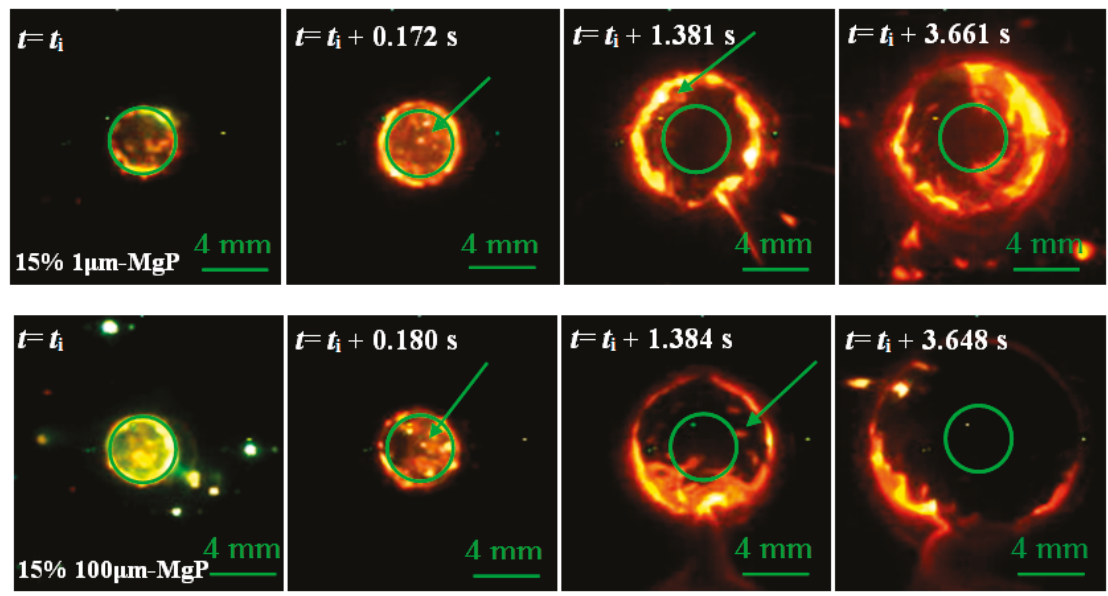

Figure 11. Combustion surface regression process of small (top) and large (bottom) magnesium particles/paraffin composite fuels.

The relationships between the regression rate and oxidizer mass flux of magnesium particles/paraffin composite fuels are illustrated in Figure 12. As the figures show, the influence of $1 \mu \mathrm{m}-\mathrm{MgP}$ on the combustion characteristics of composite fuels is quite different from that of $100 \mu \mathrm{m}-\mathrm{MgP}$. The regression rate of formulation 15\% $1 \mu \mathrm{m}-\mathrm{MgP}$ improved at high oxidizer mass flux but markedly decreased with decreasing oxidizer mass flux. On the contrary, the regression rate of formulation $15 \% 100 \mu \mathrm{m}-\mathrm{MgP}$ increased over the whole range of oxidizer mass flux and the increased percent was little dependent on the oxidizer mass flux. At $G_{0 x}=335 \mathrm{~kg} /\left(\mathrm{m}^{2} \cdot \mathrm{s}\right)$, the regression rate of formulations $15 \% 1 \mu \mathrm{m}-\mathrm{MgP}$ and $15 \% 100 \mu \mathrm{m}-\mathrm{MgP}$ increased by $163.2 \%$ and $82.1 \%$ with respect to pure paraffin, respectively, while at $100 \mathrm{~kg} /\left(\mathrm{m}^{2} \cdot \mathrm{s}\right)$, the regression rate respectively changed by $-47.6 \%$ and $49.2 \%$.
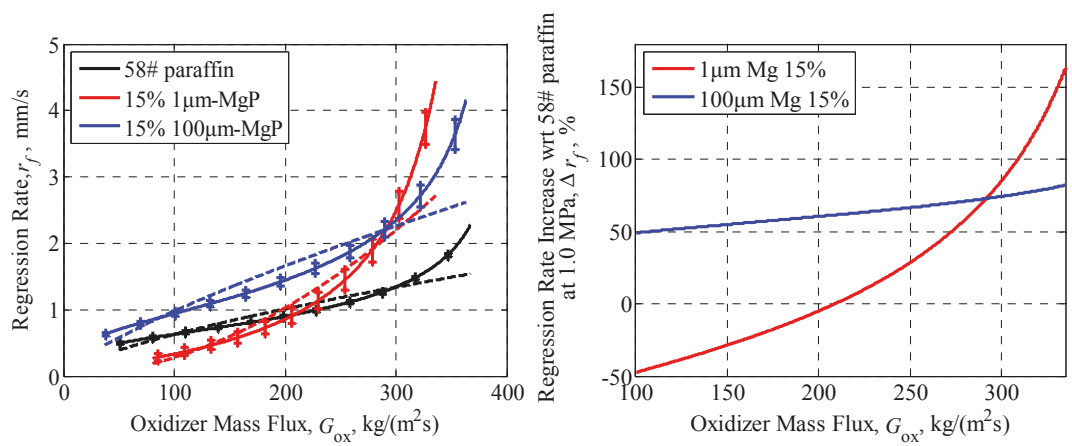

Figure 12. $r_{f}$ vs. $G_{O x}$ (left) and percentage increase in regression rate (right) of magnesium particles/paraffin composite fuels, at $1 \mathrm{MPa}$, pointing out a strong particle size effect. The dotted lines are fitting curves according to the semi-empirical formula: $\dot{r}_{f}=a_{r} G_{o x}^{n_{r}}(t)$.

The fitting results of $r_{f}$ vs. $G_{o x}$ for the two tested kinds of magnesium particles/paraffin composite fuels are shown in Table 9. The $n_{r}$ of formulation $15 \% 1 \mu \mathrm{m}-\mathrm{MgP}$ is much larger than that of formulation $15 \% 100 \mu \mathrm{m}-\mathrm{MgP}$, which indicates that the regression rate of composite fuel with small size magnesium particles is significantly affected by oxidizer mass flux. The thermal properties of magnesium particles were tested to analyze the influence of their particle size on the combustion performance of 
composite fuels. Figure 13 shows the differential scanning calorimetry/thermogravimetry (DSC/TG) (TA instruments SDT600, Lindon, UT, USA) analyses in air flow of $30 \mathrm{~mL} / \mathrm{min}$, with a heating rate of $20 \mathrm{~K} / \mathrm{min}$ : the small $\mathrm{Mg}$ particles have lower oxidation reaction temperature and active $\mathrm{Mg}$ content than the big ones, which is due to their higher specific surface areas and higher reactivity, so that they are easier to be oxidized.

Table 9. $r_{f}$ increase and fitting results of $r_{f}$ vs. $G_{o x}$ for magnesium particles/paraffin composite fuels, $15 \% \mathrm{Mg}$ showing decreasing $n_{r}$ for increasing particle size.

\begin{tabular}{cccccc}
\hline \multirow{2}{*}{ Fuel Name } & $\begin{array}{c}r_{f} \text { Increase at } \\
\left.\mathbf{3 3 5} \mathbf{~ k g} / \mathbf{m}^{\mathbf{2}} \cdot \mathbf{s}\right)\end{array}$ & $\begin{array}{c}\boldsymbol{r}_{f} \text { Increase at } \\
\left.\mathbf{1 0 0} \mathbf{~ k g} / \mathbf{( m}^{\mathbf{2}} \cdot \mathbf{s}\right)\end{array}$ & $\boldsymbol{a}_{\boldsymbol{r}}$ & $\boldsymbol{n}_{\boldsymbol{r}}$ & $\boldsymbol{R}^{\mathbf{2}}$ \\
\hline paraffin \#58 & - & - & $0.048 \pm 0.001$ & $0.566 \pm 0.004$ & 0.917 \\
$1 \mu \mathrm{m} \mathrm{MgP}$ & $163.2 \%$ & $-47.6 \%$ & $3.22 \times 10^{-5} \pm 2.66 \times 10^{-6}$ & $1.954 \pm 0.016$ & 0.907 \\
$100 \mu \mathrm{m} \mathrm{MgP}$ & $82.1 \%$ & $49.2 \%$ & $0.049 \pm 0.001$ & $0.656 \pm 0.006$ & 0.887 \\
\hline
\end{tabular}

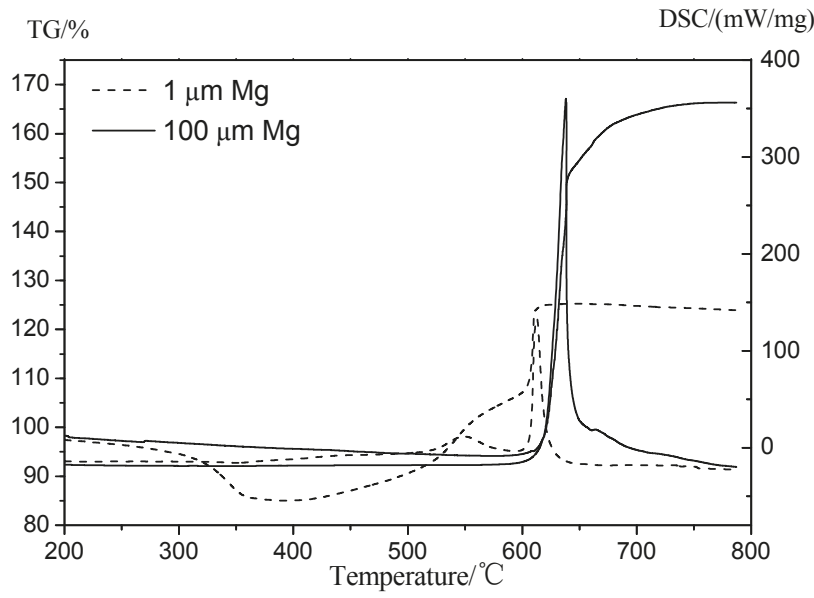

Figure 13. Differential scanning calorimetry/thermogravimetry (DSC/TG) results of small (dotted lines) and large (continuous lines) Magnesium particles showing dependence on particle size. The curves with sharp peaks are the DSC analyses.

According to combustion tests, small $\mathrm{Mg}$ particles can increase the regression rate much more than the big ones under high oxidizer mass flux, because that small $\mathrm{Mg}$ particles have higher reactivity which is beneficial to increase the thermal feedback during the combustion process. At the same time, small $\mathrm{Mg}$ particles have better diffuse distribution in the paraffin matrix so that the dissociation and fly apart phenomena are more obvious. However, with the decrease of oxidizer mass flux, the regression rate of formulation $15 \% 1 \mu \mathrm{m}-\mathrm{MgP}$ reduced sharply below that of pure paraffin under $200 \mathrm{~kg} /\left(\mathrm{m}^{2} \cdot \mathrm{s}\right)$ oxidizer mass flux. This is due to an excessive $\mathrm{MgO}$ barrier layer attached to the burning surface (see Figure 14a) and blocking combustion rather than performing the structural self-disintegration phenomenon. The above facts suggest that $100 \mu \mathrm{m}-\mathrm{MgP}$ is easier to fly into the oxygen flow during paraffin melting while $1 \mu \mathrm{m}-\mathrm{MgP}$ is conducive to form an excess $\mathrm{MgO}$ barrier layer attached to the burning surface and blocking combustion. 


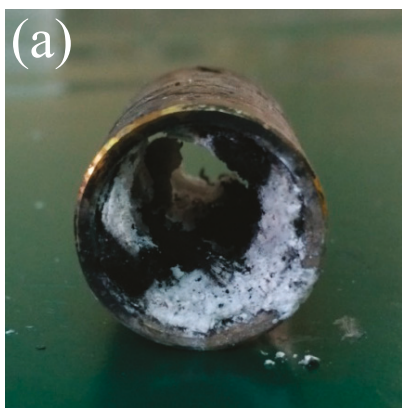

$15 \% 1 \mu \mathrm{m}-\mathrm{MgP}$

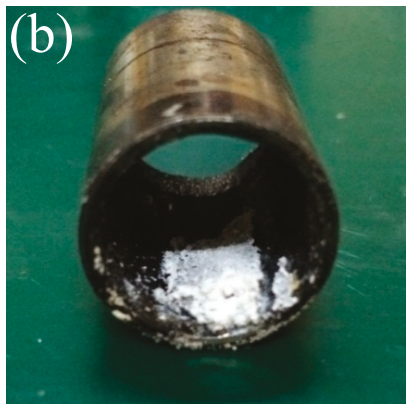

$15 \% 100 \mu \mathrm{m}-\mathrm{MgP}$

Figure 14. Samples tubes after burning of small (a) and large (b) magnesium particles/paraffin composite fuels.

\section{Porous Layer Combustion Fuels}

This is another way to increase the combustion area of the regressing solid fuel and/or the reactivity of energetic particles (see the last paragraph). In composite solid fuels, a substance filler with a low decomposition temperature to gas or a fast burning rate leaves holes in the initial matrix after decomposition or burning, thus increasing the regressing surface area. For example, Azodicarbonamide (ADCA) decomposes at $150-306^{\circ} \mathrm{C}$ while HTPB decomposes at $417-591^{\circ} \mathrm{C}$, thus forming a certain thickness of porous layer after the decomposition of ADCA particles, before HTPB melting and pyrolysis, during the burning of the composite fuel. Experimental results for ADCA/HTPB [30] point out the optimal level of $3 \%$ ADCA to enhance the regression rate and total thermal energy. Excess ADCA shifts the $\mathrm{HTPB}-\mathrm{O}_{2}$ reaction to a high temperature, as well as increases the blocking effect, hindering heat transfer from the flame zone to the fuel surface and suppressing the combustion due to low combustion heat. The ADCA $19.7 \%$ final decomposition (at $800^{\circ} \mathrm{C}$ ) residues hinders the complete combustion reaction by intercepting the heat and oxygen from the diffusive exothermic reaction of HTPB with $\mathrm{O}_{2}$. Another instance of accelerating porous layer is given by metal hydride particles, typically containing large amounts of $\mathrm{H}_{2}$ that can be released at temperatures far below the combustion temperatures. The released $\mathrm{H}_{2}$ can be used for combustion and the remaining base metal can further react and combust, thus adding more thermal energy to the system. Experimental results in Table 10 and Reference [29] confirm a previously unreported porous layer mechanism of $\mathrm{AlH}_{3}$ improving combustion. A $50 \mu \mathrm{m}$ thickness porous layer in $\mathrm{AlH}_{3} / \mathrm{HTPB}$ melting layer, which is exposed by rapidly released gas phase $\mathrm{H}_{2}$ transferring from $\mathrm{AlH}_{3}$ dehydrogenation during combustion, is determined by SEM and other techniques. The results showed that the low $\mathrm{AlH}_{3}$ content $(\leq 10 \%)$ promotes the regression rate obviously, while excessive $\mathrm{AlH}_{3}$ content $(\geq 20 \%)$ promotes only slightly as a result of aggregated $\mathrm{Al}_{2} \mathrm{O}_{3}$ attached on the burning surface, $\mathrm{AlH}_{3}$ endothermic dehydrogenation step and the blocking effect of the gaseous released $\mathrm{H}_{2}$. Nano- and/or micro-porous Al-crystals created by $\mathrm{AlH}_{3}$ dehydrogenation has a specific surface up to $15-20 \mathrm{~m}^{2} / \mathrm{g}$, promoting the combustion processes. These local porosity effects are useful for both solid and hybrid rocket propulsion, as already discussed in References [37,38]. 
Table 10. $r_{f}$ increase and fitting results of $r_{f} / G_{0 x}$ curves for $\mathrm{AlH}_{3}$ particles/HTPB composite fuels. Copyright Elsevier (2019). Reproduced with permission.

\begin{tabular}{|c|c|c|c|c|}
\hline Fuel Name & $\begin{array}{l}r_{f} \text { Increase at } \\
350 \mathrm{~kg} /\left(\mathrm{m}^{2} \cdot \mathrm{s}\right)\end{array}$ & $\begin{array}{l}r_{f} \text { Increase at } \\
150 \mathrm{~kg} /\left(\mathrm{m}^{2} \cdot \mathrm{s}\right)\end{array}$ & $\begin{array}{c}G_{o x} \text { Range from } \\
150 \text { to } 310 \mathrm{~kg} / \mathrm{m}^{2} \cdot \mathrm{s}\end{array}$ & $R^{2}$ \\
\hline Pure НТРВ & - & - & $r_{f}=0.000222 G_{0 x} 1.260$ & 0.944 \\
\hline $5 \% 40-80 \mu \mathrm{m} \mathrm{AlH} 3$ & $48.51 \%$ & $47.89 \%$ & $r_{f}=0.0035 G_{o x}^{0.806}$ & 0.971 \\
\hline $10 \% 40-80 \mu \mathrm{m} \mathrm{AlH}{ }_{3}$ & $85.75 \%$ & $9.15 \%$ & $r_{f}=0.00179 G_{o x} 0.920$ & 0.969 \\
\hline $20 \% 40-80 \mu \mathrm{m} \mathrm{AlH}$ & $3.22 \%$ & $14.79 \%$ & $r_{f}=0.00177 G_{o x} 0.886$ & 0.969 \\
\hline $5 \% 80-200 \mu \mathrm{m} \mathrm{AlH}$ & $37.70 \%$ & $21.83 \%$ & $r_{f}=0.0021 G_{o x}^{0.876}$ & 0.970 \\
\hline $10 \%$ 80-200 $\mu \mathrm{m} \mathrm{AlH}_{3}$ & $48.74 \%$ & $69.72 \%$ & $r_{f}=0.00121 G_{o x} 0.999$ & 0.968 \\
\hline $20 \%$ 80-200 $\mu \mathrm{m} \mathrm{AlH}$ & $14.71 \%$ & $16.20 \%$ & $r_{f}=0.00121 G_{o x} 0.963$ & 0.968 \\
\hline
\end{tabular}

\section{High Thermal Conductivity Fuels}

Carbon nanotubes (CNT) act as an energy transfer media, accelerating the heat transfer and combustion performance of HTPB. Multiwall carbon nanotubes HTPB/MWCNT composites create a discontinuous three-dimensional heat conducting network due to their random orientation in composites, transferring heat from the combustion zone to a melting layer. Regression rates of HTPB-based solid fuels are expected to increase thanks to the enhanced thermal conductivity.

Since MWCNT tend to cluster into microscale aggregates, a uniform and stable dispersion was obtained [31] by dispersing 20-40 nm OD of MWCNT and a surfactant in an HTPB-toluene solution through high-energy ball milling, followed by toluene evaporation and isophorone diisocyanate (IPDI) cross-linked solidification. By adding MWCNT from $0 \%$ to $3 \%$ in mass, the viscosity of the uncured fluid MWCNT/HTPB composites is increased up to $964 \%$ at $120^{\circ} \mathrm{C}$; likewise, thermal conductivity and density increase while the heat of combustion decreases. MWCNT react with oxygen at $\sim 500{ }^{\circ} \mathrm{C}$, higher than the HTPB starting reaction temperature $\left(\sim 180^{\circ} \mathrm{C}\right)$. Thus, MWCNT shifts the HTPB- $\mathrm{O}_{2}$ reaction temperature to a higher level.

Combustion tests carried out at the 2D-radial burner showed that HTPB containing $0.5 \%$, $1 \%, 2 \%$, and $3 \%$ MWCNT increase the instantaneous regression rate at $G_{0 x}=365 \mathrm{~kg} /\left(\mathrm{m}^{2} \cdot \mathrm{s}\right)$ and $G_{o x}=150 \mathrm{~kg} /\left(\mathrm{m}^{2} \cdot \mathrm{s}\right)$, respectively, as shown in Table 11 . The best fitting results are summarized in Table 11: the non-monotonic trends for both $a_{r}$ and $n_{r}$ parameters reflect the complexity of the burning process. Overall, these experimental results suggest that the optimal level of MWCNT is $1 \%$ and the addition of excessive filler $(>2 \%$ ) hinders the combustion characteristics of the HTPB/MWCNT composites by heat dissipation, high viscosity of the melting layer, low thermal energy release and high vaporization heat of excessive MWCNT agglomeration on the burning surface.

Table 11. $r_{f}$ increase and fitting results of $r_{f} / G_{0 x}$ curves for hydroxyl-terminated polybutadiene/carbon nanotubes (HTPB/CNT) composites fuels [31]. Copyright Elsevier (2019). Reproduced with permission.

\begin{tabular}{|c|c|c|c|c|}
\hline Fuel Name & $\begin{array}{l}r_{f} \text { Increase at } \\
365 \mathrm{~kg} /\left(\mathrm{m}^{2} \cdot \mathrm{s}\right)\end{array}$ & $\begin{array}{l}r_{f} \text { Increase at } \\
150 \mathrm{~kg} /\left(\mathrm{m}^{2} \cdot \mathrm{s}\right)\end{array}$ & $\begin{array}{c}G_{o x} \text { Range from } \\
150 \text { to } 350 \mathrm{~kg} / \mathrm{m}^{2} \cdot \mathrm{s}\end{array}$ & $R^{2}$ \\
\hline Pure HTPB & - & - & $r_{f}=\left(2.22 \times 10^{-4}\right) G_{O x} 1.260$ & 0.944 \\
\hline $0.5 \% \mathrm{CNT}$ & $11.2 \%$ & $27.0 \%$ & $r_{f}=\left(6.77 \times 10^{-4}\right) G_{o x}{ }^{1.092}$ & 0.947 \\
\hline $1 \% \mathrm{CNT}$ & $31.6 \%$ & $25.0 \%$ & $r_{f}=\left(2.10 \times 10^{-4}\right) G_{o x}{ }^{1.313}$ & 0.947 \\
\hline $2 \% \mathrm{CNT}$ & $-21.3 \%$ & $-26.4 \%$ & $r_{f}=\left(1.12 \times 10^{-4}\right) G_{o x}{ }^{1.331}$ & 0.947 \\
\hline $3 \% \mathrm{CNT}$ & $-39.7 \%$ & $-36.6 \%$ & $r_{f}=\left(1.85 \times 10^{-4}\right) G_{o x} 1.205$ & 0.975 \\
\hline
\end{tabular}

\section{Paraffin Fuels with Better Mechanical Properties}

Experimental results [32] show that the presence of additives usually decreases the regression rate of paraffin-based fuels. Paraffin \#58 loaded by simple blend with six different additives was investigated: stearic acid, polyethylene wax (A-C $\left.{ }^{\circledR} 6 \mathrm{~A}\right)$, ethylene-vinyl-acetate (EVA), low-density 
polyethylene (LDPE), polypropylene (PP), and high-density polyethylene (HDPE). Comparative mechanical tests, thermal performance tests (melting point and DSC), viscosity measurements and combustion tests were conducted for all fuels. With respect to pure paraffin, the presence of $5 \%$ additives caused a decrease of regression rate for all mixed formulations except the one using stearic acid. The reason is the effect of the additives augmenting the viscosity of the liquid molten paraffin, while stearic acid decreased the mix melting point from $58.3^{\circ} \mathrm{C}$ (pure paraffin) to $55.8^{\circ} \mathrm{C}$.

In order to obtain a suitable paraffin-based fuel with both good mechanical properties and combustion performance, additives should be selected not increasing the melt liquid viscosity of the compound. As a matter of fact, an exponential relation was found in Reference [39] which correlates the regression rate of liquefying fuels with their liquid viscosity. Thus, the regression rate data of the tested paraffin-based fuels could be related directly to the viscosity of the corresponding liquid fuel samples and an increase in the liquid layer viscosity resulted in a decreased regression rate [32].

If the increase of viscosity can somehow be avoided, the detrimental effect on the regression rate is canceled. This is exactly what happened for the LDPE particles tested in Section 5.3, where the SDFS technique allowed paraffin to increase and not to decrease its regression rate, being the combustion process of LDPE particles shifted from the condensed phase directly to the gas phase. A comparison of the two manufacture techniques, a simple blend of the ingredients or SDFS composites, for paraffin \#58 loaded with 5\% and 10\% LDPE, is shown in Table 12. It is seen that the SDFS samples (resorting to medium size LDPE particles) feature appreciably higher values of $a_{r}$ while $n_{r}$ is somewhat less.

Table 12. Fitting results of $r_{f}$ vs. $G_{o x}$ for LDPE/Paraffin fuels comparing $5 \%$ and $10 \%$ LDPE addition by blend and by SDFS composites ( $\mathrm{M}=$ medium particle size) [28,32]. Copyright Wiley (2017) and Begell House (2018). Reproduced with permission.

\begin{tabular}{ccccc}
\hline Name & $\begin{array}{c}\text { Average } \boldsymbol{r}_{\boldsymbol{f}} \text { Increase } \\
\left(\mathbf{1 0 0} \mathbf{3 5 0} \mathbf{~ k g} /\left(\mathbf{m}^{\mathbf{2}} \cdot \mathbf{s}\right)\right)\end{array}$ & $\boldsymbol{a}_{\boldsymbol{r}}$ & $\boldsymbol{n}_{\boldsymbol{r}}$ & $\boldsymbol{R}^{\mathbf{2}}$ \\
\hline Paraffin \#58 & - & $0.048 \pm 0.001$ & $0.566 \pm 0.004$ & 0.917 \\
5\% LDPE-M SDFS & $9.0 \%$ & $0.035 \pm 0.001$ & $0.646 \pm 0.005$ & 0.877 \\
5\% LDPE blend & $-39.0 \%$ & $0.018 \pm 0.001$ & $0.673 \pm 0.006$ & 0.879 \\
$10 \%$ LDPE-M SDFS & $22.2 \%$ & $0.044 \pm 0.001$ & $0.622 \pm 0.005$ & 0.891 \\
10\% LDPE blend & $-58.9 \%$ & $0.010 \pm 0.001$ & $0.710 \pm 0.007$ & 0.850 \\
\hline
\end{tabular}

\section{Concluding Remarks}

After almost a century of progress [1-3], hybrid rocket propulsion still suffers the severe limitations of inadequate solid fuel behavior, with conflicting trends of regression rate and mechanical properties. This penalty hinders the development of large size HRE. Several innovative techniques to increase solid fuel performance were discussed in this paper. Overlapping effects in terms of combustion phenomena (for example, porosity for the SDFS approach) were not explicitly considered but intrinsically exist and should be taken into account in developing the above techniques. Composite fuels featuring self-disintegration structure (SDFS) [26-28] appears the best candidate approach to enhance regression rates, as discussed in Section 5. A meaningful result is that illustrated in Table 12 comparing regression rates for the same paraffin with the addition of $5 \%$ or $10 \%$ LDPE: dramatically different values are measured according to the manufacturing technique (blending or SDFS composites). SDFS leaves essentially unaltered the viscosity of liquid molten paraffin and therefore allows much higher regression rates.

A common finding for the tested formulations is that the optimum mass fraction of non-active filler or additive is in general relatively low and that an excess of it becomes quickly detrimental to combustion performance. Being subject to a variety of chemical and physical effects, the influence of particle size is more involved: in general, the power $n_{r}$ decreases with increasing particle size and this affects differently the regression rate values trends under high and low oxygen mass flux. A different trend was observed for $\mathrm{DB} /$ paraffin composite fuels because of the active nature of $\mathrm{DB}$ 
particles: both the regression rate and its slope vs. $G_{0 x}$ increase with increasing DB load. Overall, best results were achieved by an SDFS structure using Mg powder in a paraffin matrix. This finding is somehow in agreement with the results obtained in small-scale hybrid engine fire tests [25], where different loads of $\mathrm{Mg}(15 \%$ metal, $15 \%$ hydride, and a mixture $15 \% \mathrm{Mg}+1 \% \mathrm{Fe})$ produced the largest increase of regression rate (about $12 \%, 14 \%$, and $48 \%$, respectively, based on total mass flux). Although encouraging results were achieved, the investigated methods are still at their initial stage. Substantial work of understanding and refinement in this paper is for producing solid fuels to fulfill the needs of hybrid rocket propulsion. At any rate, validation by fire tests in a small-scale engine is a mandatory step before attempting large-size HRE.

Funding: This work was supported by the Fundamental Research Funds for the Central Universities (Grant No. 30918011315).

Acknowledgments: Suhang Chen is grateful for the financial support received from China Scholarship Council. Thanks are due to Junzhou Pu for his help in the combustion tests. Thanks are also due for the great contribution of Luigi T. DeLuca in detailing the introduction of hybrid propulsion progress in our group, revising the language, and restructuring the entire article.

Conflicts of Interest: The authors declare no conflict of interest.

\begin{tabular}{|c|c|}
\hline \multicolumn{2}{|c|}{ Nomenclature } \\
\hline \multicolumn{2}{|c|}{ Roman and Greek symbols } \\
\hline$\Delta D$ & diameter variation of the inner burning surface \\
\hline$G_{o x}(t)$ & oxygen mass flux, $\mathrm{kg} /\left(\mathrm{m}^{2} \cdot \mathrm{s}\right)$ \\
\hline$r_{f}(t)$ & regression rate at time $t$ \\
\hline$A_{b}$ & combustion surface area \\
\hline$\rho_{f}$ & density of the fuel \\
\hline$a_{r}$ & multiplicative factor in Equation (1) \\
\hline$n_{r}$ & power in Equation (1) \\
\hline PEG & polyethylene glycol \\
\hline PE paraffin & low molecular mass polyethylene \\
\hline TIN & dibutyltin diacetate \\
\hline IPDI & isophorone diisocyanate \\
\hline PS & polystyrene \\
\hline DB & double-base \\
\hline LDPE & low-density polyethylene \\
\hline ADCA & azodicarbonamide \\
\hline $2 \mathrm{D}$ & two-dimensional \\
\hline \multicolumn{2}{|l|}{ Acronyms } \\
\hline $\mathrm{MgP}$ & Magnesium particles \\
\hline HRE & hybrid rocket engine \\
\hline НТРВ & hydroxyl-terminated polybutadiene \\
\hline TNT & 2,4,6-Trinitrotoluene \\
\hline $\mathrm{AlH}_{3}$ & Aluminum hydride \\
\hline SDFS & Self-disintegration fuel structure \\
\hline NUST & Nanjing University of Science and Technology \\
\hline OD & outer diameter \\
\hline ID & inner diameter \\
\hline IPDI & isophorone diisocyanate \\
\hline SPLab & Space Propulsion Laboratory at Politecnico di Milano \\
\hline CLSM & Confocal Laser Scanning Microscope \\
\hline SEM & scanning electron microscope \\
\hline DSC & differential scanning calorimetry \\
\hline TG & thermogravimetry \\
\hline
\end{tabular}




\section{References}

1. Altman, D. Highlights in Hybrid Rocket Propulsion, Volume 10 of IWCP 'In-Space Propulsion'; DeLuca, L.T., Sackheim, R.L., Palaszewski, B.A., Eds.; Grafiche GSS: Bergamo, Italy, 2005; Chapter 17.

2. DeLuca, L.T. Energetic Problems in Space Propulsion, Chapter 12, Hybrid Rocket Propulsion; Premani Snc: Pantigliate, Italy, 2009.

3. Chiaverini, M.J.; Kuo, K.K. Fundamentals of Hybrid Rocket Combustion and Propulsion; Progress in Astronautics and Aeronautics: Reston, VA, USA, 2007; pp. 3-4.

4. Potter, C.R. Design of Economical Upper Stage Hybrid Rocket Engine; University of Tennessee Honors Thesis Projects; University of Tennessee: Knoxville, TN, USA, May 2012; Available online: https://trace.tennessee. edu/utk_chanhonoproj/1619 (accessed on 7 March 2019).

5. Soller, S.; Maeding, C.; Preclik, D.; Martin, F.; Cretella, A.; De Amicis, P.; Orlandi, O.; Theil, D. Development roadmap and design of demonstrators for hybrid rocket propulsion in Europe. In Proceedings of the 4th European Conference for Aerospace Sciences (EUCASS), Saint Petersburg, Russia, 4-8 July 2011.

6. Schmierer, C.; Tomilin, K.; Kobald, M.; Steelant, J.; Schlechtriem, S. Analysis and preliminary design of a hybrid propulsion lunar lander. In Proceedings of the Space Propulsion Conference, Rome, Italy, 2-6 May 2016; SP2016-3124677.

7. Martin, F.; Chapelle, A.; Lemaire, F. Promising space transportation applications using hybrid propulsion. In Proceedings of the 4th European Conference for Aerospace Sciences (EUCASS), Saint Petersburg, Russia, 4-8 July 2011.

8. Wright, A.; Foley, P.; Tilahun, D.; Reason, M.; Bryant, C.; Patton, J.; Hudson, M. The effect of high concentration guanidinium azo-tetrazolate on thrust and specific impulse of a hybrid rocket. In Proceedings of the 36th AIAA/ASME/SAE/ASEE Joint Propulsion Conference and Exhibit, Las Vegas, NV, USA, 24-28 July 2000; AIAA-2000-3885.

9. George, P.; Krishnan, S.; Varkey, P.M.; Ravindran, M.; Ramachandran, L. Fuel regression rate in hydroxyl-terminated polybutadiene/gaseous-oxygen hybrid rocket motors. J. Propuls. Power 2001, 17, 35-42. [CrossRef]

10. Galfetti, L.; Merotto, L.; Boiocchi, M.; Maggi, F.; DeLuca, L.T. Experimental investigation of paraffin based fuels for hybrid rocket propulsion. In Proceedings of the 5th European Conference for Aerospace Sciences (EUCASS), Munich, Germany, 1-5 July 2011. [CrossRef]

11. Karabeyoglu, M.A.; Zilliac, G.; Castellucci, P.; Urbanczyk, P.; Stevens, J.; Inalhan, G.; Cantwell, B.J. Development of high burning rate hybrid rocket fuel flight demonstrators. In Proceedings of the 39th AIAA/ASME/SAE/ASEE Joint Propulsion Conference and Exhibit, Huntsville, AL, USA, 20-23 July 2003; AIAA-2003-5196.

12. Karabeyoglu, M.A.; Zilliac, G.; Cantwell, B.J.; Dezilwa, S.; Castellucci, P. Scale-up tests of high regression rate paraffin-based hybrid rocket fuels. J. Propuls. Power 2004, 20, 1037-1045. [CrossRef]

13. Karabeyoglu, M.A.; Altman, D.; Cantwell, B.J. Combustion of liquefying hybrid propellants: Part 1, general theory. J. Propuls. Power 2002, 18, 610-620. [CrossRef]

14. Karabeyoglu, M.A.; Cantwell, B.J. Combustion of liquefying hybrid propellants: Part 2, stability of liquid films. J. Propuls. Power 2002, 18, 621-630. [CrossRef]

15. George, P.; Krishnan, S.; Varkey, P.M.; Ravindran, M.; Ramachandran, L. Hybrid rocket fuel combustion and regression rate study. In Proceedings of the 28th Joint Propulsion Conference and Exhibit, Nashville, TN, USA, 20-23 July 1992. AAA-92-3302. [CrossRef]

16. Carmicino, C.; Sorge, A.R. Role of injection in hybrid rockets regression rate behavior. J. Propuls. Power 2005, 21, 606-612. [CrossRef]

17. Lee, C.; Na, Y.; Lee, J.W.; Byun, Y.H. Effect of induced swirl flow on regression rate of hybrid rocket fuel by helical grain configuration. Aerosp. Sci. Technol. 2007, 11, 68-76. [CrossRef]

18. Armold, D.M. Formulation and Characterization of Paraffin-Based Solid Fuels Containing Swirl Inducing Grain Geometry and/or Energetic Additives. Master's Thesis, The Pennsylvania State University, University Park, PA, USA, 11 April 2014. Available online: https://etda.libraries.psu.edu/catalog/21836 (accessed on 7 March 2019). 
19. Lips, H. Metal combustion in high performance hybrid rocket propulsion systems. In Proceedings of the 12th Propulsion Conference, Palo Alto, CA, USA, 26-29 July 1976. [CrossRef]

20. Karabeyoglu, A. Mixtures of nitrous oxide and oxygen (nytrox) as oxidizers for rocket propulsion applications. In Proceedings of the 45th AIAA/ASME/SAE/ASEE Joint Propulsion Conference and Exhibit, Denver, CO, USA, 2-5 August 2009; AIAA-2009-4966. [CrossRef]

21. Karabeyoglu, M.A. Nitrous oxide and oxygen mixtures (nytrox) as oxidizers for rocket propulsion applications. J. Propuls. Power 2015, 30, 696-706. [CrossRef]

22. Risha, G.; Boyer, E.; Wehrman, R.; Evans, B.; Kuo, K.K. Nano-sized aluminum and boron-based solid fuel characterization in a hybrid rocket engine. In Proceedings of the 39th AIAA/ASME/SAE/ASEE Joint Propulsion Conference and Exhibit, Huntsville, AL, USA, 20-23 July 2003; AIAA-2003-4593. [CrossRef]

23. Risha, G.; Boyer, E.; Wehrman, R. Performance comparison of HTPB-based solid fuels containing nano-sized energetic powder in a cylindrical hybrid rocket motor. In Proceedings of the 38th AIAA/ASME/SAE/ASEE Joint Propulsion Conference and Exhibit, Indianapolis, IN, USA, 7-10 July 2002; AIAA-2002-3576. [CrossRef]

24. Calabro, M. LOX/HTPB/AlH3 hybrid propulsion for launch vehicle boosters. In Proceedings of the 40th AIAA/ASME/SAE/ASEE Joint Propulsion Conference and Exhibit, Fort Lauderdale, FL, USA, 11-14 July 2003; AIAA-2004-3823. [CrossRef]

25. Carmicino, C.; Sorge, A.R. Experimental investigation into the effect of solid-fuel additives on hybrid rocket performance. J. Propuls. Power 2015, 31, 699-713. [CrossRef]

26. Shen, R.Q.; Tang, Y.; Chen, S.H.; Zhao, Q.; Zhang, W.; Ye, Y.; DeLuca, L.T. Self-disintegration effects on the regression rate of composite polymer particle paraffin fuel (CM3PF). In Proceedings of the 7th European Conference for Aeronautics and Space Sciences (EUCASS), Milan, Italy, 3-6 July 2017.

27. Tang, Y.; Chen, S.; Zhang, W.; Shen, R.; DeLuca, L.T.; Ye, Y. Flame Visualization and Combustion Performance of Composite Energetic Particle Paraffin-based Fuels for Hybrid Rocket Propulsion. Int. J. Energ. Mater. Chem. Propuls. 2017, 16, 49-59.

28. Tang, Y.; Zhang, W.; Chen, S.H.; Yu, H.S.; Shen, R.; DeLuca, L.T.; Ye, Y. A novel polyethylene particles/paraffin-based self-disintegration fuel for hybrid rocket propulsion. Int. J. Energ. Mater. Chem. Propuls. 2018, 17, 205-216. [CrossRef]

29. Chen, S.H.; Tang, Y.; Yu, H.S.; Bao, L.; Zhang, W.; DeLuca, L.T.; Shen, R.; Ye, Y. The rapid $\mathrm{H}_{2}$ release from $\mathrm{AlH}_{3}$ dehydrogenation forming porous layer in $\mathrm{AlH}_{3}$ /hydroxyl-terminated polybutadiene (HTPB) fuels during combustion. J. Hazard. Mater. 2019, 371, 53-61. [CrossRef] [PubMed]

30. Chen, S.H.; Tang, Y.; Zhang, W.; Shen, R.; DeLuca, L.T.; Ye, Y. Effect of azodicarbonamide particles on the regression rate of hydroxyl-terminated polybutadiene (HTPB)-based fuels for hybrid rocket propulsion. Int. J. Energ. Mater. Chem. Propuls. 2017, 16, 103-114. [CrossRef]

31. Chen, S.H.; Tang, Y.; Yu, H.S.; Guan, X.Y.; DeLuca, L.T.; Zhang, W.; Shen, R.; Ye, Y. Combustion enhancement of hydroxyl-terminated polybutadiene by doping multiwall carbon nanotubes. Carbon 2019, 144, 472-480. [CrossRef]

32. Tang, Y.; Chen, S.H.; Zhang, W.; Shen, R.; DeLuca, L.T.; Ye, Y. Mechanical modifications of paraffin-based fuels and the effects on combustion performance. Propellants Explos. Pyrotech. 2017, 16, 103-114. [CrossRef]

33. DeLuca, L.T.; Galfetti, L.; Maggi, F.; Colombo, G.; Merotto, L.; Boiocchi, M.; Paravan, C.; Reina, A.; Tadini, P.; Fanton, L. Characterization of HTPB-based solid fuel formulations: Performance, mechanical properties, and pollution. Acta Astronaut. 2013, 92, 150-162. [CrossRef]

34. DeLuca, L.T.; Galfetti, L.; Colombo, G.; Maggi, F.; Bandera, A.; Boiocchi, M.; Gariani, G.; Merotto, L.; Paravan, C.; Reina, A. Time-resolved burning of solid fuels for hybrid rocket propulsion. In Proceedings of the 3rd European Conference for Aerospace Sciences (EUCASS), Versailles, France, 6-10 July 2009. [CrossRef]

35. Zilliac, G.; Karabeyoglu, M. Hybrid rocket fuel regression rate data and modeling. In Proceedings of the 42th AIAA/ASME/SAE/ASEE Joint Propulsion Conference and Exhibit, Sacramento, CA, USA, 9-12 July 2006; AIAA-2006-4504. [CrossRef]

36. Guo, X.; Li, F.; Song, H.; Liu, G.; Kong, L.; Li, M.; Chen, W. Combustion Characteristics of a Novel Grain-Binding High Burning Rate Propellant. Propellants Explos. Pyrotech. 2008, 33, 255-260. [CrossRef]

37. DeLuca, L.T.; Galfetti, L.; Severini, F.; Rossettini, L.; Meda LMarra, G.; D'Andrea, B.; Weiser, V.; Calabro, M.; Vorozhtsov, A.B.; Glazunov, A.A.; et al. Physical and Ballistic Characterization of AlH3-Based Space Propellants. Aerosp. Sci. Technol. 2007, 11, 18-25. [CrossRef] 
38. DeLuca, L.T.; Rossettini, L.; Kappenstein, C.; Weiser, V. Ballistic Characterization of AlH3-Based Propellants for Solid and Hybrid Rocket Propulsion. In Proceedings of the 45th AIAA/ASME/SAE/ASEE Joint Propulsion Conference \& Exhibit Denver, Denver, CO, USA, 2-5 August 2009; AIAA 2009-4874.

39. Kobald, M.; Schmierer, C.; Ciezki, H.K.; Schlechtriem, S.; Toson, E.; DeLuca, L.T. Viscosity and Regression Rate of Liquefying Hybrid Rocket Fuels. J. Propuls. Power 2017, 33, 1245-1251. [CrossRef]

(C) 2019 by the authors. Licensee MDPI, Basel, Switzerland. This article is an open access article distributed under the terms and conditions of the Creative Commons Attribution (CC BY) license (http://creativecommons.org/licenses/by/4.0/). 



\title{
The Application of Computational Thermo-Fluid-Dynamics to the Simulation of Hybrid Rocket Internal Ballistics with Classical or Liquefying Fuels: A Review
}

\author{
Giuseppe Daniele Di Martino *, Carmine Carmicino, Stefano Mungiguerra and Raffaele Savino \\ Department of Industrial Engineering-Aerospace Division, University of Naples "Federico II", Piazzale Tecchio, \\ 80-80125 Naples, Italy; carmicin@unina.it (C.C.); stefano.mungiguerra@unina.it (S.M.); rasavino@unina.it (R.S.) \\ * Correspondence: giuseppedaniele.dimartino@unina.it
}

Received: 20 February 2019; Accepted: 11 May 2019; Published: 16 May 2019

\begin{abstract}
The computational fluid dynamics of hybrid rocket internal ballistics is becoming a key tool for reducing the engine operation uncertainties and development cost as well as for improving experimental data analysis. Nevertheless, its application still presents numerous challenges for the complexity of modeling the phenomena involved in the fuel consumption mechanism and its coupling with the chemically reacting flowfield. This paper presents a review of the computational thermo-fluid-dynamic models developed for the internal ballistics of hybrid rockets burning gaseous oxygen with classical polymeric or paraffin-based fuels, with a special focus on the interaction between the fluid and the solid fuel surface. With the purpose of predicting the local fuel regression rate, which is the main parameter needed for the hybrid rocket design, the model is coupled with an improved gas/surface interface treatment based on local mass, energy and mean mixture-fraction balances, combined to either a pyrolysis-rate equation in the case of classical polymers, or to an additional equation for the liquid paraffin entrainment fraction of the total fuel consumption rate. A number of experimental test cases obtained from the static firing of two different laboratory-scale rockets are simulated to determine the models' capabilities, showing very good agreement between the calculated and measured fuel regression rates with both standard pyrolyzing and liquefying fuels. The prediction of the chamber pressure measured with paraffin fuel resulted in it being more cumbersome for the single-phase flow assumption. The advantages and limitations of the models are discussed.
\end{abstract}

Keywords: hybrid rocket; fuel regression rate; internal ballistics; computational fluid dynamics

\section{Introduction}

The hybrid rocket is a chemical propulsion engine in which the fuel and oxidizer are separated in different physical states [1]. In the classical system configuration, the fuel is stored in the combustion chamber in the solid state and a liquid or gaseous oxidizer is injected into single or multiple ports obtained in the solid fuel grain. When the two propellants are ignited, a diffusive flame is formed in the boundary layer developing in the grain port, relatively far from the fuel surface, and it is fed from the outer side by the oxidizer, which is transported from the free stream by turbulent diffusion mechanisms, and, from the inner side, by the products of fuel gasification and/or liquefaction (depending on the nature of the fuel itself) which is sustained by the flame. The combusted mixture expands through an exhaust nozzle, generating the required thrust.

In the last few years, a significantly growing interest has been addressed towards hybrid rocket propulsion, thanks to its numerous advantages [2] relative to traditional solid and liquid systems, 
which include, among the main features, the re-ignition and throttling capabilities combined with the possibility of employing environmentally sustainable propellants and, most importantly, its intrinsic safety $[3,4]$. Although the potentialities of the hybrid rocket have been widely recognized to warrant the renewed research efforts that are being devoted to its development, such propulsion technology still raises challenging tasks.

Engine performance is governed by the rate at which the fuel is consumed, i.e., by the fuel regression rate, as this determines the total mass flow rate and overall oxidizer-to-fuel mixture ratio, which, for a given chamber pressure, controls the motor thrust and the ideal specific impulse. For given operating conditions, the regression rate depends on the type of solid fuel employed. Fuels typically burned in hybrid rockets are classical polymers, such as high-density polyethylene (HDPE), hydroxyl-terminated polybutadiene (HTPB), and polymethylmethacrylate (PMMA), or polymers with metal additives to improve the density impulse [5]. The fuel regression of these polymers is determined by the ratio between the heat flux to the surface and the heat of the phase change, thus, it is limited by the heat and mass transfer mechanisms occurring from the flame to the fuel wall. The blowing of the fuel from the surface decreases the velocity gradient at the wall and the convective heat transfer for the so-called blocking effect [6]. Owing to this sort of "counter-balance" between heat flux and blowing, the regression rate marginally depends on the nature of classical polymeric fuels [6], and is usually relatively small; consequently, the volumetric fuel loading efficiency may be too poor for space applications.

Compared to conventional polymers, the consumption mechanism of paraffin-based fuels is different and allows for a significantly larger regression rate. Karabeyoglu et al. [7] have shown that these fuels display regression rates up to 3-4 times higher than those achieved with traditional hybrid fuels. For this reason, a lot of studies have been focused on the type of materials that belong to the class of the so-called liquefying fuels [8]. Their intrinsic characteristic is the onset of a thin liquid layer on the fuel grain surface, which may become unstable at low viscosity and surface tension $[9,10]$, leading to the lift-off and entrainment of fuel liquid droplets or filaments into the main gas stream, increasing the fuel mass transfer rate. This mass-transfer mechanism essentially does not depend on the heat transfer to the surface and raises the fuel mass flow without entailing the consequent blocking on gaseous fuel blowing. As a result, the overall regression rate can be considered to be composed of two terms, one determined by classical fuel vaporization and the other by liquid entrainment. The entrainment phenomenon is strongly susceptible to fuel composition, its manufacturing process and the obtained thermo-mechanical properties, as well as to the engine operating conditions [11], which makes the prediction of the combustion chamber internal ballistics even harder than in the case of non-liquefying polymers. Hence, on the one hand, designers need to characterize the fuel with extended experimental campaigns $[12,13]$ and, on the other, to carry out rocket static firings to measure the achieved engine performance.

Predicting the local, time-resolved fuel regression rate is one major challenge in the design of a hybrid rocket. A number of models have been developed over the years to describe the hybrid combustion mechanism, but often they lack some important aspects and/or fail in the prediction of the experimental results [14,15]. The first hybrid combustion theory accounting for the main underlying phenomena involved in the burning of classical polymeric fuels was developed in 1963 by Marxman and Gilbert $[6,16]$ and it still remains the starting point of design calculations and experimental comparisons. Subsequent improvements [17] are, however, all based on the same assumption of a turbulent boundary layer with chemical reactions occurring over a fuel slab burning in an oxidant gas flow. Because of this fundamental hypothesis, those classical theories are unable to reproduce the effects of complex flows, such as the recirculation ensuing from the oxidizer injection, which may have a non-negligible impact even in standard-flow motors [18,19]. Analytical models later developed for liquefying fuels $[7,20]$ are essentially modifications of the classical hybrid boundary-layer combustion theory for the entrainment mass transfer from the fuel grain and, consequently, yield the same limits as the original theory. 
In this framework, the numerical modeling of the rocket internal thermo-fluid-dynamics, with predictive capabilities of the fuel regression rate and overall engine performance, is becoming a key tool both in the system design process and in the experimentally measured performance-analysis stage. In fact, affordable computational models provide a quick detailed representation of the phenomena governing the engine internal ballistics, on the one hand allowing for numerous motor optimization trials and, on the other, reducing the need of expensive experimental testing.

This paper presents the computational fluid dynamic (CFD) models defined by the authors for simulating the internal ballistics of a hybrid rocket burning either classical polymeric or paraffin-based fuels with gaseous oxygen, with an eye to their evolution. The presentation starts with an overview of the numerical techniques available in the competent literature and develops through the steps taken up to the current level. Numerical results are summarized and compared to the experimental data gathered by static firings of two laboratory-scale engines.

\section{State of the Art of CFD Techniques for Rocket Internal-Ballistics Simulation}

CFD approaches to the solution of the flowfield in the hybrid-propellant rocket chamber have been considerably developed recently [21-23], but comprehensive models to describe the complex interactions among fluid dynamics [24], solid fuel pyrolysis [25] or melting and entrainment [7], oxidizer atomization and vaporization, mixing and combustion in the gas phase [26], nozzle thermochemical erosion [27], particulate formation, and radiative characteristics of the flame [28] are still lacking and numerical simulations are often considered only as a qualitative tool to afford the thermo-fluid-dynamics of the rocket. Most of the effort has been addressed, so far, to classical non-liquefying fuels. A common strategy is solving the Reynolds averaged Navier-Stokes (RANS) equations, with suitable turbulence closure and combustion models. The latter models are essentially split into two categories: finite-rate kinetics and mixing-limited combustion mechanisms. When the former philosophy is followed, usually gas phase reactions are modeled by global reaction mechanisms because detailed chemical kinetics would include many species requiring a huge computational cost and, based on the experience gained by the authors, would probably not effectively improve the gas-surface interaction simulation $[29,30]$. Whereas, following the approach that chemistry is infinitely fast, the overall rate of the reaction is controlled by turbulent mixing and the combustion can be described, for instance, with the eddy dissipation model [31,32] or with chemical equilibrium and an interaction model between turbulence and chemistry [33].

Justified by the fact that the chemical and fluid-dynamic characteristic times are much shorter than the fuel-regression time scale, the steady-state solution of RANS equations is generally sought [34]. An acceptable method to study the hybrid rocket internal ballistics can, therefore, be simulating the flowfield at different times in the motor firing by updating the fuel port diameter [26]. Nevertheless, a single numerical simulation is often performed on the chamber geometry drawn at the time-space averaged port diameter $[29,30]$. The cases in which the port section longitudinal non-uniformity is considered are rare [35].

Depending on the specific target of the simulation, several techniques can be implemented; the fuel regression rate can be, on the one hand, an input to the numerical simulation, for instance, when one is interested in studying the propellant mixing and combustion processes in the motor [31] compared to experimental data; or, maybe in a pre-screening stage, to make trade-offs among several oxidizer injections and/or motor configurations as described by the authors in Reference [33]. In this latter case, the simple mass flow inlet boundary at the fuel wall is sufficient and the problem is simplified. On the other hand, the fuel regression rate is an outcome of the simulation and the proper fuel-surface boundary conditions are required to allow for its calculation.

In particular, with classical polymeric fuels, an energy balance at the solid fuel interface with gas, coupled with a semiempirical pyrolysis-rate relationship between the regression rate and surface temperature, is used. Typically, the regression rate is modeled with a one-step irreversible Arrhenius-type equation. Given a polymeric fuel, the laws of pyrolysis available in the open literature 
are widely scattered because of significantly different pre-exponential factors and activation energies. However, by means of a sensitivity analysis conducted with HDPE, in Reference [36], it has been demonstrated that there is no significant dependence of the calculated regression rate from the law of pyrolysis adopted, provided that each variable is consistently taken from a single set of data.

The computational domain can include the actual prechamber geometry or just a simplification of it $[29,30]$ based on the assumption that, with a gaseous oxidizer, the real dump-plenum arrangement does not have a significant impact on the results. Additionally, the postchamber can be assimilated to a cylinder that is equal to the grain port if one is not concerned with the details of mixing the upstream of the nozzle. In other cases, one may exclude the motor aft-mixing chamber and exhaust nozzle if the chamber pressure and combustion efficiency are not under investigation [35]. In that case, the pressure has to be an input to the problem and a pressure-outlet boundary condition is usually imposed.

When considering the energy balance at the wall for the regression rate calculation, other than the convective heat transfer, the heat exchanged by radiation with the combustion gases may play a non-negligible role for some polymers [36]; however, a clear conclusion has not been drawn yet.

The definition of a suitable and computational cost-effective strategy for liquefying fuels poses further complications because, in principle, to successfully simulate the paraffin-fuel consumption, two non-trivial tasks have to be accomplished. These are modeling, first, the formation, the breaking up and the entrainment of the liquid film on the melting fuel surface; and, second, the transformation of the melted fuel into a gaseous species participating in the combustion process. These demanding efforts have probably discouraged researchers, so drastic simplifications are usually introduced, such as giving the regression rate calculation away by assuming it from experiments [31,37-39], or limiting the analysis to one-dimensional integral-differential models [40]. In other cases, by observing that the melted paraffin wax is in the supercritical state under the hybrid rocket chamber characteristic conditions (thus, surface tension vanishes and the sharp distinction at droplets surface between gas and liquid phases disappear), the melted layer break up and, subsequently, the liquid paraffin injection in the flowfield is disregarded, supposing that the entrainment is part of the turbulent mixing process [41,42]. However, those models are not successfully validated, still displaying significant deviations from the experimental data which, in some cases, are around 25\% [42].

In the forthcoming sections, the physical and numerical models developed by the authors for the calculation of the regression rate of both classical and liquefying fuels are presented, and a summary of the main results obtained in several test cases is discussed with the complement of experimental data. All the numerical simulations are carried out with a commercial fluid dynamic solver with ad-hoc user-defined functions. The base numerical framework is firstly shown and, then, the details of the different wall-boundary management are reported.

\section{Physical and Numerical Models}

\subsection{Governing Equations}

The RANS equations for single-phase multicomponent turbulent reacting flows are solved; note that when dealing with liquefying fuels, the main underlying hypothesis here is that, being liquid paraffin in supercritical conditions [42], its viscosity and diffusivity are close to those of a gas and the diffusion processes are significantly faster than in the liquid phase, which, in a first approximation, may allow for neglecting the two-phase flow. Hence, in both the cases of polymers and paraffin wax burning, the fuel is supposed to enter the combustion chamber as $100 \%$ gaseous ethylene (which is expected to be the main product of both the HDPE thermal pyrolysis and paraffin-wax decomposition).

The Favre-averaged (i.e., density-weighted) equations of continuity and momentum can be expressed in Cartesian tensor form, with an understanding that repeated indices mean summation, as [43]

$$
\frac{\partial \bar{\rho}}{\partial t}+\frac{\partial}{\partial x_{j}}\left(\bar{\rho} \widetilde{u}_{j}\right)=S_{m}
$$




$$
\frac{\partial}{\partial t}\left(\bar{\rho} \widetilde{u}_{i}\right)+\frac{\partial}{\partial x_{j}}\left(\bar{\rho} \widetilde{u}_{i} \widetilde{u}_{j}\right)=-\frac{\partial \bar{p}}{\partial x_{i}}+\frac{\partial \bar{\tau}_{i j}}{\partial x_{j}}+\frac{\partial}{\partial x_{j}}\left(-\overline{\rho u_{i}^{\prime} u_{j}^{\prime}}\right)
$$

where $S_{m}$ is the mass source term, which, as will be explained later, is used to model the liquid fuel mass entrainment occurring with paraffin wax. Here, the bar denotes conventional time averaging, while the tilde denotes density-weighted averaging; $\tau_{i j}$ is the stress tensor that is defined as

$$
\tau_{i j}=\mu\left[\left(\frac{\partial u_{i}}{\partial x_{j}}+\frac{\partial u_{j}}{\partial x_{i}}\right)-\frac{2}{3} \delta_{i j} \frac{\partial u_{k}}{\partial x_{k}}\right]
$$

where $\delta_{i j}$ is the Kronecker delta. Symbols with a prime indicate the corresponding quantity fluctuation. The term $\mathcal{R}_{i j}=-\overline{\rho u_{i}^{\prime} u_{j}^{\prime}}$, originating from the averaging operation, is known as the Reynolds stress tensor, and it needs to be modeled.

The Shear Stress Transport (SST) turbulence model [44] has been employed for its improved capability of predicting flows with separated regions. This is a combination of the robust and accurate $k-\omega$ model, developed by Wilcox [45] in the near-wall region, with the standard $k-\varepsilon$ model implemented away from the wall using a blending function. With the SST model, the transport equations of the turbulence kinetic energy, $k$, and the specific dissipation rate, $\omega$, are formulated as

$$
\begin{gathered}
\frac{\partial}{\partial t}(\bar{\rho} k)+\frac{\partial}{\partial x_{i}}\left(\bar{\rho} \widetilde{u}_{i} k\right)=\frac{\partial}{\partial x_{j}}\left[\left(\mu+\mu_{t} \sigma_{k}\right) \frac{\partial k}{\partial x_{j}}\right]+\mathcal{R}_{i j} \frac{\partial \widetilde{u}_{i}}{\partial x_{j}}-\beta^{*} \bar{\rho} \omega k \\
\frac{\partial}{\partial t}(\bar{\rho} \omega)+\frac{\partial}{\partial x_{i}}\left(\bar{\rho} \widetilde{u}_{i} \omega\right)=\frac{\partial}{\partial x_{j}}\left[\left(\mu+\mu_{t} \sigma_{\omega}\right) \frac{\partial \omega}{\partial x_{j}}\right]+\bar{\rho} \frac{\alpha}{\mu_{t}} \mathcal{R}_{i j} \frac{\partial \widetilde{u}_{i}}{\partial x_{j}}-\beta \bar{\rho} \omega^{2}+2\left(1-F_{1}\right) \bar{\rho} \sigma_{\omega_{2}} \frac{1}{\omega} \frac{\partial k}{\partial x_{j}} \frac{\partial \omega}{\partial x_{j}}
\end{gathered}
$$

in which the Reynolds stress is modeled using the Boussinesq approximation

$$
\mathcal{R}_{i j}=\mu_{t}\left[\left(\frac{\partial \widetilde{u}_{i}}{\partial x_{j}}+\frac{\partial \tilde{u}_{j}}{\partial x_{i}}\right)-\frac{2}{3} \delta_{i j} \frac{\partial \widetilde{u}_{l}}{\partial x_{l}}\right]-\frac{2}{3} \bar{\rho} k \delta_{i j}
$$

The turbulent viscosity, $\mu_{t}$, is expressed as follows

$$
\mu_{t}=\frac{\bar{\rho} k}{\omega} \frac{1}{\max \left(1 ; \frac{\Omega F_{2}}{0.31 \omega}\right)}
$$

where the function $F_{2}$ is defined depending on the distance from the wall $y$, as

$$
F_{2}=\tanh \left(\Phi_{2}^{2}\right)
$$

with

$$
\Phi_{2}=\max \left(\frac{2 \sqrt{k}}{0.09 \omega y} ; \frac{500 \mu}{\bar{\rho} \omega y^{2}}\right)
$$

The coefficient $\alpha$ is given by

$$
\alpha=\gamma \frac{1 / 9+R e_{t} / 2.95}{1+R e_{t} / 2.95}
$$

where $R e_{t}=\bar{\rho} k / \mu \omega$ is the turbulent Reynolds number. The blending function $F_{1}$ takes the value of 1 on the wall and tends to zero at the boundary layer edge, being defined as

$$
F_{1}=\tanh \left(\Phi_{1}^{4}\right)
$$

with

$$
\Phi_{1}=\min \left[\max \left(\frac{\sqrt{k}}{0.09 \omega y} ; \frac{500 \mu}{\bar{\rho} \omega y^{2}}\right) ; \frac{4 \bar{\rho} \sigma_{\omega_{2}} k}{C D_{k \omega} y^{2}}\right]
$$


where $C D_{k \omega}$ is the positive part of the last term in Equation (5) (cross-diffusion term):

$$
C D_{k \omega}=\max \left(2 \bar{\rho} \sigma_{\omega_{2}} \frac{1}{\omega} \frac{\partial k}{\partial x_{j}} \frac{\partial \omega}{\partial x_{j}} ; 10^{-20}\right)
$$

The model coefficients $\sigma_{k}, \sigma_{\omega}, \beta, \gamma$ are defined by blending the corresponding coefficients of the original $k-\omega$ model, denoted with the subscript 1 , with those of the transformed $k-\varepsilon$ model that are denoted with the subscript 2:

$$
\left[\begin{array}{c}
\sigma_{k} \\
\sigma_{\omega} \\
\beta \\
\gamma
\end{array}\right]=F_{1}\left[\begin{array}{c}
\sigma_{k_{1}} \\
\sigma_{\omega_{1}} \\
\beta_{1} \\
\gamma_{1}
\end{array}\right]+\left(1-F_{1}\right)\left[\begin{array}{c}
\sigma_{k_{2}} \\
\sigma_{\omega_{2}} \\
\beta_{2} \\
\gamma_{2}
\end{array}\right]
$$

All the model constants are listed in Table 1.

Table 1. The values of the Shear Stress Transport (SST) model constants [43].

\begin{tabular}{cccc}
\hline Constant & Value & Constant & Value \\
\hline$\sigma_{k_{1}}$ & 0.850 & $\sigma_{k_{2}}$ & 1.00 \\
$\sigma_{\omega_{1}}$ & 0.500 & $\sigma_{\omega_{2}}$ & 0.856 \\
$\beta_{1}$ & 0.075 & $\beta_{2}$ & 0.0828 \\
$\gamma_{1}$ & 0.553 & $\gamma_{2}$ & 0.440 \\
$\beta^{*}$ & 0.090 & & \\
\hline
\end{tabular}

Assuming that the chemical kinetics are fast compared to the diffusion processes occurring in the motor for the typical mass fluxes and chamber pressures considered here [46], the non-premixed combustion of oxygen and gaseous ethylene is modeled by means of the Probability Density Function (PDF) approach coupled with chemical equilibrium [47]. Accordingly, the combustion is simplified to a mixing problem and the difficulties associated with closing non-linear reaction rates are avoided. In fact, under the hypothesis of equal diffusivities for all chemical species and assuming that the Lewis number is equal to 1 , the species equations can be reduced to a single equation for the transport of the mixture fraction, which represents the sum of the element mass fractions contained in the fuel stream [48], $f=1 /(1+O F)$, where $O F$ is the local oxidizer-to-fuel mass ratio for the equivalent non-burning field. The density-averaged mixture fraction equation is

$$
\frac{\partial}{\partial t}(\bar{\rho} \widetilde{f})+\frac{\partial}{\partial x_{j}}\left(\bar{\rho} \widetilde{u}_{j} \tilde{f}\right)=\frac{\partial}{\partial x_{j}}\left(\frac{\mu_{t}}{S c_{t}} \frac{\partial \widetilde{f}}{\partial x_{j}}\right)+S_{m}
$$

where $S c_{t}$ is the turbulent Schmidt number, which, for the hypothesis of the unity Lewis number, is equal to the turbulent Prandtl number, $P r_{t}$; the latter is assumed to be equal to 0.85 .

For the closure model describing the turbulence-chemistry interaction, the variance of the mean mixture fraction $\widetilde{f^{\prime 2}}$ is introduced and an additional equation for this quantity is needed, which, according to Reference [49], and making use of the relation between $\omega, k$, and $\varepsilon$, is written as

$$
\frac{\partial}{\partial t}\left(\bar{\rho} \widetilde{f^{\prime 2}}\right)+\frac{\partial}{\partial x_{j}}\left(\bar{\rho} \widetilde{u_{j}} \widetilde{f^{\prime 2}}\right)=\frac{\partial}{\partial x_{j}}\left(\frac{\mu_{t}}{P r_{t}} \frac{\partial \widetilde{f^{\prime 2}}}{\partial x_{j}}\right)+2 \frac{\mu_{t}}{P r_{t}}\left(\frac{\partial \widetilde{f}}{\partial x_{j}}\right)^{2}-2 \beta^{*} \bar{\rho} \omega \widetilde{f^{\prime 2}}
$$

The assumed shape of the PDF is based on the beta distribution [50]:

$$
\mathrm{B}(f)=\frac{f^{a-1}(1-f)^{b-1}}{\int_{0}^{1} x^{a-1}(1-x)^{b-1} d x}
$$


in which the two parameters $a$ and $b$ are functions of the mean mixture fraction and its variance

$$
\begin{gathered}
a=f\left[f\left[\frac{f(1-\tilde{f})}{\widetilde{f^{\prime 2}}}-1\right]\right. \\
b=(1-\widetilde{f})\left[\frac{\widetilde{f}(1-\widetilde{f})}{\widetilde{f^{\prime 2}}}-1\right]
\end{gathered}
$$

Finally, the energy equation needs to be solved because the system is non-adiabatic (the heat is exchanged at the fuel surface), which has an impact on the temperature and species of the reacting flow at the chemical equilibrium. The enthalpy form of the energy equation is selected for the obvious benefit connected with the equilibrium calculations; by neglecting the term of viscous dissipation (the Brinkman number, $\mathrm{Br}=U^{2} P r_{t} / \Delta H_{w}$, defined as the ratio between heat produced by viscous dissipation and heat transported by turbulent conduction at the wall, is on the order of $5 \times 10^{-3}$ ) as well as the variation of pressure (which is approximately constant all over the combustion chamber except through the discharge nozzle where, however, adiabatic walls are imposed), and combining the conduction and species diffusion terms, the latter can be written as

$$
\frac{\partial}{\partial t}(\bar{\rho} \widetilde{H})+\frac{\partial}{\partial x_{j}}\left(\bar{\rho} \widetilde{u}_{j} \widetilde{H}\right)=\frac{\partial}{\partial x_{j}}\left(\frac{\mu_{t}}{P r_{t}} \frac{\partial \widetilde{H}}{\partial x_{j}}\right)+S_{h}
$$

where the source term $S_{h}$ includes the volumetric heat of phase change. Here, the enthalpy of the fluid mixture is defined as

$$
H=\sum_{j} Y_{j}\left[H_{j}^{0}\left(T_{r e f, j}\right)+\int_{T_{r e f, j}}^{T} c_{p, j}(T) d T\right]
$$

where $Y_{j}$ is the mass fraction of the $j$-th species and $H_{j}^{0}\left(T_{r e f, j}\right)$ is its formation enthalpy at the reference temperature $T_{r e f, j}$.

Density-weighted mean temperature and individual species mole fractions are then obtained as functions of $\widetilde{f}, \widetilde{f^{\prime 2}}$ and $\widetilde{H}$ as yielded by the following equation

$$
\widetilde{\varphi}=\int_{0}^{1} \varphi(f, \widetilde{H}) \mathrm{B}(f) d f
$$

where $\varphi(f, \widetilde{H})$ represents the generic functional dependency of one of the above-mentioned parameters on the mixture fraction and enthalpy, whereas the mean density is calculated as

$$
\frac{1}{\bar{\rho}}=\int_{0}^{1} \frac{\mathrm{B}(f)}{\rho(f, \widetilde{H})} d f
$$

Note that the enthalpy turbulent fluctuations are assumed to be independent of the enthalpy level so that the selected PDF in Equation (17) does not change.

Chemical equilibrium conditions are calculated via the minimization of Gibbs free energy algorithm [51] in a number of $\widetilde{f}, \widetilde{f^{\prime 2}}$ and $\widetilde{H}$ points at a specified value of the chamber pressure (which here has been assumed equal to that measured), and a lookup table is constructed. Thus, once the mean mixture fraction, its variance and mean enthalpy are calculated at each point in the flowfield, the corresponding time-averaged species mole fractions, temperature and density are obtained by interpolating the values in the lookup table. The densities are, then, scaled with the values of the actual pressure field in the system.

Heat capacities, molecular weights, and enthalpies of formation for each species considered are extracted from the solver chemical database; the specific heat is determined via the mixing law. 
Molecular dynamic viscosities and thermal conductivities of each species are calculated as functions of local temperature, according to Reference [51].

\subsection{Computational Domain and Boundary Conditions}

Computational results presented in the following table refer to experimental firing tests performed with two laboratory-scale hybrid rocket engines, one in the 200-N thrust scale and the other in the $1-\mathrm{kN}$ class. The two engines have a similar design. They have axisymmetric combustion chambers. A conical nozzle injector, whose exit-section diameter is $6 \mathrm{~mm}$ in the case of the smaller engine and $8 \mathrm{~mm}$ in the case of the larger one, has been employed to deliver gaseous oxygen into a single-port cylindrical fuel grain. Upstream and downstream of the solid grain, a dump plenum and an aft-mixing chamber are set up respectively. In both configurations, the combustion products are accelerated through converging-diverging discharge nozzles. The main dimensions are summarized in Table 2.

Table 2. The computational domains' characteristic dimensions.

\begin{tabular}{cccccc}
\hline Engine & $\begin{array}{c}\text { Prechamber } \\
\text { Diameter }\end{array}$ & $\begin{array}{c}\text { Prechamber } \\
\text { Length }\end{array}$ & $\begin{array}{c}\text { Fuel Grain } \\
\text { Length }\end{array}$ & $\begin{array}{c}\text { Postchamber } \\
\text { Diameter }\end{array}$ & $\begin{array}{c}\text { Postchamber } \\
\text { Length }\end{array}$ \\
\hline $200-\mathrm{N}$ scale & $46 \mathrm{~mm}$ & $25 \mathrm{~mm}$ & $220 \mathrm{~mm}$ & $40 \mathrm{~mm}$ & $60 \mathrm{~mm}$ \\
$1-\mathrm{kN} \mathrm{scale}$ & $80 \mathrm{~mm}$ & $65 \mathrm{~mm}$ & $570 \mathrm{~mm}$ & $80 \mathrm{~mm}$ & $65 \mathrm{~mm}$ \\
\hline
\end{tabular}

For the flowfield axial-symmetry, numerical computations are conducted with two-dimensional grids built in the internal volume of the combustion chamber components; examples of the grids employed with two different scale engines are shown in Figure 1.

The prechamber of the smaller scale engine (Figure 1a) is subdivided into $40 \times 90$ grid cells in the axial and radial directions, respectively; the grain port into $240 \times 40$ grid cells, the postchamber into $80 \times 90$ cells and the nozzle into $60 \times 40$ cells; whereas in the larger scale engine (Figure $1 \mathrm{~b}$ ), the prechamber and the postchamber are both subdivided into $60 \times 110$ cells, the grain port into $310 \times 50$ grid cells and the nozzle into $140 \times 50$ cells. Cells are clustered towards the grain wall in such a way to ensure that the maximum value of $y^{+}$is around $1 \div 2$ at the wall-adjacent cell all along the grain length. Additional axial clustering of cells is placed in the regions near the grain inlet and outlet edges, and near the prechamber, postchamber and nozzle inner surfaces. Grid sensitivity analyses assessing the grid-convergence of the numerical results are reported in Reference [52].

On the inner surface of both the prechamber and postchamber as well as on the nozzle wall, no-slip and adiabatic boundary conditions are imposed. At the injector exit section, a mass flow boundary condition is prescribed along with the temperature (equal to $300 \mathrm{~K}$ ), the oxygen mass fraction and the turbulent quantities, and a supersonic outflow condition is set at the nozzle exit section for which all the variables are extrapolated from the domain interior. 


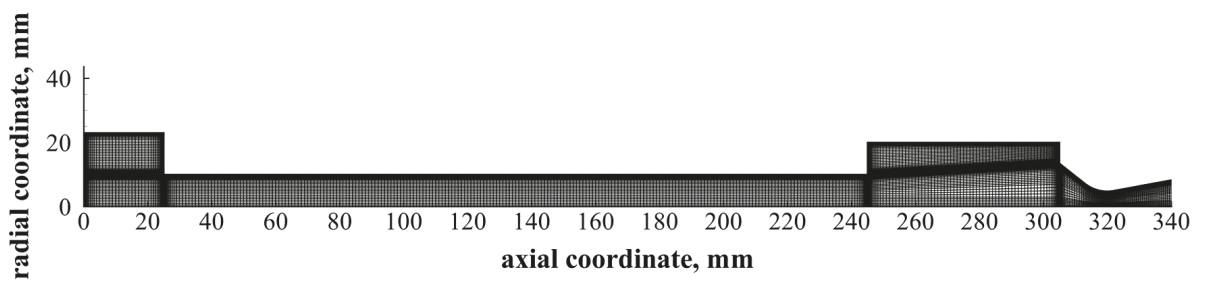

(a) 200-N class hybrid rocket engine.

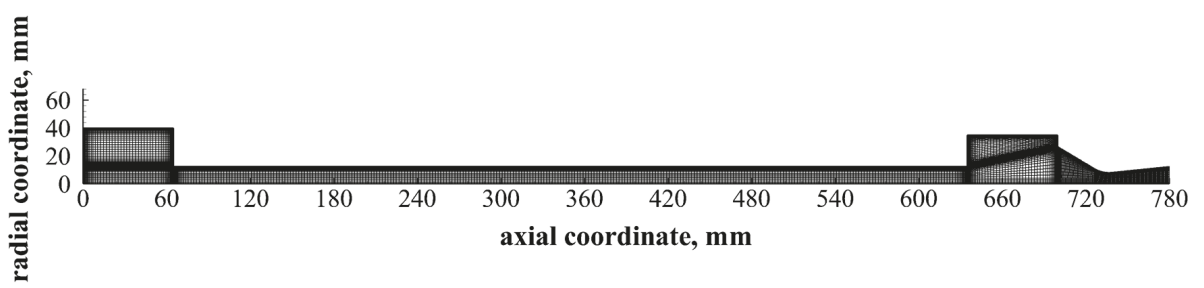

(b) 1-kN class hybrid rocket engine.

Figure 1. Examples of computational grid construction.

\subsection{Gas-Solid Fuel Interface Modeling}

The theoretical model formulation is completed by assigning the boundary conditions at the interface between the gaseous flow region and the solid fuel wall, which can properly describe the fuel consumption mechanism. The fuel surface is an inlet boundary along which the fuel mass flux, the temperature and the mixture fraction depend on the regression rate that is an unknown to be determined.

Now it is necessary to separate the case of classical polymers from the one of liquefying fuels. In fact, while regardless of the type of fuel, the gas/surface interface treatment relies on local mass, energy and mean mixture-fraction balances. In the former case, an additional pyrolysis rate equation is needed for modeling the regression rate, whereas, in the latter case, a different formulation is introduced to account for the entrainment of liquid paraffin from the unstable melt layer forming along the fuel surface, which is the dominant consumption mechanism.

\subsubsection{Classical Non-Liquefying Fuels}

In the case of pyrolyzing fuels, since no material is removed from the surface in a condensed phase (neither solid, such as in the case of fuel loaded with metal particles; nor liquid, as in the case of paraffin wax analyzed later), the mass conservation at the gas-solid interface imposes that

$$
(\rho v)_{w}=\rho_{f} \dot{r}
$$

where $\rho$ is the gas density at the wall and $v$ is the normal-to-wall velocity component due to the pyrolysis products injection; $\rho_{f}$ is the solid fuel density and $\dot{r}$ is the local regression rate.

The energy balance at the gas-solid interface, taking into account the convective heat transfer from the gas to the fuel surface, the steady heat conduction into the solid, and neglecting the radiation, leads to the following relationship between the convective heat flux to the wall, $\dot{q}_{w}$, and the regression rate [29]

$$
\dot{q}_{w}=\left(k_{g} \frac{\partial T}{\partial n}\right)_{w}=\rho_{f} \dot{r}\left[\Delta h_{p}+C_{s}\left(T_{w}-T_{a}\right)\right]
$$


where $n$ is the coordinate normal to the surface oriented from solid to gas, $k_{g}$ is the gas thermal conductivity, $C_{s}$ is the solid heat capacity per unit mass, $\Delta h_{p}$ is the so-called heat of pyrolysis, $T_{w}$ is the fuel surface temperature, and $T_{a}$ is its initial temperature (which is assumed to be equal to the one of the external surface of the fuel). The term in brackets at the right-hand side represents the effective heat of gasification of the fuel, which accounts for the heat radially conducted into the solid grain, further than for the heat of pyrolysis. Note that Equation (25), by properly defining the heat of pyrolysis, implicitly takes into account the heat transfer to the wall by the species diffusion [30].

The fuel pyrolysis is, finally, modeled with the following Arrhenius-type equation [25]:

$$
\dot{r}=A \cdot \exp \left(-\frac{E_{a}}{2 R T_{w}}\right)
$$

where $A$ is the pre-exponential factor, $E_{a}$ is the activation energy and $R$ is the universal gas constant.

The values of the constants appearing in Equations (25) and (26) considered for the HDPE fuel grains analyzed in this work are summarized in Table 3. The density, specific heat and heat of pyrolysis are taken from the work in Reference [53], while the values of the pre-exponential factor and the activation energy from Reference [25] are obtained by modifying the activation energy to match the surface temperature commonly observed in polymeric hybrid fuels (which is around $800 \mathrm{~K}$ ) [54].

Table 3. The high-density polyethylene (HDPE) fuel properties and rate constants.

\begin{tabular}{cccccc}
\hline $\begin{array}{c}\text { Density, } \\
\rho_{f}, \mathrm{~kg} / \mathrm{m}^{3}\end{array}$ & $\begin{array}{c}\text { Specific Heat, } \\
C_{s}, \mathrm{~J} / \mathrm{kg} \mathrm{K}\end{array}$ & $\begin{array}{c}\text { Heat of Pyrolysis, } \\
\Delta h_{p}, \mathrm{MJ} / \mathrm{kg}\end{array}$ & $\begin{array}{c}\text { Initial Fuel Temperature, } \\
T_{a}, \mathrm{~K}\end{array}$ & $\begin{array}{c}\text { Pre-Exponential Factor, } \\
A, \mathrm{~mm} / \mathrm{s}\end{array}$ & $\begin{array}{c}\text { Activation Energy, } \\
E_{a}, \mathrm{~kJ} / \mathrm{mol}\end{array}$ \\
\hline 950 & 2833 & 4.045 & 300 & $4.78 \cdot 10^{6}$ & 190 \\
\hline
\end{tabular}

A dedicated treatment of the mean mixture-fraction boundary condition at the fuel wall is needed as well. The fuel regression rate is typically low in hybrid rockets; therefore, on the one hand, the normal convection of the fuel at the grain surface is relatively weak compared to the gas convection in the cells near the boundary. On the other hand, the diffusive flux plays a dominant role in the mixture-fraction transport near the grain surface where the species concentrations rapidly change, so that a steep mixture-fraction gradient at the fuel wall is present. As a consequence, the simple Dirichlet boundary condition, $f_{w}=1$, on the gas-fuel interface (which is equivalent to imposing that the diffusion coefficient is equal to zero in the cells close to the fuel inlet boundary) is not adequate. In fact, first, it would imply a non-exact evaluation of the gradients in this zone, and, in particular, of the heat flux to the wall (which, for Equation (25), would produce a flawed regression rate), and, second, an extra mixture fraction would be diffused into the flow affecting the global oxidizer to fuel ratio and the chemical equilibrium properties, which eventually would lead to an incorrect estimation of the characteristic exhaust velocity and chamber pressure. The correct solution to this problem is considering an additional equation for the mean mixture-fraction balance at the gas-solid interface [55], which, by rearranging Equation (15) at the wall, can be expressed as

$$
(\rho v)_{w} f_{w}-\left(\frac{\mu_{t}}{S c_{t}} \frac{\partial f}{\partial n}\right)_{w}=\rho_{f} \dot{r}
$$

According to Equation (27), the total mass flux entering the gaseous domain due to the solid fuel regression, which appears on the right-hand side of the equation and represents the production term, is partially balanced by the convection and partially by the diffusion of the fuel mass fraction.

The balance of the mixture-fraction variance, $f^{\prime 2}$, at the wall is instead ignored and it is imposed to be zero.

Note that Equations (25)-(27), upon substitution of Equation (24) into Equation (27) for the wall mass flux, constitute a system of three algebraic equations for the three unknowns-regression rate, the 
surface temperature and the mixture fraction at the wall-which need the computation of the flowfield at each iterative step to be solved.

The required level of mesh refinement near the grain surface allows for the resolution of the viscous sub-layer, with the following boundary conditions for the turbulent kinetic energy and the specific dissipation rate, respectively:

$$
\frac{\partial k}{\partial n}=0, \omega_{w}=\frac{6 \rho}{\mu \beta}\left(\frac{u^{*}}{y^{+}}\right)^{2}
$$

where $u^{*}=\sqrt{\tau_{w} / \rho}$ is the friction velocity.

Both the source terms appearing in Equations (1), (15) and (20) are identically zero:

$$
S_{m}=S_{h}=0
$$

As we will see in the forthcoming section, they are instead calculated in the modeling of the paraffin-wax fuel combustion.

\subsubsection{Liquefying Fuels}

The boundary conditions for the turbulent quantities applied in this case are equal to those described above for polymeric fuels.

The regression rate, $\dot{r}$, can be, now, assumed composed of two terms: the vaporization fraction, $\dot{r}_{v}$, that is generated by the liquid thermal decomposition and later vaporization into the gas stream, and the entrainment fraction, $\dot{r}_{\text {ent }}$, that is related to the mechanical transfer of the liquid from the surface melt layer

$$
\dot{r}=\dot{r}_{v}+\dot{r}_{\text {ent }}
$$

A scheme of this peculiar fuel consumption mechanism is shown in Figure 2 in the case of the supercritical regime: part of the molten fuel on the solid surface undergoes pyrolysis and part leaves the surface in the form of a supercritical fluid that is entrained in the gas stream and burns farther from the wall. More precisely, the molten paraffin wax will behave like a dense fluid which, for simplicity, we call "liquid" and assume having the same properties as paraffin in the liquid state.

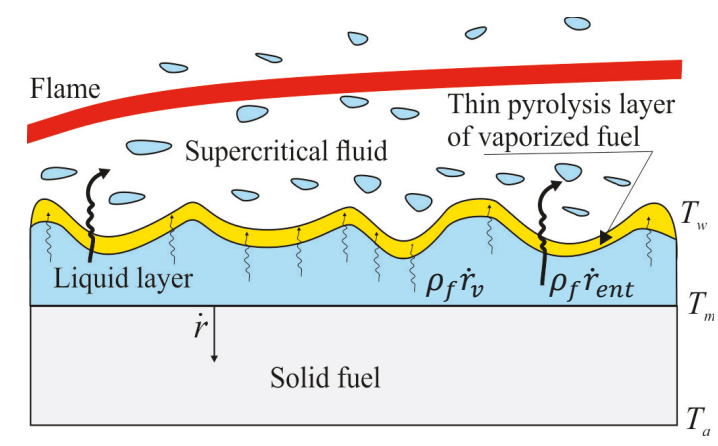

Figure 2. The liquefying fuel consumption mechanism schematic.

A set of equations is, thus, needed for the calculation of the regression rate and its two components, along with the resulting fuel mass flow rates, whose solution is to be incorporated in the fluid dynamic computation. As in the previous case, the mass, energy and mixture-fraction balances at the gas/fuel surface boundary are formulated, with a difference that an additional equation for the calculation of the entrainment component of the fuel mass flow rate is required. 
Following the arguments in References [7,54], by coupling the energy balance equations at both the liquid-solid and gas-liquid interfaces (see Figure 2), at the steady state, the following relationship of the total surface heat flux with the total and the vaporization regression rate is obtained

$$
\dot{q}_{w}=\left(k_{g} \frac{\partial T}{\partial n}\right)_{w}=\rho_{f} \dot{r}\left[C_{s}\left(T_{m}-T_{a}\right)+L_{m}+C_{l}\left(T_{w}-T_{m}\right)\right]+\rho_{f} \dot{r}_{v} L_{v}^{\prime}
$$

where $\rho_{f}$ is the solid fuel density, $C_{s}$ and $C_{l}$ are the specific heats of the solid and liquid fuels (which are considered independent from the temperature here), respectively, $T_{m}$ is the fuel melting temperature, and $L_{m}$ and $L_{v}^{\prime}$ are the fuel heat of the fusion and the heat of pyrolysis, respectively.

Equation (31) represents the fact that the total heat flux transferred from the combusting gases to the fuel surface must be equal to the sum of heat conducted into the liquid layer and the heat required for the pyrolysis of the fuel vaporized fraction (the overall term in the square brackets and last term on the right-hand side of Equation (31), respectively). Note that, for the finite thickness of the liquid layer, the conductive heat flux at the liquid-gas interface is not equal to $\rho_{f} \dot{r} C_{l}\left(T_{w}-T_{m}\right)$, which one might accidentally expect by analogy with the heat conducted in the solid appearing in Equation (25).

In the subcritical regime, the wall phenomena are governed by the evaporation process and $L_{v}^{\prime}=L_{v}$ ( $L_{v}$ being the heat of vaporization) and the wall temperature is equal to the vaporization temperature; whereas, in supercritical conditions, pyrolysis takes place on the surface. In the absence of clear paraffin pyrolysis data, for the sake of simplicity, and also in the supercritical case, the heat of vaporization reported in Reference [7] has been employed by neglecting the heat required for the thermal degradation of paraffin into gaseous ethylene monomers. The wall temperature has a significant influence on the fuel regression rate as it affects both the heat flux and the term $C_{l}\left(T_{w}-T_{m}\right)$ appearing in the wall energy balance, Equation (31). Compared to a purely pyrolyzing polymer, due to the effect of the entrainment, the surface temperature is reduced [54]; however, this parameter can be hardly determined and. in Reference [56], a sensitivity analysis was performed for which the value of $675 \mathrm{~K}$ is the one allowing for the best fit of the experimental data. An isothermal boundary condition is set all over the fuel wall.

It is worth noting that regardless of the definition of the wall temperature and enthalpy (either vaporization or pyrolysis) entailing the vaporization fraction of the regression rate, Equation (31) produces a total regression rate that is marginally sensitive to the value of the enthalpy because. If the vaporization regression rate is decreased for a larger vaporization enthalpy, the total heat flux to the wall tends to rise and so does the entrainment fraction. This sort of balance is further explained later.

Material constants appearing in Equation (31) are listed in Table 4.

Table 4. Paraffin fuel properties.

\begin{tabular}{|c|c|c|c|c|c|c|}
\hline $\begin{array}{l}\text { Solid Fuel Density, } \\
\qquad \rho_{f}, \mathrm{~kg} / \mathrm{m}^{3}\end{array}$ & $\begin{array}{c}\text { Specific } \\
\text { Heat Solid } \\
\text { Phase, } C_{s} \\
\mathrm{~J} / \mathrm{kg} \mathrm{K}\end{array}$ & $\begin{array}{c}\text { Specific } \\
\text { Heat Liquid } \\
\text { Phase, } C_{l} \\
\text { J/kg K }\end{array}$ & $\begin{array}{l}\text { Heat of Fusion, } \\
L_{m}, \mathrm{~kJ} / \mathrm{kg}\end{array}$ & $\begin{array}{l}\text { Heat of Vaporization, } \\
\qquad L_{v}, \mathrm{~kJ} / \mathrm{kg}\end{array}$ & $\begin{array}{l}\text { Melting Temperature, } \\
T_{m}, \mathrm{~K}\end{array}$ & $\begin{array}{l}\text { Surface Temperature, } \\
\qquad T_{w}, \mathrm{~K}\end{array}$ \\
\hline $920\left(^{*}\right)$ & $2030\left(^{*}\right)$ & $2920\left(^{*}\right)$ & $\left.167.2^{*}\right)$ & $\left.163.5^{*}\right)$ & $339.6\left(^{*}\right)$ & 675 \\
\hline
\end{tabular}

According to the approach described in Reference [7], the following semiempirical relationship has been considered for modeling the entrainment component of the fuel regression rate as a function of the rocket chamber operating conditions and of an entrainment parameter that lumps the liquid fuel properties together:

$$
\dot{r}_{\text {ent }}=a_{\text {ent }} \frac{G^{3}}{\dot{r}^{1.5}}
$$


where $G=4 \dot{m} / \pi D^{2}$ is the total mass flux in the local section of the grain port and $a_{e n t}$ is the entrainment factor depending on the physical properties of the selected fuel, primarily on the fuel liquid viscosity, and on the average gas density in the chamber as

$$
a_{\text {ent }} \propto \frac{1}{\mu_{l} \rho_{g}^{1.5}}
$$

Equation (33) is derived from a theoretical assessment of the surface liquid-layer fluid dynamic stability; the main result is that the susceptibility of a given fuel to the instability increases with decreasing viscosity and surface tension of the melt layer; the entrainment component of fuel regression rate is, therefore, roughly inversely proportional to viscosity at the characteristic temperature of the layer, while it depends directly on the dynamic pressure. A parametric analysis of the effect of the entrainment parameter on the regression rate components is reported in Reference [56]. From this analysis, the value of $2.1 \times 10^{-13} \mathrm{~m}^{8.5} \mathrm{~s}^{0.5} / \mathrm{kg}^{3}$ has been identified for the best fit of the experimental data in the reference Test $\mathrm{P} 4$. The latter value, considering the different densities (average gas density in Test P4 is $1.62 \mathrm{~kg} / \mathrm{m}^{3}$ ) is in good agreement with the one reported in Reference [7].

Once Equations (30)-(32) are combined, given the heat flux to the wall and the total mass flux, the three components of the fuel regression rate can be calculated. The fuel mass fluxes associated with the vaporization and entrainment components, respectively, are obtained as follows

$$
\begin{aligned}
G_{f, v} & =\rho_{f} \dot{r}_{v} \\
G_{f, e n t} & =\rho_{f} \dot{r}_{e n t}
\end{aligned}
$$

Vaporization and entrainment components are handled differently.

The vaporization component is treated equally to the case of pyrolyzing fuels, considering the mass and mixture-fraction balance equations at the grain wall, given by

$$
\begin{gathered}
(\rho v)_{w}=G_{f, v} \\
(\rho v)_{w} f_{w}-\left(\frac{\mu_{t}}{S c_{t}} \frac{\partial f}{\partial n}\right)_{w}=G_{f, v}
\end{gathered}
$$

This allows for correctly taking the blocking effect on the heat transfer to the wall into account, while, as explained in the previous section, Equation (37) is needed to ensure the mixture fraction has a global balance.

The entrainment mass flux does not contribute to the blocking effect, thus, a specific treatment is adopted for the introduction of the entrainment component into the computational domain. For the sake of simplicity, we assume that despite the entrained paraffin initially in the liquid phase immediately gasifies because of the large combustion heat release. The local entrainment contribution is uniformly assigned as a mass production term in the local volume of the grain port corresponding to the surface cell of length $\Delta x$ through which the fuel mass enters the fluid domain, $\pi D^{2} \Delta x / 4$ :

$$
S_{m}=4 \frac{G_{f, e n t}}{D}
$$

In order to satisfy the species balance, an equal production term is assigned also for the mean mixture fraction. Finally, the energy required by the pyrolysis of the liquid fuel mass flow rate is taken into account by assigning a corresponding negative energy source term in the same volume:

$$
S_{h}=-4 \frac{G_{f, e n t}}{D} L_{v}^{\prime}
$$


Additionally, in this case, as the heat flux to the surface and the total mass flux needed for the calculation of the regression rate are outputs of the flowfield resolution, which, in turn, depends on the regression rate itself, an iterative procedure is needed for the problem solution.

\subsection{Solution Strategy}

In this context, steady-state solutions to the equations presented above are searched, even in the case of transient simulations, as explained in Section 3.5. The equations are solved with a control-volume-based technique and a pressure-based solver [57], i.e., Equations (1) and (2) are not solved directly, but are combined to derive a pressure equation in such a way that the velocity field, corrected by the pressure satisfies the continuity equation. The velocity and pressure fields are simultaneously solved with a coupled algorithm. The diffusion terms are central-differenced and are second-order accurate; the convective terms are represented according to a quadratic upwind scheme. Convergence is obtained by iterating until the residuals drop by five orders of magnitude.

The solution procedure is approached through the following steps.

(1) Input the boundary conditions and a combustion pressure reference value; at the solid grain wall, trial values of the unknown required variables are used.

(2) Computation of the look-up table containing the time-averaged values of species mass fractions, density, and temperature as a function of mean mixture fraction, mixture fraction variance, and enthalpy by means of the integrations in Equations (22) and (23).

(3) The solution of the mass, momentum, turbulence and flow mixture fraction equations, Equations (1), (2), (4), (5), (16) and (20).

(4) Calculation of the spatial distribution of temperature, density and individual chemical species mass fractions by interpolating the values in the look-up table.

(5) Scaling of the density field with pressure with respect to the reference value. Dependence of the temperature and mixture composition on pressure is neglected.

(6) From the results of this simulation, the convective heat flux to the wall is evaluated, so that Equations (25)-(27) in the case of standard polymers, or Equations (30)-(32) and (37) in the other case of liquefying fuels, can be solved simultaneously to compute the new distributions of the variables along the grain surface and, accordingly, the mass flux distribution either from Equation (24), or Equations (34) and (35), respectively, along with the volume source terms in Equations (38) and (39) required in the latter case.

Steps from (3) to (6) are then iterated by adjusting the local values of the mentioned quantities until convergence is reached.

\subsection{Fuel Port Diameter Update with Time}

To capture the local regression-rate variations in the firing, transient simulations have been attempted. Here, for transient simulation, a series of steady-state computations is intended, which are carried out on different port sizes calculated over several instants in the firing by updating the fluid-solid interface boundary. A quasi-steady approach is employed by neglecting the time-dependent terms in the balance equations and boundary conditions in the time interval between two successive flow-domain updates. This assumption is based on the consideration that the fluid dynamic characteristic time, which can be assumed to be proportional to the diffusion time in the boundary layer, is shorter than the time required for the thermal profile adjustment in the solid grain due to a change in the port diameter and, thus, in the regression rate.

The displacements of the computational grid nodes are not uniform all over the grain length but, rather, they vary according to the different calculated values of the regression rate. As the regression rate is defined in the direction normal to the fuel surface, due to the local surface inclination, the displacement of a generic point occurs along both the radial and axial directions.

Starting from a given port profile at the $n$-th time-step, defined by the axial and radial coordinates $x_{i}^{n}, y_{i}^{n}$ of the grid nodes (where the subscript $i$ indicates the $i$-th node), a CFD simulation is carried out 
with the models described above in order to compute the fuel regression rate distribution $\dot{r}^{n}\left(x_{i}\right)$ at the same time step. A forward Euler integration of the local regression rate is then carried out to calculate the displacements after a fixed time-step, $\Delta t=t^{n+1}-t^{n}$, which, for the $i$-th node can be expressed as

$$
\Delta_{i}^{n}=\dot{r}^{n}\left(x_{i}\right) \Delta t
$$

By indicating with $\theta_{i}^{n}$ the local inclination of the fuel surface with respect to the axial direction in the $i$-th node, which is equal to the angle between the direction normal to the surface and the radial direction (see Figure 3), the coordinates of the node at the time $t^{n+1}$ are calculated as

$$
\begin{aligned}
& x_{i}^{n+1}=x_{i}^{n}-\Delta_{i}^{n} \cos \theta_{i}^{n} \\
& y_{i}^{n+1}=y_{i}^{n}+\Delta_{i}^{n} \sin \theta_{i}^{n}
\end{aligned}
$$

which allow reconstructing the new grain port profile.

Once the new distribution is calculated, the fluid domain geometry is consequently modified, the computational mesh is adjusted to the new geometry and the steady-state numerical simulation at the new time-step is performed.

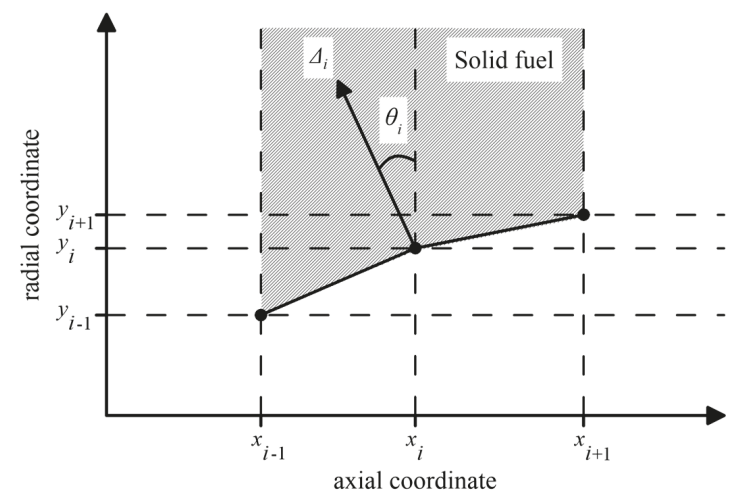

Figure 3. The schematic representation of the $i$-th node displacement calculation.

\section{Classical Polymeric Fuels: Numerical Results}

In this section, the numerical results obtained with the model described in Section 3.3.1 for the simulation of hybrid rockets burning classical polymeric fuels are presented.

\subsection{Experimental Test Cases}

Two motor firings, both performed by burning HDPE fuel grains with gaseous oxygen, are considered here as test cases.

Test HDPE-1 was performed with the subscale $200-\mathrm{N}$ thrust class rocket. The fuel grain was $220 \mathrm{~mm}$ long and the initial port diameter was equal to $D_{0}=15 \mathrm{~mm}$. The firing duration was set to $12 \mathrm{~s}$. A graphite nozzle with a 9.6- $\mathrm{mm}$ throat diameter and an area expansion ratio of 2.99 was employed.

Test HDPE-2 was performed with the $1-\mathrm{kN}$ thrust engine. The grain length was equal to $L=570 \mathrm{~mm}$ and the initial port diameter was $D_{0}=25 \mathrm{~mm}$; a $44 \mathrm{~s}$ firing duration was set in this case. The large scale motor employed a copper water-cooled nozzle with a 16-mm throat diameter and an area ratio equal to 2.5. A detailed description of the rocket engine setup and of the experimental facilities can be found in Reference [58].

All the data averaged over the firings presented in this work have been obtained with the loss mass method; the measurement uncertainty quantification followed the procedure in Reference [5]. 
The average parameters measured in the two firings are summarized in Table 5. The overall mixture ratio, $O / F$, is the ratio of the average oxygen mass flow rate to the average fuel mass flow rate in the burn; the latter was calculated as the fuel mass loss divided by the burning time.

Table 5. The test operating parameters measured with HDPE fuel grains.

\begin{tabular}{ccccccc}
\hline Test ID & $\begin{array}{c}\text { Average } \\
\text { Oxidizer } \\
\text { Mass Flozw } \\
\text { Rate, g/s }\end{array}$ & $\begin{array}{c}\text { Time-Space } \\
\text { Averaged } \\
\text { Grain Port } \\
\text { Diameter, } \\
\mathbf{m m}\end{array}$ & $\begin{array}{c}\text { Time-Space } \\
\text { Averaged } \\
\text { Oxidizer } \\
\text { Mass Flux, } \\
\mathbf{k g} / \mathbf{m}^{\mathbf{2}} \mathbf{s}\end{array}$ & $\begin{array}{c}\text { Time-Space } \\
\text { Averaged } \\
\text { Regression } \\
\text { Rate, } \mathbf{m m} / \mathbf{s}\end{array}$ & $\begin{array}{c}\text { Average } \\
\text { Overall } \\
\text { Mixture } \\
\text { Ratio, } \mathbf{O} / \mathbf{F}\end{array}$ & $\begin{array}{c}\text { Postchamber } \\
\text { Pressure, } \\
\text { Bar }\end{array}$ \\
\hline HDPE-1 & $27.0 \pm 0.04$ & $19.4 \pm 0.05$ & $92.5 \pm 0.11$ & $0.39 \pm 0.04$ & $5.44 \pm 0.4$ & $6.49 \pm 0.65$ \\
HDPE-2 $\left(^{*}\right)$ & $210.4 \pm 10$ & $55.9 \pm 0.65$ & $84.8 \pm 4.7$ & $0.693 \pm 0.013$ & $3.09 \pm 0.16$ & $24.0 \pm 0.021$ \\
\hline
\end{tabular}

* The different measurement uncertainties of the variables involving the oxidizer mass flow rate are due to the different system for measuring the mass flow rate.

\subsection{Internal Ballistics Steady Simulation: Comparison with Experimental Data}

A single numerical simulation of the Test HDPE-1 has been carried out by considering the time-spatially averaged grain port diameter measured in the burn (note that we call a steady simulation one in which the port diameter is not updated across the firing); the results are discussed in this section.

Figure 4 shows the plot of the calculated temperature contour with the streamlines overlapped on the top half (with respect to the axis of symmetry) and the fuel mass-fraction in the unburned mixture isolines drawn on the bottom half. From this picture, all the main features of the internal flowfield can be unveiled.

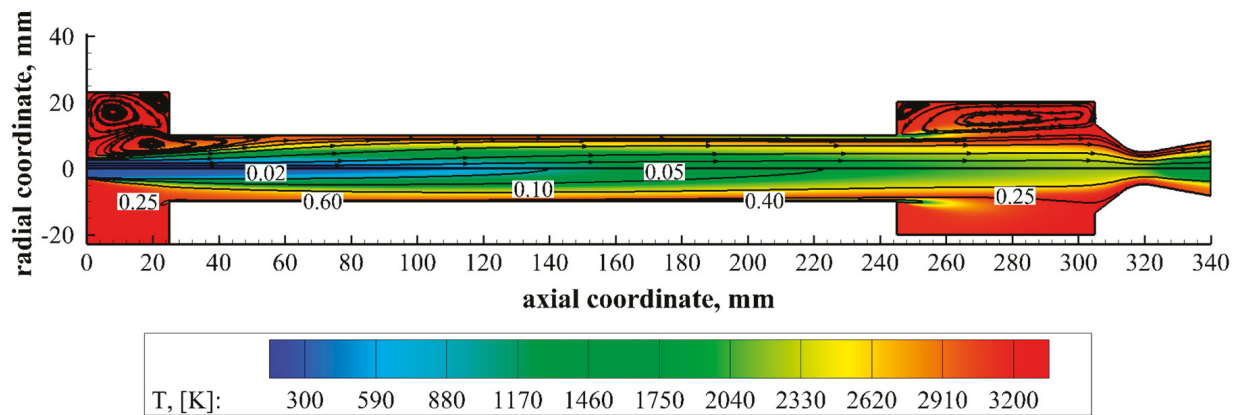

Figure 4. Test HDPE-1: The temperature contour plot with overlapped streamlines (top half) and mixture-fraction isolines (bottom half).

The combustor inlet flow is dominated by the development of the oxygen jet emerging from the axial nozzle injector (which is clearly distinguishable from the low-temperature region), which spreads almost linearly up to the impingement point on the grain surface. Upstream of the impingement point, in the entrance region of the grain, there is an extended recirculation region characterized by a main, broad counter-clockwise-rotating (if seen from the top half) vortex that is bounded, on the front side, by the zone of oxygen impingement. In the pre-combustion chamber, another large vortex, clockwise rotating, is formed by delimiting the main one on the backside. Finally, also in the aft-mixing chamber, a large trapped counter-clockwise-rotating vortex is formed, which further promotes the propellant mixing.

As a result of the flow recirculation generated at the motor head end, the propellant mixing is strongly promoted, and combustion takes place in the recirculation core; hot combustion gases are transported from the grain entrance region back to the prechamber, where the temperature is very high [5]. Downstream of the recirculation, the temperature distribution reflects the typical structure 
of a diffusion flame, with a narrow region close to the fuel surface where the near-stoichiometric conditions are reached, and the temperature shows its maximum value. Anyway, as a consequence of the relatively high turbulent kinetic energy determined by the different vortices, relatively high temperatures also characterize the core flow.

Figure 5 shows a comparison between the computed fuel regression rate axial profile and the measured data. Experimental points are obtained by sectioning the grain transversally in a number of slices, and measuring the port diameter by means of a caliper; in each transversal section, the minimum, maximum and the average of eight diameter measurements have been recorded; the corresponding time-averaged regression rate is calculated by dividing the port radius variation in the firing by the burning time.

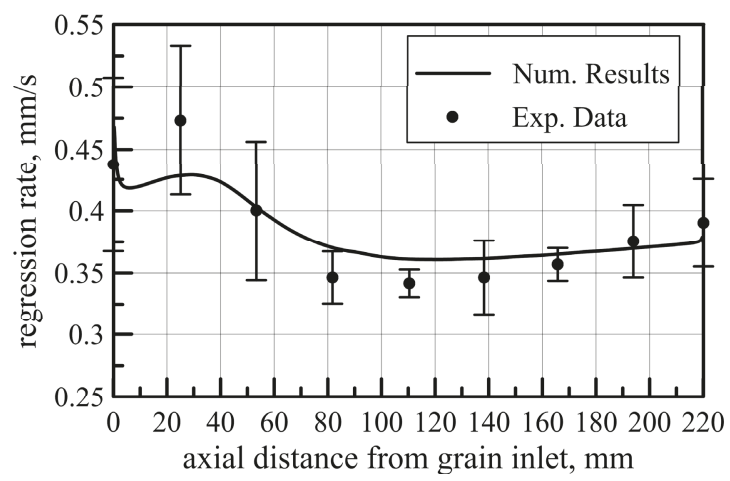

Figure 5. Test HDPE-1: The comparison between the computed and measured regression rate axial profile.

The regression rate axial trend yields a peak for the oxygen jet impingement, followed by a minimum point, after which it monotonically increases. This latter behavior is typical of the boundary layer heat transfer, for which the heat flux increase due to the mass addition down the port becomes dominant on the decrease due to the boundary layer growth from a certain axial distance.

Provided that a comparison is drawn between the regression rate calculated by simulating the flowfield at the average port diameter and experimental data retrieved from the port shape measured after the motor extinguishment, a good agreement is shown. The maximum deviation is yielded in the zone of maximum fuel consumption where, however, the experimental uncertainty is maximum because of the asymmetric consumption determined by the motor ignition device [35].

The numerical model is able to predict also the pressure measured in the aft-mixing chamber as well as an indication of the combustion efficiency. The latter is defined in terms of the characteristic exhaust velocity efficiency, $\eta$, as the ratio

$$
\eta=\frac{c^{*}}{c_{t h}^{*}}
$$

where $c^{*}$ is the characteristic exhaust velocity estimated with the pressure, total mass flow rate and nozzle throat area, $A_{t}$, either measured or numerically calculated

$$
c^{*}=\frac{p_{c} A_{t}}{\left(\dot{m}_{o x}+\dot{m}_{f}\right)}
$$

and $c_{t h}^{*}$ is the theoretical exhaust characteristic velocity obtained in adiabatic chemical equilibrium conditions at the given overall mixture ratio with the CEA code [51]. The fuel mass flow rate is calculated from the numerical simulations by integrating the fuel mass flux along with the fuel port; $\mathrm{O} / \mathrm{F}$ is, hence, obtained as the ratio between the prescribed oxidizer mass flow rate entering in the computational domain and the total fuel mass flow rate. The calculated parameters with the relative 
deviations with respect to the measured values are reported in Table 6 and an excellent agreement is obtained.

Table 6. Test HDPE-1: The computed average parameters and deviation with respect to the experimental data.

\begin{tabular}{lcc}
\hline \multicolumn{1}{c}{ Parameter } & Numerical Results & Deviation wrt Experimental Data \\
\hline Average fuel regression rate, $\mathrm{mm} / \mathrm{s}$ & 0.384 & $-1.6 \%\left(^{*}\right)$ \\
Overall mixture ratio, $O / F$ & 5.54 & $+1.6 \%$ \\
Aft-mixing chamber pressure, bar & 6.60 & $+1.7 \%$ \\
$c^{*}$ efficiency & 0.966 & $+1.1 \%$ \\
\hline
\end{tabular}

* Experimental regression rate to be intended as time-space averaged value.

\subsection{Internal Ballistics Transient Simulation: Comparison with Experimental Data}

It is clear that, although numerically simulating the flowfield in the hybrid rocket combustion chamber at the time-space average port diameter in the burn is an efficient method to provide meaningful details, as well as the time-space, averaged regression rate, a transient simulation, in the sense specified above, is required to compute the $O / F$ time shift and the fuel consumption distribution at the end of the burn, especially when the regression rate axial profile is uneven and strongly depends on the grain port geometry, as observed in Section 4.2.

The results of such a simulation carried out in the conditions of Test HDPE-2, by updating the local port diameter in 22-time-steps of $2 \mathrm{~s}$ each according to the procedure described in Section 3.4, are analysed.

Figure 6 shows the plots of the calculated temperature contours with the streamlines on the top half and the fuel mass-fraction in the unburned mixture isolines on the bottom half, which have been obtained at several time instants in the burn. First, note that the fluid domain enlarges with the time step because, of course, the port opens up and its shape changes as well for the axial regression-rate non-uniformity

In all the time frames, the oxygen jet spreading and the associated recirculation region in the grain inlet portion can also be clearly seen in this larger-scale engine configuration. The recirculation region at the motor start-up is confined to the prechamber, while, as the grain port enlarges, the oxygen jet impingement point on the fuel wall moves downstream and the recirculation region becomes larger.

The numerical results confirm that the forward axial shift of the impingement point at each time step, once the port diameter is known, can be easily estimated by referring to the characteristics of a free jet ensuing from the injector with a spreading angle of 8 degrees, as observed in Reference [59]. 

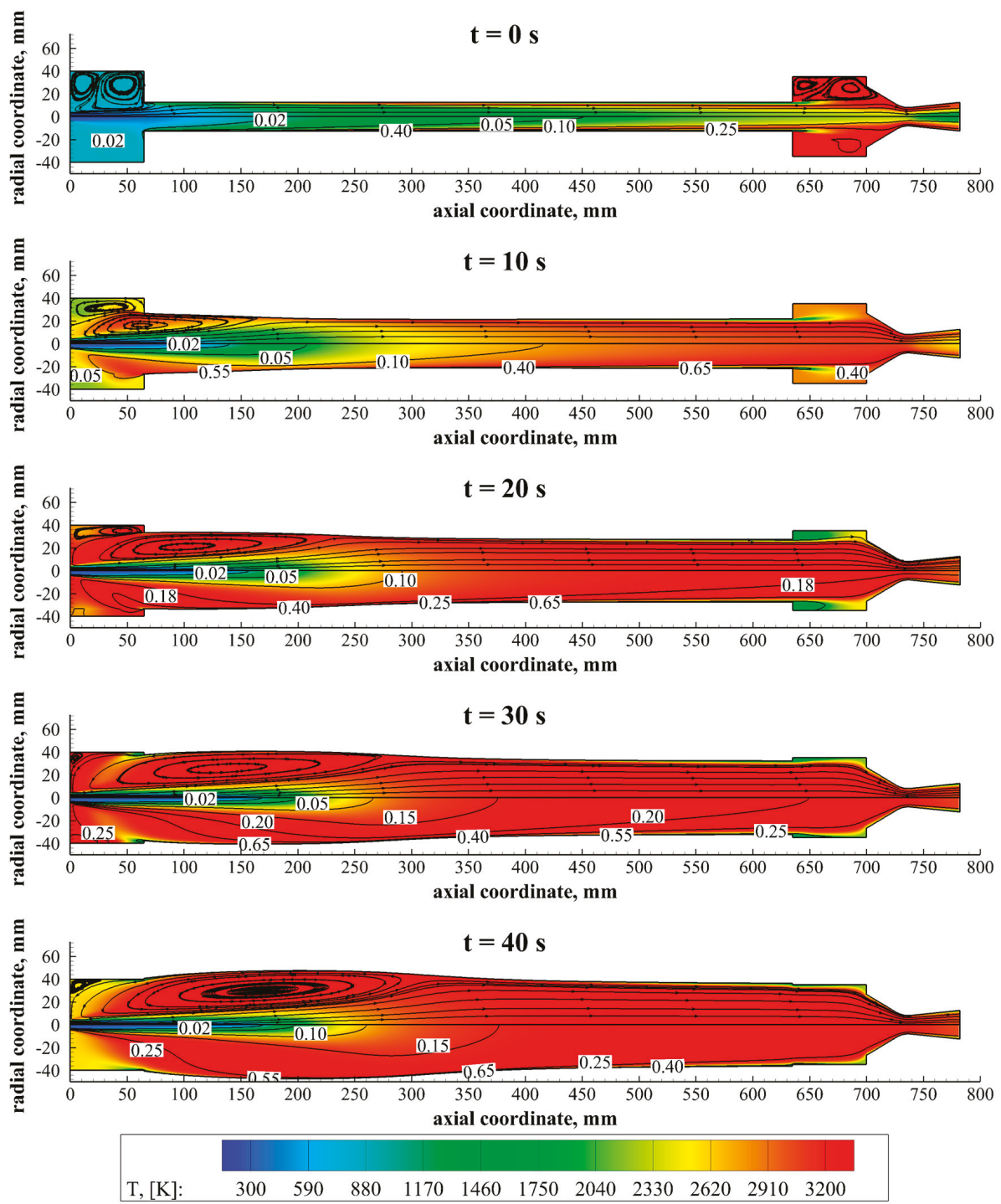

Figure 6. Test HDPE-2: The temperature contour plot with overlapped streamlines (top half) and mixture-fraction isolines (bottom half).

The lateral boundaries of the computational domain shown in Figure 6 suggest that the maximum fuel consumption is achieved in the recirculation region, nearly upstream of the impingement point. Accordingly, the axial profiles of both the regression rate and local port diameter, displayed in Figure 7, yield maximum points, which move downstream in the burn, for the jet impingement shift described above. 


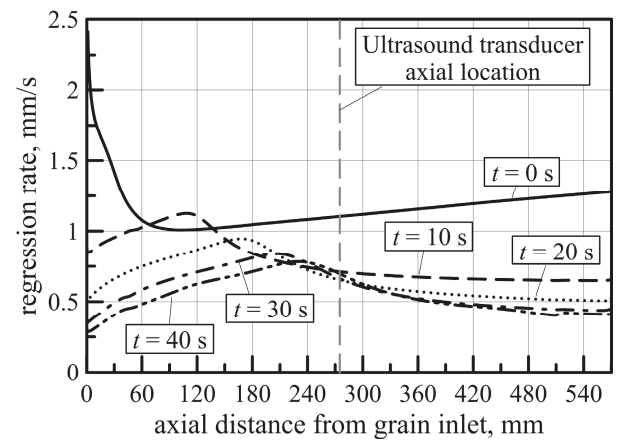

(a) Regression rate

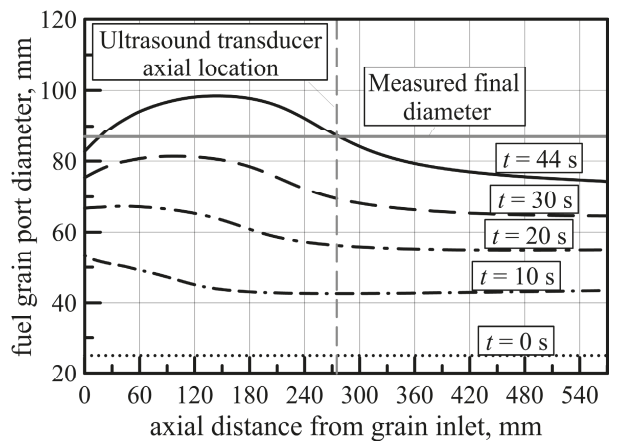

(b) Fuel grain port diameter

Figure 7. Test HDPE-2: (a) the regression-rate and (b) the fuel-grain port diameter axial profiles at several time steps in the burn.

Moreover, because of the mass flux decrease, a reduction of the average regression rate can be observed.

The average parameters computed with the numerical simulation are summarized in Table 7 along with the deviation with respect to the corresponding experimental data. A good agreement between the numerical results and experimental data has been obtained, which validates the employed model. The time-space averaged regression rate is fairly predicted with a deviation with respect to the measured value of only $1.2 \%$; note that, based on the definition of the average regression rate, the latter coincides with the error on the postburn space-averaged port diameter prediction.

The numerically predicted average values of the mixture ratio and of the chamber pressure are calculated as the ratio of the average oxidizer mass flow rate to the average fuel mass flow rate and as the arithmetic mean of the chamber pressure over all the time steps, respectively. The numerically predicted average combustion efficiency is calculated by means of Equation (44), considering the average values of the chamber pressure and mass flow rates.

The prediction of the aft-mixing chamber pressure is affected by the maximum deviation of $5 \%$, which can be explained through the combined effect of the errors in the fuel mass flow rate (and, consequently, in the overall mixture ratio) and combustion efficiency that both affect the chamber pressure. Once the comparison is made in terms of the combustion efficiency, a better agreement is obtained, with a deviation of $3.7 \%$.

Table 7. Test HDPE-2: The computed average parameters and deviation with respect to the experimental data.

\begin{tabular}{lcc}
\hline \multicolumn{1}{c}{ Parameter } & Numerical Results & Deviation wrt Experimental Data \\
\hline Postburn space-averaged port diameter, $m m$ & 85.8 & $-1.2 \%$ \\
Time-averaged overall mixture ratio & 3.18 & $+2.9 \%$ \\
Aft-mixing chamber pressure, bar & 22.9 & $-5.0 \%$ \\
$c^{*}$ efficiency & 0.953 & $-3.7 \%$ \\
\hline
\end{tabular}

Test HDPE-2 allows for an assessment of the calculated local time-resolved regression rate. The ultrasound pulse-echo technique was used to measure the local fuel web thickness and derive the regression rate. One ultrasonic transducer was located around the chamber mid-span [60], whose axial location along the grain is indicated in Figure 7.

Figure 8 a shows the time trends of the local regression rate and port diameter. The numerically computed regression rate fairly reproduces the characteristic behavior shown by the measured data for which, around $25 \mathrm{~s}$, there is a minimum consumption rate. This feature can be explained considering that the regression rate initially decreases for the mass flux reduction, then, owing to the shift of the 
oxygen jet impingement point towards the probe location, the recirculation region enlarges and the consumption rate increases (see also Figure 7a).

Additionally, despite the bias relative to the measured trend, the increase of the aft-chamber pressure over the firing, which is due to the increase of the fuel mass flow rate, is well predicted (Figure 8 b). The nearly constant difference between the numerical and experimental curves is essentially a consequence of the regression rate underestimation (see the deviation in O/F shown in Table 7), which remains constant at the different time steps.

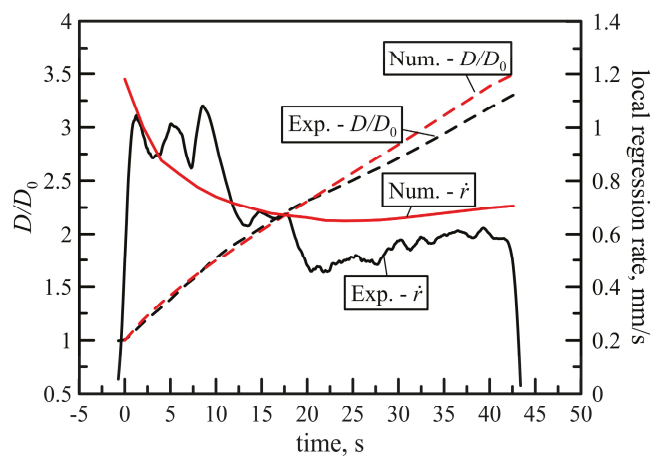

(a) Regression rate

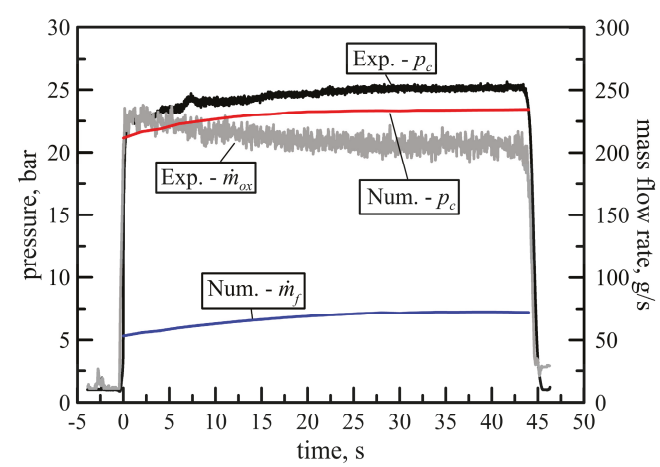

(b) Postchamber pressure

Figure 8. Test HDPE-2: The comparison between the computed and measured (a) local regression rates and port diameters; (b) the pressure and mass flow rates

\section{Paraffin-Based Fuels: Numerical Results}

The numerical model presented in Section 3.3.2 has been applied to the simulation of several test cases obtained from the static firing of the sub-scale hybrid rocket burning paraffin-based fuels; the results are discussed in this section.

\subsection{Experimental Test Cases}

Seven test cases are presented here, which were performed by varying the oxidizer mass flow rate and the firing time, with the aim of achieving a significant range of the average oxidizer mass flux, which is the main control variable in the hybrid rocket's operation (see Table 8). Paraffin-based fuel grains were made of a blending of a low-melting-point paraffin wax and a microcrystalline wax; gaseous oxygen was axially injected in the grain's single port. In all the firings, an exhaust nozzle having the throat diameter equal to $10.7 \mathrm{~mm}$ and an area expansion ratio equal to 2.4 was employed. 
The details of the test campaign from which the experimental data have been gathered can be retrieved in Reference [31].

Steady numerical simulations are carried out on the port geometry corresponding to the average diameter in the burn and the imposing oxygen mass flow rate (both reported in Table 8).

Table 8. The test operating parameters measured with paraffin-based fuel grains.

\begin{tabular}{|c|c|c|c|c|c|c|}
\hline Test ID & $\begin{array}{c}\text { Average } \\
\text { Oxidizer } \\
\text { Mass Flow } \\
\text { Rate, g/s }\end{array}$ & $\begin{array}{c}\text { Time-Space } \\
\text { Averaged } \\
\text { Grain Port } \\
\text { Diameter, } \\
\text { mm }\end{array}$ & $\begin{array}{c}\text { Time-Space } \\
\text { Averaged } \\
\text { Oxidizer } \\
\text { Mass Flux, } \\
\text { kg/m } \mathrm{m}^{2} \mathrm{~s}\end{array}$ & $\begin{array}{c}\text { Time-Space } \\
\text { Averaged } \\
\text { Regression } \\
\text { Rate, } \mathrm{mm} / \mathrm{s}\end{array}$ & $\begin{array}{c}\text { Average } \\
\text { Overall } \\
\text { Mixture } \\
\text { Ratio, } O / F\end{array}$ & $\begin{array}{c}\text { Postchamber } \\
\text { Pressure, } \\
\text { Bar }\end{array}$ \\
\hline P1 & $16.0 \pm 0.06$ & $20.5 \pm 0.29$ & $48.38 \pm 1.54$ & $1.63 \pm 0.22$ & $0.77 \pm 0.10$ & $4.9 \pm 0.005$ \\
\hline P2 & $29.0 \pm 0.68$ & $25.0 \pm 0.06$ & $59.22 \pm 1.66$ & $1.79 \pm 0.10$ & $1.03 \pm 0.07$ & $8.0 \pm 0.008$ \\
\hline P3 & $38.0 \pm 0.85$ & $26.6 \pm 0.16$ & $67.83 \pm 2.30$ & $2.04 \pm 0.13$ & $1.10 \pm 0.09$ & $11.2 \pm 0.011$ \\
\hline $\mathrm{P} 4$ & $42.0 \pm 1.35$ & $27.1 \pm 0.10$ & $72.58 \pm 2.83$ & $2.29 \pm 0.15$ & $1.08 \pm 0.10$ & $12.9 \pm 0.013$ \\
\hline P5 & $55.5 \pm 1.36$ & $29.0 \pm 0.04$ & $83.75 \pm 2.26$ & $2.41 \pm 0.17$ & $1.26 \pm 0.11$ & $16.9 \pm 0.017$ \\
\hline P6 & $59.5 \pm 1.66$ & $28.0 \pm 0.12$ & $96.76 \pm 3.48$ & $2.73 \pm 0.20$ & $1.19 \pm 0.10$ & $18.4 \pm 0.018$ \\
\hline P7 & $60.5 \pm 1.56$ & $27.1 \pm 0.10$ & $105.22 \pm 3.50$ & $2.96 \pm 0.23$ & $1.20 \pm 0.11$ & $19.1 \pm 0.019$ \\
\hline
\end{tabular}

\subsection{Role of the Regression-Rate Entrainment Component}

The primary difference of the paraffin-wax consumption mechanism with respect to the classical polymer pyrolysis relies upon the liquid layer formation and entrainment in the gas stream. With the aim of highlighting the role played by the entrainment component of regression rate on the flowfield, two numerical test cases built with the input conditions of Test P4 (Table 8) have been considered.

First, an extreme case is analyzed in which the entrainment component is assumed to be zero, so that the overall regression rate, as with a standard polymer, is only due to vaporization, i.e., $\dot{r}=\dot{r}_{v}$, (Equation (32) is, thus, not considered), and it is compared to a second case in which the entrainment term also is included in the calculations. In the second case, the entrainment parameter has been assumed to be equal to $2.1 \times 10^{-13} \mathrm{~m}^{8.5} \mathrm{~s}^{0.5} / \mathrm{kg}^{3}$; the latter is the one allowing for the best fit of the experimental data obtained in test $\mathrm{P} 4$ as discussed in Reference [56]. In addition, the calculations are repeated with an HDPE fuel grain of equal dimensions by imposing an equal oxygen mass flow rate, i.e., with the model described in Section 3.3.1.

Figure 9a shows the obtained fuel regression rates and Figure 9b, the corresponding surface heat fluxes. Total regression rate and relevant heat flux have similar axial profiles. It is worth noting that, as the heat requested for HDPE pyrolysis is larger than that used in Equation (31) for modeling paraffin melting and vaporization (about $5500 \mathrm{~kJ} / \mathrm{kg}$ against $1400 \mathrm{~kJ} / \mathrm{kg}$, respectively), in the first extreme case, without considering the entrainment contribution, the regression rate (see the dark grey continuous line in Figure 9a) is fairly higher than that obtained with HDPE (light grey continuous line in Figure 9a) at equal oxidizer mass fluxes, despite the fact that the enhanced blocking effect determines a significantly lower surface heat flux (see Figure 9b). However, the spatially-averaged regression rate obtained in this case is equal to $1.11 \mathrm{~mm} / \mathrm{s}$, which is still significantly lower than the corresponding measured value of $2.29 \mathrm{~mm} / \mathrm{s}$ (see Table 8 ).

When the entrainment component is taken into account, the calculated regression rate is more than doubled (see the black continuous line in Figure 9a) because, with the set of parameters considered here, the most significant contribution is given by the entrainment itself (black dotted line), the vaporization component (black dashed line) being smaller than the entrainment fraction. With the larger mass flux due to the entrained fuel, as entrainment does not contribute to the heat-transfer blocking, the heat flux is raised (see the black line in Figure 9b). In particular, in the fore end of the grain (up to about 80 $\mathrm{mm}$ ), where the effect of the mass addition is low, the vaporization regression rate yields values similar to the HDPE regression rate; accordingly, comparable equilibrium conditions between the heat transfer and the mass blowing at the grain wall is obtained, and the heat flux profiles are similar, whereas, 
downstream of that point, for the largely increased mass flux due to the entrainment component, the heat flux to the paraffin fuel surface significantly diverges from that achieved with HDPE.

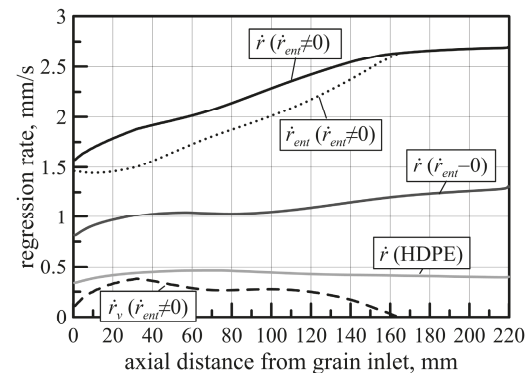

(a) Regression rate

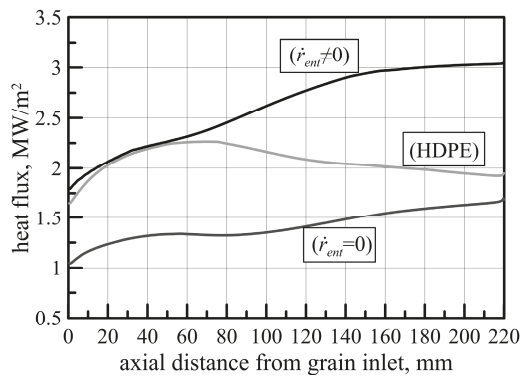

(b) Heat flux to fuel grain surface

Figure 9. The comparison between the results obtained with and without considering the entrainment.

Figure 10 shows the two contour maps of the temperatures calculated with paraffin (considering both vaporization and entrainment) and HDPE. It is worth noting that, in the case of paraffin fuel burning, the recirculation region is smaller for the modeling of entrained paraffin mass that, as mentioned above, is introduced in the port volume; the latter needs to be axially accelerated at the expense of the oxygen jet momentum, which decreases and causes a larger jet spreading. For the same reason, the hottest region in the flowfield of the HDPE grain port is attained close to the grain surface, whereas, in the paraffin-fuel port, it rapidly extends into the core flow because of the significant fuel mass addition largely due to the entrainment.
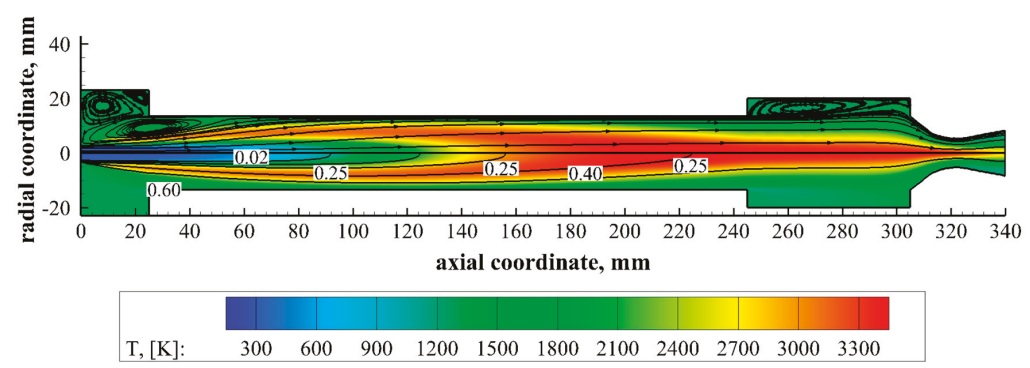

(a) Paraffin fuel

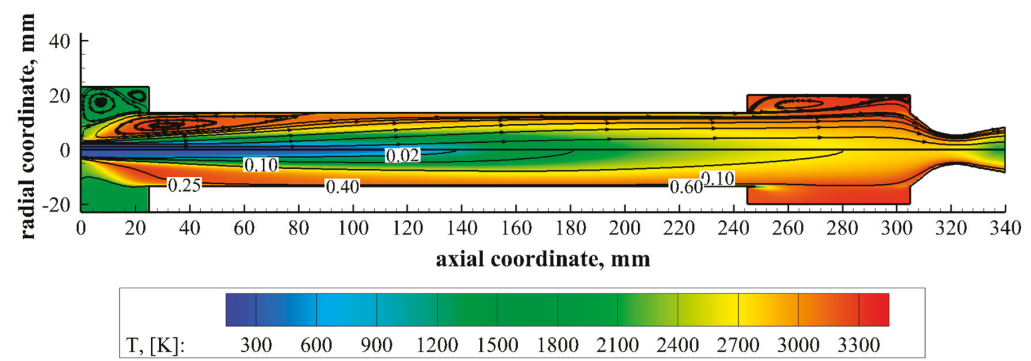

(b) HDPE fuel

Figure 10. The temperature contour plots with overlapped streamlines (top half) and mixture-fraction isolines (bottom half), (a) paraffin fuel, (b) HDPE fuel. 


\subsection{Comparison between Numerical and Experimental Results}

The firing tests presented in Section 5.1 have been simulated for the sake of model validation.

The entrainment parameter used in each test case has been obtained by scaling the reference value of $2.1 \times 10^{-13} \mathrm{~m}^{8.5} \mathrm{~s}^{0.5} / \mathrm{kg}^{3}$, identified for the best fit of the experimental data in Test P4, with the ratio $\left(\rho_{g}^{*} / \rho_{g}\right)^{1.5}$, where $\rho_{g}^{*}$ is the average gas density in the grain port calculated in Test P4 and $\rho_{g}$ is the corresponding value calculated in the analyzed test case. This allows for considering the dependence of the entrainment parameter on the average gas density as prescribed by Equation (33).

Figure 11a shows the calculated fuel regression rates averaged along the grain compared with the measured time-space averaged ones; the maximum deviation of $11 \%$ is reached at the minimum mass flux (Test P1 in Table 8). The numerical prediction improves with higher mass fluxes, showing excellent agreement at the largest mass fluxes where the deviation is only $0.3 \%$ (still lower than that accepted at the reference, Test P4).

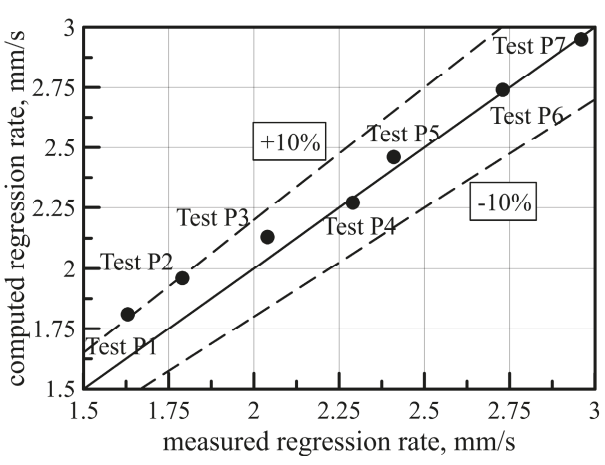

(a) Regression rate

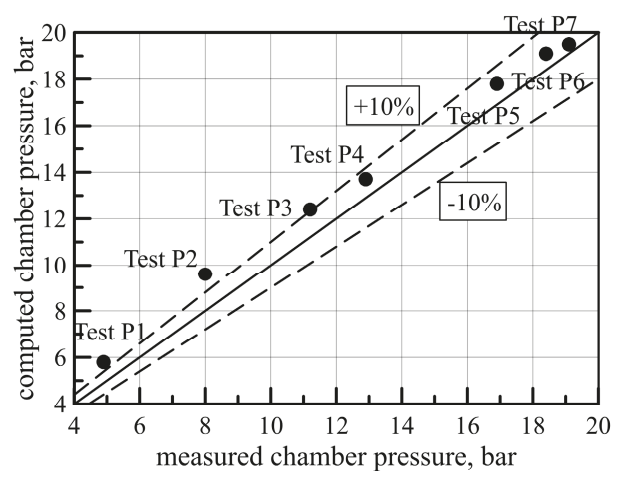

(b) Postchamber pressure

Figure 11. The comparison between computed and measured (a) regression rate, (b) pressure.

In Figure 11b, the calculated chamber pressures in the whole set of test cases are compared with the measured values; data are retrieved in the rocket aft-mixing chamber. All the calculated pressures are overestimated by at most $10 \%$ except those of Test P1 and Test P2, whose deviations from the measured data fall around $20 \%$. A detailed analysis of the factors of deviation between the computed and measured pressure is addressed in Reference [56]. However, the displayed deviation trend can be explained by observing that the critical pressure of paraffin wax is 6.5 bar [42] and that the chamber pressure attained in the test with the largest deviation is lower than the critical pressure (see Table 8). The agreement with experiments is improved as the pressure increases with the mass flux. In fact, below the critical pressure, neglecting the effects of the entrained liquid paraffin dynamics is a much less suitable assumption.

\section{Conclusions}

A review of the computational thermo-fluid-dynamic models developed by the authors for the simulation of the internal ballistics of a hybrid rocket burning either classical polymeric or paraffin-based fuels with gaseous oxygen is presented along with some basic results compared to the experimental data.

The theoretical approach consists in solving the single-phase RANS equations with two additional transport equations for the average mixture fraction and its variance combined to the PDF combustion model and thermochemical equilibrium. In addition, for the prediction of the fuel regression rate, two integrated sub-models that are able to describe the interaction between the gaseous flow and the grain 
surface depending on the nature of the fuel itself-non-liquefying or liquefying-were implemented. The gas/surface interaction modeling is based on the local mass, energy and mixture-fraction balances, regardless of the type of fuel, but specialized treatments were needed to cope with the different consumption mechanisms of classical polymers and liquefying fuels. In the former case, a pyrolysis rate equation correlating with the regression rate to the surface temperature was included for predicting the fuel burning rate; whereas, in the latter case, an equation relating to the additional regression rate fraction (which originates from the liquid entrainment phenomenon) with the total mass flux and the total regression rate through an entrainment parameter, was implemented.

A procedure for the grain port local section evolution in the burning was also defined, accounting for the regression rate axial unevenness.

A series of test cases gathered from the static firings of two different laboratory-scales hybrid rockets that were burning gaseous oxygen and HDPE or paraffin-based fuels were numerically reproduced in order to assess the numerical model capability of predicting the regression rate and chamber pressure. With this purpose, on the assumption that combustion is limited by turbulent diffusion (and, thus, the combustion efficiency is governed by the mixing of propellants), the flow recirculation occurring upstream and downstream of the fuel grain needs to be simulated; accordingly, the computational domains were built on the exact thrust chamber geometry including the real preand post-chamber configurations and the exhaust nozzle.

Two tests with HDPE were analysed: one on the small-scale engine in which a steady simulation was carried out to compare the regression rate axial profile, and another with a larger-scale rocket in which an unsteady simulation allowed for making a comparison with the local time-resolved regression rate and chamber pressure that was experimentally measured. The model has been demonstrated to capture (not only from a qualitative standpoint) the fundamental features of the motor internal ballistics, which involve flow recirculation at the grain port inlet with the characteristic heat transfer mechanism, leading to a point of maximum regression rate that shifts downstream during combustion. The calculated regression-rate time trend, despite the relatively large time step of the port diameter integration, fairly reproduces the change of the wall heat transfer consequent on the recirculation region dynamics.

Seven firing tests with paraffin-based fuels were reproduced with steady simulations to evaluate the approach formulated to model the entrainment fraction of the regression rate, which is based on two main considerations: first, at a combustion pressure larger than paraffin critical pressure, one may disregard the fact that a significant fuel fraction is injected in the main flow in the liquid state by retaining the key aspect that the entrained fuel does not contribute to the heat transfer blocking; second, the entrainment parameter given the fuel formulation only depends on the mean gas density in the port.

In all the analyzed cases, a satisfactory agreement between the calculated regression rates and chamber pressure with the relevant measured data is obtained.

In particular, the chamber pressure is predicted with lower accuracy; a more accurate prediction likely requires modeling the two-phase flow dynamics ensuing from the melted paraffin injection into the chamber, which is the subject of future developments.

Author Contributions: Conceptualization and definition of the CFD models, G.D.D.M., C.C. and R.S.; implementation of numerical models, G.D.D.M.; running of numerical simulations, G.D.D.M. and S.M.; carrying out of experimental tests, G.D.D.M. and S.M.; writing—original draft preparation, C.C. and G.D.D.M.; supervision, C.C. and R.S.

Funding: This research received no external funding.

Conflicts of Interest: The authors declare no conflict of interest. 


\section{Nomenclature}

$a_{\text {ent }}=$ entrainment parameter, $\mathrm{m}^{8.5} \mathrm{~s}^{0.5} / \mathrm{kg}^{3}$

$A \quad=$ pre-exponential factor, $\mathrm{mm} / \mathrm{s}$

$\mathrm{Br}=$ Brinkman number

$\mathrm{C} \quad=$ specific heat, $\mathrm{J} /(\mathrm{kg} \cdot \mathrm{K})$

$c^{*} \quad=$ characteristic exhaust velocity, $\mathrm{m} / \mathrm{s}$

$D \quad=$ fuel grain port diameter, $\mathrm{mm}$

$D_{0} \quad$ = fuel grain port initial diameter, $\mathrm{mm}$

$E_{a} \quad=$ activation energy, $\mathrm{J} / \mathrm{mol}$

$f=$ fuel mixture fraction

$f^{\prime 2}=$ mixture fraction variance

$\mathrm{G}=$ mass flux, $\mathrm{kg} /\left(\mathrm{m}^{2} \cdot \mathrm{s}\right)$

$\mathrm{H}=$ total specific enthalpy, $\mathrm{J} / \mathrm{kg}$

$k=$ turbulent kinetic energy, $\mathrm{m}^{2} / \mathrm{s}^{2}$

$k_{g} \quad=$ gas thermal conductivity, $\mathrm{W} /(\mathrm{m} \cdot \mathrm{K})$

$L_{m} \quad=$ heat of fusion, $\mathrm{J} / \mathrm{kg}$

$L_{v} \quad=$ heat of vaporization, $\mathrm{J} / \mathrm{kg}$

$\dot{m} \quad=$ mass flow rate, $\mathrm{kg} / \mathrm{s}$

$=$ normal-to-surface coordinate oriented

from solid to gas, $\mathrm{m}$

OF = local oxidizer-to-fuel mixture ratio

$\mathrm{O} / \mathrm{F} \quad=$ average overall mixture ratio

$p \quad=$ pressure, $\mathrm{Pa}$

$p_{c} \quad=$ chamber pressure, $\mathrm{Pa}$

$\mathrm{Pr}_{t} \quad=$ turbulent Prandtl number

$\dot{q} \quad=$ heat flux, $\mathrm{W} / \mathrm{m}^{2}$

$\dot{r}=$ regression rate, $\mathrm{mm} / \mathrm{s}$

$R=$ universal gas constant, $\mathrm{J} / \mathrm{K} \cdot \mathrm{mol}$

$\mathcal{R} \quad=$ Reynolds stress tensor, $\mathrm{kg} /\left(\mathrm{m} \cdot \mathrm{s}^{2}\right)$

$R e_{t} \quad=$ turbulent Reynolds number

$S c_{t}=$ turbulent Schmidt number

$S_{m} \quad=$ mass source term, $\mathrm{kg} /\left(\mathrm{m}^{3} \cdot \mathrm{s}\right)$

$S_{h} \quad=$ energy source term, $\mathrm{J} /\left(\mathrm{m}^{3} \cdot \mathrm{s}\right)$

$t \quad=$ time, $\mathrm{s}$

$T=$ temperature, $\mathrm{K}$

$T_{a} \quad=$ room temperature, $\mathrm{K}$

$T_{m} \quad=$ melting temperature, $\mathrm{K}$

$T_{v} \quad=$ vaporization temperature, $\mathrm{K}$

$u \quad=$ gas velocity, $\mathrm{m} / \mathrm{s}$

$U=$ gas mean velocity in the port, $\mathrm{m} / \mathrm{s}$

$=$ gas velocity component normal to surface, $\mathrm{m} / \mathrm{s}$

= generic coordinate, axial coordinate, $\mathrm{m}$

$=$ distance from the wall, $\mathrm{m}$

$y^{+} \quad=$ dimensionless wall distance

$Y_{j} \quad=$ mass fraction of the $j$-th species

\section{Greek Symbols}

B = probability density function

$\Delta \quad=$ node displacement, $\mathrm{m}$

$\Delta h_{p} \quad=$ fuel heat of pyrolysis, $\mathrm{J} / \mathrm{kg}$

$\Delta H_{w}=$ total enthalpy difference between flame and wall, $\mathrm{J} / \mathrm{kg}$

$\Delta x=$ surface cell length, $\mathrm{m}$

$\varepsilon \quad=$ turbulence dissipation rate, $\mathrm{J} /(\mathrm{kg} \cdot \mathrm{s})$

$\theta=$ fuel surface local inclination, $\mathrm{rad}$

$\mu \quad=$ viscosity, $\mathrm{kg} /(\mathrm{m} \cdot \mathrm{s})$

$\mu_{t} \quad=$ turbulent viscosity, $\mathrm{kg} /(\mathrm{m} \cdot \mathrm{s})$

$\rho \quad=$ density, $\mathrm{kg} / \mathrm{m}^{3}$

$\rho_{g} \quad=$ average gas density in the grain port, $\mathrm{kg} / \mathrm{m}^{3}$

$\tau \quad=$ stress tensor, $\mathrm{kg} /\left(\mathrm{m} \cdot \mathrm{s}^{2}\right)$

$\eta \quad=c^{*}$ efficiency

$\omega=$ turbulence specific dissipation rate, $1 / \mathrm{s}$

$\varphi \quad=$ generic function of enthalpy and mixture fraction

$\Omega \quad=$ vorticity vector magnitude, $1 / \mathrm{s}$

\section{References}

1. Altman, D.; Humble, R. Hybrid rocket propulsion systems. In Space Propulsion Analysis and Design, 1st ed.; Humble, R.W., Henry, G.N., Larson, W.J., Eds.; McGraw-Hill: New York, NY, USA, 1995; pp. 365-370.

2. Altman, D.; Holzman, A. Overview and history of hybrid rocket propulsion. In Fundamentals of Hybrid Rocket Combustion and Propulsion; Kuo, K., Chiaverini, M., Eds.; Progress in Astronautics and Aeronautics AIAA: Reston, VR, USA, 2007; Volume 218, pp. 1-36.

3. Oikine, $\mathrm{C}$. New perspectives for hybrid propulsion. In Proceedings of the 42nd AIAA/ASME/SAE/ASEE Joint Propulsion Conference \& Exhibit, AIAA 2006-4674, Sacramento, CA, USA, 9-12 July 2006. [CrossRef] 
4. Ronningen, J.E.; Husdal, J. Nammo hybrid rocket propulsion TRL improvement program. In Proceedings of the 48th AIAA/ASME/SAE/ASEE Joint Propulsion Conference \& Exhibit, AIAA 2012-4311, Atlanta, GR, USA, 30 July-1 August 2012. [CrossRef]

5. Carmicino, C.; Russo Sorge, A. Experimental investigation into the effect of solid-fuel additives on hybrid rocket performance. J. Propuls. Power 2015, 31, 699-713. [CrossRef]

6. Marxman, G.A.; Gilbert, M. Turbulent boundary layer combustion in the hybrid rocket. Symp. Int. Combust. 1963, 9, 371-383. [CrossRef]

7. Karabeyoglu, M.A.; Altman, D.; Cantwell, B.J. Combustion of liquefying hybrid propellants: Part 1 general theory. J. Propuls. Power 2002, 18, 610-620. [CrossRef]

8. Mazzetti, A.; Merotto, L.; Pinarello, G. Paraffin-based hybrid rocket engines applications: A review and a market perspective. Acta Astronaut. 2016, 126, 286-297. [CrossRef]

9. Karabeyoglu, M.A.; Cantwell, B.J. Combustion of liquefying hybrid propellants: Part 2, stability of liquid films. J. Propuls. Power 2002, 18, 621-630. [CrossRef]

10. Petrarolo, A.; Kobald, M.; Schlechtriem, S. Understanding Kelvin-Helmholtz instability in paraffin-based hybrid rocket fuels. Exp. Fluids 2018, 59, 62. [CrossRef]

11. Kobald, M.; Schmierer, C.; Ciezki, H.K.; Schlechtriem, S.; Toson, E.; De Luca, L.T. Viscosity and regression rate of liquefying hybrid rocket fuels. J. Propuls. Power 2017, 33, 1245-1251. [CrossRef]

12. Boiocchi, M.; Milova, P.; Galfetti, L.; Landro, L.A.; Golovko, A.K. A wide characterization of paraffin-based fuels mixed with styrene-based thermoplastic polymers for hybrid propulsion. Progr. Propuls. Phys. 2016, 8 , 241-262. [CrossRef]

13. Merotto, L.; Boiocchi, M.; Mazzetti, A.; Maggi, F.; Galfetti, L.; De Luca, L.T. Characterization of a family of paraffin-based solid fuels. In Proceedings of the 4th European Conference for Aerospace Sciences (EUCASS), St. Petersburg, Russia, 4-8 July 2011.

14. Green, L.J. Introductory considerations on hybrid rocket combustion. Progr. Astronaut. Rocket. 1964, 15, 451-484. [CrossRef]

15. Houser, T.; Peck, M. Research in hybrid combustion. Progr. Astronaut. Rocket. 1964, 15, 559-581. [CrossRef]

16. Marxman, G.A. Combustion in the turbulent boundary layer on a vaporizing surface. Symp. Int. Combust. 1965, 10, 1337-1349. [CrossRef]

17. Netzer, D.W. Hybrid Rocket Internal Ballistics, Naval Postgraduate School; CPIA: Monterey, CA, USA, 1972.

18. Carmicino, C.; Russo Sorge, A. Role of injection in hybrid rockets regression rate behavior. J. Propuls. Power 2005, 21, 606-612. [CrossRef]

19. Carmicino, C.; Russo Sorge, A. Performance comparison between two different injector configurations in a hybrid rocket. Aerosp. Sci. Technol. 2007, 11, 61-67. [CrossRef]

20. Kim, S.; Lee, J.; Moon, H.; Sung, H. Empirical estimation of entrainment regression rate in liquefying solid fuel for hybrid rocket motor. In Proceedings of the 47th AIAA/ASME/SAE/ASEE Joint Propulsion Conference \& Exhibit, AIAA 2011-5823, San Diego, CA, USA, 31 July-3 August 2011. [CrossRef]

21. Chen, Y.S.; Chou, T.H.; Gu, B.R.; Wu, J.S.; Wu, B.; Lian, Y.Y.; Yang, L. Multiphysics simulations of rocket engine combustion. Comp. Fluids 2011, 45, 29-36. [CrossRef]

22. Coronetti, A.; Sirignano, W.A. Numerical analysis of hybrid rocket combustion. J. Propuls. Power 2013, 29, 371-384. [CrossRef]

23. Lazzarin, M.; Barato, F.; Bettella, A.; Pavarin, D. Computational fluid dynamics simulation of regression rate in hybrid rockets. J. Propuls. Power 2013, 29, 1445-1452. [CrossRef]

24. Kim, B.; Na, Y.; Shin, K.; Lee, C. Nonlinear combustion and fluid mechanics in a hybrid rocket. J. Propuls. Power 2012, 28, 1351-1358. [CrossRef]

25. Lengellé, G. Solid-fuel pyrolysis phenomena and regression rate, Part 1: Mechanisms. In Fundamentals of Hybrid Rocket Combustion and Propulsion; Kuo, K., Chiaverini, M., Eds.; AIAA of Progress in Astronautics and Aeronautics: Reston, VA, USA, 2007; Volume 218, pp. 127-163.

26. Motoe, M.; Shimada, T. Numerical simulations of combustive flows in a swirling-oxidizer-flow-type hybrid rocket. In Proceedings of the 52nd Aerospace Sciences Meeting, AIAA 2014-0310, National Harbor, MD, USA, 13-17 January 2014. [CrossRef]

27. Bianchi, D.; Nasuti, F. Numerical analysis of nozzle material thermochemical erosion in hybrid rocket engines. J. Propul. Power 2013, 29, 547-558. [CrossRef] 
28. Leccese, G.; Bianchi, D.; Nasuti, F. Simulations of hybrid rocket flowfields including modeling of fuel pyrolysis and thermal radiation. In Proceedings of the 5th Space Propulsion Conference, Rome, Italy, 2-6 May 2016. SP2016-3125176.

29. Bianchi, D.; Betti, B.; Nasuti, F.; Carmicino, C. Simulation of gaseous oxygen/hydroxyl-terminated polybutadiene hybrid rocket flowfields and comparison with experiments. J. Propuls. Power 2015, 31, 919-929. [CrossRef]

30. Bianchi, D.; Nasuti, F.; Carmicino, C. Hybrid rockets with axial injector: Port diameter effect on fuel regression rate. J. Propuls. Power 2016, 32, 984-996. [CrossRef]

31. Di Martino, G.D.; Mungiguerra, S.; Carmicino, C.; Savino, R.; Cardillo, D.; Battista, F.; Invigorito, M.; Elia, G. Two-hundred-newton laboratory-scale hybrid rocket testing for paraffin fuel-performance characterization. J. Propuls. Power 2019, 35, 224-235. [CrossRef]

32. Cai, G.; Zhang, Y.; Tian, H.; Wang, P.; Yu, N. Effect of grain port length-diameter ratio on combustion performance in hybrid rocket motors. Acta Astronaut. 2016, 128, 83-90. [CrossRef]

33. Di Martino, G.D.; Malgieri, P.; Carmicino, C.; Savino, R. A simplified computational fluid-dynamic approach to the oxidizer injector design in hybrid rockets. Acta Astronaut. 2016, 129, 8-21. [CrossRef]

34. Sankaran, V. Computational fluid dynamics modeling of hybrid rocket flowfields. In Fundamentals of Hybrid Rocket Combustion and Propulsion; Kuo, K., Chiaverini, M., Eds.; AIAA Progress in Astronautics and Aeronautics: Reston, VA, USA, 2007; Volume 218, pp. 323-349.

35. Di Martino, G.D.; Carmicino, C.; Savino, R. Transient computational thermofluid-dynamic simulation of hybrid rocket internal ballistics. J. Propuls. Power 2017, 33, 1395-1409. [CrossRef]

36. Bianchi, D.; Leccese, G.; Nasuti, F.; Carmicino, C. Modeling of high density polyethylene regression rate in the simulation of hybrid rocket flowfields. In Proceedings of the 7th European Conference for Aeronautics and Space Sciences (EUCASS), Milan, Italy, 3-6 July 2015.

37. Lazzarin, M.; Faenza, M.; Barato, F.; Bellomo, N.; Bettella, A.; Pavarin, D. Computational fluid dynamics simulation of hybrid rockets of different scales. J. Propuls. Power 2015, 31, 1458-1469. [CrossRef]

38. Ranuzzi, G.; Cardillo, D.; Invigorito, M. Numerical investigation of a N2O-paraffin hybrid rocket engine combusting flowfield. In Proceedings of the 6th European Conference for Aeronautics and Space Sciences (EUCASS), Krakow, Poland, 29 June-3 July 2015.

39. Cardillo, D.; Battista, F.; Elia, G.; Di Martino, G.D.; Mungiguerra, S.; Savino, R. Design and testing of a paraffin-based hybrid rocket demonstrator. In Proceedings of the Space Propulsion Conference 2018, Seville, Spain, 14-18 May 2018. SP2018-00508.

40. Lestrade, J.Y.; Anthoine, J.; Lavergne, G. Liquefying fuel regression rate modeling in hybrid propulsion. Aerosp. Sci. Technol. 2015, 42, 80-87. [CrossRef]

41. Leccese, G.; Bianchi, D.; Nasuti, F.; Stober, K.J.; Pavan, N.; Cantwell, B. Simulations of paraffin-based hybrid rocket engines and comparison with experiments. In Proceedings of the 53rd AIAA/SAE/ASEE Joint Propulsion Conference, AIAA 2017-4737, Atlanta, GA, USA, 10-12 July 2017. [CrossRef]

42. Leccese, G.; Bianchi, D.; Nasuti, F. Modeling and simulation of paraffin-based hybrid rocket internal ballistics. In Proceedings of the 2018 Joint Propulsion Conference, AIAA 2018-4533, Cincinnati, OH, USA, 9-11 July 2018. [CrossRef]

43. Cebeci, T. Analysis of Turbulent Flows, 2nd ed.; Elsevier: Oxford, UK, 2004; pp. 32-38.

44. Menter, F.R. Two-equation eddy-viscosity turbulence models for engineering applications. AIAA J. 1994, 32, 1598-1605. [CrossRef]

45. Wilcox, D.C. Turbulence Modeling for CFD, 1st ed; DCW Industries, Inc.: La Canada, CA, USA, 1998; pp. 73-170.

46. Wooldridge, C.E.; Marxman, G.A.; Kier, R.J. Investigation of Combustion Instability in Hybrid Rockets; NASA contract NAS 1-7310; Stanford Research Inst.: Menlo Park, CA, USA, 1969.

47. Sivathanu, Y.R.; Faeth, G.M. Generalized state relationships for scalar properties in non-premixed hydrocarbon/airflames. Combust. Flame 1990, 82, 211-230. [CrossRef]

48. Peters, N. Nonpremixed turbulent combustion. In Turbulent Combustion (Cambridge Monographs on Mechanics); Peters, N., Ed.; Cambridge University Press: Cambridge, UK, 2000; pp. 170-236. [CrossRef]

49. Jones, W.P.; Whitelaw, J.H. Calculation methods for reacting turbulent flows: A review. Combust. Flame 1982, 48, 1-26. [CrossRef]

50. Hazewinkel, M. Beta-distribution. In Encyclopedia of Mathematics; Hazewinkel, M., Ed.; Springer: Amsterdam, The Netherlands, 2001. 
51. Gordon, S.; McBride, B.J. Computer Program of Complex Chemical Equilibrium Compositions and Applications; NASA Reference Publication 1311; NASA: Cleveland, OH, USA, 1994.

52. Di Martino, G.D. Experiments and Simulations of Hybrid Rocket Internal Flows and Material Behaviour. Ph.D. Thesis, University of Naples "Federico II", Naples, Italy, December 2018.

53. De Wilde, J.P. The Heat of Gasification of Polyethylene and Polymethylmethacrilate; Rept. PML 1988-C42, SFCC Publ. 53; Faculty of Aerospace Engineering/Prins Maurits Lab./Solid Fuel Combustion Chamber, Delft University of Technology: Delft, The Netherlands, 1988.

54. Karabeyoglu, A.; Cantwell, B.J.; Stevens, J. Evaluation of homologous series of normal-alkanes as hybrid rocket fuels. In Proceedings of the 41st AIAA/ASME/SAE/ASEE Joint Propulsion Conference \& Exhibit, AIAA 2005-3908, Tucson, AZ, USA, 10-13 July 2005. [CrossRef]

55. Di Martino, G.D.; Mungiguerra, S.; Carmicino, C.; Savino, R. Computational fluid-dynamic simulations of hybrid rocket internal flow including discharge nozzle. In Proceedings of the 53rd AIAA/SAE/ASEE Joint Propulsion Conference, AIAA 2017-5045, Atlanta, GA, USA, 10-12 July 2017. [CrossRef]

56. Di Martino, G.D.; Mungiguerra, S.; Carmicino, C.; Savino, R. Computational fluid-dynamic modeling of the internal ballistics of paraffin-fueled hybrid rocket. Aerosp. Sci. Technol. 2019, 89, 431-444. [CrossRef]

57. Chorin, A.J. Numerical solution of navier-stokes equations. Math. Comput. 1968, 22, 745-762. [CrossRef]

58. Carmicino, C.; Scaramuzzino, F.; Russo Sorge, A. Trade-off between paraffin-based and aluminium-loaded HTPB fuels to improve performance of hybrid rocket fed with N2O. Aerosp. Sci. Technol. 2014, 37, 81-92. [CrossRef]

59. Abramovich, G.N. The Theory of Turbulent Jets; Massachussets Institute of Technology Press: Cambridge, MA, USA, 1963; pp. 3-17.

60. Carmicino, C. Acoustics, vortex shedding, and low-frequency dynamics interaction in an unstable hybrid rocket. J. Propuls. Power 2009, 25, 1322-1335. [CrossRef]

(C) 2019 by the authors. Licensee MDPI, Basel, Switzerland. This article is an open access article distributed under the terms and conditions of the Creative Commons Attribution (CC BY) license (http://creativecommons.org/licenses/by/4.0/). 



\title{
Theoretical Investigation on Feedback Control of Hybrid Rocket Engines
}

\author{
Jérôme Messineo * and Toru Shimada * \\ Institute of Space and Astronautical Science, Japan Aerospace Exploration Agency, Sagamihara City 252-5210, \\ Kanagawa, Japan \\ * Correspondence: jerome.messineo@gmail.com (J.M.); shimada.toru@jaxa.jp (T.S.)
}

Received: 21 February 2019; Accepted: 10 May 2019; Published: 3 June 2019

\begin{abstract}
Despite the fact that hybrid propulsion offers significant benefits, it still suffers from some limitations such as the natural oxidizer to fuel ratio shift which induces variations of the engines' performances while operating. To overcome that issue, Japan Aerospace Exploration Agency (JAXA) has been studying an innovative concept for several years based on the combination of controlled axial and radial oxidizer injections, called altering-intensity swirling-oxidizer-flow-type engine. This type of motor is theoretically capable of managing both the thrust and the oxidizer to fuel ratio independently and instantaneously by using a feedback control loop. To be effective, such engines would require in-flight instantaneous and precise thrust and an oxidizer to fuel ratio measurements as well as an adapted feedback control law. The purpose of this study is to investigate the effect of measurement errors on the engine control and to propose a regulation law suitable for these motors. Error propagation analysis and regulation law are developed from fundamental equations of hybrid motors and applied in a case where resistor-based sensors are used for fuel regression rate measurement. This study proves the theoretical feasibility of hybrid engines feedback control while providing some methods to design the engine and regression rate sensors depending on the mission requirements.
\end{abstract}

Keywords: hybrid rocket propulsion; swirl oxidizer injection; feedback loop control; error propagation analysis; resistor-based sensors

\section{Introduction}

While new applications and constraints have arisen in the field of space transportation in the last decade, hybrid propulsion has been actively investigated worldwide for its benefits in comparison with other chemical engines. The technology is mature enough that the space industry is starting to invest and develop applications based on it. These engines offer significant advantages [1]: they are green propellant-based and have good propulsive performances with a relative simplicity and low costs. The safety level is higher than with other types of chemical engines since the propellants are non-explosive and stored separately. Moreover, hybrid engines can be throttled, reused, and ignited or extinguished several times.

Despite these benefits, hybrid engines suffer from some disadvantages [1] that can be addressed with more or less difficulty. The fundamental difference with other types of chemical propulsion comes from the nature of the combustion which occurs through a diffusion flame located in the turbulent boundary layer close to the fuel grain surface [2]. As a consequence, the fuel regression rate in hybrid motors depends on the heat transfers at the fuel grain surface, which are not directly controllable, and is a function of the mass flux in the combustion chamber. The fuel regression rate in hybrid engines is generally low, which means it may be difficult to obtain high thrust levels. The diffusion-limited combustion is the source of relatively low combustion efficiency due to a lower degree of propellants mixing. Moreover, the increase of the grain port diameter during the combustion causes an $\mathrm{O} / \mathrm{F}$ 
(oxidizer to fuel ratio) shift and some performance modifications. Finally, like all chemical rocket engines, hybrid motors can suffer from pressure oscillations and instabilities that can be provoked by different phenomena such as combustion, acoustics, hydrodynamics or hybrid intrinsic behavior.

These fundamental issues have been investigated and solutions do exist depending on the mission and engine configuration. Although a low regression rate may be interesting for long duration and low thrust missions, a higher regression rate can be obtained by using liquefying fuels $[3,4]$. By increasing the residence time of propellants and improving their mixing, swirl oxidizer injection enhances the combustion efficiency making the hybrid engine competitive regarding that aspect $[5,6]$. Moreover, the vortex motion of the flow tends to stabilize the combustion and reduces pressure oscillations. In order to overcome the $\mathrm{O} / \mathrm{F}$ shift due to the fuel regression, several options are possible such as maintaining a constant fuel-burning surface during combustion [7], adding an oxidizer aft-injection [8,9] or using an A-SOFT (Altering-intensity Swirling-Oxidizer-Flow-Type) engine [10] described in Figure 1.

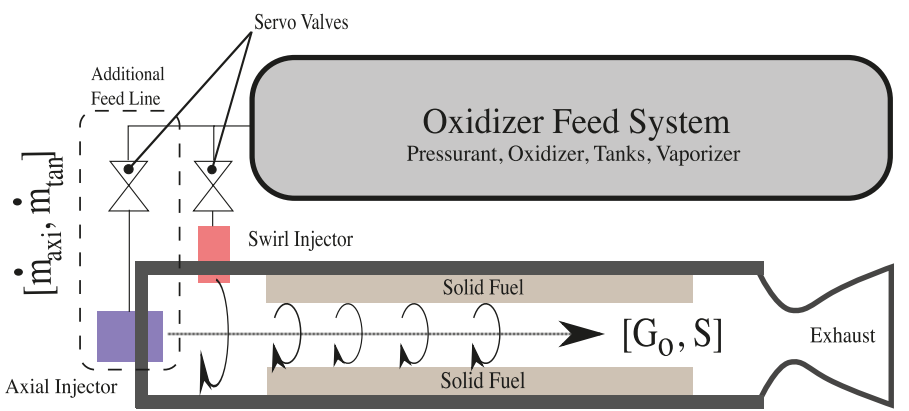

Figure 1. A-SOFT (Altering-intensity Swirling-Oxidizer-Flow-Type) concept with dual front oxidizer injections [10].

The oxidizer mass flow rate influences the regression rate but also plays a major role in the thrust of the engine. As a consequence, to properly manage both the thrust and the $\mathrm{O} / \mathrm{F}$ ratio in hybrid rockets, two control variables are required. In A-SOFT engines, these variables are the total oxidizer mass flow rate and the effective swirl number, which can be viewed as a ratio between axial and radial oxidizer momentums or more simply as the swirling intensity of the flow. In practice, these variables are manipulated through the use of axial and swirl injectors. The swirl oxidizer injection increases the regression rate compared to the non-swirled case $[6,11]$ and an A-SOFT engine uses this effect to control the fuel regression rate and hence the O/F ratio. An ideal A-SOFT motor should be capable of managing the thrust and $\mathrm{O} / \mathrm{F}$ ratio independently and instantaneously in a feedback control loop to adapt to any required thrust profile while maintaining an optimal operating $\mathrm{O} / \mathrm{F}$ ratio. The concept has been studied within the Hybrid Rocket Research Working Group in Japan and theoretical $[10,12-14]$, numerical $[15,16]$, and experimental $[5,11,17,18]$ investigations have been conducted to prove the feasibility and interest of such motors. To move forward in the development of A-SOFT engines, two major issues have to be addressed. The first one is related to sensing and measurements and the second one concerns the design of the control law.

To operate properly, an A-SOFT motor would require in-flight, instantaneous, precise, and robust measurements of the thrust and $\mathrm{O} / \mathrm{F}$ ratio that are the variables to be regulated by a feedback loop control. Although the thrust cannot be measured directly in flight conditions, it can be estimated by using external parameters such as the rocket's acceleration and trajectory and/or by using the motor's data such as the combustion chamber pressure and the propellants mass flow rates. While the oxidizer mass flow rate is not difficult to measure, it is more complicated for the fuel. The main difficulty comes from the necessity of getting instantaneous measurements that are required for the feedback control in flight conditions. One of the solutions that is currently considered is to use RBS (Resistor-Based 
Sensors) that are embedded in the fuel grain and deliver an electric voltage proportional to their length. The sensors burn at the same rate as the fuel and allow for measuring the fuel regression rate and mass flow rate while the engine is operating. The SPLab (Space Propulsion Laboratory) from Politecnico di Milano developed its own RBS technology $[19,20]$. JAXA and SPLab have initiated a collaboration in order to study these types of measurements for A-SOFT applications [21].

A closed-loop throttling of a hybrid rocket has already been demonstrated based on thrust or pressure feedback using proportional/integral control algorithms [22]. Although the results are interesting, the $\mathrm{O} / \mathrm{F}$ ratio evolution was not considered at all, which is a major difference with the A-SOFT engine concept. Regulating both the thrust and the O/F ratio instantaneously and independently significantly increases the complexity of the feedback control. Due to the complex physical phenomena and their respective interactions in a combustion chamber, an efficient feedback control law must be developed for A-SOFT hybrid motors.

In that context, the goal of this work is twofold: firstly, we investigate the effect of measurement errors on the engine control by conducting an error propagation analysis regarding the thrust and the $\mathrm{O} / \mathrm{F}$ ratio. Secondly, we develop a regulation law suitable for A-SOFT engine applications. In addition, we perform numerical simulations of a simplified hybrid motor using a feedback control loop to confirm that the regulation law is valid, in a case where RBS are used for the fuel regression rate estimation. From the error propagation analysis, we demonstrate that it is possible to determine the requirements of measurement precision to fit a desired precision on the thrust and $\mathrm{O} / \mathrm{F}$ ratio control. Moreover, we propose two methods to help design the engine and the RBS based on propagation analysis. Finally, we perform numerical simulations with simple thrust profiles and confirm the efficiency of the proposed regulation law for these configurations.

\section{Materials and Methods}

\subsection{Fundamental Equations of A-SOFT Engines}

The regression rate law proposed by Marxman [2] is largely used to analyze hybrid motors. However, in the case of a swirl oxidizer injection, an extended version of this law can be used [11] and is given by Equation (1):

$$
\dot{r}[t]=f\left[S_{e}[t]\right] g\left[G_{0}[t]\right] .
$$

While $g$ is the classical regression rate law depending on the oxidizer mass flux (Equations (2)-(4)), $f$ is an extended function depending on the effective swirl number (Equations (5) and (6)):

$$
\begin{gathered}
g\left[G_{o}[t]\right]=a G_{o}^{n}[t], \\
G_{o}[t]=\frac{4}{\pi} \frac{\dot{m}_{o}[t]}{\phi^{2}[t]}, \\
\dot{m}_{o}[t]=\dot{m}_{o A}[t]+\dot{m}_{o T}[t], \\
f\left[S_{e}[t]\right]=\left(1+S_{e}^{2}[t]\right)^{m}, \\
S_{e}[t]=S_{g} \frac{\dot{m}_{o T}^{2}[t]}{\left(\dot{m}_{o A}[t]+\dot{m}_{o T}[t]\right)^{2}} .
\end{gathered}
$$

The geometric swirl number $S_{g}$ depends on the geometry of the injectors and remains constant during the engine operation. Finally, the fuel regression rate can be expressed by Equation (7):

$$
\dot{r}[t]=4^{n} \pi^{-n} a\left(1+S_{g}^{2} \frac{\dot{m}_{o T}^{4}[t]}{\left(\dot{m}_{o A}[t]+\dot{m}_{o T}[t]\right)^{4}}\right)^{m}\left(\frac{\dot{m}_{o A}[t]+\dot{m}_{o T}[t]}{\phi^{2}[t]}\right)^{n} .
$$


The fuel mass flow rate can be expressed by Equation (8), with $C_{1}=4^{n} \pi^{1-n} a L \rho_{f}$.

$$
\dot{m}_{f}[t]=C_{1} \phi[t]\left(1+S_{g}^{2} \frac{\dot{m}_{o T}^{4}[t]}{\left(\dot{m}_{o A}[t]+\dot{m}_{o T}[t]\right)^{4}}\right)^{m}\left(\frac{\dot{m}_{o A}[t]+\dot{m}_{o T}[t]}{\phi^{2}[t]}\right)^{n}
$$

The mixture ratio is then estimated by Equation (9):

$$
\begin{aligned}
\xi[t] & =\frac{\dot{m}_{o}[t]}{\dot{m}_{f}[t]} \\
= & \frac{\dot{m}_{o A}[t]+\dot{m}_{o T}[t]}{C_{1} \phi[t]\left(1+S_{g}^{2} \frac{\dot{m}_{o T}^{4}[t]}{\left(\dot{m}_{o A}[t]+\dot{m}_{o T}[t]\right)^{4}}\right)^{m}\left(\frac{\dot{m}_{o A}[t]+\dot{m}_{o T}[t]}{\phi^{2}[t]}\right)^{n}} .
\end{aligned}
$$

The thrust is evaluated by Equation (10):

$$
\begin{aligned}
F[t] & =I_{s p}[\xi] g_{0}\left\{\dot{m}_{o A}[t]+\dot{m}_{o T}[t]+\dot{m}_{f}[t]\right\} \\
& =I_{s p}[\xi] g_{0}\left\{\dot{m}_{o A}[t]+\dot{m}_{o T}[t]+C_{1} \phi[t]\left(1+S_{g}^{2} \frac{\dot{m}_{o T}^{4}[t]}{\left(\dot{m}_{o A}[t]+\dot{m}_{o T}[t]\right)^{4}}\right)^{m}\left(\frac{\dot{m}_{o A}[t]+\dot{m}_{o T}[t]}{\phi^{2}[t]}\right)^{n}\right\} .
\end{aligned}
$$

The dependence of the specific impulse on the chamber pressure is generally of second order compared to the mixture ratio influence and has been neglected in this analysis. Consequently, the specific impulse is considered as a function of the mixture ratio only and is given by Equation (11). In that study, we consider constant nozzle and combustion efficiencies and no nozzle throat erosion:

$$
I_{s p}[\xi]=\eta_{c} \eta_{n} I_{s p, t h}[\xi]
$$

As a consequence from the previous development, it is possible to express the thrust and the mixture ratio as two functions of three independent variables: $\dot{m}_{o A}, \dot{m}_{o T}$, and $\phi$ (Equation (12)):

$$
\left(\begin{array}{l}
F \\
\xi
\end{array}\right)=\left(\begin{array}{l}
F\left[\dot{m}_{O A}, \dot{m}_{o T}, \phi\right] \\
\xi\left[\dot{m}_{O A}, \dot{m}_{o T}, \phi\right]
\end{array}\right)
$$

\subsection{Error Propagation of A-SOFT Engines}

In this study, errors refer to RMS (Root Mean Square) relative errors. The RMS relative error of a function $h$ depending on three independent variables $[x, y, z]$ is given by Equation (13) (by using the $\log$ function properties: $\partial x=x \partial \ln x$, and: $\partial h / h=\partial \ln h)$ :

$$
\begin{aligned}
e_{h}=\frac{\delta h}{h} & =\frac{\sqrt{\left(\frac{\partial h}{\partial x} \delta x\right)^{2}+\left(\frac{\partial h}{\partial y} \delta y\right)^{2}+\left(\frac{\partial h}{\partial z} \delta z\right)^{2}}}{h} \\
& =\sqrt{\left(\frac{\partial h}{\partial x} \frac{\delta x}{h}\right)^{2}+\left(\frac{\partial h}{\partial y} \frac{\delta y}{h}\right)^{2}+\left(\frac{\partial h}{\partial z} \frac{\delta z}{h}\right)^{2}} \\
& =\sqrt{\left(\frac{\partial \ln h}{\partial \ln x} e_{x}\right)^{2}+\left(\frac{\partial \ln h}{\partial \ln y} e_{y}\right)^{2}+\left(\frac{\partial \ln h}{\partial \ln z} e_{z}\right)^{2}} .
\end{aligned}
$$


By using this definition, the relative errors of thrust and $\mathrm{O} / \mathrm{F}$ ratio can be written by Equations (14) and (15):

$$
\begin{aligned}
& e_{F}=\sqrt{\left(\frac{\partial \ln F}{\partial \ln \dot{m}_{O A}} e_{\dot{m}_{O A}}\right)^{2}+\left(\frac{\partial \ln F}{\partial \ln \dot{m}_{O T}} e_{\dot{m}_{o T}}\right)^{2}+\left(\frac{\partial \ln F}{\partial \ln \phi} e_{\phi}\right)^{2}}, \\
& e_{\xi}=\sqrt{\left(\frac{\partial \ln \xi}{\partial \ln \dot{m}_{O A}} e_{\dot{m}_{O A}}\right)^{2}+\left(\frac{\partial \ln \xi}{\partial \ln \dot{m}_{o T}} e_{\dot{m}_{o T}}\right)^{2}+\left(\frac{\partial \ln \xi}{\partial \ln \phi} e_{\phi}\right)^{2}} .
\end{aligned}
$$

To simplify the relations, we will consider that the oxidizer Mass Flow Rate (MFR) errors are equal: $e_{\dot{m}_{O T}}=e_{\dot{m}_{O A}}=e_{M F R}$. Moreover, in order to perform a comparative analysis of the results, we define the thrust, $\mathrm{O} / \mathrm{F}$, diameter, and total normalized errors (Equations (16)-(19)) as the following:

$$
\begin{gathered}
\overline{e_{F}}=\frac{e_{F}}{e_{M F R}}, \\
\overline{e_{\xi}}=\frac{e_{\xi}}{e_{M F R}}, \\
\overline{e_{\phi}}=\frac{e_{\phi}}{e_{M F R}}, \\
\overline{e_{\text {total }}}=\frac{\sqrt{e_{F}^{2}+e_{\xi}^{2}}}{e_{M F R}} .
\end{gathered}
$$

Consequently, the thrust, $\mathrm{O} / \mathrm{F}$ and total normalized errors are written in Equations (20)-(22):

$$
\begin{gathered}
\overline{e_{F}}=\sqrt{\left(\frac{\partial \ln F}{\partial \ln \dot{m}_{o A}}\right)^{2}+\left(\frac{\partial \ln F}{\partial \ln \dot{m}_{o T}}\right)^{2}+\left(\frac{\partial \ln F}{\partial \ln \phi}\right)^{2}{\overline{e_{\phi}}}^{2},} \\
\overline{e_{\xi}}=\sqrt{\left(\frac{\partial \ln \xi}{\partial \ln \dot{m}_{o A}}\right)^{2}+\left(\frac{\partial \ln \xi}{\partial \ln \dot{m}_{o T}}\right)^{2}+\left(\frac{\partial \ln \xi}{\partial \ln \phi}\right)^{2}{\overline{e_{\phi}}}^{2}} \\
\overline{e_{\text {total }}}=\sqrt{{\overline{\bar{e}_{F}}}^{2}+{\overline{e_{\xi}}}^{2}} .
\end{gathered}
$$

As it can be seen, these normalized errors are functions of the ratio $\overline{e_{\phi}}=e_{\phi} / e_{M F R}$. The oxidizer mass flow rate measurements precision determines $e_{M F R}$ while $e_{\phi}$ depends on the fuel regression rate sensors' precision. We introduce the parameter $\alpha_{T}$ defined as the ratio of the oxidizer injections and varying in [0, 1] (0 if: $\dot{m}_{O T}=0$ and 1 if: $\left.\dot{m}_{O A}=0\right)$ and the function $\Phi$ in Equations (23) and (24):

$$
\begin{gathered}
\alpha_{T}=\frac{\dot{m}_{o T}}{\dot{m}_{o A}+\dot{m}_{o T}}, \\
\Phi\left[\alpha_{T}\right]=1+S_{g}^{2} \alpha_{T}^{4} .
\end{gathered}
$$

The sensitivity coefficients involved in the expressions of the normalized errors can be calculated based on Equations (9) and (10), and are given in the following relations (Equations (25)-(30)):

$$
\begin{gathered}
\frac{\partial \ln F}{\partial \ln \dot{m}_{o A}}=\left(1-\alpha_{T}\right)\left\{\frac{n+\xi}{1+\xi}-\frac{4 m S_{g}^{2} \alpha_{T}^{4}}{(1+\xi) \Phi\left[\alpha_{T}\right]}+\left(1-n+\frac{4 m S_{g}^{2} \alpha_{T}^{4}}{\Phi\left[\alpha_{T}\right]}\right) \frac{d \ln I_{s p}}{d \ln \xi}[\xi]\right\}, \\
\frac{\partial \ln F}{\partial \ln \dot{m}_{o T}}=\alpha_{T}\left\{\frac{n+\xi}{1+\xi}-\frac{4 m S_{g}^{2}\left(\alpha_{T}-1\right) \alpha_{T}^{3}}{(1+\xi) \Phi\left[\alpha_{T}\right]}+\left(1-n+\frac{4 m S_{g}^{2}\left(\alpha_{T}-1\right) \alpha_{T}^{3}}{\Phi\left[\alpha_{T}\right]}\right) \frac{d \ln I_{s p}}{d \ln \xi}[\xi]\right\}, \\
\frac{\partial \ln F}{\partial \ln \phi}=(2 n-1)\left\{-\frac{1}{1+\xi}+\frac{d \ln I_{s p}}{d \ln \xi}[\xi]\right\},
\end{gathered}
$$




$$
\begin{gathered}
\frac{\partial \ln \xi}{\partial \ln \dot{m}_{o A}}=\left(1-\alpha_{T}\right)\left\{1-n+\frac{4 m S_{g}^{2} \alpha_{T}^{4}}{\Phi\left[\alpha_{T}\right]}\right\}, \\
\frac{\partial \ln \xi}{\partial \ln \dot{m}_{o T}}=\alpha_{T}\left\{1-n+\frac{4 m S_{g}^{2}\left(\alpha_{T}-1\right) \alpha_{T}^{3}}{\Phi\left[\alpha_{T}\right]}\right\}, \\
\frac{\partial \ln \xi}{\partial \ln \phi}=2 n-1 .
\end{gathered}
$$

Finally, the specific impulse coefficient in Equations (25)-(27) is written in Equation (31):

$$
\frac{d \ln I_{s p}}{d \ln \xi}[\xi]=\frac{\xi}{I_{s p}[\xi]} \frac{d I_{s p}}{d \xi}[\xi] .
$$

The specific impulse reaches its maximum at the optimal mixture ratio $\xi_{\text {opt }}$. As a consequence, $d I_{s p} / d \xi\left[\tilde{\xi}_{o p t}\right]=0$, and $d \ln I_{s p} / d \ln \xi\left[\xi_{o p t}\right]=0$, which simplifies the previous equations if the engine operates at the optimal mixture ratio.

\subsection{Regulation Law of A-SOFT Engines}

While measuring the engine's performances at a given time during its operation, the role of the control law is to determine the commands to be sent to the servo valves to reach the desired thrust and $\mathrm{O} / \mathrm{F}$ ratio. As seen previously, the thrust and $\mathrm{O} / \mathrm{F}$ ratio depend on three independent variables which are the two oxidizer mass flow rates $\left(\dot{m}_{O A}\right.$ and $\left.\dot{m}_{O T}\right)$ and the fuel grain diameter. It is however possible to rewrite Equations (9) and (10) to highlight the role of the total oxidizer mass flow rate and effective swirl number $\left(\dot{m}_{0}\right.$ and $\left.S_{e}\right)$ which are independent variables as well (Equations (32)-(34)):

$$
\begin{gathered}
F[t]=I_{s p}[\xi] g_{0}\left\{\dot{m}_{o}[t]+C_{1} \phi[t] f\left[S_{e}[t]\right]\left(\frac{\dot{m}_{o}[t]}{\phi^{2}[t]}\right)^{n}\right\}, \\
\xi[t]=\frac{\dot{m}_{o}[t]}{C_{1} \phi[t] f\left[S_{e}[t]\right]\left(\frac{\dot{m}_{o}[t]}{\phi^{2}[t]}\right)^{n}}, \\
\left(\begin{array}{l}
F \\
\xi
\end{array}\right)=\left(\begin{array}{l}
F\left[\dot{m}_{0}, S_{e}, \phi\right] \\
\xi\left[\dot{m}_{0}, S_{e}, \phi\right]
\end{array}\right) .
\end{gathered}
$$

From these relations, we can calculate the total derivatives of the thrust and the O/F ratio (Equations (35) and (36)), which can be written in vector form in the Relation (37):

$$
\begin{aligned}
& \frac{d F}{d t}=\left(\frac{\partial F}{\partial \dot{m}_{o}}\right) \frac{d \dot{m}_{o}}{d t}+\left(\frac{\partial F}{\partial S_{e}}\right) \frac{d S_{e}}{d t}+\left(\frac{\partial F}{\partial \phi}\right) \frac{d \phi}{d t}, \\
& \frac{d \xi}{d t}=\left(\frac{\partial \xi}{\partial \dot{m}_{o}}\right) \frac{d \dot{m}_{o}}{d t}+\left(\frac{\partial \xi}{\partial S_{e}}\right) \frac{d S_{e}}{d t}+\left(\frac{\partial \xi}{\partial \phi}\right) \frac{d \phi}{d t}, \\
& \left(\begin{array}{l}
\frac{d F}{d t} \\
\frac{d \xi}{d t}
\end{array}\right)=\left[\begin{array}{ll}
\frac{\partial F}{\partial \dot{m}_{o}} & \frac{\partial F}{\partial S_{e}} \\
\frac{\partial \xi}{\partial \dot{m}_{o}} & \frac{\partial \xi}{\partial S_{e}}
\end{array}\right]\left(\begin{array}{c}
\frac{d \dot{m}_{o}}{d t} \\
\frac{d S_{e}}{d t}
\end{array}\right)+\left(\begin{array}{c}
\frac{\partial F}{\partial \phi} \\
\frac{\partial \xi}{\partial \phi}
\end{array}\right) \frac{d \phi}{d t} .
\end{aligned}
$$

In the last relation, the variation related to the port diameter is an environmental change resulting from combustion, whereas the oxidizer mass flow rate and effective swirl number are the variables to be controlled. As a consequence, it is possible to control $d F / d t$ and $d \xi / d t$ by measuring $d \phi / d t$, which is the fuel regression rate, and by manipulating $d \dot{m}_{0} / d t$ and $d S_{e} / d t$. 
As for the error propagation analysis, it is possible to calculate the partial derivative coefficients which are given in Equations (38)-(43). It should be noted that these partial derivative coefficients are related with the sensitivity coefficients by the relation: $\frac{\partial \ln h}{\partial \ln x}=\frac{\partial h}{\partial x} \frac{x}{h}$ :

$$
\begin{gathered}
\frac{\partial F}{\partial \dot{m}_{o}}=g_{0} \frac{(n+\xi)}{\xi} I_{s p}[\xi]-g_{0}(n-1)(1+\xi) \frac{d I_{s p}}{d \xi}[\xi], \\
\frac{\partial F}{\partial S_{e}}=g_{0} \dot{m}_{0} \frac{d f}{d S_{e}}\left[S_{e}\right]\left\{I_{s p}[\xi]-\xi(1+\xi) \frac{d I_{s p}}{d \xi}[\xi]\right\}\left(f\left[S_{e}\right] \xi\right)^{-1}, \\
\frac{\partial F}{\partial \phi}=g_{0}(2 n-1) \dot{m}_{o}\left\{-I_{s p}[\xi]+\xi(1+\xi) \frac{d I_{s p}}{d \xi}[\xi]\right\}(\phi \xi)^{-1}, \\
\frac{\partial \xi}{\partial \dot{m}_{o}}=(1-n) \frac{\xi}{\dot{m}_{o}}, \\
\frac{\partial \xi}{\partial S_{e}}=-\frac{\xi}{f\left[S_{e}\right]} \frac{d f}{d S_{e}}\left[S_{e}\right], \\
\frac{\partial \xi}{\partial \phi}=(2 n-1) \frac{\xi}{\phi} .
\end{gathered}
$$

Equation (37) can be solved so that the derivatives of the controlled variables are given in Equation (44). This system defines the regulation laws that could be used in an A-SOFT engine feedback loop control and which will be analyzed by numerical simulations:

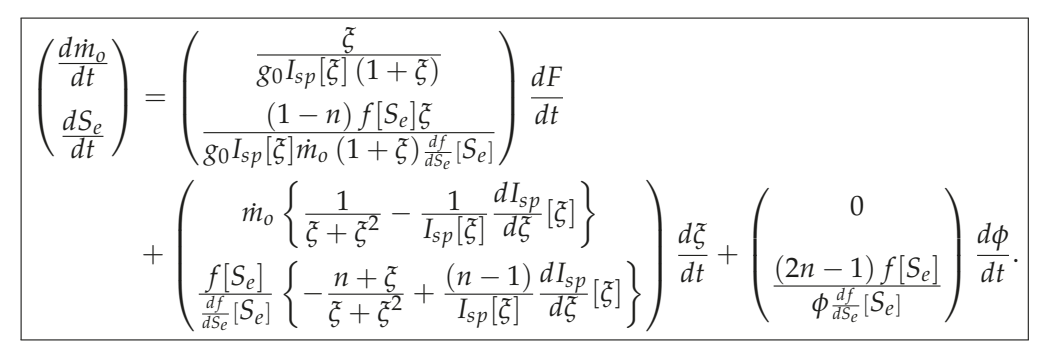

\subsection{Simplified Modeling of A-SOFT Engines for Simulations}

The main objective of performing numerical simulations is to analyze how an A-SOFT engine would operate autonomously with a feedback loop control system including resistor-based sensors for the fuel regression rate measurement. In particular, the control law and the RBS integration are the major points to be discussed to evaluate the feasibility of such a system.

In order to perform numerical simulations, the A-SOFT engine has been simplified and separated into six distinguishable subsystems as listed below and presented in Figure 2:

(1) Oxidizer feed system,

(2) Servo valves,

(3) Injection,

(4) Engine,

(5) Measurements,

(6) Feedback control system. 


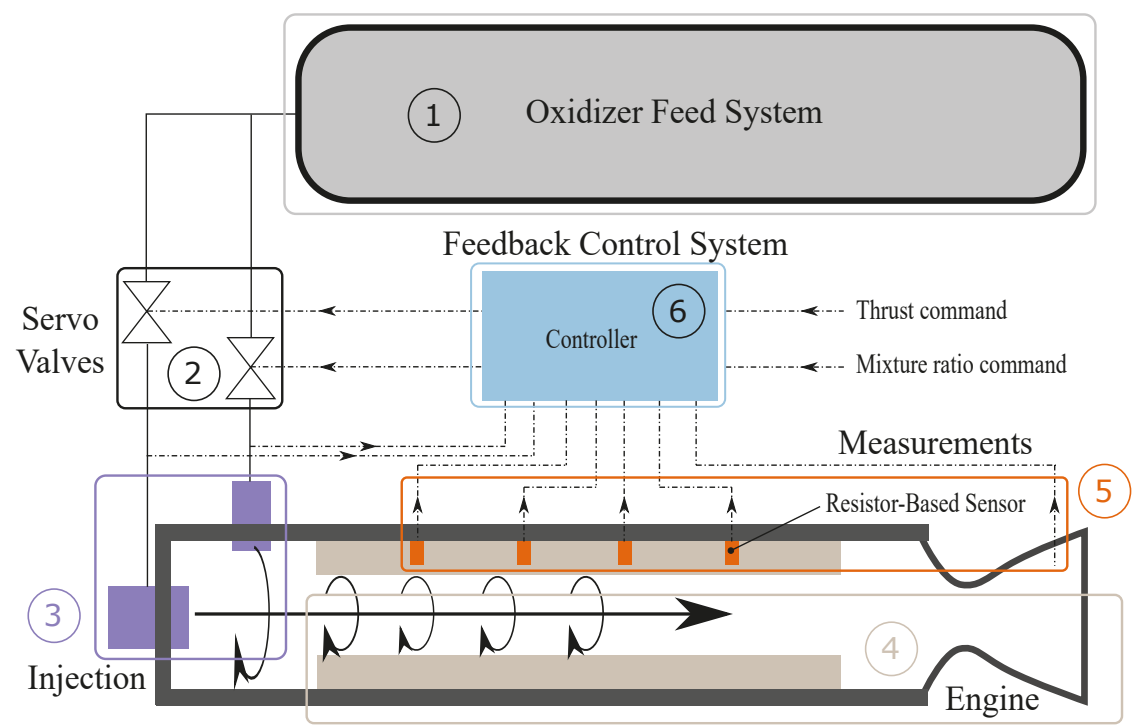

Figure 2. A-SOFT engine simplification for the simulations.

The oxidizer feed system (1) is not modeled in this study. More precisely, we suppose that the tank is maintained pressurized and that there is no issue regarding the liquid level either.

Ideal servo valves (2) would deliver an oxidizer mass flow rate proportional to the percentage of maximum valve travel (or valve opening). However, some types of valves may have a highly nonlinear relationship between the effective valve flow area (or mass flow rate) and the valve opening [22]. In order to take into account this effect that would be observed in an experimental setup, Equations (45) and (46) provide the relationships between the oxidizer mass flow rate and the corresponding valve opening if a circular port ball valve is used [22]. These relations are both valid for the axial or tangential injectors $(\mathrm{A}-\mathrm{T})$ if the same valves are used:

$$
\begin{gathered}
\dot{m}_{o, A-T}[t]=c_{1}\left(1-e^{-\frac{v o_{A-T}[t]-c_{2}}{c_{3}}}\right), \\
v o_{A-T}[t]=-c_{3} \ln \left(1-\frac{\dot{m}_{o, A-T}[t]}{c_{1}}\right)+c_{2} .
\end{gathered}
$$

$c_{1}, c_{2}$, and $c_{3}$ are the valves' parameters and are given in Table 1 . This set of parameters has been chosen theoretically so that it induces a maximum oxidizer mass flow rate of $100 \mathrm{~g} \cdot \mathrm{s}^{-1}$ as illustrated in Figure 3. In this example, the valve remains essentially closed until a valve opening vo around $20 \%$, then increases rapidly up to vo close to $50 \%$. Finally, the mass flow rate is only slightly changed as the valve opening approaches $100 \%$. These parameters should be determined experimentally for a real application.

Table 1. Valves' parameters used in this study.

\begin{tabular}{ccc}
\hline$c_{1}\left(\mathrm{~kg} \cdot \mathrm{s}^{-1}\right)$ & $c_{2}(-)$ & $c_{3}(-)$ \\
\hline 0.1 & 0.2 & 0.15 \\
\hline
\end{tabular}




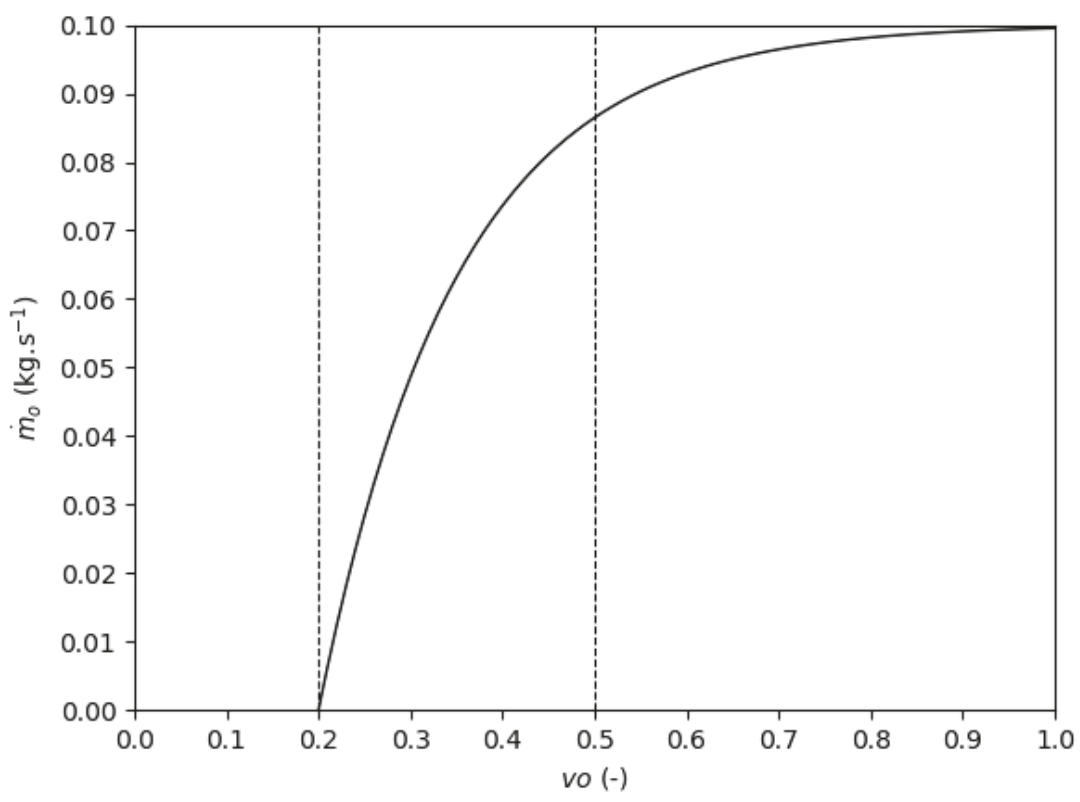

Figure 3. Mass flow rate profile in function of the valve opening.

The injection subsystem (3) deals with the axial and tangential oxidizer injectors. Both the total oxidizer mass flow rate and the effective swirl number have been defined previously by Equations (4) and (6) and these definitions are used for the simulations. The total oxidizer mass flow rate is the sum of the axial and tangential oxidizer mass flow rates, and the effective swirl number depends on the geometric swirl number.

The engine (4) simulation is based on three specific calculations which are the fuel regression rate, the combustion chamber and nozzle ejection pressures, and the generated thrust. To consider the most general case possible, the fuel regression rate law is assumed to be slightly different than the one used for the previous sections and is given in Equation (47):

$$
\dot{r}[x, t]=a\left(1+S_{e}^{2}[t]\right)^{m}\left(\frac{4 \dot{m}_{t o t}[x, t]}{\pi \phi[x, t]^{2}}\right)^{n} x^{b} .
$$

The interest of this relation is that it includes the influence of the local mass flux, of the swirl oxidizer injection, and of the axial location along the fuel grain. The coefficients $m$ and $n$ were chosen identical to the one obtained experimentally and used in previous studies [15]. The $b$ coefficient has been chosen arbitrarily with a small value to introduce an explicit dependency on the axial position along the fuel grain. Finally, the $a$ coefficient has been calculated so that this regression rate law is consistent with experimental data obtained with an A-SOFT engine [11]. For a real application, these coefficients should be determined carefully and based on experimental results. The total and fuel mass flow rates are given in Equations (48) and (49):

$$
\begin{gathered}
\dot{m}_{t o t}[x, t]=\dot{m}_{o}[t]+\dot{m}_{f}[x, t], \\
\dot{m}_{f}[x, t]=\int_{x^{\prime}=0}^{x} \rho_{f} \dot{r}\left[x^{\prime}, t\right] \pi \phi\left[x^{\prime}, t\right] d x^{\prime} .
\end{gathered}
$$


The mixture ratio is given by Equation (50):

$$
\xi[t]=\frac{\dot{m}_{o}[t]}{\dot{m}_{f}[L, t]} .
$$

The combustion chamber pressure is estimated by using a thermochemical code, in our case a free version of RPA software [23] (Rocket Propulsion Analysis). The pressure is given by Equation (51), and calculated by an iterative resolution at every time step. A pressure error $p_{\text {error }}$ is then chosen as a convergence parameter. The theoretical isentropic coefficient $\gamma$ is also determined during that process:

$$
p_{c}[t]=\eta_{c} c_{t h}^{*}[t] \dot{m}_{t o t}[L, t] A_{t}^{-1} .
$$

The nozzle exit pressure is then determined by solving Equation (52), in our case using a simple dichotomy algorithm:

$$
\frac{A_{e}}{A_{t}}=\left(\frac{p_{e}[t]}{p_{c}[t]}\right)^{-1 / \gamma[t]} \sqrt{\left(\frac{2}{\gamma[t]+1}\right)^{\frac{\gamma[t]+1}{\gamma[t-1}}}\left(\sqrt{\frac{2}{\gamma[t]-1}\left\{1-\left(\frac{p_{e}[t]}{p_{c}[t]}\right)^{\frac{\gamma[t]-1}{\gamma[t]}}\right\}}\right)^{-1} .
$$

Finally, the thrust is estimated through Equation (53):

$$
F[t]=A_{t} p_{c}[t] \sqrt{\frac{2 \gamma[t]^{2}}{\gamma-1}\left(\frac{2}{\gamma[t]+1}\right)^{\frac{\gamma[t]+1}{\gamma(t)-1}}\left\{1-\left(\frac{p_{e}[t]}{p_{c}[t]}\right)^{\frac{\gamma[t]-1}{\gamma[t]}}\right\}}+\left(p_{e}[t]-p_{a}\right) A_{e}
$$

The feedback loop control is based on getting instantaneous data from the measurement subsystem (5) on the engine's parameters which need to be regulated. In the A-SOFT case, these parameters are the thrust and the mixture ratio. The objective of the simulations is to study the RBS compatibility with an actual control system. To simplify the modeling, we will consider that the thrust and the oxidizer mass flow rate measurements are ideal (Equations (54) and (55)), which means the measured values are the real ones:

$$
\begin{aligned}
F_{m}[t] & =F[t], \\
\dot{m}_{o, m}[t] & =\dot{m}_{o}[t] .
\end{aligned}
$$

The fuel mass flow rate measurement is realized by embedding RBS in the fuel grain. Figure 4 presents the sensors' principle and the modeling which is adopted in the simulations for a sensor with four resistors. 


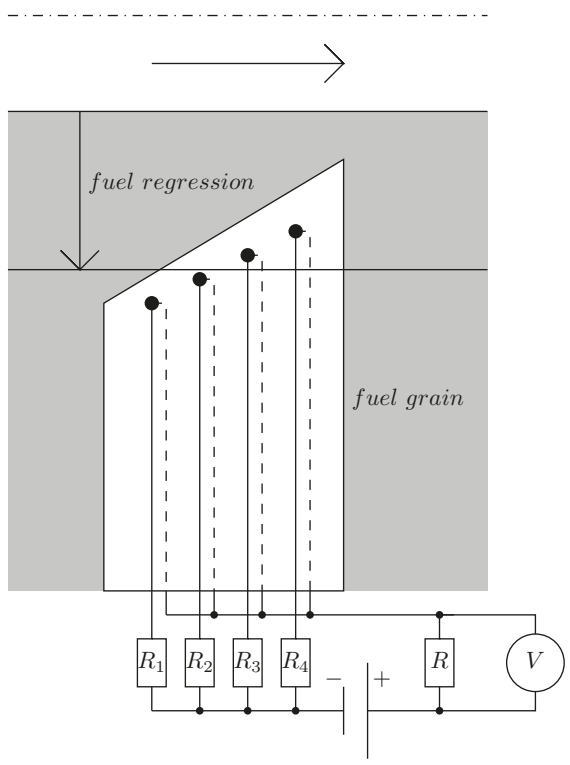

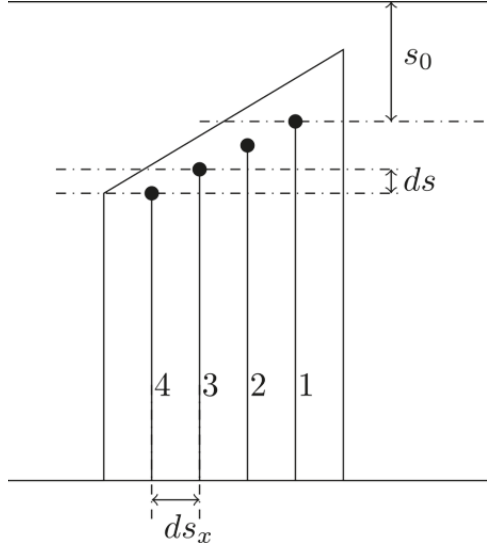

(b)

(a)

Figure 4. (a) resistor-based sensor principle and (b) modeling.

While the fuel grain burns and its surface moves, parallel resistors are successively disconnected which modifies the electric voltage at the terminals of the sensor. As a consequence, the measured fuel grain diameter is a constant piece-wise function over time and so are the fuel regression and mass flow rates that are calculated based on it.

As a simplification, we consider that the sensors are ideal: a resistor is disconnected when the fuel surface reaches it. Moreover, we suppose that the resistors are located at the same axial position along the fuel grain length: $d s_{x}=0$. We consider that if a resistor is connected it delivers a voltage of $1 \mathrm{~V}$ and $0 \mathrm{~V}$ if not connected. This simplification does not remove any physical sense but avoids the calculation depending on real electric resistance values. The total electric voltage delivered by a sensor is then the sum of resistors' voltages. Applying this rule, the resistors' and sensor's electric voltage are calculated based on Equations (56) and (57). Examples of results describing the sensors' behavior are given in Section 3.2.3.

$$
\begin{array}{cr}
\forall s \in\left[0, N_{s}-1\right] \quad \forall r \in\left[0, N_{r}-1\right], & \\
\begin{aligned}
\text { if }: \phi\left[x_{s}, t\right]<\phi_{0}+2 s_{0}+2 r d s & \operatorname{sr}[s, r, t]=1, \\
\text { else } & \operatorname{sr}[s, r, t]=0,
\end{aligned} \\
\forall s \in\left[0, N_{s}-1\right], \quad s s[s, t]=\sum_{r=0}^{N_{r}-1} \operatorname{sr}[s, r, t] .
\end{array}
$$

$N_{s}$ and $N_{r}$ are respectively the number of sensors in the grain and the number of resistors in a single sensor, $x_{s}$ is the axial location of a sensor $s, s s$ and $s r$ are respectively the electric signals of a sensor and a resistor. 
The measured fuel grain diameter $\phi_{m, s}$ for a given sensor is then calculated based on the electric signal in Equation (58):

$$
\begin{aligned}
\forall s \in\left[0, N_{s}-1\right], & & \\
\text { if }: s s[s, t]>N_{s}-1 & \phi_{m, s}[t] & =\phi_{0}, \\
\text { else }: & \phi_{m, s}[t] & =\phi_{0}+2 s_{0}+2 d s\left(N_{r}-s s[s, t]-1\right) .
\end{aligned}
$$

Based on these measurements, the local regression rate can only be evaluated when the sensor's signal changes. By knowing the distance between two resistors and the delay for the fuel surface to move from one to another, Equation (59) provides the calculation method for the measured local regression rate:

$$
\begin{aligned}
\forall s \in\left[0, N_{s}-1\right], & \\
\text { if }: \phi_{m, s}[t]=\phi_{m, s}\left[t^{\prime}\right] & \dot{r}_{m, s}[t]=\dot{r}_{m, s}\left[t^{\prime}\right], \\
\text { if }: \phi_{m, s}[t]>\phi_{m, s}\left[t^{\prime}\right] & \dot{r}_{m, s}[t]=\frac{\phi_{m, s}[t]-\phi_{m, s}\left[t^{\prime}\right]}{2\left(t-t^{\prime}\right)} .
\end{aligned}
$$

In this relation, $t^{\prime}$ and $t$ are two successive times when the sensor's signal has changed and $t>t^{\prime}$. The fuel regression rate is constant when the measured diameter is not changed. As a consequence, the fuel regression rate can only be evaluated once the first resistor has been reached by the fuel surface.

The local and total fuel mass flow rates are then evaluated by Equations (60) and (61):

$$
\begin{gathered}
\forall s \in\left[0, N_{s}-1\right], \quad \dot{m}_{f, m, s}[t]=\pi \rho_{f} \dot{r}_{m, s}[t] \phi_{m, s}[t] \frac{L}{N_{s}}, \\
\dot{m}_{f, m}[t]=\sum_{s=0}^{N_{s}-1} \dot{m}_{f, m, s}[t] .
\end{gathered}
$$

Finally, the measured mixture ratio based on RBS is written in Equation (62):

$$
\xi_{m}[t]=\frac{\dot{m}_{o, m}[t]}{\dot{m}_{f, m}[t]} .
$$

The last subsystem to be modeled is the feedback control system (6). It can be divided into two parts: the first part concerns the use of a PID (Proportional Integral Derivative) controller and the second part is the integration of the control law. As demonstrated in the previous section, Equation (44) allows for calculating the derivatives of the oxidizer mass flow rate and effective swirl number in function of the thrust and mixture ratio derivatives and the regression rate. In the case of the simulation, the feedback control system must follow the thrust and mixture ratio profiles which are given. The control law is used to determine the targeted total oxidizer mass flow rate and effective swirl number to satisfy the control command. As a consequence, the required terms appearing in the control law are given by Equations (63)-(65):

$$
\begin{gathered}
\frac{d F}{d t}[t]=\frac{F_{\text {command }}[t+d t]-F_{m}[t]}{d t}, \\
\frac{d \xi}{d t}[t]=\frac{\xi_{\text {command }}[t+d t]-\xi_{m}[t]}{d t} \\
\frac{d \phi}{d t}[t]=2 \dot{r}_{m}[t] .
\end{gathered}
$$


In the last relation, $\dot{r}_{m}$ represents the measured regression rate and is averaged based on the local values provided by the sensors. Based on these calculations, we can evaluate the targeted oxidizer mass flow rate and swirl number to satisfy the commands through Equations (66) and (67):

$$
\begin{gathered}
\dot{m}_{0, \text { target }}[t+d t]=\frac{d \dot{m}_{0}}{d t}[t] d t+\dot{m}_{0, m}[t], \\
S_{e, \text { target }}[t+d t]=\frac{d S_{e}}{d t}[t] d t+S_{e, m}[t] .
\end{gathered}
$$

The targeted axial and tangential oxidizer mass flow rates are then determined based on Equations (4) and (6), and the targeted valves opening by Equation (46). The PID error is then calculated (Equation (68)):

$$
e_{\text {pid }}[t]=v o_{A-T, \text { target }}[t+d t]-v o_{A-T}[t] .
$$

The PID correction to be applied to the controlled valve is then given by Equation (69):

$$
\delta v o_{A-T}[t+d t]=k_{P} e_{p i d}[t]+k_{I} \int_{0}^{t} e_{p i d}\left[t^{\prime}\right] d t^{\prime}+k_{D} \frac{d e_{p i d}}{d t}[t] .
$$

Finally, the commands to be sent to the servo valves are given as follows (Equation (70)):

$$
v o_{A-T}[t+d t]=v o_{A-T}[t]+\delta v o_{A-T}[t+d t] .
$$

Regarding the numerical parameters, time derivatives are calculated based on an Euler explicit scheme and integrals are estimated through the trapezoids' method. In order to consider the latency that occurs in real experimental setup regarding the valves response times, pipeline delays, controller calculation time, etc., an artificial regulation frequency $f_{\text {reg }}$ has been added for the numerical simulations. This frequency has been fixed to $2 \mathrm{~Hz}$ for all the simulations, meaning that the feedback control system sends new orders to the valves every $0.5 \mathrm{~s}$. This value has been chosen to be realistic but is not based on experimental results or calculations. It is an additional parameter and its influence could be investigated in a future work. The numerical parameters that have been used for the simulations are summarized in Table 2.

Table 2. Numerical parameters used for the simulations.

\begin{tabular}{cccc}
\hline$d x(\mathrm{~m})$ & $d t(\mathrm{~s})$ & $p_{\text {error }}(\%)$ & $f_{\text {reg }}(\mathrm{Hz})$ \\
\hline 0.0001 & 0.01 & 0.1 & 2.0 \\
\hline
\end{tabular}

\section{Results}

\subsection{Error Propagation Analysis}

The A-SOFT concept is based on an innovative idea which is to use the swirl influence on the fuel regression rate to control the thrust and the $\mathrm{O} / \mathrm{F}$ ratio at the same time but independently. However, other concepts exist to achieve this objective [7-9]. Among them, the AOA (Aft-end Oxidizer Addition) hybrid engine $[8,9]$ is somehow similar to the A-SOFT engine. Unlike A-SOFT that uses dual front injections, the AOA engine combines a front injection with an aft-end oxidizer addition in order to regulate the thrust and the mixture ratio (Figure 5). 


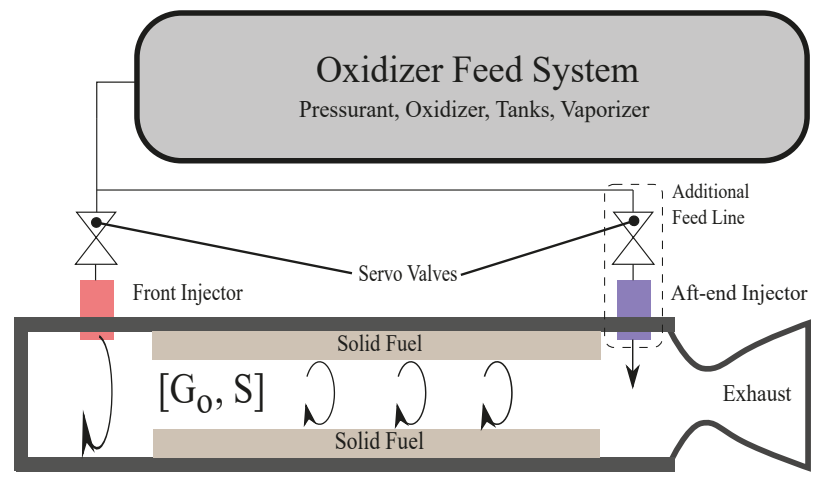

Figure 5. AOA (Aft-end Oxidizer Addition) concept with front and aft-end oxidizer injections.

Despite the concepts being different, both use two servo valves to regulate the thrust and the $\mathrm{O} / \mathrm{F}$ ratio and it seems logical to compare their respective interests. To do so, the relative error analysis has also been conducted for the AOA engines and will be compared to the one conducted for the A-SOFT engines. Because the mathematical development is very similar to the one presented in the previous section and does not bring any additional information, materials and methods related to the AOA engines are given in Appendix A. An important point to note is the difference regarding the $\alpha_{T}$ parameter: in the case of A-SOFT, it represents the ratio between the tangential and the total oxidizer mass flow rates (Equation (23)), while it represents the ratio between the front and the total oxidizer mass flow rates in the case of AOA (Equation (A15)).

\subsubsection{Optimal Mixture Ratio}

To evaluate the error propagation, the study case is chosen based on experimental results [18]. We will consider an optimal mixture ratio configuration with corresponding parameters given below:

- $\quad$ mixture ratio: $\xi=\xi_{\text {opt }}=2$,

- regression rate: $a=0.029 \times 10^{-3} \mathrm{~m} \cdot \mathrm{s}^{-1}, m=0.166, n=0.650$,

- $\quad$ specific impulse: $d \ln I_{s p} / d \ln \xi=0$.

Figure 6 presents the normalized thrust error for three given $e_{\phi} / e_{M F R}$ ratios. As we can first notice, the $e_{\phi} / e_{M F R}$ ratio plays a major role in the error propagation. The thrust normalized error will increase with the ratio which means that, for a given oxidizer mass flow rate measurement error, the increase of the diameter measurement error will increase the thrust error as well. It is also clear that the geometric swirl number $S_{g}$ has an influence on the A-SOFT errors. For $S_{g} \neq 0$, the minimal thrust errors are obtained for: $\alpha_{T} \approx 0.35$ both for A-SOFT and AOA. If $S_{g}=0$, the error profiles are symmetric around $\alpha_{T}=0.5$ for A-SOFT since there is no swirl effect. We can notice that A-SOFT and AOA errors are very similar for $\alpha_{T} \geq 0.5$ and become equal if $\alpha_{T}=1$. In that case, the A-SOFT engine uses only the tangential injection and the AOA engine uses only the front injection (which is also tangential), the engines are consequently strictly identical. Finally, the AOA engine generates less thrust propagation errors than the A-SOFT engine for low values of $\alpha_{T}$. However, this result must be contrasted since operating in these conditions would imply that most of the oxidizer mass flow rate would come from the aft-end injection and may result in combustion troubles that are not considered at all in this analysis.

Figure 7 presents the normalized $\mathrm{O} / \mathrm{F}$ ratio error for the three same $e_{\phi} / e_{M F R}$ ratios. Some of the previous conclusions are naturally shared with the previous ones: the A-SOFT symmetric profile when $S_{g}=0$, the equal errors for A-SOFT and AOA when $\alpha_{T}=1$ and the $e_{\phi} / e_{M F R}$ influence are also true regarding the mixture ratio. We can notice that the increase of $e_{\phi} / e_{M F R}$ ratio from 0 to 5 can multiply the relative errors on $\mathrm{O} / \mathrm{F}$ ratio by a factor 6 while this factor was limited to a value lower 
than 1.5 in the case of thrust error propagation. Naturally, the mixture ratio errors are much more sensitive to the precision of the fuel diameter measurements since there is a direct relation between $\mathrm{O} / \mathrm{F}$ ratio and fuel grain diameter. In contrast to the thrust error, the mixture ratio error for A-SOFT is significantly lower than for AOA (especially when $\alpha_{T} \leq 0.5$ ). The main reason to explain this effect is that A-SOFT engines are more flexible and efficient to regulate the mixture ratio. Indeed, both oxidizer injections influence the fuel regression rate in A-SOFT while only the front injection can do it in AOA. At this point, the overall conclusions comparing A-SOFT and AOA are not evident, but Figure 8 which describes the total errors will help to conclude.

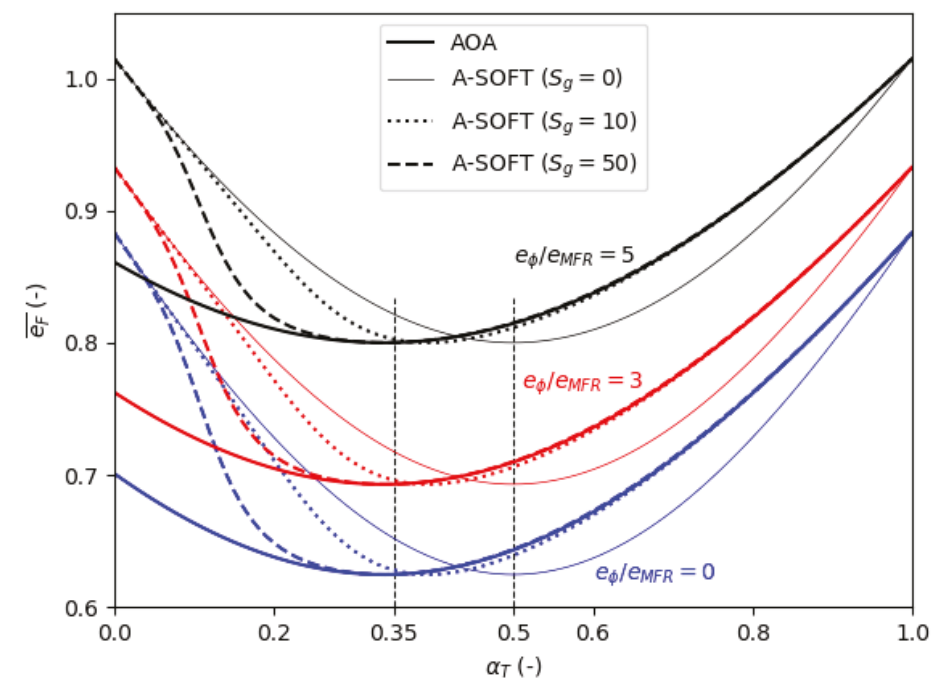

Figure 6. Normalized thrust errors.

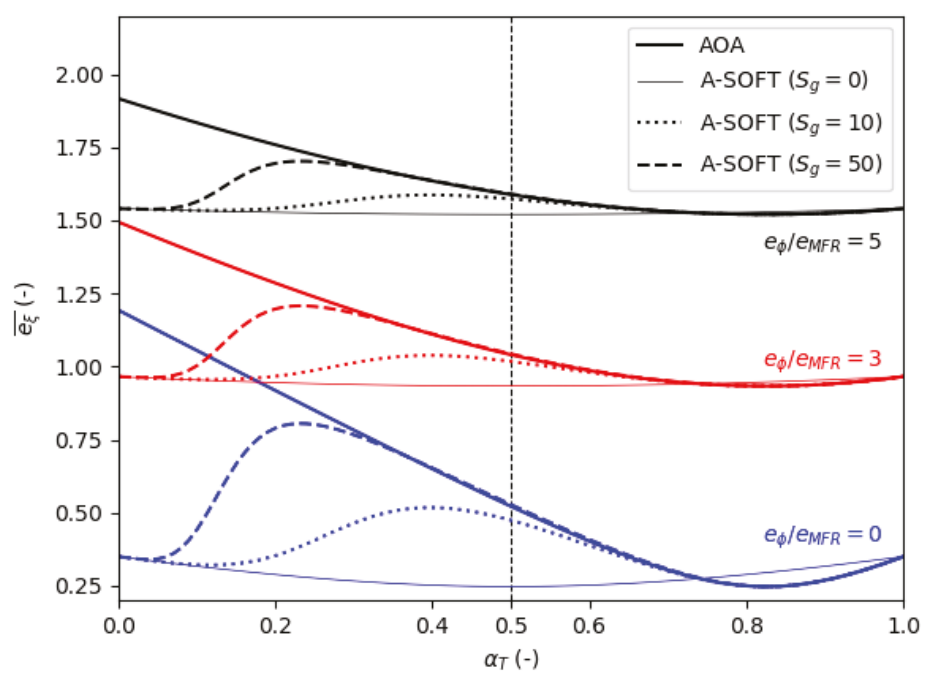

Figure 7. Normalized mixture ratio errors. 
Once again, some of the previous conclusions regarding the thrust and the mixture ratio are shared with the total error propagation (symmetry, case when $\alpha_{T}$ is high, influence of $e_{\phi} / e_{M F R}$ ratio). From the total error point of view, it can be said that the AOA engine's error is generally larger than the A-SOFT one, especially for low $\alpha_{T}$ (mainly due to the mixture ratio error) even though these differences tend to decrease as the diameter error increases. Based on these results, the A-SOFT concept seems more interesting regarding the total error propagation and the following discussions will concern A-SOFT engines only.

As visible in Figure 8, the A-SOFT total error increases as $S_{g}$ increases, regardless of the $e_{\phi} / e_{M F R}$ ratio. Figure 9 focuses on this effect and presents the results for $S_{g}$ ranging from 0 to $50\left(S_{g}=\right.$ $0,2,4,6,8,10,12,15,20,30,40,50)$ at a given $e_{\phi} / e_{M F R}$ ratio. The case where $S_{g}=0$ leads to the lowest errors but is purely theoretical and presents no interest since there would be no swirl control which is the basic principle of A-SOFT engines. For $\alpha_{T}$ lower than 0.1 and higher than 0.9 , the geometric swirl number has almost no influence on the results; however, these $\alpha_{T}$ values generate the highest errors for low $S_{g}$ and are the extreme operating conditions of the engine. The first local minimum at $\alpha_{T}=0.5$ for $S_{g}=0$ is constantly shifted to lower $\alpha_{T}$ values and the error continues to increase with $S_{g}$. At the same time, a second local minimum appears around $\alpha_{T}=0.68$ if $S_{g} \geq 6$. Unlike the first one, this second local minimum error tends to an asymptotic limit and stops increasing even for high geometric swirl numbers. Moreover, as $S_{g}$ increases, a local maximum appears for which the induced error can become larger than the extremity errors if $S_{g}$ exceeds 30 . These results tend to indicate that, for a given geometric swirl number, the total error can be minimized by operating close to optimal $\alpha_{T}$ values, but also that some other values of $\alpha_{T}$ may lead to significant errors and should be avoided. As $\alpha_{T}$ varies during the engine operation, additional analysis is required to design and choose the optimal geometric swirl number.

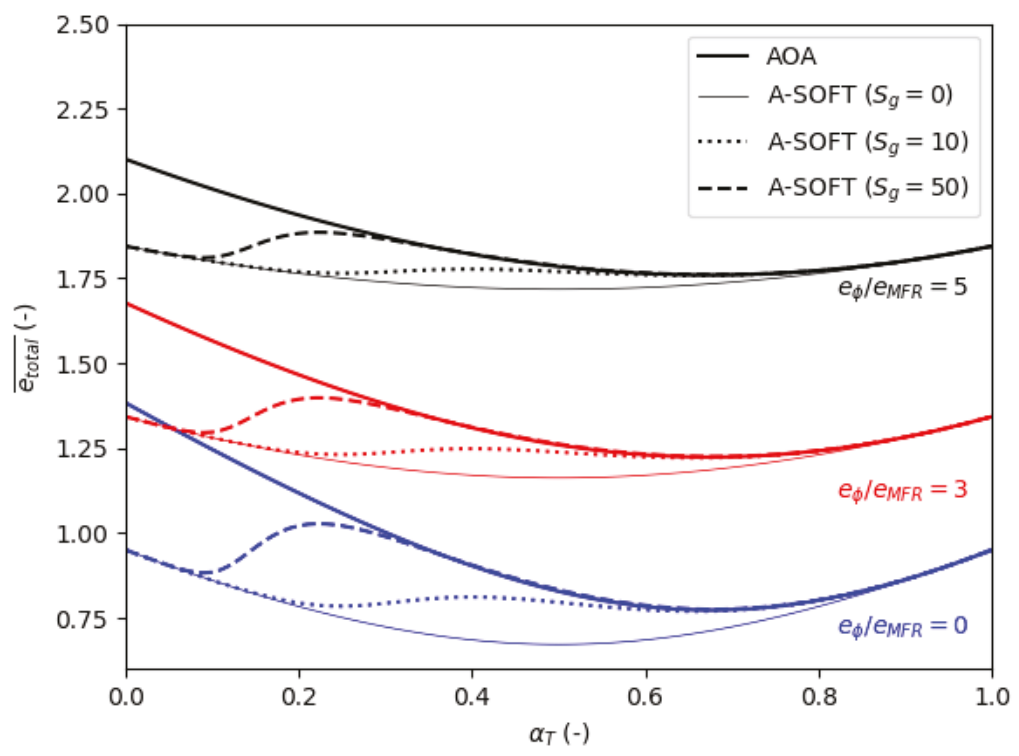

Figure 8. Normalized total errors. 


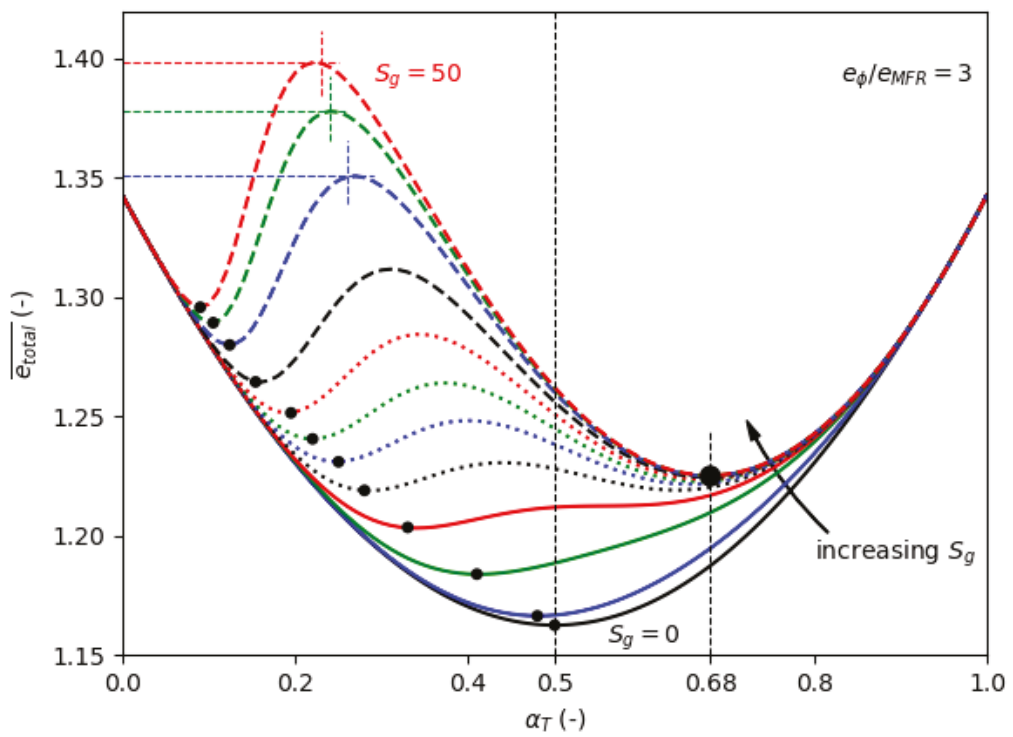

Figure 9. Influence of the geometric swirl number on the normalized total errors in A-SOFT engines for $S_{g}$ values in $[0,2,4,6,8,10,12,15,20,30,40,50]$.

\subsubsection{Optimal Design of the Geometric Swirl Number}

As we have seen, the geometric swirl number has a significant influence on the error propagation in an A-SOFT engine. For a given $S_{g}$, there are optimal operating $\alpha_{T}$ to minimize the errors but other values of $\alpha_{T}$ could drastically increase these errors. In practice, the fuel regression rate is influenced by the effective swirl number provided by the oxidizer's injections and these parameters are related by Equation (71):

$$
S_{e}[t]=S_{g} \alpha_{T}^{2}[t] .
$$

For a given mission, the effective swirl number will be modified to fit as much as possible the thrust and the $\mathrm{O} / \mathrm{F}$ commands while the grain is burning and the fuel port diameter is modified. The range of effective swirl number will obviously depend on the mission profiles, on the fuel and oxidizer combination, on the regression rate coefficients, and on other parameters like the feedback loop control system. As a consequence, it is not possible to chose a geometric swirl number which would be optimal unless the mission is known.

That being said, we could propose a method for designing the optimal geometric swirl number for a given mission, based on the error propagation results. As a study case, let us consider a lab-scale engine operating during one minute with the regression rate coefficients given earlier in the paper, and with a constant target thrust of $250 \mathrm{~N}$. This should lead to an operating effective swirl number ranging between 10 and 50 . Moreover, we will assume that $\alpha_{T}$ increases linearly with time, which is the simplest evolution possible. Finally, we consider the case of an optimal mixture ratio:

- $\quad$ mixture ratio: $\xi=\xi_{\text {opt }}=2$,

- regression rate: $a=0.029 \times 10^{-3} \mathrm{~m} \cdot \mathrm{s}^{-1}, m=0.166, n=0.650$,

- effective swirl number: $S_{e}[t] \in[10,50], \alpha_{T}$ increases linearly with time (Equation (73)).

Once the effective swirl number range is known, there is an infinite number of combinations of possible geometric swirl number and operating $\alpha_{T}$. By definition, $S_{g} \geq S_{e, \max }$ since $\alpha_{T}$ never exceeds 1 (Equation (71)). Table 3 summarizes a few examples: 
Table 3. Examples of possible geometric swirl number and $\alpha_{T}$ combinations for a given effective swirl number range.

\begin{tabular}{ccc}
\hline$S_{e}$ Range (-) & $S_{g}(-)$ & $\alpha_{T}$ Range (-) \\
\hline $10-50$ & 50 & $0.44-1.00$ \\
$10-50$ & 60 & $0.41-0.91$ \\
$10-50$ & 70 & $0.38-0.85$ \\
$10-50$ & 80 & $0.35-0.79$ \\
\hline
\end{tabular}

In order to find a potential optimal geometric swirl number regarding the total error propagation, two natural criteria could be defined: the first one could be to ensure that the error will never exceed a certain level at a given time (C1), and the second one could be to minimize the error over the entire operation duration (C2). If an optimal geometric swirl number $S_{g, o p t}$ does exist, these criteria could be expressed by:

- $\quad \mathrm{C} 1: \forall \alpha_{T}[t]: \overline{e_{\text {total }}}\left[\alpha_{T}[t], S_{g, o p t}\right] \leq \overline{e_{\text {total,max }}}$,

- $\quad \mathrm{C} 2: \forall S_{g}: \int_{t} \overline{e_{\text {total }}}\left[\alpha_{T}[t], S_{g, o p t}\right] \leq \int_{t} \overline{e_{\text {total }}}\left[\alpha_{T}[t], S_{g}\right]$.

As an example, Figure 10 shows that using a geometric swirl number of 40 could lead to a total error exceeding the upper limit if the engine has to operate with $\alpha_{T}$ close to 0.3. Figure 11 illustrates the calculation of the integrated errors. For a given range of effective swirl number (here from 10 to 50), modifying the geometric swirl number will change the integrated error. The blue and red areas respectively represent the integrated errors for $S_{g}=50$ and 80 . The integrated error between two values of effective swirl numbers, denoted by $S_{e, 1}$ and $S_{e, 2}$, is written in Equation (72):

$$
\overline{E_{\text {total }}}\left[S_{e, 1}, S_{e, 2}, S_{g}, \alpha_{T}[t]\right]=\int_{t=0}^{t=t_{f}} \overline{e_{\text {total }}}\left[\alpha_{T}[t], S_{g}\right] d t
$$

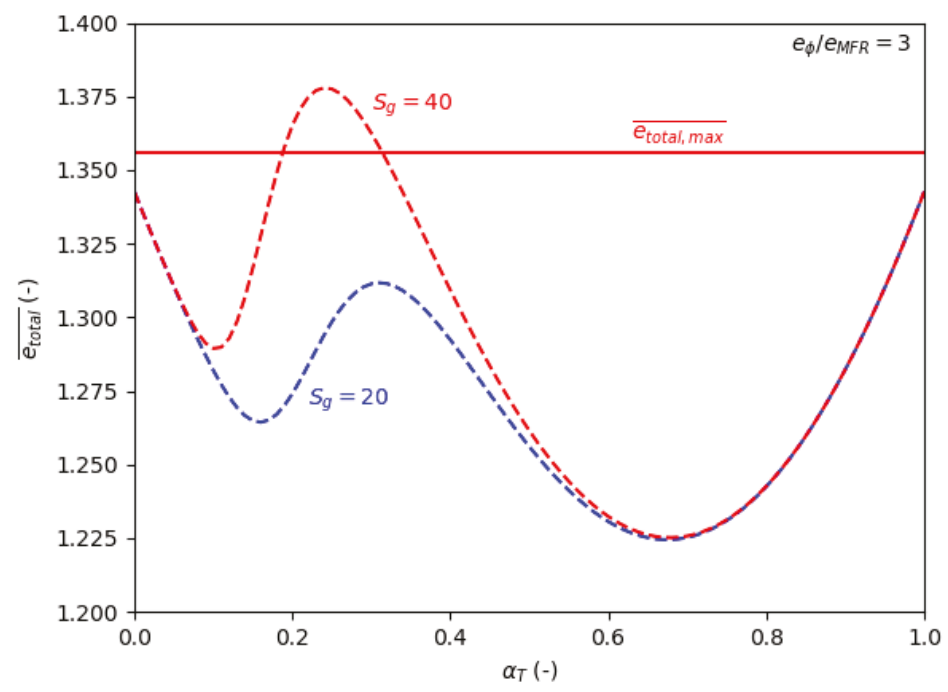

Figure 10. Illustration of the $\mathrm{C} 1$ criterion. 


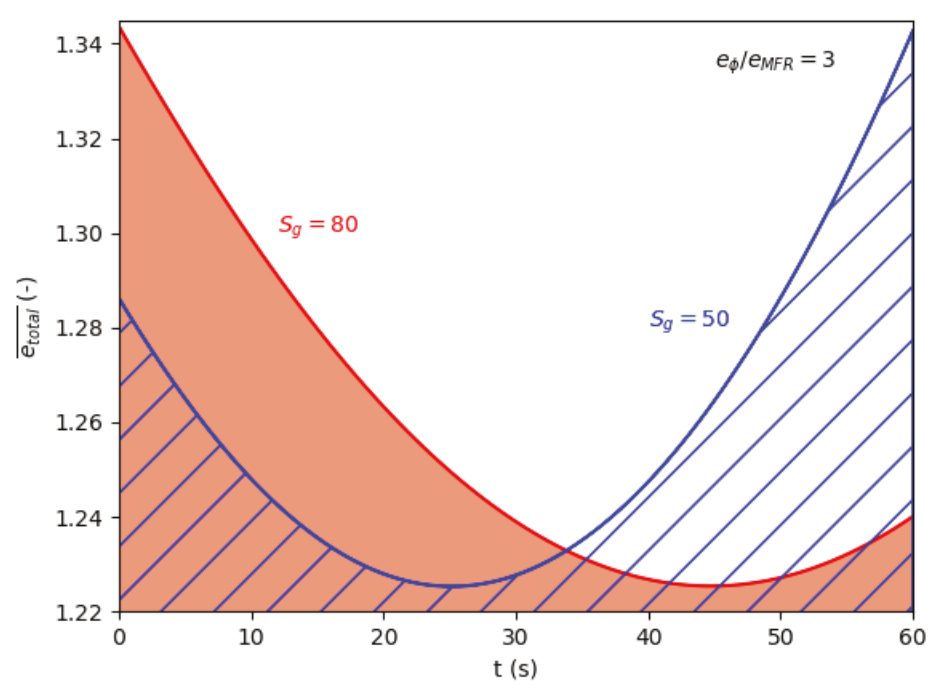

Figure 11. Illustration of the $\mathrm{C} 2$ criterion.

If the effective swirl number range is fixed, the $\alpha_{T}$ evolution depends on $S_{g}$ and can be written by Equation (73) (where $t_{f}$ is the firing duration).

$$
\alpha_{T}[t]=\sqrt{\frac{S_{e, 1}}{S_{g}}}+\frac{\sqrt{\frac{S_{e, 2}}{S_{g}}}-\sqrt{\frac{S_{e, 1}}{S_{g}}}}{t_{f}} t .
$$

Figure 12 provides the evolution of the integrated error by varying the geometric swirl number in the configuration described earlier. As it can be seen, an optimal geometric swirl number does exist in that case and is around 62.5 .

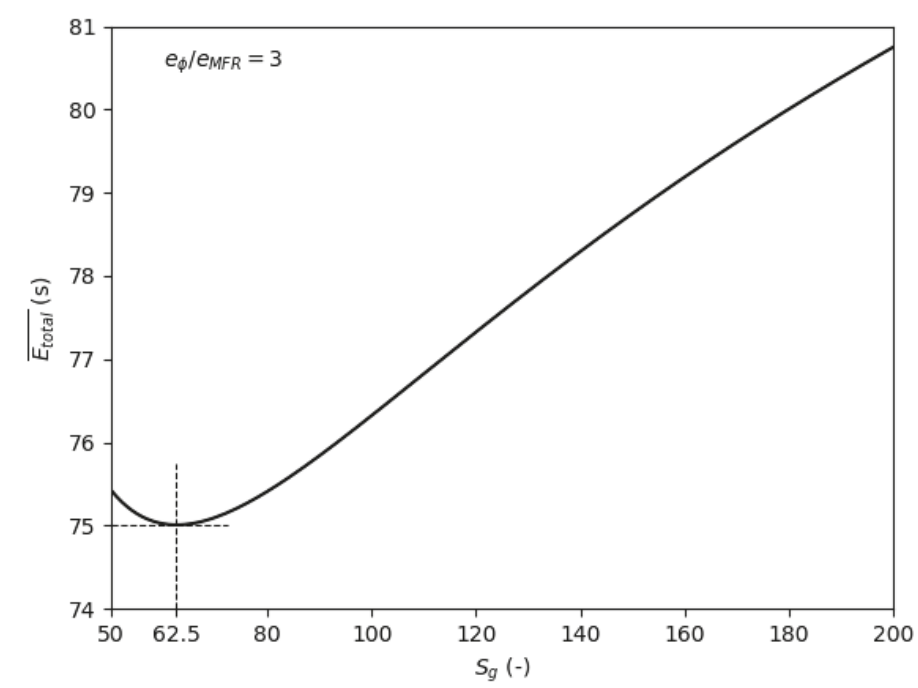

Figure 12. Integrated error evolution depending on the geometric swirl number. 
This optimal value minimizes the integral error over the firing test duration, but, additionally, its maximum instantaneous error is lower than the ones for $S_{g}=50$ or 100 (Figure 13). Consequently, it seems that choosing $S_{g}=62.5$ for this firing configuration would be an interesting choice regarding the error's criteria.

As a partial conclusion, we proposed a method based on the error propagation analysis to choose an optimal geometric swirl number choice regarding the defined criteria. For a given engine configuration, an optimal $S_{g}$ was found by minimizing the integrated total error over the firing duration.

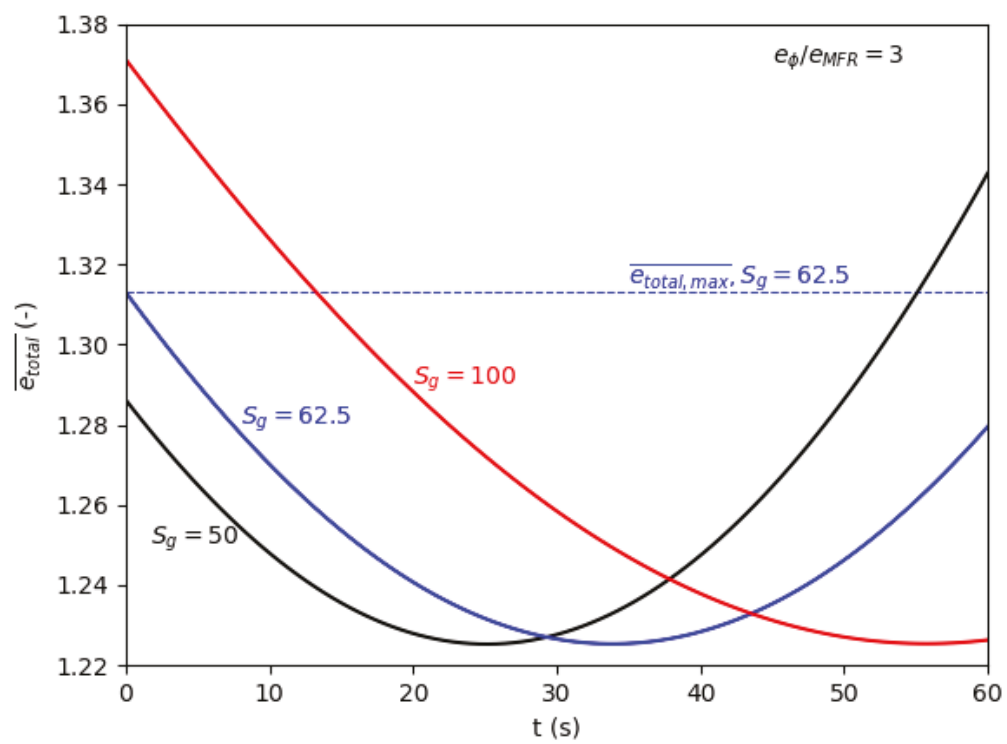

Figure 13. Instantaneous total error evolution.

\subsubsection{Optimal Design of the Resistor-Based Regression Rate Sensors}

In the previous sections, the ratio $e_{\phi} / e_{M F R}$ was arbitrarily fixed to study different cases. In practice, this parameter depends on the precision of the fuel regression and oxidizer mass flow rates measurement sensors. In this study, we consider using RBS to control an A-SOFT through a feedback loop system (Figures 2 and 4). Such sensors have various possible designs and one of the most important parameter is the radial distance between two resistors. Each time the fuel grain surface reaches a resistor, this resistor is burnt and the electric voltage provided by the sensor is modified. As a consequence, the fuel grain diameter measured by the sensor is a piece-wise constant function. In this discussion, we will consider that the sensors are ideal: the resistors are burnt as soon as the fuel surface reaches them. Moreover, we assume the resistors are axially aligned, and the initial distance grain surface-first resistor is equal to the distance between two resistors:

- $d s_{x}=0$,

- $s_{0}=d s$.

In this section, the error propagation analysis will be used to determine the design of RBS to satisfy given conditions. As a starting point, the error due to the oxidizer mass flow measurement must be assumed. Depending on the type of measurement system and sensor, this error generally ranges from $0.2 \%$ to $5 \%$. As an intermediate possibility, we will consider an error of $2 \%$. The next 
errors to determine are the maximum acceptable errors regarding the thrust and the mixture ratio. In this example, we will consider that these errors are equal to $2 \%$ as well:

- $\quad e_{M F R}=0.02$,

- $e_{F, \max }=0.02$,

- $e_{\xi, \max }=0.02$.

These values lead to the calculation of the maximum tolerable normalized errors: $\overline{e_{F, \max }}=1$, $\overline{e_{\xi, \max }}=1$ and: $\overline{e_{\text {total,max }}}=\sqrt{2}$. Based on the previous relations (Equations (20)-(22)), we can define a maximum normalized error on the diameter by Equation (74):

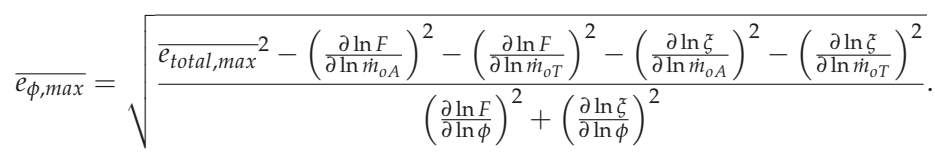

According to this relation, there may be no solution if the maximum total error $\overline{e_{\text {total, } \max }}$ is too small since it would conduct to a negative term in the square root. Figure 14 presents the results for several $S_{g}$ values and for the optimal mixture ratio case.

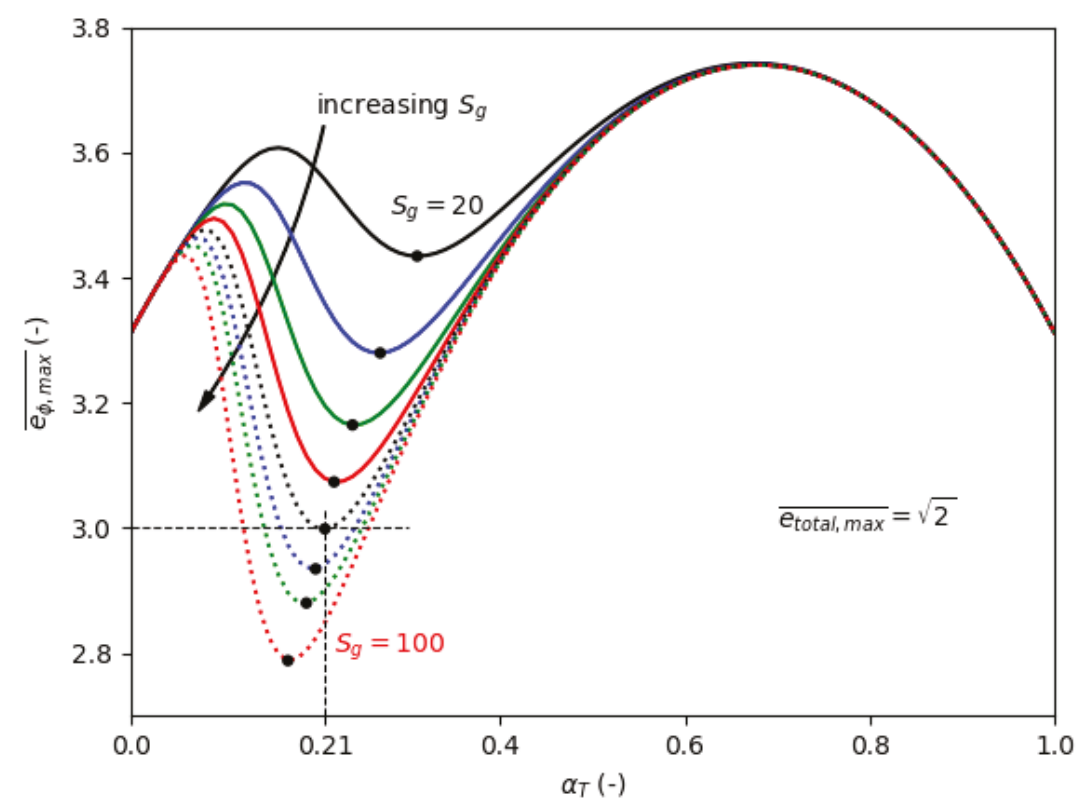

Figure 14. Maximum normalized diameter error for $S_{g}$ values in $[20,30,40,50,60,70,80,100]$.

As it can be seen, there is a local minimum if $\alpha_{T}$ varies around 0.2 or 0.3 depending on the geometric swirl number. The value of $\overline{e_{\phi, \max }}$ tends to be reduced while the geometric swirl number increases. In other words, the higher $S_{g}$ is, the more the constraint on the fuel regression rate sensors is severe and the more the relative error needs to be reduced. As an example, let us consider a geometric swirl number of 60: the maximum normalized error on the diameter would be around 3 if the engine operates at $\alpha_{T}$ close to 0.21 according to the previous result. In the case of ideal RBS, the maximum error on the diameter could be evaluated by Equation (75):

$$
e_{\phi}=\frac{\delta \phi}{\phi}=\frac{2 d s}{\phi} .
$$


Since the fuel grain diameter will naturally increase during the combustion, the diameter relative error will decrease with time. Consequently, to estimate the required $d s$ parameter, the initial fuel grain diameter should be chosen to calculate the maximum diameter relative error possible. In the current case, if we choose an initial fuel port diameter $\phi_{i n i}=40 \mathrm{~mm}$, this would lead to the following results:

- $\overline{e_{\phi, \max }}=3.0$,

- $e_{\phi, \max }=\overline{e_{\phi, \max }} \cdot e_{M F R}=0.06$,

- $d s_{\max }=\frac{1}{2} e_{\phi, \max } \cdot \phi_{\text {ini }}=1.2 \mathrm{~mm}$.

In this configuration and by using the propagation error analysis, we could conclude that using a RBS radial distance lower than $1.2 \mathrm{~mm}$ between resistors would limit the thrust and mixture ratio relative errors to less than $2 \%$. Naturally, increasing the tolerance regarding these errors would lead to increase the $d s$ parameter, which may be required by practical manufacturing issues or other external factors.

\subsubsection{Non-Optimal Mixture Ratio}

A-SOFT hybrid engines ideally operate close to the optimal mixture ratio to reach the best performance possible. However, while operating, the $\mathrm{O} / \mathrm{F}$ ratio can become higher or smaller than the optimal value due to the feedback loop control efficiency/latency or due to other limiting factors like a long burning duration resulting in a too large fuel grain diameter. Additional analyses have been carried out for two other operating conditions, one dealing with an oxidizer-rich combustion and one dealing with a fuel-rich configuration. The results regarding the total error and the geometric swirl number influence are very similar to the optimal mixture ratio case and are reported in Appendix B. These results reveal that operating at non-optimal mixture ratio does not change the tendencies and conclusions which were given in the optimal case. Consequently, the $\mathrm{O} / \mathrm{F}$ ratio variations from optimal to non-optimal values and vice versa under feedback control will have no significant impact on the errors' propagation.

\subsection{Numerical Simulations}

\subsubsection{Study Case}

Several numerical simulations have been performed and the results are given in this section. Table 4 provides the parameters that have been used and are common to all the simulations. Numerical parameters can be seen in Table 2. Thermochemical data were obtained from RPA for an engine operating with gaseous oxygen as oxidizer and high density polyethylene as fuel.

Table 4. Numerical simulations' parameters.

\begin{tabular}{|c|c|c|}
\hline$L(\mathrm{~m})$ & $\phi_{0}(\mathrm{~m})$ & $\phi_{\text {out }}(\mathrm{m})$ \\
\hline 0.330 & 0.040 & 0.100 \\
\hline$t_{f}(\mathrm{~s})$ & $p_{a}(\mathrm{~Pa})$ & $g_{0}\left(\mathrm{~m} \cdot \mathrm{s}^{-2}\right)$ \\
\hline 60.0 & $0.1 \times 10^{6}$ & 9.81 \\
\hline$\phi_{t}(\mathrm{~m})$ & $\Sigma(-)$ & $S_{g}(-)$ \\
\hline 0.010 & 3.0 & 50.0 \\
\hline$\rho_{f}\left(\mathrm{~kg} \cdot \mathrm{m}^{-3}\right)$ & $\eta_{c}(-)$ & $a\left(\mathrm{~m} \cdot \mathrm{s}^{-1}\right)$ \\
\hline 905.0 & 0.9 & $2.0 \times 10^{-5}$ \\
\hline$m(-)$ & $n(-)$ & $b(-)$ \\
\hline 0.1392 & 0.64 & -0.10 \\
\hline$v o_{A, 0}(-)$ & $v o_{T, 0}(-)$ & $\xi_{\text {command }}(-)$ \\
\hline 0.30 & 0.30 & 2.3 \\
\hline$\dot{m}_{o A, 0}\left(\mathrm{~kg} \cdot \mathrm{s}^{-1}\right)$ & $\dot{m}_{o T, 0}\left(\mathrm{~kg} \cdot \mathrm{s}^{-1}\right)$ & $\alpha_{T, 0}(-)$ \\
\hline 0.049 & 0.049 & 0.5 \\
\hline
\end{tabular}


In all the simulations, the servo valves are open at 30\% at the initial instant. Figures 15 and 16 respectively show the natural evolution of the thrust and the mixture ratio of the engine without any type of feedback control and illustrate the performances' shift that was mentioned earlier in the paper. In this configuration, the engine was not designed to operate around optimal conditions regarding the thrust and the mixture ratio.

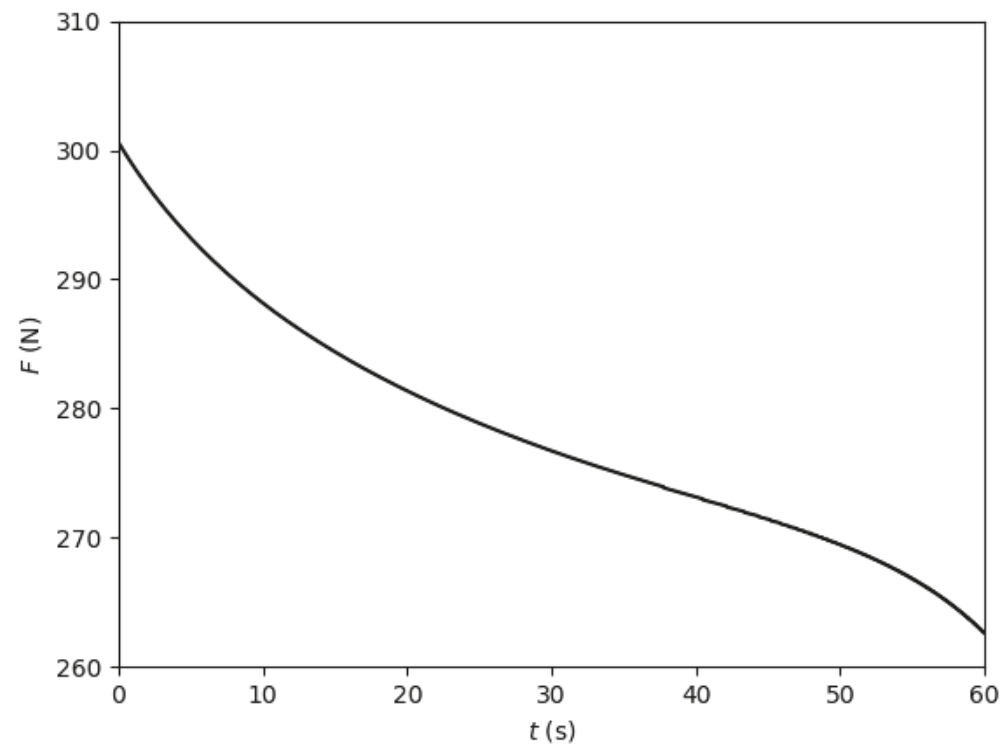

Figure 15. Thrust profile without feedback control.

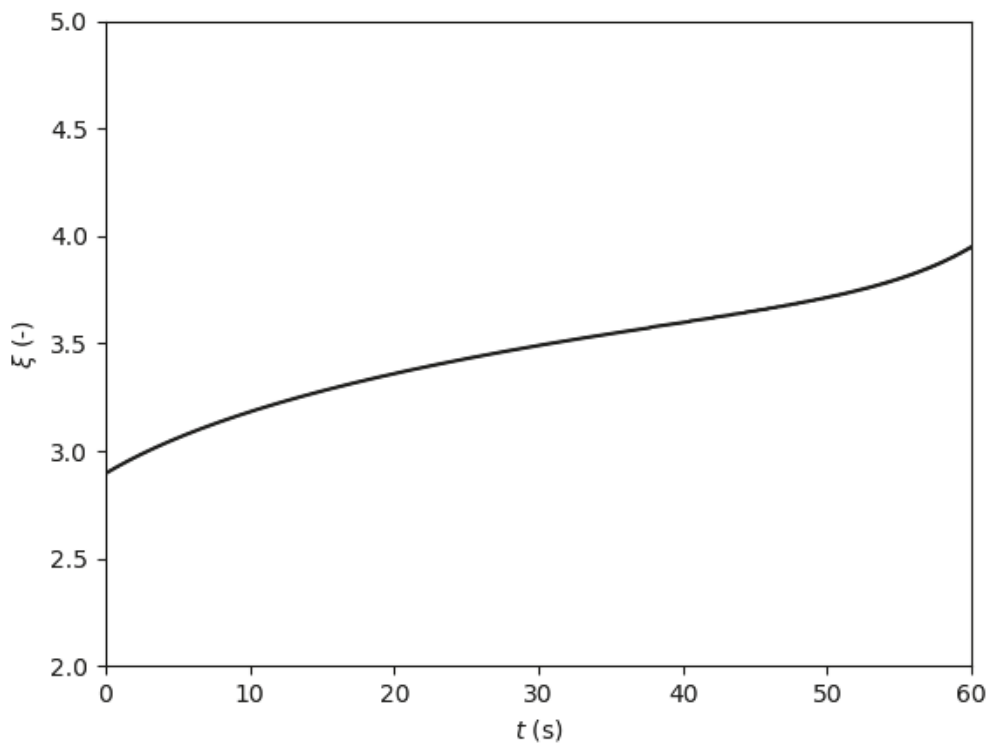

Figure 16. Mixture ratio profile without feedback control. 


\subsubsection{Feedback Control Law Efficiency}

In the following discussion, three thrust profiles have been considered: constant, piece-wise constant, and linearly decreasing thrust. In order to operate at the optimal mixture ratio, the $\mathrm{O} / \mathrm{F}$ command has been fixed constant for all cases and equal to the optimal value. In order to evaluate the efficiency of the regulation law that was developed in the previous section (complete regulation law), a second regulation law based on a very simple proportional rule has been tested (downgraded regulation law). The following list sums up the performed simulations:

- case 1: constant thrust,

- case 2: piece-wise constant thrust,

- case 3: linearly decreasing thrust,

- regulation law: complete or downgraded.

The second regulation law that has been tested simply considers that the thrust is proportional to the oxidizer mass flow rate (downgraded regulation law). Rather than using Equations (66) and (67), the target oxidizer mass flow rate is calculated based on Equation (76) and the effective swirl number is then deduced to fit to the targeted mixture ratio:

$$
\dot{m}_{o, \text { target }}[t+d t]=\dot{m}_{o}[t] \frac{F_{\text {command }}[t+d t]}{F_{m}[t]} .
$$

The resistor-based sensors parameters are given in Table 5 . The sensors are distributed uniformly along the fuel grain.

Table 5. RBS (Resistor-Based Sensors) parameters for the simulations.

\begin{tabular}{cccc}
\hline$s_{0}(\mathrm{~m})$ & $d s(\mathrm{~m})$ & $N_{r}(-)$ & $N_{s}(-)$ \\
\hline 0.001 & 0.001 & 30 & 3 \\
\hline
\end{tabular}

Regarding the PID controller, a proportional controller has been evaluated in this study and was sufficient to obtain interesting results. The PID parameters were obtained experimentally based on the simulations' results and are given in Table 6. This set of parameters simplifies the PID control law (Equation (69)), which is consequently written by Equation (77).

Table 6. PID (Proportional Integral Derivative) parameters for the simulations.

$$
\begin{array}{ccc}
\hline k_{P}(-) & k_{I}(-) & k_{D}(-) \\
\hline 0.45 & 0.0 & 0.0 \\
\hline \delta v o_{A-T}[t+d t]= & k_{P} e_{\text {pid }}[t] .
\end{array}
$$

Figures 17-20 respectively present the thrust, the mixture ratio, the effective swirl number, and the total oxidizer mass flow rate results of the simulation case 1 with the complete regulation law. As it can be seen, the initial thrust is higher than the thrust command. The initial value of the valve opening was voluntarily fixed arbitrarily to evaluate the capacity of the feedback law to rapidly match the command. The instantaneous values of the valves opening are calculated autonomously by the algorithm after the first iteration and according to the regulation law. The thrust reaches the command after $5 \mathrm{~s}$ of combustion and the feedback control is able to maintain the thrust level at $260 \mathrm{~N} \pm 1 \mathrm{~N}$ until $55 \mathrm{~s}$. After $55 \mathrm{~s}$, it can be seen that the thrust starts to decrease, which will be explained by other results given later in the paper.

The estimation of the fuel mass flow and regression rates can only be done once the first resistor of the RBS has been reached by the fuel surface. As a consequence, the feedback loop control starts 
when the RBS provide the required measurements, which can take several seconds depending on the sensors' parameters and the fuel regression rate. With this set of RBS parameters and these test conditions, this delay is around $1.2 \mathrm{~s}$. The initial mixture ratio is equal to 2.9 and rapidly changes to 2.4 after $3.5 \mathrm{~s}$ of combustion. After $4.5 \mathrm{~s}$, the $\mathrm{O} / \mathrm{F}$ ratio reaches 2.35 and is maintained between this value and the target 2.3 until $50 \mathrm{~s}$. Similarly to the thrust, the engine cannot reach the target at the end of the test. Once the RBS are providing fuel regression rate measurements, the difference between the real mixture ratio and the measured one is kept lower than 0.04 which corresponds to $1.74 \%$ of the command value until $50 \mathrm{~s}$. At this time, a significant part of the fuel grain has burnt and the sensors no longer provide measurements, which leads to an increasing error on the mixture ratio evaluation. Finally, we can notice that the measured $\mathrm{O} / \mathrm{F}$ ratio is always slightly higher than the command and varies from 2.3 to 2.34 , which leads to an oxidizer-rich combustion by the feedback control.

After a rapid regulation at the beginning of the test, the effective swirl number increases linearly until reaching 50. In this configuration, the geometric swirl number was fixed at 50, which means that $S_{e}$ cannot be higher than this value. Around $55 \mathrm{~s}$, the swirl number is limited to 50 and the feedback law is no longer able to follow the thrust and the $\mathrm{O} / \mathrm{F}$ ratio commands due to the operating conditions and the fuel grain combustion after more than $50 \mathrm{~s}$. It explains why the target values are not reached at the end of the test. The oxidizer mass flow rate is rapidly reduced after the ignition because the initial valve opening value was providing a higher thrust than the target. After this phase, the oxidizer mass flow rate is maintained approximately constant until $55 \mathrm{~s}$, when the conditions no longer allow to follow the commands.

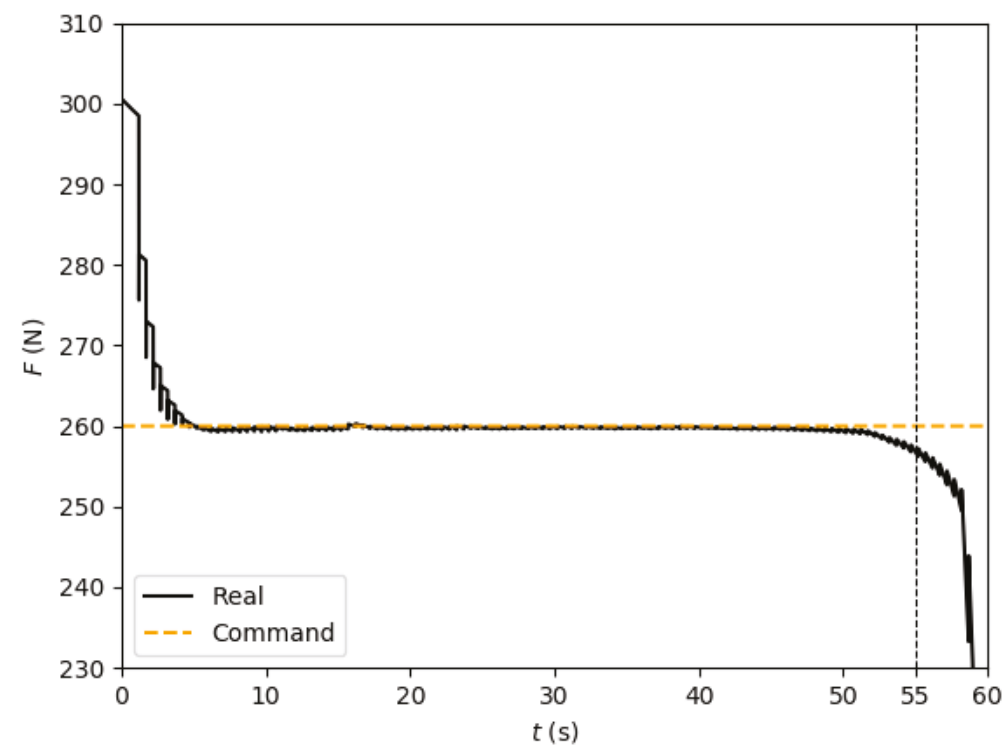

Figure 17. Thrust profile, feedback with complete regulation law, case 1. 


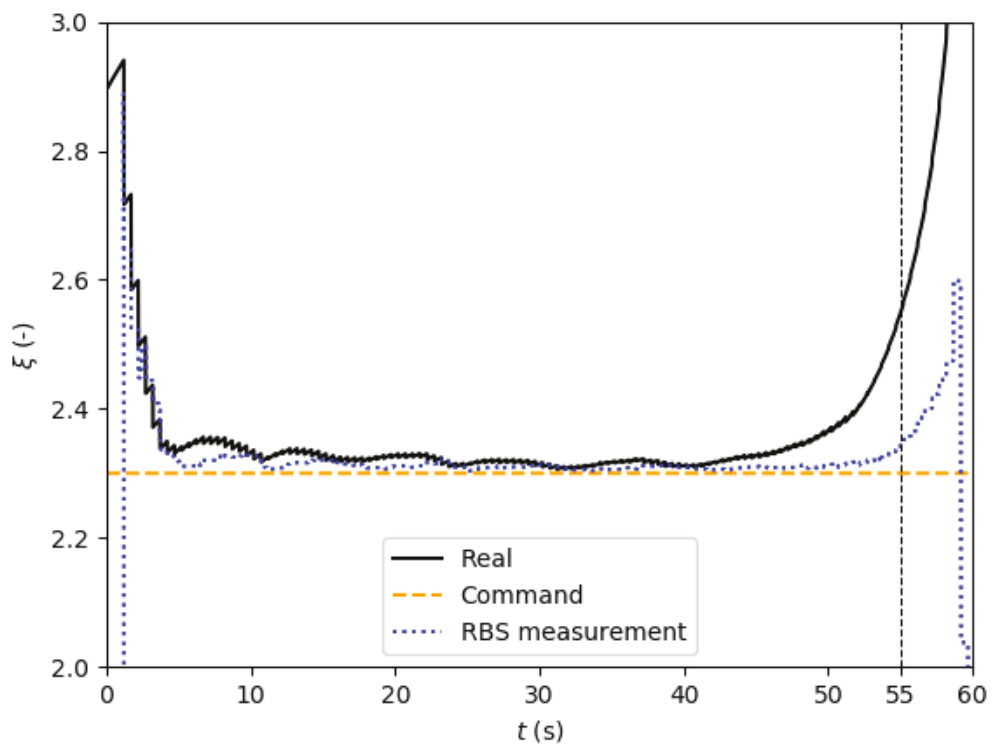

Figure 18. Mixture ratio profile, feedback with complete regulation law, case 1.

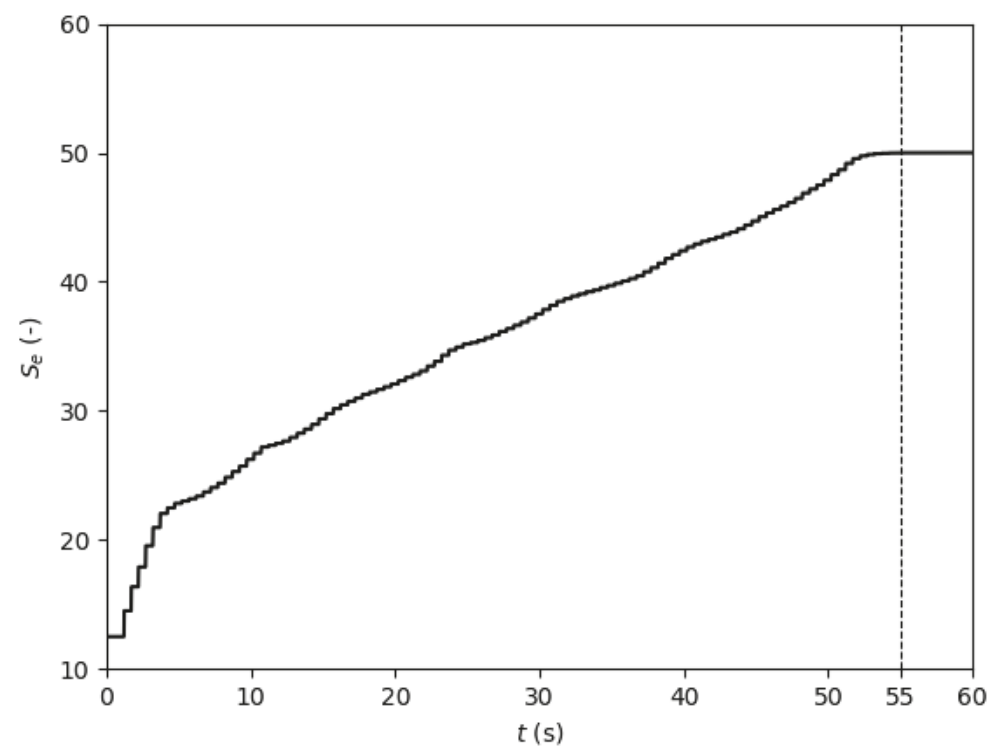

Figure 19. Effective swirl number profile, feedback with complete regulation law, case 1. 


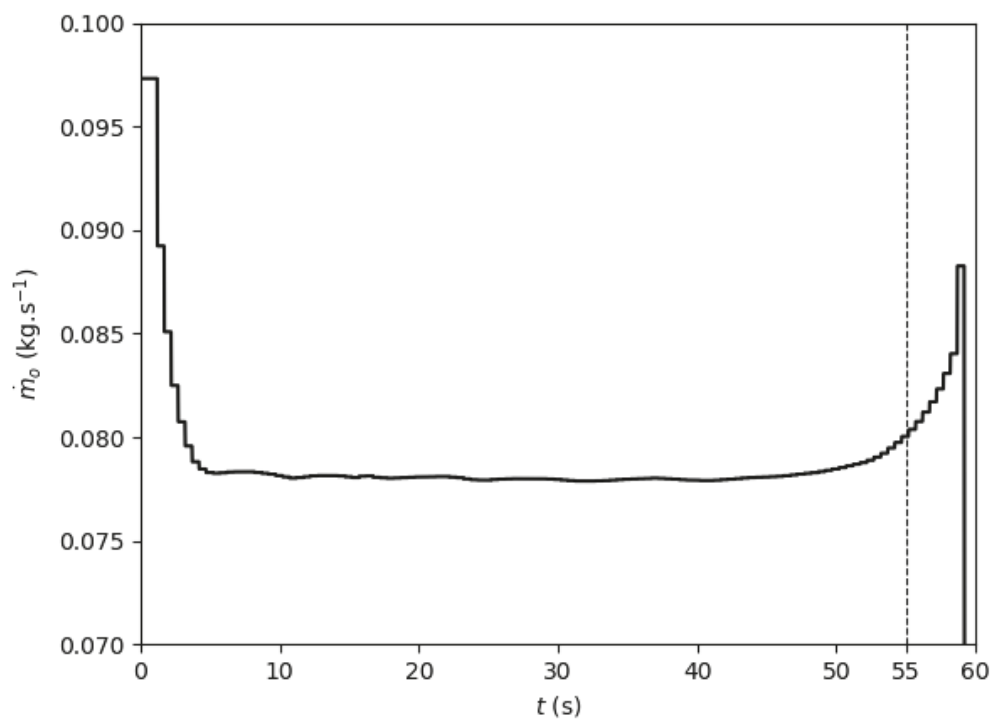

Figure 20. Oxidizer mass flow rate profile, feedback with complete regulation law, case 1.

Figures 21-24 present the results of the simulation case 2 with the complete regulation law. The main conclusions are similar to the previous case and the engine is able to follow the thrust and the $\mathrm{O} / \mathrm{F}$ ratio commands. The simulations configuration are identical between case 1 and case 2 until $30 \mathrm{~s}$ and the thrust and the $\mathrm{O} / \mathrm{F}$ ratio evolution are the same. At $30 \mathrm{~s}$, the thrust command suddenly increases from 260 to $280 \mathrm{~N}$ and the engine's thrust reaches the new target in approximately $2 \mathrm{~s}$. From $32 \mathrm{~s}$ to $48.5 \mathrm{~s}$, the thrust level varies around $280 \mathrm{~N} \pm 1 \mathrm{~N}$. After $48.5 \mathrm{~s}$, the thrust starts to decrease as it is the case in the previous simulation but earlier than it (it was after $55 \mathrm{~s}$ ).

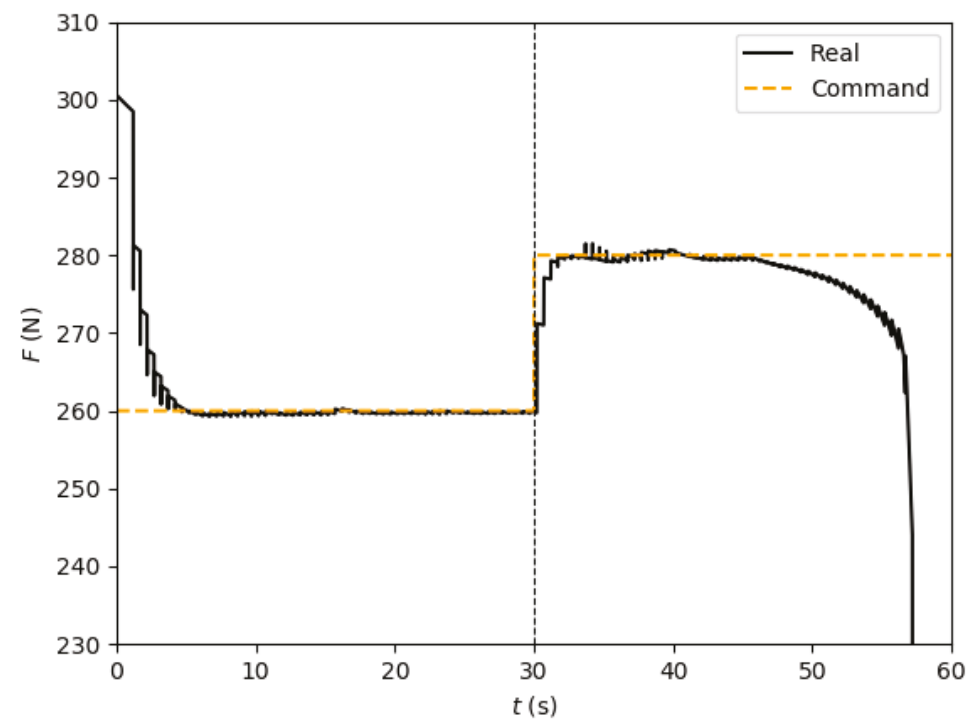

Figure 21. Thrust profile, feedback with complete regulation law, case 2. 


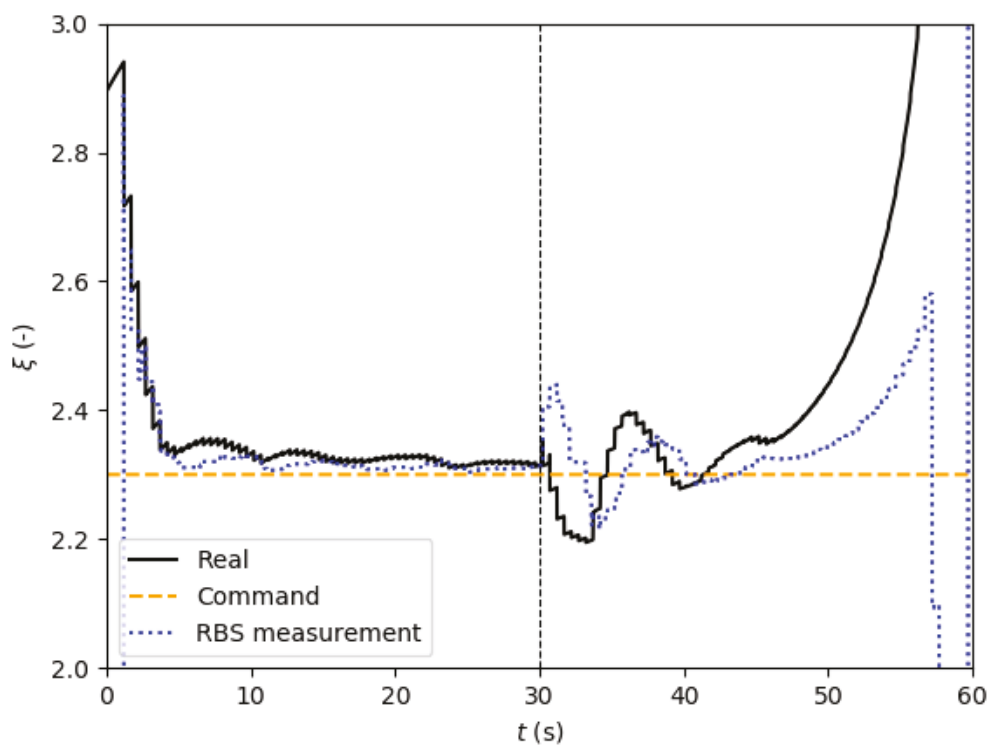

Figure 22. Mixture ratio profile, feedback with complete regulation law, case 2.

We can notice small oscillations of the $\mathrm{O} / \mathrm{F}$ ratio after the change of the thrust level at $30 \mathrm{~s}$, the regulation requiring some time to fit the new conditions. The mixture ratio slightly oscillates around $2.3 \pm 0.1$ until $50 \mathrm{~s}$ when it starts to increase significantly. Because the second level of thrust target is higher than the first target, the total oxidizer mass flow rate needs to be increased and the fuel grain is burnt quicker than in case 1 . As a consequence, the thrust and the $\mathrm{O} / \mathrm{F}$ ratio start to move away from the targeted values earlier than in the previous case.

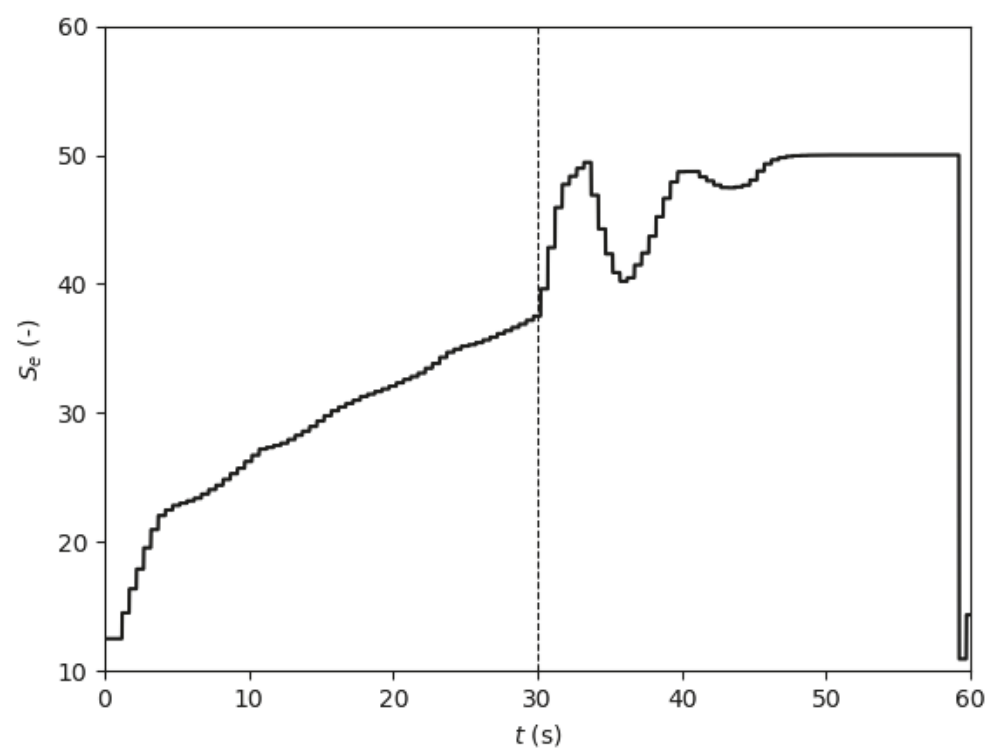

Figure 23. Effective swirl number profile, feedback with complete regulation law, case 2. 


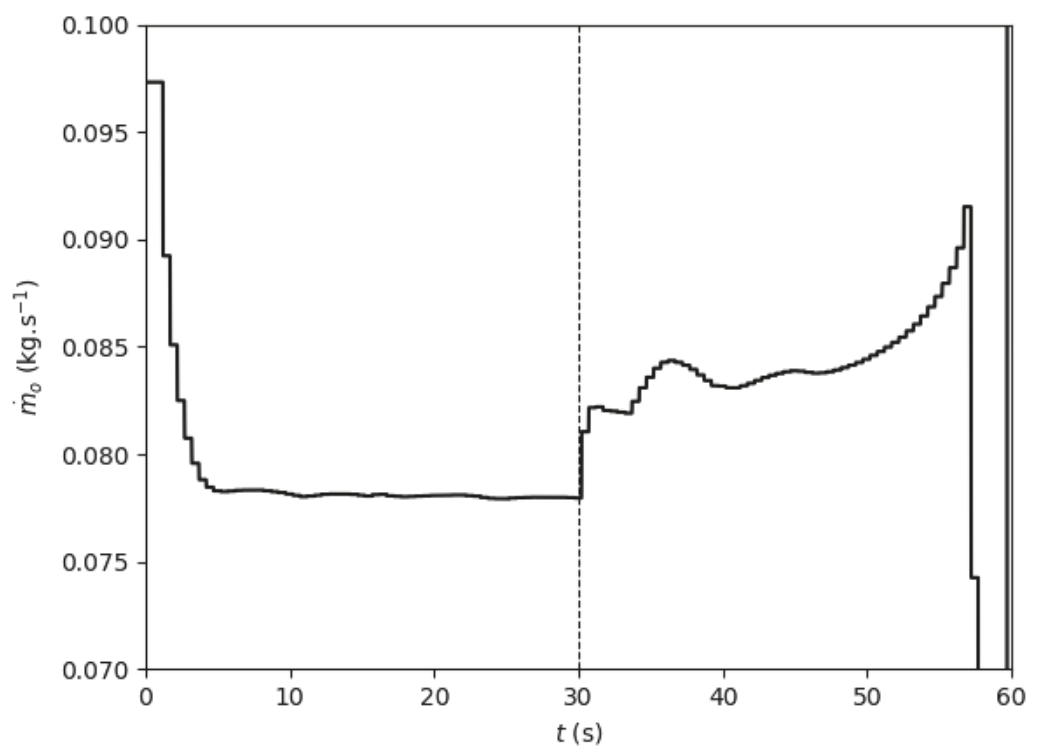

Figure 24. Oxidizer mass flow rate profile, feedback with complete regulation law, case 2.

Figures 25-28 present the results of the simulation case 2 with the downgraded regulation law. The results are similar than the previous ones, even if the regulation law is strongly downgraded in terms of precision. However, we can observe more oscillations regarding the thrust and the mixture ratio. The thrust reaches the command after $5 \mathrm{~s}$ of combustion and the feedback control maintains the thrust level at $260 \mathrm{~N} \pm 4 \mathrm{~N}$ until $30 \mathrm{~s}$ with small oscillations. At $30 \mathrm{~s}$, the engine's thrust reaches the new target in approximately $2 \mathrm{~s}$. From $32 \mathrm{~s}$ to $50 \mathrm{~s}$, the thrust level varies around $280 \mathrm{~N} \pm 5 \mathrm{~N}$. The thrust starts to decrease after $50 \mathrm{~s}$.

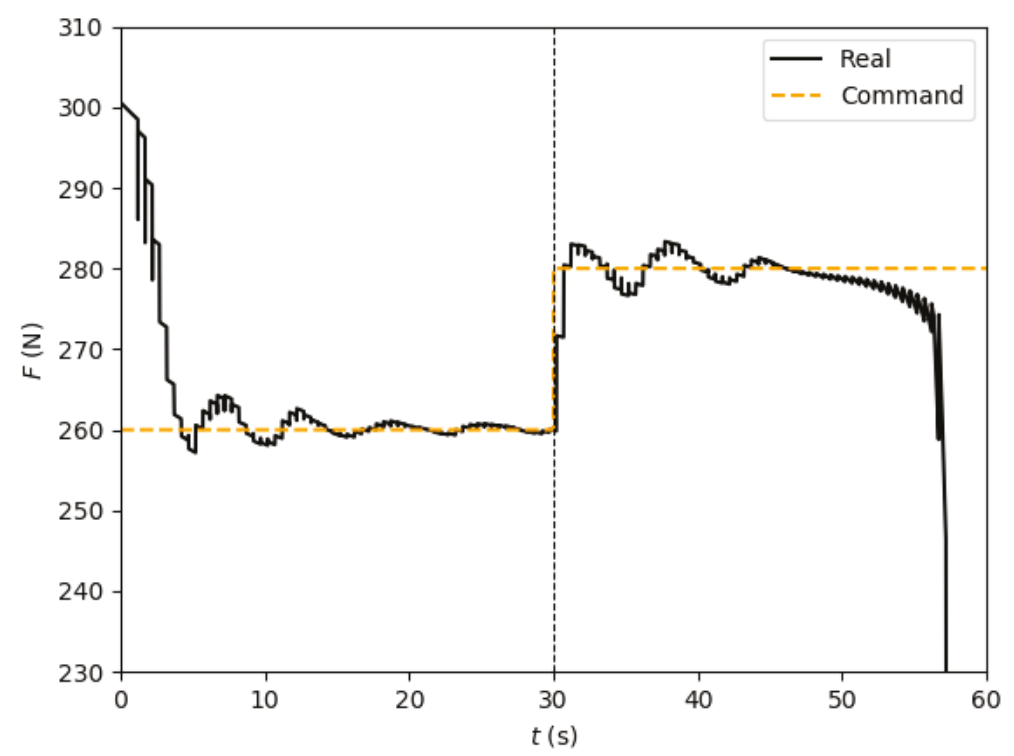

Figure 25. Thrust profile, feedback with downgraded regulation law, case 2 . 


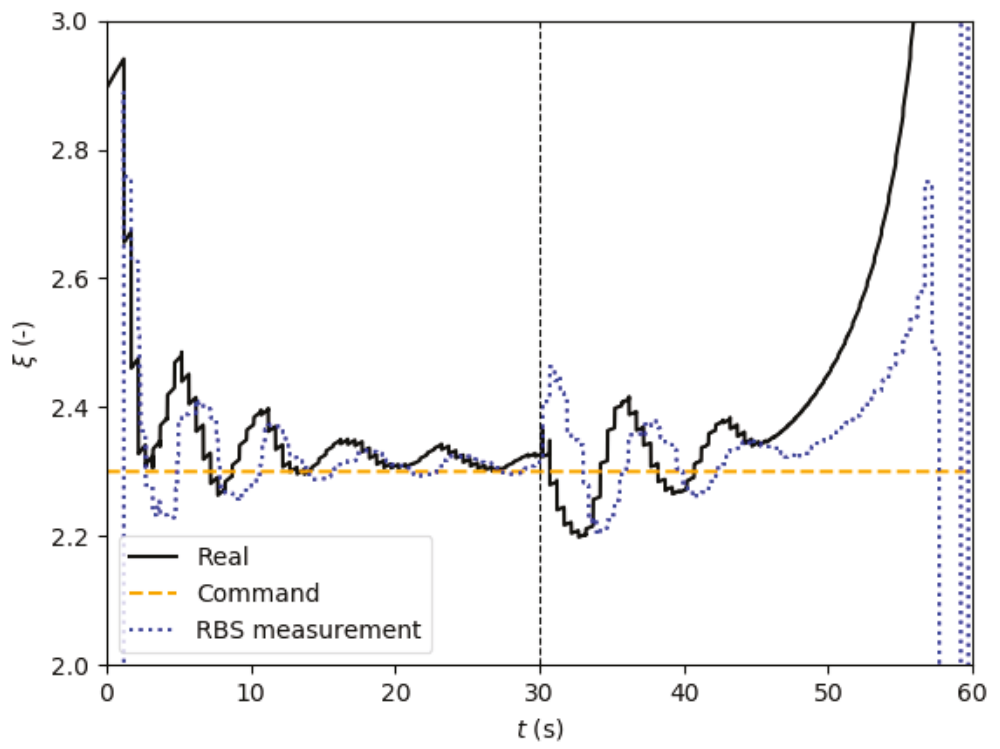

Figure 26. Mixture ratio profile, feedback with downgraded regulation law, case 2.

The initial mixture ratio is equal to 2.9 and rapidly changes to 2.4 after $3.5 \mathrm{~s}$ of combustion. After that time, the $\mathrm{O} / \mathrm{F}$ ratio oscillates between 2.27 and 2.48 until $30 \mathrm{~s}$, the amplitude of the oscillations decreasing with time. After $30 \mathrm{~s}$, the oscillations' amplitude increases again due to the change of the thrust command, and the mixture ratio varies between 2.18 and 2.44 until $50 \mathrm{~s}$. Results regarding cases 1 and 3 for the downgraded regulation law are given in Appendix $C$.

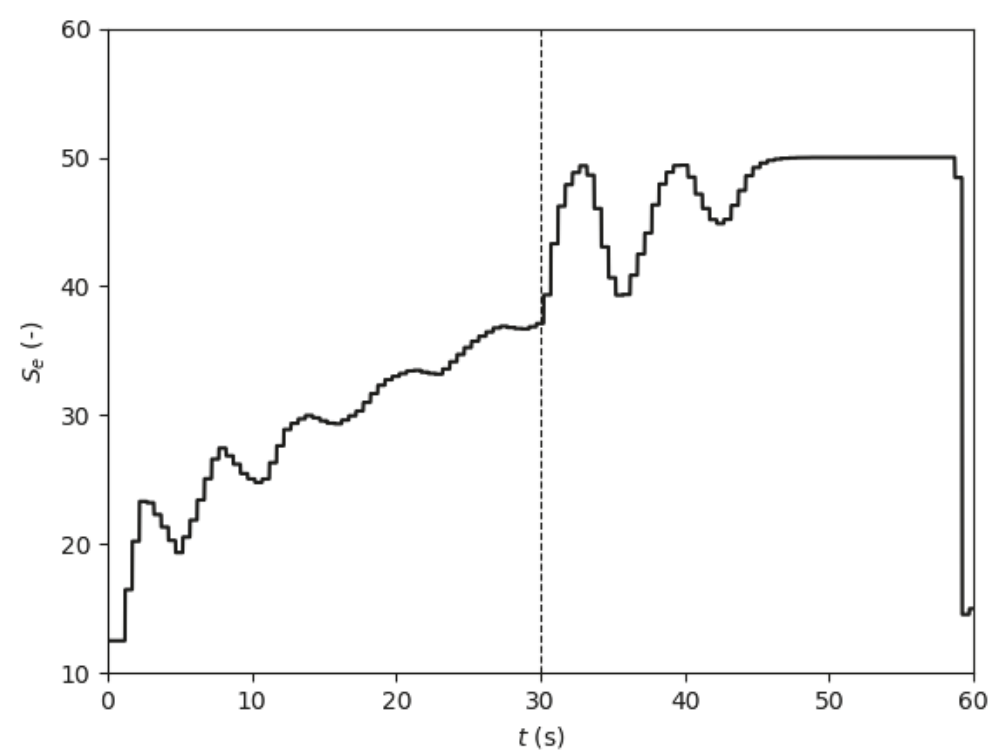

Figure 27. Effective swirl number profile, feedback with downgraded regulation law, case 2. 


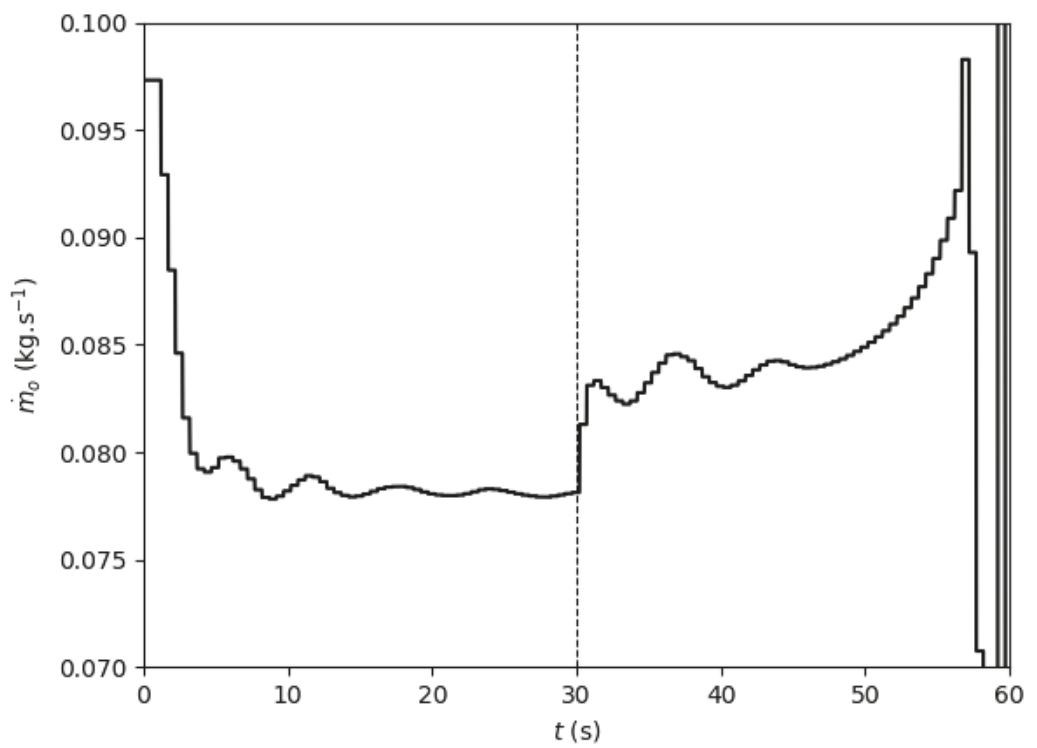

Figure 28. Oxidizer mass flow rate profile, feedback with downgraded regulation law, case 2.

Figures 29-32 present the results of the simulation case 3 with the complete regulation law. The thrust reaches the command after $5 \mathrm{~s}$ and is maintained close to the target with a difference lower than $1 \mathrm{~N}$ during all the test. The mixture ratio varies between 2.3 and 2.36 from $5 \mathrm{~s}$ to $50 \mathrm{~s}$.

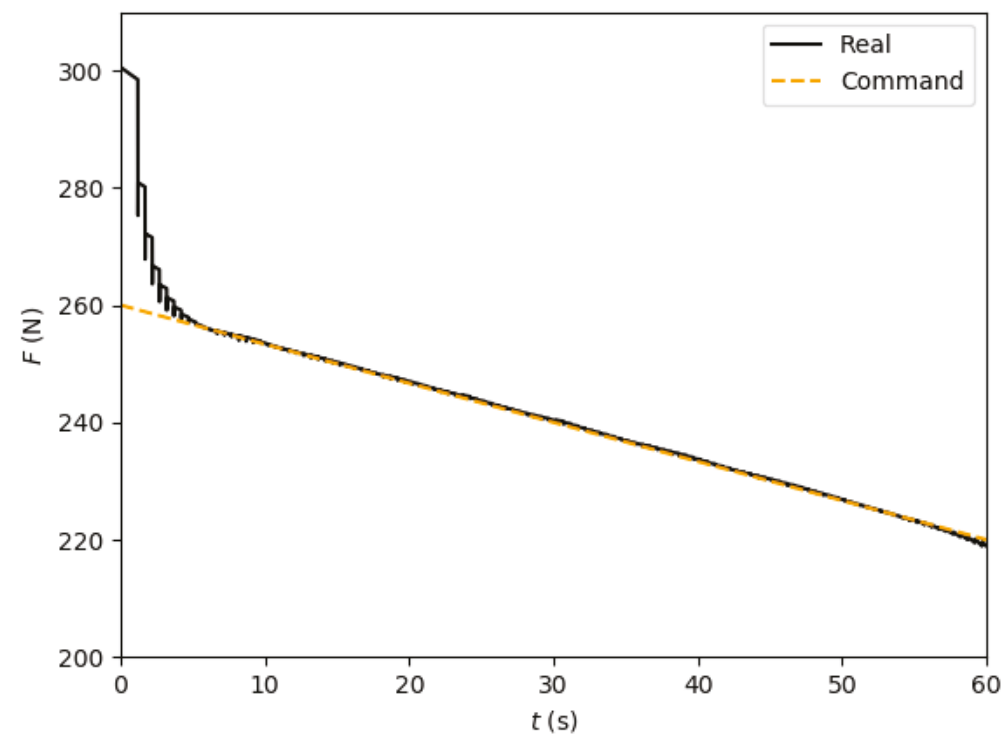

Figure 29. Thrust profile, feedback with complete regulation law, case 3. 


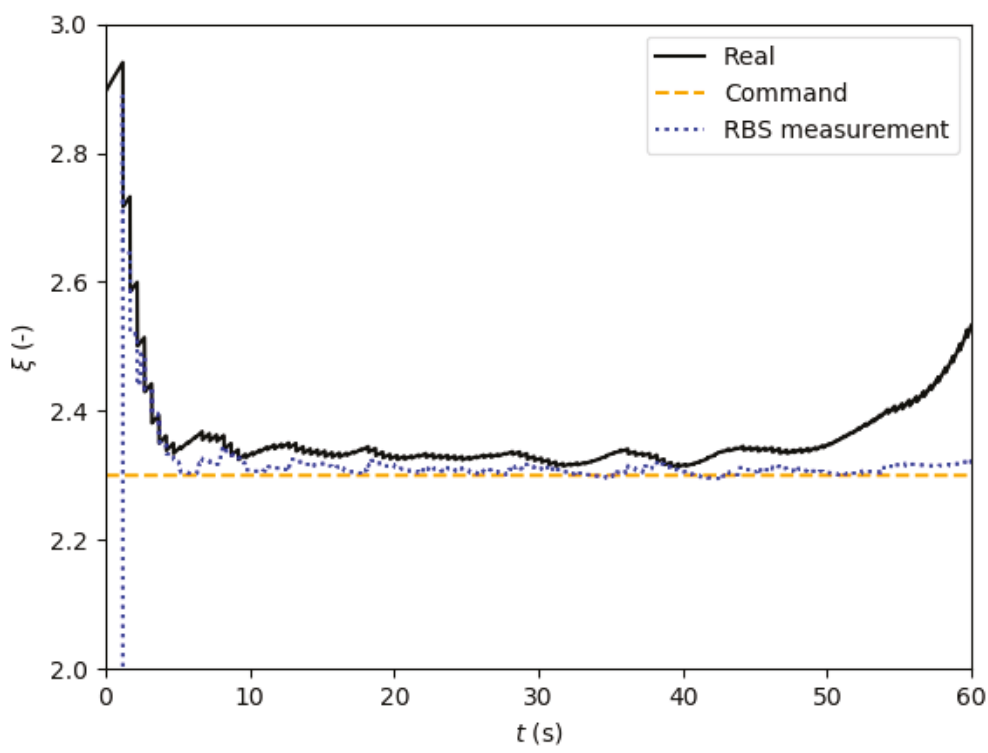

Figure 30. Mixture ratio profile, feedback with complete regulation law, case 3.

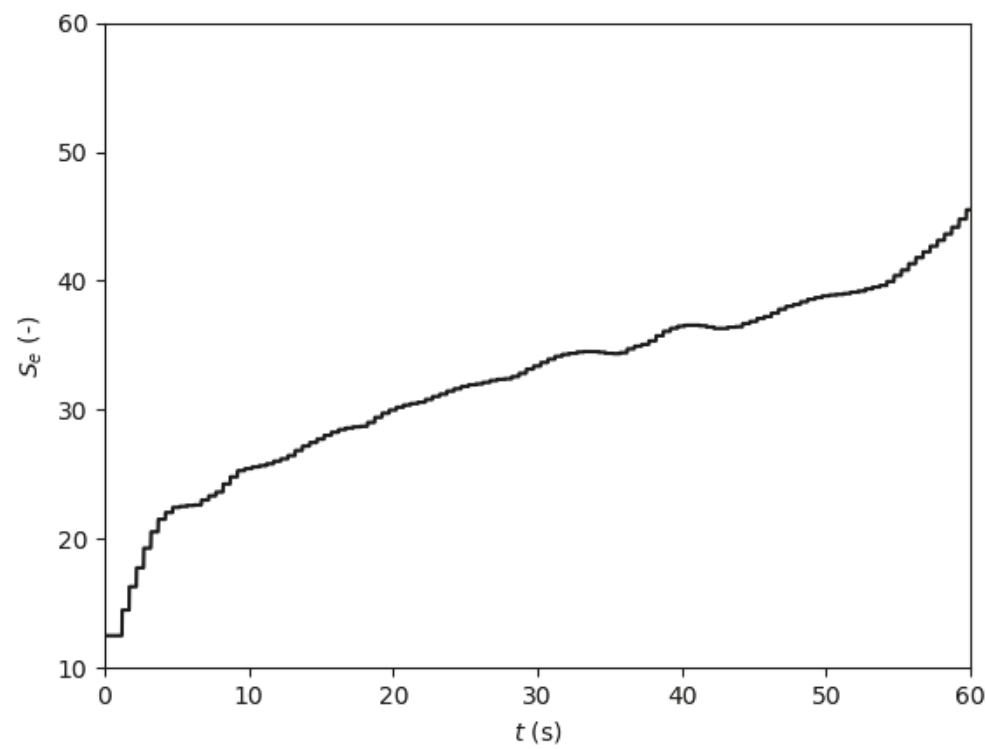

Figure 31. Effective swirl number profile, feedback with complete regulation law, case 3. 


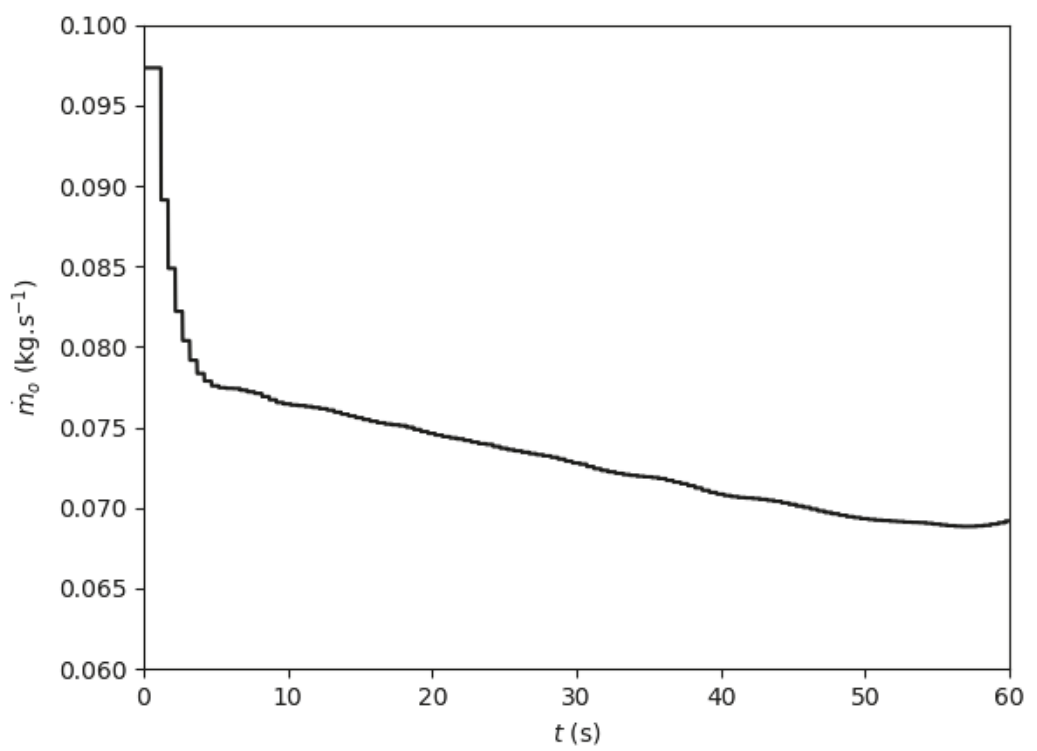

Figure 32. Oxidizer mass flow rate profile, feedback with complete regulation law, case 3.

\subsubsection{Resistor-Based Sensors' Influence}

Resistor-based sensors play a major role in the engine feedback control, Figure 33 provides the sensors' signals in case 2 with the complete regulation law. The signals are piece-wise functions and the regression and fuel mass flow rates are re-evaluated every time the signals are modified.

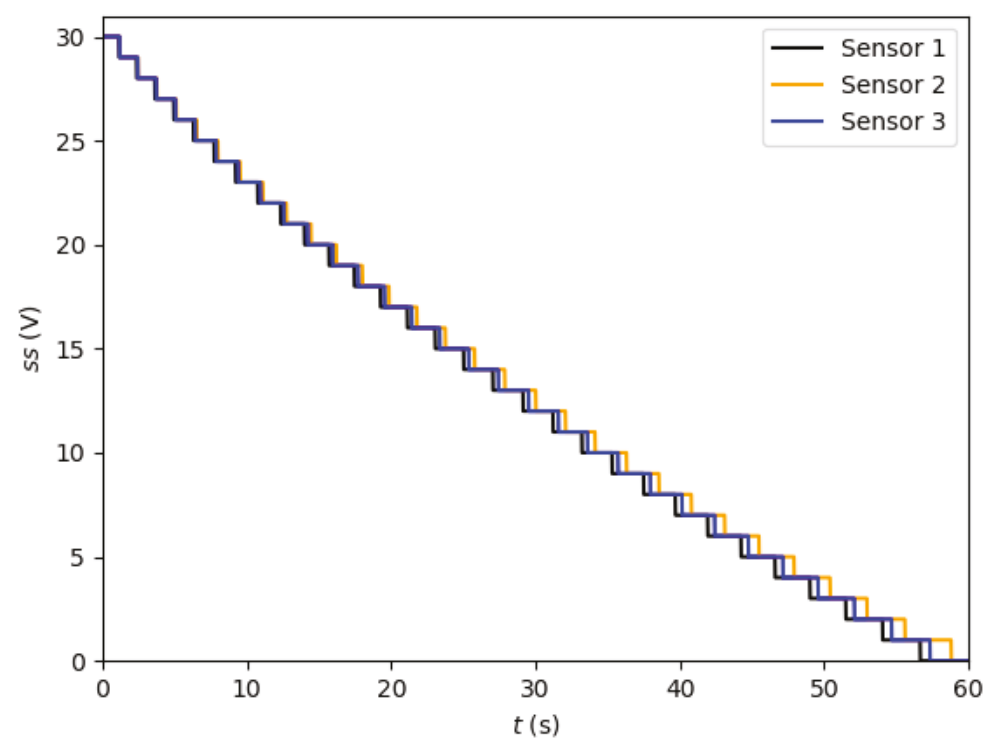

Figure 33. RBS signals, feedback with complete regulation law, case 2. 
The previous simulations were performed with sensors equipped with a large number of resistors $\left(N_{r}=30\right)$, which may be difficult to manufacture. In order to study another configuration, this number was changed to 10 and the distance between resistors was increased from $1 \mathrm{~mm}$ to $3 \mathrm{~mm}$ (Table 7); the number of sensors was not changed.

Table 7. New RBS parameters for the simulations.

\begin{tabular}{cccc}
\hline$s_{0}(\mathrm{~m})$ & $d s(\mathrm{~m})$ & $N_{r}(-)$ & $N_{s}(-)$ \\
\hline 0.003 & 0.003 & 10 & 3 \\
\hline
\end{tabular}

Figures 34-36 present the results of the simulation case 2 with the complete regulation law and the new RBS parameters.

The thrust reaches the command after $7.5 \mathrm{~s}$ and then slightly oscillates around $260 \mathrm{~N} \pm 2 \mathrm{~N}$ until $30 \mathrm{~s}$. At that time, the target level is changed and $2 \mathrm{~s}$ are needed to fit to the new command value. From $32 \mathrm{~s}$ to $48 \mathrm{~s}$, the thrust oscillates around $280 \mathrm{~N} \pm 3 \mathrm{~N}$ and then starts to decrease at the end of the simulation. The initial delay required to reach the first resistor of the sensors and to start the feedback control was changed from $1.2 \mathrm{~s}$ to $3.8 \mathrm{~s}$ with the new set of RBS parameters. This delay is multiplied by a factor close to 3 which approximately corresponds to the modification from $1 \mathrm{~mm}$ to $3 \mathrm{~mm}$ of the initial distance between the first resistor and the fuel grain surface. The mixture ratio is never completely stabilized during the test and varies within 2.1 and 2.6 after $5 \mathrm{~s}$ of combustion. The maximum error between the real $\mathrm{O} / \mathrm{F}$ ratio and the measured value is close to 0.1 corresponding to $4.3 \%$ error before $30 \mathrm{~s}$, but can reach up to 0.3 after $30 \mathrm{~s}$, which corresponds to $13 \%$ of the target value. The error is multiplied by a factor 3 compared to the previous simulation which corresponds to the change of the distance between resistors. Although the thrust remains relatively close to the target, it is clear that the new set of sensors' parameters generated more oscillations and that the engine is more difficult to control. It is particularly true regarding the mixture ratio which directly depends on the RBS measurements.

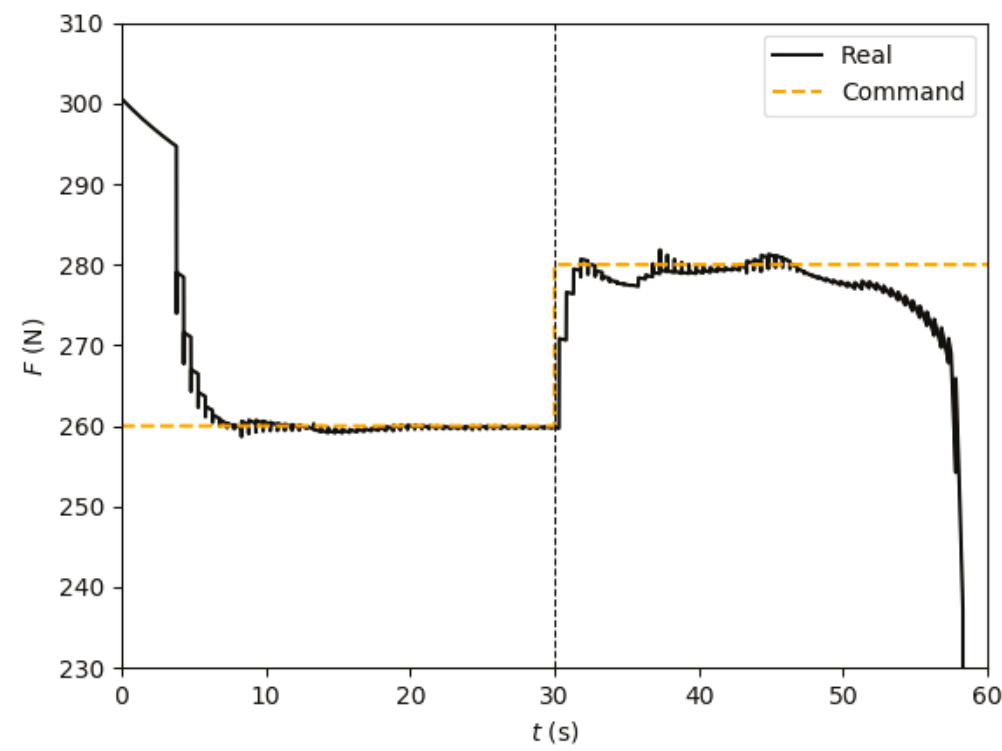

Figure 34. Thrust profile, feedback with complete regulation law, case $2, d s=3 \mathrm{~mm}$. 


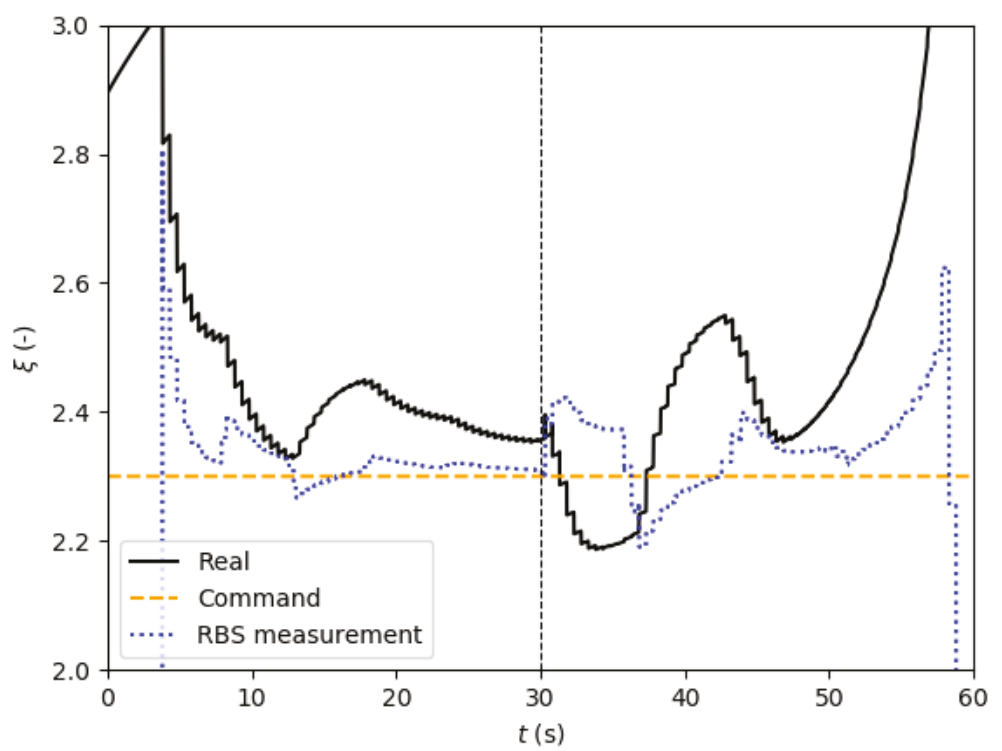

Figure 35. Mixture ratio profile, feedback with complete regulation law, case 2, $d s=3 \mathrm{~mm}$.

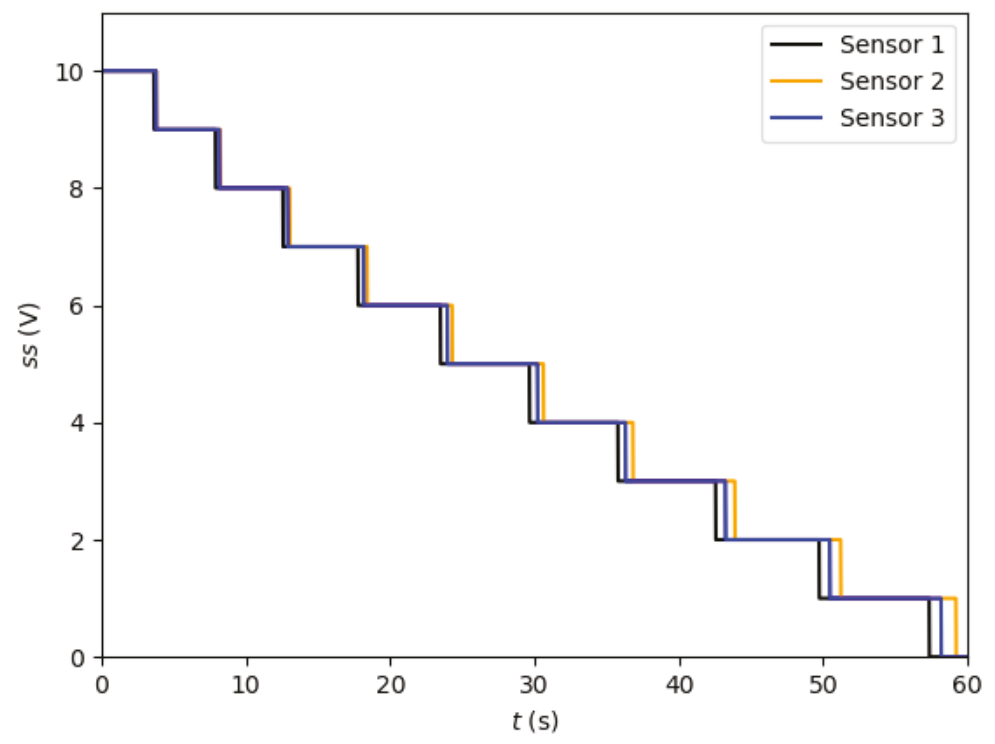

Figure 36. RBS signal, feedback with complete regulation law, case $2, d s=3 \mathrm{~mm}$.

Finally, Figures 37 and 38 present the results of the simulation case 2 with the downgraded regulation law and the new RBS parameters. 


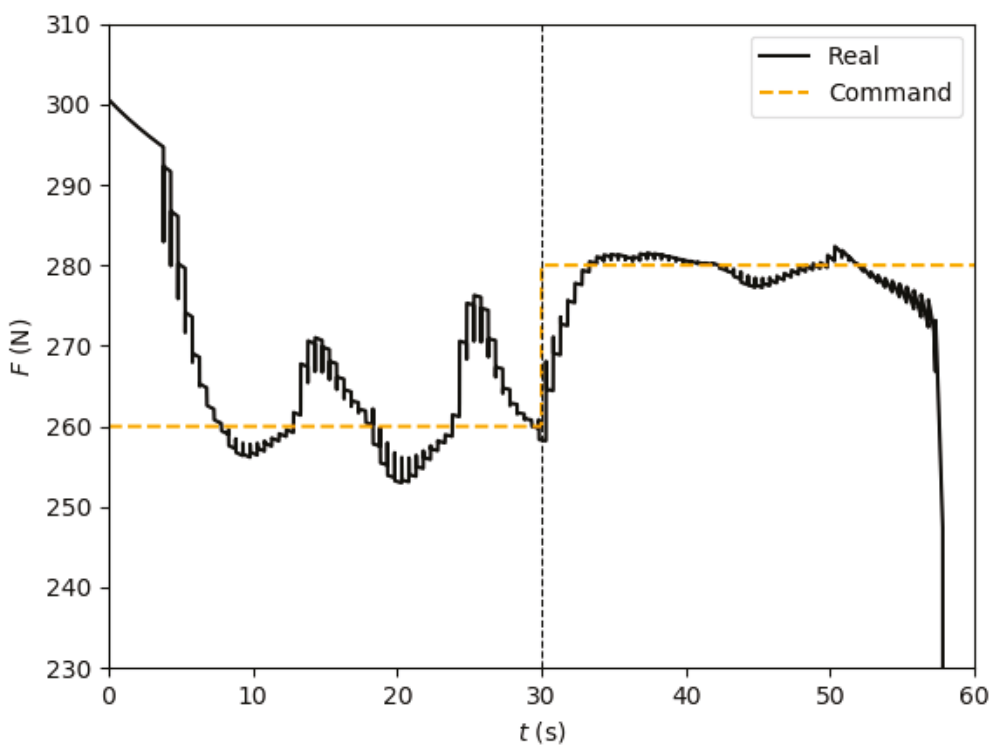

Figure 37. Thrust profile, feedback with downgraded regulation law, case $2, d s=3 \mathrm{~mm}$.

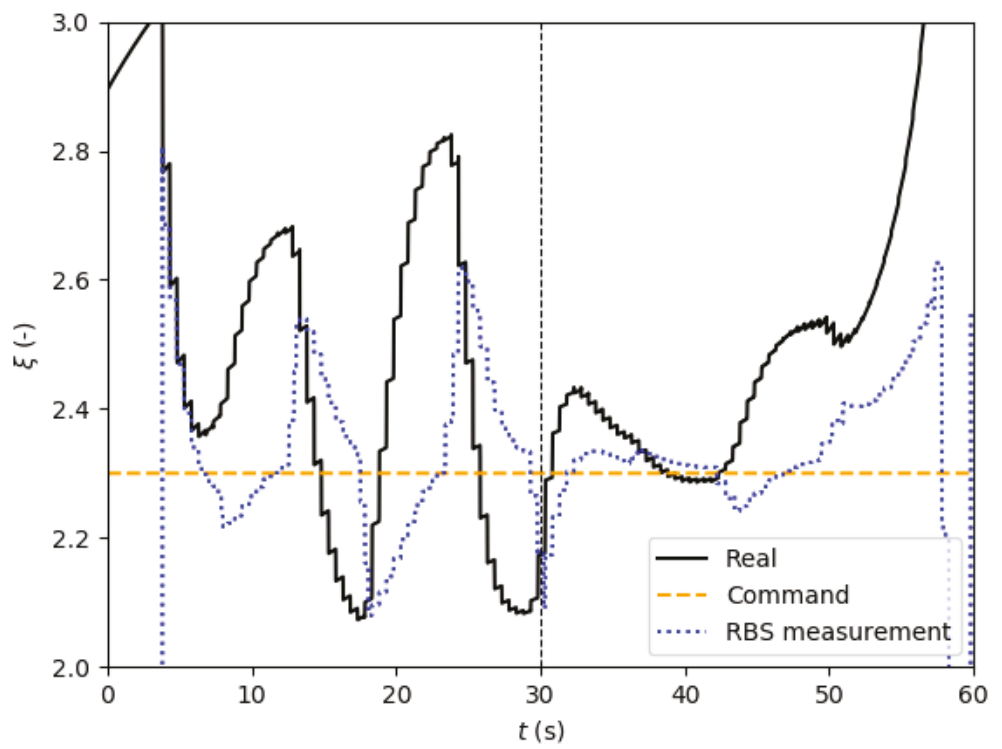

Figure 38. Mixture ratio profile, feedback with downgraded regulation law, case $2, d s=3 \mathrm{~mm}$.

The thrust reaches the command after $7.5 \mathrm{~s}$ and then oscillates significantly between 255 and $275 \mathrm{~N}$ until $30 \mathrm{~s}$. The change to the second thrust level seems to stabilize the engine which operates around $280 \mathrm{~N} \pm 2 \mathrm{~N}$ until $55 \mathrm{~s}$. The mixture ratio is also oscillating strongly and varies between 2.1 and 2.7 before $30 \mathrm{~s}$. It becomes more stable in the second phase of the test. These simulations clearly demonstrate that the complete regulation law provides significantly better results than the 
downgraded one, especially if the RBS precision is not very high, and also illustrated the importance of the sensors design for a proper feedback control.

\section{Discussion}

\subsection{Error Propagation Analysis}

The error propagation analysis has been performed in order to study the influence and importance of the measurements errors on the thrust and mixture ratio control. Two similar concepts (called A-SOFT and AOA) based on a dual oxidizer injection have been investigated and compared in terms of thrust, mixture ratio, and total relative errors. This analysis allows for calculating the errors propagation produced by measurements errors and could be used to help design future A-SOFT hybrid engines for real missions. Even though the AOA engine seems more interesting for limiting the thrust error propagation, we have seen that this theoretical result was not guaranteed. Indeed, it would suppose that the AOA engine has to operate at low values of $\alpha_{T}$ which would induce other operating troubles that are not taken into account in this study. Despite the fact that the benefit of using an A-SOFT engine rather than an AOA engine in terms of thrust error propagation could be open to debate, there is no ambiguity regarding the mixture ratio and the total relative errors. Because both of the two oxidizer injections have an influence on the fuel regression rate in the A-SOFT engines (only one in the AOA engines), this type of motor is more flexible and efficient to control the mixture ratio compared to the AOA engines. Consequently, the A-SOFT motors significantly reduce the total error propagation and are more interesting than the AOA motors in this regard. The principal assumptions to conduct this study were to neglect the combustion chamber pressure influence on the specific impulse and to suppose a constant combustion efficiency. These hypotheses lead to a specific impulse that is a function of the mixture ratio only. Since the A-SOFT and AOA engines use a swirled oxidizer injection, the combustion efficiency should keep a high value during the operating duration. As a consequence, assuming a constant combustion efficiency is not a very strong hypothesis and should not affect the described results. The error propagation analysis has been conducted and applied to propose two design methods, described as follows:

(1) The first design method deals with the choice of an optimal geometric swirl number for the A-SOFT engine. As we have seen thanks to the analysis, an augmentation of the geometric swirl number directly increases the total relative error. As a consequence, we could conclude that the lowest $S_{g}$ possible may be the most interesting choice. However, the error propagation increase due to the geometric swirl number augmentation is more or less significant depending on the engine operation condition $\left(\alpha_{T}\right)$. Consequently, the design of an optimal geometric swirl number is not trivial and depends on other factors. Even though different choices could be made, we considered two optimal criteria in this study: the first one was to never exceed a maximal error limit and the second one was to minimize the total error for the entire firing test duration. We applied these criteria on a specific case where the effective swirl number varies between 10 and 50, and the analysis conducted to an optimal geometric swirl number of 62.5, different from 50 that would have been the minimal $S_{g}$ possible. We demonstrated that the error propagation analysis could be used to define the geometric swirl number of an A-SOFT engine if the mission profile is known.

(2) The objective of the second method arising from the error analysis was to help define the resistor-based sensors. In particular, the radial distance between resistors is the most crucial parameter for the mixture ratio evaluation and must be chosen carefully. If the maximum total error which is tolerable and the oxidizer mass flow rate measurement error are known, the propagation analysis can be used to define the requirements for the radial distance between resistors, as demonstrated in this paper. Further analysis may be required to design other parameters such as the axial distance between resistors, the number of sensors, and their location along the grain. However, in order to study all the parameters, the theoretical analysis should be coupled with dedicated numerical simulations and experimental firing 
tests. Although the specific case of resistor-based sensors has been considered in this study, the method is interesting since it could be used to analyze other type of fuel regression rate measurements.

In this paper, we proposed and conducted independently two methodologies regarding the choice of the geometric swirl number and the design of resistor-based sensors. However, these parameters are not independent and should be designed in parallel. In practice, a few iterations of the described methods may be required to design the ideal swirl injector geometry and the corresponding fuel regression sensors in order to minimize the error propagation in an A-SOFT engine.

\subsection{Numerical Simulations}

The main objective of the numerical simulations was to evaluate the regulation law derived from fundamental A-SOFT equations and to apply it with the use of resistor-based sensors for the fuel regression rate measurement. The results described in the article showed that the A-SOFT engine feedback control could be performed in order to manage the thrust and mixture ratio independently and at the same time, and that using RBS was a promising solution for the mixture ratio evaluation. Since the sensors' principle is simple, it is possible to model their behavior and to study their implementation using simplified simulations. Although the simulations revealed interesting features of the feedback control, several assumptions have been made and deserve some discussion:

(1) As in the error propagation analysis, a constant combustion efficiency has been assumed to perform the simulations and particularly to calculate the engine performances. Because of the swirled oxidizer injection, this hypothesis is not very strong and the combustion efficiency should keep a high value in a real configuration. However, it would be possible to remove this assumption by using, for example, an empirical law giving the efficiency in function of the swirl number and the combustion chamber geometry. Detailed experimental analysis would then be required to obtain such an empirical relation.

(2) In our model, the regulation by the PID controller starts a few seconds after ignition since it needs the data from the resistor-based sensors and an initial configuration for the servo-valves is consequently required. In this study, we used an arbitrary $30 \%$ valve opening to check if the regulation law and controller were able to rapidly match the commands, even if the initial thrust value was far from the target one. In a real system, the initial thrust command is known and the initial valve opening would be chosen in order to reach satisfying conditions from the very beginning of the engine's operation. Depending on the RBS parameters and the type of the regulation law, 5 to $7.5 \mathrm{~s}$ were needed to reach the thrust target in the simulations. Indeed, if the distance between the first resistor and the fuel grain surface is increased, the required time to start measuring the regression rate is increased and the beginning of the regulation is delayed.

(3) In order to integrate a valve response time in the simulations, a regulation frequency of $2 \mathrm{~Hz}$ has been used. This value seems realistic but could be changed if the valve and other sources of delays are known.

(4) The mass flow rate and the thrust measurements have been considered as ideal in the simulations. As we have seen in the error propagation analysis, the mass flow rate precision is an important parameter for the efficiency of the regulation and to limit the errors' propagation. Since the thrust is one of the quantities to be regulated, its estimation is naturally essential for the feedback loop control. Further analysis regarding these measurements should be integrated in future work. For example, artificial noise could be added and numerical filtering used to reproduce real in-flight analogical filtering. The noisy-filtered signals should then be used as measured thrust and oxidizer mass flow rate into the feedback regulator.

(5) Several aspects should be highlighted and discussed regarding the resistor-based sensors. In our study, we considered perfect sensors meaning that the resistors were disconnected as soon as the fuel surface reaches them. In practice, it would imply that the RBS do regress at the exact same rate as the fuel, and that the connectors for the resistors are destroyed properly at the same time. This assumption will be studied more in detail in future work within an experimental firing test campaign 
including RBS. Even if the sensors are not ideal in reality, it would be possible to consider some realistic conditions in the simulations. For example, to consider a potential difference between the fuel and the sensors' regression rates, a specific evaluation of the sensors' burning rate could be added. The simulations have been performed to analyze the influence of the sensors' radial distance between the resistors. However, other parameters could also be investigated such as the number of sensors and their location. The axial distance between resistors has been considered as null for the simulations but a more realistic value could be used. This work has not been performed yet, but the numerical code developed for this study could be used in its current form in future work to analyze such parameters of the RBS. As we have seen, the initial distance between the first resistor of the sensors and the fuel grain surface plays a major role in the responsiveness of the feedback control at the beginning of the combustion. For real applications, this distance should be minimized as much as possible.

(6) In the current version of the simulation code and feedback law, the fuel regression rate is supposed to be constant between two re-evaluations. Similarly, the regression rate is supposed to be piece-wise constant along the fuel grain. These hypotheses have been used as a first step since they allowed a simple evaluation of the fuel regression and mass flow rates, but they could be modified easily and improved by using interpolations.

(7) The PID controller has been limited to a proportional one and was sufficient to provide interesting results in the case of the simple configurations that have been analyzed in this study. The PID coefficients depend on the characteristics of the system and experimental tests would be required to determine them.

Throughout the discussion of this study, the combustion chamber pressure was not considered for the feedback control. In practice, the pressure should be measured and used for several purposes. Firstly, the regulation law should include safety procedures in which the chamber pressure would play a major role. Secondly, the combustion chamber pressure could be used to evaluate the combustion efficiency of the operating engine by combining the other measurements. The knowledge of the combustion efficiency could be taken into account in the regulation law. Finally, the thrust measurements provided by an accelerometer could be completed by an estimation based on the chamber pressure and the propellants' mass flow rates.

Despite the few limitations and possible improvements that have just been discussed, the current version of the code allowed for obtaining interesting results regarding the A-SOFT feedback control based on RBS measurements. Three thrust command profiles have been investigated (constant, piece-wise constant, and linear evolution) with a constant optimal mixture ratio for the $\mathrm{O} / \mathrm{F}$ ratio command. For these configurations, the regulation law derived from the A-SOFT fundamental equations has been compared to a downgraded law based on a simple proportionality relation. Although the downgraded law exhibited lower performances than the full regulation law in terms of precision and oscillations around the target value, the behavior of the engine could be considered as acceptable with this simple law if the sensors' precision is high. Additionally, two sets of parameters for the RBS have been tested. The importance of the sensors design regarding the feedback control have been demonstrated as well as the advantage of using the complete regulation law. In particular, if the precision of the RBS is reduced, the feedback control of the A-SOFT engine remains efficient with limited oscillations if the full regulation law is used while the engine becomes difficult to control in the case where a downgraded law is used.

\section{Conclusions}

The error propagation analysis has been performed in order to study the influence of the measurements errors on the thrust and mixture ratio control in hybrid engines. The A-SOFT and AOA concepts are based on a dual oxidizer injection and have been investigated and compared in terms of thrust, mixture ratio, and total relative errors. It has been demonstrated that the A-SOFT engines were more interesting to limit the errors propagation compared to the AOA motors. The influence of the geometric swirl number has been highlighted, and a possible method for its design was 
proposed based on the error propagation results. The importance of the fuel and the oxidizer mass flow rates measurements has also been discussed and the use of resistor-based sensors has been investigated. Moreover, a design methodology has been proposed to make these sensors suitable for A-SOFT applications based on the error propagation results. A control law has been derived from the fundamental equations of hybrid engines and the A-SOFT concept and has been validated by numerical simulations. We demonstrated the interest of using this law to obtain precise feedback control of the engine compared to a simpler proportional regulation law. Besides the regulation law, the numerical simulations have been used to study the integration of the resistor-based sensors in the control of the engine. This study successfully demonstrated the feasibility of A-SOFT feedback loop control by using resistor-based sensors for the fuel regression rate measurement and represents an additional step towards the development of such hybrid rocket engines.

Author Contributions: Conceptualization, J.M. and T.S.; methodology, J.M. and T.S.; software, J.M.; validation, J.M.; formal analysis, J.M. and T.S.; investigation, J.M. and T.S.; resources, T.S.; data curation, J.M. and T.S.; writing — original draft preparation, J.M.; writing—review and editing, J.M. and T.S.; visualization, J.M. and T.S.; supervision, J.M. and T.S.; project administration, T.S.; funding acquisition, J.M. and T.S.

Funding: This research was funded by KAKENHI Grant No. JP16H04594 and supported by the External Funding Acquisition Incentive System provided by ISAS/JAXA.

Acknowledgments: The authors are grateful to their colleagues from SPLab (Politecnico di Milano), and particularly to Christian Paravan, for their collaboration on this research.

Conflicts of Interest: The authors declare no conflict of interest. The funders had no role in the design of the study; in the collection, analyses, or interpretation of data; in the writing of the manuscript, or in the decision to publish the results.

\section{Nomenclature}

\begin{tabular}{|c|c|}
\hline$\alpha_{T}$ & ratio of the oxidizer injections, - \\
\hline$\dot{m}_{f, m, s}$ & local measured fuel mass flow rate, $\mathrm{kg} \cdot \mathrm{s}^{-1}$ \\
\hline$\dot{m}_{f, m}$ & total measured fuel mass flow rate, $\mathrm{kg} \cdot \mathrm{s}^{-1}$ \\
\hline$\dot{m}_{f}$ & fuel mass flow rate, $\mathrm{kg} \cdot \mathrm{s}^{-1}$ \\
\hline$\dot{m}_{o, m}$ & measured oxidizer mass flow rate, $\mathrm{kg} \cdot \mathrm{s}^{-1}$ \\
\hline$\dot{m}_{o A f t}$ & aft-end oxidizer mass flow rate, $\mathrm{kg} \cdot \mathrm{s}^{-1}$ \\
\hline$\dot{m}_{o A}$ & axial oxidizer mass flow rate, $\mathrm{kg} \cdot \mathrm{s}^{-1}$ \\
\hline$\dot{m}_{\text {oFront }}$ & front oxidizer mass flow rate, $\mathrm{kg} \cdot \mathrm{s}^{-1}$ \\
\hline$\dot{m}_{o T}$ & tangential oxidizer mass flow rate, $\mathrm{kg} \cdot \mathrm{s}^{-1}$ \\
\hline$\dot{m}_{0}$ & total oxidizer mass flow rate, $\mathrm{kg} \cdot \mathrm{s}^{-1}$ \\
\hline$\dot{m}_{t o t}$ & total mass flow rate, $\mathrm{kg} \cdot \mathrm{s}^{-1}$ \\
\hline$\dot{r}$ & regression rate, $\mathrm{m} \cdot \mathrm{s}^{-1}$ \\
\hline$\dot{r}_{m, s}$ & local measured regression rate, $\mathrm{m} \cdot \mathrm{s}^{-1}$ \\
\hline$\dot{r}_{m}$ & measured regression rate, $\mathrm{m} \cdot \mathrm{s}^{-1}$ \\
\hline$\eta_{c}$ & combustion efficiency, - \\
\hline$\eta_{n}$ & nozzle efficiency, - \\
\hline$\gamma$ & isentropic coefficient, - \\
\hline$\overline{E_{\text {total }}}$ & integrated total error, s \\
\hline $\bar{e}$ & normalized relative error, - \\
\hline$\phi$ & fuel port diameter, $\mathrm{m}$ \\
\hline$\phi_{0}$ & initial fuel port diameter, $\mathrm{m}$ \\
\hline$\phi_{m, s}$ & local measured diameter, $\mathrm{m}$ \\
\hline$\phi_{\text {out }}$ & outer fuel grain diameter, $\mathrm{m}$ \\
\hline$\phi_{t}$ & nozzle throat diameter, $\mathrm{m}$ \\
\hline$\rho_{f}$ & fuel grain density, $\mathrm{kg} \cdot \mathrm{m}^{-3}$ \\
\hline
\end{tabular}


$\Sigma$

$\xi$

$\xi_{m}$

a

$A_{e}$

$A_{t}$

b

$c_{\text {th }}^{*}$

ds

$d s_{x}$

$d t$

$d x$

$e$

$e_{\text {pid }}$

F

$F_{m}$

$f_{\text {reg }}$

$g_{0}$

$\mathrm{G}_{0}$

$I_{s p, t h}$

$I_{s p}$

$k_{D}$

$k_{I}$

$k_{P}$

L

m

n

$N_{r}$

$N_{S}$

$p_{a}$

$p_{c}$

$p_{\text {error }}$

$p_{e}$

$s_{0}$

$S_{e, m}$

$S_{e}$

$S_{g}$

sr

ss

$t$

$t_{f}$

vo

$x$

$x_{S}$

nozzle expansion ratio, -

oxidizer to fuel ratio, -

measured oxidizer to fuel ratio, -

regression rate coefficient, $\mathrm{m} \cdot \mathrm{s}^{-1}$

nozzle exit area, $\mathrm{m}^{2}$

nozzle throat area, $\mathrm{m}^{2}$

regression rate coefficient, -

theoretical characteristic velocity, $\mathrm{m} \cdot \mathrm{s}^{-1}$

radial distance between resistors, $m$

axial distance between resistors, $\mathrm{m}$

numerical time step, s

numerical space step, $\mathrm{m}$

relative error, -

PID error, -

thrust, $\mathrm{N}$

measured thrust, $\mathrm{N}$

numerical regulation frequency, $\mathrm{Hz}$

ground gravitational acceleration, $\mathrm{m} \cdot \mathrm{s}^{-2}$

oxidizer mass flux, $\mathrm{kg} \cdot \mathrm{m}^{-2} \cdot \mathrm{s}^{-1}$

theoretical specific impulse, $\mathrm{s}$

specific impulse, $\mathrm{s}$

PID derivative coefficient, -

PID integral coefficient, -

PID proportional coefficient, -

fuel grain length, $\mathrm{m}$

regression rate coefficient, -

regression rate coefficient, -

number of resistors, -

number of sensors, -

ambient pressure, $\mathrm{Pa}$

combustion chamber pressure, $\mathrm{Pa}$

pressure convergence parameter, $\%$

nozzle exit pressure, $\mathrm{Pa}$

initial distance grain surface - resistor, $\mathrm{m}$

measured effective swirl number, -

effective swirl number, -

geometric swirl number, -

resistor signal, $\mathrm{V}$

sensor signal, $\mathrm{V}$

time, $\mathrm{s}$

firing duration, $\mathrm{s}$

servo valves opening, -

axial position, $\mathrm{m}$

sensor axial position, $\mathrm{m}$ 


\section{Abbreviations}

The following abbreviations are used in this manuscript:

AOA Aft-end Oxidizer Addition

A-SOFT Altering-intensity Swirling-Oxidizer-Flow-Type

MFR Mass Flow Rate

O/F Oxidizer to Fuel ratio

PID Proportional Integral Derivative

RBS Resistor-Based Sensor

RMS Root Mean Square

RPA Rocket Propulsion Analysis

SPLab Space Propulsion Laboratory

\section{Appendix A. Materials and Methods Related to AOA Engines}

\section{Appendix A.1. Fundamental Equations of AOA Engines}

The AOA engines can be somehow compared to the A-SOFT engines since they combine two oxidizer injections in order to control the thrust and the $\mathrm{O} / \mathrm{F}$ ratio [8,9]. The major difference comes from the positions of the injectors: in AOA engines, a front injection is combined with an aft-end injection (Figure 5). The swirled front injection generates the fuel regression and the thrust and the $\mathrm{O} / \mathrm{F}$ ratio are then regulated by the oxidizer addition from the aft-end injection.

In AOA engines, the fuel regression rate only depends on the front injection. The swirl number provided by this injection is constant since its geometry does not change during the engine operation. The fuel regression rate can be written by Equations (A1)-(A4):

$$
\begin{gathered}
\dot{r}[t]=g\left[G_{o}[t]\right], \\
g\left[G_{o}[t]\right]=a\left(1+S_{g}^{2}\right)^{m} G_{o}^{n}[t], \\
G_{o}[t]=\frac{4}{\pi} \frac{\dot{m}_{\text {oFront }}[t]}{\phi^{2}[t]} .
\end{gathered}
$$

We obtain:

$$
\dot{r}[t]=4^{n} \pi^{-n} a\left(1+S_{g}^{2}\right)^{m}\left(\frac{\dot{m}_{o F r o n t}[t]}{\phi^{2}[t]}\right)^{n} .
$$

The oxidizer and the fuel mass flow rates are defined by Equations (A5) and (A6) with: $C_{2}=$ $4^{n} \pi^{1-n} a\left(1+S_{g}^{2}\right)^{m} L \rho_{f}$ :

$$
\begin{gathered}
\dot{m}_{o}[t]=\dot{m}_{\text {ofront }}[t]+\dot{m}_{o A f t}[t], \\
\dot{m}_{f}[t]=C_{2} \phi[t]\left(\frac{\dot{m}_{o \text { Front }}[t]}{\phi^{2}[t]}\right)^{n} .
\end{gathered}
$$

Finally, the O/F ratio can be estimated by Equation (A7):

$$
\xi[t]=\frac{\dot{m}_{o \text { Front }}[t]+\dot{m}_{o A f t}[t]}{C_{2} \phi[t]\left(\frac{\dot{m}_{o \text { Front }}[t]}{\phi^{2}[t]}\right)^{n}} .
$$

The thrust can be evaluated by Equation (A8):

$$
\begin{aligned}
F[t] & =I_{s p}[\xi] g_{0}\left\{\dot{m}_{\text {oFront }}[t]+\dot{m}_{o A f t}[t]+\dot{m}_{f}[t]\right\} \\
& =I_{s p}[\xi] g_{0}\left\{\dot{m}_{\text {oFront }}[t]+\dot{m}_{o A f t}[t]+C_{2} \phi[t]\left(\frac{\dot{m}_{\text {oFront }}[t]}{\phi^{2}[t]}\right)^{n}\right\} .
\end{aligned}
$$


Similarly to A-SOFT engines, it is possible to express the thrust and the mixture ratio as function of the three independent variables $\dot{m}_{o F r o n t}, \dot{m}_{o A f t}$, and $\phi$ (Equation (A9)):

$$
\left(\begin{array}{l}
F \\
\xi
\end{array}\right)=\left(\begin{array}{l}
F\left[\dot{m}_{\text {oFront }}, \dot{m}_{o A f t}, \phi\right] \\
\xi\left[\dot{m}_{\text {oFront }}, \dot{m}_{o A f t}, \phi\right]
\end{array}\right) \text {. }
$$

Appendix A.2. Error Propagation of AOA Engines

The relative errors of thrust and $\mathrm{O} / \mathrm{F}$ ratio in AOA engines can be written by Equations (A10) and (A11):

$$
\begin{gathered}
e_{F}=\sqrt{\left(\frac{\partial \ln F}{\partial \ln \dot{m}_{\text {ofront }}} e_{\dot{m}_{o \text { Front }}}\right)^{2}+\left(\frac{\partial \ln F}{\partial \ln \dot{m}_{o A f t}} e_{\dot{m}_{\text {oAft }}}\right)^{2}+\left(\frac{\partial \ln F}{\partial \ln \phi} e_{\phi}\right)^{2}}, \\
e_{\xi}=\sqrt{\left(\frac{\partial \ln \xi}{\partial \ln \dot{m}_{\text {ofront }}} e_{\dot{m}_{o \text { Front }}}\right)^{2}+\left(\frac{\partial \ln \xi}{\partial \ln \dot{m}_{o A f t}} e_{\dot{m}_{o A f t}}\right)^{2}+\left(\frac{\partial \ln \xi}{\partial \ln \phi} e_{\phi}\right)^{2}} .
\end{gathered}
$$

Like for A-SOFT engines, we will consider that the oxidizer mass flow rate errors are equal: $e_{m_{o T}}=$ $e_{\dot{m}_{o A}}=e_{\dot{m}_{o F r o n t}}=e_{\dot{m}_{o A f t}}=e_{M F R}$. The relative errors are also normalized by $e_{M F R}$. As a consequence, the thrust, the $\mathrm{O} / \mathrm{F}$ ratio and the total normalized errors for AOA are written in Equations (A12)-(A14):

$$
\begin{gathered}
\overline{e_{F}}=\sqrt{\left(\frac{\partial \ln F}{\partial \ln \dot{m}_{\text {ofront }}}\right)^{2}+\left(\frac{\partial \ln F}{\partial \ln \dot{m}_{o A f t}}\right)^{2}+\left(\frac{\partial \ln F}{\partial \ln \phi}\right)^{2}{\overline{e_{\phi}}}^{2}} \\
\overline{e_{\xi}}=\sqrt{\left(\frac{\partial \ln \xi}{\partial \ln \dot{m}_{o F r o n t}}\right)^{2}+\left(\frac{\partial \ln \xi}{\partial \ln \dot{m}_{o A f t}}\right)^{2}+\left(\frac{\partial \ln \xi}{\partial \ln \phi}\right)^{2}{\overline{e_{\phi}}}^{2}} \\
\overline{e_{\text {total }}}=\sqrt{{\overline{\bar{e}_{F}}}^{2}+{\overline{e_{\xi}}}^{2}} .
\end{gathered}
$$

Similarly to A-SOFT engines, the $\alpha_{T}$ parameter indicates the ratio of the oxidizer injections. It varies in $[0,1]\left(0\right.$ if: $\dot{m}_{o \text { Front }}=0$ and 1 if: $\left.\dot{m}_{o A f t}=0\right)$ and is given by Equation (A15):

$$
\alpha_{T}=\frac{\dot{m}_{o \text { Front }}}{\dot{m}_{\text {ofront }}+\dot{m}_{o A f t}} .
$$

The sensitivity coefficients can be calculated based on Equations (A7) and (A8), and are given in the following relations (Equations (A16)-(A21)):

$$
\begin{gathered}
\frac{\partial \ln F}{\partial \ln \dot{m}_{\text {ofront }}}=\frac{n+\alpha_{T} \xi}{1+\xi}+\left(\alpha_{T}-n\right) \frac{d \ln I_{s p}}{d \ln \xi}, \\
\frac{\partial \ln F}{\partial \ln \dot{m}_{o A f t}}=\left(1-\alpha_{T}\right)\left\{\frac{\xi}{1+\xi}+\frac{d \ln I_{s p}}{d \ln \xi}\right\}, \\
\frac{\partial \ln F}{\partial \ln \phi}=(2 n-1)\left\{-\frac{1}{1+\xi}+\frac{d \ln I_{s p}}{d \ln \xi}\right\}, \\
\frac{\partial \ln \xi}{\partial \ln \dot{m}_{o F r o n t}}=\alpha_{T}-n, \\
\frac{\partial \ln \xi}{\partial \ln \dot{m}_{o A f t}}=1-\alpha_{T},
\end{gathered}
$$




$$
\frac{\partial \ln \xi}{\partial \ln \phi}=2 n-1
$$

The specific impulse coefficient in Equations (A16)-(A18) is the same as for A-SOFT and is given in Equation (31).

\section{Appendix B. Error Propagation Results of A-SOFT Engines Operating at Non-Optimal Mixture Ratio}

Appendix B.1. Oxidizer-Rich Combustion

The following case is considered:

- $\quad$ mixture ratio: $\xi=4$,

- regression rate: $a=0.029 \times 10^{-3} \mathrm{~m} \cdot \mathrm{s}^{-1}, m=0.166, n=0.650$,

- $\quad$ specific impulse: $I_{s p}=275 \mathrm{~s}$ and $d I_{s p} / d \xi=-12.5 \mathrm{~s}$.

The results are given in Figures A1 and A2.

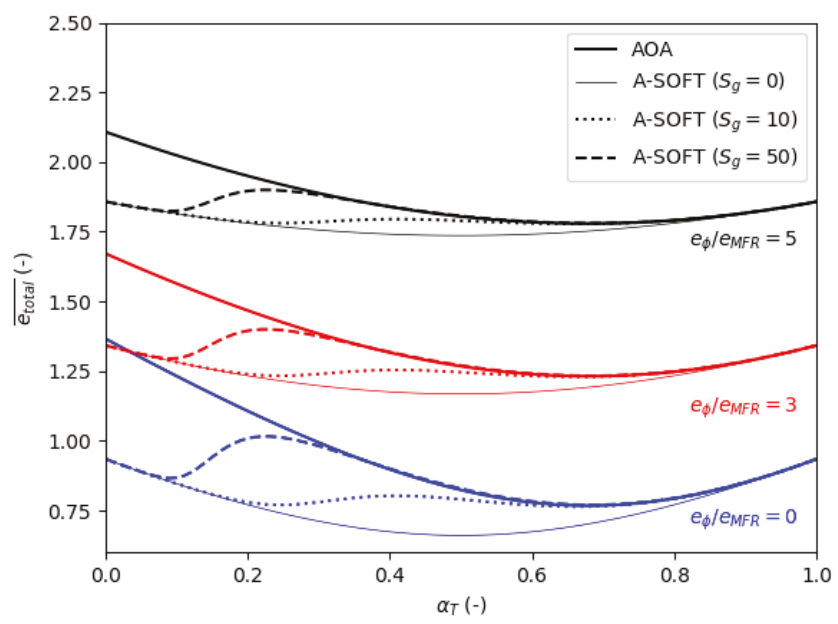

Figure A1. Normalized total error (oxidizer rich combustion).

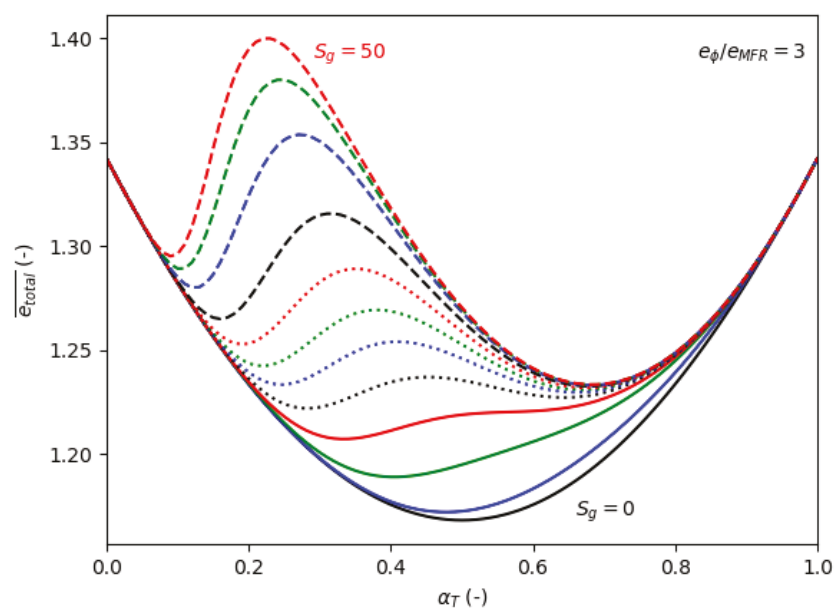

Figure A2. Influence of the geometric swirl number (oxidizer rich combustion) for $S_{g}$ values in $[0,2,4,6,8,10,12,15,20,30,40,50]$. 


\section{Appendix B.2. Fuel-Rich Combustion}

The following case is considered:

- $\quad$ mixture ratio: $\xi=1$,

- regression rate: $a=0.029 \times 10^{-3} \mathrm{~m} \cdot \mathrm{s}^{-1}, m=0.166, n=0.650$,

- $\quad$ specific impulse: $I_{s p}=225 \mathrm{~s}$ and $d I_{s p} / d \xi=50 \mathrm{~s}$.

The results are given in Figures A3 and A4.

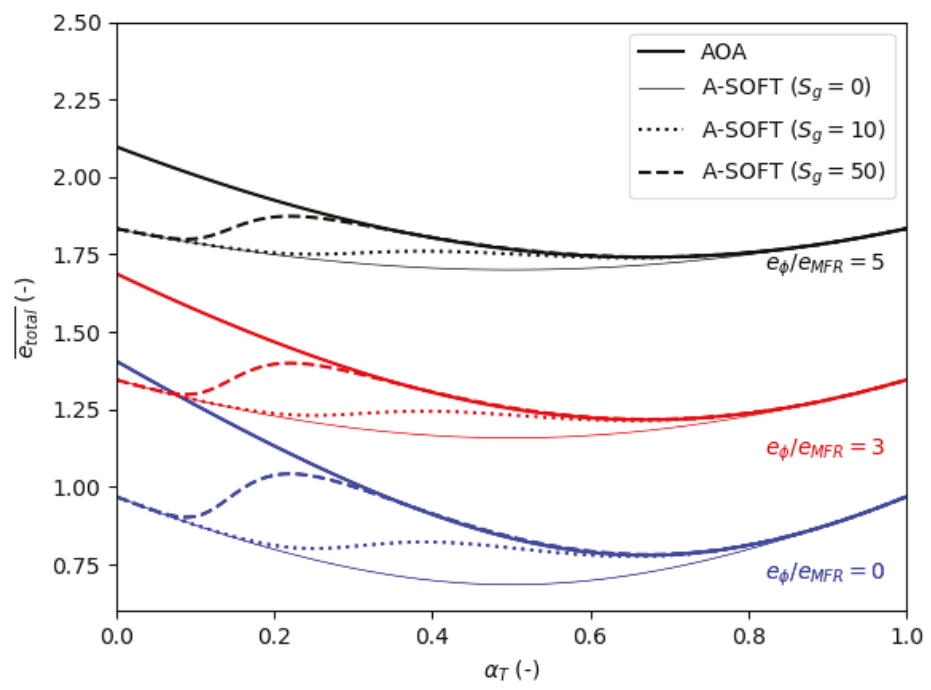

Figure A3. Normalized total error (fuel rich combustion).

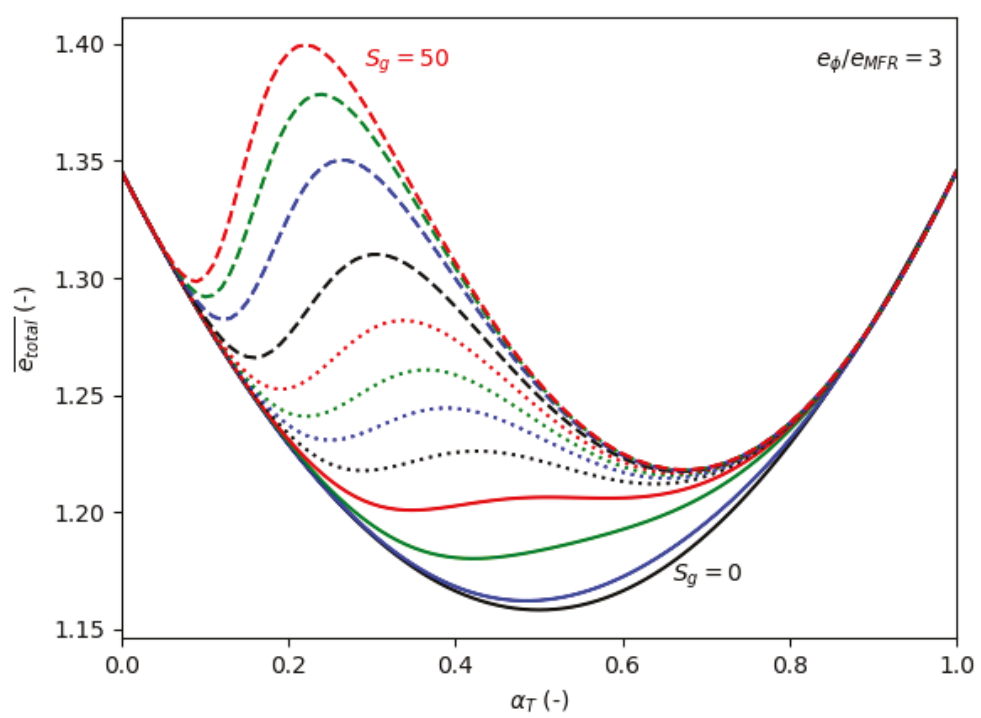

Figure A4. Influence of the geometric swirl number (fuel rich combustion) for $S_{g}$ values in $[0,2,4,6,8,10,12,15,20,30,40,50]$. 
Appendix C. Numerical Results with Downgraded Feedback Regulation Law

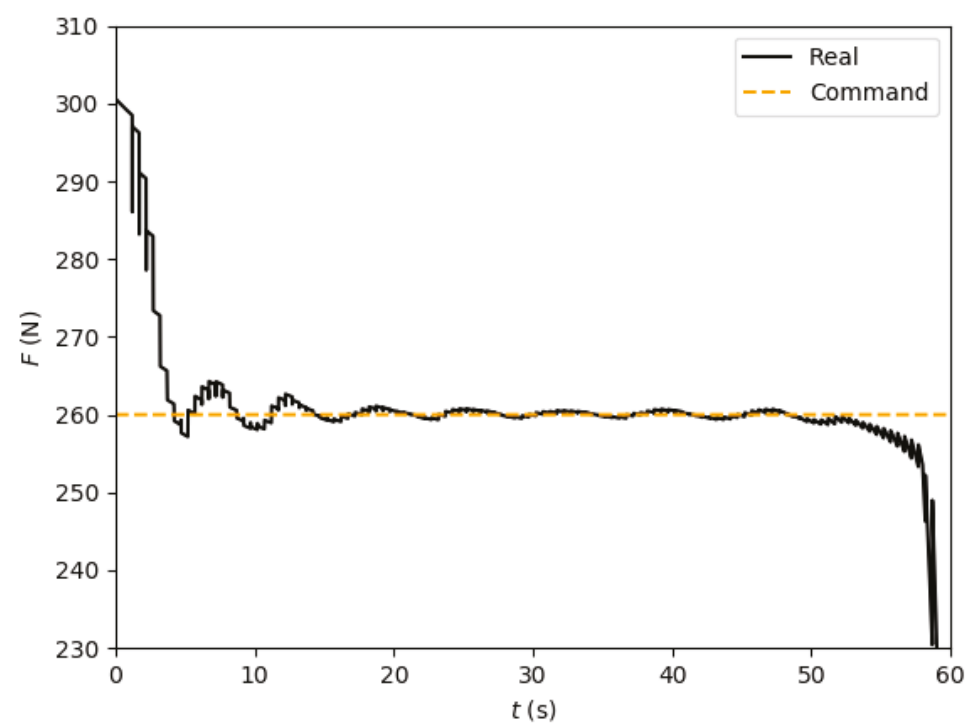

Figure A5. Thrust profile, feedback with downgraded regulation law, case 1.

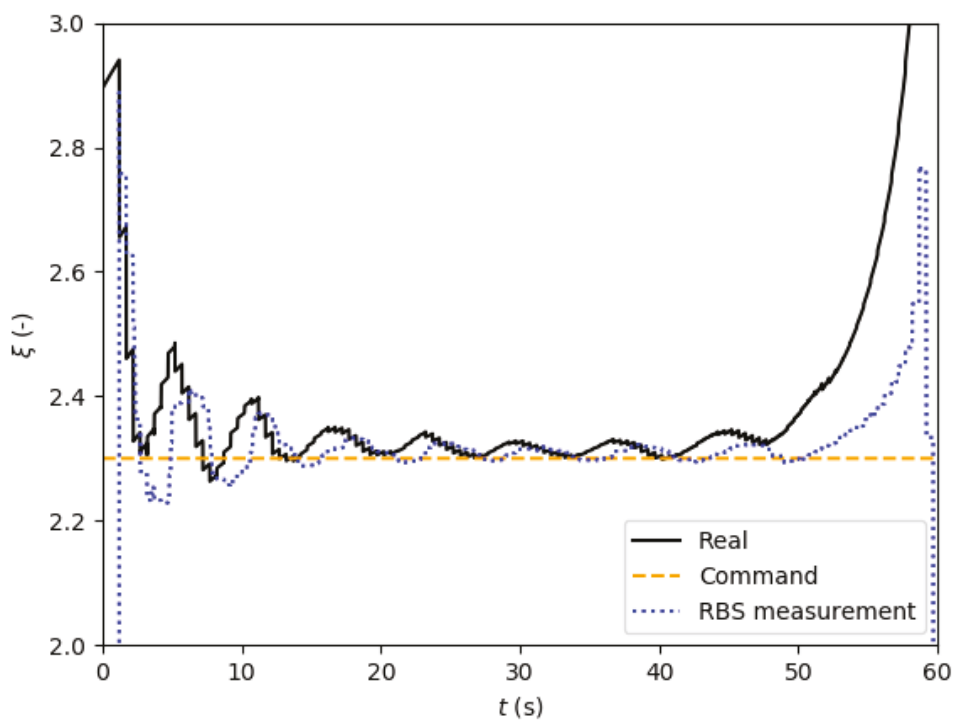

Figure A6. Mixture ratio profile, feedback with downgraded regulation law, case 1. 


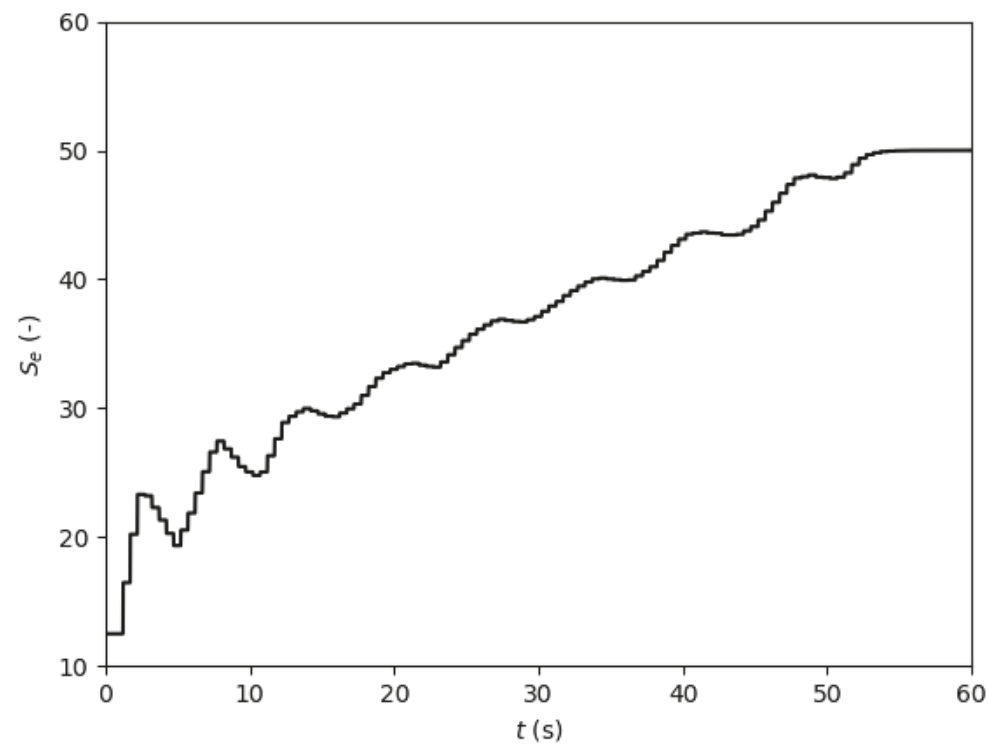

Figure A7. Effective swirl number profile, feedback with downgraded regulation law, case 1.

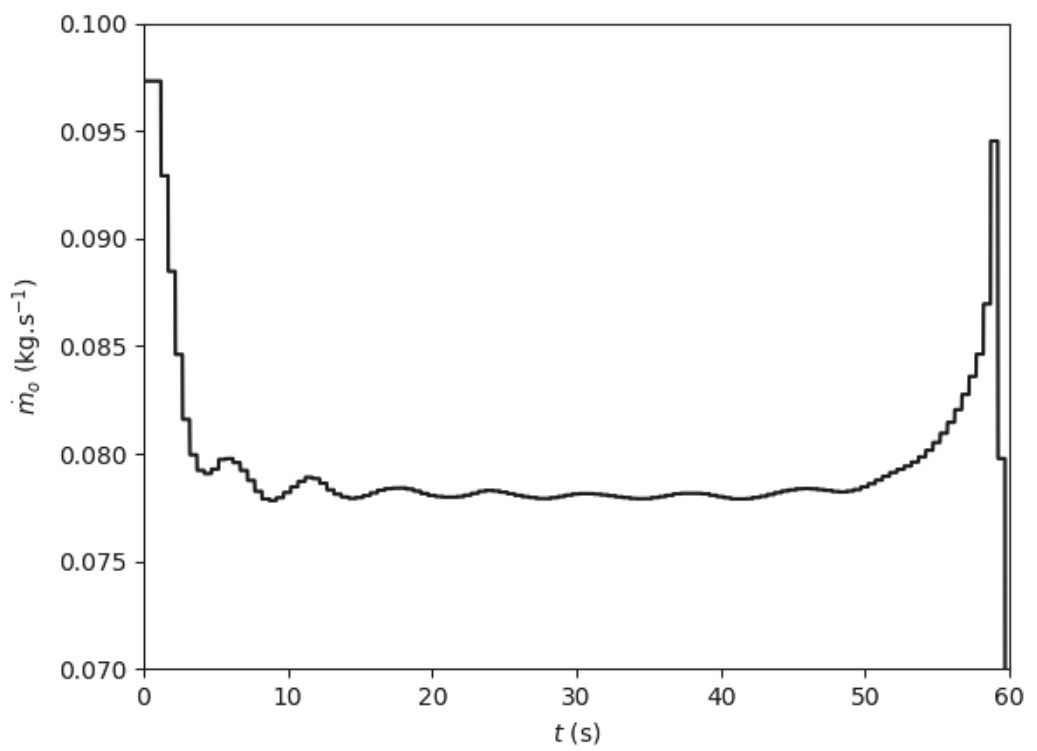

Figure A8. Oxidizer mass flow rate profile, feedback with downgraded regulation law, case 1. 


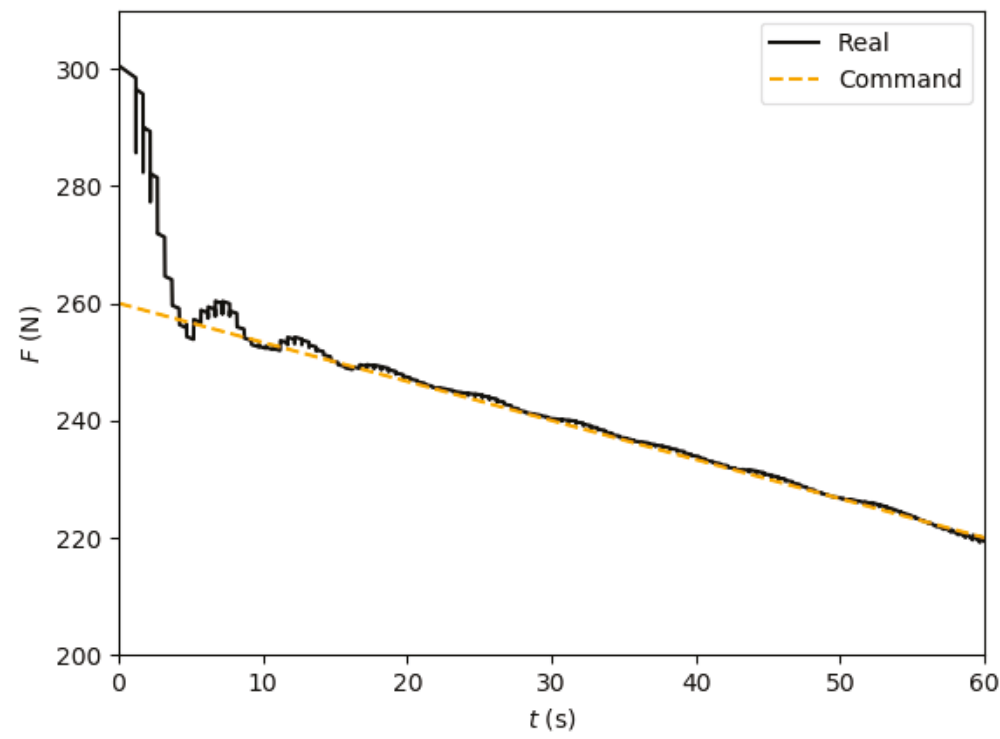

Figure A9. Thrust profile, feedback with downgraded regulation law, case 3.

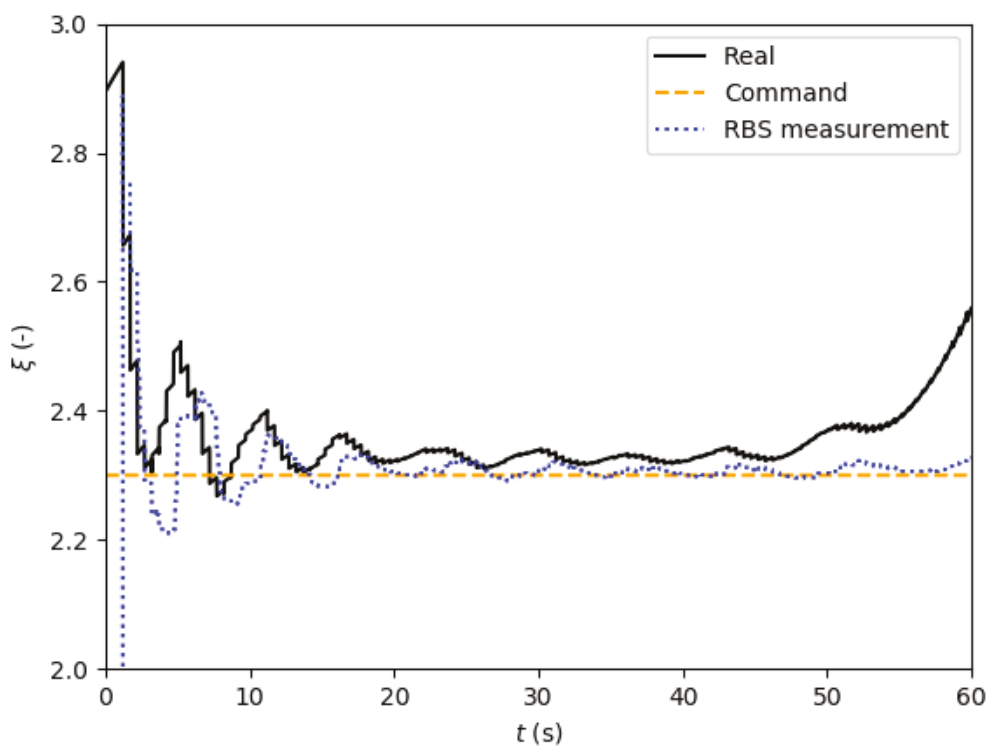

Figure A10. Mixture ratio profile, feedback with downgraded regulation law, case 3. 


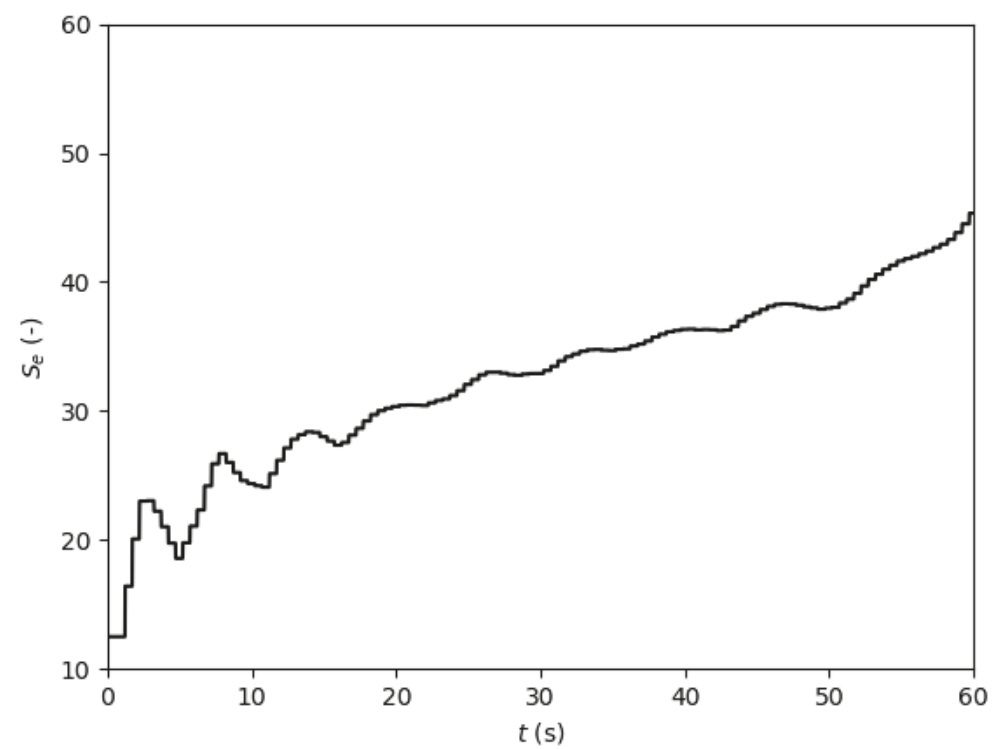

Figure A11. Effective swirl number profile, feedback with downgraded regulation law, case 3.

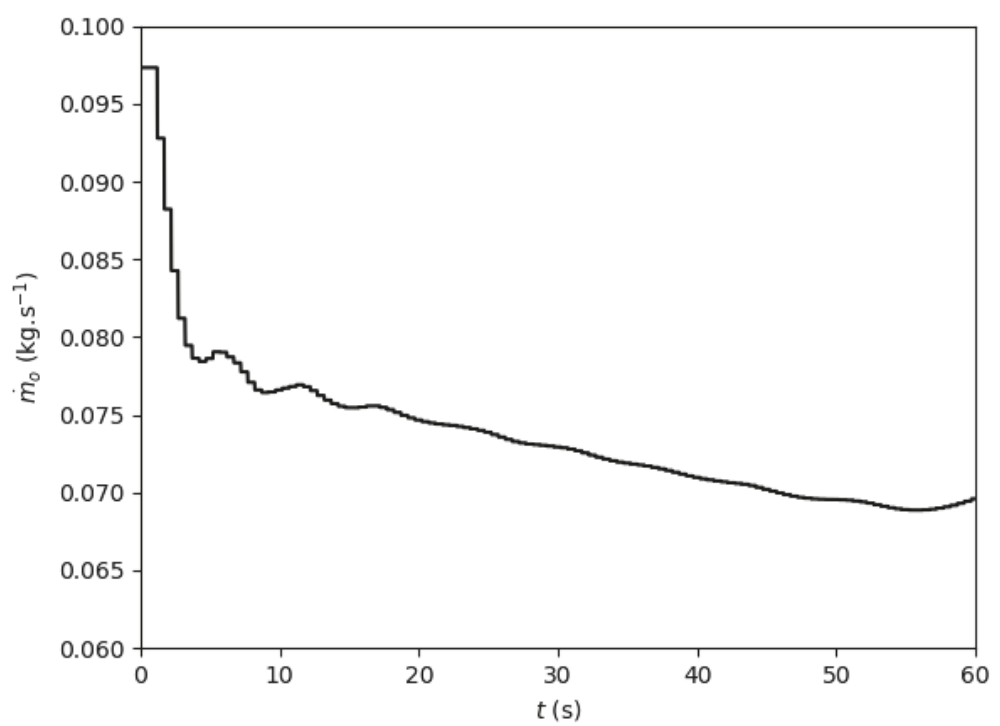

Figure A12. Oxidizer mass flow rate profile, feedback with downgraded regulation law, case 3.

\section{References}

1. Altman, D.; Holzman, A. Overview and History of Hybrid Rocket Propulsion. In Fundamentals of Hybrid Rocket Combustion and Propulsion; Chiaverini, M.J., Kuo, K.K., Eds.; Progress in Astronautics and Aeronautics; American Institute of Aeronautics and Astronautics, Inc.: Reston, VA, USA, 2007; Volume 218, pp. 1-36.

2. Marxman, G.A.; Gilbert, M. Turbulent Boundary Layer Combustion in the Hybrid Rocket. In Proceedings of the 9th International Symposium on Combustion, New York, NY, USA, 27August-1 September 1962; Volume 9, pp. 371-383. 
3. Karabeyoglu, A.; Zilliac, G.; Cantwell, B.J.; DeZilwa, S.; Castellucci, P. Scale-up Tests of High Regression Rate Paraffin-Based Hybrid Rocket Fuels. J. Propuls. Power 2004, 20, 1037-1045. [CrossRef]

4. Lestrade, J.-Y.; Anthoine, J.; Lavergne, G. Liquefying Fuel Regression Rate Modeling in Hybrid Propulsion. J. Aerosp. Sci. Technol. 2015, 42, 80-87. [CrossRef]

5. Kitagawa, K.; Yuasa, S.; Sakurai, T.; Hatagaki, S.; Shiraishi, N.; Ando, H.; Yagishita, T.; Suzuki, N.; Takayama, A.; Yui, R.; et al. Development of Test Facilities for 5 kN-Thrust Hybrid Rocket Engines and a Swirling-Oxidizer-Flow-Type Hybrid Rocket Engine for Technology Demonstration. Int. J. Energetic Mater. Chem. Propuls. 2016, 15, 435-451. [CrossRef]

6. Messineo, J.; Lestrade, J.-Y.; Hijlkema, J.; Anthoine, J. 3D MILES Simulation of a Hybrid Rocket with Swirl Injection. In Proceedings of the 5th Space Propulsion Conference, Roma, Italy, 2-6 May 2016.

7. Lestrade, J.-Y.; Messineo, J.; Anthoine, J.; Musker, A.; Barato, F. Development and Test of an Innovative Hybrid Rocket Combustion Chamber. In Proceedings of the 7th European Conference for Aeronautics and Space Sciences (EUCASS), Milan, Italy, 3-6 July 2017.

8. Culver, D.W. Comparison of Forward and Aft Injected Hybrid Rocket Boosters. In Proceedings of the 27th AIAA/SAE/ ASME Joint Propulsion Conference, Sacramento, CA, USA, 24-26 June 1991.

9. Boardman, T.A.; Porter, L.G.; Brasfield, F.W. An Ultrasonic Fuel Regression Rate Measurement Technique for Mixture Ratio Control of a Hybrid Motor. In Proceedings of the 31st AIAA/ASME/SAE/ASEE Joint Propulsion Conference and Exhibit, San Diego, CA, USA, 10-12 July 1995.

10. Usuki, T.; Shimada, T. Improvement on Thrust Profile Flexibility by Oxidiwer-to-Fuel Ratio Feedback Control in Hybrid Rocket. In Proceedings of the 66th International Astronautical Congress (IAC), Jerusalem, Israel, 12-16 October 2015.

11. Ozawa, K.; Usuki, T.; Mishima, G.; Kitagawa, K.; Yamashita, M.; Mizuchi, M.; Katakami, K.; Maji, Y.; Aso, S.; Tani, Y.; et al. Static Burning Tests on a Bread Board Model of Altering-intensity Swirling-Oxidizer-Flow-Type Hybrid Rocket Engine. In Proceedings of the 52nd AIAA/SAE/ASEE Joint Propulsion Conference, Salt Lake City, UT, USA, 25-27 July 2016.

12. Ozawa, K.; Shimada, T. A Theoretical Prediction of Regression Rates in Swirl Injection Hybrid Rocket Engines. In Proceedings of the 5th European Conference for Aeronautics and Space Sciences (EUCASS), Munich, Germany, 1-5 July 2013.

13. Shimada, T.; Usuki, T. Conceptual Study on Flight Demonstration of Mixture-Ratio-Controlled Throttling of Hybrid Rocket. In Proceedings of the 67th International Astronautical Congress (IAC), Guadalajara, Mexico, 26-30 September 2016.

14. Shimada, T.; Usuki, T, Takahashi, A.; Kitagawa, K.; Ozawa, K. Mission Requirements for Highly-Functional Hybrid Rocket Demonstration. In Proceedings of the 5th Space Propulsion Conference, Roma, Italy, 2-6 May 2016.

15. Ozawa, K.; Shimada, T. Flight Performance Simulations of Vertical Launched Sounding Rockets Using Altering-Intensity Swirling-Oxidizer-Flow-Type Hybrid Motors. In Proceedings of the 51st AIAA/SAE/ASEE Joint Propulsion Conference, Orlando, FL, USA, 27-29 July 2015.

16. Motoe, M.; Shimada, T. Numerical Simulations of Combustive Flows in a Swirling-Oxidizer-Flow-Type Hybrid Rocket. In Proceedings of the 52nd AIAA Aerospace Sciences Meeting, National Harbor, MD, USA, 13-17 January 2014.

17. Sakurai, T.; Yuasa, S.; Ando, H.; Kitagawa, K.; Shimada, T. Performance and Regression Rate Characteristics of 5-kN Swirling-Oxidizer-Flow-Type Hybrid Rocket Engine. J. Propuls. Power 2017, 33, 891-901. [CrossRef]

18. Obata, K.; Shimada, T.; Kitagawa, K. Imaging Analysis of Boundary Layer Combustion with Tangential and Radial Oxidizer Injection in a Cylinder. In Proceedings of the 7th European Conference for Aeronautics and Space Sciences (EUCASS), Milan, Italy, 3-6 July 2017.

19. Maggi, F.; Galfetti, L.; Colombo, G. Regression Sensor for a Solid Material. International Patent Publication Number WO2015011100A1, 29 January 2015.

20. Tadini, P.; Paravan, C.; Maggi, F.; Boiocchi, M.; Colombo, G; De Luca, L.T. Regression Rate Measurements in Lab-Scale Hybrid Burners. In Proceedings of the 5th European Conference for Aeronautics and Space Sciences (EUCASS); Munich, Germany, 1-5 July 2013.

21. Messineo, J.; Kitagawa, K.; Shimada, T. O/F Ratio Measurement for Hybrid Rocket Engine Feedback Control. In Proceedings of the 15th International Conference on Flow Dynamics (ICFD), Sendai, Japan, 9-13 July 2018. 
22. Whitmore, S.A.; Peterson, Z.W.; Eilers, S.D. Closed-Loop Precision Throttling of a Hybrid Rocket Motor. J. Propuls. Power 2014, 30, 325-336. [CrossRef]

23. Ponomarenko, A. RPA-Tool for Rocket Propulsion Analysis. In Proceedings of the 4th Space Propulsion Conference, Cologne, Germany, 19-22 May 2014.

(c)

(C) 2019 by the authors. Licensee MDPI, Basel, Switzerland. This article is an open access article distributed under the terms and conditions of the Creative Commons Attribution (CC BY) license (http://creativecommons.org/licenses/by/4.0/). 

Review

\title{
Review of Classical Diffusion-Limited Regression Rate Models in Hybrid Rockets
}

\author{
Timothy Marquardt and Joseph Majdalani * \\ Department of Aerospace Engineering, Auburn University, Auburn, AL 36849, USA; tam0013@auburn.edu \\ * Correspondence: joe.majdalani@auburn.edu; Tel.: +1-344-844-6800
}

Received: 13 March 2019; Accepted: 15 June 2019; Published: 20 June 2019

\begin{abstract}
In this article, we undertake a concise review of several milestone developments in classical regression rate models of hybrid rocket motors. After a brief description of the physical processes entailed in hybrid rocket combustion, Marxman's diffusion-limited theory is re-constructed and discussed. Considerations beyond the scope of basic convection-driven models, which address disparate forms of the blowing correction, variable fluid properties, and pressure and radiation effects, are also given. Finally, a selection of kinetically-limited models is presented, with the aim of comparing the characteristics of several competing theories that become applicable under particular circumstances.
\end{abstract}

Keywords: hybrid; rocket; regression; Marxman; diffusion-limited; blowing factor

\section{Introduction}

In the early 1960s, the advent of the space race played a key role in renewing commercial interest in hybrid rockets as a viable propulsion alternative. Previous experimentation in 1947 by the Pacific Rocket Society had established that the chamber pressure of a hybrid rocket was not strongly influenced by the exposed fuel surface area and that hybrid motors were insensitive to cracks in their fuel grains [1]; this safety feature essentially nullified the risk of sudden deflagrations caused by defects in motor fabrication and presented hybrids as an attractive alternative to the more universally-accepted solid propellant systems. Nonetheless, several elements of the physical processes associated with the operation of hybrid rockets remained elusive, including the details of the combustion process and the effects of scaling on motor performance. The search for answers, both quantitative and qualitative, to these fundamental questions was undertaken throughout the 1960s at the United Technology Center (UTC) in California, where Marxman, Gilbert, Wooldridge, and Muzzy carried out several elegant experimental and theoretical investigations into the nature of hybrid motor burning. Although this group was, by no means, alone in its line of inquiry, its members established themselves at the forefront of the field by conducting a series of landmark studies which are routinely cited to this day. For a more thorough perspective on the efforts of this and other contemporaneous groups, the historical exposé provided by Altman [1], who led the hybrid research group at UTC, is highly recommended.

The effort to describe the internal ballistics of hybrid motors continues to the present day, especially as novel engine configurations that cannot be accurately described by the more traditional models are becoming commonplace. Furthermore, the continuous growth in modern computing power and numerical techniques has allowed for computational investigations into hybrid rocket combustion in greater detail than ever before. The subject of classical diffusion-limited regression rate models is cursorily reviewed in the introductory parts of various articles on hybrid rockets, but these treatments are naturally not intended to elucidate the finer points of the models in question. Although other comprehensive reviews of the subject matter exist [2,3], they tend to have a generally broader focus. While their values remain unquestionable, they can require greater effort on the part of the reader to 
gather all the details necessary for the complete re-construction of Marxman's model compared to the present treatment.

This article is intended for researchers who have not been acquainted with the history of analytical, boundary-layer-based regression rate modeling in hybrid rockets. It aims to complement the existing literature by providing the reader with a concise, but sufficiently detailed, review of classical diffusion-limited regression models, in order to set a solid foundation upon which future related research could be based. In this vein, the starting point of this review will be a brief discussion of the physical processes involved in hybrid rocket combustion.

\section{Physical Processes in Hybrid Rockets}

Hybrid rockets are thrust-producing chemical propulsion systems that store their fuel and oxidizers separately in different phases; a typical hybrid rocket consists of a liquid oxidizer, which is injected into a thrust chamber whose walls are composed of a solid fuel. Such a design concept offers several unique advantages over traditional solid and liquid rocket configurations. Hybrids are less complex and, therefore, less costly than liquids because they require half of the plumbing and turbo-machinery. They offer the flexibility of throttling and are, thus, capable of non-destructive aborts, which are out of reach for solid motors. Hybrid engines are capable of generating specific impulse values that generally fall between those of solids and those of liquids. However, when designed properly, hybrids can match the performance of some liquid systems, such as LOX-RP1 [4]. In addition to their insensitivity to cracks during flight, hybrids offer unique safety advantages during all phases of their life cycle, including fabrication, storage, and handling. On the other hand, the physical separation of the fuel and oxidizer interfaces leads to a less vigorous combustion response, when compared to the quasi-pre-mixed flame character observed in solid and liquid rocket engines.

Hybrid combustion depends on the balancing act of several aerodynamic, chemical, and thermal processes. An illustrative sketch of a diffusion-limited hybrid flame is provided in Figure 1.

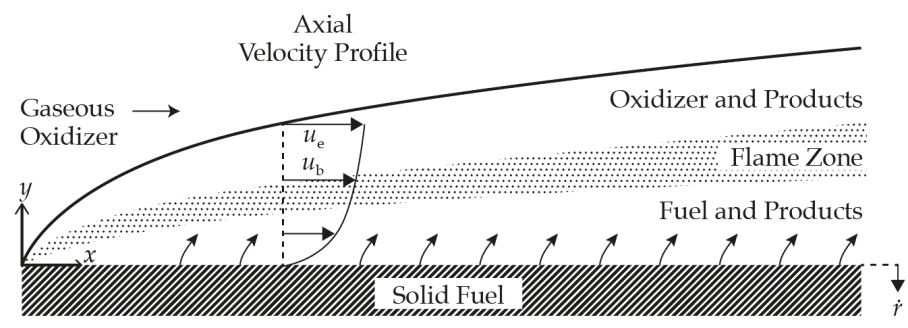

Figure 1. Illustrative sketch of the diffusion-limited combustion process in hybrid rockets.

When an oxidizer is injected uniformly into a combustion chamber, a turbulent boundary layer forms as the ensuing flowfield sweeps over the solid fuel grain. It should be noted that, in nearly all real hybrids, oxidizer injection is not uniform and injector effects dominate at the head end. These effects tend to greatly increase the local regression rate and often demand special care be taken in preventing burn-through of the grain and casing near the injectors. When injection is not uniform, the boundary layer assumes its classical structure downstream of the flowfield generated by the injectors. Heat from an ignition source reaches the wall and vaporizes a thin layer of fuel, which then infiltrates the boundary layer region. At some small distance from the wall, the local mixture ratio, due to species diffusion between the oxidizer and the fuel, proves conducive to combustion and so a thin flame appears. This location is situated approximately $10-20 \%$ of the boundary layer thickness $(\delta)$ from the wall and may be around $0.1 \delta$ thick [5].

Interestingly, chemical combustion in the boundary layer of hybrids was observed in some early experiments to occur at fuel-rich conditions [5-7], thus leading to flame temperatures that were 
considerably lower than the adiabatic flame temperature of the reacting propellants. This situation is not universal and its report immediately generated significant debate [8] which, while informative regarding the validity of diffusion-limited models, falls beyond the scope of this review. A fraction of the vaporized fuel flows axially below the flame zone and, in some configurations, this fuel is mixed with excess oxidizer in a post-combustion chamber in an effort to recover the full performance potential of the hybrid motor. Once the motor is ignited, the combustion process becomes self-sustaining and somewhat self-regulating. Increasing the oxidizer flow rate enhances the convective heat transfer to the wall and, as such, the vaporization rate of the fuel. Naturally, the increased mass of fuel blowing outwardly from the grain has a blocking effect on the inward convective heat transport across the flame, thereby reducing the vaporization rate until an equilibrium state is achieved. For this reason, and because increasing the oxidizer flow pushes the motor further away from an ideal oxidizer-to-fuel ratio, throttling the oxidizer alone as a means of controlling a hybrid motor has proven to be more complicated than throttling both propellants together as in a liquid rocket engine. Too much oxidizer "too fast" will blow the engine out, as the overall mixture ratio shifts into a region that can no longer support combustion; in the extreme case, residence times become insufficient for chemical reactions to complete. Conversely, an insufficient amount of oxidizer can lead to flame extinction. Operating just above the lower flammability limit can "cook" the motor, as low temperatures cause charring instead of complete combustion, specifically when low heat-transfer and regression rates allow the heat to penetrate deeper into the fuel grain to the extent of altering its mechanical properties. Although pressure-sensitive chemical kinetics can be quite important in either of these extremes, combustion is conventionally accepted to be a diffusion-limited process at the moderate operating conditions that are typical of most hybrid motors.

\section{Marxman's Diffusion-Limited Model}

We now turn our attention to a review of the regression rate model developed at UTC by Marxman and his associates. Their model describes the heat transfer pathways within a hybrid motor and leans heavily on the earlier work of Lees [9], who performed a similar analysis in a chemically reactive environment with blowing. The model assumes that the flow is developing along the entire length of the grain (i.e., the boundary layers on either side of the motor have not yet merged at the center-line and a core flow of nearly pure oxidizer exists). Thus, the model does not accurately portray motors whose flow becomes fully developed in the port or is dominated by injector effects over a large region of the head end. The foundational relationship of Marxman's model consists of an equivalence between the heat transferred from the gas to the wall and the energy absorbed in vaporizing the solid fuel:

$$
\dot{Q}_{\mathrm{W}}=\dot{m}_{\mathrm{f}}^{\prime \prime} h_{v}=\rho_{\mathrm{f}} \dot{r} h_{v}
$$

where $\dot{Q}_{\mathrm{w}}, \dot{m}_{\mathrm{f}}^{\prime \prime}, h_{v}$, and $\rho_{\mathrm{f}}$ represent the total heat flux at the wall, the fuel mass flux leaving the surface, the effective heat of gasification (which combines the heat of vaporization and melting, the heating of the solid fuel grain, and the heat of reaction associated with polymer degradation), and the fuel density, respectively. Note that the fuel is assumed to pyrolyze and vaporize, as is the case with most polymers, and that Marxman's model is not accurate for liquefying fuels (such as paraffin wax) that characteristically form a low-viscosity melt layer at the surface. The grain regression rate is represented by $\dot{r}$, in keeping with most other authors, despite some later simplifications that assume a planar configuration. Implicit in the relation specified in Equation (1) is the assumption that there are no heat losses through the grain to the motor casing or outside environment. Experiments and theories have both shown that such conditions provide a reasonable portrayal of normal operations for most hybrid motors, with the thermal waves penetrating only a short distance below the grain surface at moderate regression rates [6]. Next, a more specific expression for the heat transferred from the gas to the wall is given by

$$
\dot{Q}_{\mathrm{c}}=-\left(\frac{k}{c_{p}} \frac{\partial h}{\partial y}\right)_{\mathrm{w}},
$$


where $\dot{Q}_{c}, k, c_{p}, h$, and $y$ represent the convective heat flux (including the effect of partial enthalpies transport by species diffusion), thermal conductivity of the gas, specific heat of the gas, enthalpy of the gas, and normal distance into the flow from the fuel surface, respectively. Here the subscript ' $w$ ' refers to properties evaluated at the wall. Equation (2) appears as a conductive expression, although it is not explicitly labeled as such in Marxman and Gilbert's [5] original paper. It should be noted that applying such a simple conductive heat transfer relation to combusting flow implies the assumption that $\mathrm{Le}=\operatorname{Pr}=1$ and that the Reynolds analogy is valid for turbulent flow. As for the radiative component of the total heat flux, it represents a smaller contribution than convection in most hybrids. Whether the effect of radiation is small enough to be safely neglected, as in Equation (2), depends on the propellants and motor operating conditions: Although the polymethyl methacrylate (PMMA)- $\mathrm{O}_{2}$ systems studied at UTC display a weak radiation dependence, several hybrid motors behave differently. The importance of radiation is discussed at greater length in a later section.

The first of the two major hurdles in describing the heat transfer pathway has now been reached; namely, the challenge of determining the amount of convective heat flux at the wall, based on the known characteristics of the turbulent flow. The Stanton number is introduced, for this purpose, as it represents the ratio of the heat transferred into a fluid to the thermal capacity of that fluid:

$$
\text { St }=\frac{\dot{Q}_{\mathrm{c}}}{\rho_{\mathrm{b}} u_{\mathrm{b}} \Delta h^{\prime}}
$$

where the difference in gas-sensible enthalpy $\Delta h$ is evaluated between the flame zone, denoted by a subscript $b$, and the gas at the wall. In the above, the axial velocity of the burned gas is designated as $u_{\mathrm{b}}$. Writing the wall energy balance, Equation (1), in terms of the Stanton number, we have

$$
\rho_{\mathrm{f}} \dot{r}=\frac{\dot{Q}_{\mathrm{c}}}{h_{v}}=\frac{\dot{Q}_{\mathrm{w}}}{h_{v}}=\operatorname{St} \rho_{\mathrm{b}} u_{\mathrm{b}} \frac{\Delta h}{h_{v}},
$$

so that $\dot{Q}$, which is unknown and difficult to estimate directly, is eliminated.

At this juncture, the Reynolds analogy may be invoked to establish an approximate relationship between the unknown heat flux and the better-understood shear stress within the boundary layer. In this process, the Prandtl and Lewis numbers between the wall and the flame zone are taken to be of order unity. It should be noted that, since real hybrids often experience conditions that differ from the idealized assumptions made here, the Chilton-Colburn analogy may replace the Reynolds analogy for cases where $\operatorname{Pr} \neq 1$ or in the presence of an axial pressure gradient. In fact, it may be shown that the difference in the final regression rate relation is rather small when these effects are taken into account (see Marxman [7]). Accordingly, the thermal and molecular diffusion mechanisms associated with the energy and momentum transfers within the boundary layer may be assumed to be driven by similar turbulent mixing processes. Subsequently, the Reynolds analogy may be written as an equivalence between the ratio of the heat flux to the radial gradient of total enthalpy $h_{T}$ and the ratio of the shear stress $\tau$ to the radial gradient of the axial velocity:

$$
-\frac{\dot{Q}}{\partial h_{T} / \partial y}=\frac{\tau}{\partial u / \partial y}
$$

This expression may be integrated from the wall to the flame zone, since the object of invoking the analogy is to link properties at these two points. Direct integration yields

$$
\frac{\dot{Q}_{\mathrm{c}}}{\Delta h_{T}}=\frac{\tau_{\mathrm{w}}}{u_{\mathrm{b}}} .
$$

If no combustion occurs in the low-speed region below the flame, $\Delta h=\Delta h_{T}$ and dividing by $\rho_{\mathrm{b}} u_{\mathrm{b}}$ leads to an alternate expression for the Stanton number, in terms of the wall shear stress $\tau_{\mathrm{w}}$ or the skin friction coefficient $C_{f}$, by 


$$
\mathrm{St}=\frac{\tau_{\mathrm{w}}}{\rho_{\mathrm{b}} u_{\mathrm{b}}^{2}}=\frac{1}{2} C_{f} \frac{\rho_{\mathrm{e}} u_{\mathrm{e}}^{2}}{\rho_{\mathrm{b}} u_{\mathrm{b}}^{2}} .
$$

Finally, substituting Equation (7) into Equation (4) enables us to write

$$
\rho_{\mathrm{f}} \dot{r}=\frac{1}{2} C_{f} \rho_{\mathrm{e}} u_{\mathrm{e}} \frac{u_{\mathrm{e}}}{u_{\mathrm{b}}} \frac{\Delta h}{h_{v}} .
$$

Note that $\dot{Q}$ has now been replaced in the expression by $C_{f}$, which is more straightforward to estimate. The first hurdle has been cleared.

Next, the skin friction coefficient may be calculated using a suitable empirical relation. Marxman used the well-known expression for turbulent flow over a flat-plate with no blowing (Schlichting [10]),

$$
\frac{C_{f_{0}}}{2}=0.03 \operatorname{Re}_{x}^{-0.2}
$$

where the reference flat-plate value for the skin friction coefficient is given the zero subscript, in order to distinguish it from the value applicable to the combusting flowfield. The local Reynolds number in this expression is given in terms of the freestream properties at the boundary layer edge, denoted here with a subscript 'e,' and the axial distance $x$ from the grain leading edge, specifically

$$
\operatorname{Re}_{x} \equiv \frac{\rho_{\mathrm{e}} u_{\mathrm{e}} x}{\mu_{\mathrm{e}}} .
$$

Making use of this relation entails two principal assumptions: First, that the presence of blowing does not change the nature of the flow so much that a standard value of the friction coefficient modified by a correction factor would be invalidated and, second, that the result for a flat plate will adequately describe the motion in a typical cylindrical and center-perforated grain. The validity of the first assumption largely depends on the complexity or degrees of freedom offered by the correction factor applied. The flat-plate assumption is generally accepted for axially-injected hybrid motors-the grain curvature becomes inconsequential to the analysis in the absence of swirl (i.e., when the motion lacks a significant tangential component). In such configurations, the boundary layer length-scales remain at least one order of magnitude smaller than those associated with the radius of curvature, so that the combustion process may be safely modeled using a flat-plate assumption. The combined effect of both assumptions has been examined in the context of swirl-driven motors by at least one researcher [11].

Substituting the skin friction coefficient expression given by Equation (9) into the energy balance in Equation (8) produces

$$
\rho_{\mathrm{f}} \dot{r}=0.03 \rho_{\mathrm{e}} u_{\mathrm{e}} \operatorname{Re}_{x}^{-0.2} \frac{C_{f}}{C_{f_{0}}} \frac{u_{\mathrm{e}}}{u_{\mathrm{b}}} \frac{\Delta h}{h_{v}} .
$$

All that remains is to find a "blowing correction," $C_{f} / C_{f_{0}}=\mathrm{St} / \mathrm{St}_{0}$, that will account for the difference in heat flux between the combusting (i.e., blowing) and non-combusting cases. For convenience, the blowing parameter $B$ is first defined as

$$
B \equiv \frac{u_{\mathrm{e}}}{u_{\mathrm{b}}} \frac{\Delta h}{h_{v}}
$$

so that

$$
\rho_{f} \dot{r}=0.03 \rho_{\mathrm{e}} u_{\mathrm{e}} \frac{C_{f}}{C_{f_{0}}} \operatorname{Re}_{x}^{-0.2} B
$$

The blowing parameter, thus, appears as a thermochemical parameter, which depends on the flame location within the boundary layer and which can be specified for a particular propellant combination. While it is clear that the heat of gasification and difference in enthalpies are determined by the propellants and mixture ratio, the dependence of the velocity ratio $u_{\mathrm{e}} / u_{\mathrm{b}}$, which is closely 
linked to the flame height, on these same parameters is less evident. This dependence may be verified by integrating the oxidizer and fuel mass fluxes through a pair of control volumes separated by the flame zone and comparing the results to the integral momentum equation. One obtains

$$
\frac{u_{\mathrm{e}}}{u_{\mathrm{b}}}=\frac{K_{\mathrm{ox}_{\mathrm{e}}}+\left(\mathrm{O} / \mathrm{F}+K_{\mathrm{ox}_{\mathrm{e}}}\right)\left(\Delta h / h_{v}\right)}{(\mathrm{O} / \mathrm{F})\left(\Delta h / h_{v}\right)},
$$

where $K_{\mathrm{ox}_{\mathrm{e}}}$ is the oxidizer concentration in the free stream and $\mathrm{O} / \mathrm{F}$ is the local oxidizer to fuel ratio at the flame [6]. For the equi-diffusive case, the blowing parameter also proves to be a similarity parameter of the boundary layer. In other words, when $\operatorname{Le}=\operatorname{Pr}=1$ and $B=$ const., the velocity, species concentration, and enthalpy profiles become similar everywhere [2,6]. In a follow-up paper, Marxman [7] derived the following expression for the blowing correction

$$
\frac{C_{f}}{C_{f_{0}}}=\left[\frac{\ln (1+B)}{B}\right]^{0.8}\left[\frac{1+\frac{13}{10} B+\frac{4}{11} B^{2}}{(1+B)\left(1+\frac{1}{2} B\right)^{2}}\right]^{0.2},
$$

which he simplified, using curve fitting, into

$$
\frac{C_{f}}{C_{f_{0}}}=1.2 B^{-0.77} \text {. }
$$

Much academic debate has surrounded the proper representation of the blowing correction. In his original presentation, Marxman [7] acknowledged the form derived in Lees [9] using thin-film theory; particularly,

$$
\frac{C_{f}}{C_{f_{0}}}=\frac{\ln (1+B)}{B} .
$$

Marxman concluded that, since mass addition is discounted in the construction of Equation (17), it can only be expected to produce reasonable results for low values of the blowing parameter $B$. Marxman [7] then derived the equations most often used in the context of hybrids-namely, Equations (15) and (16)—using Prandtl's mixing length hypothesis combined with von Kármán's momentum integral analysis. Details of this derivation may be found in Appendix A. These expressions lead to better agreement with experiments at high mass injection rates. The improvement in accuracy when moving from Equation (17) to Equation (15) is illustrated in Figure 2 using the experimental data reported by Mickley and Davis [12] as well as Tewfick [13].

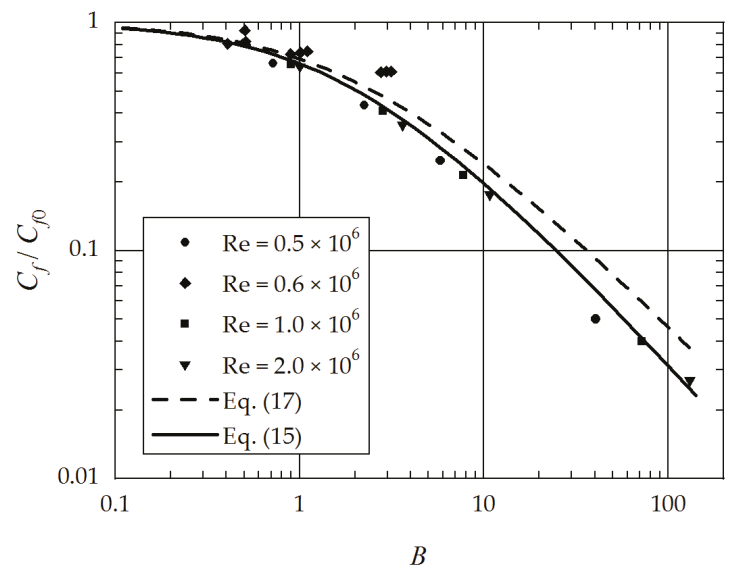

Figure 2. Comparison of blowing correction formulations expressed over a range of blowing parameters at several Reynolds numbers. Data taken from [12,13]. 
A decade later, Lengelle [14] revisited some of Marxman's assumptions on eddy diffusivity and developed a modified form of the blowing correction. Both derivations were later shown to display inconsistencies with experimental measurements, as well as an exact solution developed by Karabeyoglu [15] under similar conditions. Specifically, Marxman's expression for the skin friction coefficient did not reduce to the accepted value in the absence of blowing, although his analysis yielded a blowing correction trend that stood in better agreement with experimental measurements. Meanwhile, Lengelle's expressions fell closer to the analytical solution for skin friction but overestimated the blowing correction when compared to experiments. Based on this realization, Karabeyoglu set about deriving a new expression that did not rely on the same assumptions drawn from Prandtl's mixing length hypothesis. He arrived at an expression for the blowing correction that was nearly identical to Equation (15) of Marxman [7], albeit with a more self-consistent expression for the skin friction coefficient. In the end, Karabeyoglu suggested the use of a simplified exponential expression and pointed out that Altman [4] had already shown that a simple power-law relation remained a more accurate representation than Equation (16) over a practical range of interest. This relation is simply

$$
\frac{C_{f}}{C_{f_{0}}}=B^{-0.68} .
$$

Nonetheless, by substituting Marxman's simplified blowing correction, Equation (16), into the regression rate expression given by Equation (13), one gets

$$
\rho_{\mathrm{f}} \dot{r}=0.036 \rho_{\mathrm{e}} u_{\mathrm{e}} \operatorname{Re}_{x}^{-0.2} B^{0.23}
$$

or, in terms of the mass flux $G \equiv \rho_{\mathrm{e}} u_{\mathrm{e}}$,

$$
\rho_{\mathrm{f}} \dot{r}=0.036 G\left(\frac{G x}{\mu_{\mathrm{e}}}\right)^{-0.2} B^{0.23} .
$$

We, thus, arrive at Marxman's form of the local regression rate in the absence of radiation, although a more commonly seen result may be obtained by consolidating the constant properties in Equation (20) to produce the compact relation

$$
\dot{r}=A G^{0.8} x^{-0.2},
$$

where $A$ remains approximately invariant for a given propellant. Note that the space-time averaged equivalent of Equation (21) is often used by researchers reporting experimental measurements, specifically,

$$
\dot{r}=m G^{n} .
$$

In the above, combining $B$ with the other constants is justified by noting that, as long as $\Delta h / h_{v}$ does not change with $\operatorname{Re}_{x}$, then the distribution of the regression rate will be such that $B$ remains spatially uniform [6]. Even when this is not strictly the case, $B$ is expected to display very small variations along the length of the grain. The small exponent of $B$ further ensures that even large changes in $\Delta h$ or $h_{v}$ will have negligible effects on the overall regression rate. Another consequence of this relation is that values of $B$ calculated for one propellant can be extended, with reasonable accuracy, to other propellants, so long as they share similar compositions and conditions that justify ignoring radiation effects. The relation also suggests that any possible oxidative reactions at the fuel surface that alter $\Delta h / h_{v}$ from its idealized value will have a minor effect on the regression rate (see the reply to Rosner's comment in [7] for context and elaboration on the significance of this implication).

Before leaving this subject, it may be helpful to reflect on several regression rate characteristics that may be gleaned from Equation (20). First and foremost, one notes the absence of any pressure dependence in the expression for $\dot{r}$. While this behavior remains contingent on discarding radiative effects, an assumption which will be later examined in more depth, it proves to be surprisingly 
accurate for many systems. Physically, the lack of a pressure dependence may be attributed to the diffusion-flame burning response, which stands as the single most defining characteristic for hybrids. In fact, one may argue that a mass flux-controlled motor offers distinct advantages over a pressure-dependent solid motor. Designers are now permitted more freedom in choosing a motor operating pressure which helps to achieve a desired target performance or meet certain safety requirements, which can be particularly useful in research and adoption in academic settings. Another feature captured in Equation (20) consists of the axial variation in regression rate, which encompasses both a positive correlation with axial distance as fuel injection increases the local mass flux (i.e., the flux increases at locations further downstream as more fuel mass is added to the flow), and a negative correlation with axial distance, which may be associated with boundary layer growth and the corresponding decrease in the skin friction coefficient and, thus, the heat flux, in conformance with the Reynolds analogy. These competing factors result in an equilibrium location for the minimum regression rate that shifts downstream with the passage of time.

\section{Radiation}

Up to this point, several pressure-dependent mechanisms that can influence the regression rate in a hybrid motor have been ignored. Foremost among them is radiative heat transfer, which can contribute significantly to the overall fuel regression behavior, depending on the motor operating conditions and fuel composition. Although some researchers have found that the purely convective heat transport equations remain the most reliable for hydrocarbon fuels [16], Marxman and Gilbert recognized the potential importance of radiative heat transfer immediately and included a crude treatment of the subject in their initial investigation [5], specifically by adding a grey-body radiation term to the regression rate expression described above. They wrote

$$
\rho_{\mathrm{f}} \dot{r}=0.036 G\left(\frac{G x}{\mu_{\mathrm{e}}}\right)^{-0.2} B^{0.23}+\frac{\sigma \epsilon_{\mathrm{w}}\left(\epsilon_{\mathrm{g}} T_{\mathrm{b}}^{4}-T_{\mathrm{w}}^{4}\right)}{h_{v}},
$$

where $\epsilon_{\mathrm{g}}$ denotes the emissivity of the gas, while $\epsilon_{\mathrm{W}}$ refers to the emissivity of the wall and $\sigma$ stands for the Stefan-Boltzmann constant. However, since this expression neglects the strong coupling between radiation and the blocking effect, it does not agree well with experiments. Marxman et al. [6] addressed this coupling by representing the radiative contribution in two equivalent ways: On one hand, applying a correction factor to the blowing parameter in Equation (20) results in the modifier $\left(B_{\mathrm{rad}} / B\right)^{0.23}$ that accounts for the entire effect of radiation in one simple term:

$$
\rho_{\mathrm{f}} \dot{r}=0.036 G\left(\frac{G x}{\mu_{\mathrm{e}}}\right)^{-0.2} B^{0.23}\left(\frac{B_{\mathrm{rad}}}{B}\right)^{0.23} .
$$

On the other hand, the effect of radiation may be included more explicitly by recognizing that, to account for coupling, Equation (23) need only be adjusted by modifying the blowing correction in the convective term, such that

$$
\rho_{\mathrm{f}} h_{v} \dot{r}=\dot{Q}_{\mathrm{c}}\left(\frac{B_{\mathrm{rad}}}{B}\right)^{-0.77}+\dot{Q}_{\mathrm{rad}}
$$

where

$$
\dot{Q}_{\mathrm{c}}=0.036 G h_{v} R e_{x}^{-0.2} B^{0.23} \quad \text { and } \quad \dot{Q}_{\mathrm{rad}}=\sigma \epsilon_{\mathrm{w}}\left(\epsilon_{\mathrm{g}} T_{\mathrm{b}}^{4}-T_{\mathrm{w}}^{4}\right) .
$$

Combining Equations (24) and (25) leads to the correction factor

$$
\frac{B_{\mathrm{rad}}}{B}=1+\frac{\dot{Q}_{\mathrm{rad}}}{\dot{Q}_{\mathrm{c}}}\left(\frac{B_{\mathrm{rad}}}{B}\right)^{0.77}
$$


Although this equation cannot be solved explicitly, its solution can be approximated adequately using an expression provided in [6],

$$
\frac{B_{\mathrm{rad}}}{B}=\exp \left(\frac{1.3 \dot{Q}_{\mathrm{rad}}}{\dot{Q}_{\mathrm{c}}}\right)
$$

which, when substituted back into Equation (25), yields a simple, closed-form approximation, specifically

$$
\rho_{\mathrm{f}} \dot{r}=\left[\dot{Q}_{\mathrm{c}} \exp \left(-\dot{Q}_{\mathrm{rad}} / \dot{Q}_{\mathrm{c}}\right)+\dot{Q}_{\mathrm{rad}}\right] / h_{v}
$$

If more accuracy is desired, a three-term asymptotic approximation may be used; namely,

$$
\frac{B_{\mathrm{rad}}}{B}=\exp \left[\left(\frac{\dot{Q}_{\mathrm{rad}}}{\dot{Q}_{\mathrm{c}}}\right)+0.27\left(\frac{\dot{Q}_{\mathrm{rad}}}{\dot{Q}_{\mathrm{c}}}\right)^{2}+0.06768\left(\frac{\dot{Q}_{\mathrm{rad}}}{\dot{Q}_{\mathrm{c}}}\right)^{3}\right]
$$

Equation (29) proves to be in closer agreement with the exact solution than Equation (27). If simplicity is desired, an alternate one-term approximation may be used that remains more accurate than Equation (27) as long as radiation contributes no more than two-thirds of the convective heat flux. This expression is

$$
\frac{B_{\mathrm{rad}}}{B}=\exp \left(\frac{1.13727 \dot{Q}_{\mathrm{rad}}}{\dot{Q}_{\mathrm{c}}}\right)
$$

In the interest of clarity, the different solutions for the radiation correction are plotted in Figure 3 along with the numerical solution to Equation (26). Note that each approximation has its own merits: While being the least accurate, Equation (27) remains adequate over the entire domain and results in unitary coefficients, as per Equation (28). Equation (29) remains the most accurate over the entire domain, and Equation (30) remains nearly indiscernible from the numerical solution for smaller radiative contributions while remaining simple to apply, as long as $\dot{Q}_{\mathrm{rad}} / \dot{Q}_{\mathrm{c}} \leq 2 / 3$. All these expressions, however, correspond to Marxman's curve-fitted model for the blowing correction given by Equation (16). Naturally, a different form of $C_{f} / C_{f_{0}}$ would lead to a slightly dissimilar set of expressions for $B_{\mathrm{rad}} / B$.
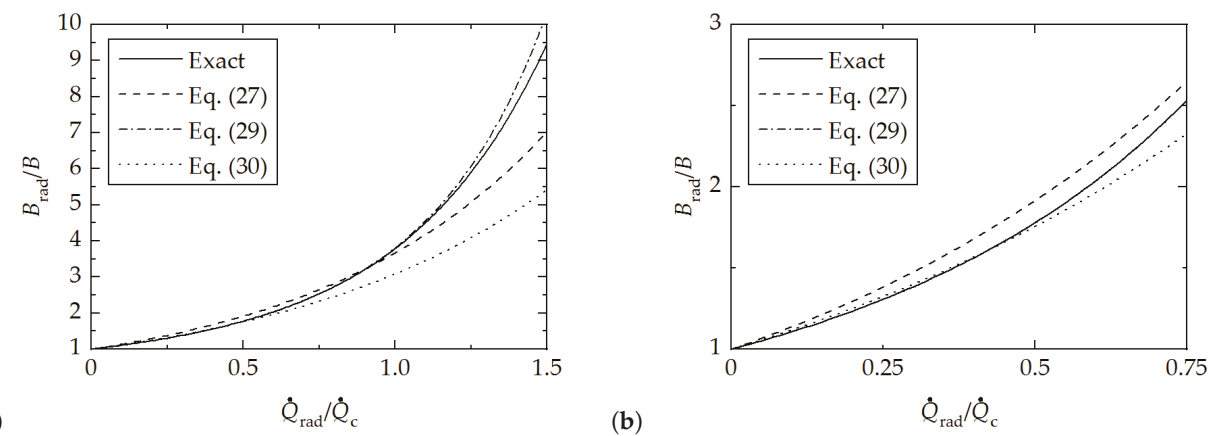

Figure 3. Comparison of different expressions for the radiation correction shown (a) over a wide range of heat flux ratios, and (b) over a more practical range.

For systems with low values of $\dot{Q}_{\text {rad }} / \dot{Q}_{c}$, Marxman et al. [6] note that the tradeoff between the new terms in Equation (28) is "nearly exact, and $\dot{r}$ can be calculated with little error by using $\dot{Q}_{\mathrm{c}}$ alone". If $\dot{Q}_{\text {rad }}=\dot{Q}_{c}$, then Equation (28) predicts that approximately three quarters of the heat actually transferred to the wall will be due to radiation but that $\dot{r}$ will be only $35 \%$ higher than the case with no radiation. While useful for illustrative purposes, this result should be treated with care: If the majority of heat transferred is radiative, the dependencies on geometry will no longer be the same as 
those already considered for the purely convective case. Furthermore, the accuracy of Equation (28) degrades rapidly once radiative heat flux overtakes that due to convection, as evidenced in Figure 3.

In a paper published three years later, Marxman [17] added further insight into the radiation problem. He proceeded to classify three general situations where radiation becomes appreciable:

1. Grains containing particles that react incompletely in the flame to the extent of producing solid or liquid products (e.g., metallized fuels);

2. grains that naturally produce solid or liquid products beyond the flame (e.g., carbon-heavy or sooty fuels); and

3. propellants whose gas-phase combustion products produce appreciable radiation.

All three cases may be described by similar expressions, with the primary difference being in how the number density of radiating particles must be treated. Furthermore, the heat transfer in all three cases becomes dependent on the chamber pressure through the particle number density. Marxman noted that, for the first two cases, the most appropriate effective optical path length will likely be the distance from the surface to the flame zone, since the particle phase absorptivity will be high beyond that point. For the third case, he posited that the optical path length will depend on a characteristic chamber dimension, such as the diameter. Although Marxman's group worked primarily with PMMA-O $\mathrm{O}_{2}$ systems with negligible radiation, other experiments with hydroxyl-terminated polybutadiene (HTPB) grains have confirmed that radiation effects indeed become significant at higher motor pressures and lower oxidizer mass fluxes. To further illustrate this behavior, Figure 4 is used to display the radiation dependence obtained in one such investigation by Chiaverini et al. [18]. Note that test 15 was performed at a higher pressure than test 17 and thus displayed a stronger variance from the values predicted by Marxman's diffusion-limited, convection-driven model. Both data sets agree with the theory at higher mass fluxes, where convection dominates the heat transfer process to the extent of justifying the use of a non-radiative model.

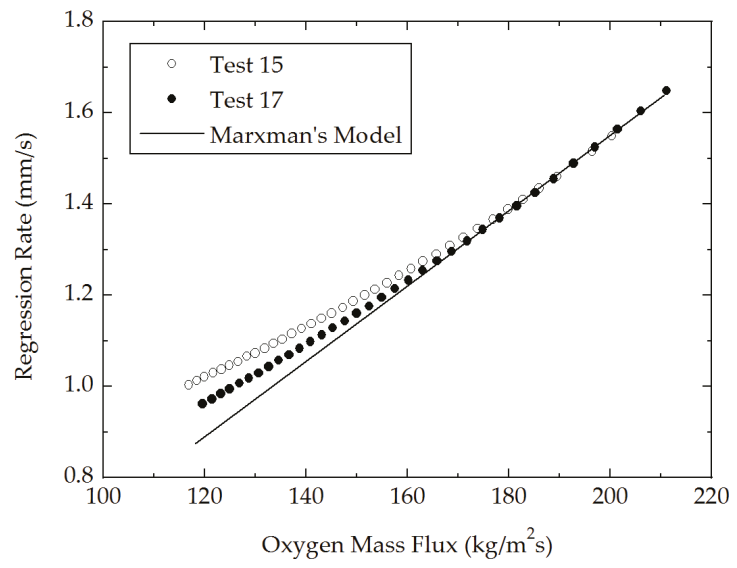

Figure 4. Effect of thermal radiation on hydroxyl-terminated polybutadiene (HTPB) regression-rate behavior. Data taken from [18].

\section{Other Non-Ideal Considerations}

In his 1965 paper, Marxman [7] provided a derivation for the regression rate, which parallels that of Marxman et al. [6], just described in detail, where the fundamental work of Lees [9] was revisited by 
relaxing the assumption that $\operatorname{Pr}=1$ to that of $\operatorname{Pr}=$ const. In this case, the thermochemical parameter $B$ becomes different from the aerodynamic similarity parameter $B^{\prime}$, according to

$$
B=B^{\prime} \operatorname{Pr}^{0.67}
$$

at the outset, Equation (20) becomes

$$
\rho_{\mathrm{f}} \dot{r}=0.036 G\left(\frac{G x}{\mu_{\mathrm{e}}}\right)^{-0.2} B^{0.23} \operatorname{Pr}^{-0.15} .
$$

As Pr is close to unity for most gases, even at high temperatures, the effect of $\operatorname{Pr}^{-0.15}$ remains sufficiently small to render the correction unnecessary in most cases. The same study by Marxman [7] separately addressed the assumption of uniform gas density between the flame and the wall by using a Howarth-Dorodnitsyn variable transformation to relate the actual, variable-property turbulent boundary layer to an equivalent incompressible boundary layer, through

$$
\rho_{\mathrm{f}} \dot{r}=0.036 \mathrm{G}\left(\frac{\rho_{0}}{\rho_{\mathrm{e}}}\right)^{0.6}\left(\frac{G x}{\mu_{\mathrm{e}}}\right)^{-0.2} B^{0.23},
$$

where the reference state ratio $\rho_{0} / \rho_{\mathrm{e}}$ is determined from a semi-empirical relation, described in terms of previously defined parameters and the boundary layer thickness $\delta$, which must be measured experimentally. Due to the added complexity, the variable-density correction is seldom used.

\section{Kinetics-Limited Models}

The importance of kinetics, both homogeneous and heterogeneous, has long been the subject of debate. As previously mentioned, Marxman [7] received criticism for neglecting heterogeneous surface reactions but argued that the effects of reactions below the flame on the values of $\Delta h$ and $h_{v}$ remained of secondary importance. However, several researchers have developed models and correlations in which kinetics played a major role. A selection of these, loosely grouped according to their dominant physical mechanism, is overviewed here as a starting point for further reading. This cursory coverage is not intended to be comprehensive, in scope nor in detail.

The first group of researchers are those who were primarily concerned with gas-phase kinetics. For example, Wooldridge et al. [19] studied pressure sensitivity at low pressures in an attempt to characterize the stability characteristics of hybrids. This effort entailed the development of an analytical expression for the regression rate as a function of several kinetic parameters and length-scales. Miller [20] developed a model that incorporated both the fuel diffusion rate and the chemical reaction rates, which he used to successfully correlate data taken by Smoot and Price [21-23]. Kosdon and Williams [24] later noted that Miller's analysis was only applicable to systems with low pressure and moderate oxidizer fluxes and derived a new expression that incorporated a flame zone of finite thickness. Wooldridge and Muzzy [25] examined the effects of scaling and pressure on motor performance in the context of throttling. In his 1972 article, Muzzy [26] pointed out that even simple PMMA-O 2 motors behaved differently at low pressures and that as the motor approached a flooding condition, combustion likely became kinetically-limited. By reviewing existing test data, he formed an explicitly pressure-dependent correlation which could be likened to that of Wooldridge et al. [19], specifically

$$
\dot{r} \propto P^{0.5} G^{0.3} x^{-0.2} .
$$

Another group of researchers focused more on heterogeneous surface reactions as the source of kinetics dependence. Smoot and Price [21-23] performed numerous tests using a slab burner and found that, above a threshold value of $G$, the regression rate became nearly independent of $G$ and instead varied with $P$ and the oxidizer composition. These researchers defined a low mass flux regime where the normal $G^{0.8}$ dependence held, an intermediate mass flux regime where both $P$ and $G$ played 
a role, and a third regime at very high mass fluxes where the regression rate varied with pressure, as in the case of a solid rocket propellant; namely,

$$
\dot{r} \propto P^{n} .
$$

Kumar and Stickler [27] cited discrepancies in hybrid test data and pyrolysis tests in an inert environment for PMMA to support their hypothesis that heterogeneous reactions must play a significant role in regression rate estimations. They additionally argued that the gas-phase kinetics could not be sufficiently slow to play a consequential role. They developed a correlation that normalized the regression rate by its maximum value before pressure effects appeared in a manner to make it possible to predict whether a particular set of conditions was kinetically or diffusively limited, in addition to successfully matching experimental data. More recently, Favaro et al. [28] performed experiments designed to illuminate the role of heterogeneous reactions, thus leading to the development of a semi-empirical model.

Despite the number of investigations into kinetically-limited operating regimes, Marxman's model and the assumptions that it prescribed have become the de facto standard for normal applications. This does not mean, however, that experts in the field have reached a definitive agreement on how to properly model regression in all situations. While modern computational studies are increasingly contributing to the understanding of diffusion-limited combustion in ways that may be less restrictive, Marxman's model is still commonly used to guide the design of hybrid systems and as a basis for the development of further analytical treatments of geometrically or thermophysically more complex hybrids, such as those incorporating swirl or liquefying fuels.

\section{Summary}

The key points that would be useful to remember by those interested in hybrid rocket regression rate modeling and its practical implementation may be summarized as follows:

- Marxman's theory is based on the assumption that the diffusion of oxidizer and fuel into the flame establishes the rate-limiting process in hybrid combustion and leads to adequate predictions at the moderate pressures and mass fluxes that accompany the steady-state operation of most hybrid motors.

- For classical, non-metallized hybrids, the fuel regression rate depends on $G^{0.8}$, but not on pressure.

- Radiation appears as a secondary correction, but should not be neglected at low mass fluxes or for metallized or other heavily sooting fuels.

- Most of the improvements to the initial diffusion-limited model, besides radiation treatment, lead to rather small corrections to the end result and may typically be ignored.

- Numerous alternate regression rate models have been formulated to be more accurate for kinetically-limited operating conditions.

For those seeking more information or a deeper understanding of regression rate models, the seminal reviews of the subject by Netzer [2] and Chiaverini [3] are suggested, along with Marxman's 1965 paper [7]. Improvements and extensions to regression rate models in novel and emerging hybrid configurations continue today and it is hoped that this cursory review will prove useful to researchers entering the field and seeking to describe the internal workings of these new and exciting hybrid motors.

Author Contributions: Writing-Original Draft Preparation, T.M.; Writing-Review and Editing, J.M.

Funding: This project was funded in part by the National Science Foundation and, in part, by NASA JPL RSA No. 1573777, Task No. R.17.021.119.

Acknowledgments: The authors wish to thank Dr. Elizabeth T. Jens and Dr. Ashley C. Karp of the NASA Jet Propulsion Laboratory for supporting elements of this work.

Conflicts of Interest: The authors declare no conflict of interest. 


\section{Nomenclature}

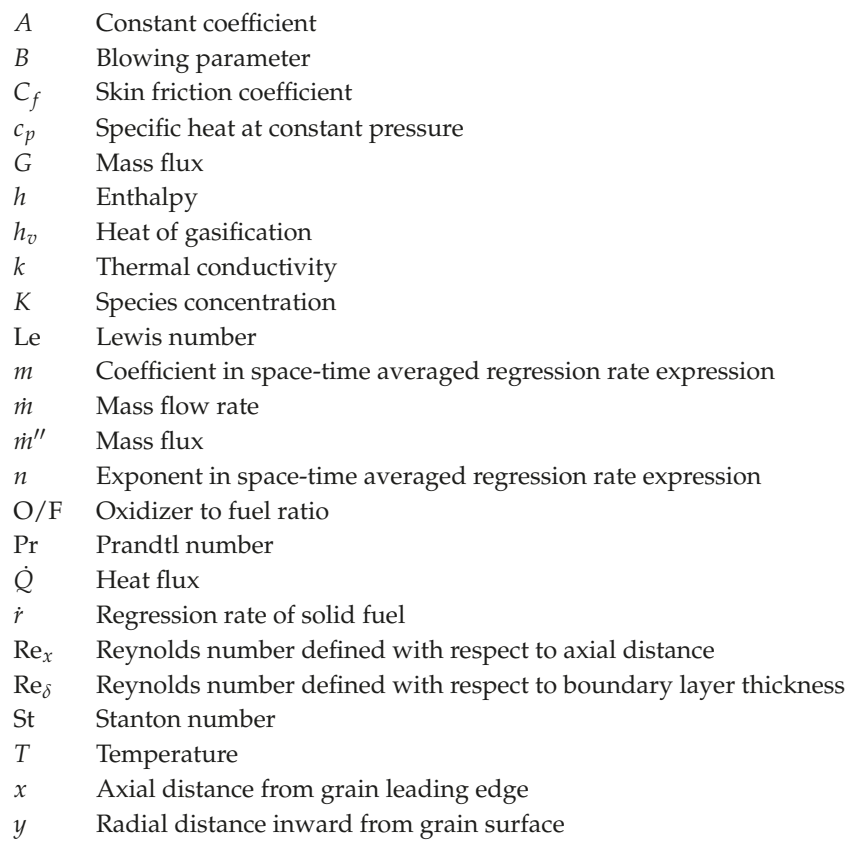

\section{Greek Symbols}

$\delta \quad$ Boundary layer thickness

$\epsilon \quad$ Emissivity or turbulent eddy diffusivity

$\eta \quad$ Nondimensional radial distance in boundary layer

$\theta$ Momentum thickness of boundary layer

$\mu \quad$ Dynamic viscosity

$\rho$ Density

$\sigma$ Stefan-Boltzmann constant

$\tau$ Shear stress

$\phi \quad$ Nondimensional axial velocity in boundary layer

\section{Subscripts}

b Burned gas (flame zone) value

c Convective

e Boundary layer edge (core flow) value

f Solid fuel value

ox Oxidizer value

rad Radiative

T Total

w Wall value

o Reference value 


\section{Appendix A. Derivation of the Blowing Correction}

Close to the wall, $u \approx 0$ and $\partial u / \partial x \approx 0$, so that continuity becomes $\rho v=\rho_{\mathrm{w}} v_{\mathrm{w}}$ and, in the absence of an axial pressure gradient, the momentum equation can be written as

$$
\tau=\tau_{\mathrm{w}}+\rho_{\mathrm{w}} v_{\mathrm{w}} u
$$

Substitution of $B=2\left(\rho_{\mathrm{w}} v_{\mathrm{w}}\right) /\left(\rho_{\mathrm{e}} u_{\mathrm{e}} C_{f}\right)$ and $\tau_{\mathrm{w}}=\frac{1}{2} C_{f} \rho_{\mathrm{e}} u_{\mathrm{e}}^{2}$ into Equation (A1) leads to

$$
\tau=\tau_{\mathrm{w}}(1+B \phi),
$$

where $\phi \equiv u / u_{\mathrm{e}}$. Next, we equate Equation (A2) to the definition of shear stress for a turbulent boundary layer; namely,

$$
\tau=(\mu+\rho \epsilon) \frac{\partial u}{\partial y}
$$

where $\epsilon$ represents the turbulent eddy diffusivity. This produces

$$
\tau_{\mathrm{w}}(1+B \phi)=(\mu+\rho \epsilon) \frac{\partial u}{\partial y} .
$$

At this juncture, switching to non-dimensional forms may be achieved using $\operatorname{Re}_{\delta} \equiv \rho_{\mathrm{e}} u_{\mathrm{e}} \delta / \mu$, $\eta=y / \delta, \phi$, and $\tau_{\mathrm{w}}$. We get

$$
\frac{1}{2} C_{f}(1+B \phi)=\operatorname{Re}_{\delta}^{-1}\left(1+\frac{\rho \epsilon}{\mu}\right) \frac{\partial \phi}{\partial \eta} .
$$

According to Prandtl's mixing length concept, $\epsilon \propto \eta^{2} \partial \phi / \partial \eta$, where, for the purpose of estimating $\epsilon$, a power law profile (such as $\phi=\eta^{n}$ ) may be used for the range of Reynolds numbers typical of hybrid motors [15]. Then, $\partial \phi / \partial \eta$ may be approximated as $n \eta^{n-1}$. Furthermore, since $n$ is usually small $(\sim 1 / 7)$, the eddy diffusivity becomes

$$
\epsilon \approx c \eta^{1+n} \approx c \eta,
$$

where $c$ represents a constant that is proportional to the mixing length-its exact value proves immaterial to this analysis. Substituting Equation (A6) into Equation (A5) yields

$$
\frac{1}{2} C_{f}(1+B \phi)=\operatorname{Re}_{\delta}^{-1}\left(1+\frac{\rho}{\mu} c \eta\right) \frac{\partial \phi}{\partial \eta} .
$$

Note that integration from the wall to the boundary layer edge (i.e., from 0 to 1 for both $\eta$ and $\phi$ ), leads to an expression for the skin friction coefficient as a sole function of $\operatorname{Re}_{\delta}$ and $B$ :

$$
\frac{1}{2} C_{f}=g\left(\operatorname{Re}_{\delta}\right) \frac{\ln (1+B)}{B} .
$$

Marxman [7] argued that, since the Reynolds number and blowing parameter dependencies can be distinctly separated in the expression for $C_{f}$, the functional form of $g\left(\operatorname{Re}_{\delta}\right)$ may be obtained by comparing Equation (A8) to known results in the absence of blowing; namely,

$$
\frac{1}{2} C_{f_{0}}=g\left(\operatorname{Re}_{\delta}\right)=0.0225 \operatorname{Re}_{\delta}^{-0.25},
$$

where a suitable empirical expression from Schlichting [10] is used. To generalize, we have

$$
\frac{1}{2} C_{f}=0.0225 \operatorname{Re}_{\delta}^{-0.25} \frac{\ln (1+B)}{B} .
$$


Evaluating Equation (A10) for the case of no blowing enables us to write

$$
\frac{C_{f}}{C_{f_{0}}}=\left(\frac{\delta_{0}}{\delta}\right)^{0.25} \frac{\ln (1+B)}{B},
$$

where $\left(\delta_{0} / \delta\right)$ accounts for the thickening effect of mass addition and differentiates Marxman's expression from that of Lees in Equation (17).

At this point, one is left with the task of determining a relation to describe $\delta$ in terms of $B$ in Equation (A11). Such a relation may be obtained by performing a control volume analysis of the boundary layer to deduce an appropriate momentum integral expression. A sketch of a suitable control volume is provided in Figure A1. In this context, a statement of mass conservation (per unit thickness) across the control volume leads to

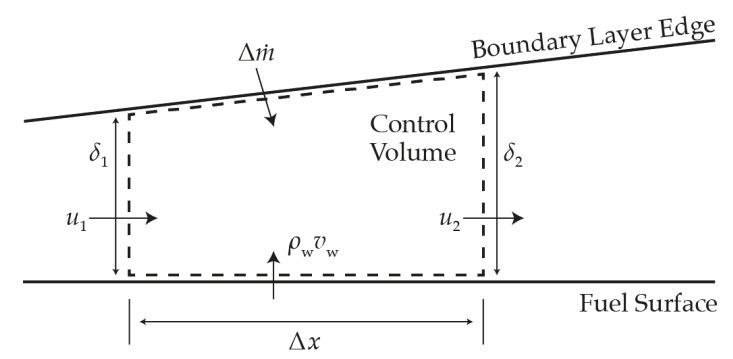

Figure A1. Sketch of the control volume for the momentum integral analysis.

$$
\Delta \dot{m}+\int_{0}^{\delta_{1}} \rho u_{1} \mathrm{~d} y-\int_{0}^{\delta_{2}} \rho u_{2} \mathrm{~d} y+\rho_{\mathrm{w}} v_{\mathrm{w}} \Delta x=0 .
$$

Similarly, a statement of axial momentum conservation yields

$$
u_{\mathrm{e}} \Delta \dot{m}+\int_{0}^{\delta_{1}} \rho u_{1}^{2} \mathrm{~d} y-\int_{0}^{\delta_{2}} \rho u_{2}^{2} \mathrm{~d} y-\tau_{\mathrm{w}} \Delta x=0 .
$$

Re-arranging and non-dimensionalizing different terms may be used to produce

$$
\begin{aligned}
& \text { Mass: } \quad \Delta \dot{m}+\frac{1}{2} C_{f} \rho u_{\mathrm{e}} B \Delta x=\rho u_{\mathrm{e}} \delta_{2} \int_{0}^{1} \phi \mathrm{d} \eta-\rho u_{\mathrm{e}} \delta_{1} \int_{0}^{1} \phi \mathrm{d} \eta \\
& \frac{\Delta \dot{m}}{\rho u_{\mathrm{e}} \Delta x}+\frac{1}{2} C_{f} B=\frac{\delta_{2}-\delta_{1}}{\Delta x} \int_{0}^{1} \phi \mathrm{d} \eta \text {. } \\
& \text { Momentum: } \quad u_{\mathrm{e}} \Delta \dot{m}+\frac{1}{2} C_{f} \rho u_{\mathrm{e}}^{2} \Delta x=\rho u_{\mathrm{e}}^{2} \delta_{2} \int_{0}^{1} \phi^{2} \mathrm{~d} \eta-\rho u_{\mathrm{e}}^{2} \delta_{1} \int_{0}^{1} \phi^{2} \mathrm{~d} \eta \\
& \frac{\Delta \dot{m}}{\rho u_{\mathrm{e}} \Delta x}-\frac{1}{2} C_{f}=\frac{\delta_{2}-\delta_{1}}{\Delta x} \int_{0}^{1} \phi^{2} \mathrm{~d} \eta \text {. }
\end{aligned}
$$

By combining the expressions for conservation of mass, Equation (A14), and momentum, Equation (A15), we collect

$$
\frac{\delta_{2}-\delta_{1}}{\Delta x}=\frac{1}{2} C_{f} \frac{1+B}{\int_{0}^{1} \phi(1-\phi) \mathrm{d} \eta}=\frac{1}{2} C_{f} \frac{1+B}{\beta},
$$


where $\beta=\int_{0}^{1} \phi(1-\phi) \mathrm{d} \eta$ is related to the momentum thickness of the boundary layer by $\beta=\theta / \delta$. In the limit as $\Delta x \rightarrow 0$, the control volume becomes infinitesimal, such that

$$
\frac{\mathrm{d} \delta}{\mathrm{d} x}=\frac{1}{2} C_{f} \frac{1+B}{\beta} .
$$

Substituting the expression for the skin friction, found earlier as Equation (A10), into Equation (A17), we get

$$
\frac{\mathrm{d} \delta}{\mathrm{d} x}=0.0225 \operatorname{Re}_{\delta}^{-0.25} \frac{(1+B) \ln (1+B)}{\beta B}=0.0225 \frac{(1+B) \ln (1+B)}{\beta B}\left(\frac{\rho u_{\mathrm{e}}}{\mu}\right)^{-0.25} \delta^{-0.25} .
$$

Subsequent integration over the boundary layer yields

$$
\frac{\delta}{x}=\left[0.02813 \frac{(1+B) \ln (1+B)}{\beta B}\right]^{0.8} \operatorname{Re}_{x}^{-0.2}
$$

and so

$$
\frac{\delta_{0}}{\delta}=\left[\frac{\beta}{\beta_{0}} \frac{B}{(1+B) \ln (1+B)}\right]^{0.8} .
$$

Finally, combining Equation (A20) with Equation (A11) leads to an expression for the blowing correction in terms of the blowing parameter, specifically

$$
\frac{C_{f}}{C_{f_{0}}}=\left[\frac{\beta}{\beta_{0}} \frac{B}{(1+B) \ln (1+B)}\right]^{0.2} \frac{\ln (1+B)}{B}=\left[\frac{\beta}{\beta_{0}} \frac{1}{(1+B)}\right]^{0.2}\left[\frac{\ln (1+B)}{B}\right]^{0.8},
$$

where $\beta$ depends on the velocity profile $\phi$, which may be approximated relatively easily. Starting from Equation (A4), it is trivial to show that

$$
\frac{\partial \phi}{\partial \eta}=\frac{\tau_{\mathrm{w}} \delta}{u_{\mathrm{e}}(\mu+\rho \epsilon)}(1+B \phi)=f(y, B)(1+B \phi) .
$$

When $\beta=0$, we may again assume a power law profile $\phi=\eta^{n}$ such that $\partial \phi / \partial \eta=n \eta^{n-1}$, which simplifies the unknown function $f(y, B)$ to the assumed form $f(B) n \eta^{n-1}$ with the requirement that $f(0)=1$. For $n=1 / 7$, Equation (A22) becomes

$$
\frac{\partial \phi}{\partial \eta}=f(B) \eta^{-6 / 7}\left(1+B \eta^{1 / 7}\right) .
$$

Integrating Equation (A23) and evaluating the result at the boundaries, as with Equation (A7), leads to

$$
\phi=\frac{\eta^{1 / 7}\left(1+\frac{1}{2} B \eta^{1 / 7}\right)}{1+\frac{1}{2} B} .
$$

Finally, the expression for $\phi$ allows for the evaluation of $\beta$ from its definition:

$$
\beta=\frac{7\left(1+\frac{13}{10} B+\frac{4}{11} B^{2}\right)}{72\left(1+\frac{1}{2} B\right)^{2}} .
$$


It is clear that, in the case with no blowing $(B=0), \beta$ reduces to $\beta_{0}=7 / 72$, such that

$$
\frac{\beta}{\beta_{0}}=\frac{\left(1+\frac{13}{10} B+\frac{4}{11} B^{2}\right)}{\left(1+\frac{1}{2} B\right)^{2}} \text {. }
$$

\section{References}

1. Altman, D. Hybrid Rocket Development History. In Proceedings of the 27th AIAA/ASME/SAE/ASEE Joint Propulsion Conference and Exhibit, Sacramento, CA, USA, 24-26 June 1991. [CrossRef].

2. Netzer, D.W. Hybrid Rocket Internal Ballistics; Technical Report; Chemical Propulsion Information Agency: Silver Spring, MD, USA, 1972.

3. Chiaverini, M.J. Review of Solid-Fuel Regression Rate Behavior in Classical and Nonclassical Hybrid Rocket Motors. In Fundamentals of Hybrid Rocket Combustion and Propulsion; Chiaverini, M.J., Kuo, K.K., Eds.; American Institute of Aeronautics and Astronautics: Reston, VA, USA, 2007; Chapter 2; Volume 218, pp. 37-126.

4. Altman, D.; Humble, R. Space Propulsion Analysis and Design; McGraw-Hill Book Company: New York, NY, USA, 1995; Chapter 7; pp. 365-442.

5. Marxman, G.; Gilbert, M. Turbulent Boundary Layer Combustion in the Hybrid Rocket. Ninth Symp. Combust. 1963, 9, 371-383. [CrossRef]

6. Marxman, G.A.; Wooldridge, C.E.; Muzzy, R.J. Fundamentals of Hybrid Boundary Layer Combustion. Prog. Aeronaut. Astronaut. 1964, 15, 485-522. [CrossRef]

7. Marxman, G.A. Combustion in the Turbulent Boundary Layer on a Vaporizing Surface. Tenth Symp. Combust. 1965, 10, 1337-1349. [CrossRef]

8. Marxman, G.A.; Wooldridge, C.E. Research on the Combustion Mechanism of Hybrid Rockets. In Advances in Tactical Rocket Propulsion, AGARD Conference Proceedings No. 1; Technivisions Services: London, UK, 1968; pp. 421-477.

9. Lees, L. Convective Heat Transfer with Mass Addition and Chemical Reactions. In Combustion and Propulsion-Third AGARD Colloquium: Noise-Shock Tubes-Magnetic Effects-Instability and Mixing; Pergamon Press: New York, NY, USA, 1958; p. 451.

10. Schlichting, H. Boundary-Layer Theory, 7th ed.; Kestin, J., Trans.; McGraw-Hill Book Company: New York, NY, USA, 1979.

11. Knuth, W.; Chiaverini, M.; Gramer, D.; Sauer, J. Solid-Fuel Regression Rate and Combustion Behavior of Vortex Hybrid Rocket Engines. In Proceedings of the 35th AIAA/ASME/SAE/ASEE Joint Propulsion Conference and Exhibit, Los Angeles, CA, USA, 20-24 June 1999. [CrossRef]

12. Mickley, H.S.; Davis, R.S. Momentum Transfer for Flow Over a Flat Plate with Blowing; Technical Report, NACA TN-4017; National Advisory Committee for Aeronautics: Washington, DC, USA, 1957.

13. Tewfick, O.E. Some Characteristics of the Turbulent Boundary Layer with Air Injection. AIAA J. 1963, 1, 1306-1312. [CrossRef]

14. Lengelle, G. Model Describing the Erosive Combustion and Velocity Response of Composite Propellants. AIAA J. 1975, 13, 315-322. [CrossRef]

15. Karabeyoglu, M.A. Transient Combustion in Hybrid Rockets. Ph.D. Thesis, Stanford University, Stanford, CA, USA, 1998.

16. Estey, P.; Altman, D.; McFarlane, J. An Evaluation of Scaling Effects for Hybrid Rocket Motors. In Proceedings of the 27th AIAA/ASME/SAE/ASEE Joint Propulsion Conference, Sacramento, CA, USA, 24-26 June 1991. [CrossRef]

17. Marxman, G.A. Boundary Layer Combustion in Propulsion. Elev. Symp. Combust. 1967, 11, 269-289. [CrossRef]

18. Chiaverini, M.J.; Serin, N.; Johnson, D.K.; Lu, Y.C.; Kuo, K.K.; Risha, G.A. Regression Rate Behavior of Hybrid Rocket Solid Fuels. J. Propuls. Power 2000, 16, 125-132. [CrossRef]

19. Wooldridge, C.E.; Marxman, G.A.; Kier, R.J. Investigation of Combustion Instability in Hybrid Rockets; Technical Report; National Aeronautics and Space Administration, Langley Research Center: Hampton, VA, USA, 1969. 
20. Miller, E. Hybrid Rocket Combustion Regression Rate Model. AIAA J. 1966, 4, 752-753. [CrossRef]

21. Smoot, L.D.; Price, C.F. Regression Rates of Nonmetalized Hybrid Fuel Systems. AIAA J. 1965, 3, 1408-1413. [CrossRef]

22. Smoot, L.D.; Price, C.F. Regression Rates of Metalized Hybrid Fuel Systems. AIAA J. 1966, 4, 910-915. [CrossRef]

23. Smoot, L.D.; Price, C.F. Pressure Dependence of Hybrid Fuel Regression Rates. AIAA J. 1966, 5, $102-106$. [CrossRef]

24. Kosdon, F.J.; Williams, F.A. Pressure Dependence of Nonmetalized Hybrid Fuel Regression Rates. AIAA J. 1967, 5, 774-778. [CrossRef]

25. Wooldridge, C.E.; Muzzy, R.J. Internal Ballistic Considerations in Hybrid Rocket Design. J. Spacecr. 1967, 4, 255-262. [CrossRef]

26. Muzzy, R.J. Applied Hybrid Combustion Theory. In Proceedings of the 8th Joint Propulsion Specialist Conference, New Orleans, LA, USA, 29 November-1 December 1972. [CrossRef]

27. Kumar, R.N.; Stickler, D.B. Polymer-Degradation Theory of Pressure-Sensitive Hybrid Combustion. Thirteen. Symp. Combust. 1971, 13, 1059-1072. [CrossRef]

28. Favaro, F.M.; Sirignano, W.A.; Manzoni, M.; DeLuca, L.T. Solid-Fuel Regression Rate Modeling for Hybrid Rockets. J. Propuls. Power 2013, 29, 205-215. [CrossRef]

(C) 2019 by the authors. Licensee MDPI, Basel, Switzerland. This article is an open access article distributed under the terms and conditions of the Creative Commons Attribution (CC BY) license (http:/ / creativecommons.org/licenses/by/4.0/). 
Article

\title{
Small-Scale Static Fire Tests of 3D Printing Hybrid Rocket Fuel Grains Produced from Different Materials
}

\author{
Mitchell McFarland and Elsa Antunes* \\ College of Science and Engineering, James Cook University, 1 James Cook Drive, Townsville, \\ QLD 4811, Australia \\ * Correspondence: elsa.antunes1@jcu.edu.au
}

Received: 1 March 2019; Accepted: 10 July 2019; Published: 15 July 2019

\begin{abstract}
The last decade has seen an almost exponential increase in the number of rocket launches for sounding missions or for delivering payloads into low Earth orbits. The emergence of new technologies like rapid prototyping, including 3D printing, is changing the approach to rocket motor design. This project conducted a series of small-scale static fire tests of fused deposition manufacturing hybrid rocket motors that were designed to explore the performance of a variety of commonly available fused deposition manufacturing materials. These materials included acrylonitrile butadiene styrene, acrylonitrile styrene acrylate, polylactic acid (PLA), polypropylene, polyethylene terephthalate glycol, Nylon, and AL (PLA with aluminum particles). To test the performance of small-scale fuel grains, a modular apparatus with a range of sensors fitted to it was designed and manufactured. The small-scale testing performed static burns on two fuel grains of each material with initial dimensions of $100 \mathrm{~mm}$ long and $20 \mathrm{~mm}$ in diameter with a $6 \mathrm{~mm}$ straight circular combustion port. The focus of this study was mainly on the regression rates of each material of fuel grains. Acrylonitrile styrene acrylate and Nylon showed the highest regression rates, while the polyethylene terephthalate glycol regression rates were relatively poor. Also, the acrylonitrile butadiene styrene and acrylonitrile styrene acrylate demonstrating relatively high regression rates when compared to existing hybrid fuels like hydroxyl-terminated polybutadiene.
\end{abstract}

Keywords: 3D printing; fuel grain; hybrid combustion; regression rate

\section{Introduction}

In the last decade, interest and investment in space exploration has increased dramatically. This has led to greater demand for satellites, and subsequently, more companies are investing significant resources into lowering the cost of space launches. Previously, the manufacture of a Hybrid Rocket Motor (HRM) was costly and labor-intensive, and the traditional 'cast and cure' method limited the geometry of internal combustion ports [1,2]. In an effort to overcome the manufacturing limitations of hybrid rocket fuel grains, researchers have looked to emerging technology and novel techniques to produce innovative solutions that have previously been unattainable. The most notable of these has been the impressive advancement in rapid prototyping, especially fused deposition manufacturing (FDM), commonly known as 3D printing [3]. FDM has enabled designers to incorporate complex combustion ports into HRMs and has opened up an entirely new set of materials for the fabrication thereof.

The process of the 3D printing involves the laying down of successive layers of material on top of each other until a 3D shape has been created [4]. The material is generally supplied in the form a thin strand, or filament, that is wound onto spools to be fed into a heating reservoir and extrusion nozzle assembly. The nozzle assembly is controlled by a computer numerically controlled (CNC) mechanism and has the ability to travel both horizontally and vertically. This means that the only limitations placed on the size of the object being printing is the size of the print area of the particular printer being 
used. However, some companies have already created printers that can essentially print large parts, such as a house, in a short period of time (less than a day) [3]. This is achieved by printing the part length-ways on a conveyor belt, with each new layer being added on a $45^{\circ}$ angle, allowing a full-sized hybrid rocket fuel grain to be completed in a single print. Other companies, such as Gilmour Space Technologies, have created both a proprietary printing method and material [5].

Traditionally, the materials used in 3D printing are generally polymers, such as acrylonitrile butadiene styrene (ABS), polylactic acid (PLA), acrylonitrile styrene acrylate (ASA), or polypropylene $(\mathrm{PP})$, but can include anything from metals, ceramics, food, and concrete to living stem cells $[3,6,7]$. More recently, the commercial market has seen a rapid increase in the number of filaments with metal particles in its composition, such as brass, copper and aluminum. Although it is possible to 3D print metallic materials [8], the filaments discussed herein have a PLA binding structure, with a fine powder of the desired metal finish added during the manufacturing process.

Favorable comparison of ABS against hydroxyl-terminated polybutadiene (HTPB) as a hybrid rocket fuel suggests that FDM has the potential to revolutionize the fabrication of hybrid rocket fuel grains, and has led a groundbreaking push for the development and enhancement of 3D-printed fuel grains at the Utah State University [9]. This study, carried out at the Utah State University, demonstrated that not only did ABS have an acceptable (although slightly reduced) performance when compared to HTPB, but that it also showed much higher burn-to-burn consistency which was attributed to the FDM process [9]. Similar work has been carried out at Purdue University and at the University of Tennessee [10,11].

The introduction of FDM into the manufacturing process of HRM fuel grains has also allowed for the creation of far more complex combustion port geometries. The traditional method of 'cast and cure,' which is used for materials such as HTPB and paraffin, requires a mold and internal tooling that can be quite complex depending on the desired geometry of the fuel grain [12]. Although recent work has been done on the use of disposable tooling structures, it is generally accepted that once the fuel mixture has cured, the internal tooling must be removed before the motor is ready for use. This has limited the complexity of the design of HRM grain ports, in so far as they must be able to allow for the removal of the tooling after the manufacturing process, generally resulting in straight ports that run the length of the motor. In contrast to this, FDM lays down each successive layer of material on top of the last, which, in components like HRM fuel grains, rarely requires the use of internal support. Even if support structure is required, this is added in the same manner as the fuel and is manufactured from water soluble material that is easily removed after manufacture [13].

Several recent studies have explored the use of rapid prototyping in the design and fabrication of HRM fuel grains. The bulk of research in this field has been carried out at the Utah State University and has centered around the exploration of the effects of a helical fuel grain on the performance of the HRM regression rate [12,14-17]. It has been shown that the helical combustion port significantly increased the regression rate when compared to straight cylindrical ports. It has been posited that this is a result of both an increase in the local skin friction, and the suppression of the radial wall blowing effect is due to the centrifugal forces introduced by the helical flow [16]. These centrifugal forces cause compression of the boundary layer, forcing the flame front closer to the fuel port wall and significantly increasing the convective heat transfer. The use of aluminum particles to increase performance of solid and HRMs has long been studied [18-20]. It has been shown that the addition of aluminum particles can increase specific impulse, volumetric heat of oxidation, and radiative heat transfer [21], as well as increase regression rates in HTBP rocket motors by up to $40 \%$ [22]. It is generally understood that this increase in regression rate is due to improvement of radiative heat flux from the diffusion flame zone to the fuel surface area via the radiating metallic particles and higher gas-phase temperature $[23,24]$.

However, it has also been demonstrated that the addition of aluminum particles does not necessarily always result in an increase in regression rates [25]. Regression rate improvement can also be a result of the release of energy during metal oxidation, but the higher temperatures required to melt the aluminum oxide layer often mean that the fuel binder is melted and burnt before the 
aluminum [21,24]. The result is that the aluminum particles are ejected from the motor without significantly contributing to the regression rate increase [26], and can form slag build-up on the nozzle wall, as well as drastically increase nozzle throat ablation [20]. A key contributing factor to the performance enhancement abilities of the added aluminum is the size of the particles. It has been shown that nanosized particles perform better than micro-sized particles, resulting in higher combustion efficiencies, better heat transfer, and reduced burn time [23].

This research project conducted a series of small-scale static fire tests of FDM HRMs that were designed and 3D-printed to explore the performance of a variety of commonly available FDM materials. These materials included ABS, ASA, PLA, PP, PETG (Polyethylene terephthalate glycol), Nylon, and AL (PLA with aluminum particles). The main objective of this research study was to analyze and compare the performance of these materials in terms of the mass flux and regression rate.

\section{Methodology}

\subsection{Material Selection}

The initial series of fuel grains tested were manufactured from commercially available ABS, ASA, PLA, AL, PETG, Nylon, and PP. ABS has been selected as it has been shown to perform as well as HTBP at lab-scale static fire tests [2,9], while ASA was selected due to its similarities to ABS. The PLA was selected as a benchmark with which to test the $\mathrm{Al}$, as there is currently no commercially available aluminum-doped ABS. PETG and Nylon were selected due to their excellent mechanical properties, which would be beneficial if they were used as a structural component of the rocket, and finally, PP was selected due to its low price and because it is a highly crystalline material. Table 1 describe the main characteristics of the seven materials tested in this study.

Table 1. Structural, thermal, and mechanical properties of test materials [27].

\begin{tabular}{ccccc}
\hline Material & Structure & $\boldsymbol{\rho} \mathbf{( g \cdot \mathbf { m } ^ { - 3 } )}$ & $\begin{array}{c}\text { Tensile Yield } \\
\text { Strength }(\mathbf{M P a})\end{array}$ & $\begin{array}{c}\text { Printing } \\
\text { Temperature }\left({ }^{\circ} \mathbf{C}\right)\end{array}$ \\
\hline ABS & Non-crystalline, amorphous & 1010 & 55 & $220-260$ \\
ASA & Non-crystalline, amorphous & 1000 & 40 & $220-250$ \\
PLA & Moderate degree of crystallinity & 1225 & 63 & $190-220$ \\
PLA-Al & Moderate degree of crystallinity & 1330 & 70 & $200-220$ \\
PETG & Moderate degree of crystallinity & 1230 & 50 & $230-250$ \\
Nylon & Moderate degree of crystallinity & 1150 & 55 & $220-260$ \\
PP & High degree of crystallinity & 980 & 40 & $230-260$ \\
\hline
\end{tabular}

\subsection{Design and Manufacture of Fuel Grains}

The small-scale fuel grains were designed to be $100 \mathrm{~mm}$ long and $20 \mathrm{~mm}$ in diameter with a $6 \mathrm{~mm}$ diameter combustion port. It is typical for HRM fuel grains to have high aspect ratios in order to achieve the desired oxidizer to fuel $(\mathrm{O} / \mathrm{F})$ ratios, and it was believed that this combination of port diameter and fuel grain length would provide that. A straight, circular combustion port was selected for the small-scale motor case verification testing and for the material selection testing, as this is the simplest and easiest to manufacture, as well as the simplest to evaluate. A series of ABS grains were printed using a Prusa i3 MK2 FDM 3D printer (Prusa Research s.r.o., Prague, Czech Republic) that were used to verify the operation of the test stand and small-scale motor case. Once the performance of the apparatus was confirmed, two fuel grains of each of the seven materials being tested (ABS, ASA, PLA, AL, PETG, Nylon, and PP) were 3D-printed using the printing temperature range described in Table 1. All fuel grains were designed to have the same dimensions. However, it was expected that due to the manufacturing process, some post-production machining was required for some materials. 


\subsection{Testing of Fuel Grains}

The testing regime was separated into two main rounds conducted at small-scale. Within these tests, there was an apparatus verification component and a fuel grain testing component. The first round of small-scale testing was designed to confirm the operation of the existing small motor casing and to verify the operation of the newly constructed test stand (Figure 1), sensors, and data logger. Several verification burns were conducted using circular, straight port ABS fuel grains. The purpose of these initial tests was to ensure that motor and nozzle were operating in the choked condition and that the chamber pressure was within the operating limits of the motor case and associated plumbing. These initial tests also allowed the data logger code to be tested and modified as required, and the correct operation of the sensors to be verified. The fuel grains of the seven different materials were then subjected to a single, three-second burn each. The testing regime consisted of:

- Measuring and recording the initial weight of the fuel grain

- Measuring and recording the initial weight of the oxidizer bottle

- Placing the fuel grain in the testing apparatus and subjecting it to a three-second burn

- $\quad$ Removing the fuel grain and measuring and recording the final weight

- Measuring and recording the final weight of the oxidizer bottle

These measurements allowed for the determination of the fuel and oxidizer and total mass flow rates, $\mathrm{O} / \mathrm{F}$ ratio, and the fuel regression rate.

To measure chamber pressure, an analogue gauge was initially mounted to a brass outlet tube plumbed to the mixing chamber at the fore of the motor case. Once the correct operation of the rocket motor was confirmed, a digital pressure transducer was used in place of the analogue gauge, allowing much more accurate measurement and recording, as well as the option to plot the pressure chamber throughout the duration of each burn. A Honeywell heavy duty, 10 BAR ratiometric pressure sensor was chosen for its robust construction and represented an excellent compromise between performance and price. Protection from over pressurization was via a 1.0 MPa pressure relief valve that was installed in-line with both the analogue gauge and pressure transducer. Temperature protection was provided via the installation of a thermocouple below the pressure measurement devices. This thermocouple provided real-time feedback on the temperatures being experienced by gauges, allowing the burn to be stopped prematurely if temperatures approached or exceeded the maximum operating temperatures of the unit.

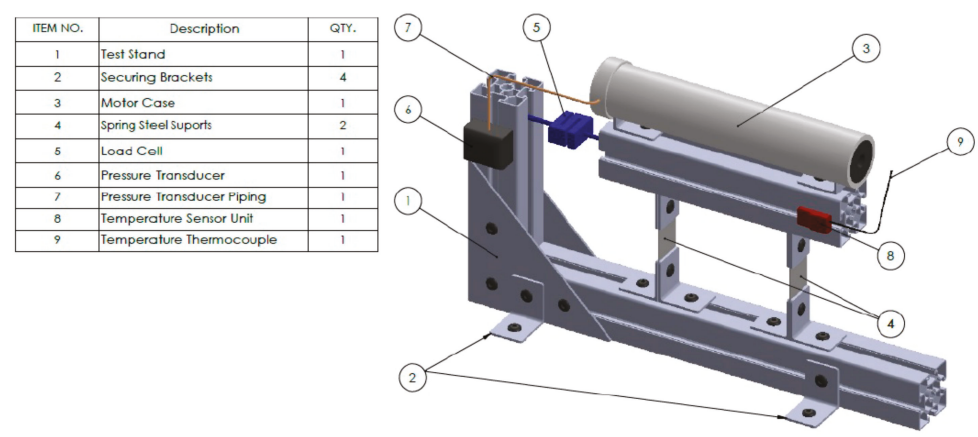

Figure 1. Schematic of the laboratory-rocket motor test stand with sensors. 


\section{Results}

\subsection{Testing}

Fuel grains were designed based on literature values and also taking in account limits of available 3D printers [28]. Preliminary tests were carried out with ABS fuel grains to analyze integrity of fuel grains and thrust. All of the experiments (preliminary and others) were carried out in triplicates.

In the initial testing, a burn of four seconds resulted in the fuel grain losing structural integrity, and subsequently, allowing its outer surface (the surface of the grain in contact with the motor casing) to begin to vaporize and contribute to the overall combustion as well. Not only is this undesirable from an operational perspective, it also renders the regression rate calculations invalid. These calculations rely on the principle that any fuel removed from the grain via combustion occurred solely in the combustion port. Without the ability to differentiate between the mass removed from the combustion and the mass removed from the outer surface, the calculations significantly over-estimate the combustion port regression rate.

Once the burning time was reduced to three seconds, the structural integrity of the fuel grain was far better preserved with combustion of fuel being limited to within the combustion port. Three further tests were then conducted with $100 \mathrm{~mm} \times 20 \mathrm{~mm}$ ABS fuel grains to ensure the repeatability of the test stand chamber pressure measurement.

The results of the thrust validation show excellent consistency across the three burns, with very similar profiles demonstrated in each run. The precise moment of motor cut-off can be seen across all three tests at the four-second mark.

The seven fuel grains 3D-printed with different materials are shown in Figure 2.

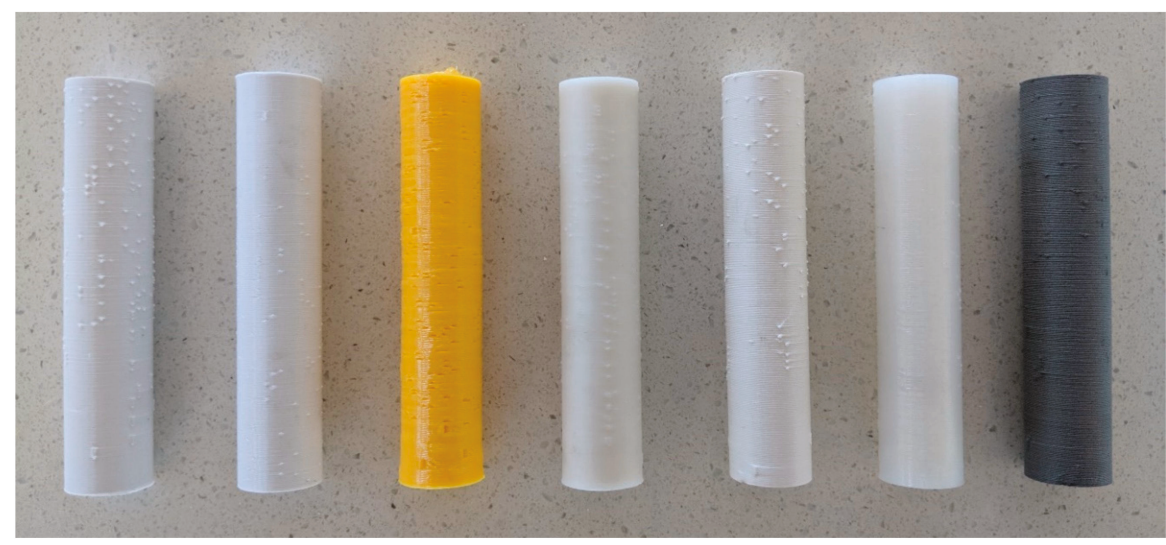

Figure 2. Small-scale fuel grains, left to right: ABS, PLA, PETG (Polyethylene terephthalate glycol), PP, ASA, Nylon, and AL (PLA with aluminum particles).

Although the majority of the fuel grains were 3D-printed ready to burn, several printing settings were modified slightly to ensure the structural integrity of the layers and to minimize any surface defects that could negatively impact the fuel grain performance. In some materials, these settings resulted in small blobs, known as "zits," on the outer surface of the print, and in some cases, extreme oozing, or "stringing," across the combustion chamber. For complex internal geometries, these settings would need to be refined to ensure completely homogeneous layers, a smooth exterior, and clean combustion port. However, the experiments were focused on material selection rather than port geometry, and as such, a straight circular port was used, meaning that the external surface and combustion port could simply be machined to the correct dimensions as required. 
Once the machining had been completed, two fuel grains for each material were subjected to a single three second burn each (Figure 3). Although all fuel grains performed reasonably well, as indicated by the size and shape of the exhaust plume exiting the nozzle, there was a noticeable difference between the better performing materials and those that did not perform well. Visual inspection of each burn suggested that the ABS and ASA performed very well, and it was also noted that the PETG burn was significantly hotter than any of the other burns. Of all the materials tested, the AL appeared to have performed extremely well, with an incredibly energetic combustion. However, inspection of the regression rate data, outlined in Section 3.2, shows that it was, in fact, one of the worst performing materials.

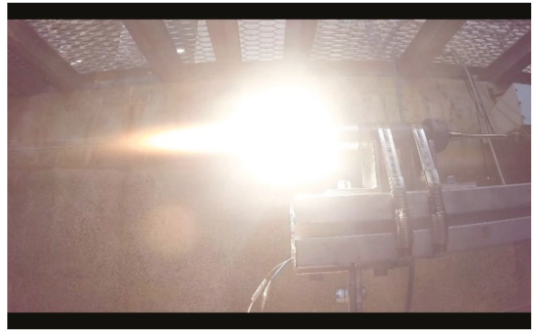

(a) ABS

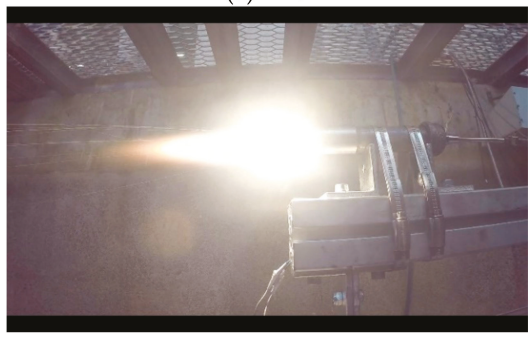

(c) PETG

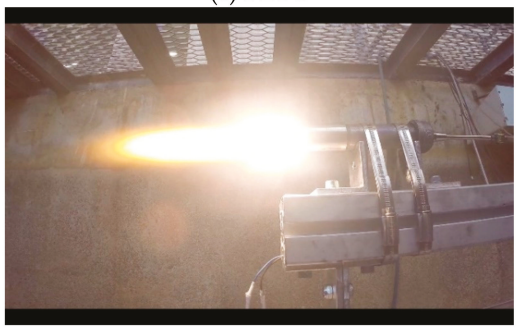

(e) ASA

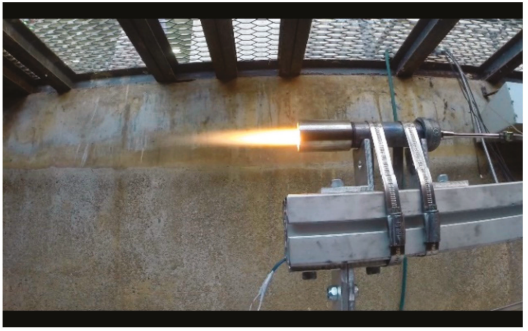

(b) PLA

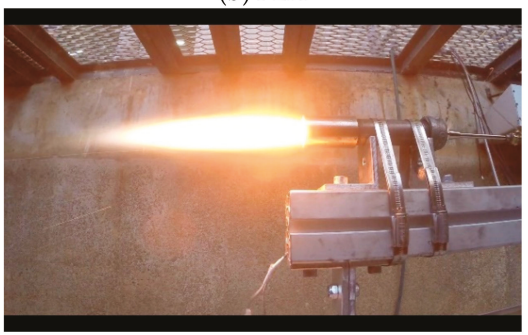

(d) PP

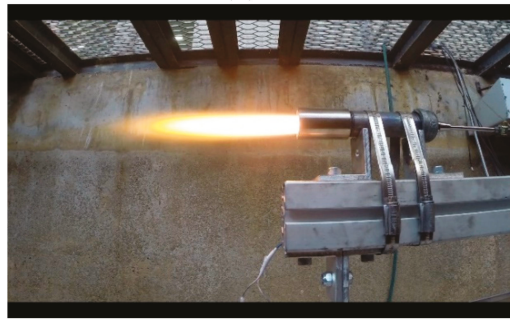

(f) Nylon

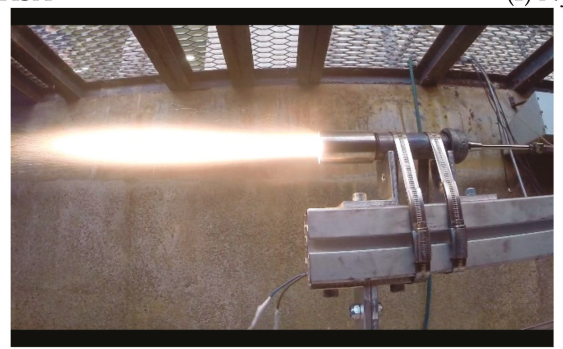

(g) AL

Figure 3. Small-scale testing of fuel grains 3D-printed from different fused deposition manufacturing (FDM) materials. 


\subsection{Regression Rate}

The time-averaged longitudinal fuel regression rate $\overline{\dot{r}}$ for each of the fuel grains can be calculated (Equation (1)) using the mass flow of the fuel grain [17]:

$$
\overline{\dot{r}}=\frac{\dot{m}_{\text {fuel }}}{\rho_{\text {fuel }} \cdot 2 \cdot \pi \cdot \bar{r}_{\text {port }} \cdot L}
$$

where $\dot{m}_{f u e l}$ is the time-average mass flow rate of the fuel, $\rho_{\text {fuel }}$ is the density of the fuel, $\bar{r}_{\text {port }}$ is the mean fuel combustion chamber radius, and $L$ is the length of the fuel combustion port. $\dot{m}_{f u e l}$ can be calculated using the measured fuel mass loss $(\Delta M)$ and dividing by the burning time $\left(t_{b}\right)$. The time-averaged fuel port radius can be determined by Equation (2) [24]:

$$
\bar{r}_{\text {port }}=\sqrt{r_{0}^{2}+\frac{\Delta M}{\rho_{\text {fuel }} \cdot \pi \cdot L}}
$$

where $r_{0}$ is the initial fuel port radius. Once the time-averaged fuel port radius has been calculated, it is then possible to determine the total mass flux of the fuel grain using Equation (3):

$$
\bar{G}_{\text {total }}=\frac{\dot{m}_{\text {total }}}{A_{\text {port }}}
$$

where $\dot{m}_{\text {total }}$ is the total mass flow rate through the combustion port and $A_{\text {port }}$ is the area of the combustion port. Substituting Equation (2) into Equation (3) yields Equation (4):

$$
\bar{G}_{\text {total }}=\frac{\dot{m}_{o x}+\dot{m}_{f u e l}}{\pi \cdot\left(r_{0}^{2}+\frac{\Delta M}{\rho_{f u e l} \cdot \pi \cdot L}\right)}
$$

where $\dot{m}_{o x}$ is the mass flow rate of the oxidiser and $\dot{m}_{f u e l}$ is the mass flow rate of the fuel. Table 2 shows

\begin{tabular}{|c|c|c|c|c|c|c|}
\hline Material & $\rho\left(\mathrm{kg} \mathrm{m}^{-3}\right)$ & $\dot{m}_{o x}\left(\mathrm{~kg} \mathrm{~s}^{-1}\right)$ & $\dot{m}_{f u e l}\left(\mathrm{~kg} \mathrm{~s}^{-1}\right)$ & O/F Ratio & $\dot{m}_{\text {total }}\left(\mathrm{kg} \mathrm{s}^{-1}\right)$ & $\overline{\dot{r}}\left(\mathrm{~mm} \mathrm{~s}^{-1}\right)$ \\
\hline ABS & 1010 & 0.0100 & 0.0023 & 4.35 & 0.0123 & 1.05 \\
\hline ASA & 1000 & 0.0125 & 0.0030 & 4.17 & 0.0155 & 1.59 \\
\hline $\mathrm{AL}$ & 1330 & 0.0108 & 0.0025 & 4.32 & 0.0133 & 1.20 \\
\hline PLA & 1225 & 0.0100 & 0.0025 & 4.00 & 0.0125 & 1.23 \\
\hline PETG & 1230 & 0.0108 & 0.0035 & 3.09 & 0.0143 & 0.94 \\
\hline Nylon & 1150 & 0.0100 & 0.0027 & 3.70 & 0.0127 & 1.51 \\
\hline $\mathrm{PP}$ & 890 & 0.0100 & 0.0023 & 4.35 & 0.0123 & 1.23 \\
\hline
\end{tabular}
the average data from the small-scale testing as well as the results of time-averaged longitudinal fuel regression rate of the burn calculated using Equation (1).

Table 2. Results of the small-scale test fire of 3D-printed fuel grains.

The material with the highest average regression rate was found to be ASA, followed closely by Nylon, while PETG had the lowest average regression rate. It was observed that the fuel port radius of the ASA grain increased by the greatest amount. Despite the expected energetic combustion of the AL fuel grain, it was found to have a regression rate similar to the PLA without the addition of aluminum powder. This shows that the aluminum powder did not impact on the regression rate, perhaps due to its particle size and surface area. The regression rate of ABS fuel grain was one of the lowest of the materials tested. A similar value to ASA was expected due to their similar chemical and mechanical properties. This unexpected value can be explained by the low oxidizer mass flux for ABS. 
Inspection of the exhaust end of each fuel grain reveals similar results. Figure 4 shows the exit of the combustion port for each different material, and while it is difficult to make accurate measurements of combustion port diameters, certain observations can be made. It is clear that the PLA and AL (PLA with aluminum particles) have significantly smaller diameters than the other materials, which correlate well with the results in Table 2. Conversely, ABS and PP appear to have relatively large combustion port diameters, which was expected for ABS but surprising for the PP grain. It is worth noting at this point that the Nylon grain could not be removed from the motor case without significant damage, and as it could not be examined in this way, it was not further evaluated in this study.

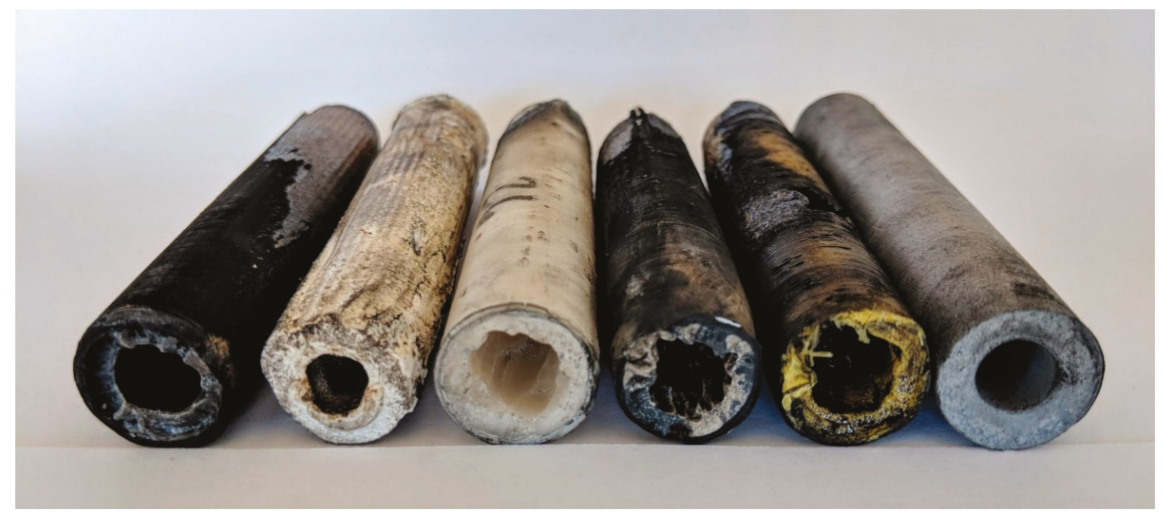

Figure 4. Combustion port comparisons, left to right: ABS, PLA, PP, ASA, PTEG, and AL.

To further validate these results, each fuel grain was cut in half longitudinally, allowing for a more comprehensive view of the ablation of the combustion chamber as seen in Figure 5 . It is from this cross-sectional view that it is possible to see from where the increased regression rate for ASA occurred. Examination of the exhaust end of the combustion port of the ASA grain suggests that it may have had similar regression rates to the AL grain. However, it is clear that significant regression occurred throughout the length of the chamber, with the exhaust and inlets end of the combustion port potentially dilating post-burn. Inspection of Figure 5 also reveals that combustion within the AL fuel grain did not occur at the beginning of the combustion port. This resulted in a small section of fuel grain that was not contributing to the regression rate, yet still being taken into account for calculations involving grain weight and port length. This may have contributed somewhat to the poor performance of the AL, but not to the levels seen in this experiment.

The poor performance of the AL fuel grain is believed to be due to the size of the aluminum particles added to the base PLA. It has been shown that the optimum particle size required to significantly increase fuel regression rates is between 0.05 to $0.1 \mathrm{~mm}$ [22]. This size range represents an incredibly fine powder, with an associated cost that would be above an economically feasible option for commonly available 3D printing filaments for some conventional applications. Thus, it could be proposed that the aluminum powder did not actively improve the radiative heat flux from the flame zone to the fuel surface, nor release significant energy during metal oxidation. The latter could be largely due to the larger particle size, which would result in the particles being ejected from the motor prior to the melting of their aluminum oxide coating. Inspection of Figure $3 \mathrm{~g}$ reveals a large number of highly energized particles being ejected from the motor during the burn, which correlates well with this hypothesis. 


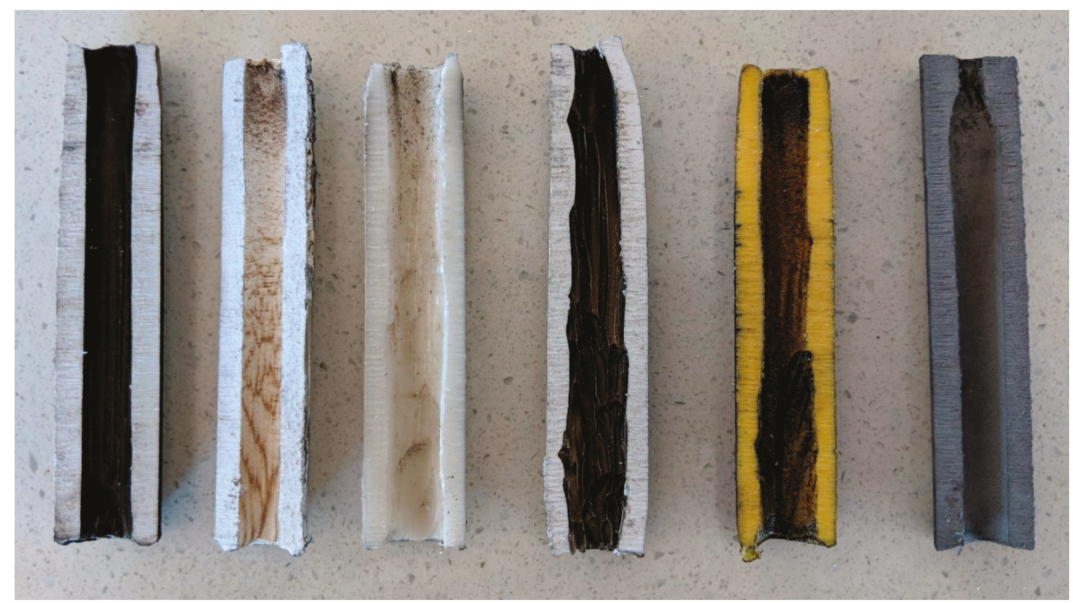

Figure 5. Cross section of each different small-scale fuel grain, left to right: ABS, PLA, PP, ASA, PTEG, and AL.

\section{Conclusions}

This research explored the viability of using commercially available 3D printing filament materials in the manufacture of hybrid rocket fuel grains. Preliminary research has demonstrated the suitability of ABS for use in mid-sized sounding rockets, and this study aimed to build on this by conducting a small-scale testing regime to determine the suitability of seven different commonly available 3D printing materials: ABS, PLA, ASA, PETG, PP, Nylon, and AL (PLA with aluminum particles). In this study, the ASA and Nylon fuel grains showed the highest regression rates, while PETG regression rates were relatively poor. PLA and AL fuel grains showed similar regression rates, showing that the aluminum particles had no contribution to the regression rates measured. It was speculated that the poor performance of the AL fuel grain was largely due to the size, shape and surface area of aluminum particles. Despite this poor performance in this research, the impact of Al particles on fuel grains performance should be analyzed, especially the contribution of different variables, such as, size, shape, and surface area of aluminum particles. The experiment design of this study was very simple as the main objective was to select the FDM materials for a large-scale test, and it has to be considered as a first step toward the realization of the larger scale engine firing campaign.

Author Contributions: M.M. and E.A. conceived the presented research idea and designed of experiments. M.M. carried out and analyzed experiments under E.A. supervision. All authors contributed to the final manuscript.

Funding: This research received no-external funding.

Conflicts of Interest: The authors declare no conflicts of interest.

\section{Nomenclature}

ABS acrylonitrile butadiene styrene

AL PLA with aluminum particles

ASA acrylonitrile styrene acrylate

CNC computer numerically controlled

FDM fused deposition manufacturing

HRM Hybrid Rocket Motor

HTPB Hydroxyl-terminated polybutadiene

PETG Polyethylene terephthalate glycol

PLA polylactic acid

PP polypropylene 
$L \quad$ length of the fuel combustion port

$\mathrm{O} / \mathrm{F} \quad$ oxidizer to fuel ratio

$A_{\text {port }} \quad$ the area of the combustion port

$\dot{m}_{\text {fuel }} \quad$ mass flow rate of fuel

$\dot{m}_{o x} \quad$ mass flow rate of the oxidizer

$\dot{m}_{\text {total }} \quad$ total mass flow rate through the combustion port

$\rho_{\text {fuel }} \quad$ density of fuel

$\bar{r} \quad$ fuel regression rate

$r_{0} \quad$ initial fuel port radius

$\bar{r}_{\text {port }} \quad$ mean fuel combustion chamber radius

$t_{b} \quad$ burning time

$\Delta M \quad$ solid-fuel mass loss

\section{References}

1. Krishnan, S. Hybrid rocket technology: An overview. In Proceedings of the 3rd International High Energy Materials Conference and Exhibit, Thiruvanthapuram, India, 6-8 December 2000.

2. Wright, P.K. 21st Century Manufacturing; Prentice Hall: Upper Saddle River, NJ, USA, 2001; Volume 1.

3. Ngo, T.D.; Kashani, A.; Imbalzano, G.; Nguyen, K.T.; Hui, D. Additive manufacturing (3D printing): A review of materials, methods, applications and challenges. Compos. B Eng. 2018, 143, 172-196. [CrossRef]

4. Wang, X.; Jiang, M.; Zhou, Z.; Gou, J.; Hui, D. 3D printing of polymer matrix composites: A review and prospective. Compos. B Eng. 2017, 110, 442-458. [CrossRef]

5. Technologies, G.S. Launch Vehicles. Available online: https://www.gspacetech.com/launch-vehicles (accessed on 11 January 2019).

6. Gu, Q.; Tomaskovic-Crook, E.; Wallace, G.G.; Crook, J.M. 3D bioprinting human induced pluripotent stem cell constructs for in situ cell proliferation and successive multilineage differentiation. Adv. Healthc. Mater. 2017, 6, 1700175. [CrossRef] [PubMed]

7. Tofail, S.A.; Koumoulos, E.P.; Bandyopadhyay, A.; Bose, S.; O’Donoghue, L.; Charitidis, C. Additive manufacturing: Scientific and technological challenges, market uptake and opportunities. Mater. Today 2018, 21, 22-37. [CrossRef]

8. Gibson, M.A.; Mykulowycz, N.M.; Shim, J.; Fontana, R.; Schmitt, P.; Roberts, A.; Myerberg, J.S. 3D printing metals like thermoplastics: Fused filament fabrication of metallic glasses. Mater. Today 2018, 21, 697-702. [CrossRef]

9. Whitmore, S.A.; Peterson, Z.W.; Eilers, S.D. Comparing Hydroxyl Terminated Polybutadiene and Acrylonitrile Butadiene Styrene as Hybrid Rocket Fuels. J. Propuls. Power 2013, 29, 582-592. [CrossRef]

10. Creech, M.; Crandell, A.; Eisenhauer, N.; Marx, S.; Busari, T.; Link, A.; Pourpoint, T.L. 3D Printer for Paraffin Based Hybrid Rocket Fuel Grains. In Proceedings of the 53rd AIAA Aerospace Sciences Meeting, Kissimmee, FL, USA, 5-9 January 2015.

11. Lyne, J.E.; Brigham, A.; Savery, R.; Karcher, K.; Pyron, J.; Adams, L.; Melendez, L. The Use of a 3-D Printed, Polymer Matrix Containing Pulverized Fuel in a Hybrid Rocket. In Proceedings of the 2018 Joint Propulsion Conference, Cincinnati, OH, USA, 9-11 July 2018.

12. Whitmore, S.A. Advantages of Using Additive Manufacturing to Build "Green" Fuels for Hybrid Propulsion. Int. J. Astronaut. Aeronaut. Eng. 2017, 2, 1-12. [CrossRef]

13. Whitmore, S.A. Three-Dimensional Printing of "Green" Fuels for Low-Cost Small Spacecraft Propulsion Systems. J. Spacecr. Rocket. 2017, 55, 13-26. [CrossRef]

14. Spurrier, Z.S. Throttleable GOX/ABS Launch Assist Hybrid Rocket Motor for Small Scale Air Launch Platform. Ph.D. Thesis, Utah State University, Logan, UT, USA, 2016.

15. Walker, S.D. High regression rate hybrid rocket fuel grains with helical port structures. In Proceedings of the 50th AIAA/ASME/SAE/ASEE Joint Propulsion Conference, Cleveland, OH, USA, 28-30 July 2014.

16. Whitmore, S.A.; Sobbi, M.; Walker, S. High Regression Rate Hybrid Rocket Fuel Grains with Helical Port Structures. J. Propuls. Power 2015, 31, 1727-1738. [CrossRef]

17. Walker, S.A.; Walker, S.D. Engineering model for hybrid fuel regression rate amplification using helical ports. J. Propuls. Power 2017, 33, 398-407. 
18. Lips, H. Metal combustion in high performance hybrid rocket propulsion systems. In Proceedings of the 12th Propulsion Conference, Palo Alto, CA, USA, 26-29 July 1976.

19. King, M.K. Aluminum combustion in a solid rocket motor environment. Proc. Combust. Inst. 2009, 32, 2107-2114. [CrossRef]

20. Farbar, E.; Louwers, J.; Kaya, T. Investigation of metallized and nonmetallized hydroxyl terminated polybutadiene/hydrogen peroxide hybrid rockets. J. Propuls. Power 2007, 23, 476-486. [CrossRef]

21. Thomas, J.C.; Petersen, E.L.; Desain, J.D.; Brady, B. Hybrid Rocket Enhancement by Micro-and Nano-Scale Additives in HTPB Fuel Grains. In Proceedings of the 51st AIAA/SAE/ASEE Joint Propulsion Conference, Orlando, FL, USA, 27-29 July 2015.

22. Chiaverini, M.J.; Kuo, K.K.; Peretz, A.; Harting, G.C. Regression-rate and heat-transfer correlations for hybrid rocket combustion. J. Propuls. Power 2001, 17, 99-110. [CrossRef]

23. Kuo, K.K.; Risha, G.A.; Evans, B.J.; Boyer, E. Metals, Energetic Additives, and Special Binders Used in Solid Fuels for Hybrid Rockets. In Fundamentals of Hybrid Rocket Combustion and Propulsion; American Institute of Aeronautics and Astronautics, Inc.: Reston, VA, USA, 2007.

24. Carmicino, C.; Russo Sorge, A. Experimental investigation into the effect of solid-fuel additives on hybrid rocket performance. J. Propuls. Power 2014, 31, 699-713. [CrossRef]

25. Lips, H.R. Heterogeneous combustion of highly aluminized hybrid fuels. AIAA J. 1977, 15, 777-778. [CrossRef]

26. Sun, X.; Tian, H.; Yu, N.; Cai, G. Regression rate and combustion performance investigation of aluminum metallized HTPB/98HP hybrid rocket motor with numerical simulation. Aerosp. Sci. Technol. 2015, 42, 287-296. [CrossRef]

27. Prospector, U. Plastics. Available online: https://plastics.ulprospector.com (accessed on 11 May 2019).

28. Veale, K.; Adali, S.; Pitot, J.; Brooks, M. A review of the performance and structural considerations of paraffin wax hybrid rocket fuels with additives. Acta Astronaut. 2017, 141, 196-208. [CrossRef]

(C) 2019 by the authors. Licensee MDPI, Basel, Switzerland. This article is an open access article distributed under the terms and conditions of the Creative Commons Attribution (CC BY) license (http://creativecommons.org/licenses/by/4.0/). 



\title{
Modeling of High Density Polyethylene Regression Rate in the Simulation of Hybrid Rocket Flowfields
}

\author{
Daniele Bianchi ${ }^{1, *}$, Giuseppe Leccese ${ }^{1}$, Francesco Nasuti ${ }^{1}$, Marcello Onofri ${ }^{1}$ and \\ Carmine Carmicino ${ }^{2}$ \\ 1 Department of Mechanical and Aerospace Engineering, Sapienza University of Rome, Via Eudossiana 18, \\ 00184 Rome, Italy \\ 2 Department of Industrial Engineering, University of Naples "Federico II", Piazzale Tecchio 80, \\ 80125 Naples, Italy \\ * Correspondence: daniele.bianchi@uniroma1.it
}

Received: 21 April 2019; Accepted: 31 July 2019; Published: 9 August 2019

\begin{abstract}
Numerical analysis of hybrid rocket internal ballistics is carried out with a Reynolds-averaged Navier-Stokes solver integrated with a customized gas-surface interaction wall boundary condition and coupled with a radiation code based on the discrete transfer method. The fuel grain wall boundary condition is based on species, mass, and energy conservation equations coupled with thermal radiation exchange and finite-rate kinetics for fuel pyrolysis modeling. Fuel pyrolysis is governed by the convective and radiative heat flux reaching the surface and by the energy required for the propellant grain to heat up and pyrolyze. Attention is focused here on a set of static firings performed with a lab-scale GOX/HDPE motor working at relatively low oxidizer mass fluxes. A sensitivity analysis was carried out on the literature pyrolysis models for HDPE, to evaluate the possible role of the uncertainty of such models on the actual prediction of the regression rate. A reasonable agreement between the measured and computed averaged regression rate and chamber pressure was obtained, with a noticeable improvement with respect to solutions without including radiative energy exchange.
\end{abstract}

Keywords: hybrid rocket engines; modeling and simulation; computational fluid dynamics

\section{Introduction}

Hybrid Rocket Engines (HRE) are identified as promising propulsion systems for space transportation application [1,2]. Their performance is comparable to that of storable or semi-cryo liquid rocket engines, and they exhibit appealing features of both solid rocket motors and liquid rocket engines. Moreover, they are safer and less expensive than solid and liquid rockets and are more environmentally friendly than solid rockets and storable-liquid rockets. Therefore, many research programs have been focusing on HRE development for applications that range from Earth-suborbital flights to space exploration. Despite the above-mentioned advantages, some issues hinder the success of HRE: low regression rates of commonly used polymeric fuels, reduced combustion efficiency, mixture ratio shifts, the uncertainty of regression rate law, and its scalability are the most challenging problems. Uncertainty in the regression rate and its scalability remain some of the most challenging issues, since the regression rate is a parameter that strongly drives the design process and heavily influences the performance of the entire propulsion system.

To date, research efforts in developing reliable hybrid propulsion systems have strongly depended on firing tests and experimental trials and errors, which are expensive and time-consuming. In particular, as concerns the estimate of the fuel regression rate, empirical correlations based on linear interpolation in the logarithmic plane are mostly used, as well as surface energy balances, which include the simplified boundary layer correlations based on bulk transfer coefficients to evaluate the 
convective heat flux to the solid fuel. Such approaches are particularly useful during the preliminary design and analysis process, providing a simpler and faster estimation of the rocket performance, but they rely on a very simplified modeling of the mutually-interacting and complex physico-chemical phenomena involved. Therefore, they should be carefully calibrated relying on the availability of specific experimental data existing for each analyzed motor. Consequently, such models are limited to providing a qualitative analysis of the motor trends, but they are insufficient for providing the kind of quantitative data that are required for motor final design and optimization. Indeed, the extension of those models to new motors that can be different in geometry, scale, etc., is hardly possible without the availability of existing experimental data for each motor. For these reasons, there is a renewed interest in the development of more accurate and advanced models based on Computational Fluid Dynamics (CFD) [3-15] that are capable of representing more accurately the physico-chemical phenomena involved. The numerical modeling of the fluid dynamics and the combustion process in the fuel port area and nozzle of a hybrid rocket is a challenging task as it involves strongly-interacting multiphysics processes such as fluid dynamics, fuel pyrolysis [16,17], atomization and vaporization of the oxidizer, mixing and combustion in the gas phase [11-13,18], thermochemical erosion of the nozzle [3,19], particulate formation, and the radiative characteristics of the flame. Commercial CFD tools are generally not optimized to this task, as they are typically less flexible for the treatment of fluid/solid boundary conditions, which are typically prescribed as constant temperature or heat flux with no feedback with the mass transfer mechanisms (pyrolysis, sublimation, etc.). To obtain an adequate tool for the analysis of the flowfield of hybrid rocket burning classical non-liquefying fuels, CFD codes should take into account spatially-varying heat flux, surface temperature, and fuel regression rate, realistic surface multispecies mass and energy balances, thermal soak into the fuel grain, radiative energy exchange, and finite-rate Arrhenius kinetics for fuel pyrolysis modeling or, in the case of commercial solvers, a number of user-defined functions need to be built and integrated in the numerical framework $[8,14,20]$. In the most general case, an in-house code has to be used.

The goal of this study is the high-fidelity simulation of the internal ballistics of a hybrid rocket, including the pre- and post-combustion chamber and nozzle. The reference configuration was a 1-kN-class lab-scale hybrid rocket tested at the University of Naples "Federico II" and equipped with an axial subsonic injector nozzle that feeds gaseous oxygen into the port of High-Density Polyethylene (HDPE) grains. With such an injector arrangement, the regression rate results in being appreciably larger than what expected from the literature [21], in particular at the very low mass fluxes (around $10 \mathrm{~kg} / \mathrm{m}^{2} \cdot \mathrm{s}$ ) that exhibit a 2.4-fold increase [18]; it also shows a lower dependence on the mass flux, and at a given mass flux, an increase with the port diameter is achieved. Furthermore, combustion efficiency and motor stability are both favored.

The numerical simulations presented here were carried out by solving the Reynolds-averaged Navier-Stokes equations for multicomponent, single-phase, turbulent reacting flows [22,23], including the sub-models required in order to describe the homogeneous combustion in the gaseous phase, the radiative energy exchange, and the gas-surface interaction in the combustion chamber (fuel pyrolysis model) for HDPE grains. The in-house computational tool used for the simulations, and its gas-surface interaction capability has been validated for high-speed re-entry flows [24], for the analysis of Hydroxyl-Terminated Polybutadiene (HTPB) fuel grains [3,4,25], and for hybrid rocket nozzle thermal protection systems' ablation $[3,19,26]$. Recent literature works focusing on HDPE fuel grains in hybrid rockets either used commercial CFD tools with user-defined functions to compute the fuel mass flux as a function of the wall heat flux [8,14] or in-house CFD code [15] with imposed fuel mass flux using the mean regression rates measured during the firing test. Thermal radiation was not taken into account in $[14,15]$. Interestingly, the work in [8] showed that the regression rate was underestimated by $30 \%$ for high-density polyethylene grains when radiative heat exchange was not accounted for. The authors concluded stating that no clear information exists regarding the relative importance of radiative heat flux with respect to the convective one for HDPE grains at relatively low gaseous oxygen mass fluxes. The current work also aims at providing some insight into the effect 
of thermal radiation for HDPE regression rate predictions. Two main contributions to the field of hybrid rocket internal ballistics numerical simulation are addressed in this paper: First, a sensitivity analysis of the regression rate and chamber pressure to the pyrolysis models for HDPE available in the literature is carried out, highlighting the main effect of the heat of pyrolysis against the much lower influence of the pyrolysis kinetics parameters, leading to the conclusion that the former has a key role in the correct motor performance prediction. Second, an evaluation of the possible contribution of the radiative energy exchange between combustion gases and the solid fuel on the regression rate calculation, which is often neglected in simulations, is conducted with a novel approach, showing that radiation seems to play a significant role, especially with low oxidizer mass fluxes. This represents a first step towards a complete modeling of radiative contribution, which should also include emission from soot particles.

\section{Theoretical and Numerical Model}

The study of HRE flowfields requires a suitable modeling of the motor internal ballistics including both gas-surface interaction (fuel pyrolysis) and gas phase reactions' (pyrolyzed fuel and oxidizer combustion) sub-models. Concerning the former aspect, a detailed gas-surface interaction sub-model based on surface mass and energy balances for fuel pyrolysis, including thermal radiation exchange and finite-rate Arrhenius kinetics, was coupled with a chemically-reacting three-dimensional CFD code and a gray/non-scattering two-dimensional axisymmetric radiation code.

The CFD tool is a finite-volume solver for three-dimensional compressible multicomponent turbulent reacting flows [22,23], with temperature-variable thermodynamic and transport properties. The thermodynamic properties of individual species were approximated by seventh-order polynomials of temperature, while the transport properties were approximated by fourth-order polynomials [27]. Mixture properties for viscosity and thermal conductivity were derived from Wilke's mixing rule [28]. The species diffusion model was based on a single effective diffusion coefficient obtained assuming a constant Schmidt number. The Spalart-Allmaras one-equation turbulence model [29] was used to compute the turbulent viscosity. The turbulent thermal conductivity and the turbulent species diffusivity were computed from the turbulent viscosity, the specific heat at constant pressure, and the turbulent Prandtl and Schmidt number. The gas-phase chemistry was modeled assuming a zero-dimensional, perfectly-stirred reactor model. The numerical code solved the time-dependent conservation equations of species, mass, momentum, and energy for the chemical nonequilibrium flowfield by adopting a standard finite-volume Godunov-type formulation. It used multi-block structured meshes and was second-order accurate in space. An explicit Runge-Kutta integration scheme was adopted to advance in time the ordinary differential equation system, resulting from the time-discretization.

Radiation energy exchange from the hot combustion gases to the pyrolyzing fuel surface was accounted for through a separate code for generic axisymmetric gray/diffuse boundaries and inhomogeneous gray/non-scattering media, based on the Discrete Transfer Method (DTM) [30]. The equations of the problem under scrutiny were written in finite form by discretizing the solid angle, at each node, and the path length, for each ray. The discretized equations were then integrated by means of a summation over the whole path length along each ray, in order to evaluate the corresponding radiative intensity, and over the whole solid angle at each surface node location, to evaluate the associated radiative wall heat flux. Finally, the field and wall local state parameters needed by the radiation tool were retrieved from the CFD simulations, with which the DTM computations were coupled. A suitable geometric ray-tracing procedure was also implemented in the DTM radiation code.

\subsection{Gas-Surface Interaction Model for Pyrolyzing Fuels}

To complete the formulation of the theoretical model, suitable boundary conditions that describe the physics of the surface phenomena must be specified at the gas-surface interface (i.e., "wall"). 
Such wall boundary conditions that are applicable to pyrolyzing fuels (such as HDPE) are detailed in the following.

Assuming that no material is being removed in a condensed phase (either solid or liquid), then the general conservation equations at the gas-surface interface over a pyrolyzing fuel wall can be written as follows [31]. The overall surface mass balance is:

$$
\dot{m}_{b}=(\rho v)_{w}=\rho_{s} \dot{r}
$$

where $\dot{m}_{b}$ is the overall mass flux of the injected fuel pyrolysis products per unit area of the wall surface, $\rho$ and $v$ are the mixture gas density at the wall and its normal-to-wall bulk velocity component, and finally, $\rho_{s}$ and $\dot{r}$ are the solid fuel density and its regression rate, respectively. The surface mass balance for each generic species is:

$$
\rho D_{i m} \frac{\partial y_{i}}{\partial \eta}+\dot{\omega}_{i}=(\rho v)_{w} y_{i} \quad i=1,2, \ldots, N
$$

where $D_{i m}$ is the $i^{\text {th }}$ species-to-mixture effective diffusion coefficient, $y_{i}$ the gas phase mass fraction of the $i^{\text {th }}$ species at the wall, $N$ the total number of species, $\dot{\omega}_{i}$ is the rate of production per unit surface area of gas-phase species $i$ due to fuel pyrolysis, and $\eta$ is the coordinate axis normal to the surface and oriented from the solid to gas. The overall surface energy balance is:

$$
k \frac{\partial T}{\partial \eta}+\sum_{i=1}^{N} h_{i} \rho D_{i m} \frac{\partial y_{i}}{\partial \eta}+\dot{m}_{b} h_{s}+\dot{q}_{\mathrm{rad}_{\mathrm{abs}}}=\dot{m}_{b} h_{w}+\dot{q}_{\mathrm{cond}}^{s s}+\dot{q}_{\mathrm{rad}_{\mathrm{em}}}
$$

where $h_{w}$ represents the enthalpy of the gas mixture at the wall, $h_{i}$ the enthalpy of the single gas species at the wall temperature, $h_{s}$ the enthalpy of the solid fuel grain at the wall temperature, $k$ the gas mixture thermal conductivity, $T$ the gas temperature, and $\dot{q}_{\text {cond }}^{\text {ss }}$ the solid conduction heat flux inside the fuel grain. Note that the term $\dot{m}_{b} h_{s}$ represents the energy flux entering the surface due to fuel grain regression. Finally, radiation emitted from the hot combustion products and absorbed by the fuel grain, as well as emitted radiation from the fuel surface were included in the surface energy balance, Equation (3). The terms $\dot{q}_{\mathrm{rad}_{\mathrm{abs}}}$ and $\dot{q}_{\mathrm{rad}_{\mathrm{em}}}$ represent the absorbed and emitted radiation from the fuel grain surface, respectively. The absorbed radiative heat flux, which is the radiative heat flux coming from the gas and absorbed by the wall, is:

$$
\dot{q}_{\mathrm{rad}}=\alpha_{w s} \dot{q}_{\mathrm{rad}}=\epsilon_{w} \dot{q}_{\mathrm{rad}}
$$

where $\dot{q}_{\text {rad }}$ represents the incident radiative heat flux that is evaluated from the radiation model. Note that, according to Kirchhoff's law, the wall absorptivity $\alpha_{w}$ is equal to the wall emissivity $\epsilon_{w}$. The emitted radiative heat flux, which is the radiative heat flux emitted from the wall because of its temperature $T_{w}$, can be expressed as:

$$
\dot{q}_{\mathrm{rad}_{\mathrm{em}}}=\epsilon_{w} \sigma T_{w}^{4}
$$

where $\sigma$ is the Stefan-Boltzmann constant, $\sigma=5.67 \times 10^{-8} \mathrm{Wm}^{-2} \cdot \mathrm{K}^{-4}$.

As concerns the energy transfer into the solid fuel, it was assumed that the heat conduction was dominant in the direction normal to the fuel surface. Although axial temperature gradients certainly existed along the fuel surface, they were generally small if compared with those in the radial direction due to the negligible temperature variation along the fuel surface. Hence they could be assumed to represent a second-order effect. In a local coordinate system that is moving with the receding fuel surface, the steady-state conduction term $\dot{q}_{\text {cond }}^{\text {ss }}$ can be expressed as:

$$
\dot{q}_{\text {cond }}^{s s}=k_{s} \frac{\partial T_{s}}{\partial \eta}=\rho_{s} \dot{r} c_{s}\left(T_{w}-T_{s i}\right)=\dot{m}_{b} c_{s}\left(T_{w}-T_{s i}\right)
$$


where $c_{S}$ and $k_{s}$ indicate the fuel heat capacity per unit mass and the fuel thermal conductivity, respectively, and $T_{w}$ and $T_{s i}$ are the fuel grain wall temperature and initial temperature, respectively. It is worth noting that the steady-state conduction heat flux, Equation (6), is affected by the fuel specific heat, but not by its thermal conductivity, which only influences the in-depth fuel grain temperature profile. The steady-state assumption appears as a reasonable approximation as long as the thermal lag in the solid phase is sufficiently short, which actually occurs in the hybrid rocket operating conditions to be simulated, i.e., moderately high fuel regression rates and low fuel thermal diffusivity.

The surface energy balance equation can be recast using Equation (2), in order to emphasize the contributions due to convection from the gas phase, net radiation, pyrolysis surface reactions, and conduction into the solid fuel grain by recalling that, by the definition of the mixture enthalpy at the wall, $h_{w}=\sum_{i=1}^{N} y_{i} h_{i}$ :

$$
\underbrace{k \frac{\partial T}{\partial \eta}}_{\text {convection }}+\underbrace{\epsilon_{w}\left(\dot{q}_{\mathrm{rad}}-\sigma T_{w}^{4}\right)}_{\text {net radiation }}=\underbrace{\sum_{i=1}^{N} \dot{\omega}_{i} h_{i}-\dot{m}_{b} h_{s}}_{\text {pyrolysis }}+\underbrace{\dot{q}_{\text {cond }}^{s s}}_{\text {solid conduction }}
$$

The chemical heat flux due to pyrolysis surface reactions can be expressed as:

$$
\dot{q}_{\mathrm{chem}}=\dot{m}_{b}\left(\sum_{i=1}^{N} \frac{\dot{\omega}_{i}}{\dot{m}_{b}} h_{i}-h_{s}\right)=\dot{m}_{b} \Delta h_{p}
$$

where the term between parentheses represents the heat absorbed by the pyrolysis surface reactions, and hence, it is the so-called heat of pyrolysis (more correctly, a heat of thermal degradation or depolymerization), $\Delta h_{p}$. Note that, upon substitution of Equations (6) and (8) into Equation (7), one obtains the final form of the surface energy balance:

$$
k \frac{\partial T}{\partial \eta}+\epsilon_{w}\left(\dot{q}_{\mathrm{rad}}-\sigma T_{w}^{4}\right)=\dot{q}_{\mathrm{conv}}+\dot{q}_{\mathrm{rad}} \mathrm{det}_{\mathrm{n}}=\rho_{s} \dot{r}\left[\Delta h_{p}+c_{s}\left(T_{w}-T_{s i}\right)\right]
$$

which, by defining $h_{v}=\left[\Delta h_{p}+c_{s}\left(T_{w}-T_{s i}\right)\right]$ as the effective heat of gasification of the solid fuel, coincides with the classical regression rate expression derived by Marxman and Gilbert [32]:

$$
\dot{r}=\frac{\dot{q}_{\mathrm{conv}}+\dot{q}_{\mathrm{rad}_{\mathrm{net}}}}{\rho_{\mathrm{s}} h_{v}}
$$

which shows that the fuel regression rate is proportional to the incoming heat flux (convective plus radiative) and is inversely proportional to the fuel density and its effective heat of gasification.

At each time step, the gas-surface interface boundary condition iteratively solves the overall mass balance, Equation (1), the species mass balance, Equation (2), and the energy balance, Equation (7), in order to compute the mixture composition at the fuel surface, the injected pyrolysis mass flux, and the wall temperature of the fuel grain.

\subsection{HDPE Pyrolysis Model}

The rate of production per unit surface area of gas-phase species $i, \dot{\omega}_{i}$, appearing in Equations (2) and (7), has to be estimated based on the fuel pyrolysis model. The kinetics and energetics of fuel thermal degradation can be investigated with various experimental techniques, such as thermogravimetry, typically under much lower heating rates than those of hybrid propulsion. In particular, these techniques have been applied to HDPE, a fuel often considered for application to lab-scale hybrid motors with simple perforations. Surface regression (often called linear pyrolysis) experiments have been performed by different authors [33-36] in order to measure the regression rate and the surface temperature of the fuel, as well as its heat of pyrolysis (or of degradation). Under the effect of an external heating source, the fuel grain is subjected to an abrupt temperature rise and, 
only very close to the surface, undergoes a thermal decomposition into gases [37]. The fuel grain thermal behavior is usually separated in two zones: the conduction zone, where heat is penetrating into the fuel, and the superficial degradation zone, where the fuel thermal degradation occurs. The latter is typically more than one order of magnitude smaller than the former. According to Lengelle [37], assuming a first-order reaction, the superficial degradation zone is described by the conservation of the non-degraded (virgin) material along the material thickness, $x$, as:

$$
\rho_{s} \dot{r} \frac{d Y_{p}}{d x}=\rho_{s} Y_{p} A \cdot \exp \left(-E_{a} / R T_{w}\right)
$$

where $Y_{p}$ is the mass fraction of the virgin polymer. The terms $A$ and $E_{a}$ are the pre-exponential factor (in $\mathrm{sec}^{-1}$ ) and the activation energy (in $\mathrm{kJ} / \mathrm{mol}$ ) of the fuel pyrolysis law, respectively, and $R$ is the universal gas constant. With the details of the complete explicit calculation given by Lengelle [34], the relation between fuel regression rate and surface temperature is obtained,

$$
\dot{r}=\sqrt{A \cdot \exp \left(-E_{a} / R T_{w}\right) \frac{1}{E_{a} / R T_{w}} a_{s} \mathcal{R}^{2}}
$$

with $a_{S}$ being the fuel thermal diffusivity and $\mathcal{R}$ being a non-dimensional regression rate of the form:

$$
\mathcal{R}^{2}=\frac{1}{\left[-\ln \left(Y_{p w}\right)\left(1-T_{s i} / T_{w}+\Delta h_{p} / c_{s} T_{w}\right)-\Delta h_{p} / c_{s} T_{w}\right]}
$$

Equation (12) expresses that as the regression rate increases, the surface temperature has to increase in order to accelerate the degradation process to allow for the complete decomposition of the material into gases. The term $Y_{p w}$, i.e., the mass fraction of the virgin polymer at the fuel surface, was assumed to be 0.01. Equation (12) can be expressed in the form:

$$
\dot{r}=\hat{A} \cdot \exp \left(-E_{a} / 2 R T_{w}\right)
$$

with:

$$
\hat{A}=\mathcal{R} \sqrt{\frac{A a_{s}}{E_{a} / R T_{w}}}
$$

where the term $\hat{A}$ is expressed in $\mathrm{m} / \mathrm{s}$ and is varying with temperature. A simpler Arrhenius-type pyrolysis law of the form:

$$
\dot{r}=\bar{A} \cdot \exp \left(-E_{a} / 2 R T_{w}\right)
$$

is often used for correlating the regression rate with surface temperature [38,39], where the term $\bar{A}$ represents the Arrhenius pre-exponential constant obtained from curve fitting of experimental data. Arrhenius-type pyrolysis laws of the form of Equation (16) can be obtained for various fuel formulations by plotting the fuel regression rate (in millimeters per second) vs. the reciprocal surface temperature $1 / T_{w}$ (in degrees Kelvin). Experimental sets of data generally follow the same trend and fit quite well to a single straight line on the semilog plot represented by the Arrhenius-type expression of the form of Equation (16) [40]. Note that Equation (16) is given in the form of the pyrolysis law based on bulk kinetics (Equation (14)) where the term $E_{a} / 2$ represents the value of the activation energy of the Arrhenius-type data fit [40]. The values assumed for these coefficients are reported in Table 1, according to [34]. Note that, because of the large value of the activation energy $E_{a}$ (Table 1), relevant regression rate changes can be generated with minor changes of the fuel surface temperature.

In this work, as a single one-step irreversible Arrhenius-type equation was adopted to model $\dot{r}=\dot{m}_{b} / \rho_{s}$, it was assumed that the only pyrolysis product of HDPE was the monomer ethylene $\left(\mathrm{C}_{2} \mathrm{H}_{4}\right)$. 
Table 1. Arrhenius rate constants from curve fitting for HDPE [34].

\begin{tabular}{lcc}
\hline Surface Reaction & $\bar{A}, \mathbf{m} / \mathbf{s}$ & $E_{a}, \mathbf{k J} / \mathbf{m o l}$ \\
\hline $\mathrm{HDPE} \rightarrow \mathrm{C}_{2} \mathrm{H}_{4}$ & $4.78 \times 10^{3}$ & 251.04 \\
\hline
\end{tabular}

Hence, according to [6], the species production rate per unit surface area, $\dot{\omega}_{i}$, is equal to $\dot{m}_{b}$ for the species $\mathrm{C}_{2} \mathrm{H}_{4}$, while it is equal to zero for all the remaining gaseous species. As concerns the heat of pyrolysis, it could be directly evaluated from Equation (8); nevertheless, since the assumption that the pyrolysis gas is only composed by the monomer ethylene is necessarily an approximation, here, the heat of pyrolysis was rather derived from the available experimental data. According to [34], a value of $2.72 \mathrm{MJ} / \mathrm{kg}$ was assumed for the heat of pyrolysis of HDPE. All the HDPE properties used for the simulations are listed in Table 2.

Table 2. HDPE properties [34].

\begin{tabular}{llll}
\hline $\begin{array}{l}\text { Density } \rho_{s} \\
\mathrm{~kg} / \mathrm{m}^{3}\end{array}$ & $\begin{array}{l}\text { Specific Heat } c_{s} \\
\mathrm{~J} /(\mathbf{k g} \cdot \mathrm{K})\end{array}$ & $\begin{array}{l}\text { Thermal Conductivity } k_{s} \\
\mathrm{~W} /(\mathrm{m} \cdot \mathrm{K})\end{array}$ & $\begin{array}{l}\text { Heat of Pyrolysis } \Delta h_{p} \\
\mathbf{M J} / \mathbf{k g}\end{array}$ \\
\hline 960 & 1255.2 & 0.1549 & 2.72 \\
\hline
\end{tabular}

Note that, although ethylene was assumed to be the only pyrolysis product, the gaseous mixture at the fuel surface was not solely composed of $\mathrm{C}_{2} \mathrm{H}_{4}$, as the other gaseous species (i.e., oxygen and the combustion gases) can actually reach/leave the surface due to diffusion and convection induced by wall blowing, as shown in Equation (2). The gaseous mixture composition at the HDPE surface, in fact, can be determined by solving the species surface mass balance, Equation (2), coupled with the surface energy balance, Equation (7), to guarantee that the correct amount of gaseous fuel is injected into the flowfield from the grain surface.

\subsection{Gas Phase Reactions}

Finite-rate gas phase reactions were modeled using a global reaction mechanism, because detailed chemical kinetics mechanisms [41] would include several species and many reactions and would be, on the one hand, computationally intensive and, on the other hand, beyond the scope of the present study, whose major purpose is to focus on the gas-surface interaction and its coupling with the internal ballistics. Therefore, gas phase reactions were modeled using a simplified two-step global reaction mechanism. Within this mechanism, the first irreversible global reaction step involved $\mathrm{C}_{2} \mathrm{H}_{4}$, the fuel pyrolysis product, and molecular oxygen to form $\mathrm{CO}$ and $\mathrm{H}_{2} \mathrm{O}$ and was considered first-order in both the fuel and oxidizer. The second global step was reversible and accounted for the formation of $\mathrm{CO}_{2}$.

$$
\begin{aligned}
\mathrm{C}_{2} \mathrm{H}_{4}+1.5 \mathrm{O}_{2} & \Rightarrow 2 \mathrm{CO}+\mathrm{H}_{2} \mathrm{O}+\mathrm{H}_{2} \\
\mathrm{CO}+0.5 \mathrm{O}_{2} & \Leftrightarrow \mathrm{CO}_{2}
\end{aligned}
$$

According to [5,42], the net reaction rates of these two global steps can be expressed as follows, where $[\cdot]$ indicates the species concentration:

$$
\begin{aligned}
& \dot{w}_{1}=k_{f_{1}}\left[\mathrm{C}_{2} \mathrm{H}_{4}\right]\left[\mathrm{O}_{2}\right] \\
& \dot{w}_{2}=k_{f_{2}}[\mathrm{CO}]\left[\mathrm{H}_{2} \mathrm{O}\right]^{0.5}\left[\mathrm{O}_{2}\right]^{0.25}-k_{b_{2}}\left[\mathrm{CO}_{2}\right]\left[\mathrm{H}_{2} \mathrm{O}\right]^{0.5}\left[\mathrm{O}_{2}\right]^{-0.25}
\end{aligned}
$$


The resulting rates of production and destruction of species $i$ per unit volume, $\dot{w}_{i}$, were obtained from the net reaction rates, $\dot{w}_{j}$, and the reaction stoichiometry:

$$
\begin{aligned}
\dot{w}_{\mathrm{C}_{2} \mathrm{H}_{4}} & =-M_{\mathrm{C}_{2} \mathrm{H}_{4}} \dot{w}_{1} \\
\dot{w}_{\mathrm{O}_{2}} & =M_{\mathrm{O}_{2}}\left\{-1.5 \dot{w}_{1}-0.5 \dot{w}_{2}\right\} \\
\dot{w}_{\mathrm{CO}} & =M_{\mathrm{CO}}\left\{2 \dot{w}_{1}-\dot{w}_{2}\right\} \\
\dot{w}_{\mathrm{H}_{2} \mathrm{O}} & =M_{\mathrm{H}_{2} \mathrm{O}} \dot{w}_{1} \\
\dot{w}_{\mathrm{H}_{2}} & =M_{\mathrm{H}_{2}} \dot{w}_{1} \\
\dot{w}_{\mathrm{CO}_{2}} & =M_{\mathrm{CO}_{2}} \dot{w}_{2}
\end{aligned}
$$

where $M_{\ldots}$ is the molar mass of the generic species indicated as the subscript. The forward and backward reaction rates, $k_{f}$ and $k_{b}$, for the two reaction steps are expressed as Arrhenius functions in the usual form $k=A_{k} T^{n_{k}} \exp \left(-E_{a, k} / R T\right)$, where the values of the constants used in this work are tabulated in Table 3.

Table 3. Reaction rate constants for global reactions (17) and (18).

\begin{tabular}{lccc}
\hline Reaction Rate & $A_{\boldsymbol{k}}$ & $n_{\boldsymbol{k}}$ & $\boldsymbol{E}_{\boldsymbol{a}, \boldsymbol{k}} / \boldsymbol{R}, \mathbf{K}$ \\
\hline$k_{f_{1}}[5]$ & $4.9486 \times 10^{9}$ & 0.0 & 15200 \\
$k_{f_{2}}[42]$ & $2.2400 \times 10^{6}$ & 0.0 & 5032.7 \\
$k_{b_{2}}[42]$ & $1.1000 \times 10^{13}$ & -0.97 & 39456.5 \\
\hline
\end{tabular}

The first global step rate constants were taken from the work of [5], while the second step was taken from [42], which is a modified version of the reaction used in [5,6] to account for oxy-fuel combustion conditions.

\subsection{Radiation Model}

The radiation heat flux is usually recognized to play a role in determining the fuel regression rate, especially when burning either metalized or carbon black solid fuels. Furthermore, according to [39], in addition to convection, radiation from soot and variable fluid properties across the boundary layer can have significant effects on the regression rate, even with pure polymers. Radiation from the gas phase is, instead, typically considered smaller than convection and soot radiation. Despite soot radiation being generally considered more important than radiation from combustion products in the gaseous phase, soot radiation modeling still involved much more uncertainties than gas radiation modeling. For this reason, as a first step towards complete modeling of radiative heat flux for the present gas-surface interaction model, only the radiative contribution from hot gases was taken into account and discussed. Marxman and Gilbert [32] and Muzzy [43] proposed the first fuel regression model incorporating the effect of the radiative heat flux of the gas phase to the wall. By means of their boundary layer diffusion-controlled regression theory, they showed that a small radiation heat flux would not significantly affect the regression rate for the well-known convective heat transfer blockage due to the increased fuel blowing. As expected, the radiation contribution grows with the engine scale, i.e., with fuel port diameter. However, it has been established that the radiant heat flux is relatively more significant under low mass flux and low oxidizer-to-fuel ratio conditions. Here, a separate model for the calculation of the radiation heat flux from the gas phase is presented. The basic hypotheses adopted include gray/diffuse wall and gray/non-scattering medium. The gray/diffuse wall assumption was deemed acceptable since thermal radiation from the wall is not a major contribution. The gray/non-scattering medium assumption, on the other hand, allows us to reduce the computational time enormously, thanks to a spectrally-averaged treatment. It was also assumed that the radiation energy exchange did not affect the flowfield significantly [44,45], a hypothesis that is justified in view of the relatively small weight ( $1 \%$ at most for the analyzed test 
cases) of total power lost through heat transfer and the radiative one in particular, as compared to the thermal power generated within the thrust chamber. This assumption reasonably allows avoiding the calculation of the radiative heat flux at each cell volume in the flowfield and, hence, to neglect its contribution to the energy conservation equation. The radiative heat flux was therefore only evaluated at selected nodes on the wall boundary.

The incident radiative wall heat flux, $\dot{q}_{\text {rad }}$, reaching a specific wall location and coming from other wall locations through the gas and from the gas, is defined by the following integral expression of the radiative intensity at the wall, $\mathcal{I}_{w}$, over the hemispherical solid angle that is facing the incoming radiation, $\Omega$ :

$$
\dot{q}_{\mathrm{rad}}=\int_{2 \pi} \mathcal{I}_{w} \sin \theta d \Omega=\int_{0}^{2 \pi} \int_{0}^{\frac{\pi}{2}} \mathcal{I}_{w} \sin \theta \cos \theta d \theta d \psi
$$

The radiative intensity at the wall from each generic line-of-sight can be computed by integrating the Radiative Transfer Equation (RTE) along the entire radiation path length. The RTE represents the balance of the radiative intensity along a generic direction, including contributions due to emission/absorption and, potentially, in-/out-scattering. Under the hypothesis of gray/non-scattering medium, it reduces to the form:

$$
\frac{d \mathcal{I}}{d s}=j^{e}-\kappa \mathcal{I}=\kappa \mathcal{I}_{b}-\kappa \mathcal{I}
$$

where $j^{e}$ is the power emitted from the gas per unit volume, which can be expressed as proportional to black-body radiative intensity $\mathcal{I}_{b}=\sigma T^{4} / \pi$ through a proportionality constant for emission, which is the same as the absorption coefficient $\kappa$.

The RTE formal solution is then given by:

$$
\mathcal{I}_{w}=\mathcal{I}_{w, 0} \exp \left(-\int_{s_{0}}^{s_{w}} \kappa d s\right)+\int_{s_{0}}^{s_{w}} j^{e} \exp \left(-\int_{s}^{s_{w}} \kappa d s^{\prime}\right) d s
$$

where it was assumed that the line-of-sight is originating from another wall point. The radiative intensity $\mathcal{I}_{w}$ reaching a generic wall point from a given line-of-sight was hence due to the contributions $\mathcal{I}_{w, 0}$ from the origin of the line-of-sight and $j^{e}$ from each point in the medium along the line-of-sight. The exponential terms account for the radiation absorption by the medium from the origin (first term in Equation (21)) and through the medium itself (second term in Equation (21)). Equations (19) and (21) require the knowledge of the radiative intensity at the origin, $\mathcal{I}_{w, 0}$, and of the absorption coefficient, $\kappa$, in order to be solved.

The radiative intensity at the origin, under the hypothesis of a gray/diffuse wall, is evaluated as:

$$
\mathcal{I}_{w, 0}=\frac{\epsilon_{w, 0} \sigma T_{w, 0}^{4}+r_{w, 0} \dot{q}_{\mathrm{rad}, 0}}{\pi}
$$

taking into account both the radiative intensity that is emitted and reflected by the wall at the line-of-sight origin, where surface emissivity and reflectivity are $\epsilon_{w, 0}$ and $r_{w, 0}$, respectively. Assuming the wall as opaque, the radiation balance yields the relation $r_{w}+\alpha_{w}=1$ between reflectivity and absorptivity. Since such a boundary condition also depends on the radiative heat flux reflected by the wall, which in turn depends on the incoming radiative heat flux, $\dot{q}_{\mathrm{rad}, 0}$, an iterative procedure is hence required for the more general case of gray reflecting walls.

The absorption coefficient of the gas mixture was derived by means of a global model, which is typically used for high-temperature combustion gas mixtures under vibrational equilibrium conditions,

$$
\kappa=p \sum_{i=1}^{N_{\mathrm{rad}}} X_{i} \kappa_{p, i}
$$


indicating that the absorption coefficient of radiative energy is proportional to the pressure, $p$, and to the absorption coefficients of the $N_{\text {rad }}$ participating species, weighted with their molar fraction, $X_{i}$. In particular, water vapor, carbon monoxide, and carbon dioxide $[46,47]$ are the most relevant species in the process of thermal radiation exchange. The present absorption coefficients, also known as Planck-mean absorption coefficients, were averaged over the whole wavelength spectrum. Their temperature dependence was given up to $5000 \mathrm{~K}$ and at atmospheric pressure in [48], by fitting and extending the curves reported in $[46,47]$. This model is adopted here, although not explicitly accounting for high-pressure effects, admittedly leaving some uncertainty, which is deemed of a weight comparable to those implied by other aspects of the model.

In the present work, the RTE was integrated by DTM using an in-house software for generic inhomogeneous gray/non-scattering media and axisymmetric gray/diffuse boundaries. The software was suitably developed and validated in $[49,50]$.

\section{Motor Configuration and Firing Tests}

A basic description of the lab-scale motor and of the test facility that was employed to carry out the firing tests referred to in this work is reported here; more exhaustive data can be found in [51].

The lab-scale rocket had an axisymmetric combustion chamber; the main dimensions needed in the numerical simulations are reported in Figure 1 for the sake of the reader's convenience. Several static firings were performed with this motor configuration using HDPE grains. All the experimental firing test data used in this paper were obtained with an axial injector configuration that employs a subsonic converging conical nozzle whose exit diameter was $8 \mathrm{~mm}$. A stainless steel pre-chamber and a thermally insulated aft-mixing chamber were arranged upstream and downstream of the fuel grain, respectively. Gaseous oxygen was supplied to the motor feeding line through a pressure regulator and was measured by means of a calibrated Venturi tube. A water-cooled conical converging-diverging nozzle made of copper alloy with a 2.4 expansion ratio and a $16 \mathrm{~mm}$ throat diameter ensured long-duration firings without throat erosion. Chamber pressure was measured by using two capacitive transducers arranged in the pre-chamber and in the aft-mixing chamber.

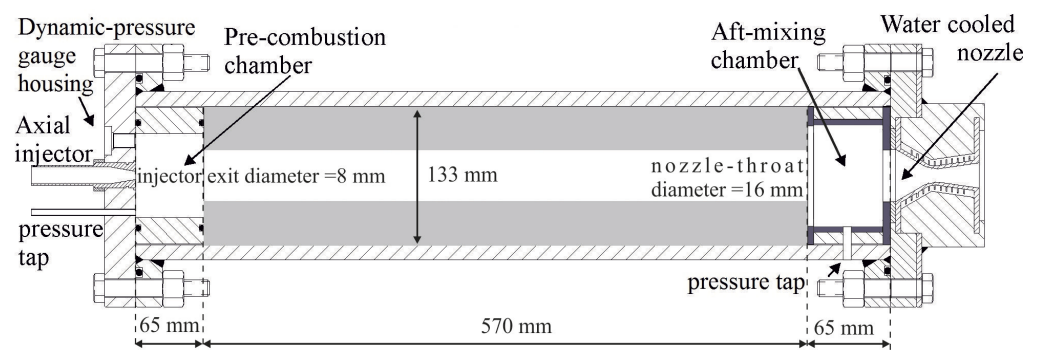

Figure 1. Lab-scale hybrid rocket engine schematic.

Cylindrical HDPE grains with a single circular port were employed with a fixed length, and four different initial inner diameters $(16,25,50$, and $75 \mathrm{~mm}$ ) were used to achieve a wide range of grain length to diameter ratios and of average mass fluxes at a limited expense of oxidizer mass flow rate (the maximum achievable oxygen flow rate being $350 \mathrm{~g} / \mathrm{s}$ ). The selected experimental test cases that were used for model sensitivity analysis and validation in this work are summarized in Table 4, which indicates the average parameters measured over the different firing tests. More details can be found in [51]. The firing test parameters were derived as follows. 
Table 4. Firing test average experimental results (HDPE) [51].

\begin{tabular}{ccccccc}
\hline Test No. & $\boldsymbol{t}_{\boldsymbol{b}}, \mathbf{s}$ & $\bar{G}_{\boldsymbol{o x}}, \mathbf{k g} / \mathbf{m}^{2} \cdot \mathbf{s}$ & $\overline{\mathbf{D}}, \mathbf{m m}$ & $\overline{\boldsymbol{r}}, \mathbf{m m} / \mathbf{s}$ & $\bar{p}_{\boldsymbol{c}}$, bar & $\overline{\mathrm{O} / \mathrm{F}}$ \\
\hline 2 & 54.9 & 27.68 & 75.84 & 0.47 & 17.07 & 2.05 \\
4 & 43.0 & 87.14 & 43.48 & 0.64 & 15.84 & 2.70 \\
9 & 31.5 & 28.41 & 89.84 & 0.47 & 22.91 & 2.50 \\
10 & 21.2 & 126.00 & 42.33 & 0.82 & 20.52 & 3.02 \\
13 & 26.2 & 28.19 & 60.04 & 0.38 & 10.22 & 2.05 \\
18 & 44.1 & 78.07 & 40.32 & 0.55 & 11.94 & 2.64 \\
19 & 44.3 & 54.03 & 47.19 & 0.50 & 11.26 & 2.37 \\
\hline
\end{tabular}

The time- and space-averaged fuel regression rate was calculated via the fuel-mass loss method:

$$
\overline{\dot{r}}=\frac{\overline{\dot{m}}_{f}}{\rho_{s} \pi \bar{D} L}
$$

where $\overline{\dot{m}}_{f}$ represents the time-averaged fuel mass flow rate that is obtained by dividing the measured fuel mass loss, $\Delta M$, by the burning time, $t_{b}$ :

$$
\overline{\dot{m}}_{f}=\frac{\Delta M}{t_{b}}
$$

The time-averaged (over the entire burning) port diameter, $\bar{D}=\left(D_{0}+\widehat{D}_{2}\right) / 2$, was computed from the initial port diameter, $D_{0}$, and the space-averaged final one, $\widehat{D}_{2}$. $\widehat{D}_{2}$ was determined by means of the consumed fuel mass as follows:

$$
\widehat{D}_{2}=\sqrt{D_{0}^{2}+\frac{4 \Delta M}{\pi \rho_{s} L}}
$$

The determination of the burning time, $t_{b}$, derived from the pressure-time trace, following a well-assessed procedure [51].

Finally, the time- and space-averaged mass flux was computed based on the average port diameter and the time-averaged mass flow rate (either the oxidizer or the total one):

$$
\bar{G}=\frac{4}{\pi} \frac{\overline{\dot{m}}}{\bar{D}^{2}}
$$

This averaging definition was demonstrated to be the most accurate and reliable in describing the classical power law of the regression rate over mass flux [52].

\section{Domain Discretization and Boundary Conditions}

In order to simulate the motor operating conditions and predict the internal ballistics in terms of motor regression rate and chamber pressure that were experimentally measured, the flow domain was discretized into a grid. The computational domain analyzed represented a simplified geometrical representation of the physical domain in which the pre-chamber, the fuel grain, and the post-chamber were all schematized with a constant cross-section (see Figure 2). Based on the available experimental data, the inflow boundary condition enforced the oxidizer static temperature and mass flow rate. More specifically, a single value for the oxygen inflow temperature ( $T=300 \mathrm{~K}$ ) was imposed in all simulations, whereas different values for the oxygen mass flow rate were enforced according to the firing test (see Table 4). It is worth noting that the oxidizer mass flow rate was controlled by the choked Venturi injector and was held constant during the firing, regardless of the grain diameter and chamber pressure variations over time. The pyrolysis gas that was injected from the fuel wall was $\mathrm{C}_{2} \mathrm{H}_{4}$, and its amount was determined first by iteratively solving Equation (7) for the wall temperature and, then, 
by updating the gaseous chemical composition at the grain surface through Equations (2) and (16). Incoming net radiative heat flux was computed from the DTM radiation code as a post-processing of the CFD solution. The CFD code and radiation code were coupled until convergence was reached. Some iterations between the two codes were required to reach convergence as the radiation computed by the DTM code affected the fuel regression rate, which, in turn, affected the CFD solution. All the simulation presented here were axisymmetric and at the steady-state condition reached by iterating in time until the residuals dropped by five orders of magnitude. For each value of enforced inflow oxygen mass flow rate, the CFD solution provided a prediction of the fuel regression rate axial distribution (from the surface balances), as well as of the chamber pressure value in the motor arising from the choked condition at the nozzle throat. Hence, the chamber pressure level attained in the chamber depended on both the fuel mass flow rate and the mixing and combustion process.

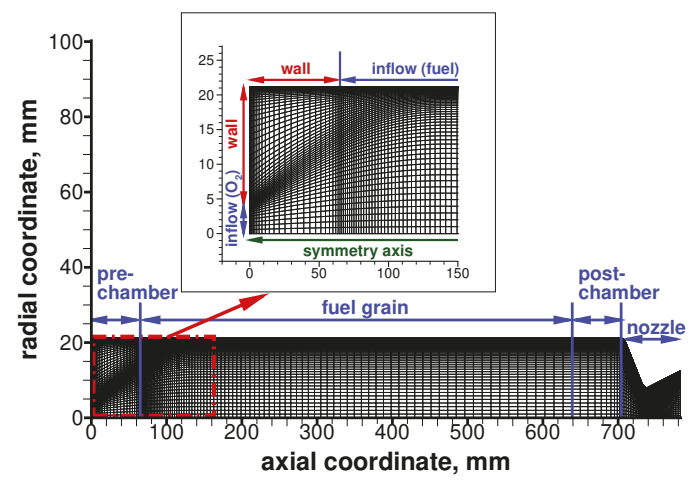

Figure 2. Numerical grid schematic (not to scale).

Figure 2 shows the numerical grid for Firing Test \#10 (see Table 4) at the average port diameter condition. An enlargement of the injection region is also shown that highlights the adopted grid refinement needed to capture the strong recirculation region induced by the axial injector. The computational domain was divided into $170 \times 60$ grid volumes in the axial and radial directions, respectively. Volume cells were clustered towards the surface so as to ensure a value of $y^{+}$of about one at the wall-adjacent cell to accurately describe the boundary layer up to the wall all along the motor length. The dimension of the wall-adjacent cell at the fuel surface was of the order of $10 \mu \mathrm{m}$. Additional axial clustering of cell volumes was placed in the region near the grain leading and trailing edges, as well as in the nozzle region. In the numerical simulations, the details of the leading- and trailing-edge regions of the fuel and the corresponding pre- and post-chamber cavities were omitted. Those simplifications of the actual geometrical details (Figure 1) allowed a reasonable computational time and accuracy in order to perform parametric analyses. A grid sensitivity analysis and a grid convergence analysis was carried out to ensure that the results were grid independent and to assess that the grid size and refinement was sufficient to obtain an accurate solution, respectively. A finer $340 \times 120$ mesh, made by doubling the grid volumes in both axial and radial directions with respect to the reference one, was also used for Firing Test \#10. Results computed by the two grids showed a difference in the average chamber pressure and in the average regression rate of approximately $1 \%$ and $2 \%$, respectively. Hence, the reference $170 \times 60$ grid was considered sufficiently refined for the present analysis.

\section{Results and Discussion}

For each experimental firing, which was characterized by a given oxygen mass flow rate (constant over the firing) and by an evolving port diameter due to the fuel regression, a single computation 
was performed at the average port diameter (known from measurements and reported in Table 4) and enforcing the experimentally-determined oxygen mass flow rate. Performing a single numerical simulation for each experimental firing is indeed a simplification of the actual test condition as the port diameter inevitably changes during the burning time. However, previous results [4] have shown that the time- and space-averaged regression rate computed through several computations performed at different grain geometries (each corresponding to a specific phase of the burn) only deviates by a few percent from the space-averaged regression rate derived from a single simulation computed at the average port diameter. Hence we assumed, here and in the following, that the space-averaged values computed from the simulations carried out at the average port diameter were representative of time- and spatially-averaged values measured in the tests. Moreover, the oxidizer mass flux and the port diameter of each simulation, respectively indicated with $\bar{G}_{0 x}$ and $\bar{D}$, were to be considered as averaged values. As an example, Figure 3 shows the resulting temperature field at the average port diameter of Firing Test \#19 with an oxygen mass flow rate of $94.5 \mathrm{~g} / \mathrm{s}$. The simulation showed that the flowfield in the motor entrance region was dominated by a strong recirculation region generated by the oxidizer axial injection. This hot recirculation region was instrumental in enhancing fuel and oxidizer mixing and combustion. Figure 3 also shows the cold region $(<1000 \mathrm{~K})$ close to the motor axis in the proximity of the axial oxygen injector.

Before comparing the computed results in terms of predicted fuel regression rate and motor chamber pressure with respect to the experimental measurements, a thorough sensitivity analysis of the fuel pyrolysis model was performed.

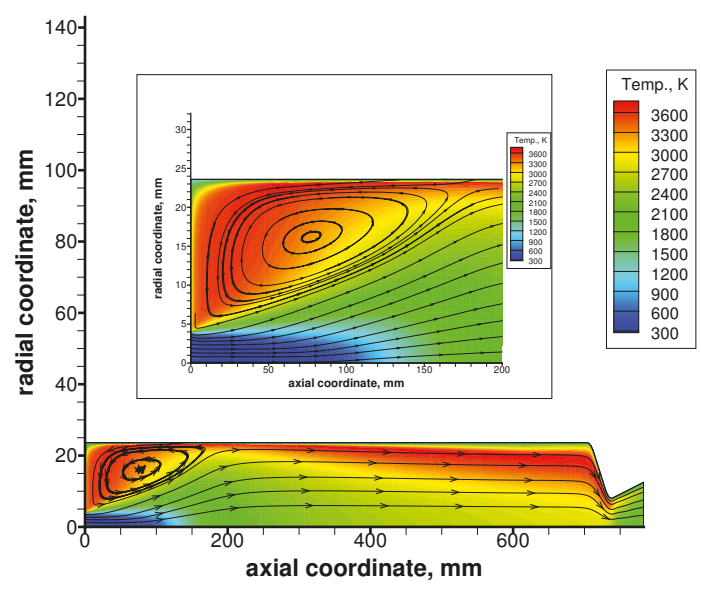

Figure 3. Temperature field and detail of the recirculation zone for Firing Test \#19 (not to scale).

\subsection{Sensitivity Analysis of the Pyrolysis Model}

In order to understand how the law of pyrolysis and the heat of pyrolysis used in the surface energy balance can affect the regression rate and chamber pressure prediction, a sensitivity analysis was performed. In fact, aside from the reference model presented in Section 2.2 (see Table 1), other pyrolysis models are available in the literature. Hence, before comparing the obtained predictions with the experimental measurements, it was important to quantify the effect of the fuel pyrolysis model uncertainties on such predictions. For such a study, Firing Test \#19 was taken as the reference because of its intermediate port diameter $(47.19 \mathrm{~mm})$ and oxidizer mass flux $\left(54.03 \mathrm{~kg} / \mathrm{m}^{2} \cdot \mathrm{s}\right)$ with respect to the operating conditions of the considered firing tests (Table 4).

The laws of pyrolysis available in the literature are widely scattered because of significantly different pre-exponential constants and activation energies. At a given temperature, this leads to significantly different values of regression rate, as is shown in Figure 4, where five different laws of 
pyrolysis are compared for HDPE fuel grain temperatures ranging approximately from 750 to $950 \mathrm{~K}$. The five analyzed laws of pyrolysis were taken from different references in the open literature, and they were expressed either in the form of Equation (14) or in the form of Equation (16). In fact, when the pyrolysis reaction rates were obtained from thermogravimetric analyses, they were expressed in terms of reaction speeds with a pre-exponential factor $A$ in $\mathrm{sec}^{-1}$, while the pre-exponential constant $\bar{A}$ of Equation (16) was expressed in $\mathrm{m} / \mathrm{s}$ and was obtained from curve fitting of surface regression experiments. When the form of Equation (14) was used, a pre-exponential term $\hat{A}$, which varied with temperature, could be computed from the pre-exponential factor, the fuel thermal diffusivity, and the non-dimensional regression rate from Equation (15).

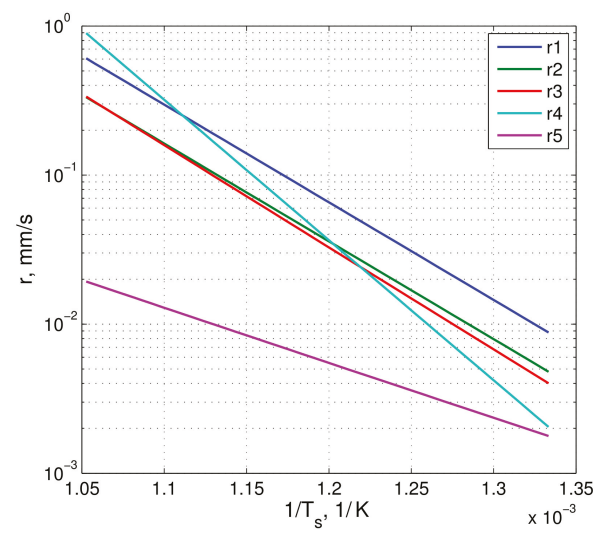

Figure 4. HDPE regression rate vs. temperature from various pyrolysis laws.

Tables 5 and 6 list the activation energy and the pre-exponential constant (see Table 5) or the pre-exponential factor (see Table 6) for the different HDPE pyrolysis laws. As shown in Figure 4, a significant scattering was evidenced between the various laws. Note that the r5 pyrolysis law, according to its authors [36], was obtained with a very low heating rate of about $20 \mathrm{~K} / \mathrm{min}$ that was several orders of magnitude lower than the heating rate of the fuel in actual hybrid rocket combustion chamber conditions, which was estimated to be around $10^{5} \mathrm{~K} / \mathrm{s}$ [36]. For this reason, the r5 pyrolysis law was discarded from this analysis. The behavior of the $\mathrm{r} 1, \mathrm{r} 2$, and $\mathrm{r} 3$ laws showed a very similar slope (Figure 4) due to the fact that the activation energies were practically the same. Differently, the r4 pyrolysis law showed a significantly higher activation energy. Finally, although the regression rate of the $\mathrm{r} 1, \mathrm{r} 2, \mathrm{r} 3$, and $\mathrm{r} 4$ pyrolysis laws were comparable, they could show differences in $\dot{r}$ at the same temperature up to $40 \%$. Therefore, it was of importance to analyze the effect of different activation energies and pre-exponential constants on the regression rate prediction in actual hybrid rocket conditions.

Starting from the reference law of pyrolysis r1 (blue curve in Figure 4), the following parameters were changed: first, the pre-exponential constant was modified by using the corresponding (lower) value from the law r2 (green curve in Figure 4), and second, the activation energy was modified by using the corresponding (higher) value from the law r4 (cyan curve in Figure 4). This was in order to analyze separately the effect of a change in each of the parameters $A$ and $E_{a}$ of the pyrolysis law.

Table 5. Activation energies and pre-exponential constants for different HDPE pyrolysis laws (Equation (16)).

\begin{tabular}{lccc}
\hline Pyrolysis Law & Source & $\bar{A}, \mathrm{~m} / \mathbf{s}$ & $E_{a}, \mathbf{k J} / \mathbf{m o l}$ \\
\hline r1 (reference) & {$[34,51]$} & $4.78 \times 10^{3}$ & 251.04 \\
r2 & {$[33]$} & $2.68 \times 10^{3}$ & 251.20 \\
\hline
\end{tabular}


Table 6. Activation energies and pre-exponential factors for different HDPE pyrolysis laws (Equation (14)).

\begin{tabular}{lccc}
\hline Pyrolysis Law & Source & $\boldsymbol{A}, \mathbf{1} / \mathbf{s}$ & $\boldsymbol{E}_{\boldsymbol{a}}, \mathbf{k J} / \mathbf{m o l}$ \\
\hline r3 & {$[34]$} & $2.00 \times 10^{16}$ & 251.04 \\
r4 & {$[35]$} & $4.80 \times 10^{22}$ & 349.00 \\
r5 & {$[36]$} & $7.57 \times 10^{6}$ & 130.00 \\
\hline
\end{tabular}

\subsubsection{Sensitivity to the Pyrolysis Law Parameters}

Figure 5 shows the effect of a $44 \%$ reduction in the pre-exponential constant (from $4.78 \times 10^{3}$ to $2.68 \times 10^{3} \mathrm{~m} / \mathrm{s}$ ) on both the fuel regression rate and the fuel temperature. Table 7 shows the results in terms of the space-averaged regression rate and motor chamber pressure.

Table 7. Computed average results for Firing Test \#19 with the effect of a change in the pre-exponential constant, Case \#19a.

\begin{tabular}{lll}
\hline Firing Test & $\bar{p}_{c}$, Bar & $\overline{\dot{r}}, \mathbf{m m} / \mathbf{s}$ \\
\hline$\# 19$ & 9.78 & 0.320 \\
$\# 19 \mathrm{a}$ & $9.69(-0.9 \%)$ & $0.316(-1.3 \%)$ \\
\hline
\end{tabular}

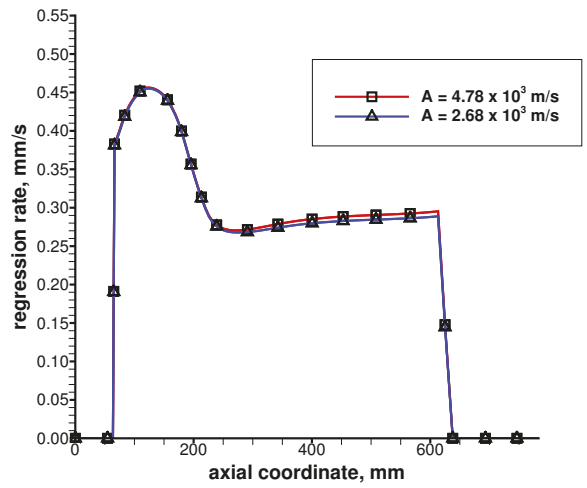

(a)

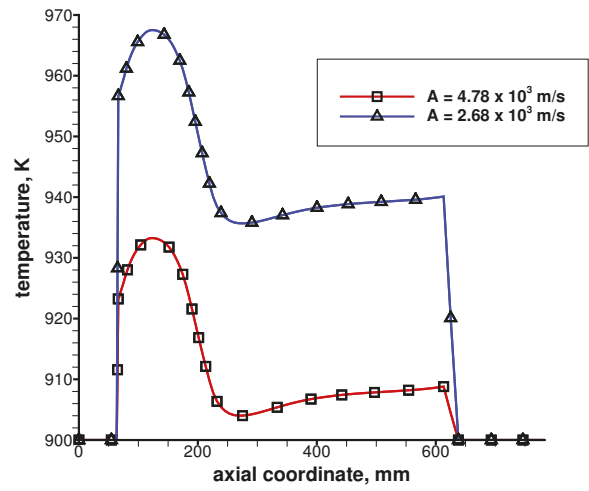

(b)

Figure 5. Effect of the pre-exponential constant on the fuel regression rate and the fuel temperature: (a) fuel regression rate; (b) fuel temperature.

Not surprisingly, once the widely-different pre-exponential constants were used in the gas-surface interaction wall boundary condition, very similar values of fuel regression rate were found, with a difference of the order of $1 \%$. This was due to the fact that the wall temperature behaved as a compensating factor, while the regression rate was dominantly dictated by the incoming heat flux, Equation (10). In fact, when a lower pre-exponential constant was used, a higher wall temperature resulted from the energy balance (see Figure $5 b$ ), leading to a computed regression rate that remained practically unaffected (see Figure 5a). Thus, the pyrolysis process was certainly controlled by the heating of the fuel surface, with reaction kinetics only playing a minor role [32]. Hence, as shown in Figure 5 and in Table 7, the reduction in the pre-exponential constant of the pyrolysis law was almost completely compensated by the increased grain wall temperature (of the order of 30-40 K), leading to negligibly small variations of both regression rate and motor chamber pressure.

Figure 6 shows the effect of a 39\% increase in the activation energy (from 251.04 to $349.00 \mathrm{~kJ} / \mathrm{mol}$ ) on both the fuel regression rate and the fuel temperature. Table 8 shows the results in terms of space-averaged regression rate and motor chamber pressure. 


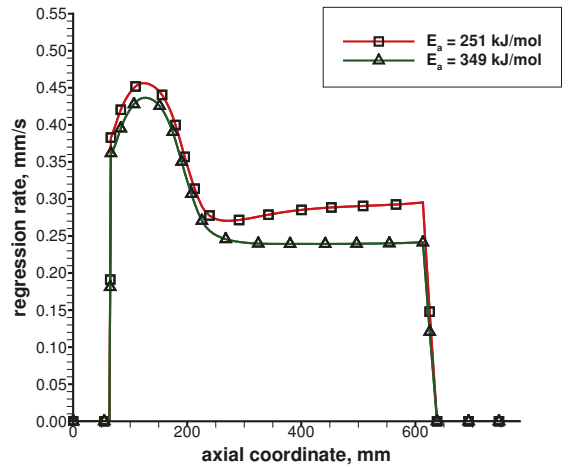

(a)

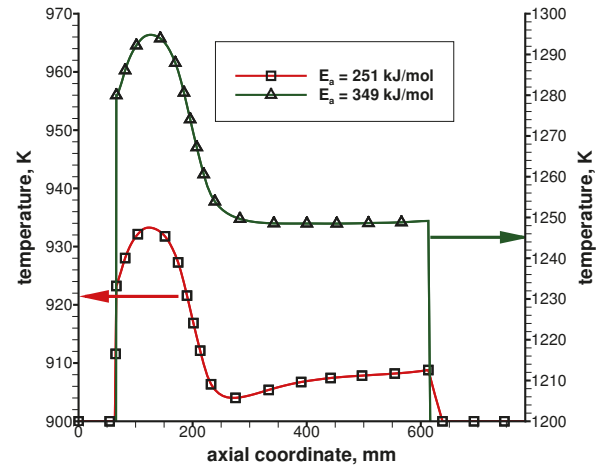

(b)

Figure 6. Effect of the activation energy on the fuel regression rate and the fuel temperature: (a) fuel regression rate; (b) fuel temperature.

Table 8. Computed average results for Firing Test \#19 with the effect of a change in the activation energy, Case \#19b.

\begin{tabular}{lll}
\hline Firing Test & $\overline{p_{c}}$, Bar & $\overline{\dot{r}}, \mathbf{m m} / \mathbf{s}$ \\
\hline$\# 19$ & 9.78 & 0.320 \\
$\# 19 \mathrm{~b}$ & $9.25(-5.4 \%)$ & $0.283(-11.6 \%)$ \\
\hline
\end{tabular}

The effect of the increase of the activation energy was qualitatively similar to the effect of a decrease in the pre-exponential constant, although quantitative results were significantly different. As shown in Figure 6 and in Table 8, the increase in the activation energy was only partially compensated by the increased grain wall temperature, causing limited, but non-negligible variations of both the regression rate and chamber pressure. Although the analyzed percentage variations in the pre-exponential constant and activation energy were similar ( $44 \%$ and $39 \%$, respectively), the effect of the activation energy on the overall results was much stronger than that of the pre-exponential constant, due to its exponential effect on the pyrolysis law. It must be stressed that the grain temperature increase was also much higher (of the order of 300-350 K) than in the previous case, thus causing a more significant reduction of the convective heat flux that translated into a regression rate reduction. Finally, it has to be noted that the present sensitivity analysis was carried out for the sake of investigation to understand the specific role of the two parameters $\left(A\right.$ and $\left.E_{a}\right)$ of the pyrolysis law. However, pyrolysis laws should be obviously used taking all the relevant parameters from a single reference, avoiding mismatches between parameters taken from different data. Therefore, a direct comparison between $\mathrm{r} 1$ and $r 4$ pyrolysis laws will be later presented. We conclude this sensitivity analysis highlighting the fact that both the pre-exponential constant and the activation energy had a direct effect on the grain wall temperature, while they were only mildly affecting the grain regression rate and, hence, the motor chamber pressure. This was especially true for the pre-exponential constant.

\subsubsection{Sensitivity to the Heat of Pyrolysis}

The last sensitivity that was analyzed, before comparing directly one complete set of pyrolysis law data to another complete set (r1 vs. r4), was the effect of the heat of pyrolysis, $\Delta h_{p}$. A significant scattering was found in the literature also for the heat of pyrolysis of HDPE. For such a reason, the heat of pyrolysis was reduced by an arbitrary amount of $30 \%$ with respect to the reference value $(2.72 \mathrm{MJ} / \mathrm{kg})$, listed in Table 2 , in order to measure the direct effect of its change on the results in terms of regression rate and chamber pressure. The results presented in Figure 7 and Table 9 showed a strong 
sensitivity to the value assumed for the heat of pyrolysis. A modest rise of the grain wall temperature (of the order of 6-12 K) was also found because of the increased available energy associated with the lower heat of pyrolysis. However, different from the two previous cases (\#19a and \#19b), the effect of the heat of pyrolysis on the grain wall temperature was minimal (of the order of $1 \%$ ), while the effect on the fuel regression rate (and hence, on the chamber pressure) was definitely more pronounced ( $+21 \%$ on the regression rate).

Table 9. Computed average results for Firing Test \#19 with the effect of a change in the heat of pyrolysis, Case \#19c.

\begin{tabular}{lll}
\hline Firing Test & $\overline{p_{c}}$, Bar & $\overline{\dot{r}}, \mathbf{m m} / \mathbf{s}$ \\
\hline$\# 19$ & 9.78 & 0.320 \\
$\# 19 \mathrm{c}$ & $10.37(+6.0 \%)$ & $0.387(+20.9 \%)$ \\
\hline
\end{tabular}

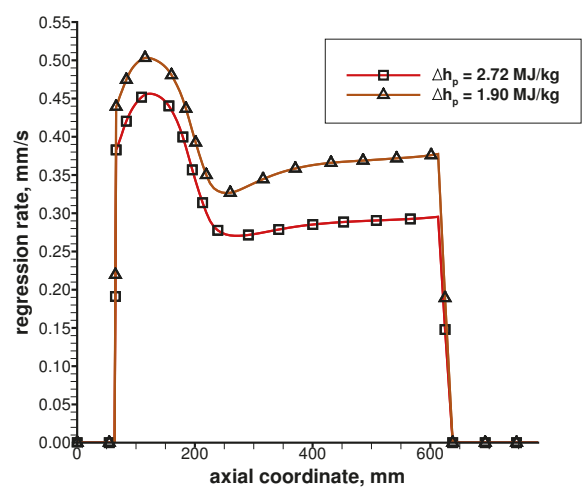

(a)

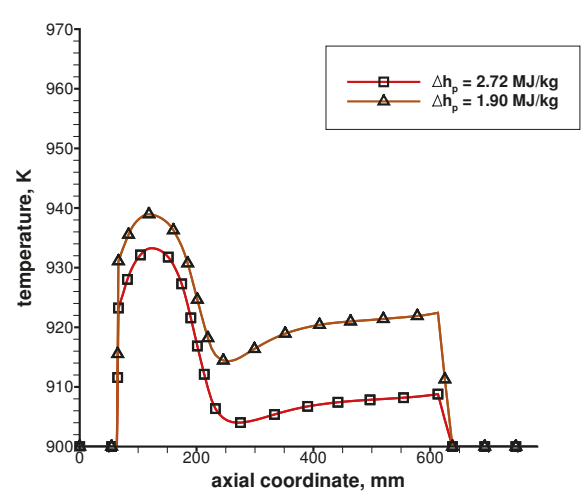

(b)

Figure 7. Effect of the heat of pyrolysis on the fuel regression rate and the fuel temperature:

(a) fuel regression rate; (b) fuel temperature.

This, again, confirmed that the regression rate was dictated by the energy balance at the grain surface and by the competition between incoming heat fluxes (convection and radiation) and outgoing heat fluxes (fuel pyrolysis and solid grain heating), as shown in Equation (10). A modification of the heat of pyrolysis without any change in the pyrolysis law (pre-exponential constant and activation energy), hence, caused a direct effect on the fuel regression rate, which was increased as the heat of pyrolysis was decreased and generated only a very limited effect on the grain temperature.

According to the results of the previous sensitivity analyses (Cases \#19a, \#19b, and \#19c), it can be concluded that the heat of pyrolysis was the parameter most affecting the fuel regression rate, followed by the activation energy with a relatively weak effect, and the pre-exponential constant showing negligibly small variations.

\subsubsection{Sensitivity to the Pyrolysis Law Dataset}

This last sensitivity analysis was performed comparing directly one complete set of pyrolysis law data to another complete set ( $\mathrm{r} 1$ in Table 5 vs. $\mathrm{r} 4$ in Table 6). The heat of pyrolysis $\Delta h_{p}$ associated with $\mathrm{r} 1$ law was $2.72 \mathrm{MJ} / \mathrm{kg}$, and the corresponding average specific heat $c_{s}$ was $1255.2 \mathrm{~J} /(\mathrm{kg} \cdot \mathrm{K})$ [34], while the heat of pyrolysis associated with $\mathrm{r} 4$ law was $1.138 \mathrm{MJ} / \mathrm{kg}$, and the corresponding average specific heat was $3428.5 \mathrm{~J} /(\mathrm{kg} \cdot \mathrm{K})$ [53]. For such a study, Firing Tests \#2 and \#10 were analyzed because they were characterized by the minimum and maximum oxidizer mass flux, respectively (see Table 4).

As shown in Table 10, the results in terms of the space-averaged regression rate and motor chamber pressure were moderately affected by a change in the adopted law of pyrolysis and associated 
heat of pyrolysis. In conclusion, it can be stated that a significant dependence of the solution from the law of pyrolysis adopted was not evidenced, provided that each dataset was consistently taken from a single reference.

Table 10. Computed average results for Firing Tests \#2 and \#10 with the effect of a change in the pyrolysis law data (r1 vs. r4), Cases \#2d and \#10d.

\begin{tabular}{lll}
\hline Firing Test & $\bar{p}_{c}$, Bar & $\overline{\dot{r}}, \mathbf{m m} / \mathbf{s}$ \\
\hline$\# 2(\mathrm{r} 1)$ & 12.78 & 0.248 \\
$\# 2 \mathrm{~d}(\mathrm{r} 4)$ & $13.23(+3.5 \%)$ & $0.265(+6.9 \%)$ \\
$\# 10(\mathrm{r} 1)$ & 16.26 & 0.541 \\
$\# 10 \mathrm{~d}(\mathrm{r} 4)$ & $17.01(+4.6 \%)$ & $0.589(+8.9 \%)$ \\
\hline
\end{tabular}

\subsection{Comparison with Experimental Data}

All the firing tests are considered in this section in order to understand the ability of the numerical setup to reproduce the experimental data in terms of fuel regression rate and motor chamber pressure. For such a comparison, at first, all simulations were performed neglecting radiative energy exchange and either imposing the experimental fuel regression rate or solving for the surface balances, which enabled the determination of the fuel temperature and regression rate as part of the flowfield solution. The corresponding deviation between measured and predicted data of chamber pressure and regression rate over all the firing tests are summarized in Figure 8, with both the imposed or calculated fuel regression rate. When the fuel regression rate was computed by solving the gas-surface interaction wall boundary condition (neglecting radiative energy exchange), results (see Figure 8a) indicated a quite evident underestimation of the averaged regression rate for all firing tests. This underestimation in the regression rate reflected more or less directly (depending on the average $\mathrm{O} / \mathrm{F}$ ratio and on the corresponding theoretical $c^{*}$ of the firing test) an underestimation of the motor operating chamber pressure (see Figure $8 b$ ). In particular, the regression rate was underestimated between 31 and $47 \%$, while the chamber pressure was underestimated between 13 and 25\%. Table 11 shows the space-averaged results in terms of regression rate, chamber pressure, and convective heat flux when solving the gas-surface interaction wall boundary condition. It is important to underline here that the predicted chamber pressure was not only the result of the fuel regression rate, but also of the mixing and combustion process within the combustion chamber, which can strongly affect the characteristic exhaust velocity. Hence, a correct prediction of the fuel regression rate did not necessarily grant that the chamber pressure was correctly predicted. If the mixing and combustion processes were not correctly modeled, in fact, a mismatch between predicted and measured chamber pressure would result.

Since the previously discussed sensitivity analysis showed that the results could be affected by the law of pyrolysis adopted just for a few percentage points (less than $10 \%$ for regression rate and less than $5 \%$ for the chamber pressure), a study to evaluate the goodness of the combustion model was carried out. To check that the mixing and combustion processes were adequately modeled in the current approach, simulations for five firing tests (namely \#2, \#4,\#10,\#13, and \#19) were computed by directly imposing the experimentally-measured averaged regression rate at the fuel boundary condition. Results are shown in Figure $8 \mathrm{~b}$ in terms of motor chamber pressure and show that, once the experimentally-measured regression rate value was imposed in the simulation, a very good prediction of motor pressure (hence of the characteristic velocity) was obtained. This confirmed that mixing and combustion processes were adequately modeled in the present approach. Nevertheless, when the fuel regression rate was predicted from the gas-surface interaction wall boundary condition, the averaged regression rate values from the various experimental firing tests were always underestimated (by $31 \%$ up to $47 \%$ ). Considering the analyses that were carried out so far with respect to the fuel pyrolysis model uncertainties, it is clear that such a significant underestimation must be attributed to another source of error, as the absence of the radiative contribution to the wall heating. The regression rate general trend (Figure 8a), in fact, seemed to suggest the lack of radiation modeling, 
whose effects are known to be more substantial at the lower oxidizer mass fluxes. Chiaverini et al. [38] showed that radiation effects are relevant for oxygen mass fluxes lower than $\approx 160 \mathrm{~kg} / \mathrm{m}^{2} \cdot \mathrm{s}$ and for chamber pressures in the range 1.5-2.5 MPa or higher. The experimental conditions analyzed here were inside the indicated pressure range, and all fall well below the $160 \mathrm{~kg} / \mathrm{m}^{2} \cdot \mathrm{s}$ for the oxygen mass flux, with three firing tests (\#2,\#13, and \#9) reaching average values below $30 \mathrm{~kg} / \mathrm{m}^{2} \cdot \mathrm{s}$. Hence, the analyzed tests were expected to show significant radiation effects.

Table 11. Computed average results (gas-surface interaction $\mathrm{w} / \mathrm{o}$ radiation).

\begin{tabular}{cccc}
\hline Test No. & $\overline{\dot{r}}_{,} \mathbf{~ m m} / \mathbf{s}$ & $\bar{p}_{c}$, Bar & $\overline{\dot{q}}_{\text {conv }}, \mathbf{M W} / \mathbf{m}^{2}$ \\
\hline 2 & 0.25 & 12.80 & 0.83 \\
4 & 0.42 & 12.51 & 1.43 \\
9 & 0.28 & 17.97 & 0.94 \\
10 & 0.54 & 16.21 & 1.86 \\
13 & 0.22 & 8.61 & 0.75 \\
18 & 0.38 & 9.91 & 1.28 \\
19 & 0.32 & 9.77 & 1.07 \\
\hline
\end{tabular}

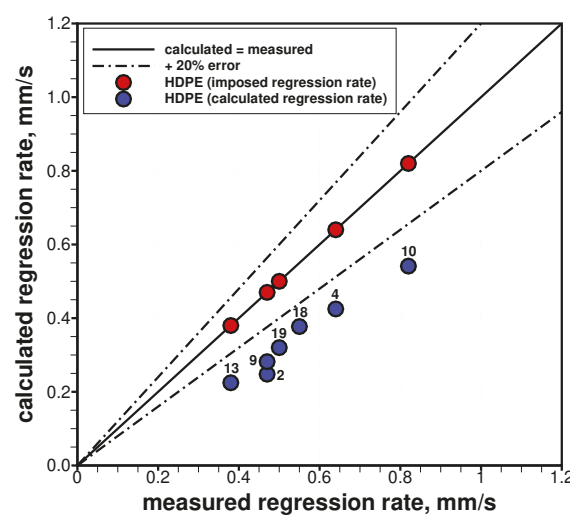

(a)

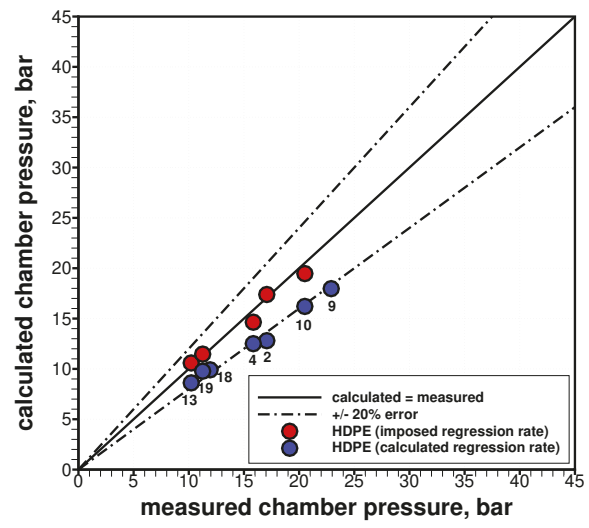

(b)

Figure 8. Comparison of computed values from simulations (with the calculated or imposed regression rate) with measured data from the experimental firings: (a) time- and space-averaged regression rates; (b) time-averaged chamber pressures.

\subsection{Effect of Thermal Radiation Exchange}

In order to analyze the effect of radiative energy exchange, CFD simulations were coupled to radiation heat transfer calculations to take into account the net incoming radiative heat flux to the fuel grain and its effect on the fuel regression rate, through Equation (10). A wall emissivity equal to 0.85 for the HDPE grain was assumed according to typical values for polymers exposed to flames. The wall and flowfield local conditions required by the radiative simulation were taken from the CFD solution, with which the radiative solution was coupled until convergence was attained. The resulting deviation between computed (with and without radiative energy exchange) and measured data of chamber pressure and regression rate over all the firing tests is summarized in Figure 9.

Table 12 shows the space-averaged results with coupled radiative energy exchange in terms of regression rate, chamber pressure, convective heat flux, and absorbed radiative heat flux. The larger port diameter firing tests (Firing Tests \#2, \#9, and \#13) were those for which the absorbed radiative heat flux was larger than the convective heat flux, denoting the highly non-linear contribution from thermal radiation exchange for varying operating conditions. Moreover, it is worth noting that the results obtained with coupled thermal radiation exchange (see Table 12) showed a convective heat flux 
that was lower than the corresponding case without radiation (see Table 11). This confirmed that, due to the well-known convective heat transfer blockage, the regression rate increase due to radiation was mitigated by a corresponding convective heat flux decrease, induced by the increased fuel blowing.

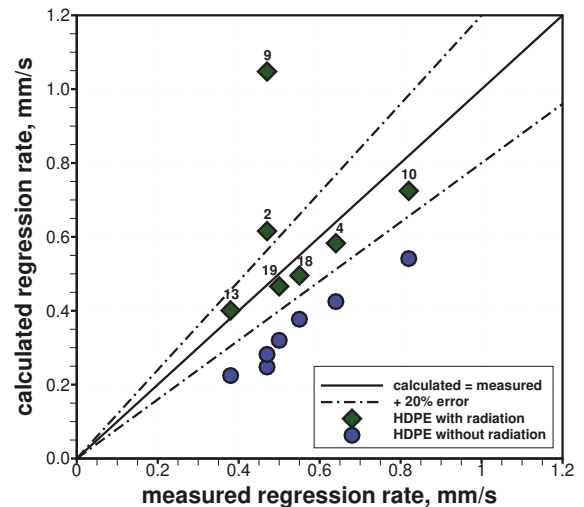

(a)

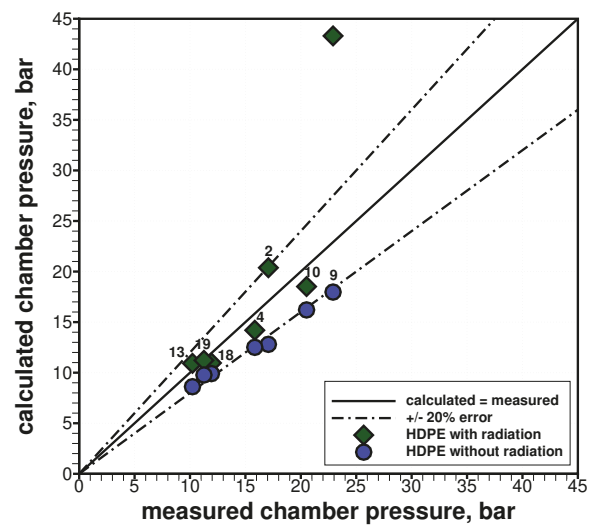

(b)

Figure 9. Comparison of computed values from simulations (with and without radiative energy exchange) with measured data from the experimental firings: (a) time- and space-averaged regression rates; (b) time-averaged chamber pressures.

Table 12. Computed average results (gas-surface interaction with radiation).

\begin{tabular}{ccccc}
\hline Test No. & $\overline{\dot{r}}, \mathbf{~ m m} / \mathbf{s}$ & $\bar{p}_{c}$, Bar & $\overline{\dot{q}}_{\text {conv }}, \mathbf{M W} / \mathbf{m}^{2}$ & $\overline{\dot{q}}_{\text {rad }_{\text {abs }},} \mathbf{M W} / \mathbf{m}^{2}$ \\
\hline 2 & 0.62 & 20.38 & 0.50 & 1.59 \\
4 & 0.58 & 14.20 & 1.34 & 0.64 \\
9 & 1.05 & 43.31 & 0.38 & 3.22 \\
10 & 0.72 & 18.52 & 1.74 & 0.73 \\
13 & 0.40 & 10.92 & 0.59 & 0.76 \\
18 & 0.50 & 10.96 & 1.22 & 0.46 \\
19 & 0.47 & 11.22 & 0.96 & 0.61 \\
\hline
\end{tabular}

Looking at the numerical results obtained with the radiation modeling, the errors previously found without including thermal radiation were much mitigated. Despite a noticeable improvement with respect to previous solutions without radiation, the regression rate and chamber pressure for firing tests characterized by the largest port diameters (Firing Tests \#2 and \#9) were evidently overestimated when including thermal radiation. In particular, Firing Test \#9 showed a large overestimation with respect to measured data on both the regression rate and chamber pressure data, requiring a deeper analysis of the port diameter effect on the net radiative heat flux to the grain surface. The rest of the analyzed tests, however, fell within a $\pm 12 \%$ error on both regression rate and chamber pressure.

In particular, Figures 10 and 11 show the axial distributions of convective, radiative, and total heat flux together with the fuel regression rate for Firing Test \#13 (for which $\bar{q}_{\text {rad }}$ abs was larger than $\bar{q}_{\text {conv }}$ ) and for Firing Tests \#18 (for which $\bar{q}_{\text {rad }}$ abs was smaller than $\bar{q}_{\text {conv }}$ ), respectively. The results highlight the coupling between radiation and the blocking effect, showing a reduction of the convective heat flux when radiation was included in the calculation. However, the effect was much stronger in the first one-third of the grain as the extra fuel injection was increasing the overall mass flux in the port, hence counteracting the blockage effect toward the aft-end of the grain. The analysis of the axial profiles also revealed that the peak of radiation heating was shifted further downstream with respect to that of convective heating, hence flattening the total incoming wall heat flux and, correspondingly, the regression rate axial distribution. 


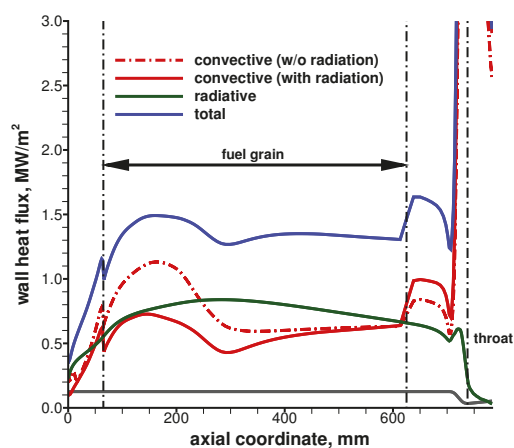

(a)

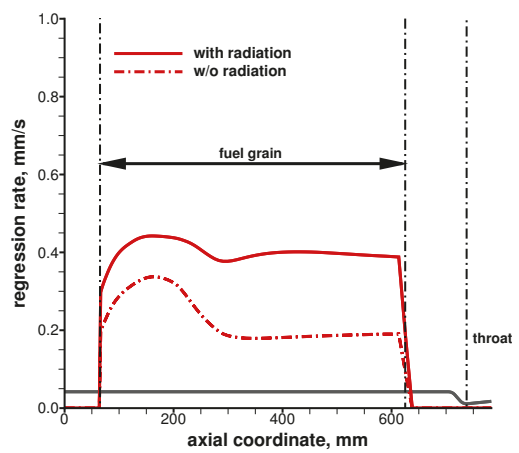

(b)

Figure 10. Axial distributions (with and without radiative energy exchange) of surface parameters for Firing Test \#13: (a) convective and radiative heat flux; (b) regression rate.

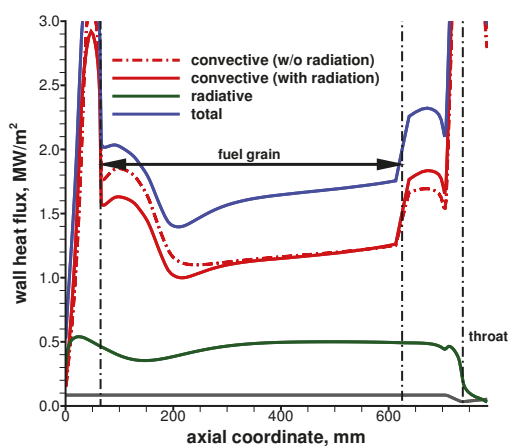

(a)

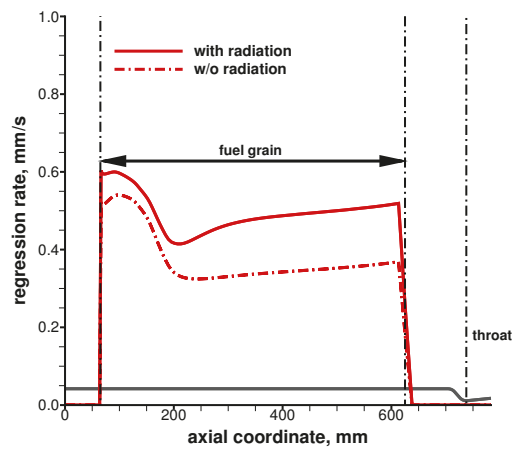

(b)

Figure 11. Axial distributions (with and without radiative energy exchange) of surface parameters for Firing Test \#18: (a) convective and radiative heat flux; (b) regression rate.

For Firing Test \#9, the radiative wall heat flux was found to be much higher with respect to the other test cases as a consequence of the high value of the product between chamber pressure and port diameter. One of the causes for this significant error on the computed thermal radiation at the wall could be the simplified chemical mechanism, which lacked radicals and hence tended to overestimate both flame temperature and radiation-emitting species concentrations. Therefore, to reduce the error for the high-pressure high-diameter tests, more detailed chemical reaction mechanisms including radical species should be included to have a better prediction of the radiative heat flux. Probably, this error was also amplified in the coupling procedure between CFD and radiative simulations. In fact, as the radiation enhanced the regression rate, the mixture ratio tended to shift from an oxidizer- to a fuel-rich condition, hence increasing flame temperature and radiation-emitting species' concentrations, which further increased the radiation contribution. Moreover, it has to be recalled that a single simulation was computed at the average port diameter, which was assumed representative of the whole firing test. This assumption might not be fully justified for firing tests characterized by a more relevant radiation exchange, which has been shown to be highly non-linear. In addition, it has been confirmed by radiative simulations [49] that the value assumed for the wall emissivity has a differential impact on the net computed radiative wall heat flux, strictly depending on the medium optical thickness, i.e., the product between chamber pressure and port diameter. In particular, for high values of the medium optical thickness, as in the case Firing Test \#9, a high sensitivity to the wall emissivity was found, hence requiring more carefulness in the selection of the HDPE wall emissivity value. 


\section{Conclusions}

A CFD approach for the internal ballistics of GOX/HDPE hybrid rocket engines integrated with gas-surface interaction modeling capabilities was presented. The fluid dynamic equations were the Reynolds-averaged Navier-Stokes equations with additional transport equations for turbulence and chemical species. Suitable sub-models were included in order to describe the homogeneous combustion in the gaseous phase, the radiative energy exchange toward the fuel surface, and the fluid-surface interaction in the fuel combustion port (fuel pyrolysis model). The gas-phase equations were solved coupled to the solid fuel-phase by using customized surface balances of energy and mass, which enabled the determination of the fuel surface temperature and regression rate as part of the flowfield solution. An experimental lab-scale test case, represented by a $1 \mathrm{kN}$-class hybrid rocket engine burning gaseous oxygen and HDPE, was simulated. A thorough sensitivity analysis was carried out on the literature pyrolysis models for HDPE. The obtained results confirmed that both the pre-exponential constant and the activation energy of the pyrolysis law had a direct effect on the grain wall temperature, while they were only mildly affecting the grain regression rate and, hence, the motor chamber pressure. This was due to the fact that the fuel temperature behaved as a compensating factor, while the regression rate was dominantly dictated by the surface energy balance. Differently, the obtained results showed a much stronger sensitivity of the fuel regression rate and chamber pressure to the value assumed for the heat of pyrolysis, which directly affected the surface energy balance. On the other hand, the effect of the heat of pyrolysis on the grain wall temperature appeared to be minimal. Thus, the pyrolysis process appeared to be definitely controlled by the energy balance at the grain surface and thus by the competition between incoming heat fluxes (convection and radiation) and outgoing heat fluxes (fuel pyrolysis and solid grain heating), with reaction kinetics only playing a minor role. The heat of pyrolysis was the parameter most affecting the fuel regression rate, followed by the activation energy with a relatively weak effect and the pre-exponential constant inducing negligibly small variations.

The numerical approach was validated by direct comparison of experimental and numerical data, in terms of time- and space-averaged measurements. The comparison revealed that, despite the adopted simplifying assumptions, the CFD approach was fairly able to capture the main features of the motor internal ballistics both in terms of regression rate and average chamber pressure trends with oxidizer mass flux and port diameter. When the experimentally-measured regression rate was imposed as a boundary condition in the simulation, the model was correctly able to predict the motor chamber pressure; hence, the mixing and combustion process were adequately modeled, with errors ranging from $+4 \%$ to $-8 \%$. When the regression rate was computed from the gas-surface interaction wall boundary condition, however, the regression rate was underestimated between 31 and 47\%, while the chamber pressure was underestimated between 13 and 25\%. Considering the sensitivity analyses that were carried out on the fuel pyrolysis model, such a significant underestimation must be attributed to another source of error, as the absence of the radiative contribution to the wall heat flux. When a thermal radiation model was included, the errors previously found were much mitigated. Despite a noticeable improvement with respect to solutions without radiation, regression rate and chamber pressure for firing tests characterized by the larger port diameters were overestimated when including thermal radiation effects. To improve predictions, a more detailed combustion modeling including radical species was deemed necessary, as well as a deeper analysis of the port diameter and grain wall emissivity effects on the net radiative heat flux to the fuel surface. The rest of the analyzed tests, however, fell within a $\pm 12 \%$ error on both the regression rate and chamber pressure when the radiative contribution to grain heating was included. Hence, the importance of including thermal radiation effects in the prediction of fuel regression rate and motor internal ballistics was highlighted for firing tests characterized by low oxidizer mass fluxes. Finally, the results also highlighted the coupling between radiation and the blocking effect, showing a reduction of the convective heat flux when radiation was included in the calculation, although the effect was much stronger in the first 
one-third of the grain and was much mitigated toward the aft-end of the grain due to the increase of the overall mass flux in the port.

Author Contributions: Conceptualization of theoretical models, D.B., G.L., F.N., M.O. and C.C.; definition of CFD models, D.B, G.L. and F.N.; implementation of CFD models, D.B. and G.L.; running of numerical simulations, D.B. and G.L.; writing-original draft preparation, D.B. and C.C.; supervision, F.N, M.O. and C.C.

Funding: This research received no external funding.

Conflicts of Interest: The authors declare no conflict of interest.

\section{Nomenclature}

a thermal diffusivity, $\mathrm{m}^{2} / \mathrm{s}$

c heat capacity, $\mathrm{J} / \mathrm{kg} \cdot \mathrm{K}$

$D \quad$ port diameter, $\mathrm{m}$

$D_{i m} \quad$ effective diffusion coefficient, $\mathrm{m}^{2} / \mathrm{s}$

G mass flux, $\mathrm{kg} / \mathrm{m}^{2} \cdot \mathrm{s}$

$h \quad$ enthalpy, $\mathrm{J} / \mathrm{kg}$

I radiative intensity, $\mathrm{W} / \mathrm{m}^{2} \cdot \mathrm{sr}$

$k$ thermal conductivity, $\mathrm{W} / \mathrm{m} \cdot \mathrm{K}$

$L \quad$ grain length, $\mathrm{m}$

in mass flow rate, $\mathrm{kg} / \mathrm{s}$

$\dot{m}_{b} \quad$ mass blowing rate per unit area, $\mathrm{kg} / \mathrm{m}^{2} \cdot \mathrm{s}$

M molar mass of the species, $\mathrm{kg} / \mathrm{kmole}$

$N \quad$ number of species

O/F $\quad \dot{m}_{o x} / \dot{m}_{f}$, oxidizer to fuel ratio of the motor

$p \quad$ pressure, $\mathrm{N} / \mathrm{m}^{2}$

$\dot{q} \quad$ heat flux, $\mathrm{W} / \mathrm{m}^{2}$

$r \quad$ reflectivity

$\dot{r} \quad$ regression rate, $\mathrm{m} / \mathrm{s}$

$R \quad$ universal gas constant, $\mathrm{J} / \mathrm{mol} \cdot \mathrm{K}$

$S \quad$ abscissa along a line-of-sight, $\mathrm{m}$

$T$ temperature, $\mathrm{K}$

$t_{b} \quad$ burning time, $\mathrm{s}$

$v \quad$ velocity component normal to surface, $\mathrm{m} / \mathrm{s}$

$\dot{w}_{i} \quad$ source term of species $i$ in the control volume, $\mathrm{kg} / \mathrm{m}^{3} \cdot \mathrm{s}$

$\dot{w}_{j} \quad$ net reaction rate of reaction $j, \mathrm{kmole} / \mathrm{m}^{3} \cdot \mathrm{s}$

$X_{i} \quad$ mole fraction of species $i$

$y_{i} \quad$ mass fraction of species $i$

Greek

$\alpha \quad$ absorptivity

$\epsilon \quad$ emissivity

$\theta \quad$ line-of-sight elevation angle, rad

$\eta \quad$ inward (from solid to gas) coordinate normal to the surface

$\kappa \quad$ absorption coefficient, $\mathrm{m}^{-1}$

$\kappa_{p} \quad$ Planck-mean absorption coefficient, $\mathrm{m}^{-1} \mathrm{~atm}^{-1}$

$\rho \quad$ density, $\mathrm{kg} / \mathrm{m}^{3}$

$\sigma \quad$ Stefan-Boltzmann constant, $\mathrm{W} / \mathrm{m}^{2} \cdot \mathrm{K}^{4}$

$\psi \quad$ line-of-sight azimuth angle, rad

$\dot{\omega}_{i} \quad$ source term of species $i$ in the control surface, $\mathrm{kg} / \mathrm{m}^{2} \cdot \mathrm{s}$

$\Omega \quad$ solid angle, sr 


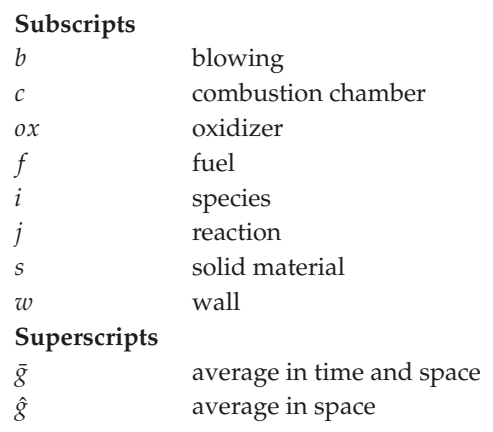

\section{Abbreviations}

The following abbreviations are used in this manuscript:

$\begin{array}{ll}\text { CFD } & \text { Computational Fluid Dynamics } \\ \text { DTM } & \text { Discrete Transfer Method } \\ \text { GOX } & \text { Gaseous Oxygen } \\ \text { HDPE } & \text { High-Density Polyethylene } \\ \text { HRE } & \text { Hybrid Rocket Engines } \\ \text { HTPB } & \text { Hydroxyl-Terminated Polybutadiene } \\ \text { RTE } & \text { Radiative Transfer Equation }\end{array}$

\section{References}

1. Kuo, K.K.; Houim, R.W. Theoretical Modeling and Numerical Simulation Challenges of Combustion Processes of Hybrid Rockets. AIAA Paper 2011-5608. In Proceedings of the 47th AIAA/ASME/SAE/ASEE Joint Propulsion Conference \& Exhibit, San Diego, CA, USA, 31 July-3 August 2011.

2. Altman, D.; Holzman, A. Overview and History of Hybrid Rocket Propulsion. In Fundamentals of Hybrid Rocket Combustion and Propulsion; Kuo, K., Chiaverini, M., Eds.; Progress in Astronautics and Aeronautics; AIAA: Reston, VA, USA, 2007; Volume 218, pp. 1-36.

3. Bianchi, D.; Urbano, A.; Betti, B.; Nasuti, F. CFD Analysis of Hybrid Rocket Flowfields Including Fuel Pyrolysis and Nozzle Erosion. AIAA Paper 2013-3637. In Proceedings of the 49th AIAA/ASME/SAE/ASEE Joint Propulsion Conference, San Jose, CA, USA, 15-17 July 2013. [CrossRef]

4. Bianchi, D.; Betti, B.; Nasuti, F.; Carmicino, C. Simulation of Gaseous Oxygen/Hydroxyl-Terminated Polybutadiene Hybrid Rocket Flowfields and Comparison with Experiments. J. Propuls. Power 2015, 31, 919-929. [CrossRef]

5. Cheng, G.C.; Farmer, R.C.; Jones, H.S.; McFarlane, J.S. Numerical Simulation of the Internal Ballistics of a Hybrid Rocket Motor. AIAA Paper 94-0554. In Proceedings of the 32nd Aerospace Sciences Meeting and Exhibit, Reno, NV, USA, 10-13 January 1994. [CrossRef]

6. Sankaran, V. Computational Fluid Dynamics Modeling of Hybrid Rocket Flowfields. In Fundamentals of Hybrid Rocket Combustion and Propulsion; Kuo, K., Chiaverini, M., Eds.; Progress in Astronautics and Aeronautics; AIAA: Reston, VA, USA, 2007; Volume 218, pp. 323-349.

7. Chen, Y.S.; Chou, T.H.; Gu, B.R.; Wu, J.S.; Wu, B.; Lian, Y.Y.; Yang, L. Multiphysics simulations of rocket engine combustion. Comput. Fluids 2011, 45, 29-36. [CrossRef]

8. Lazzarin, M.; Barato, F.; Bettella, A.; Pavarin, D. Computational Fluid Dynamics Simulation of Regression Rate in Hybrid Rockets. J. Propuls. Power 2013, 29, 1445-1452. [CrossRef]

9. Gariani, G.; Maggi, F.; Galfetti, L. Simulation Code for Hybrid Rocket Combustion. AIAA Paper 2010-6872. In Proceedings of the 46th AIAA/ASME/SAE/ASEE Joint Propulsion Conference, Nashville, TN, USA, 25-28 July 2010.

10. Coronetti, A.; Sirignano, W.A. Numerical Analysis of Hybrid Rocket Combustion. J. Propuls. Power 2013, 29, 371-384. [CrossRef] 
11. Motoe, M.; Shimada, T. Numerical Simulations of Combustive Flows in a Swirling-Oxidizer-Flow-Type Hybrid Rocket. AIAA Paper 2014-0310. In Proceedings of the 52nd Aerospace Sciences Meeting, National Harbor, MD, USA, 13-17 January 2014. [CrossRef]

12. Bellomo, N.; Lazzarin, M.; Barato, F.; Bettella, A.; Pavarin, D.; Grosse, M. Investigation of Effect of Diaphragms on the Efficiency of Hybrid Rockets. J. Propuls. Power 2014, 30, 175-185. [CrossRef]

13. Kumar, C.P.; Kumar, A. Effect of Diaphragms on Regression Rate in Hybrid Rocket Motors. J. Propuls. Power 2013, 29, 559-572. [CrossRef]

14. Di Martino, G.D.; Carmicino, C.; Savino, R. Transient Computational Thermofluid-Dynamic Simulation of Hybrid Rocket Internal Ballistics. J. Propuls. Power 2017, 33, 1395-1409. [CrossRef]

15. Messineo, G.; Lestrade, J.Y.; Hijlkema, J.; Anthoine, J. Vortex Shedding Influence on Hybrid Rocket Pressure Oscillations and Combustion Efficiency. J. Propuls. Power 2016, 32, 1386-1394. [CrossRef]

16. Arisawa, H.; Brill, T.B. Flash Pyrolysis of Hydroxyl Terminated Poly-Butadiene (HTPB) II: Implications of the Kinetics to Combustion of Organic Polymers. Combust. Flame 1996, 106, 131-143. [CrossRef]

17. Ramohalli, K.; Yi, J. Hybrids Revisited. AIAA Paper 90-1962. In Proceedings of the AIAA/SAE/ASME/ASEE 26th Joint Propulsion Conference, Orlando, FL, USA, 16-18 July 1990. [CrossRef]

18. Carmicino, C.; Russo Sorge, A. Influence of a Conical Axial Injector on Hybrid Rocket Performance. J. Propuls. Power 2006, 22, 984-995. [CrossRef]

19. Bianchi, D.; Nasuti, F. Numerical Analysis of Nozzle Material Thermochemical Erosion in Hybrid Rocket Engines. J. Propuls. Power 2013, 29, 547-558. [CrossRef]

20. Di Martino, G.D.; Mungiguerra, S.; Carmicino, C.; Savino, R. Computational Fluid-dynamic Simulations of Hybrid Rocket Internal Flow Including Discharge Nozzle. AIAA Paper 2017-5045. In Proceedings of the 53rd AIAA/SAE/ASEE Joint Propulsion Conference, Atlanta, GA, USA, 10-12 July 2017.

21. Sutton, G.P.; Biblarz, O. Rocket Propulsion Elements; John Wiley and Sons, Inc.: New York, NY, USA, 2001.

22. Betti, B.; Nasuti, F.; Martelli, E. Numerical Evaluation of Heat Transfer Enhancement in Rocket Thrust Chambers by Wall Ribs. Numer. Heat Transf. Part A Appl. 2014, 66, 488-508. [CrossRef]

23. Betti, B.; Bianchi, D.; Nasuti, F.; Martelli, E. Chemical Reaction Effects on Heat Loads of $\mathrm{CH}_{4} / \mathrm{O}_{2}$ and $\mathrm{H}_{2} / \mathrm{O}_{2}$ Rockets. AIAA J. 2016, 54, 1693-1703. [CrossRef]

24. Bianchi, D.; Nasuti, F.; Paciorri, R.; Onofri, M. Computational Analysis of Hypersonic Flows Including Finite Rate Ablation Thermochemistry. In Proceedings of the 7th European Workshop on Thermal Protection Systems and Hot Structures, ESA-ESTEC, Noordwijk, The Netherlands, 8-10 April 2013.

25. Bianchi, D.; Nasuti, F.; Carmicino, C. Hybrid Rockets with Axial Injector: Port Diameter Effect on Fuel Regression Rate. J. Propuls. Power 2016, 32, 984-996. [CrossRef]

26. Bianchi, D.; Kamps, L.; Nasuti, F.; Nagata, H. Numerical and Experimental Investigation of Nozzle Thermochemical Erosion in Hybrid Rockets. AIAA Paper 2017-4640. In Proceedings of the 53rd AIAA/SAE/ASEE Joint Propulsion Conference, Atlanta, GA, USA, 10-12 July 2017. [CrossRef]

27. Gordon, S.; McBride, B.J. Computer Program for Calculation of Complex Chemical Equilibrium Compositions and Applications. NASA RP 1311. 1994. Available online: https://ntrs.nasa.gov/archive/ nasa/casi.ntrs.nasa.gov/19950013764.pdf (accessed on 21 April 2019).

28. Wilke, C.R. A Viscosity Equation for Gas Mixtures. J. Chem. Phys. 1950, 18. [CrossRef]

29. Spalart, P.R.; Allmaras, S.R. A One-Equation Turbulence Model for Aerodynamic Flows. Rech. Aérosp. 1994, $1,5-21$.

30. Lockwood, F.C.; Shah, N.G. A New Radiation Solution Method for Incorporation in General Combustion Prediction Procedures. Symp. Int. Combust. 1981, 18, 1405-1414. [CrossRef]

31. Kendall, R.M.; Bartlett, E.P.; Rindal, R.A.; Moyer, C.B. An Analysis of the Chemically Reacting Boundary Layer and Charring Ablator. Part I: Summary Report; NASA CR 1060; NASA: Washington, DC, USA, 1968.

32. Marxman, G.; Gilbert, M. Turbulent Boundary Layer Combustion in the Hybrid Rocket. Symp. Int. Combust. 1963, 9, 371-383. [CrossRef]

33. Li, C.; Cai, G.; Tian, H. Numerical analysis of combustion characteristics of hybrid rocket motor with multi-section swirl injection. Acta Astronaut. 2016, 123, 26-36. [CrossRef]

34. Lengelle, G.; Fourest, B.; Godon, J.; Guin, C. Condensed-phase behavior and ablation rate of fuels for hybrid propulsion. AIAA Paper 1993-2413. In Proceedings of the 29th Joint Propulsion Conference and Exhibit, Monterey, CA, USA, 28-30 June 1993. 
35. Stoiliarov, S.I.; Crowley, S.; Lyon, R.E.; Linteris, G.T. Prediction of the burning rates of non-charring polymers. Combust. Flame 2009, 156, 1068-1083. [CrossRef]

36. Gascoin, N.; Fau, G.; Gillard, P.; Mangeot, A. Experimental flash pyrolysis of high density polyethylene under hybrid propulsion conditions. J. Anal. Appl. Pyrolysis 2013, 101, 45-52. [CrossRef]

37. Lengelle, G. Solid-Fuel Pyrolysis Phenomena and Regression Rate, Part 1: Mechanisms. In Fundamentals of Hybrid Rocket Combustion and Propulsion; Kuo, K., Chiaverini, M., Eds.; Progress in Astronautics and Aeronautics; AIAA: Reston, VA, USA, 2007; Volume 218, pp. 127-165.

38. Chiaverini, M.J.; Serin, N.; Johnson, D.K.; Lu, Y.C.; Kuo, K.K.; Risha, G.A. Regression Rate Behavior of Hybrid Rocket Solid Fuels. J. Propuls. Power 2000, 16, 125-132. [CrossRef]

39. Chiaverini, M.J.; Kuo, K.K.; Peretz, A.; Harting, G.C. Regression-Rate and Heat-Transfer Correlations for Hybrid Rocket Combustion. J. Propuls. Power 2001, 17, 318-326. [CrossRef]

40. Chiaverini, M. Review of Solid-Fuel Regression Rate Behavior in Classical and Nonclassical Hybrid Rocket Motors. In Fundamentals of Hybrid Rocket Combustion and Propulsion; Kuo, K., Chiaverini, M., Eds.; Progress in Astronautics and Aeronautics; AIAA: Reston, VA, USA, 2007; Volume 218, pp. 37-125.

41. Ciottoli, P.P.; Malpica Galassi, R.; Lapenna, P.; Leccese, G.; Bianchi, D.; Nasuti, F.; Creta, F.; Valorani, M. CSP-based chemical kinetics mechanisms simplification strategy for non-premixed combustion: An application to hybrid rocket propulsion. Combust. Flame 2017, 186, 83-93. [CrossRef]

42. Andersen, J.; Rasmussen, C.L.; Giselsson, T.; Glarborg, P. Global Combustion Mechanisms for Use in CFD Modeling under Oxy-Fuel Conditions. Energy Fuels 2009, 23, 1379-1389. [CrossRef]

43. Muzzy, R. Applied Hybrid Combustion Theory. In Proceedings of the 8th Joint Propulsion Specialist Conference, New Orleans, LA, USA, 29 November-1 December 1972. [CrossRef]

44. Göbel, F.; Mundt, C. Implementation of the P1 Radiation Model in the CFD Solver NSMB and Investigation of Radiative Heat Transfer in the SSME Main Combustion Chamber. AIAA Paper 2011-2257. In Proceedings of the 17th AIAA International Space Planes and Hypersonic Systems and Technologies Conference, San Francisco, CA, USA, 11-14 April 2011. [CrossRef]

45. Jiwen, L.; Tiwari, S.N. Radiative Heat Transfer Effects in Chemically Reacting Nozzle Flows. J. Thermophys. Heat Transf. 1996, 10, 436-444. [CrossRef]

46. Modest, M.F. Radiative Heat Transfer; Academic Press: San Diego, CA, USA, 2013; pp. 288-410.

47. Tien, C.L. Thermal Radiation Properties of Gases. In Advances in Heat Transfer; Irvine, T.F., Hartnett, J.P., Eds.; Academic Press: New York, NY, USA, 1968; Volume 5, pp. 253-324.

48. Rivière, P.; Soufiani, A. Updated Band Model Parameters for $\mathrm{H}_{2} \mathrm{O}, \mathrm{CO}_{2}, \mathrm{CH}_{4}$ and $\mathrm{CO}$ Radiation at High Temperature. Int. J. Heat Mass Transf. 2012, 55, 3349-3358. [CrossRef]

49. Leccese, G.; Bianchi, D.; Betti, B.; Lentini, D.; Nasuti, F. Convective and Radiative Wall Heat Transfer in Liquid Rocket Thrust Chambers. J. Propuls. Power 2018, 34, 318-326. [CrossRef]

50. Leccese, G.; Bianchi, D.; Nasuti, F.; Stober, K.J.; Narsai, P.; Cantwell, B.J. Experimental and numerical methods for radiative wall heat flux predictions in paraffin-based hybrid rocket engines. Acta Astronaut. 2019, 158, 304-312. [CrossRef]

51. Carmicino, C.; Russo Sorge, A. Role of Injection in Hybrid Rockets Regression Rate Behavior. J. Propuls. Power 2005, 21, 606-612. [CrossRef]

52. Karabeyoglu, M.A.; Cantwell, B.J.; Zilliac, G. Development of Scalable Space-Time Averaged Regression Rate Expressions for Hybrid Rockets. J. Propuls. Power 2007, 23, 737-747. [CrossRef]

53. Stoiliarov, S.I.; Walters, R.N. Determination of the heats of gasification of polymers using differential scanning calorimetry. Polym. Degrad. Stab. 2008, 93, 422-427. [CrossRef] 


\title{
Design and Testing of a Paraffin-Based $1000 \mathrm{~N}$ HRE Breadboard
}

\author{
Francesco Battista ${ }^{1, *, \dagger}$, Daniele Cardillo ${ }^{1, \dagger}$, Manrico Fragiacomo ${ }^{1}$, \\ Giuseppe Daniele Di Martino ${ }^{2}$, Stefano Mungiguerra ${ }^{2}$ and Raffaele Savino ${ }^{2}$ \\ 1 CIRA, Italian Aerospace Research Centre, 81043 Capua, Italy \\ 2 Department of Industrial Engineering-Aerospace Division, University of Naples "Federico II", \\ Piazzale Tecchio, 80, 80125 Naples, Italy \\ * Correspondence: f.battista@cira.it; Tel.: +39-0823-623378 \\ + These authors contributed equally to this work.
}

Received: 17 June 2019; Accepted: 4 August 2019; Published: 12 August 2019

\begin{abstract}
The paper presents some relevant achievements in hybrid rocket propulsion carried out by the Italian Aerospace Research Centre. On the basis of the experimental results obtained on a $200 \mathrm{~N}$ thrust class engine, a $1000 \mathrm{~N}$ class breadboard, fed with gaseous oxygen coupled with a paraffin-based fuel grain, was designed and experimentally tested in different conditions. The breadboard exhibited a stable combustion in all the firing test conditions; the testing campaign allowed the acquisition of different experimental data, as pre and post-combustion chamber pressure, throat material temperature, pre-combustion chamber temperature. The new breadboard was characterized by higher measured regression rate values with respect to corresponding data obtained with the smaller scale one, highlighting that the oxidizer mass flux is not the only operating quantity affecting the fuel consumption behavior, which could be also influenced by scale parameters, such as the grain port diameter, and other operating conditions, such as the mixture ratio.
\end{abstract}

Keywords: hybrid propulsion; paraffin-based fuel; oxygen; combustion; testing

\section{Introduction}

Numerous interesting features give to hybrid rocket propulsion technology potential advantage over both solid and liquid propulsion systems, such as safety, low development costs, minimal environmental impact, re-ignition and throttling capabilities. A thorough explanation of these aspects can be found in the work by Altman and Holzman [1].

Nevertheless, to effectively demonstrate its advantages over the actual consolidated technologies, the TRL of HRE systems should be further increased for a carefully selected class of missions, as indicated by Karabeyoglu [2]. In fact, the hybrid engine development has not still achieved the same level of maturity as solid and liquid traditional systems.

Some disadvantages of the HRE technology include the low fuel regression rate, the combustion efficiency and the oxidizer to fuel mixture ratio shift.

In the last years, paraffin-based fuels gained much attention, due to higher regression rates with respect to classical polymeric fuels [3]. Specifically, in addition to the classical fuel regression rate due to evaporation, in this class of polymers there is a further contribution to regression rate due to entrainment. The formation of a low-viscosity unstable melt layer, on the burning surface, leads to fuel liquid droplets entrainment into the main gas stream, which significantly increases the solid regression rate.

Detailed studies have been presented by Karabeyoglu [4,5], where it is demonstrated that the fuel composition and its thermo-mechanical properties strongly affect the liquid layer instability and, therefore, the fuel regression rate. 
In this scenario, the present research was mainly focused on the investigation of the fuel characteristics, in order to ensure high performance in terms of regression rate but maintaining good mechanical properties. In a previous authors' work, results of an experimental test campaign, performed at subscale level on a $200 \mathrm{~N}$ breadboard, demonstrated very good performances and mechanical properties of the analyzed paraffin-wax formulation [6].

Based on these results, the main objective of the present work is the scale-up of the fuel grain, adopting the same fuel formulation, and the design of a new breadboard, moving towards the $1000 \mathrm{~N}$ thrust class. A new experimental test campaign was carried out on the $1000 \mathrm{~N}$ breadboard, allowing for the investigation of the paraffin-based fuel blend behavior on a larger scale. In particular, with respect to subscale experiments, the oxidizer to fuel mixture ratio range was extended. Numerous data were acquired, including chamber pressure, thrust, temperature of the flow in the pre-chamber and temperature inside the graphite nozzle material. The latter parameter allows for the estimation of the convective heat transfer coefficient in the nozzle region, which is strictly linked to the graphite nozzle thermo-chemical erosion. This is an extremely important parameter to be evaluated, since the throat area enlargement directly affects the motor performances [7].

The results of the experimental test campaign show that hybrid rocket engine can operate, with good efficiency and stability, in a wide range of operating conditions, confirming some of the advantages over both solid and liquid technologies often mentioned in the relevant literature $[1,2,8,9]$.

\section{Methodology and Design}

\subsection{Design Logic}

The procedure, adopted for the design of the $1000 \mathrm{~N}$ hybrid breadboard, is based on an iterative process in time, which is schematically represented in Figure 1, and was implemented in a relatively simple and fast design tool (named HDC code). The HDC code, once selected the propellants and given the required engine performance in terms of average thrust $(\mathrm{T})$ and the minimum total impulse $\left(\mathrm{I}_{\text {tot }}\right)$, provides the design oxidizer mass flow rate and the total burning time. After that, for the chosen propellants, the regression rate law can be selected (see Section 2.2), which is integrated in time for the fuel grain sizing; in particular, fuel grain internal diameter and length are optimized to ensure a thrust profile with maximum deviation of $10 \%$, on thrust, during firing.

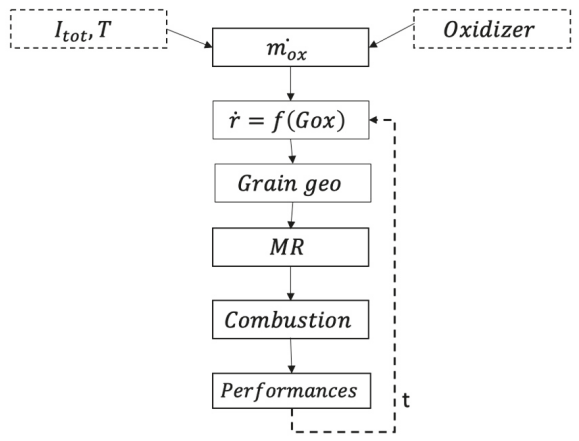

Figure 1. HDC code simplified design loop.

Combustion equilibrium data, obtained from the RPA (Rocket Propulsion Analysis) lite software [10], are used. Combustion properties, derived from RPA, are interpolated as a function of chamber pressure and mixture ratio.

$$
T c=f\left(M R, p_{c}\right), \quad M W=f\left(M R, p_{c}\right), \quad \gamma\left(M R, p_{c}\right)
$$


From these quantities, rocket performance at each time step are calculated considering a combustion efficiency and nozzle efficiency according to [11].

The tool gives as output the baseline design of the injection system (given the oxidizer composition and its physical state), the case thickness, the throat regression, the pre and post-combustion chamber layout, providing the preliminary design of the configuration.

The demonstrator was preliminarily designed, with the exposed procedure that provides the main thruster geometrical characteristics and performances. The nominal target data for the design of the breadboard, in terms of minimum total impulse and nominal thrust, are $10^{4} \mathrm{~N} \cdot \mathrm{s}$ and $1000 \mathrm{~N}$, respectively. In order to take into account uncertainties in regression rate law, a total impulse of $2 \times 10^{4} \mathrm{~N} \cdot \mathrm{s}\left(>10^{4} \mathrm{~N} \cdot \mathrm{s}\right)$ was considered as input data for the HDC code. This is to avoid total consumption of the fuel grain before the end of the firing, which could lead to expose the breadboard case to high thermal loads. The regression rate selected for design will be analyzed later, the dependence of regression rate on the axial coordinate has been preliminary taken into account according to [12]. The results of the preliminary design process are reported in Figure 2a (profiles of pressure and thrust over time) and Figure $2 \mathrm{~b}$ (thruster configuration and expected grain recession), the expected mean fuel grain regression rate is $1.9 \mathrm{~mm} / \mathrm{s}$. It is worth to notice that, in the first $8 \mathrm{~s}$, thrust (Figure 2a) remains almost constant; this because the change in the thrust coefficient, deriving from the thermodynamic properties variation with the fast varying oxidizer to fuel ratio, compensates the pressure decrease. The port diameter has been optimized in order to have the optimum mean thrust (considering $20 \mathrm{~s}$ of firing time) once fixed the grain length. Post and pre-chamber play an important role in hybrid rocket engine combustion efficiency, they have been dimensioned according to the principles reported in [13]. The injectors diameters have been selected according to the criteria reported in [14] for gaseous injectors considering an oxygen nominal mass flow rate of $0.260 \mathrm{~kg} / \mathrm{s}$. Detailed design analyses on this breadboard configuration can be found in [15].

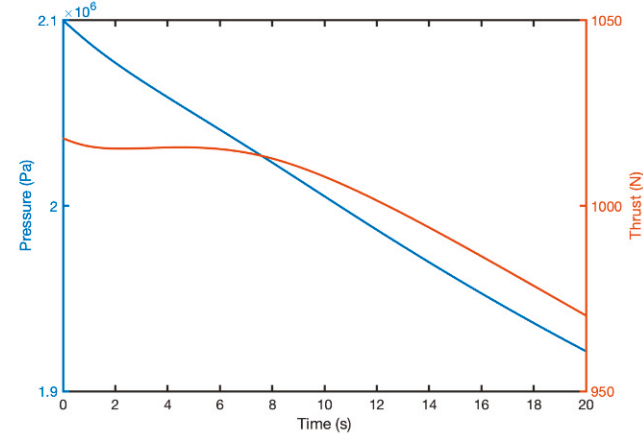

(a)

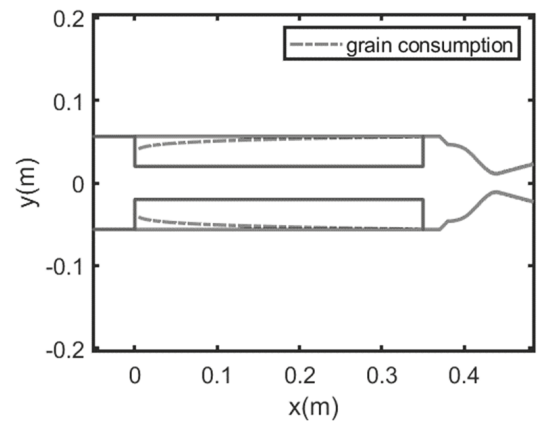

(b)

Figure 2. Pressure and thrust profiles in time (a) and thruster configuration from HDC (b).

\subsection{Fuel Regression Rate Formulation}

The fuel regression rate law, considered in the present work, is referred to paraffin-based fuel grains burning with gaseous oxygen. The fuel formulation is a blend of paraffin waxes, which mainly include microcrystalline paraffin commercialized by SASOL ${ }^{\circledR}$ (labelled with the trade code 0907—see Table 1). Previous investigations by CIRA [16] showed high fracture toughness and workability of the SASOL 0907. Other paraffin-waxes and additives are included in the adopted formulation to enhance both mechanical characteristics and performances. They include a refined low melting point $\left(58-62^{\circ} \mathrm{C}\right)$ paraffin, relatively high melting point microcrystalline wax (about $100^{\circ} \mathrm{C}$ ) and stearic acid. A small amount of a blackening additive was added to the melted wax, to increase the thermal radiation absorption at the fuel surface improving the regression rate $[5,17]$. 
Table 1. SASOL ${ }^{\circledR} 0907$ paraffin wax properties.

\begin{tabular}{cccc}
\hline Melting Point $\left[{ }^{\circ} \mathrm{C}\right]$ & Congealing Point $\left[{ }^{\circ} \mathrm{C}\right]$ & Oil Content $[\%]$ & Penetration at $25{ }^{\circ} \mathrm{C}[\mathbf{1} / \mathbf{1 0} \mathbf{~ m m}]$ \\
\hline $88-102$ & $83-94$ & $0-1.5$ & $4-10$ \\
\hline
\end{tabular}

The formulation was tested on a $200 \mathrm{~N}$ subscale breadboard, in different operating conditions, in order to evaluate the regression rate and the mechanical behavior [6]. Several tests were performed up to $60 \mathrm{~g} / \mathrm{s}$ oxygen mass-flow rate, corresponding to $1.9 \mathrm{MPa}$ for chamber pressure and about $200 \mathrm{~N}$ for thrust. Since the employed breadboard was optimized for operation with classical polymeric fuels (in the range of typical obtainable mass flow rates and pressures), tests with paraffin-based fuels were characterized by relatively low mixture ratios, up to 1.2. Results in terms of fuel regression rate against oxidizer mass-flux are reported in Figure 3 and compared with well-known literature results.

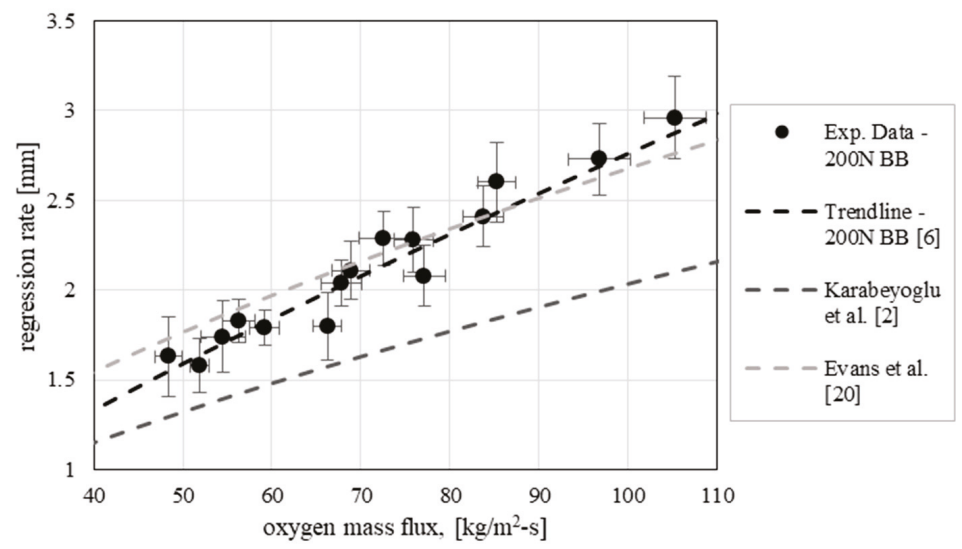

Figure 3. Regression rate versus oxidizer mass-flux for the $200 \mathrm{~N}$ breadboard [6].

The paraffin-based fuel showed good mechanical properties and performances in terms of regression rate. The following correlation of the fuel regression rate as a function of the oxidizer mass flux was determined, based on the data acquired during the testing campaign.

$$
\overline{\dot{r}}=a \overline{\mathrm{G}}_{o x}^{n} \quad a=0.071 ; \quad n=0.795 ; \quad\left(R^{2}=0.922\right)
$$

where the regression rate is expressed in $\mathrm{mm} / \mathrm{s}$ and mass flux in $\mathrm{kg} / \mathrm{m}^{2}$-s. Due to these results, this paraffin formulation was adopted for scaling-up the propellant grain to a higher thrust class breadboard (1000 N). In order to design the new test article, the correlation exposed in Equation (2) has been used.

\section{Breadboard Architecture and Experimental Setup}

\subsection{Breadboard Description}

The $1000 \mathrm{~N}$ breadboard completely assembled is depicted in Figure 4a, while details on the configuration are shown in Figure $4 \mathrm{~b}$.

The breadboard injection system is very simple and it is based on a showerhead architecture. The system is characterized by seven injectors (one on the breadboard axis see Figure $4 \mathrm{~b}$ ), of constant diameter, which ensure a more uniform axial flow at grain port inlet with respect to a single injector element with the same area. The injector plate is designed such that it can be easily replaced for testing other injector configurations. The external shell of the breadboard is made of stainless steel and contains the pre-chamber, the propellant grain, post combustion chamber and nozzle. The pre-chamber 
has a thermal protection system based on graphite and hosts a pressure transducer. Nozzle pre and post combustion chamber are protected by graphite; the post combustion chamber hosts a further pressure transducer. An embedded thermo-couple is located in proximity of the nozzle throat in order to monitor temperature of the zone, as shown in Figure 4. The ignition of the breadboard is assured by a spark plug located in the pre-chamber that ignites a mixture of oxygen and methane injected in the pre-chamber.

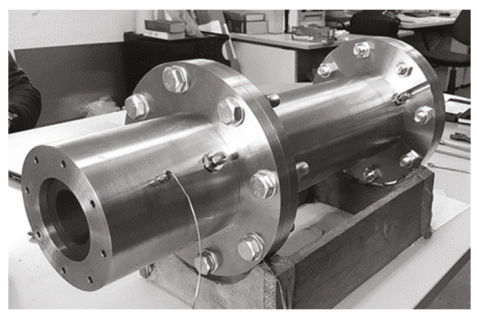

(a)

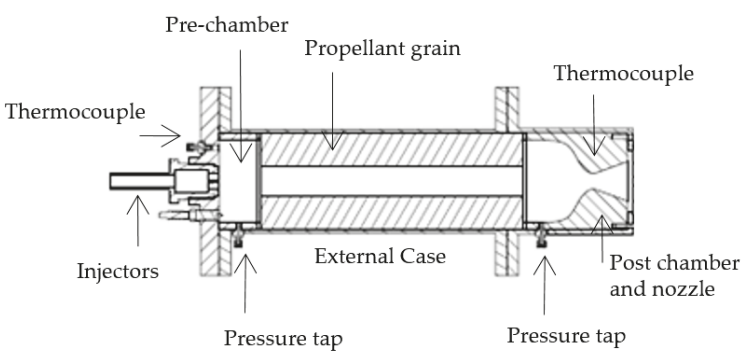

(b)

Figure 4. CIRA Hybrid $1000 \mathrm{~N}$ breadboard assembled (a), general arrangement (b).

\subsection{Test Rig Description and Test Setup}

The test facility is a versatile experimental setup primarily designed for firing hybrid rocket engines of several sizes [6].

The piping and instrumentation schematic of the test rig is shown in Figure 5. Gaseous oxygen is supplied by a reservoir consisting of eight cylinders, while pressure regulators set the operating pressure along the breadboard feed line. Oxygen mass flow rate is evaluated through gas temperature and pressure measurements upstream of the throat of a chocked Venturi tube. The Venturi can be dismounted and replaced depending on the requested oxidizer mass-flow rate. For testing the $1000 \mathrm{~N}$ breadboard a $5 \mathrm{~mm}$ throat Venturi tube was adopted in order to guarantee the flow rate requested.

Nitrogen is purged into the chamber for the burn out and in case of an emergency shutdown.

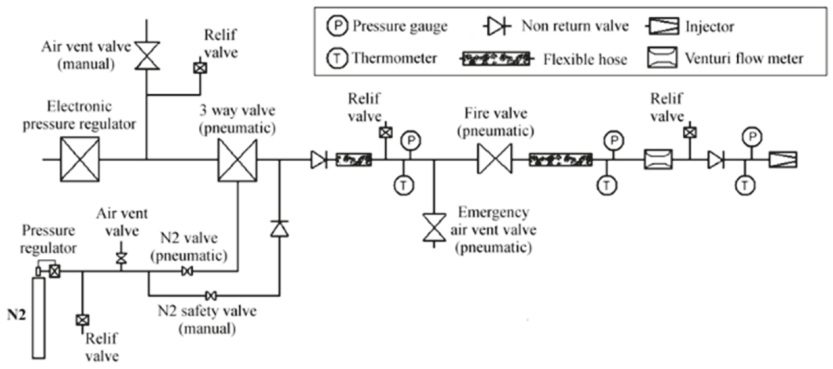

Figure 5. Test rig layout.

Figure 6 shows the $1000 \mathrm{~N}$ breadboard integrated on the test bench. First, a leakage test was performed, using pressurized nitrogen at $0.8 \mathrm{MPa}$ for five minutes, in order to verify the absence of any leak from internal interfaces. 


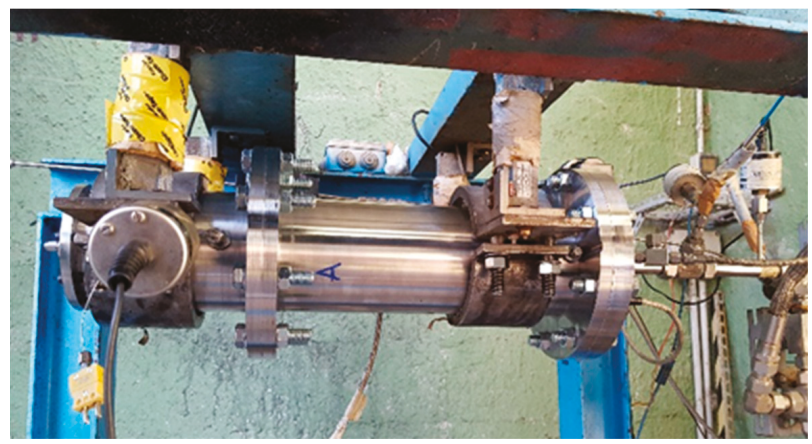

Figure 6. $1000 \mathrm{~N}$ breadboard integrated on the test bench.

Firing tests have been conducted with an incremental logic, being the hardware a new design, in order to evaluate the behavior of the breadboard from lower pressure/duration to higher ones.

The operating conditions of each test are reported in Table 2.

Table 2. Nominal operating conditions.

\begin{tabular}{cccccc}
\hline Operating Condition & Test L1 & Test L2 & Test L3 & Test L4 & Test L5 \\
\hline Oxidizer mass-flow rate $(\mathrm{kg} / \mathrm{s})$ & 0.120 & 0.200 & 0.200 & $0.140-0.225$ & 0.260 \\
Firing time $(\mathrm{s})$ & 6 & 8 & 12 & $5+1$ (transient) +4 & 10 \\
\hline
\end{tabular}

According to the incremental logic, the first test L1 was conducted with about the $50 \%$ oxidizer mass-flow rate for a shorter time $(6 \mathrm{~s})$ with respect to nominal one. In the second L2 test both oxidizer mass-flow rate and time were increased. In the L3 test the nominal firing time was further increased. Firing test L4 was made to demonstrate throttling capabilities of the breadboard. Test L5 was finally performed with the nominal oxidizer mass-flow rate.

The following telemetry set-up was adopted (see also Figure $4 b$ ):

- Two Setra C206 pressure transducers, specifically in pre- and post-chamber, which can measure pressures up to 70 bar with an accuracy of \pm 0.1 bar and an acquisition frequency of $2.5 \mathrm{kHz}$;

- A thermocouple (type $\mathrm{K}$ ) in the breadboard pre-chamber directly in contact with the hot flow;

- An embedded thermocouple (type K) in proximity of the nozzle throat (see Figure 7);

- Four load cells for thrust measurement, with an overall accuracy of $\pm 1.5 \mathrm{~N}$.

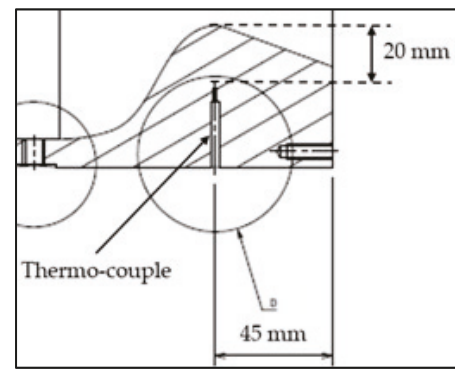

Figure 7. Embedded thermocouple positioning.

Moreover, the oxidizer temperature and mass-flow rate were acquired, for all the firing tests. 


\section{Experimental Test Campaign: Results and Discussion}

In this section the experimental results of the test campaign are presented and discussed.

\subsection{Test Execution and Hardware Inspection}

All the planned tests were successfully performed. No damage in any parts of the breadboard was detected. The experimental oxidizer mass-flow rates were in line with the test plan reported in Table 2, with deviations between nominal and experimental measured values below $10 \%$.

Figure 8 shows pictures of the breadboard and the exhaust during firing test L1 (a) and firing test L3 (b). A remarkable difference stands out in both flame shape and brightness, due to different test conditions. In particular, in the higher oxidizer mass-flow rate condition of test L3, both exhaust velocity and flame temperature were higher, as will be later discussed.

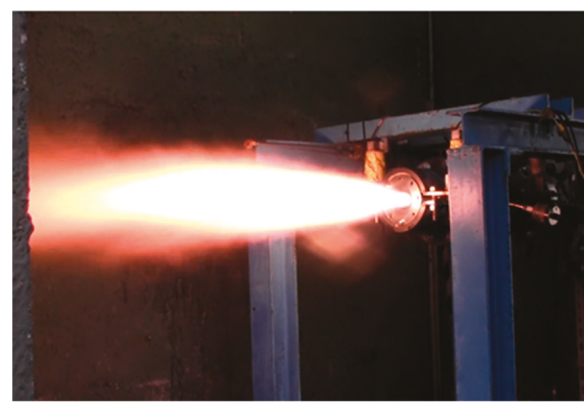

(a)

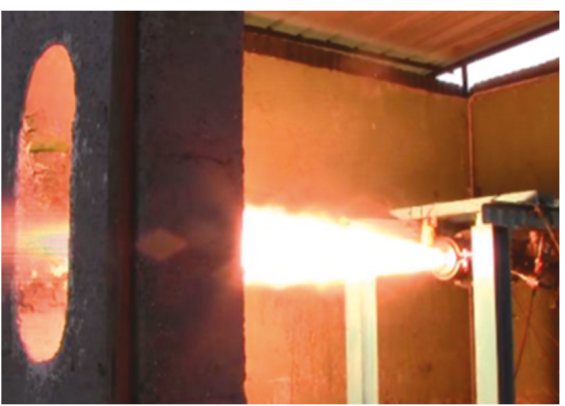

(b)

Figure 8. Breadboard during the firing test L1 (a) and L3 (b).

The paraffin-wax grain, used before burning, is shown in Figure 9a, while Figure 9b shows the grain after firing test L3. A uniform consumption of the solid fuel grain was observed in radial direction, while axially there was a slightly higher regression at grain exit.

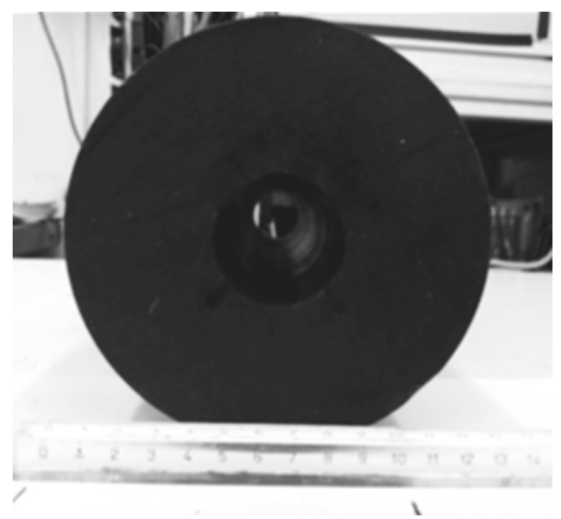

(a)

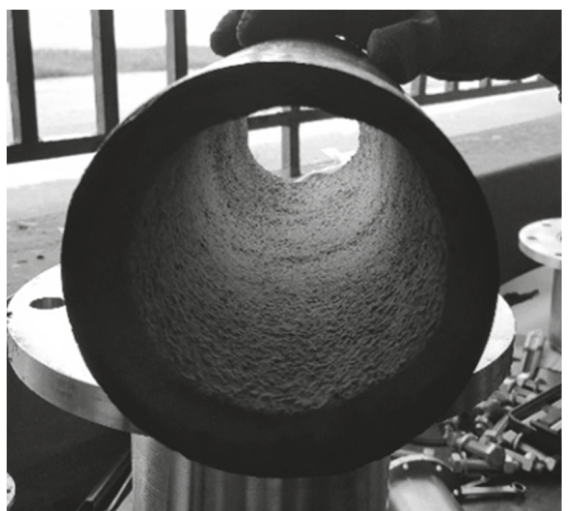

(b)

Figure 9. Paraffin grain before test (a) and after firing test L3 (b).

The weight of each propellant grain was measured after the firing tests for estimating the respective time-averaged fuel mass-flow rate and the corresponding time-averaged mixture ratio. Moreover, the mass loss method has been employed to estimate the space-averaged final port diameters, allowing computing the time-space averaged oxidizer mass-fluxes and fuel regression rates. For a detailed 
description of the data reduction technique refer to [6]. Propellant grains used for Test L4 and Test L5 reported an anomalous consumption in the inlet port diameter that shows a slightly larger diameter with respect to the medium one. Probably this is due to some delay experienced in the ignition sequence causing an initial overheating of the propellant. In any case no significant deviation in terms of thrust and pressure have been recorded during tests. No ablation occurred in the graphite nozzle, since the throat diameter remained unchanged during all the test campaign.

\subsection{Experimental Regression Rate Considerations}

The main measured quantities, for the different firing tests, are summarized in Table 3.

Table 3. Experimental data acquisitions.

\begin{tabular}{cccccc}
\hline Data & Test L1 & Test L2 & Test L3 & Test L4 & Test L5 \\
\hline Effective firing time $(\mathrm{s})$ & 5.6 & 7.5 & 11.4 & $9.7(5.3+4.4)$ & 9.0 \\
Effective oxidizer mass-flow rate $(\mathrm{kg} / \mathrm{s})$ & 0.110 & 0.195 & 0.192 & $0.142-0.215$ & 0.243 \\
Time-averaged fuel mass-flow rate $(\mathrm{kg} / \mathrm{s})$ & 0.984 & 0.152 & 0.172 & 0.177 & 0.2039 \\
Time-averaged mixture ratio $(-)$ & 1.12 & 1.29 & 1.11 & - & 1.20 \\
Time-space-averaged ox mass-flux $\left(\mathrm{kg} / \mathrm{m}^{2} \mathrm{~s}\right)$ & 55.35 & 72.35 & 52.37 & 53.3 & 69.94 \\
Time-space averaged fuel regression rate $(\mathrm{mm} / \mathrm{s})$ & 1.91 & 2.53 & 2.49 & 2.6 & 2.99 \\
\hline
\end{tabular}

The experimental data point in terms of fuel regression rate as function of the oxidizer mass flux are reported in Figure 10 along with the data point measured on the subscale $200 \mathrm{~N}$-class breadboard. Also, trends from relevant literature are reported for comparison. It is quite evident that, although the fuel grain formulation was the same, the regression rates experienced with the $1000 \mathrm{~N}$ breadboard, which range between about $1.9 \mathrm{~mm} / \mathrm{s}$ (test L1) and $3 \mathrm{~mm} / \mathrm{s}$ (test L5), are significantly higher with respect to the values obtained with the subscale breadboard at equal oxidizer mass flux. In fact, Table 4 reports the relative deviations between the expected values of the regression rate on the basis of the regression rate law as function of the oxidizer mass flux only (Equation (2)) and the corresponding measured data, which range between around $10 \%$ and $40 \%$ in module.

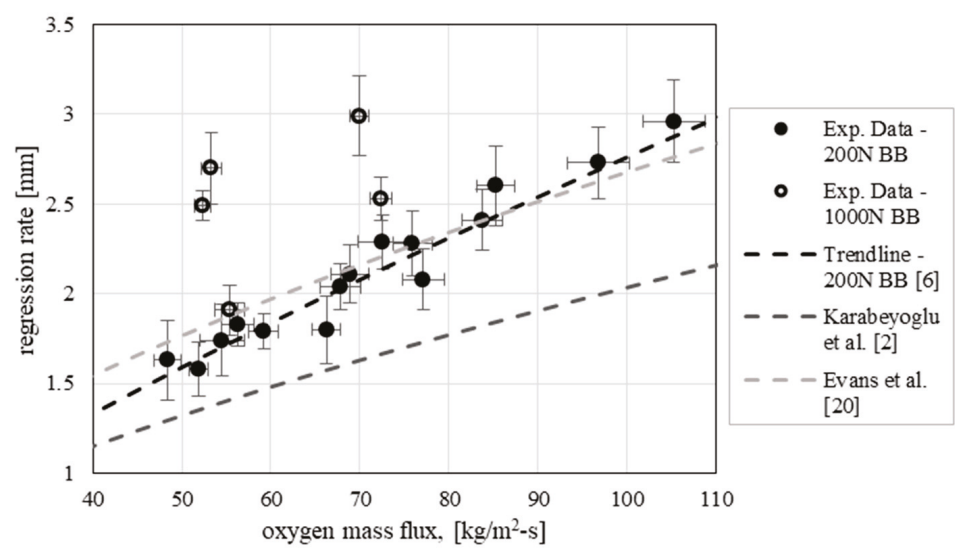

Figure 10. Regression rate versus oxidizer mass flux for all the tests. 
Table 4. Expected regression rate obtained with Equation (2) and deviation with respect to experimental data.

\begin{tabular}{ccc}
\hline Test ID & Expected Regression Rate with Equation (2) & Relative Deviation w.r.t. Experimental Data \\
\hline Test L1 & 1.73 & $-9.6 \%$ \\
Test L2 & 2.14 & $-15.6 \%$ \\
Test L3 & 1.65 & $-33.7 \%$ \\
Test L4 & 1.67 & $-38.0 \%$ \\
Test L5 & 2.08 & $-30.5 \%$ \\
\hline
\end{tabular}

The above discussed results show that, while Equation (2) represents a sufficiently good fit of experimental data obtained with the $200 \mathrm{~N}$ breadboard, it leads to a non-negligible underestimation of the regression rate in the case of the larger scale engine. Consequently, in the scale-up of hybrid rockets it should be taken into account that the oxidizer mass flux is not the only quantity affecting the fuel regression rate. This is also confirmed by the fact that the experimental data for the $1000 \mathrm{~N}$ breadboard themselves do not show a clear trend of regression rate with oxygen mass flux. Therefore, other significant operating parameters must play a significant role, which should be properly investigated and assessed. In particular, the possible effect of two main parameters, i.e., the grain port diameter and the mixture ratio, on the fuel consumption behavior is discussed in the following and will be the subject of the future experimental investigations.

Recalling the main idea of previous authors' work [6], the grain port diameter is a first quantity which could have an effect on the regression rate, as a consequence of the oxidizer injection flow pattern. In the case of axially injected oxidizer, a flow recirculation is created in the entrance region of the grain port where the oxidizer jet spreads up to the impingement point on the grain surface, enhancing the heat transfer to the wall. The extension of the vortex increases with the port diameter and, therefore, macroscopic result is that the fuel regression rate could be influenced by the port diameter other than by the mass flux [18]. For this reason, in Ref. [6] a correlation of the fuel regression rate with the oxidizer mass flux and the time-space averaged port diameter has been obtained which is expressed by

$$
\overline{\dot{r}}=a \overline{\mathrm{G}}_{o x}^{n} \bar{D}^{m} \quad a=0.029 ; \quad n=0.697 ; \quad m=0.398 \quad\left(R^{2}=0.95\right)
$$

where the regression rate is expressed in $\mathrm{mm} / \mathrm{s}$, mass flux in $\mathrm{kg} / \mathrm{m}^{2} \mathrm{~s}$ and the port diameter is in $\mathrm{mm}$.

Although it should be taken into account that the different injector and pre-chamber design limit the application of the above described correlation, the law of Equation (3) has been applied to calculate new values of the expected fuel regression rate and the results are reported in Table 5 .

Table 5. Expected regression rate obtained with Equation (3) and deviation with respect to experimental data.

\begin{tabular}{ccc}
\hline Test ID & Expected Regression Rate with Equation (3) & Relative Deviation w.r.t. Experimental Data \\
\hline Test L1 & 2.26 & $18.4 \%$ \\
Test L2 & 2.90 & $14.5 \%$ \\
Test L3 & 2.45 & $-1.3 \%$ \\
Test L4 & 2.41 & $-10.6 \%$ \\
Test L5 & 2.98 & $-0.5 \%$ \\
\hline
\end{tabular}

Although the deviations are reduced in almost all cases with respect to the basic formulation, the errors are still relatively high, so it can be concluded that combustion chamber conditions (i.e., MR, $\mathrm{p}_{\mathrm{c}}$ ) directly affect the regression rate. In particular, as reported in [2], the regression rate could be significantly affected by the oxidizer-to-fuel ratio. In fact, from Figure 11, which shows the adiabatic flame temperature variation with mixture ratio at fixed chamber pressure $\left(2 \times 10^{6} \mathrm{~Pa}\right)$, it can be observed that in the mixture ratio range between 1 and 2 a strong temperature increase of the flame is expected. The temperature increase determines both increase of the heat transfer to the gas-liquid interface 
and variation of the gas properties, which in turn affect both the vaporization and the entrainment contributions to the fuel regression rate.

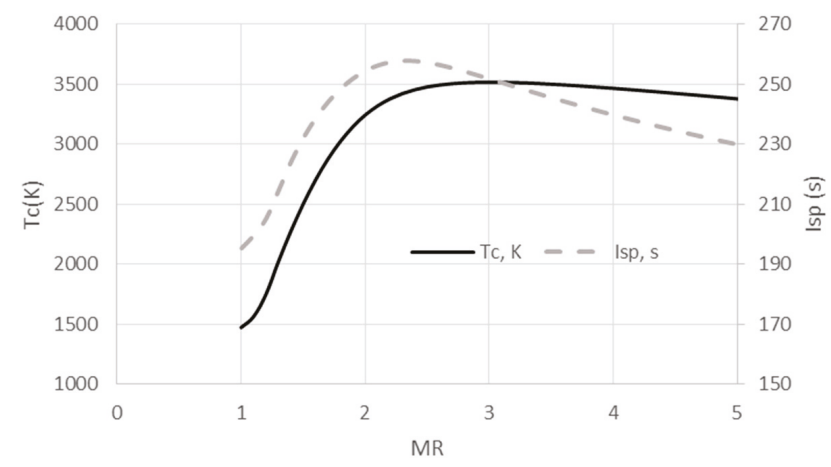

Figure 11. Specific impulse and chamber temperature vs. MR at $2 \times 10^{6} \mathrm{~Pa}$.

In particular, the vaporization contribution is given by the classical hybrid theory, which describes the regression rate of hybrid fuels in the absence of entrainment. The widely accepted formula due to Marxman et al. [19] shows the dependence of the vaporization regression rate with the gas properties (the gas viscosity in particular) and the radiative to convective heat transfer ratio.

$$
\dot{r}_{c l}=C_{f, r e f}\left(\frac{2 \mu_{g}}{\mathrm{~L}}\right)^{0.2} C_{B 1}\left(1+\frac{\dot{Q}_{r}}{\dot{Q}_{c}}\right)\left(\frac{B}{\rho_{s}}\right) G^{0.8}
$$

where $C_{f, r e f}$ is the reference shear stress (generally assumed equal to 0.03 ) and $C_{B 1}$ is a corrective factor of the surface friction [5]. It is evident that, once chosen the fuel propellant and fixed the oxidizer mass flux, the evaporation regression rate increases with the flame temperature due to both increase of gas viscosity and radiative to convective heat transfer ratio.

The entrainment contributions, on the two different breadboards, is more complex to define and requires deeper investigations. However, this mechanism is meanly related to the fuel properties, which remain unchanged. According to [5], the general empirical expression for the entrainment rate, in terms of the relevant properties of the hybrid breadboard, is

$$
\dot{r}_{e} \propto \frac{\left(C_{f} P_{d}\right)^{\alpha} h^{\beta}}{\sigma^{\gamma} \mu^{\delta}}
$$

where $\alpha$ is approximately $1.5, \beta$ is approximately 2 and $\gamma$ and $\delta$ are approximately $1 . P_{d}$ is the dynamic pressure of the gas flow in the port and $C_{f}$ has the same formulation of Equation (4), therefore depending on $\mu_{g}^{0.2}$. Once the mass flux through the port is fixed, a higher flame temperature leads to an increase of both skin friction coefficient (proportional to gas viscosity) and dynamic pressure $\left(\propto \rho V^{2}\right)$. The melt layer thickness is more difficult to quantify, but if a slight variation is assumed, then it can be asserted that also the entrainment contribution to regression rate is higher for the $1000 \mathrm{~N}$ breadboard with respect to the subscale.

Therefore, observing that the tests with the $1000 \mathrm{~N}$ breadboard are characterized by higher average mixture ratio if compared to the test performed with the subscale engine at similar oxidizer mass flux, the previous considerations could explain the higher measured values of the regression rate. Moreover, the regression rate in test L3 is higher than that of test L1 although the average oxidizer mass fluxes are similar (and similarly regression rate in test L5 is higher with respect to that of test L2) because the 
higher oxygen mass flow rate determines higher initial value of the mixture ratio which, as said before, is the cause of an enhanced fuel consumption.

The above discussed considerations lead to the conclusion that the regression rate law derived with subscale testing (Equation (2)) is not suitable when the motor operating parameters move away from those associated with the $200 \mathrm{~N}$ campaign. In fact, considering Test L1 of the $1000 \mathrm{~N}$ breadboard the Equation (2) remains still applicable, but for the other tests its application leads to a regression rate underestimation (since Test L2 to L5 are characterized by higher value of the oxidizer to fuel mixture ratio). A general expression of the fuel regression rate should include other operating parameters, beyond the oxidizer mass-flux, as for instance, mixture ratio. Dedicated experimental test campaign will be addressed to further investigate this question and, once more data would be available, an upgraded formulation of the Equations (2) and (3) for the regression rate will be derived.

\subsection{Data Assessment and Performances Considerations}

This section presents main data recorded by the experimental test campaign along with their preliminary assessment with consideration about general performance parameters. Figure 12 provides the temporal evolution of chamber pressure (a) and thrust (b) acquired for the different firing tests. Chamber pressure was not affected by throat diameter increase, since negligible ablation was measured after the firing tests. It is interesting noticing that pressure slightly increases and tends to a constant value during firing time; this behavior is opposite with respect to the previsions by HDC, since they were based on the regression rate law of the small-scale breadboard (Equation (2)). Due to higher measured regression rates with respect to the law presented in [6], even if on one side the temperature decreases in time due to decrease of MR from the optimum (see Figure 11), the higher paraffin mass-flow rate compensates this effect and the result is a nearly constant chamber pressure.

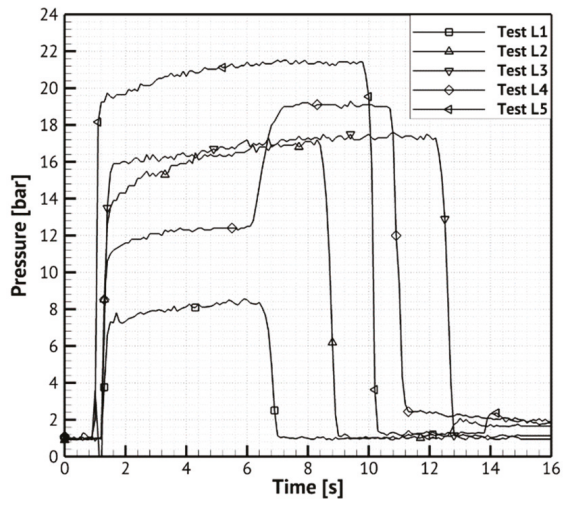

(a)

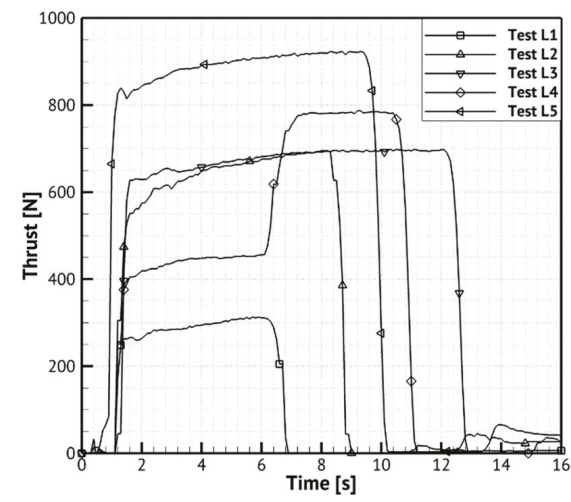

(b)

Figure 12. Measured pressure in the post-chamber (a) and thrust (b) over firing time.

Figure 13 shows temperature acquisitions by a thermocouple located in the breadboard pre-chamber (a) and an embedded thermocouple in proximity of the nozzle throat (b) - (for details see Figure 7). For test L3, the pre-chamber TC did not acquire due to a paraffin occlusion of the hole.

The experimental nozzle material temperature levels recorded during test, in Figure 13b, justify the absence of ablation phenomena. 


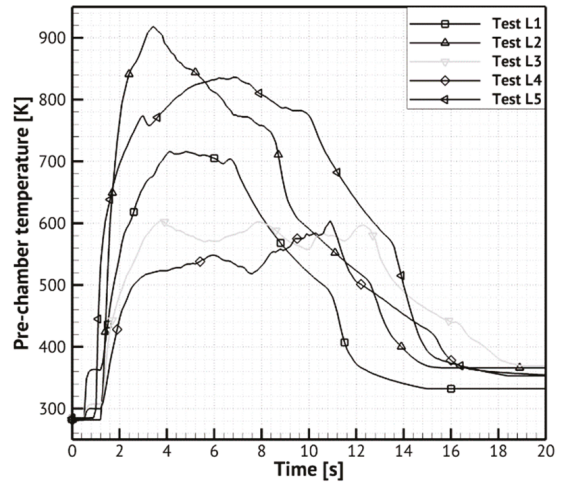

(a)

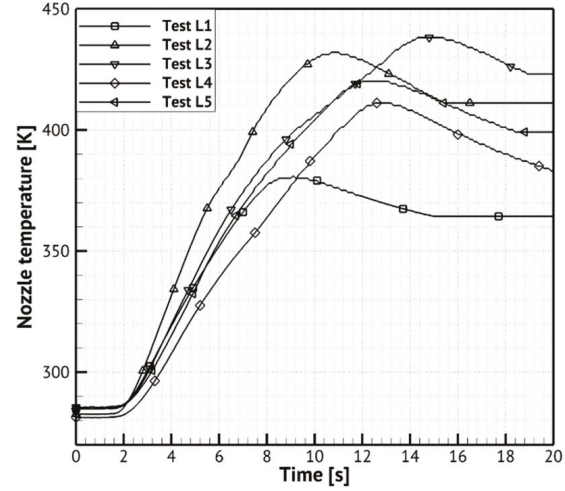

(b)

Figure 13. Measured pre-chamber temperature (a) and nozzle temperature (b) over firing time.

Table 6 reports the experimental breadboard performances. The time-averaged specific impulse is in line with the prevision made by the equilibrium calculation for each test conditions and thrust level is almost coherent with design predictions. It is important pointing out the behavior of the breadboard in the throttling test L4 (Figure 12), where thrust and pressure levels are steady for both the operating conditions and the transition appears stable without spikes and oscillations.

The combustion efficiency has been highlighted only for test L5, being, this operative point very close to the nominal one in terms of oxidizer mass flow. The estimation is based on the mean mixture ratio and has been calculated as the ratio between real and ideal values:

$$
\eta_{c *}=\frac{c_{r}^{*}}{c_{i d}^{*}}
$$

Table 6. Experimental $1000 \mathrm{~N}$ breadboard performances.

\begin{tabular}{cccccc}
\hline Quantity & Test L1 & Test L2 & Test L3 & Test L4 & Test L5 \\
\hline Time-averaged chamber pressure $(\mathrm{MPa})$ & 0.79 & 1.59 & 1.67 & $1.23-1.90$ & 2.09 \\
Time-averaged thrust $(\mathrm{N})$ & 291 & 650 & 679 & $446-782$ & 893 \\
Time-averaged specific impulse $(\mathrm{s})$ & 142 & 191 & 190 & 173 & 204 \\
Time averaged $\eta_{\mathrm{c}^{*}}$ & - & - & - & - & 0.94 \\
\hline
\end{tabular}

\section{Conclusions}

The design and testing activities of a novel $1000 \mathrm{~N}$ HRE breadboard have been presented. The breadboard, fed with gaseous oxygen and a paraffin-based fuel, showed a robust design and good performances. In particular, a stable combustion occurred in all the testing conditions including a wide range of pressure and mixture ratio. No significant chamber pressure oscillation was observed. The breadboard was also tested in order to verify throttling capabilities, oxidizer mass-flow rate was increased of about $35 \%$ during one of the firing test, leading to thrust increase of about $40 \%$. Numerous experimental data were collected, including temperature of the flow in the pre-combustion chamber and temperature inside the nozzle material. The experimental input variables-e.g., oxidizer mass-flow rates-were in agreement with the test plan; the breadboard performances, in terms of thrust and pressure, were almost in line with test objectives. A higher fuel regression rate was experimentally found with respect to subscale results, due to different operating conditions which affected both gas properties and heat transfer to the fuel surface. At the end of the test campaign, the graphite nozzle throat showed no significant erosion, in contrast with the expectations associated with the pre-testing 
numerical predictions and literature investigations. The result was due to lower than expected wall temperatures and, therefore, heat transfer rate.

Further investigations are still required for a thorough assessment of the collected data. Other test campaigns will be necessary, in order to better assess the role of the mixture ratio on the fuel regression rate.

Author Contributions: Conceptualization, F.B.; methodology, F.B. and G.D.D.M.; formal analysis, D.C.; investigation, F.B., D.C., G.D.D.M., and S.M.; data curation; D.C.; writing-original draft preparation, D.C.; writing-review and editing, F.B., G.D.D.M.; visualization, D.C. and M.F.; supervision, R.S.; project administration, F.B.

Funding: This research was founded by the Italian Ministry of University of Research (MIUR), whose support is much appreciated, financed this project.

Acknowledgments: The authors would like to thank all the HYPROB-NEW hybrid development line project team in particular Giuseppe Spaziani, Valeria De Simone, Marco Invigorito, Gianpaolo Elia, Vito Salvatore, whose effort was significant for the advancements of the project. Moreover, we would like to express our gratitude to all the UniNA colleagues, in integration and testing activities, for their precious cooperation and Marotta Srl for the manufacturing of the components.

Conflicts of Interest: The authors declare no conflict of interest. The funders had no role in the design of the study; in the collection, analyses, or interpretation of data; in the writing of the manuscript, or in the decision to publish the results.

\section{Nomenclature}

$a$

$A_{t}$

$B$

$C^{*}$

$C_{B 1}$

$C_{f}$

$C_{f, r e f}$

G

h

$h_{g}$

L

$m$

$n$

$p_{c}$

$P_{d}$

$\dot{Q}_{c}$

$\dot{Q}_{r}$

r

$\dot{r} \cdot$

$\dot{r}_{e}$

$R^{2}$

T

$T_{w a}$

$T_{w h}$

Greek Symbols

$\eta \quad$ combustion efficiency [-]

$\mu \quad$ gas molecular viscosity [kg/m-s]

$\rho \quad$ density $\left[\mathrm{kg} / \mathrm{m}^{3}\right]$

$\sigma \quad$ surface tension $[\mathrm{N} / \mathrm{m}]$

regression rate factor [-]

nozzle throat area $\left[\mathrm{m}^{2}\right]$

blowing number [-]

characteristic velocity $[\mathrm{m} / \mathrm{s}]$

blowing correction coefficient [-]

friction coefficient [-]

reference friction coefficient [-]

port average mass flux $\left[\mathrm{kg} / \mathrm{m}^{2}-\mathrm{s}\right]$

melt layer thickness [m]

fuel grain length $[\mathrm{m}]$

chamber pressure exponent

oxidizer mass flux exponent

chamber pressure $[\mathrm{Pa}]$

dynamic pressure $[\mathrm{Pa}]$

convective heat flux $\left[\mathrm{W} / \mathrm{m}^{2}\right]$

radiative heat flux $\left[\mathrm{W} / \mathrm{m}^{2}\right]$

surface regression rate $[\mathrm{m} / \mathrm{s}]$

classical theory regression rate $[\mathrm{m} / \mathrm{s}]$

regression rate due to entrainment $[\mathrm{m} / \mathrm{s}]$

coefficient of determination [-]

temperature [K]

adiabatic wall temperature $[\mathrm{K}]$

hot gas wall temperature $[\mathrm{K}]$

convective heat transfer coefficient $[\mathrm{W} / \mathrm{m}-\mathrm{K}]$ 


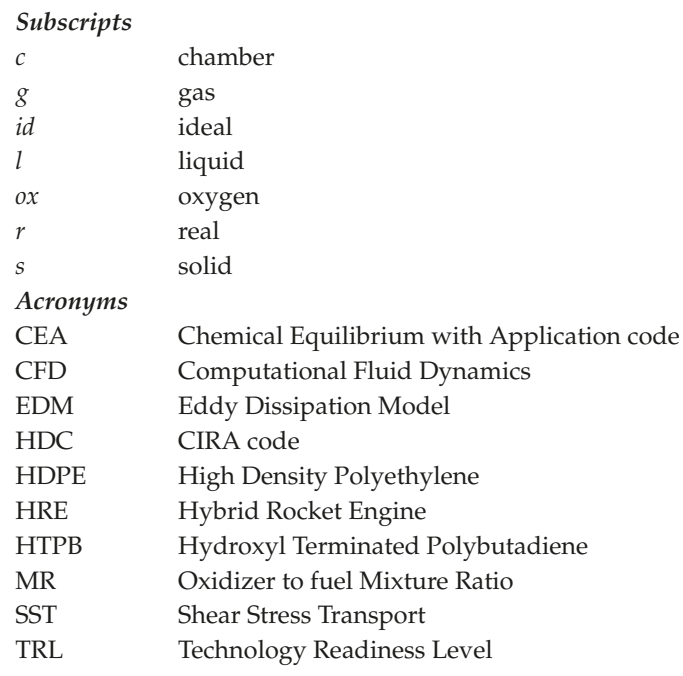

\section{References}

1. Altman, D.; Holzman, A. Overview and History of Hybrid Rocket Propulsion. In Fundamentals of Hybrid Rocket Combustion and Propulsion; Kuo, K., Chiaverini, M., Eds.; Progress in Astronautics and Aeronautics; AIAA: Reston, VA, USA, 2007; pp. 1-36.

2. Karabeyoglu, A. Scale-up tests of high regression rate paraffin-based hybrid rocket fuels. J. Propuls. Power 2004, 20. [CrossRef]

3. Carmicino, C.; Scaramuzzino, F.; Sorge, A. Trade-off Between Paraffin-Based and Aluminium-Loaded HTPB Fuels to Improve Performance of Hybrid Rocket Fed with $\mathrm{N}_{2} \mathrm{O}$. Aerosp. Sci. Technol. 2014, 37, 81-92. [CrossRef]

4. Kobald, M.; Schmierer, C.; Ciezki, H.K.; Schlechtriem, S.; Toson, E.; De Luca, L.T. Viscosity and Regression Rate of Liquefying Hybrid Rocket Fuels. J. Propuls. Power 2017, 33, 1245-1251. [CrossRef]

5. Karabeyoglu, M.A.; Cantwell, B.J.; Altman, D. Development and Testing of Paraffin-Based Hybrid Rocket Fuels. In Proceedings of the 37th AIAA/ASME/SAE7ASEE Joint Propulsion Conference and Exhibit, Salt Lake City, UT, USA, 8-11 July 2001.

6. Di Martino, G.D.; Mungiguerra, S.; Carmicino, C.; Savino, R.; Cardillo, D.; Battista, F.; Invigorito, M.; Elia, G. Two-Hundred-Newton Laboratory-Scale Hybrid Rocket Testing for Paraffin Fuel-Performance Characterization. J. Propuls. Power 2018, 35. [CrossRef]

7. Bianchi, D.; Nasuti, F. Numerical Analysis of Nozzle Material Thermochemical Erosion in Hybrid Rocket Engines. J. Propuls. Power 2013, 29, 547-558. [CrossRef]

8. Sutton, G.P.; Biblarz, O. Hybrid Propellant Rockets, Rocket Propulsion Elements, 7th ed.; John Wiley \& Sons: Hoboken, NJ, USA, 2001; pp. 585-593.

9. Karabeyoglu, A. Advanced Hybrid Rockets for Future Space Launch. In Proceedings of the Fifth European Conference for Aeronautics and Space Sciences, Munich, Germany, 1-5 July 2013.

10. Ponomarenko, A. RPA Tool for Rocket Propulsion Analysis. In Proceedings of the Space Propulsion Conference, Cologne, Germany, 19-22 May 2014.

11. Hill, P.G.; Peterson, C.R. Mechanics and Thermodynamics of Propulsion; Addison-Wesley: Boston, MA, USA, 1965.

12. David, W. Hybrid Rocket Internal Ballistics; Chemical Propulsion Information Agency: Monterey, CA, USA, 1972.

13. Altman, D.; Humble, R. Hybrid Rocket Propulsion Systems. In Space Propulsion Analysis and Design; Humble, R.W., Henry, G.N., Larson, W.J., Eds.; The McGraw-Hill Companies, Inc., Primis Custom Publishing: New York, NY, USA, 1995; pp. 403-441.

14. Yang, V. Liquid Rocket Thrust Chambers: Aspects of Modeling, Analysis, and Design; AIAA: Reston, VT, USA, 2004; ISBN 1563472236. 
15. Cardillo, D.; Battista, F.; Elia, G.; Di Martino, G.D.; Mungiguerra, S.; Savino, R. Design and Testing of a Paraffin-Based Hybrid Rocket Demonstrator. In Proceedings of the Space Propulsion Conference, Seville, Spain, 14-18 May 2018.

16. Saccone, G.; Piscitelli, F.; Gianvito, A.; Cosentino, G.; Mazzola, L. Manufacturing Processes of Paraffin Grains as Fuel for Hybrid Rocket Engines. In Proceedings of the 51st AIAA/ASME/SAE/ASEE Joint Propulsion Conference, Orlando, FL, USA, 27-29 July 2015.

17. Kumar, C.P.; Kumar, A. A Numerical Study on the Regression Rate of Hybrid Rocket Motors Using a Combination of Enhancement Techniques. In Proceedings of the 48th AIAA/ASME/SAE/ASEE Joint Propulsion Conference Exhibit, Atlanta, GA, USA, 30 July-1 August 2012.

18. Carmicino, C.; Sorge, R.A. Role of Injection in Hybrid Rockets Regression Rate Behavior. J. Propuls. Power 2005, 21, 606-612. [CrossRef]

19. Marxman, G.A.; Wooldridge, C.E.; Muzzy, R.J. Fundamentals of Hybrid Boundary Layer Combustion. Prog. Astronaut. Aeronaut. 1964, 15, 485-522.

(C) 2019 by the authors. Licensee MDPI, Basel, Switzerland. This article is an open access article distributed under the terms and conditions of the Creative Commons Attribution (CC BY) license (http://creativecommons.org/licenses/by/4.0/). 



\title{
Nano-Sized and Mechanically Activated Composites: Perspectives for Enhanced Mass Burning Rate in Aluminized Solid Fuels for Hybrid Rocket Propulsion
}

\author{
Christian Paravan \\ Space Propulsion Laboratory (SPLab), Aerospace Science and Technology Department, Politecnico di Milano, 34, \\ via La Masa, 20156 Milan, Italy; christian.paravan@polimi.it; Tel.: +39-02-2399-8068
}

Received: 31 May 2019; Accepted: 18 November 2019; Published: 25 November 2019

\begin{abstract}
This work provides a lab-scale investigation of the ballistics of solid fuel formulations based on hydroxyl-terminated polybutadiene and loaded with Al-based energetic additives. Tested metal-based fillers span from micron- to nano-sized powders and include oxidizer-containing fuel-rich composites. The latter are obtained by chemical and mechanical processes providing reduced diffusion distance between $\mathrm{Al}$ and the oxidizing species source. A thorough pre-burning characterization of the additives is performed. The combustion behaviors of the tested formulations are analyzed considering the solid fuel regression rate and the mass burning rate as the main parameters of interest. A non-metallized formulation is taken as baseline for the relative grading of the tested fuels. Instantaneous and time-average regression rate data are determined by an optical time-resolved technique. The ballistic responses of the fuels are analyzed together with high-speed visualizations of the regressing surface. The fuel formulation loaded with $10 \mathrm{wt} . \%$ nano-sized aluminum (ALEX-100) shows a mass burning rate enhancement over the baseline of $55 \% \pm 11 \%$ for an oxygen mass flux of $325 \pm 20 \mathrm{~kg} /\left(\mathrm{m}^{2} \cdot \mathrm{s}\right)$, but this performance increase nearly disappears as combustion proceeds. Captured high-speed images of the regressing surface show the critical issue of aggregation affecting the ALEX-100-loaded formulation and hindering the metal combustion. The oxidizer-containing composite additives promote metal ignition and (partial) burning in the oxidizer-lean region of the reacting boundary layer. Fuels loaded with $10 \mathrm{wt}$.\% fluoropolymer-coated nano-Al show mass burning rate enhancement over the baseline $>40 \%$ for oxygen mass flux in the range 325 to $155 \mathrm{~kg} /\left(\mathrm{m}^{2} \cdot \mathrm{s}\right)$. The regression rate data of the fuel composition loaded with nano-sized $\mathrm{Al}$-ammonium perchlorate composite show similar results. In these formulations, the oxidizer content in the fuel grain is $<2 \mathrm{wt} . \%$, but it plays a key role in performance enhancement thanks to the reduced metal-oxidizer diffusion distance. Formulations loaded with mechanically activated ALEX-100-polytetrafluoroethylene composites show mass burning rate increases up to $140 \% \pm 20 \%$ with metal mass fractions of $30 \%$. This performance is achieved with the fluoropolymer mass fraction in the additive of $45 \%$.
\end{abstract}

Keywords: nano-sized aluminum; micron-sized aluminum; fluoropolymer; mechanically activated aluminum; coated aluminum; fuel-rich composite powder; aluminum aggregation; aluminum agglomeration; regression rate; mass burning rate

\section{Introduction}

Hybrid rocket engines (HREs) feature a diffusion-limited combustion process between the atomized liquid (or gaseous) oxidizer and the pyrolyzed/vaporized solid fuel. The turbulent boundary layer combustion is ruled by convective heat transfer, with the $r_{f}$ showing relatively strong dependence on $G$ [1-5]. In the complex boundary layer combustion mechanism, oxidizer-lean conditions are encountered in the region extending from the regressing surface to the flame. Conventional polymer-based solid fuels feature relatively slow $r_{f}$, in turn yielding low thrust levels with simple 
grain geometries (i.e., cylindrical grains with single central port perforation). This issue can be partially overcome by multi-port grains enabling increased $\dot{m}_{f}$ thanks to the enlarged burning surface area. Such a solution requires complex implementation, with possible system dry mass increases and structural integrity issues [6]. Liquefying fuels show faster regression rates than conventional polymeric formulations thanks to the entrainment of melted fuel droplets [7,8]. On the other hand, liquefying fuels such as paraffin wax feature weak mechanical properties and are unsuitable for effective exploitation in operating systems. Different studies are currently ongoing to obtain reinforced fuel grains combining ballistic and mechanical properties. Paraffin wax blending with reinforcing polymers is a strategy to achieve this purpose [9-14]. The improved mechanical properties of the blended formulations are accompanied by increased viscosity of the melted phase; this lessens the entrainment mass transfer, thus decreasing $r_{f}$ and $\dot{m}_{f}$. In spite of differences in the combustion mechanism of polymeric and liquefying fuels, both kinds of formulations can achieve increased ballistic performance thanks to the use of metal-based energetic additives [15-19].

Metal-based energetic additives aim at providing enhanced flame temperatures and/or increased radiation heat transfer to promote faster $r_{f}$ of the solid fuel grain and increased $\dot{m}_{f}$. Moreover, augmented radiation heat transfer reduces the $r_{f}(G)$ dependence, enabling solid fuel ballistic response tailoring [20]. Aluminum is an attractive candidate as an energetic filler, thanks to its oxidation enthalpy [21] and its commercial availability at different dispersity levels (from micron-sized particles to the nanoscale), enabling its use as it is, or as an ingredient for composite additives [22-24].

Conventional $\mathrm{Al}$ powders feature micron-sized spherical particles [25]. The $\mu \mathrm{Al}$ is typically air-passivated, thus showing a core-shell particle structure with the metal core surrounded by an amorphous $\mathrm{Al}_{2} \mathrm{O}_{3}$ layer [26-28]. The micrometric size, together with the particle morphology and the specific surface area (SSA) $<1 \mathrm{~m}^{2} / \mathrm{g}$, yields $\mu \mathrm{Al}$ high metal content (typically $>95 \mathrm{wt} . \%$ ), relatively low reactivity, and inherent safety (i.e., high ignition temperature, reduced dispersion in air, and limited aging influence) [28-31]. The reactivity of $\mu \mathrm{Al}$ can be enhanced by reducing the particle size down to the nanoscale [32-35] and by activation processes [23,24,36-39]. Nano-sized Al is characterized by SSA $\sim 10 \mathrm{~m}^{2} / \mathrm{g}$ (or higher, depending on the average powder particle size and size distribution). These powders show marked reactivity at temperatures lying below the Al melting point $(933 \mathrm{~K})[17,34,35,40]$. Nano-sized $\mathrm{Al}$ is typically passivated by air, although special chemicals may be used to shield the metal from the external environment $[34,35,40]$. With respect to $\mu \mathrm{Al}$, air-passivated nAl exhibits reduced $C_{A l}$ and increased sensitivity to storage conditions [28]. Due to the reduced size, the nAl powders feature cold-cohesion phenomena. Clusters of particles exhibit SSA reduction with respect to the single particles. Thus, special manufacturing procedures are typically implemented to disperse nAl in energetic compositions [41-43]. Moreover, the use of nano-sized particles is complicated by their effects on propellant/fuel slurry rheology $[25,44]$, and stricter safety requirements than the micron-sized counterpart. Activated $\mathrm{Al}$ powders typically feature reactivity tailoring of the starting material by chemical, mechanical, and mechano-chemical methods [22-24]. Activation techniques modify the powder surface characteristics (such as SSA and/or composition), and/or the particle morphology, size, and composition [22-24,36-38,45-48]. In particular, activation processes enable the creation of composite additives such as dual-metal $[38,48]$ or metal-oxidizer compositions $[45,48]$, offering wide possibilities for additive reactivity tailoring. Focusing on mechanical activation (i.e., ball milling), it is worth noting that the additives produced from micron-sized granules typically feature flake shape. For this particle morphology, thickness is the only nanoscale dimension, while complex surface texture (typically providing increased SSA with respect to conventional $\mu \mathrm{Al}$ ) is usually achieved. Thanks to this, these materials offer increased reactivity while reducing the issues related to the difficult handling of nano-sized materials [48]. The creation of composite additives eases the propellant/fuel manufacturing, enabling the use of a single (dual) ingredient, instead of adding/handling different materials.

The presence of metal additives, including $\mathrm{Al}$, implies the possible insurgence of performance losses due to incomplete metal combustion, two-phase flow expansion, and residual accumulation in the combustion chamber/nozzle walls [17-22]. All these issues are of particular relevance in HRE 
combustion due to the diffusion-limited mechanism and the relatively low combustion efficiency of these systems. The mitigation of the low combustion efficiency requires specific implementations such as mixing chambers and/or the design of energetic additives featuring tailored composition and reactivity to lessen the detrimental effects related to metal combustion.

Current open literature includes several experimental studies on the $r_{f}$ behavior of metallized solid fuels for hybrid rocket propulsion [15-17,22,49-56]. In these works, differences in additive characterization approaches, experimental operating conditions, and data reduction techniques possibly yield difficult interpretations of the effects of the investigated materials when comparing different studies.

This work investigates the effects of different Al-based energetic additives on the ballistic response of polymer-based fuels for hybrid rocket propulsion. The objectives of this work are (i) evaluating the $r_{f}$ and $\dot{m}_{f}$ enhancement offered by innovative Al-based additives, and (ii) analyzing the surface phenomena occurring during the combustion of metallized formulations to link the additive composition effects to the solid fuel ballistic response. Hydroxyl-terminated polybutadiene (HTPB) is considered as a binder in the solid fuel formulations, in light of its suitable mechanical properties [42], making it an interesting candidate for the lab-scale study, as well as for possible applications requiring high volumetric efficiency pursued by high filler loads. The information gained by insight into the burning behavior of polymer-based fuels can provide useful suggestions for the development of energetic additives suitable for paraffin-based fuels reinforced by blending with thermoplastic polymers, in which the entrainment reduction due to the increased melt layer viscosity could be compensated for by using energetic fillers. The investigated additives range from conventional $\mu \mathrm{Al}$ to $\mathrm{nAl}$, and the test matrix includes mechanical-activated powders. The tested $\mathrm{nAl}$ powders feature different passivation layers and are either uncoated or coated. Investigated mechanical-activated powders are based on $\mu \mathrm{Al}$ and nAl. Composite powders are fuel-rich formulations including ammonium perchlorate (AP), a copolymer of vinylidene fluoride and hexafluoropropylene (VFHFP) [57], and polytetrafluoroethylene (PTFE) as oxidizers. Composite additives are produced by chemical methods (i.e., particle coating) and mechanical activation. Both approaches aim at reducing the diffusion distances between the $\mathrm{AP} /$ fluoropolymer and the metal fuel particles. The composites are formulated to deliver the oxidizing species close to the metal fuel during the combustion. The pursued effects are a prompter Al ignition and combustion in the oxidizer-lean region close to the gasifying fuel surface. The proposed strategy differs from mixed fuel approaches previously presented in the open literature [58,59]. In the current work, the oxidizer is localized in the composite additive, and fluoropolymers are selected as oxidizers due to their relatively high thermal stability joined with reactivity in the presence of metals such as $\mathrm{Al}$. In general, the oxidizer-loaded solid fuels possibly imply a loss of some of the HRE peculiarities such as faint $r_{f}$ dependence on the $p_{c}$ (in turn simplifying throttling operations and granting combustion tolerance to grain cracks), stop-restart capability, and reduced environmental impact. The current work focuses on the combustion behavior of metallized solid fuels, and on the analysis of the surface phenomena involved in this process; therefore, the abovementioned effects are not considered in this study. On the other hand, it is worth noting that the operating flexibility of HREs is not lost in the presence of solid fuel grains embedding oxidizing substances, unless the resulting formulation is burnt above its pressure deflagration limit (PDL). The latter is the lowest pressure a solid propellant/mixed hybrid can exhibit self-sustained combustion at. If a mixed hybrid is burning at $p_{c}<$ PDL, the combustion can be stopped and restarted without differences from what occurs in an equivalent fuel composition with no oxidizer load [59].

The work starts with pre-burning characterization of the investigated materials, and then focuses on the burning behavior of the HTPB-based fuels. The ballistic responses of the fuel formulations are characterized by time-resolved $r_{f}$ and $\dot{m}_{f}$ measurements and by high-speed visualizations of the burning surface.

This work aims at providing a detailed view of the combustion process of aluminized fuel formulations. Pre-burning characterization of the metal additives is analyzed together with 
time-resolved ballistics of the loaded fuels. In the open literature, solid fuel combustion behavior is typically evaluated by time- and space-averaged data reduction techniques based on pre- and post-firing measurements of relevant parameters. The time-resolved technique for $r_{f}$ and $\dot{m}_{f}$ tracks the combustion evolution from ignition to the final condition, providing the fuel ballistic response over a range of $G_{o x}$ with a single test. High-speed visualizations of the burning surface phenomena offer detailed insight into the effects of the metal additives on the fuel combustion behavior, and they enable evaluating the impact of aggregation/agglomeration phenomena on the combustion of Al-based additives. These effects are not widely investigated for solid fuel formulations. The combination of ballistic analyses and surface visualizations contributes to clarifying the reasons for some contrasting results observed in the open literature for the combustion of aluminized fuels, in particular when nAl formulations are considered.

\section{Background}

A background of the current work is hereby presented considering open literature studies focused on (i) the fundamentals of the solid fuel combustion process, (ii) the influence of metal additives on the solid fuel ballistics, and (iii) a discussion of $\mathrm{Al}$ aggregation and agglomeration phenomena (with particular emphasis on solid fuel formulations).

\subsection{Fundamentals of Solid Fuel Combustion Process}

In hybrid rockets, the flame has a diffusive nature, and it is set in the boundary layer generated by the oxidizer flow over the grain surface. Fundamentals of the HRE combustion mechanism were reviewed by Chiaverini [4] and Marquardt and Majdalani [5]. A survey on solid fuel thermal decomposition (a phenomenon playing a central role in HRE combustion) was given by Lengellé [60]. The behavior of non-metallized polymeric fuel formulations is dominated by convective heat transfer, and it is subject to heat transfer blockage due to mass blowing from the surface [1-3]. Under these conditions, theoretical modeling of the combustion mechanism yields $r_{f}(G)=a_{r} \cdot G^{n_{r}}$ (or $\left.r_{f}\left(G_{o x}\right)=a_{r} \cdot G_{o x}^{n_{r}}\right)$, with $n_{r}=0.8$ [1-3]. Experimental investigations typically yield $r_{f}(G)$ power law approximations with $n_{r}$ in the range 0.6 to 0.8 [4]. Radiation heat transfer plays a role as convective phenomena are lessened $[4,20,61,62]$. The effects of radiation are due to gas radiation (playing a minor role), to soot, and (if present) to radiating metal particles [1-5,20,61-63]. Under the influence of radiation heat transfer, $r_{f}$ may exhibit $p_{c}$ dependence $[4,20]$, and the $r_{f}(G)$ power law approximation shows $n_{r}<0.8$ [1-5,20,61-63]. Liquefying fuels show a regression rate dependence on $G$ similar to the polymeric formulations, although their $r_{f}$ exhibits percentage increases of $300-400 \%$ with respect to reference HTPB [7,8]. This enhanced performance is achieved thanks to an instability of the melt fuel layer formed at the regressing surface. If this layer features low viscosity and reduced surface tension, its interaction with the oxidizer stream yields the formation of waves from which melt fuel droplets are detached $[7,8]$. Therefore, in liquefying fuels the overall $r_{f}$ is the sum of the vaporization and of the entrainment contributions. The latter does not affect the convective heat transfer blockage; moreover, the entrained mass flow rate does not require vaporization enthalpy absorption at the regressing surface $[7,8]$. Current liquefying fuels offer low mechanical properties. Typical mechanical properties reinforcement techniques yield a reduction of the entrainment effect due to the augmented viscosity of the melted fuel layer $[10-14,19]$. Recent efforts proposed innovative reinforcing strategies exerting minor effects on the liquid phase viscosity such as self-disintegrating structures [64], as well as the use of three-dimensional (3D) printed cellular structures $[65,66]$.

The diffusion-limited combustion of HREs implies low combustion efficiency, thus requiring specific countermeasures such as enhanced oxidizer-fuel vapors mixing in the aft-combustion chamber and/or the use of diaphragms [67], non-conventional oxidizer injection implementations (playing a role in $r_{f}$ enhancement) [68-70], and/or design of special engine configurations [71]. 


\subsection{Regression Rate Performance and Metal Additives}

For both polymer- and paraffin-based fuel formulations, augmented or tailored, $r_{f}$ performance is typically pursued by loading the fuel grains with energetic additives such as oxidizer, metal, and hydride powders. A comprehensive review of the early steps of this approach was given by Risha et al. in [15]. First studies on the combustion of metal-loaded fuels investigated the effects of micron-sized additives (such as $\mathrm{Al}$ and $\mathrm{Mg}$ ). In these analyses, the achieved $r_{f}$ enhancements were mainly due to the augmented radiation heat transfer caused by the presence of metal particles, the effect on the flame temperature being limited by the high ignition temperatures, and relatively long burning times of these conventional additives $[15,20,61,62]$. Different analyses showed that $\mu \mathrm{Al}$ exerts no marked influence on the $r_{f}$ by particle sizes in the range of 30 to $250 \mu \mathrm{m}[15,20,61,62]$. Thanks to their enhanced reactivity over the micron-sized counterpart, nanometric additives disclose new opportunities for solid fuel performance enhancement. In general, the lower ignition temperatures and the shorter combustion times of nano-sized particles should promote increased $r_{f}[15-18,40,41,50-54]$, as well as relatively high combustion efficiencies [55]. On the other hand, the open literature shows contrasting results for fuel formulations loaded with $\mathrm{nAl}$. The ballistic responses of HTPB-based fuels doped with different nano-sized ingredients was extensively investigated by Risha et al. [50-52]. In these studies, $r_{f}$ variations were observed for different sets of formulations featuring similar compositions [50-52]. These effects were probably due to different manufacturing procedures (without/with fuel slurry sonication). Results for HTPB + $13 \mathrm{wt} . \% \mathrm{nAl}$ (air-passivated, nominal size 100-150 nm, SSA = 13 $\left.\mathrm{m}^{2} / \mathrm{g}\right)$, exhibited a $r_{f}$ enhancement over the HTPB baseline of $105 \%\left(G_{o x}=112 \mathrm{~kg} /\left(\mathrm{m}^{2} \cdot \mathrm{s}\right)\right)[52]$. In the same dataset, formulations loaded with Viton-A-coated $\mathrm{nAl}$ and $\mu \mathrm{Al}$ showed an $r_{f}$ increase (relative to the baseline) of $123 \%$ and $142 \%$, respectively [52]. Experimental evidences suggest an easier ignition and a more complete combustion of the fluoropolymer coated powder with respect to the other tested additives. The data presented in References [55,56] show moderate (if any) $r_{f}$ enhancement for nAl-loaded formulations. In the work of Thomas et al. [56], the absence of marked effects of the $\mathrm{nAl}$ on the solid fuel ballistic response of HTPB-based fuels was probably due to the limited additive mass fraction (5 wt.\%). In a recent study, Connell et al. reported a maximum $r_{f}$ increase of $20 \%$ over a non-metallized baseline for HTPB + $10 \mathrm{wt}$. $\% \mathrm{nAl}$ [72]. In the same study, results on innovative Ti-Al-B nanopowders were presented. These new additives showed $r_{f}$ effects similar to the aforementioned $\mathrm{nAl}$, but with a reduced $G_{0 x}$ sensitivity [72]. The ballistic response of a paraffin-based fuel formulation loaded with micron-sized $\mathrm{Al}$ flakes (with nano-metric thickness) was presented in Reference [18]. The metallized fuel promoted an $r_{f}$ enhancement over a pure paraffin formulation, although slight differences in operating conditions prevented a precise relative grading. Galfetti et al. reported significant performance benefits achieved by the use of $\mathrm{nAl}$ (nominal sizes of $50 \mathrm{~nm}$ and $100 \mathrm{~nm}$ ) in entrainment-producing fuels tested in a lab-scale slab burner [19]. The solid fuel $r_{f}$ increase over an HTPB baseline was $186 \%$ for pure solid wax fuel, while it reached $210 \%$ for $n A l$-doped compositions.

Fuel formulations loaded with AP produce $r_{f}$ increases over an HTPB-baseline up to $300 \%$ for a solid oxidizer mass fraction of $25 \mathrm{wt} . \%$ [59]. Reference [73] discussed the characteristics and the performance of a solid fuel formulation with $80 \mathrm{wt} . \%$ PTFE + $20 \mathrm{wt} \% \mathrm{~B}$ that is considered for specific hybrid propulsion applications.

Mechanical, chemical, and mechano-chemical activation processes offer breakthrough opportunities for the development of new energetic composites [22-24,36-39,45-48]. The use of mechanical and mechano-chemical activation processes enables coupling ingredients featuring relatively low compatibility [24,45]. Detailed discussions on the design strategy for mechanically activated powders were presented in Reference [24]. Micron-sized Al-rich composites containing PTFE and produced by mechanical activation were presented in Reference [74]. In the analysis, a paraffin and ethylene-vinyl acetate blend was loaded with an Al + PTFE composite and featured a 300\% $r_{f}$ increase over the non-metallized counterpart. 


\subsection{Aluminized Solid Fuel Formulations: Aggregation and Agglomeration}

Aluminum combustion under solid/hybrid rocket motor conditions implies the presence of condensed combustion products (CCPs) [75-78]. Condensed products feature different compositions, morphologies, and sizes, depending on their genesis conditions (i.e., solid propellant/fuel composition details and operating conditions). Following the nomenclature introduced in Reference [77], CCPs include (i) smoke-oxide particles (SOP), and (ii) agglomerates. The SOP consist of fine particles $(<1 \mu \mathrm{m})$ formed by condensation of gaseous reaction products and combustion of non-agglomerated particles [77]. Agglomerates are composed by $\mathrm{Al}$ and $\mathrm{Al}_{2} \mathrm{O}_{3}$ (with the eventual presence of binder decomposition products). Agglomerates feature enlarged size with respect to the original $\mathrm{Al}$ powder particles loaded in the solid propellant/fuel grain. This size growth is caused by aggregation of single particles at the burning propellant surface/subsurface, and by the coalescence of agglomerates (possibly followed by particle breakup during expansion) [79]. A larger agglomerate particle size and mass fraction results in higher performance losses due to incomplete combustion and two-phase flow [17]. Experimental and numerical studies on the $\mathrm{Al}$ combustion in solid propellant formulations were discussed in References [75-83].

The burning behavior of HTPB-based formulations doped with high mass fractions of $\mu \mathrm{Al}$ was presented in Reference [61]. In this study by Strand et al., $7 \mathrm{~kg} /\left(\mathrm{m}^{2} \cdot \mathrm{s}\right) \leq G_{0 x} \leq 35 \mathrm{~kg} /\left(\mathrm{m}^{2} \cdot \mathrm{s}\right)$, and the investigated fuel featured $30 \mathrm{wt} \% \mathrm{Al}$ powder and $30 \mathrm{wt} \%$ coal (added to the formulation to segregate the metal particles). Experimental results showed a layered combustion. The formation of an HTPB-coal melt containing Al was observed. Filigrees/flakes protruded from the regressing surface to the reaction zone, and large-size particles $(\sim 1 \mathrm{~mm})$ were ejected by the regressing surface. During motor firings on different lab-scale set-ups, Risha et al. noted different $r_{f}$ performance from similar aluminized formulations loaded with Al flakes with nanometric thickness [15]. SEM images of extinguished fuel grains showed the more abundant $\mathrm{Al}$ melt layer accumulation at the regressing surface of the larger motor and causing the lower $r_{f}$. Observation of aggregation and incipient agglomeration of $\mu \mathrm{Al}$ - and nAl-loaded fuels and mixed hybrid compositions were reported in References [17,84] where high-speed and high-magnification visualizations were performed. The captured high-speed images showed localized particle aggregation phenomena affecting the investigated fuels that were tested under low $G_{o x}\left(10 \mathrm{~kg} /\left(\mathrm{m}^{2} \cdot \mathrm{s}\right)\right)$ with the use of $60 \mathrm{wt} . \% \mathrm{O}_{2}+40 \mathrm{wt} . \% \mathrm{~N}_{2}$ as oxidizer.

\section{Investigated Materials}

\subsection{Aluminum and Al-Based Powders}

An overview of the tested $\mathrm{Al}$ powders and Al-based composites is reported in Table 1. A conventional micron-sized $\mathrm{Al}(\mu \mathrm{Al} 15)$ was taken as the reference for relative grading of the additives. Two different families of Al-based energetic materials are presented: (a) nAl passivated by different methods and without/with coating, and (b) mechanically activated metal powders and fuel-rich composites based on both $\mu \mathrm{Al}$ and nAl. AP, VFHFP, and PTFE were included in nano- and/or micron-sized fuel-rich composites. Composite powders exploited the oxidizing species release from the oxidizer/fluoropolymer decomposition to trigger metal ignition and to provide (partial) metal oxidation in the fuel-rich conditions encountered close to the solid grain regressing surface. Table 2 provides the relative grading of $T_{f l}$ for $\mathrm{Al}$ composites (heat of formation for VFHFP and PTFE from References [85-87], respectively). The Al + AP combustion at $1.0 \mathrm{MPa}$ for oxidizer content $\geq 15 \mathrm{wt} \%$ yielded a $T_{f l}$ exceeding the melting point of $\mathrm{Al}_{2} \mathrm{O}_{3}(\sim 2300 \mathrm{~K})$. The VFHFP offered reduced calculated flame temperature with respect to PTFE due to the lower F content (66 wt.\% vs. 76 wt.\%) [57,87]. Focusing on the oxidizer densities, PTFE featured the highest value. This characteristic is of interest for the design of composites with high densities for enhanced $\dot{m}_{f}$. 
Table 1. Investigated powders: nomenclature, description, and production details (LE: low energy; HE: high energy; MA: mechanical activation; PTFE: polytetrafluoroethylene; ALEX: aluminum exploded).

\begin{tabular}{|c|c|c|c|}
\hline Powder Id. & $\begin{array}{l}\text { Powder Description } \\
\text { and Notes }\end{array}$ & $\begin{array}{l}\text { Particle Surface } \\
\text { Composition }\end{array}$ & $\begin{array}{l}\text { Producer and } \\
\text { References }\end{array}$ \\
\hline$\mu \mathrm{Al15}$ & $\begin{array}{l}\text { Air-passivated } \mu \mathrm{Al} \text { with } \\
\text { nominal size of } 15 \mu \mathrm{m}\end{array}$ & \multirow[b]{2}{*}{$\mathrm{Al}_{2} \mathrm{O}_{3}$} & \multirow[b]{2}{*}{ AMG Alpoco (UK) [25] } \\
\hline$\mu \mathrm{Al} 7.5$ & $\begin{array}{l}\text { Air-passivated } \mu \mathrm{Al} \text { with } \\
\text { nominal size of } 7.5 \mu \mathrm{m}\end{array}$ & & \\
\hline ALEX-100 & $\begin{array}{l}\text { Air-passivated nAl with } \\
\text { nominal size of } 100 \mathrm{~nm}\end{array}$ & \multirow[b]{2}{*}{$\mathrm{Al}_{2} \mathrm{O}_{3}$} & \multirow{6}{*}{$\begin{array}{c}\text { Advanced Powder } \\
\text { Technology LLC (Tomsk, } \\
\text { Russia) }[40,54]\end{array}$} \\
\hline ALEX-50 & $\begin{array}{l}\text { Air-passivated } \mathrm{nAl} \text { with } \\
\text { nominal size of } 50 \mathrm{~nm}\end{array}$ & & \\
\hline ALEX-100B & $\begin{array}{l}\text { ALEX-100 coated with } \\
0.2 \text { wt.\% pyrocatechol } \\
\left(\mathrm{C}_{6} \mathrm{H}_{6} \mathrm{O}_{2}\right)\end{array}$ & $\mathrm{C}_{6} \mathrm{H}_{6} \mathrm{O}_{2}$ & \\
\hline L-ALEX-100 & $\begin{array}{l}\text { Stearic acid coated } \\
\text { ALEX-100 }\end{array}$ & $\mathrm{C}_{17} \mathrm{H}_{35} \mathrm{COOH}$ & \\
\hline VF-ALEX-100F & $\begin{array}{l}\text { Fluorotelomer } \\
\text { alcohol-derived } \\
\text { ester-coated ALEX-100 } \\
\text { (coating solvent removed } \\
\text { by filtration) }\end{array}$ & \multirow{2}{*}{$\begin{array}{l}\text { Vinylidene } \\
\text { fluoride-hexafluoropropylen } \\
\text { (70:30) copolymer } \\
\text { (VFHFP) + ester }\end{array}$} & \\
\hline VF-ALEX-50E & $\begin{array}{l}\text { Fluorotelomer } \\
\text { alcohol-derived } \\
\text { ester-coated ALEX-50 } \\
\text { (coating solvent removed } \\
\text { by evaporation) }\end{array}$ & & \\
\hline AP15-ALEX-100B & $\begin{array}{l}\text { AP }(15 \text { wt. } \%)+ \\
\text { ALEX-100B }(85 \text { wt. } \%) \text {, } \\
\text { ALEX-100B clustered } \\
\text { and coated by AP } \\
\text { (solvent removal by } \\
\text { evaporation) }\end{array}$ & $\begin{array}{c}\mathrm{Al}_{2} \mathrm{O}_{3}+\mathrm{C}_{6} \mathrm{H}_{6} \mathrm{O}_{2}+ \\
\mathrm{NH}_{4} \mathrm{ClO}_{4}\end{array}$ & SPLab \\
\hline LE- $\mu$ Al15 & Low-energy MA, $\mu$ Al15 & \multirow{6}{*}{$\mathrm{Al}_{2} \mathrm{O}_{3}+\left(\mathrm{C}_{2} \mathrm{~F}_{4}\right)_{\mathrm{n}}$} & \multirow{6}{*}{$\begin{array}{c}\text { SPLab } \\
\text { (MA procedure) }[23,24]\end{array}$} \\
\hline LE- $\mu$ Al15-T10 & $\begin{array}{l}\text { Low-energy MA, } \mu \text { A15 } \\
\text { (90wt. } \%)+ \text { PTFE (10 } \\
\text { wt. } \%)\end{array}$ & & \\
\hline LE- $\mu$ Al15-T30 & $\begin{array}{l}\text { Low-energy MA, } \mu \text { Al15 } \\
\text { (70 wt. } \%)+ \text { PTFE ( } 30 \\
\text { wt. } \%)\end{array}$ & & \\
\hline LE- $\mu \mathrm{Al} 7.5-\mathrm{T} 30$ & $\begin{array}{l}\text { Low-energy MA, } \mu \mathrm{Al} 7.5 \\
(70 \text { wt. } \%)+\text { PTFE ( } 30 \\
\text { wt. } \%)\end{array}$ & & \\
\hline HE- $\mu$ Al15-T45 & $\begin{array}{l}\text { High-energy MA, } \mu \text { Al15 } \\
(55 \text { wt. } \% \text { ) + PTFE ( } 45 \\
\text { wt. } \%)\end{array}$ & & \\
\hline HE-ALEX-50-T45 & $\begin{array}{l}\text { High-energy MA, } \\
\text { ALEX-50 (55 wt.\%) + } \\
\text { PTFE (45 wt.\%) }\end{array}$ & & \\
\hline
\end{tabular}

Table 2. $T_{f l}$ for $\mathrm{Al}$ reaction with different fluoropolymers and ammonium perchlorate (AP) as a function of the oxidizer mass fraction in the composite (NASA Chemical Equilibrium with Applications (CEA) software, $p_{c}=1.0 \mathrm{MPa}$ ).

\begin{tabular}{cccccc}
\hline Oxidizer & Density, $\mathbf{k g} / \mathbf{m}^{\mathbf{3}}$ & $\mathbf{1 0} \mathbf{w t} . \%$ & $\mathbf{1 5} \mathbf{w t .} \%$ & $\mathbf{3 0} \mathbf{w t .} \%$ & $\mathbf{4 5} \mathbf{w t} . \%$ \\
\hline AP & 1950 & $1754 \mathrm{~K}$ & $2327 \mathrm{~K}$ & $2656 \mathrm{~K}$ & $3524 \mathrm{~K}$ \\
VFHFP & 1800 & $933.6 \mathrm{~K}$ & $1304 \mathrm{~K}$ & $1752 \mathrm{~K}$ & $1975 \mathrm{~K}$ \\
PTFE & 2200 & $1013 \mathrm{~K}$ & $1440 \mathrm{~K}$ & $1853 \mathrm{~K}$ & $2095 \mathrm{~K}$ \\
\hline
\end{tabular}


Air-passivated ALEX powders with nominal sizes of $100 \mathrm{~nm}$ and $50 \mathrm{~nm}$ were considered in the study. The nominal coating mass fraction was $10 \%$ for all the coated powders but AP15-ALEX-100B (see Table 1). Production details for the starting $\mathrm{nAl}$ powders and for the variants coated by stearic acid and fluoropolymer are reported elsewhere [40]. The AP15-ALEX-100B was produced by dispersing $\mathrm{nAl}$ in an acetone solution of AP. The ingredients were mixed during ultrasound irradiation of the bath. Solvent was gradually removed by evaporation at $318 \mathrm{~K}$. Powders were dried for $72 \mathrm{~h}$ in an oven at $333 \mathrm{~K}$ before grinding. Activated powders were produced by low-energy (LE) and high-energy (HE) mechanical activation (MA) processes, both developed at SPLab. Details on the former process are provided in dedicated works [24,48], while the latter procedure is undergoing patenting. Mechanical activation enables the coupling of different materials, removing some of the limitations of other techniques, such as the chemical compatibility required for efficient coating.

\subsection{Tested Formulations}

Tested fuel formulations are reported in Table 3. Relative ballistic grading was performed considering a non-metallized HTPB baseline (for formulation details, see Reference [63]). The investigated compositions were all produced at lab-scale level. Formulations F2 to F7 featured a 10 wt.\% load of $\mathrm{Al}$ powders, and they were produced by a special manufacturing procedure aiming at inter-particle cold-cohesion mitigation (i.e., ultrasound irradiation [41-43]). The solid fuel compositions from F8 to F14 featured the same metal molar content as F2, and they were prepared without special procedures for the additive dispersion in the matrix. The effects of the addition to the HTPB binder of PTFE and of (separated) Al and PTFE powders were investigated using F15 and F16. High-energy mechanical milling was used to produce the additives tested in the fuel formulations with the highest metal mass fractions (F17 to F19), which were designed to explore the possibility of significantly augmented $\dot{m}_{f}$ using high loads of Al-based composites with PTFE. Fuels F16 to F18 were formulated granting the same HTPB to PTFE ratio as the F15. Actual fuel densities were evaluated by a high-precision balance measuring sample dry weight and buoyancy. For all the tested fuel formulations, the difference between the actual density and the theoretical maximum density (TMD) was lower than $3 \%$.

Table 3. Tested fuel formulations: nomenclature, composition, and details. ID: identifier; HTPB: hydroxyl-terminated polybutadiene; TMD: theoretical maximum density.

\begin{tabular}{|c|c|c|c|c|}
\hline Solid Fuel ID & HTPB, wt.\% & Energetic Additive ID, wt.\% & TMD, kg/m ${ }^{3}$ & Notes \\
\hline F1 & 100 & - & 920 & Relative grading baseline \\
\hline $\mathrm{F} 2$ & 90.0 & $\mu \mathrm{Al} 15,10.0$ & 980 & - \\
\hline F3 & 90.0 & $\mu \mathrm{Al} 17.5,10.0$ & 980 & - \\
\hline F4 & 88.0 & ALEX-100, 10.0 & 992 & \multirow{4}{*}{$\begin{array}{l}\text { Formulation includes } 2 \mathrm{wt} . \% C B \\
\text { additive dispersion by ultrasound } \\
\text { irradiation during mixing }\end{array}$} \\
\hline F5 & 88.0 & L-ALEX-100, 10.0 & 992 & \\
\hline F6 & 88.0 & VF-ALEX-100F, 10.0 & 992 & \\
\hline F7 & 88.0 & VF-ALEX-50E, 10.0 & 992 & \\
\hline F8 & 88.7 & ALEX-100B, 11.3 & 906 & \multirow{7}{*}{$\begin{array}{l}\text { Formulation } \mathrm{Al} \text { content is equimolar } \\
\text { to } \mathrm{F} 2 \text {, no additive dispersion by } \\
\text { ultrasound irradiation during mixing }\end{array}$} \\
\hline F9 & 87.4 & AP $1.9 \%+$ ALEX-100B $10.7 \%$ a & 999 & \\
\hline F10 & 87.4 & AP15-ALEX-100B, 12.6 & 999 & \\
\hline F11 & 89.9 & LE- $\mu \mathrm{A} 115,10.1$ & 980 & \\
\hline F12 & 89.0 & LE- $\mu$ Al15-T10, 11.0 & 986 & \\
\hline $\mathrm{F} 13$ & 85.5 & LE- $\mu$ Al15-T30, 14.5 & 986 & \\
\hline F14 & 84.9 & LE- $\mu \mathrm{A} 17.5-\mathrm{T} 30,15.1$ & 1006 & \\
\hline F15 & 65.0 & PTFE, 35.0 & 1150 & Evaluation of HTPB + PTFE ballistics \\
\hline \multirow[b]{2}{*}{ F16 } & \multirow[b]{2}{*}{45.5} & PTFE, 24.5 & & \multirow{2}{*}{$\begin{array}{c}\text { Evaluation of the effects of HTPB + } \\
\text { PTFE on nAl combustion (no } \\
\text { mechanical activation) }\end{array}$} \\
\hline & & ALEX-50, 30.0 & 1389 & \\
\hline F17 & 45.5 & HE- $\mu$ Al15-T45, 54.5 & 1389 & \multirow{3}{*}{$\begin{array}{l}\text { HE activation effects evaluation with } \\
\text { respect to LE, increased PTFE content }\end{array}$} \\
\hline F18 & 45.5 & HE-ALEX-50-T45, 54.5 & 1389 & \\
\hline F19 & 30.0 & HE-ALEX-50-T45, 70.0 & 1630 & \\
\hline
\end{tabular}




\section{Experimental Methods}

\subsection{Aluminum and Aluminum-Based Additives Pre-Burning Characterization}

The pre-burning characterization of the metal additives included particle size distribution (PSD) by laser diffraction (Malvern Mastersizer 2000 with dry/liquid dispersion unit for micron- and nano-sized particles, respectively), SSA determination by $\mathrm{N}_{2}$ adsorption/desorption, scanning electron microscopy (SEM), transmission electron microscopy (TEM), and transmission energy dispersion (TED) imaging. The $C_{A l}$ of the powders was determined by $\mathrm{Al}+\mathrm{H}_{2} \mathrm{O}$ reaction in a basic solution (10 wt. $\% \mathrm{NaOH}$ ) The reactivity of the powders was investigated by slow heating rate thermogravimetry (TG). The TG relative grading of the additives was performed in air, at $10 \mathrm{~K} / \mathrm{min}$, considering the reactivity parameters identified by Ilyin et al. [34]. The reactivity data evaluated on the TG trace are illustrated in Figure 1. The $\mathrm{Al} \rightarrow \mathrm{Al}_{2} \mathrm{O}_{3}$ conversion factor was evaluated as follows:

$$
\alpha(T)=\frac{\Delta m(T)}{0.89 \cdot C_{A l}}
$$

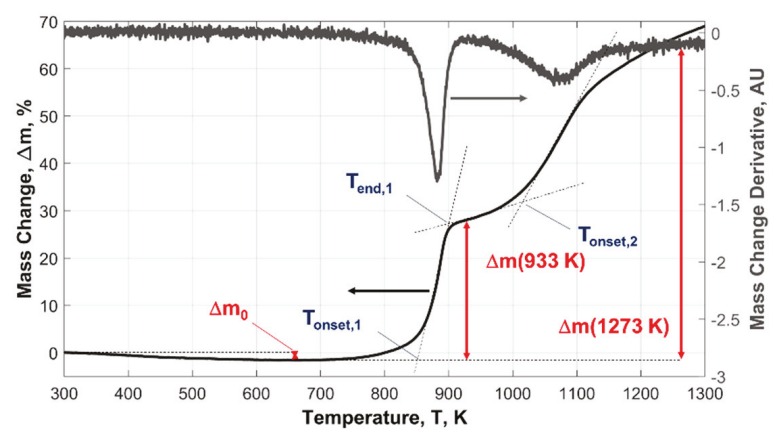

Figure 1. Reactivity parameters for thermogravimetry (TG) analysis [34]. The determination of onset and end points was performed based on the differential TG (DTG) curve. Percent mass change was evaluated with respect to the initial mass of the specimen.

\subsection{Burning Behavior Investigation}

\subsubsection{Lab-Scale Two-Dimensional (2D) Radial Micro-Burner}

The SPLab research team designed and developed a 2D radial burner for the relative ballistic grading of solid fuel formulations at the lab-scale level. Cylindrical-shaped fuel grains with a length of $30 \mathrm{~mm}$, outer case diameter of $20 \mathrm{~mm}$, and central port diameter $\left(D_{0}\right)$ of $4 \mathrm{~mm}$ are typically tested. The SPLab facility features peculiar characteristics such as the visualization of the burning specimen head-end, and a quasi-steady $p_{c}$ during the combustion [63]. In the 2D radial burner, the $p_{c}(t)$ is monitored by a piezo-resistive pressure transducer and controlled by a pressure regulator commanding exhaust electro-valves. These servo-actuators are opened/closed depending on the sensed pressure difference between actual and threshold values. The latter was selected for the test (and it could be easily changed between different runs, if needed). The $\dot{m}_{o x}(t)$ was monitored and controlled by a digital flowmeter. The $\dot{m}_{o x}(t)$ and the $p_{c}(t)$ could be controlled independently. Oxidizer injection was performed by standard (non-swirled) flow. Tested strand ignition was achieved by a primer charge whose combustion was initiated by a laser beam impinging on it. A scheme of the experimental set-up is shown in Figure 2, while its peculiarities were discussed elsewhere [63]. In the current study, gaseous oxygen (GOX) was taken as the oxidizer, and combustion tests were performed with $\dot{m}_{o x}$ of $5 \mathrm{~g} / \mathrm{s}$ and 6 $\mathrm{g} / \mathrm{s}$ (the two operating conditions were identified as A and B, respectively), with $p_{c}=1.0 \mathrm{MPa}$. 


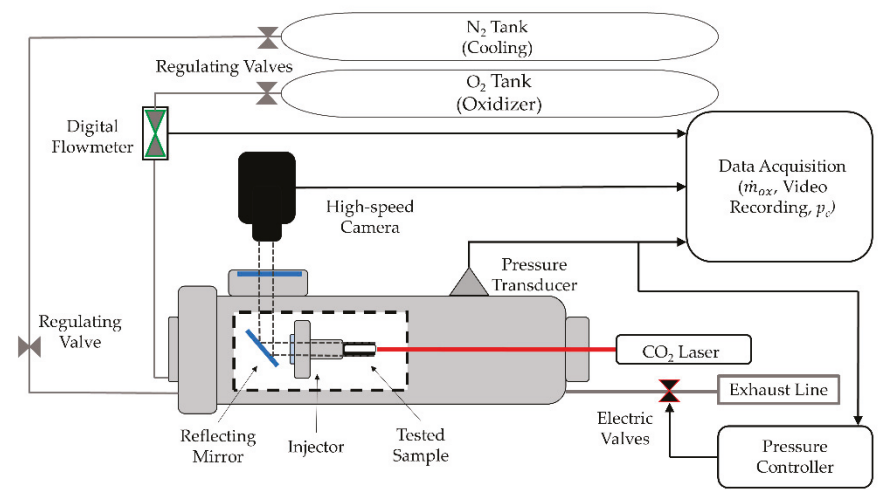

Figure 2. Lab-scale two-dimensional (2D) radial burner scheme.

\subsubsection{Time-Resolved Regression Rate}

An optical, time-resolved technique for $r_{f}$ measurement was developed and validated at SPLab [16, 53,63]. The time-resolved $r_{f}$ was evaluated by central port diameter sampling during the combustion (see Figure 3). Regression surface tracking started from the frame when the head-end central port diameter became visible (after primer charge ignition). Data sampling extended until the end of the combustion (depending on visualization quality). Diameter sampling was performed with the maximum possible frequency $(5-20 \mathrm{~Hz}$ under the investigated conditions, with higher frequencies in the earlier phases of the burning process). During the combustion, central port diameter was sampled along different radial directions (depending on combustion uniformity). The local diameters were averaged to provide $\bar{D}_{i}$. This diameter was considered uniform for the whole specimen length. The $\bar{D}_{i}\left(t_{i}\right)$ was a discrete information in time, which was successively fitted to achieve a continuous $\bar{D}(t)$, as shown in Equation (2).

$$
\bar{D}(t)-D_{0}=a_{D} \cdot\left(t-t_{0}\right)^{n_{D}}, t \geq t_{i g n}>t_{0}=0 .
$$

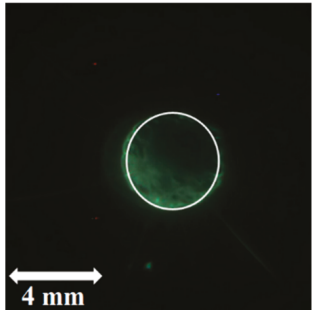

(a) $t_{i g n}$

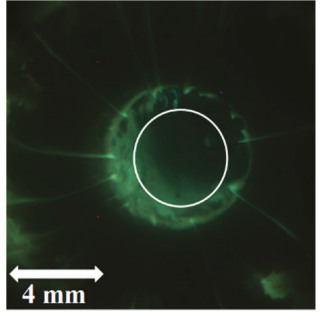

(b) $t_{i g n}+1 \mathrm{~s}$

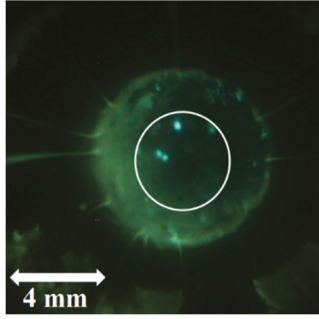

(c) $t_{i g n}+2 \mathrm{~s}$

Figure 3. Typical head-end visualization of a combustion run in the $2 \mathrm{D}$ radial burner (F3-GOX, $p_{c}=1.0$ $\mathrm{MPa})$ : (a) sample ignition, $(\mathbf{b}, \mathbf{c})$ central port diameter enlargement in time due to solid fuel regression (the initial port diameter $\left(D_{0}\right)$ is highlighted by the white circle).

In time-resolved techniques, the function describing the diameter evolution in time was valid starting from $t_{i g n}$. The latter parameter was ad hoc defined as the one enabling the maximization of the data fitting $\left(R^{2}\right)$ of the $\bar{D}(t)-D_{0}$ based on $a_{D}$ and $n_{D}$ (Equation (2)). In the implemented technique, the $t_{i g n}$ values were in agreement with convective ignition time estimated by open literature models, as discussed in References [53,63]. The ballistic data of a fuel formulation tested under the selected conditions were determined starting from Equation (2) [63]. The time derivative of the $\bar{D}(t)-D_{0}$ 
provided the $r_{f}(t)$, while the $G_{0 x}(t)$ could be determined from the diameter history once the operating condition (and, in particular, $\left.\dot{m}_{o x}\right)$ was defined [63]. The $r_{f}\left(G_{o x}\right)$ history in time was approximated by a power law as follows:

$$
r_{f}\left(G_{o x}\right)=a_{r} G_{o x}^{n_{r}}(t), t \geq t_{i g n}>t_{0} .
$$

The $r_{f}(t)$ and the $\bar{D}(t)$ were used to evaluate the mass burning rate.

$$
\dot{m}_{f}(t)=\rho_{f}\left[\pi \bar{D}(t) L_{f}\right] r_{f}(t), t \geq t_{i g n}>t_{0}
$$

Time-resolved data could be used to determine the time-averaged ballistics of the investigated formulation [63]. In particular, the time-averaged values of the $r_{f}$ and of the $G_{0 x}$ were evaluated as follows:

$$
\begin{gathered}
\left\langle r_{f}(t)\right\rangle=\frac{1}{t-t_{\text {ign }}} \int_{t_{\text {ign }}}^{t} r_{f}(t) d t, t_{\text {end }} \geq t \geq t_{\text {ign }}>t_{0} . \\
\left\langle G_{o x}(t)\right\rangle=\frac{1}{t-t_{\text {ign }}} \int_{t_{\text {ign }}}^{t} G_{o x}(t) d t, t_{\text {end }} \geq t \geq t_{\text {ign }}>t_{0} .
\end{gathered}
$$

As extensively discussed in References [16,63], Equations (5) and (6) can be used to compare the overall time-averaged data derived from Equation (2) to thickness over time (TOT) results of a test. The typical experimental output obtained by this data reduction technique is shown in Figure 4, where time-resolved instantaneous and time-averaged data for a HTPB-GOX run are reported and compared with the corresponding TOT-based $r_{f}$ and $G_{O x}$, which were defined as follows:

$$
\begin{aligned}
\left\langle r_{f}\right\rangle_{T O T}= & \frac{1}{2} \frac{\bar{D}\left(t_{\text {end }}\right)-\bar{D}\left(t_{\text {ign }}\right)}{t_{\text {end }}-t_{\text {ign }}}, t_{\text {end }}>t_{\text {ign }}>t_{0} . \\
\left\langle G_{\text {Ox }}\right\rangle_{T O T}= & \frac{4 \dot{m}_{\text {ox }}}{\pi\left[\frac{\bar{D}\left(t_{\text {end }}\right)+\bar{D}\left(t_{\text {ign }}\right)}{2}\right]^{2}}, t_{\text {end }}>t_{\text {ign }}>t_{0}
\end{aligned}
$$

Equations (5) and (6) and the TOT data typically show percent differences of less than 5\% (see References $[16,63]$ for details). Under the investigated conditions, the time-averaged and the instantaneous data featured minor differences at a given oxidizer mass flux (see Figure 4).

For a given experimental condition $\left(D_{0}, \dot{m}_{0 x}\right.$, and $\left.p_{c}\right)$, single tests for the same fuel formulation yielded the definition of ensemble average curves, enabling the relative grading of the different compositions $[16,63]$. Ensemble average curves were completed by error bars considering the data dispersion of the performed combustion tests. Single-test diameter histories resulting from the application of Equation (2) were fitted together in the ensemble curve of the same kind. The resulting $\bar{D}(t)-D_{0}$ was then used to determine the relevant ballistic parameters. Thus, following the same logical steps performed on a single test, $r_{f}\left(G_{o x}\right)$ and $\dot{m}_{f}\left(G_{o x}\right)$ could be evaluated. The uncertainties on the ensemble average curve were evaluated by considering confidence intervals with $95 \%$ accuracy. A minimum of three tests were considered for error bar definition. 


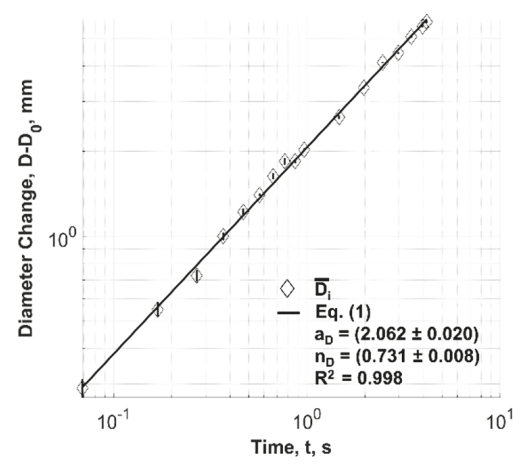

(a)

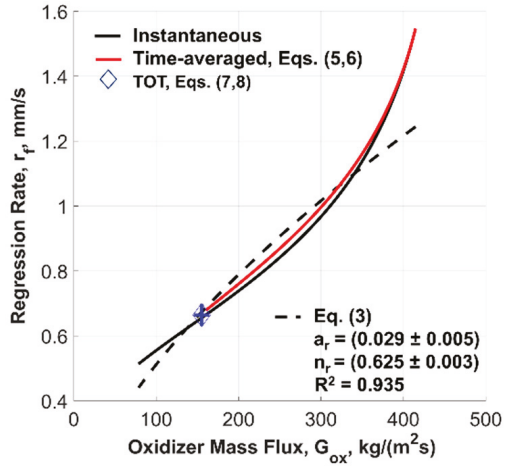

(b)

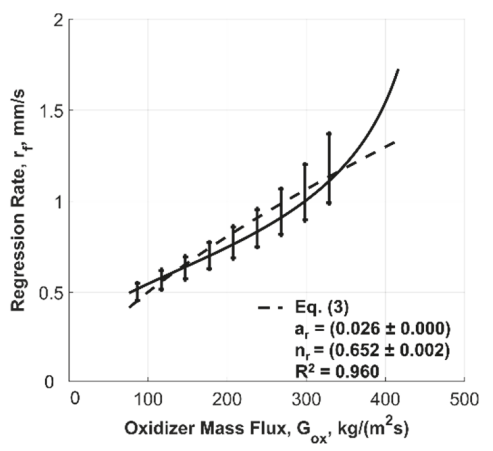

(c)

Figure 4. Time-resolved regression rate determination (HTPB-GOX, $p_{c}=1.0 \mathrm{MPa}, \dot{m}_{o x}=6 \mathrm{~g} / \mathrm{s}$ ): (a) sampled diameter interpolation in time (Equation (1)), (b) instantaneous $r_{f}\left(G_{o x}\right)$ with its power law approximation (Equation (3)), time-averaged history (Equations (5) and (6)), and thickness over time (TOT) data, (c) ensemble average data with error bars.

\subsubsection{Lab-Scale Micro-Slab Motor}

Combustion surface visualizations were performed in a windowed micro-slab burner $[17,84]$. The implemented set-up featured the same general layout as the radial burner shown in Figure 2. Burning fuel strand images were captured by side-window. Recorded images showed the solid fuel slab and the flame zone. A typical combustion frame is shown in Figure 5. The burning strands had a length of $15 \mathrm{~mm}$ and a square cross section of $3 \times 3 \mathrm{~mm}$. The ignition of the samples was achieved by a non-aluminized propellant primer charge (in turn ignited by a hot wire). The facility enabled the exploration of different $p_{c}$ by changing the throat area of the implemented nozzle. The burning surface visualization runs were intended to provide qualitative insight into the phenomena involved in the combustion of metallized fuel formulations, while no detailed quantitative information could be captured by these tests. The visualizations were performed in GOX, with $p_{c}=0.4 \mathrm{MPa}$, and 145 $\mathrm{kg} /\left(\mathrm{m}^{2} \cdot \mathrm{s}\right) \leq G_{0 x} \leq 160 \mathrm{~kg} /\left(\mathrm{m}^{2} \cdot \mathrm{s}\right)$. The frame rate of the captured videos was in the range 5000-10,000 frames per second (fps), depending on the investigated fuel $r_{f}$ and observable phenomena. 


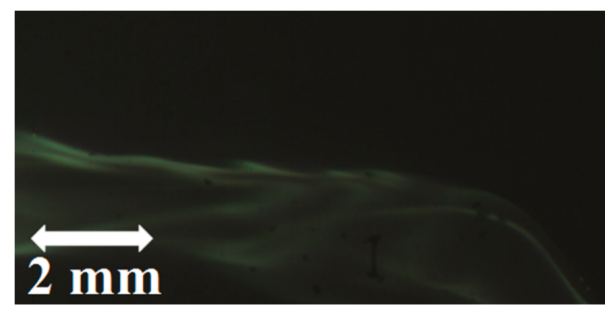

Figure 5. Combustion visualization with slab configuration (F1-GOX, $p_{c}=0.4 \mathrm{MPa}$ ). The oxidizer flows from right to left. Note the diffusion flame over the solid fuel grain.

\section{Experimental Results and Discussion}

\subsection{Additives Pre-Burning Characterization}

The PSD, SSA, and $C_{A l}$ data for the tested additives are reported in Table 4. Selected SEM and TED images of the additives are shown in Figure 6. Slow heating rate reactivity parameters from the TG tests are reported in the Table 5. In this work, the implemented LE and HE activation procedures aimed at reducing the diffusion distance between the PTFE and the Al. When considering metal-loaded fuel formulations for hybrid rocket applications, the pursued effects of additives are increased $r_{f}$ and $\dot{m}_{f}$. The achievement of enhanced ballistic performance requires materials with fast reactivity, while additive metal content exerts limited effects on performance (particularly when $\mathrm{O}_{2}$ is used as oxidizer) [25].

Table 4. Particle size distribution (PSD), specific surface area (SSA), and $C_{A l}$ data for the tested additives, $\mathrm{AP}$, and PTFE.

\begin{tabular}{|c|c|c|c|c|c|c|}
\hline Powder ID & $D_{43}, \mu \mathrm{m}$ & $D_{(0.1)}, \mu \mathrm{m}$ & $D_{(0.5), \mu \mathrm{m}}$ & $D_{(0.9)}, \mu \mathrm{m}$ & $\mathrm{SSA}, \mathrm{m}^{2} / \mathrm{g}$ & $C_{A l}$, wt. $\%$ \\
\hline$\mu \mathrm{Al} 15$ & 19.8 & 10.0 & 17.9 & 32.3 & $<0.1$ & $99.5 \pm 0.4$ \\
\hline$\mu \mathrm{Al} 7.5$ & 6.6 & 2.99 & 6.08 & 11.0 & $<0.1$ & $95.3 \pm 0.2$ \\
\hline ALEX-100 & 0.138 & 0.105 & 0.135 & 0.176 & $11.8 \pm 0.4$ & $89.0 \pm 5.0$ \\
\hline ALEX-50 & - & - & - & - & $15.5 \pm 0.1$ & $86.3 \pm 4.1$ \\
\hline ALEX-100B & 0.141 & 0.104 & 0.138 & 0.182 & $11.3 \pm 0.1$ & $88.0 \pm 1.5$ \\
\hline L-ALEX-100 & - & - & - & - & $9.1 \pm 0.3$ & $70.3 \pm 4.3^{a}$ \\
\hline VF-ALEX-100F & - & - & - & - & $6.9 \pm 0.2$ & $78.3 \pm 4.4$ \\
\hline VF-ALEX-50E & - & - & - & - & $10.9 \pm 0.3$ & $78.4 \pm 4.4$ \\
\hline AP15-ALEX-100B & 6.73 & 0.42 & 1.66 & 16.0 & - & $76.0 \pm 1.3$ \\
\hline LE- $\mu$ Al15 & 123 & 15.9 & 90.8 & 284 & $<1.0$ & $98.2 \pm 0.1$ \\
\hline LE- $\mu$ Al15-T10 & 28.4 & 12.0 & 25.1 & 49.5 & $<1.0$ & $90.5 \pm 0.1$ \\
\hline LE- $\mu$ Al15-T30 & 31.2 & 5.46 & 21.7 & 59.4 & $<1.0$ & $68.6 \pm 0.6$ \\
\hline LE- $\mu \mathrm{Al} 7.5-\mathrm{T} 30$ & 13.5 & 2.67 & 8.13 & 29.2 & $<1.0$ & $65.8 \pm 0.4$ \\
\hline HE- $\mu$ Al15-T45 & 52.2 & 4.05 & 22.6 & 141 & $1.5 \pm 0.1$ & $53.7 \pm 1.2$ \\
\hline HE-ALEX-50-T45 & 85.2 & 3.41 & 41.7 & 237 & $5.9 \pm 0.1$ & $47.3 \pm 1.7$ \\
\hline $\mathrm{AP}$ & 10.2 & 0.68 & 5.74 & 26.4 & - & - \\
\hline PTFE & 7.24 & 0.36 & 0.88 & 8.28 & - & - \\
\hline
\end{tabular}

${ }^{\mathrm{a}}$ Active metal content underestimation due to side-effects in the $\mathrm{Al}+\mathrm{H}_{2} \mathrm{O}$ reaction [40]. 


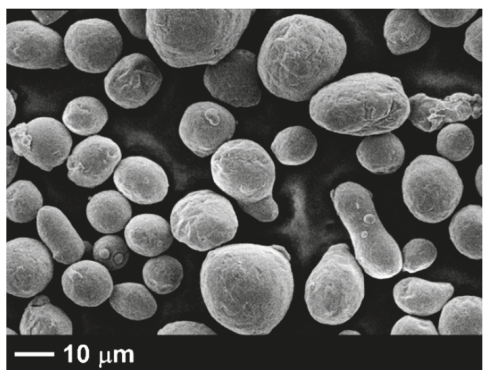

(a)

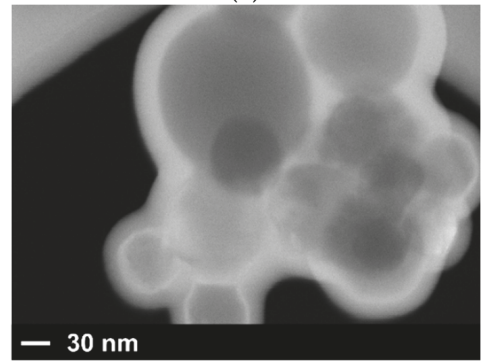

(c)

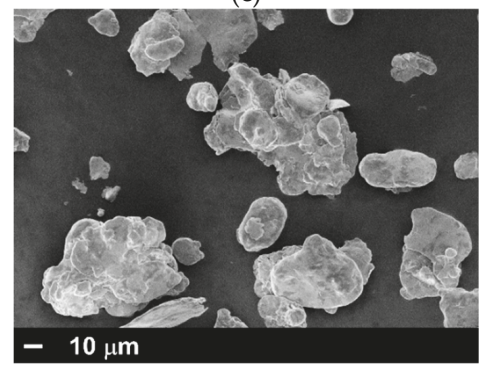

(e)

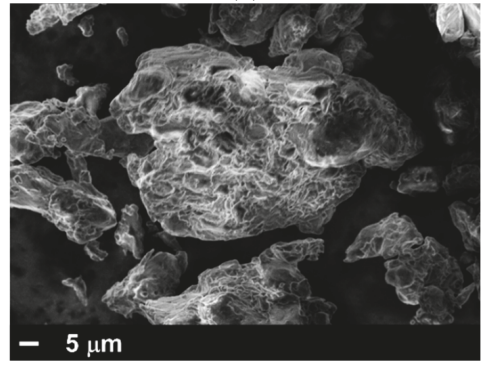

(g)

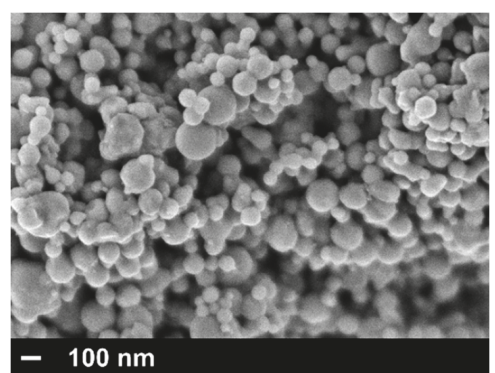

(b)

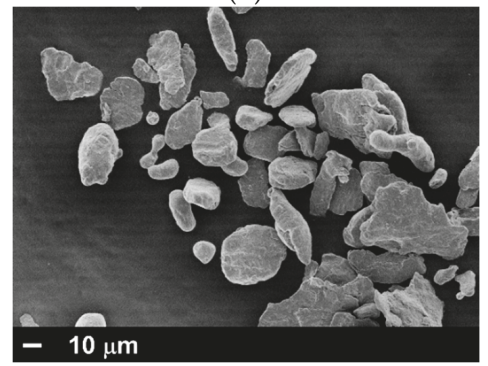

(d)

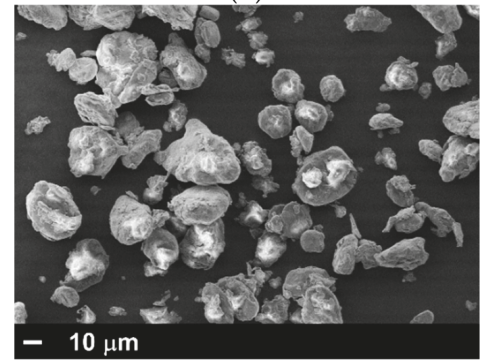

(f)

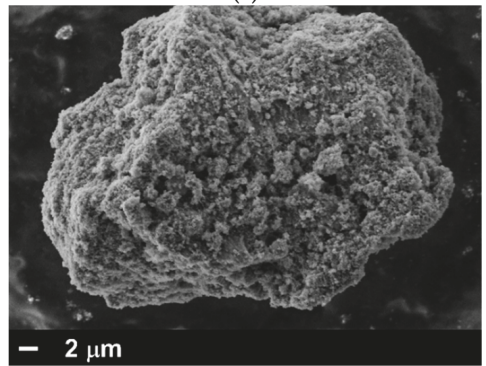

(h)

Figure 6. Electron microscopy images of the investigated powders: (a) SEM of $\mu$ Al15, (b) SEM of ALEX-100, (c) transmission energy dispersion (TED) of VF-ALEX-100F, (d) SEM of LE- $\mu$ Al15, (e) SEM of LE- $\mu$ Al15-T10, (f) SEM of LE- $\mu$ Al15-T30, (g) SEM of HE- $\mu$ Al15-T45, and (h) SEM of HE-ALEX-50-T45.

The $\mu$ Al15 featured spherical/spheroid particles, with (apparent) smooth texture (see Figure 6a). The PSD data reported in Table 4 show a monomodal distribution of the measured diameters. The powder showed a limited reactivity under slow heating rates $(\alpha(933 \mathrm{~K})=1.1 \%, \alpha(1273 \mathrm{~K})=8 \%)$ due to the low SSA. The $\mu$ Al7.5 featured a small volumetric fraction of sub-micrometric particles $(\sim 2 \%)$, as 
testified by the low $D_{(0.1)}$ reported in Table 4 . The TG reactivity of the powder was similar to the one of the coarser counterpart (see Table 5). In spite of the SSA difference (see Table 4), ALEX-100 and ALEX-50 exhibited similar $T_{\text {on,1 }}$. ALEX-50 showed the highest $\alpha(933 \mathrm{~K})$ and $\alpha(1273 \mathrm{~K})$ in the dataset. The pyrocatechol-coated powder showed no marked SSA and reactivity differences with respect to the ALEX-100 (see Tables 4 and 5). This was due to the reduced coating mass fraction (0.2 wt.\%), limiting coating-related effects such as particle clustering. Other coated powders featured higher SSA reduction (i.e., particle size increase) due to coating gluing of originally separated particles and because of cluster coverage by coating deposition. The AP15-ALEX-100B showed micron-sized particles that were clusters of the original ALEX-100B glued and (at least partially) covered by AP. Fluoropolymer coating reduced the reactivity of the powders at slow heating rates, yielding increased $T_{\text {on, } 1}$ and lowered $\alpha(1273 \mathrm{~K})$. This was probably induced by carbonaceous residuals from coating degradation that partially shielded the particles from oxidation. The effect was lowered for VF-ALEX-50E, which was prepared from a powder featuring higher reactivity than the 100-nm counterpart. In spite of the composition differences between the hydrocarbon coating and the AP composite, AP15-ALEX-100B and the L-ALEX-100 showed nearly the same reactivity as the original powders, due to the degradation behavior of their surface layers featuring vaporization/sublimation for temperatures $<670 \mathrm{~K}$.

Table 5. Powder reactivity: slow heating rate thermogravimetric (TG) data (air, $10 \mathrm{~K} / \mathrm{min}, 0.1 \mathrm{MPa}$ ).

\begin{tabular}{|c|c|c|c|c|c|}
\hline Powder ID & $\Delta m_{0}$, wt. $\%$ & $T_{o n, 1}, \mathrm{~K}$ & $\alpha(933 \mathrm{~K}), \%$ & $T_{o n, 2}, \mathrm{~K}$ & $\alpha(1273 \mathrm{~K}), \%$ \\
\hline$\mu \mathrm{Al15}$ & -0.1 & 845 & 1.1 & 1173 & 8.0 \\
\hline$\mu \mathrm{Al} 7.5$ & -0.2 & 843 & 2.2 & 1203 & 13.7 \\
\hline ALEX-100 & -1.0 & 850 & 40.4 & 983.2 & 83.3 \\
\hline ALEX-50 & -1.0 & 850 & 52.1 & 1000 & 90.9 \\
\hline ALEX-100B & -1.3 & - & 36.3 & - & $79.3^{a}$ \\
\hline L-ALEX-100 & -3.1 & 858 & $45.1^{\mathrm{b}}$ & 985.0 & $103.5^{\mathrm{b}}$ \\
\hline VF-ALEX-100F & -5.8 & 888 & 33.9 & 1088 & 76.8 \\
\hline VF-ALEX-50E & -5.1 & 883 & 39.4 & 1091 & 88.5 \\
\hline AP15-ALEX-100B & -12.3 & 858 & 39.8 & 993.0 & 84.0 \\
\hline LE- $\mu$ Al15 & -0.1 & 831 & 3.9 & 1198 & 14.5 \\
\hline LE- $\mu$ Al15-T10 & -7.1 & 899 & 2.5 & 1216 & 18.1 \\
\hline LE- $\mu \mathrm{Al} 15-\mathrm{T} 30$ & -24.1 & 902 & 0.8 & 1175 & 9.8 \\
\hline LE- $\mu$ Al7.5-T30 & -26.7 & 902 & 1.7 & 1174 & 41.0 \\
\hline HE- $\mu$ Al15-T45 & -36.5 & 1148 & 0.0 & - & 27.4 \\
\hline HE-ALEX-50-T45 & -34.2 & 893 & 43.0 & 988 & $61.0^{\mathrm{a}}$ \\
\hline
\end{tabular}

${ }^{\mathrm{a}}$ Limiting temperature of $1223 \mathrm{~K} .{ }^{\mathrm{b}}$ Active metal content underestimation due to side-effects in the $\mathrm{Al}+\mathrm{H}_{2} \mathrm{O}$ reaction $[40]$.

The non-isothermal oxidation of LE- $\mu$ Al15 showed an anticipated $T_{\text {on,1 }}$ and higher $\alpha$ (933 K) and $\alpha(1273 \mathrm{~K})$ than the original micron-sized powder. Activated powders derived from $\mu$ Al15 showed irregular shape and texture (Figure 6e,f). PTFE friction and wearing properties were the likely explanation for the size reduction of these powders with respect to LE- $\mu$ Al15 (see Table 4) [88]. The PTFE-based composites showed $C_{A l}$ decrease with respect to $\mu$ Al15. The metal content reduction was $9 \% \pm 1 \%$ for LE- $\mu$ Al15-T10 and $31 \% \pm 1 \%$ for LE- $\mu$ Al15-T30 and LE- $\mu$ Al7.5-T30. The $\Delta m_{0}$ data of Table 5 show a general agreement with these values, thus confirming the quality of the produced powders. All the LE-activated powders featured SSA $<1 \mathrm{~m}^{2} / \mathrm{g}$. The SEM images of LE- $\mu$ Al15 showed flake particles with limited surface roughness, and the presence of some spheroid particles due to the low-energy process (Figure 6d). The laser diffraction measurement showed an increase in the particle size (see Table 4). The PSD data were evaluated considering spherical and smooth-texture particles (Malvern Mastersizer 2000 data reduction algorithm); thus, considering the flake morphology of mechanically activated particles (Figure $6 \mathrm{~d}-\mathrm{h}$ ) achieved results should be considered for the relative grading of milled particles only. LE- $\mu$ Al15 showed a reduced $C_{A l}$ with respect to $\mu$ Al15 due to the crack-healing of the alumina shell during the activation process. The activation volume was filled with air; thus, the $\mathrm{Al}_{2} \mathrm{O}_{3}$ shell ruptures induced by the mechanical stresses yielded $\mathrm{Al}$ core exposure and oxidation with consequent $C_{A l}$ reduction. The oxidation onset of the LE PTFE-containing composites 
occurred at higher temperatures with respect to $\mu \mathrm{Al15}$ and LE-Al15. Under the investigated conditions, LE- $\mu$ Al7.5-T30 was the LE-activated composite featuring the highest $\alpha$ (T). This result was probably due to the reduced particle size of the initial powder, in turn yielding a final product with reduced $D_{43}$ and $D_{0.5}$ with respect to other $\mu$ Al15-based composites. Both HE-activated composites featured increased SSA with respect to the LE-activated powders, as shown in Table 4. HE- $\mu$ Al15-T45 showed particles with rough texture and irregular shape (see Figure 6g). The HE-ALEX-50-T45 SSA reduction with respect to the original ALEX was due to the creation of nAl + PTFE granules with a complex structure but increased size (see Figure 6h). Data for $\mathrm{Al} \rightarrow \mathrm{Al}_{2} \mathrm{O}_{3}$ conversion of the two powders showed $\alpha$ (1273 $\mathrm{K})=27.5 \%$ for the $\mu$ Al15-based composite, and $\alpha(1223 \mathrm{~K})=61.0 \%$ for HE-ALEX-50-T45.

\subsection{Burning Behavior}

\subsubsection{Time-Resolved Regression Rate}

Relative ballistic grading results are reported in Figures 7 and 8 and Table 6 . All the ballistic data were defined by ensemble average curves summarizing, at least four tests per formulation. All the fuels but F3, F8-F10, and F15-F19 were tested with $\dot{m}_{o x}$. $=5 \mathrm{~g} / \mathrm{s}$; thus, their performance was evaluated taking F1A as baseline. For the tests with $\dot{m}_{0 x}=6 \mathrm{~g} / \mathrm{s}, \mathrm{F} 1 \mathrm{~B}$ was considered as the reference for the relative grading. Data reported in Table 6 feature relatively low $R^{2}$ due to the typical trend captured by time-resolved approaches, as originally reported by Evans et al. [18] (see Figure 4b,c). The time-averaged data of Figure 8 were evaluated using Equations (5) and (6) at $\left\langle G_{o x}\right\rangle=(250 \pm 15)$ $\mathrm{kg} /\left(\mathrm{m}^{2} \cdot \mathrm{s}\right)$. The latter value was selected since it was common to all the tested formulations.

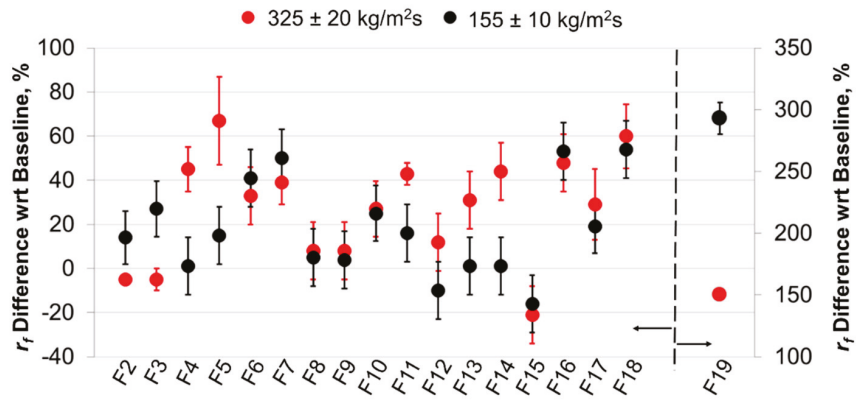

(a)

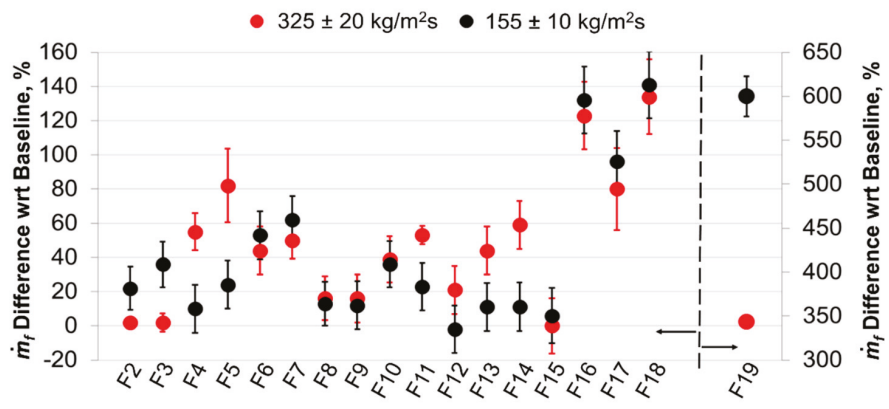

(b)

Figure 7. Relative ballistic grading of the tested fuel formulations (GOX, $p_{c}=1.0 \mathrm{MPa}$, time-resolved instantaneous data): (a) $r_{f}$ and (b) $\dot{m}_{f}$ differences with respect to the baseline. 


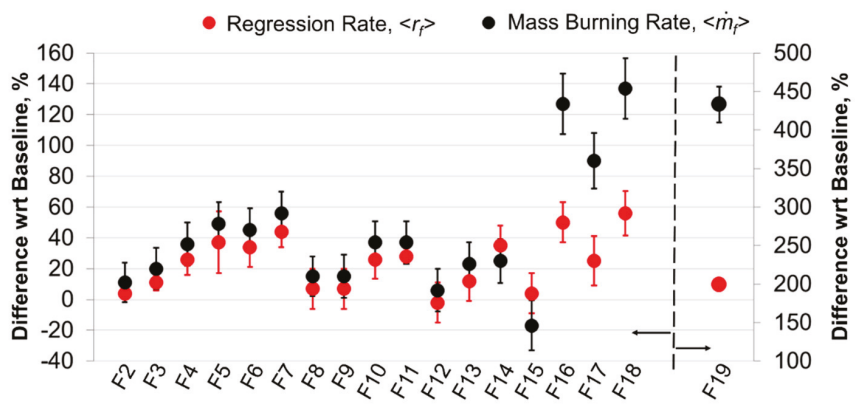

Figure 8. Relative ballistic grading of the tested formulations (GOX, $p_{c}=1.0 \mathrm{MPa}$, time-averaged data) for $\left\langle G_{0 x}\right\rangle=(250 \pm 15) \mathrm{kg} /\left(\mathrm{m}^{2} \cdot \mathrm{s}\right):\left\langle r_{f}\right\rangle$ and $\left\langle\dot{m}_{f}\right\rangle$ differences with respect to the baseline.

Table 6. Power law approximation of time-resolved $r_{f}\left(G_{0 x}\right)$ (Equation (3)) for the investigated formulations (F1A and F1B were used as baselines for the relative grading with $\dot{m}_{o x}$ of $5 \mathrm{~g} / \mathrm{s}$ and $6 \mathrm{~g} / \mathrm{s}$ respectively).

\begin{tabular}{cccc}
\hline Solid Fuel & \multicolumn{2}{c}{$r_{f}\left(G_{o x}\right)$ Power Law Approximation (Equation (3)) } \\
\hline ID & $a_{r}$ & $n_{r}$ & $R^{2}$ \\
\hline F1A & $0.018 \pm 0.001$ & $0.680 \pm 0.003$ & 0.88 \\
F1B & $0.026 \pm 0.000$ & $0.652 \pm 0.002$ & 0.96 \\
F2 & $0.059 \pm 0.001$ & $0.477 \pm 0.001$ & 0.95 \\
F3 & $0.064 \pm 0.001$ & $0.494 \pm 0.001$ & 0.90 \\
F4 & $0.005 \pm 0.001$ & $0.959 \pm 0.022$ & 0.85 \\
F5 & $0.007 \pm 0.001$ & $0.905 \pm 0.012$ & 0.96 \\
F6 & $0.052 \pm 0.003$ & $0.535 \pm 0.010$ & 0.91 \\
F7 & $0.041 \pm 0.003$ & $0.595 \pm 0.014$ & 0.85 \\
F8 & $0.017 \pm 0.000$ & $0.630 \pm 0.002$ & 0.95 \\
F9 & $0.016 \pm 0.000$ & $0.643 \pm 0.002$ & 0.92 \\
F10 & $0.019 \pm 0.000$ & $0.902 \pm 0.003$ & 0.91 \\
F11 & $0.006 \pm 0.000$ & $0.908 \pm 0.002$ & 0.93 \\
F12 & $0.006 \pm 0.000$ & $0.897 \pm 0.002$ & 0.95 \\
F13 & $0.006 \pm 0.000$ & $0.954 \pm 0.002$ & 0.95 \\
F14 & $0.006 \pm 0.000$ & $0.491 \pm 0.001$ & 0.96 \\
F15 & $0.065 \pm 0.000$ & $0.869 \pm 0.002$ & 0.94 \\
F16 & $0.011 \pm 0.000$ & $0.815 \pm 0.003$ & 0.94 \\
F17 & $0.012 \pm 0.002$ & $0.769 \pm 0.002$ & 0.88 \\
F18 & $0.021 \pm 0.002$ & $0.053 \pm 0.000$ & 0.95 \\
F19 & $1.969 \pm 0.002$ & & 0.88 \\
\hline
\end{tabular}

In spite of the (minor) differences in the operating conditions, solid fuel formulations loaded with $\mu \mathrm{Al}$ (F2 and F3) featured similar ballistic responses (see Figures 7 and 8). The $r_{f}\left(G_{o x}\right)$ of F3 is shown in Figure 9a. Fuels loaded with $\mu \mathrm{Al}$ exhibited no significant performance enhancement over the baseline for $G_{o x}>200 \mathrm{~kg} /\left(\mathrm{m}^{2} \cdot \mathrm{s}\right)$. This reflected the relatively low TG reactivity of these additives (see Table 5), yielding limited heat transfer enhancement for high oxidizer mass fluxes (long burning/reaction time). Faint $r_{f}$ and $\dot{m}_{f}$ increases were achieved for $G_{0 x}=155 \mathrm{~kg} /\left(\mathrm{m}^{2} \cdot \mathrm{s}\right)$, thanks to the radiation heat transfer that mitigated the effects of convective heat transfer decrease (as testified by the $n_{r} \sim 0.5$ in Table 6 ).

The F4 showed marked $r_{f}$ and $\dot{m}_{f}$ increases over the baseline for relatively high $G_{o x}$ (see Figure 7). This performance was quickly lost as the convection decreased, as testified by the $r_{f}\left(G_{o x}\right)$ power law approximation reported in Table 6, and by the low $G_{0 x}$ data of Figure 7. The time-averaged data of Figure 8 captured this effect, as testified by $\left\langle r_{f}\right\rangle=26 \% \pm 10 \%$, in turn yielding $\left\langle\dot{m}_{f}\right\rangle=36 \% \pm 14 \%$. In the high- $G_{0 x}$ region, the augmented flame temperature obtained thanks to ALEX-100 combustion increased the convective heat transfer, and the emitting particles enhanced the radiation contribution. Figure 10 
shows images captured during F4 combustion. In the earlier phases of the combustion, small and bright slivers detached from the regressing surface and from the strand head-end (Figure 10a,b). As the burning proceeded (Figure 10c,d), the formation of a surface crust layer was observed at the head-end (but likely also on the regressing surface). The creation of this surface layer was not observed in $\mu$ Al-loaded fuels (see Figure 3). A more detailed discussion of this phenomenon is given in Section 5.2, where $\mathrm{Al}$ aggregation/agglomeration phenomena are discussed.

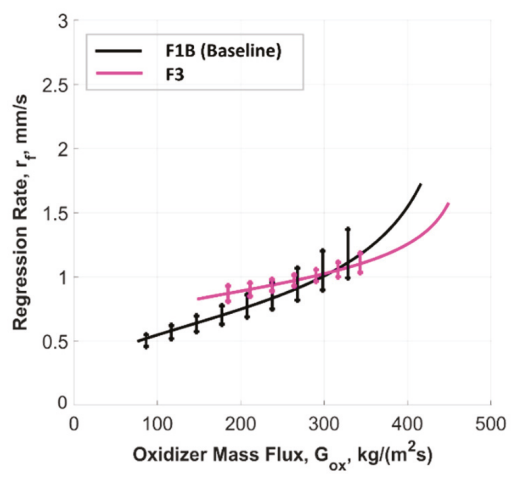

(a)

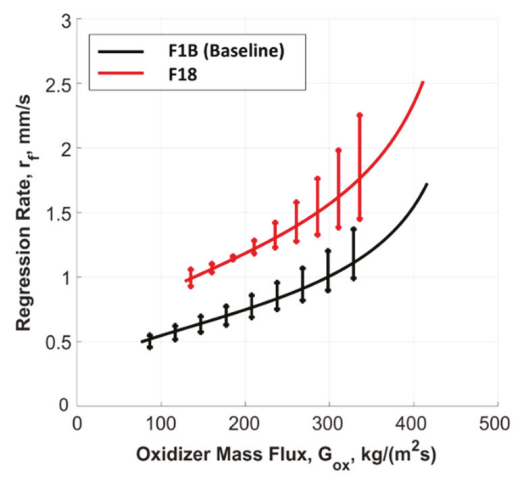

(b)

Figure 9. Time-resolved $r_{f}\left(G_{o x}\right)$ in GOX with $p_{c}=1.0 \mathrm{MPa}$ (a) F3 and (b) F18.

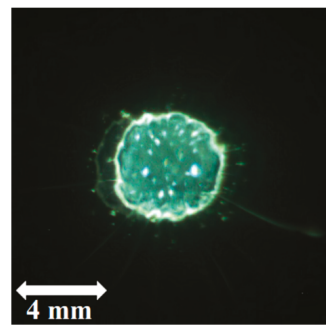

(a) $G_{o x}=265 \pm 16 \mathrm{~kg} /\left(\mathrm{m}^{2} \cdot \mathrm{s}\right)$

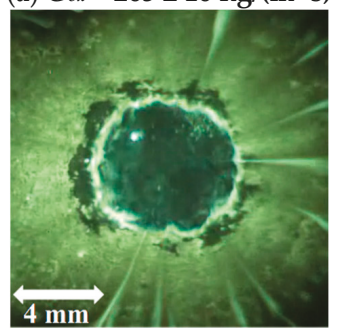

(c) $G_{o x}=165 \pm 10 \mathrm{~kg} /\left(\mathrm{m}^{2} \cdot \mathrm{s}\right)$

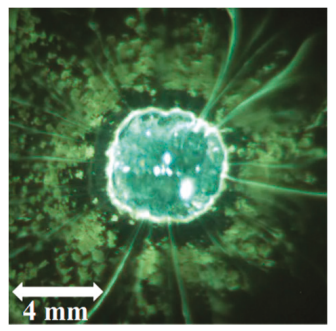

(b) $G_{o x}=230 \pm 14 \mathrm{~kg} /\left(\mathrm{m}^{2} \cdot \mathrm{s}\right)$

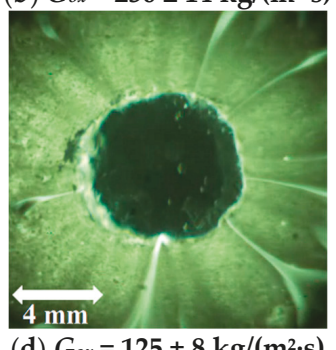

(d) $G_{o x}=125 \pm 8 \mathrm{~kg} /\left(\mathrm{m}^{2} \cdot \mathrm{s}\right)$

Figure 10. Head-end visualization of F4 burning in GOX at $p_{c}=1.0 \mathrm{MPa}:(\mathbf{a}, \mathbf{b})$ bright slivers detached from the regressing surface and from the visible strand end, (c,d) a surface crust layer is formed. Note that no fuel fragments and condensed combustion products are released from the sample toward the end of the combustion.

Interestingly, the nAl-loaded formulation manufactured without additive dispersion techniques (F8) showed no performance enhancement over the corresponding baseline probably due to the metal particle clustering inhibiting Al combustion (see Figures 7 and 8). The F5 showed $r_{f}$ performance and $G_{0 x}$ sensitivity similar to F4, as testified by the data reported in Figure 7 (note the relatively 
high error bars for $\left.G_{0 x}=325 \mathrm{~kg} /\left(\mathrm{m}^{2} \cdot \mathrm{s}\right)\right)$ and Table 6 . The stearic acid coating exerted no marked influence on the powder characteristics (Table 4) and reactivity (Table 5); this surface treatment was implemented mainly for storage purposes. The solid fuel formulations F6 and F7 exhibited a limited $r_{f}$ dependence on $G_{o x}$ (see Table 6), while providing interesting instantaneous and time-averaged $\dot{m}_{f}$. In particular, these formulations featured a nearly uniform $\dot{m}_{f}$ enhancement over the baseline for the whole investigated $G_{o x}$ range. This suggests that, at high heating rates, the metal reaction with fluoropolymer decomposition products may enhance the metal combustion performance close to/at the regressing surface in both high- and low- $G_{0 x}$ regimes. Such an effect was not observed at slow heating rate (see Table 5). Data reported in Figure 7 showed that, for $G_{o x}=155 \mathrm{~kg} /\left(\mathrm{m}^{2} \cdot \mathrm{s}\right)$, the average $\dot{m}_{f}$ increase over the baseline of F6 and F7 were $53 \% \pm 14 \%$ and $62 \% \pm 14 \%$, respectively, with marked differences with respect to the ALEX-loaded fuel.

The ballistic responses of fuel formulations loaded with VF-ALEX showed similarities with the burning behavior of F10. The AP-ALEX-100B-loaded formulation featured an average $\dot{m}_{f}$ increase over the baseline of $\sim 37 \%$ over the whole investigated $G_{0 x}$ range (see Figure 7). The AP content in the solid fuel was $\sim 2 \mathrm{wt} . \%$; however, locating it in the composite powder enabled a maximization of the oxidizer impact formulation ballistic response. This was testified by the fact that F9 (same nominal composition of F10, but with AP and nAl that were added separately) showed no significant performance enhancement over the baseline (see Figures 7 and 8). The performance difference between F10 and the VF-ALEX-100-loaded fuel was partially due to the higher SSA and reduced particle size of the latter, promoting the additive reactivity at fast heating rates and compensating for the higher flame temperature of $\mathrm{Al}+\mathrm{AP}$ (see Table 2).

Data for F11 burning with $G_{0 x}=325 \mathrm{~kg} /\left(\mathrm{m}^{2} \cdot \mathrm{s}\right)$ showed that the $\dot{m}_{f}$ enhancement of $\mu$ Al15 was improved by the LE mechanical activation (see Figures 7 and 8). Similarly to F4, F11 showed reduced $r_{f}$ and $\dot{m}_{f}$ performance as $G_{o x}$ decreased. The TG analyses suggested a slightly improved reactivity of LE- $\mu \mathrm{Al} 15$ with respect to the starting $\mu \mathrm{Al}$ (see $\alpha(933 \mathrm{~K}$ ) and $\alpha(1273 \mathrm{~K})$ in Table 5), although the SSA of the powders featured no significant differences. The morphology of LE- $\mu$ Al15 was the likely reason for the augmented reactivity of the powder at the high heating rates encountered in the combustion process. In addition to this, the irregular shape of particles of LE- $\mu$ Al15 may augment the gasifying surface roughness when protruding from the regressing fuel grain. As an effect, the increased surface roughness may have played a minor role in the $r_{f}$ enhancement of F11.

The ballistic effects of the fuels loaded with Al + PTFE composites obtained from LE mechanical activation showed no differences for $G_{0 x}=155 \mathrm{~kg} /\left(\mathrm{m}^{2} \cdot \mathrm{s}\right)$. On the other hand, for $G_{0 x}=325 \mathrm{~kg} /\left(\mathrm{m}^{2} \cdot \mathrm{s}\right)$, the ballistic response of F14 exhibited a $r_{f}$ increase over the baseline of $44 \% \pm 13 \%$. This corresponded to an $\dot{m}_{f}$ enhancement of $59 \% \pm 15 \%$. In Figure 7 , the uncertainty interval of F14 data at high $G_{o x}$ partially overlapped those of F12 and F13. In spite of this, it should be noted that the average $r_{f}$ and $\dot{m}_{f}$ increases over the baseline of the fuels loaded with PTFE- $\mu \mathrm{Al}$ composites from LE activation showed higher values as the fluoropolymer content was risen and the particle size of the starting $\mathrm{Al}$ was reduced (see Tables 4 and 5 and Figure 7). Under the investigated conditions, the presence of a particle fraction of sub-micrometric size probably played a key role in the effectiveness of the activation process of $\mu$ Al7.5, compensating for the lubricant effect of the PTFE.

The use of an HE process enabled the production of $\mathrm{Al}+$ PTFE composites by more intense activation stresses. These, in turn, permitted higher fluoropolymer mass fractions in the composites than the LE procedure. Under the investigated conditions, the solid fuels loaded with additives produced by HE mechanical activation featured the highest $\dot{m}_{f}$ performance enhancements (see Figures 7 and 8). F15 showed no significant $\dot{m}_{f}$ enhancement over the baseline, since the exploitation of PTFE as the oxidizer required the presence of additional metal ingredients (acting as fluorine scavengers $[10,24,87])$. Formulations in which the Al-PTFE reaction was exploited showed enhanced ballistic performance over non-loaded HTPB in terms of both $r_{f}$ and $\dot{m}_{f}$. For F17, $r_{f}$ enhancements were mainly observed for $G_{o x}<250 \mathrm{~kg} /\left(\mathrm{m}^{2} \cdot \mathrm{s}\right)$, due to a relatively low impact of the $\mathrm{Al}+\mathrm{PTFE}$ reaction on the metal combustion under intense convection. This was possibly related to the slow heating rate 
behavior of HE- $\mu$ Al15-T45 (see Table 5). This powder showed a relatively low $\alpha(1273 \mathrm{~K})$, and a higher $T_{o n, 1}$ with respect to LE- $\mu$ Al7.5-T30 and nano-sized additives featuring strong $r_{f}$ increases at high $G_{o x}$. The F16 and F18 formulations featured the same composition, although the former was loaded with mixed ALEX-50 + PTFE (separated and not mechanically activated powders), while the second was loaded with HE-ALEX-50-T45. The ballistic response of F18 was similar to that of F16, as reported in Figure 7. Both formulations featured strong data dispersion in the early phases of the combustion, with ensemble uncertainty intervals close to those of the baseline formulation. For this reason, in Figure 7 , the data for $r_{f}$ and $\dot{m}_{f}$ increases of $\mathrm{F} 18$ at $325 \pm 20 \mathrm{~kg} /\left(\mathrm{m}^{2} \cdot \mathrm{s}\right)$ are presented without error bars. As combustion proceeded, data scattering was reduced, and F18 showed percentage $r_{f}$ enhancements over the baseline of $54 \% \pm 13 \%$ at $155 \pm 10 \mathrm{~kg} /\left(\mathrm{m}^{2} \cdot \mathrm{s}\right)$. Under these operating conditions, the uncertainties in the performance enhancement were as for the other formulations, showing interesting performance with an $\dot{m}_{f}$ increase over the baseline of $141 \% \pm 20 \%$ (see Figure 7). F16 and F18 showed similar ballistic responses. This was partially due to the high PTFE and nAl loads providing good fluoropolymer and metal dispersion (fuel slurry was treated by ultrasound irradiation, thus mitigating metal particle clustering). Thus, the relative grading was performed in conditions favoring F16. In addition to this, three points should be highlighted to understand the advantages offered by F18: (i) the implemented HE activation procedure parameters used in this study aimed at a simple diffusion distance reduction between $\mathrm{Al}$ and PTFE, without pursuing further reactivity increases, (ii) the preparation of this fuel formulation proceeded in a simpler way than that of F16 since the micron-sized HE-ALEX-50-T45 was added to the formulation as a single ingredient that did not require ultrasound irradiation for effective dispersion and reduced the fuel formulation viscosity (see PSD and SSA data in Table 4), and (iii) the use of a micron-sized composite based on $\mathrm{nAl}$ reduced the risks of particle suspension in air due to the use of a nano-sized ingredient. In spite of the high metal load, neither F16 nor F18 showed evidence of regressing surface phenomena inhibiting the nAl combustion. F19 showed a ballistic response that was not dependent on $G_{0 x}$, while, for the other PTFE-loaded formulations, the $r_{f}\left(G_{o x}\right)$ power law approximations yielded $n_{r} \sim 0.8$ (see Table 6). Thanks to this, the $\dot{m}_{f}$ enhancement over the baseline of F19 exceeded $600 \%$ for $G_{o x}=155 \mathrm{~kg} /\left(\mathrm{m}^{2} \cdot \mathrm{s}\right)$. These interesting results show the possibilities offered by fuel-rich composite additives, although the optimization of their performance requires future investigations to evaluate the impact of the high $\mathrm{Al}+$ PTFE mass fraction on the nature of the formulation combustion behavior and, in particular, on its PDL.

\subsubsection{Combustion Surface Visualization}

Combustion surface visualizations were performed on selected fuel formulations (F1, F3, F4, F8, F17, F18, and F19). The F1 was tested to provide details on the non-metallized baseline behavior, while the surface behavior of F3 provided insight into the solid fuel surface phenomena in the presence of the standard $\mu \mathrm{Al}$. A comparative analysis of F4 and F8 enabled a comparison of the burning behavior of nAl-loaded fuels with and without sonication for additive dispersion. The PTFE-containing formulations F17, F18, and F19 were tested to evaluate the surface phenomena characterizing the fuels featuring the highest metal powder load and ballistic performance.

Figure 5 shows a representative frame for the combustion of F1. The burning proceeded uniformly along the visible sample length. High-speed visualization of the HTPB binder burning with 100 $\mathrm{kg} /\left(\mathrm{m}^{2} \cdot \mathrm{s}\right) \leq G_{0 x} \leq 400 \mathrm{~kg} /\left(\mathrm{m}^{2} \cdot \mathrm{s}\right)$ revealed the detachment of small fragments from the fuel grain [63]. This phenomenon was not observed under the investigated conditions. The image sequences reported in Figure 11 and in Figure 12 show the combustion of F3 and F4, respectively. The fuel loaded by the micron-sized additive featured a combustion surface similar to that of F1, but with the detachment of small elements of intense brightness (see Figure 11). These were Al particles or agglomerates released by the burning surface. The composition of these elements included $\mathrm{Al}_{1} \mathrm{Al}_{2} \mathrm{O}_{3}$, and (probably) binder decomposition products. The image sequence of Figure 11e-g shows the protrusion from the regressing surface of an apparently non-ignited spherical aggregate (apparent size of $\sim 200 \mu \mathrm{m}$ ). The latter was 
then inflamed during its flow in the boundary layer, as testified by the growth of its brightness and by the appearance of a diffusion trail.

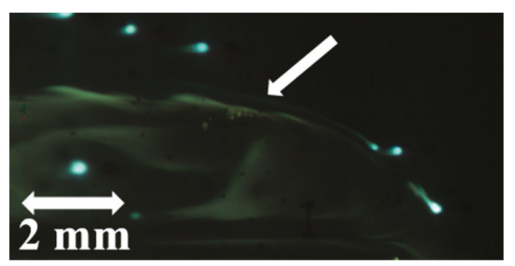

(a) $t_{i g n}+497.8 \mathrm{~ms}$

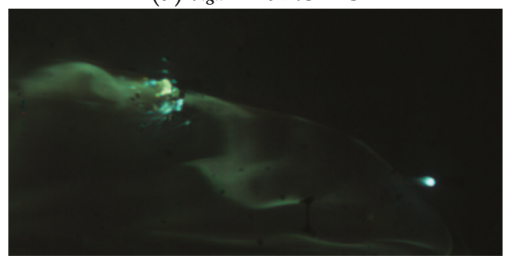

(c) $t_{i g n}+500.3 \mathrm{~ms}$

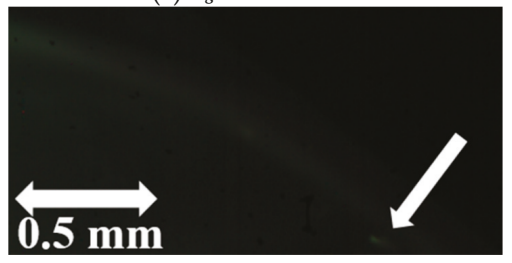

(e) $t_{i g n}+606.9 \mathrm{~ms}$

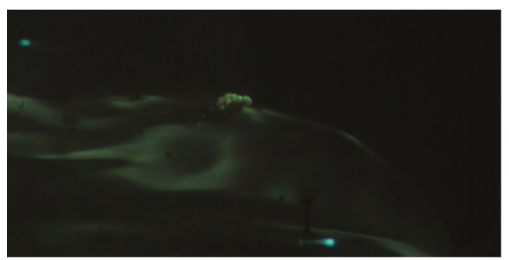

(b) $t_{i g n}+499.4 \mathrm{~ms}$

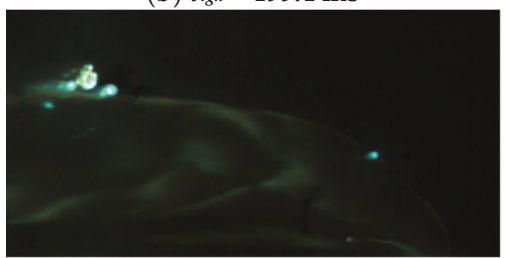

(d) $t_{i g n}+500.8 \mathrm{~ms}$

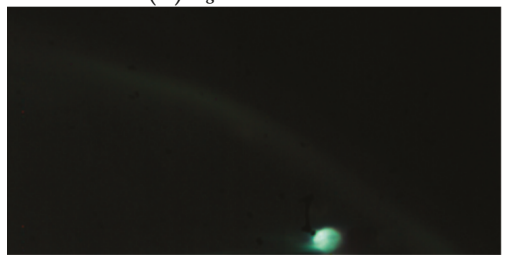

(f) $t_{i g n}+608.9 \mathrm{~ms}$

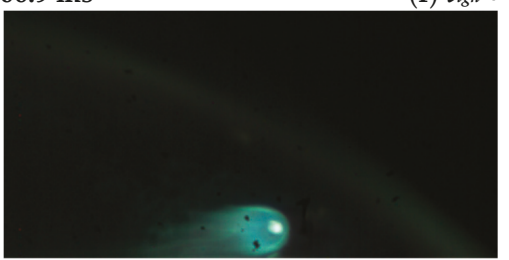

(g) $t_{i g n}+609.7 \mathrm{~ms}$

Figure 11. Combustion visualization of F2 fuel $\left(G_{o x}, p_{c}=0.4 \mathrm{MPa}\right.$, oxidizer flow from right to left): (a-d) overall view with details of metal particles blown from the surface; $(\mathbf{e}-\mathbf{g})$ details of agglomerate combustion after release from the solid fuel grain. 

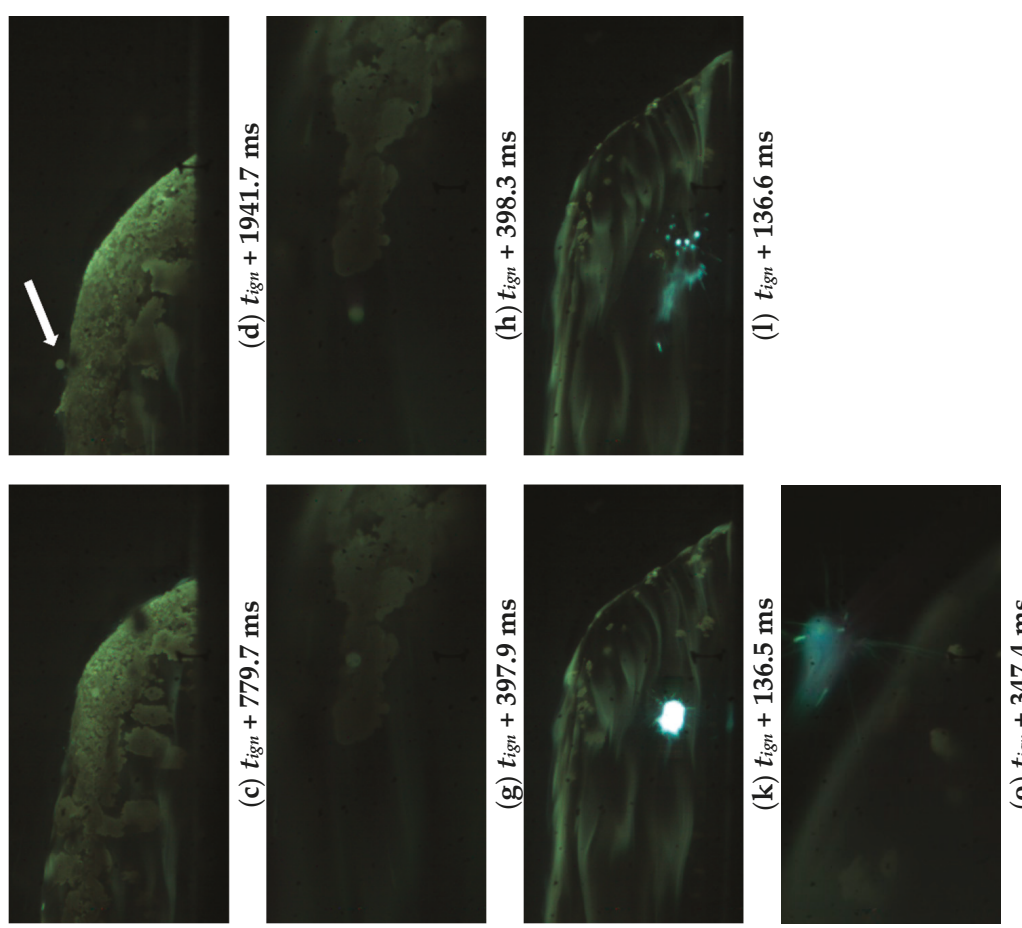

융 ్ㅗㅇ

节

क

एँ.

穾
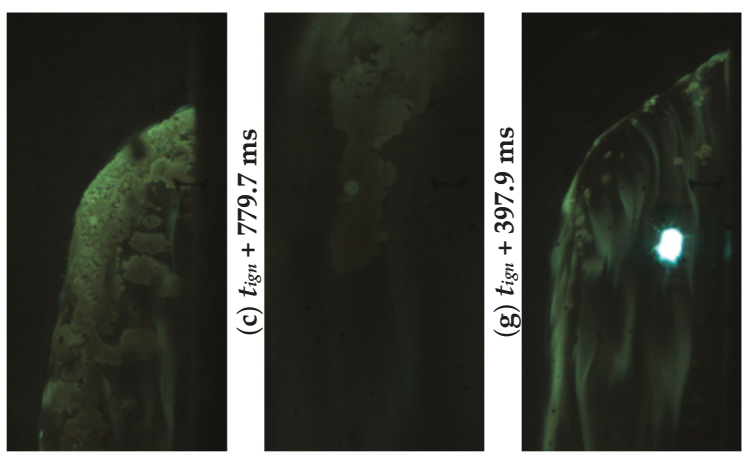

范

की

iิ :듀

茟告

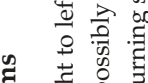

g

म.

मे

+3 ह

50
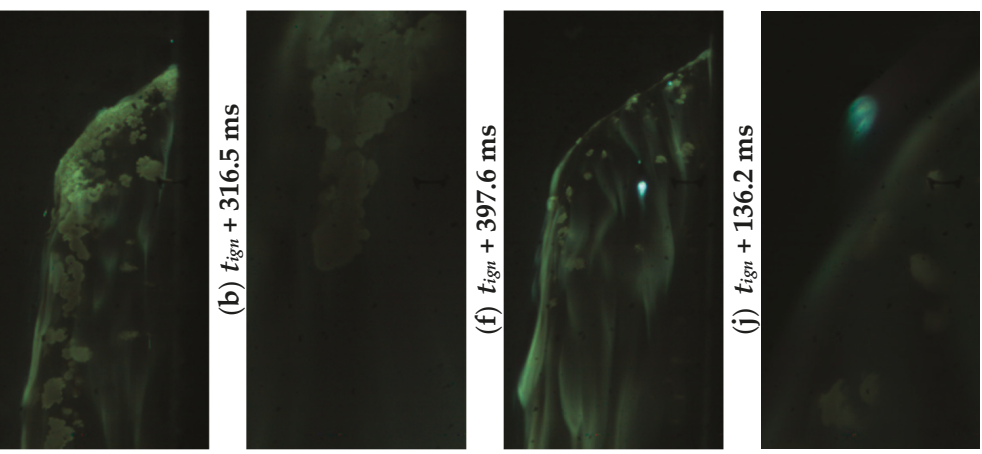

范

政

의

$\stackrel{I}{*}$

质哭

॥I $\Xi$

능

है छ

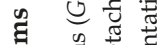

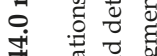

孚 焉

+ घ

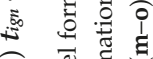

$\Xi$

$\underset{\infty}{2}$ ह

政

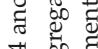

壮

岁

ㄷํㅇ

च च च

1

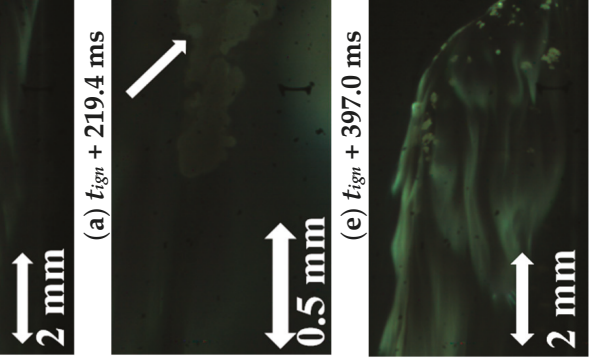

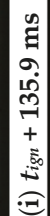

$\infty$ 可

छ 5

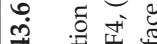

年

में ले

+ ह

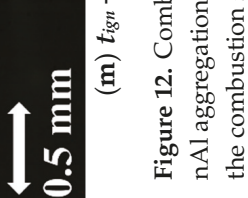


Under the investigated conditions, the velocity of the gaseous mass blown from the regressing surface was relatively slow (approximately one order of magnitude lower than in solid propellant formulations), while the surface layer exerted relatively intense retention forces due to the viscosity of the pyrolyzing fuel. As a consequence, $\mu \mathrm{Al}-7.5$ showed a faint activity at the gasifying surface (where temperature should be in the range of the $\mathrm{Al}$ melting point). On the one hand, this low activity limited the particle aggregation at the regressing surface and, in particular, the creation of a crust layer hampering the combustion. On the other hand, the metal powder characteristics hindered the additive enthalpy release close to the regressing surface once the particle was captured by the oxidizer stream. The condensed products leaving the regressing surface featured an apparent particle size of 100-200 $\mu \mathrm{m}$ (this observed size is not statistically relevant, and it should be taken as a rough estimation). Such a particle size range suggested relatively long burning times with energy release occurring far from the fuel grain (see Figure 11a and, for a convenient comparison with the burning tests, Figure 3). F4 showed a different burning behavior with respect to both F1 and F3. In the early phases of the combustion, the gasifying surface of HTPB + ALEX-100 showed the insurgence of a marked nAl aggregation (see Figure 12a-d, with the white arrow in Figure 12a highlighting the point the surface layer formation started at). This phenomenon probably began in the sub-surface layer of the pyrolyzing fuel. The insurgence of this surface layer was the likely cause of the fast decrease of the $r_{f}$ and $\dot{m}_{f}$ performance observed in the F4 combustion tests (see Figure 7, Figure 8, and Figure 12). Under the investigated conditions, the time-resolved $r_{f}$ of F8 showed no significant performance enhancement with respect to the baseline. Observing the image sequence reported in Figure 12i-l, a reduction in the aggregation phenomena characterizing F4 was noted for this fuel. The similarities between the high-speed surface visualization of F3 and F8 captured the effect of the reduction of $\mathrm{nAl}$ reactivity due to clustering, with a limited $r_{f}$ effect caused by the relatively large size of the $\mathrm{Al}$ agglomerates detaching from the surface (see Figure 12j).

Under the tested $G_{0 x}$ conditions, these aggregates were not detached from the fuel grain and gradually covered the entire gasifying surface of the port. As a result, the vaporization surface was shielded from the flame by a layer of unreacted (or partially oxidized) metal. This condition is shown in Figure 12d. Under these circumstances, the heat feedback to the solid fuel grain was reduced by the missed/incomplete metal oxidation.

The highly loaded fuel compositions with $\mathrm{Al}$ and PTFE featured a regressing surface with a glowing appearance (see Figure 13). This was a possible effect of the reaction between $\mathrm{Al}$ and the fluoropolymer. Independently from the micro- or nano-metric size of the Al particles embedded in the formulation, F17, F18, and F19 featured a surface layer that was apparently weaker than that formed in F4. This surface layer was also easily exfoliated by the oxidizer flow in the (relatively low) $G_{o x}$ conditions tested in the high-speed visualizations. The weakness of the surface layer limited (or avoided) the inhibition of heat feedback to the solid fuel grain encountered with F4. As a result, metal combustion occurred in more favorable conditions and, therefore, $r_{f}$ enhancement was achieved. 

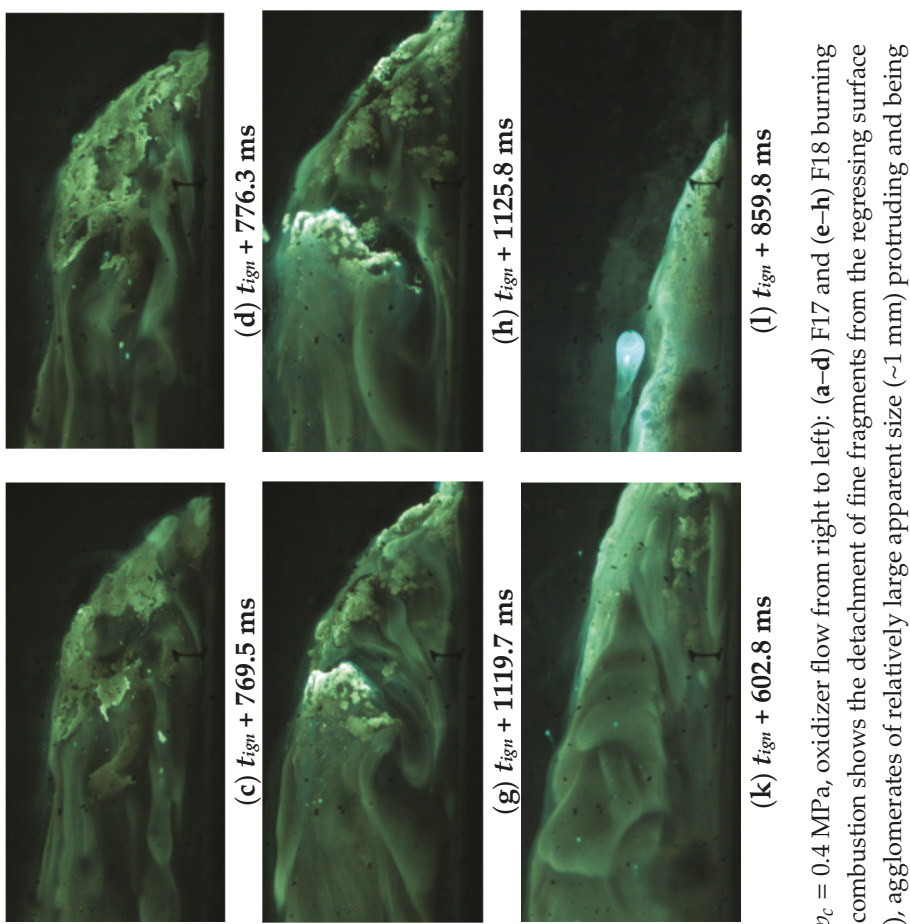

욜

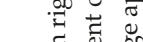

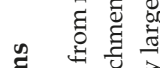

I 3 齐

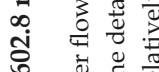

○. \ّ

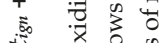

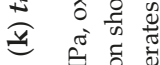

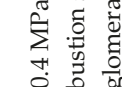

॥ है

ำ के

论
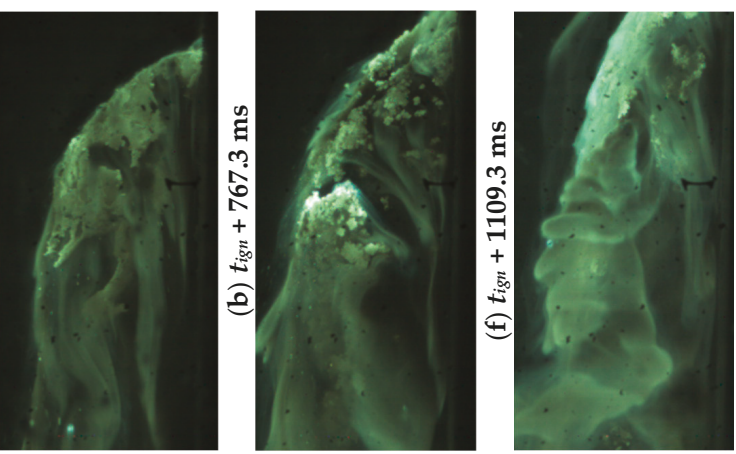

œ. I \&

营

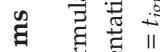

ㄱ. 월

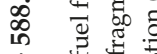

+ के

ثี ए

今

๙

压

氠

峲 :
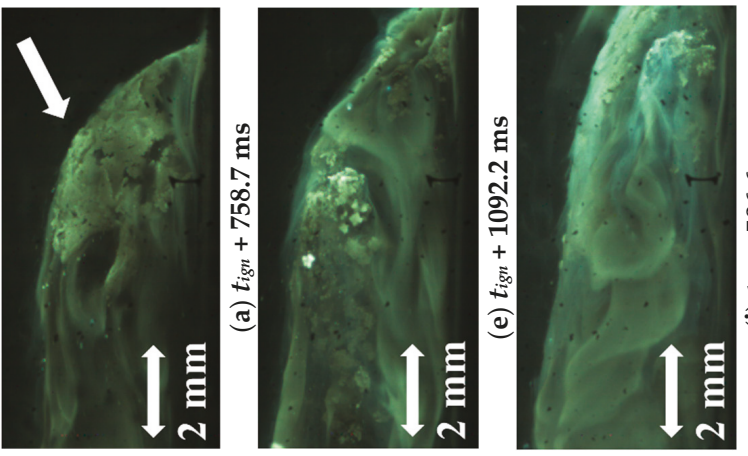

웡

글

ฮั 원

๘

b. छี

¿̊.

+ है ปิ่

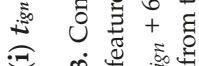

ल

总离 


\subsubsection{Concluding Remarks}

The screening of different fuel formulations was performed starting from the pre-burning characterization of a variety of Al-based energetic fillers. Composite additives were investigated together with air-passivated $\mu \mathrm{Al}$ and $\mathrm{nAl}$. Effects of ingredients as $\mathrm{AP}$ and fluoropolymers on the metal ignition and combustion in the oxidizer-lean conditions encountered at/close to the regressing surface of burning fuel formulations were investigated.

Under the investigated conditions, F4 showed an $r_{f}$ enhancement over the non-metallized baseline of $59 \% \pm 10 \%$ at $350 \mathrm{~kg} /\left(\mathrm{m}^{2} \cdot \mathrm{s}\right)$ and a marked $r_{f}\left(G_{o x}\right)$ sensitivity. The ballistic performance of the $\mathrm{nAl}$-load formulation worsened as the oxidizer mass flux decreased. The $r_{f}$ increase over the baseline reduced to $45 \% \pm 10 \%$ at $325 \mathrm{~kg} /\left(\mathrm{m}^{2} \cdot \mathrm{s}\right)$ and were absent at $155 \mathrm{~kg} /\left(\mathrm{m}^{2} \cdot \mathrm{s}\right)$. The resulting $\left\langle\dot{m}_{f}\right\rangle$ at $\left\langle G_{0 x}\right\rangle=250 \pm 15$ $\mathrm{kg} /\left(\mathrm{m}^{2} \cdot \mathrm{s}\right)$ was $36 \% \pm 14 \%$, and the power law approximation of $r_{f}\left(G_{0 x}\right)$ yielded $n_{r}=0.959 \pm 0.022$. The latter value highlighted other effects on the combustion evolution than the convective heat transfer decrease. High-speed visualizations of the burning surface suggested that F4 burning performance loss was mainly caused by metal aggregation occurring at the regressing surface/subsurface. This phenomenon yielded the formation of a shield of unreacted/partially oxidized $\mathrm{Al}$ that limited the heat feedback toward the surface. Combustion tests results and surface visualizations supported the idea that high $G_{o x}$ promoted $\mathrm{Al}$ particle/aggregate removal from the surface (and their combustion), as shown by Figures 10 and 12. On the other hand, under $145 \mathrm{~kg} /\left(\mathrm{m}^{2} \cdot \mathrm{s}\right) \leq G_{o x} \leq 160 \mathrm{~kg} /\left(\mathrm{m}^{2} \cdot \mathrm{s}\right)$, the aggregates resided on the fuel surface, creating a crust of accumulated material. This was a likely effect of subsurface aggregation creating a relatively strong web with good cohesion (see Figures 10 and 12). Such behavior was not observed for fuels loaded with $\mu \mathrm{Al}(\mathrm{F} 2-\mathrm{F} 3$ ) and for F8 (that was prepared without nAl dispersion procedures). These fuels exhibited no significant $r_{f}$ and $\dot{m}_{f}$ enhancements over the baseline at high $G_{o x}$, while at $155 \mathrm{~kg} /\left(\mathrm{m}^{2} \cdot \mathrm{s}\right), \mu \mathrm{Al}$-loaded fuels showed increased performance over both F4 and F8. For F2-F3, F4, and F8, relatively large aggregates were observed to detach from the surface. The use of AP- and fluoropolymer-containing composites based on Al contrasted the $r_{f}$ detriment observed for ALEX-100, thanks to the metal reaction with the oxidizer/coating decomposition products. In spite of an increased additive (and metal) mass fraction with respect to the other investigated fuels, combustion surface visualizations of F18 showed the build-up of a surface metal layer of reduced cohesion. This was the likely effect of the partial reaction between the metal and the PTFE. The resulting surface layer was easily exfoliated by the oxidizer flow, and faster $r_{f}$ was, therefore, achieved by a combination of convective and radiation effects (see Figures 7 and 8).

\section{Conclusions and Future Developments}

This work focused on the lab-scale investigation of the combustion behaviors of solid fuel formulations based on hydroxyl-terminated polybutadiene loaded with Al-based energetic additives. Tested metal-based fillers spanned from micron- to nano-sized powders and included oxidizer-containing fuel-rich composites. The latter were obtained by chemical and mechanical processes applied to the starting Al powders. The aim of these processes was the reduction of the diffusion distance between the metal and the oxidizing species source, and/or the modification of the particle characteristics (i.e., morphology, size). The chemical and mechanical processes pursued easier $\mathrm{Al}$ ignition and combustion in the oxidizer-lean conditions characterizing the region between the diffusion flame and the regressing surface of the burning grains. The combustion behaviors of the tested formulations were analyzed considering the solid fuel regression rate and the mass burning rate as the main parameters of interest. A non-metallized formulation was taken as baseline for the relative grading of the tested fuels. Instantaneous and time-average regression rate $\left(r_{f}\right)$ and mass burning rate $\left(\dot{m}_{f}\right)$ data were determined using an optical time-resolved technique. The ballistic responses of the fuels were analyzed together with high-speed visualizations of the regressing surface.

The ballistic responses of fuel formulations loaded with uncoated air-passivated nano-sized $\mathrm{Al}$ (nominal size $100 \mathrm{~nm}$, ALEX-100) showed a strong dependence on the fuel manufacturing procedure. The dispersion of nano-sized $\mathrm{Al}$ down to the nanoscale requires special manufacturing procedures to 
mitigate the clustering of particles. In the study, a fuel formulation prepared by ultrasound irradiation was contrasted with a similar composition prepared without any effort toward a clustering reduction. For oxygen mass fluxes $>250 \mathrm{~kg} /\left(\mathrm{m}^{2} \cdot \mathrm{s}\right)$, the fuels loaded with ALEX-100 and prepared by nanopowder dispersion procedures showed increased $r_{f}$ and $\dot{m}_{f}$ with respect to formulations where the same additive was included without special treatments. The polymeric fuel loaded with ALEX-100 and prepared by additive dispersion techniques exhibited instantaneous mass burning rate enhancement over the baseline of $55 \% \pm 11 \%$ for an oxidizer mass flux of $325 \pm 20 \mathrm{~kg} /\left(\mathrm{m}^{2} \cdot \mathrm{s}\right)$. This ballistic performance was lost as the oxidizer mass flux decreased, and, at $155 \pm 10 \mathrm{~kg} /\left(\mathrm{m}^{2} \cdot \mathrm{s}\right)$, no significant $\dot{m}_{f}$ increase over the baseline was identified. Under the same operating conditions, a formulation prepared without special procedures for ALEX-100 dispersion yielded a $\dot{m}_{f}$ increase over the baseline of $15 \% \pm 13 \%$ at $325 \pm 20 \mathrm{~kg} /\left(\mathrm{m}^{2} \cdot \mathrm{s}\right)$, and this performance remained nearly unchanged over the whole investigated oxidizer mass flux range.

Combustion surface visualizations performed on ALEX-100-loaded solid fuel slabs burning at oxygen mass fluxes in the range of 145 to $160 \mathrm{~kg} /\left(\mathrm{m}^{2} \cdot \mathrm{s}\right)$ suggested that the observed ballistic responses were due to different metal aggregation/agglomeration phenomena (in turn induced by the different manufacturing procedures). In fuel formulations prepared by special manufacturing procedures for ALEX-100 dispersion, for oxygen mass flux $<160 \mathrm{~kg} /\left(\mathrm{m}^{2} \cdot \mathrm{s}\right)$, intense aggregation of the metal particles was noted at the burning surface. This phenomenon caused the formation of a crust of sintered and (possibly) partially oxidized metal, inhibiting the combustion process and canceling the performance enhancement over the baseline of the ALEX-100-loaded fuel. Such phenomena were probably limited at relatively high oxygen mass fluxes, thanks to the more intense convection that yielded more effective combustion conditions for ALEX-100. No marked aggregation/agglomeration surface phenomena were observed during the combustion of the ALEX-100-loaded fuel prepared without procedures for additive dispersion. In this latter formulation, clusters of metal particles were ejected from the regressing surface and burned relatively far from it, with a combustion mechanism similar to that observed in fuels loaded with micron-sized Al. This was a consequence of the combustion of relatively large clusters of particles featuring a reduced specific surface area compared to the smaller clusters or single nanoparticles (and, therefore, a lower reactivity). With heat release occurring far from the regressing surface, and a likely incomplete combustion of the clusters, the fuel loaded with ALEX-100 and prepared without additive dispersion procedures yielded a lower performance enhancement at high oxygen fluxes, and a limited $\dot{m}_{f}$ with reduced oxygen mass fluxes.

Composite Al-rich additives limited the negative effects of ALEX-100 aggregation/agglomeration on combustion, while providing increased regression rate and mass burning rate performance over the baseline. The fuel loaded with air-passivated nano-sized Al coated with a copolymer of vinylidene fluoride and hexafluoropropylene showed a mass burning rate enhancement over the baseline of $45 \%$ $\pm 14 \%$ at $155 \mathrm{~kg} /\left(\mathrm{m}^{2} \cdot \mathrm{s}\right)$, with a limited sensitivity of this result with regard to the oxidizer mass flux changes. A similar ballistic response was achieved by a composite featuring nano-sized Al as fuel, and ammonium perchlorate (AP) as oxidizer. In this case, in spite of the presence of large clusters of nano-sized $\mathrm{Al}$ and $\mathrm{AP}$, the $\dot{m}_{f}$ enhancement over the baseline was $39 \% \pm 14 \%$ at $325 \mathrm{~kg} /\left(\mathrm{m}^{2} \cdot \mathrm{s}\right)$ and $36 \% \pm 13 \%$ at $155 \mathrm{~kg} /\left(\mathrm{m}^{2} \cdot \mathrm{s}\right)$. In both the latter formulations, the oxidizer content in the final fuel was $<2$ wt.\%; thus, a crucial role was played by the limited diffusion distance between the metal and the oxidizing species source. The ballistic responses of the fuels prepared with nAl-based composites produced by chemical methods suggested an interaction between $\mathrm{Al}$ and the oxidizing species in the additive during the combustion. This interaction promoted the metal ignition and combustion, and it lessened the effects of the nano-sized aluminum aggregation under the oxidizer-lean conditions encountered at/close to the regressing surface. This observation was supported by combustion surface visualizations of solid fuels containing mechanically activated composites with nano-sized $\mathrm{Al}$ and polytetrafluoroethylene (PTFE) as oxidizer. This fuel formulation featured an additive load of 54.4 wt.\%, with a composition of the energetic filler that was $55 \mathrm{wt}$ \% air-passivated nAl (nominal size 50 $\mathrm{nm}$ ) and $45 \mathrm{wt}$ \% PTFE. In spite of a relatively high nano-sized Al mass fraction (30\% of the whole 
formulation), the fuel loaded with this additive did not show evidence of the formation of a surface layer of aggregated metal hindering the combustion process. The nAl reaction with PTFE favored the metal ignition and combustion at/close to the regressing surface, as in the case of the composites containing fluoropolymer/AP produced by chemical methods. The fuel formulation loaded with the mechanically activated nano-sized $\mathrm{Al}$ and PTFE exhibited augmented performance with respect to the baseline, with a mass burning rate increase of $141 \% \pm 20 \%$ at $155 \mathrm{~kg} /\left(\mathrm{m}^{2} \cdot \mathrm{s}\right)$. Yet, when considering the data scattering between different combustion tests, the performance of the composite additive produced by mechanical activation did not differ significantly from that of fuels containing a simple mix of nano-sized $\mathrm{Al}$ and polytetrafluoroethylene. This result was mainly due to two reasons. Firstly, in this work the mechanical activation procedure for the realization of the nano-sized Al-PTFE composite was not optimized. Secondly, the relatively high PTFE content in the formulation (24.5 wt. \%) favored its dispersion in the binder matrix and also reduced the distance between fluoropolymer and metal particles in the case of non-activated, simply mixed ingredients.

The achieved results provide a better understanding of the effects of the use of $\mathrm{nAl}$ in solid fuel formulations, showing the possible detrimental effects due to the metal aggregation observed at relatively low oxidizer mass fluxes. On the other hand, the ballistic responses of fuel formulations loaded with nAl-based composite additives testify that the behavior of the nano-sized metal powders can be tailored to mitigate possible shortcomings due to the oxidizer-lean conditions locally encountered in the reacting boundary layer. Moreover, the use of composite powders promises simplified manufacturing procedures for the production of nAl-containing fuels, since chemical and mechanical activation processes may be designed to provide additives with tailored reactivity and suitable particle size distribution. All these aspects show the attractive perspectives offered by fuel-rich composite additives.

Future activities for augmented $r_{f}$ and $\dot{m}_{f}$ of solid fuels on nAl-based composite additives should focus on (i) combustion efficiency of the fuel formulations loaded with composite additives, (ii) suitability of the proposed approach to liquefying fuel formulations, (iii) impacts of green oxidizers on the preparation of the composites, and (iv) determination of the PDL of the fuel formulations, to evaluate the effects of composite additives on stop/restart and throttling capabilities of solid fuel grains loaded with these additives.

Funding: This research received no external funding.

Acknowledgments: The author thanks Stefano Dossi (ReActive-Powder Technology srl, Milan, Italy) for the work done on the development of procedures for the mechanical activation processes and helpful discussions. The efforts of Marco Castagna and Marco DiLorenzo, who helped with the experimental data collection during their MSc activities at the SPLab of Politecnico di Milano, are highly appreciated. Special thanks go to Gianluigi Marra (Istituto ENI Donegani, Novara, Italy) for the work done on the SEM, TEM, and SSA data collection.

Conflicts of Interest: the author declares no conflicts of interest.

\section{Nomenclature}

Roman and Greek Symbols

- $\quad$ space-averaged value

〈.) time-averaged value

$\alpha(\mathrm{T}) \quad \mathrm{Al} \rightarrow \mathrm{Al}_{2} \mathrm{O}_{3}$ conversion factor, \%

$\Delta m_{0} \quad$ mass change before the first intense oxidation onset temperature, $\%$

$\Delta m(T) \quad$ mass change at the temperature $T, \%$

$\mu \mathrm{Al} \quad$ micron-sized $\mathrm{Al}$ powder

$\rho_{A l} \quad$ Al density, $\mathrm{kg} / \mathrm{m}^{3}$

$\rho_{f} \quad$ fuel density, $\mathrm{kg} / \mathrm{m}^{3}$

$a_{D} \quad$ pre-exponential coefficient in diameter change power law fitting, $\mathrm{mm} / \mathrm{s}^{n D}$

$a_{r} \quad$ pre-exponential coefficient in regression rate power law approximation, $\mathrm{mm} / \mathrm{s} /\left(\mathrm{kg} /\left(\mathrm{m}^{2} \cdot \mathrm{s}\right)\right)^{n r}$

$a_{S} \quad$ particle size derived from the specific surface area, $\mathrm{nm}$

$C_{A l} \quad$ active $\mathrm{Al}$ content, wt.\% 
$D_{(0.1)} \quad$ particle diameter below which $10 \%$ of the particles lay, $\mu \mathrm{m}$

$D_{(0.5)} \quad$ particle diameter below which $50 \%$ of the particles lay, $\mu \mathrm{m}$

$D_{(0.9)} \quad$ particle diameter below which $90 \%$ of the particles lay, $\mu \mathrm{m}$

$D_{32} \quad$ surface-based mean particle diameter, $\mu \mathrm{m}$

$D_{43} \quad$ volume-based mean particle diameter, $\mu \mathrm{m}$

$D_{i} \quad i$-th sampled port diameter, $\mathrm{mm}$

G total mass flux $\left(G=G_{o x}+G_{f}\right), \mathrm{kg} /\left(\mathrm{m}^{2} \cdot \mathrm{s}\right)$

$G_{f} \quad$ fuel mass flux, $\mathrm{kg} /\left(\mathrm{m}^{2} \cdot \mathrm{s}\right)$

Gox oxidizer mass flux, $\mathrm{kg} /\left(\mathrm{m}^{2} \cdot \mathrm{s}\right)$

$L_{f} \quad$ fuel grain length, $m$

$\dot{m}_{f} \quad$ fuel mass burning rate, $\mathrm{kg} / \mathrm{s}$

$\dot{m}_{o x} \quad$ oxidizer mass flow rate, $\mathrm{kg} / \mathrm{s}$ (except where otherwise stated)

$n A l \quad$ nano-sized $\mathrm{Al}$ powder

$n_{D} \quad$ exponent in diameter change power law fitting

$n_{r} \quad$ exponent in regression rate power law approximation

$p_{c} \quad$ combustion chamber pressure, $\mathrm{MPa}$

$R^{2} \quad$ data fitting parameter

$r_{f} \quad$ solid fuel regression rate, $\mathrm{mm} / \mathrm{s}(\mathrm{m} / \mathrm{s}$ in Equation (4))

T temperature, $\mathrm{K}$

$t$ time, s

$t_{\text {end }} \quad$ end time, $\mathrm{s}$

$T_{f l} \quad$ calculated flame temperature, $\mathrm{K}$

$t_{i} \quad$ time of the $i$-th diameter sampling, $\mathrm{s}$

$t_{\text {ign }} \quad$ ignition time, $\mathrm{s}$

$T_{\text {on, } i} \quad i$-th intense oxidation onset temperature, $\mathrm{K}$

Acronyms

ALEX

AP

CB

$\mathrm{CCP}$

DTG

GOX

HE

HRE

HTPB

LE

MA

PDL

PTFE

SEM

SSA

SOP

TED

TEM

TOT

TG

TMD

VFHFP

wrt

aluminum exploded (nAl produced by electrical explosion of wires, typically air-passivated) ammonium perchlorate

carbon black

condensed combustion product

differential of the thermogravimetry trace

gaseous oxygen

high energy (mechanical activation)

hybrid rocket engine

hydroxyl-terminated polybutadiene

low energy (mechanical activation)

mechanical activation

pressure deflagration limit, $\mathrm{MPa}$

polytetrafluoroethylene

scanning electron microscopy

specific surface area, $\mathrm{m}^{2} / \mathrm{g}$

small oxide particles

transmission energy dispersion

transmission electron microscopy

thickness over time

thermogravimetry

theoretical maximum density, $\mathrm{kg} / \mathrm{m}^{3}$

vinylidene fluoride hexafluoropropylene copolymer

with respect to

\section{References}

1. Marxman, G.A.; Gilbert, M. Turbulent boundary layer combustion in the hybrid rocket. In 9th International Symposium on Combustion; Academic Press, Inc.: New York, NY, USA, 1963; pp. 371-383. 
2. Marxman, G.A. Boundary layer combustion in propulsion. In 11th Symposium (International) on Combustion; The Combustion Institute: Pittsburg, PA, USA, 1964; pp. 269-289.

3. Marxman, G.A.; Wooldridge, C.E. Research on the Combustion Mechanism of Hybrid Rockets. In Advances in Tactical Rocket Propulsion; Penner, S.S., Ed.; AGARD Conference Proceedings No. 1; Technivision Services: London, UK, 1968; pp. 421-477.

4. Chiaverini, M.J. Review of solid fuel regression rate behavior in classical and non-classical hybrid rocket motors. In Fundamentals of Hybrid Rocket Combustion and Propulsion; Chiaverini, M.J., Kuo, K.K., Eds.; AIAA Progress in Astronautics and Aeronautics: Reston, VA, USA, 2007; Volume 218, pp. 37-125.

5. Marquardt, T.; Majdalani, J. Review of Classical Diffusion-Limited Regression Rate Models in Hybrid Rockets. Aerospace 2019, 6, 75. [CrossRef]

6. Pastrone, D. Approaches to low fuel regression rates in hybrid rocket engines. Int. J. Aerosp. Eng. 2012, 2012, 649753. [CrossRef]

7. Karabeyoglu, M.A.; Altman, D.; Cantwell, B.J. Combustion of liquefying hybrid propellants: Part 1, general theory. J. Propul. Power 2002, 18, 610-620. [CrossRef]

8. Karabeyoglu, M.A.; Cantwell, B.J. Combustion of liquefying hybrid propellants: Part 2, stability of liquid films. J. Propul. Power 2002, 18, 621-630. [CrossRef]

9. Kim, S.; Lee, J.; Moon, H.; Sung, H.; Kim, J.; Cho, J. Effect of paraffin-LDPE blended fuel in hybrid rocket motor. In Proceedings of the 46th AIAA. ASME/SAE/ASEE Joint Propulsion Conference and Exhibit, Nashville, TN, USA, 25-28 July 2010. AIAA Paper No. 2010-7031.

10. Boiocchi, M.; Paravan, C.; Dossi, S.; Maggi, F.; Colombo, G.; Galfetti, L. Paraffin-Based Fuels and Energetic Additives for Hybrid Rocket Propulsion. In Proceedings of the 51st AIAA/SAE/ASEE Joint Propulsion Conference, Orlando, FL, USA, 27-29 July 2015. AIAA Paper No. 2015-4042.

11. Paravan, C.; Galfetti, L.; Maggi, F. A Critical Analysis of Paraffin-based Fuel Formulations for Hybrid Rocket Propulsion. In Proceedings of the 53rd AIAA/SAE/ASEE Joint Propulsion Conference, Atlanta, GA, USA, 10-12 July 2017. AIAA Paper No. 2017-4830.

12. Paravan, C.; Bisin, R.; Piscaglia, F.; Galfetti, L. Combustion Processes in Hybrid Propulsion. Int. J. Energ. Mater. Chem. Propuls. 2019, 18, 255-286.

13. Veale, K.; Adali, S.; Pitot, J.; Brooks, M. A Review of the Performance and Structural Considerations of Paraffin Wax Hybrid Rocket Fuels with Additives. Acta Astronaut. 2017, 141, 196-208. [CrossRef]

14. Tan, Y.; Chen, S.; Zhang, W.; Shen, R.; DeLuca, L.T.; Ye, Y. The Effects of Mechanical Modification Additives on the Regression Rate of Paraffin-Based Fuels for Hybrid Rocket. In Proceedings of the 7th European Conference for Aeronautics and Space Sciences, Milan, Italy, 1-4 July 2017.

15. Risha, G.A.; Evans, B.J.; Boyer, E.; Kuo, K.K. Metals, energetic additives and special binders used in solid fuels for hybrid rockets. In Fundamentals of Hybrid Rocket Combustion and Propulsion; Chiaverini, M.J., Kuo, K.K., Eds.; AIAA Progress in Astronautics and Aeronautics: Reston, VA, USA, 2007; Volume 218, pp. $413-456$.

16. Paravan, C. Ballistics of Innovative Solid Fuel Formulations for Hybrid Rocket Engines. Ph.D. Thesis, Politecnico di Milano, Aerospace Science and Technology Department, Milan, Italy, 2012.

17. De Luca, L.T.; Galfetti, L.; Maggi, F.; Colombo, G.; Paravan, C.; Reina, A.; Dossi, S.; Fassina, M.; Sossi, A. Characterization and combustion of aluminum nanopowders in energetic systems. In Metal Nanopowders Production, Characterization, and Energetic Applications; Gromov, A.A., Teipel, U., Eds.; Wiley VCH: Weinheim, Germany, 2014; pp. 299-408.

18. Evans, B.; Favorito, N.A.; Boyer, E.; Risha, G.A.; Wehrman, R.B.; Kuo, K.K. Characterization of Nano-sized Energetic Particle Enhancement of Solid Fuel Burning Rates in an X-Ray Transparent Hybrid Rocket Engine. In Proceedings of the 40th AIAA/ASME/SAE/ASEE Joint Propulsion Conference and Exhibit (AIAA), Fort Lauderdale, FL, USA, 11-14 July 2004. AIAA Paper No. 2004-3821.

19. Galfetti, L.; Merotto, L.; Boiocchi, M.; Maggi, F.; DeLuca, L.T. Ballistic and Rheological Characterization of Paraffin-based Fuels for Hybrid Rocket Propulsion. In Proceedings of the 47th AIAA/ASME/SAE/ASEE Joint Propulsion Conference \& Exhibit (AIAA), San Diego, CA, USA, 31 July-3 August 2011. AIAA Paper No. 2011-5680.

20. Strand, L.D.; Ray, R.L.; Anderson, F.; Cohen, N.S. Hybrid rocket fuel combustion and regression rate study. In Proceedings of the 28th Joint Propulsion Conference and Exhibit, Nashville (AIAA), Nashville, TN, USA, 6-8 July 1992. AIAA Paper No. 1992-3302.

21. Grosse, A.V.; Conway, J.B. Combustion of metals in oxygen. Ind. Eng. Chem. 1958, 50, 663-672. [CrossRef] 
22. DeLuca, L.T.; Maggi, F.; Dossi, S.; Fassina, M.; Paravan, C.; Sossi, A. Prospects of Aluminum Modifications as Energetic Fuels in Chemical Rocket Propulsion. In Chemical Rocket Propulsion: A Comprehensive Survey of Energetic Materials; DeLuca, L.T., Shimada, T., Sinditskii, V.P., Calabro, M., Eds.; Springer: Berlin, Germany, 2017; pp. 191-233.

23. Dossi, S. Mechanically Activated Al Fuels for High Performance Solid Rocket Propellants. Ph.D. Thesis, Politecnico di Milano, Aerospace Science and Technology Department, Milan, Italy, 2014.

24. Dossi, S.; Paravan, C.; Maggi, F.; Galfetti, L. Enhancing micrometric aluminum reactivity by mechanical activation. In Proceedings of the 51st AIAA/SAE/ASEE Joint Propulsion Conference, Orlando, FL, USA, 27-29 July 2015. AIAA Paper No. 2015-4221.

25. Paravan, C.; Dossi, S.; Maggi, F.; Galfetti, L.; Marra, G. Pre-burning characterization of nano-sized aluminum in condensed energetic systems. In Energetic Nanomaterials: Synthesis, Production, and Characterization; Gromov, A.A., Zarko, V.E., Eds.; Elsevier: Amsterdam, The Netherlands, 2016; pp. 341-368.

26. Juergens, L.P.H.; Sloof, W.G.; Tichelaar, F.D.; Mittemeijer, E.J. Structure and morphology of aluminum oxide films formed by thermal oxidation of aluminum. Thin Solid Films 2002, 418, 89-101. [CrossRef]

27. Trunov, M.A.; Schoenitz, M.; Zhu, X.; Dreizin, E.L. Effect of polymorphic phase transformations on $\mathrm{Al}_{2} \mathrm{O}_{3}$ film on oxidation kinetics of aluminum powders. Combust. Flame 2005, 140, 310-318. [CrossRef]

28. Paravan, C.; Verga, A.; Maggi, F.; Galfetti, L. Accelerated ageing of aluminum powders: Metal content, composition, and non-isothermal oxidation reactivity. Acta Astronaut. 2019, 158, 397-406. [CrossRef]

29. Schoenitz, M.; Patel, B.; Agboh, O.; Dreizin, E.L. Oxidation of aluminum powders at high heating rates. Thermochim. Acta 2010, 507, 115-122. [CrossRef]

30. Trunov, M.; Schoenitz, M.; Dreizin, E.L. Ignition of aluminum powders under different experimental conditions. Propellants Explos. Pyrotech. 2005, 30, 36-43. [CrossRef]

31. Sundaram, S.D.; Puri, P.; Yang, V. A general theory of ignition and combustion of nano- and micron-sized aluminum particles. Combust. Flame 2016, 169, 94-109. [CrossRef]

32. Gen, M.Y.; Frolov, Y.V.; Storozhev, V.B. On combustion of particles of subdispersed aluminum. Combust. Explos. Shock Waves 1978, 14, 153-155. [CrossRef]

33. Ivanov, G.V.; Tepper, F. Activated aluminum as stored energy source for propellants. In Challenges in Propellants and Combustion 100 Years after Nobel; Kuo, K.K., Ed.; Begell House: New York, NY, USA, 1997; pp. 636-645.

34. Ilyin, A.P.; Gromov, A.A.; An, V.; Faubert, F.; de Izarra, C.; Espagnacq, A.; Brunet, L. Characterization of aluminum powders I. Parameters of reactivity of aluminum powders. Propellants Explos. Pyrotech. 2002, 27, 361-364. [CrossRef]

35. Gromov, A.A.; Ilyin, A.P.; Förther-Barth, U.; Teipel, U. Characterization of aluminum powders: II. Aluminum nanopowders passivated by non-inert coatings. Propellants Explos. Pyrotech. 2006, 31, 401-409. [CrossRef]

36. Hahma, A.; Gany, A.; Palovuori, K. Combustion of activated aluminum. Combust. Flame 2006, 145, 464-480. [CrossRef]

37. Yogodnikov, D.A.; Andreev, E.A.; Vorob'ev, V.S.; Glotov, O.G. Ignition, combustion, and agglomeration of encapsulated aluminum particles in a composite solid propellant. I. Theoretical study of the ignition and combustion of aluminum with fluorine-containing coatings. Combust. Explos. Shock Waves 2006, 42, 153-155. [CrossRef]

38. Dreizin, E.; Schoenitz, M. Reactive and Metastable Nanomaterials Prepared by Mechanical Milling. In Metal Nanopowders Production, Characterization, and Energetic Applications; Gromov, A.A., Teipel, U., Eds.; Wiley VCH: Weinheim, Germany, 2014; pp. 227-278.

39. Maggi, F.; Dossi, S.; Paravan, C.; DeLuca, L.T.; Liljedhal, M. Activated aluminum powders for space propulsion. Powder Technol. 2015, 270, 46-52. [CrossRef]

40. Sossi, A.; Duranti, E.; Paravan, C.; DeLuca, L.T.; Vorozhtsov, A.B.; Gromov, A.A.; Pautova, Y.I.; Lerner, M.I.; Rodkevich, N.G. Non-isothermal oxidation of aluminum nanopowder coated by hydrocarbons and fluorohydrocarbons. Appl. Surf. Sci. 2013, 271, 337-343. [CrossRef]

41. Paravan, C.; Reina, A.; Sossi, A.; Manzoni, M.; Massini, G.; Rambaldi, G.; Duranti, E.; Adami, A.; Seletti, E.; DeLuca, L.T. Time-resolved Regression Rate of Innovative Solid Fuel Formulations. In Advances in Propulsion Physics; DeLuca, L.T., Bonnal, C., Haidn, O., Frolov, S., Eds.; Torus Press: Moscow, Russia, 2013; Volume 4, pp. 75-98. 
42. DeLuca, L.T.; Galfetti, L.; Maggi, F.; Colombo, G.; Merotto, L.; Boiocchi, M.; Paravan, C.; Reina, A.; Tadini, P.; Fanton, L. Characterization of HTPB-based solid fuel formulations: Performance, mechanical properties, and pollution. Acta Astronaut. 2013, 92, 150-162. [CrossRef]

43. Zare, A.; Harriman, T.A.; Lucca, D.A.; Roncalli, S.; Kosowski, B.; Paravan, C.; DeLuca, L.T. Mapping of aluminum particle dispersion in solid rocket fuel formulations. In Chemical Rocket Propulsion: A Comprehensive Survey of Energetic Materials; DeLuca, L.T., Shimada, T., Sinditskii, V.P., Calabro, M., Eds.; Springer: Berlin, Germany, 2017; pp. 673-688.

44. Teipel, U.; Förter-Barth, U. Rheology of nano-aluminum suspensions. Propellants Explos. Pyrotech. 2001, 26, 268-272. [CrossRef]

45. Sippel, T.; Son, S.F.; Groven, L.J. Altering the reactivity of aluminum with selective inclusion of polytetrafluoroethylene through mechanical activation. Propellants Explos. Pyrotech. 2013, 38, $286-295$. [CrossRef]

46. Sippel, T.R.; Son, S.F.; Groven, L.J.; Zhang, S.; Dreizin, E.L. Exploring mechanisms for agglomerate reduction in composite solid propellants with polyethylene inclusion modified aluminum. Combust. Flame 2015, 162, 846-854. [CrossRef]

47. Terry, B.C.; Son, S.F.; Groven, L.J. Altering combustion of silicon/polytetrafluoroethylene with two-step mechanical activation. Combust. Flame 2015, 162, 1350-1357. [CrossRef]

48. Dossi, S.; Paravan, C.; Maggi, F.; Di Lorenzo, M.; Ardalic, J.; Galfetti, L. Novel activated metal powders for improved hybrid fuels and green solid propellants. In Proceedings of the 52nd AIAA/SAE/ASEE Joint Propulsion Conference, Salt Lake City, UT, USA, 25-27 July 2016. AIAA Paper No. 2016-4596.

49. Chiaverini, M.J.; Serin, N.; Johnson, D.; Lu, Y.C.; Kuo, K.K.; Risha, G.A. Regression Rate Behavior of Hybrid Rocket Solid Fuels. J. Propul. Power 2000, 16, 125-132. [CrossRef]

50. Risha, G.A.; Ulas, A.; Boyer, E.; Kumar, S.; Kuo, K.K. Combustion of HTPB-Based Solid Fuels for Containing Nano-Sized Energetic Powder in a Hybrid Rocket Motor. In Proceedings of the 37th Joint Propulsion Conference and Exhibit, Salt Lake City, UT, USA, 8-11 July 2001. AIAA Paper No. 2001-3535.

51. Risha, G.A.; Boyer, E.; Wehrman, R.B.; Kuo, K.K. Performance Comparison of HTPB-Based Solid Fuels Containing Nano-Sized Energetic Powder in a Cylindrical Hybrid Rocket Motor. In Proceedings of the 38th AIAA/ASME/SAE/ASEE Joint Propulsion Conference \& Exhibit, Indianapolis, IN, USA, 7-10 July 2002. AIAA Paper No. 2002-3576.

52. Risha, G.A.; Evans, B.; Boyer, E.; Wehrman, R.B.; Kuo, K.K. Nano-Sized Aluminum and Boron-Based Solid Fuel Characterization in a Hybrid Rocket Engine. In Proceedings of the 39th AIAA/ASME/SAE/ASEE Joint Propulsion Conference and Exhibit, Huntsville, AL, USA, 20-23 July 2003. AIAA Paper No. 2003-4593.

53. Fanton, L.; Paravan, C.; DeLuca, L.T. Testing and modeling fuel regression rate in a miniature hybrid burner. Int. J. Aerosp. Eng. 2012, 2012, 673838. [CrossRef]

54. Sossi, A.; Duranti, E.; Manzoni, M.; Paravan, C.; DeLuca, L.T.; Vorozhtsov, A.B.; Lerner, M.I.; Rodkevich, N.G.; Gromov, A.A.; Savin, N.L. Combustion of HTPB-based solid fuels loaded with coated nanoaluminum. Combust. Sci. Technol. 2013, 185, 17-36. [CrossRef]

55. Carmicino, C.; Sorge, A.R. Experimental Investigation into the Effect of Solid-Fuel Additives on Hybrid Rocket Performance. J. Propul. Power 2015, 31, 699-713. [CrossRef]

56. Thomas, J.; Petersen, E.L.; DeSain, J.D.; Brady, B.B. Enhancement of Regression Rates in Hybrid Rockets with HTPB Fuel Grains by Metallic Additives. In Proceedings of the 51st AIAA/SAE/ASEE Joint Propulsion Conference, Orlando, FL, USA, 27-29 July 2015. AIAA Paper No. 2015-4041.

57. 3M Material Safety Datasheet. Available online: http://www.machichemicals.com/pdf/3M_FC-2175.pdf (accessed on 19 July 2019).

58. George, P.; Krishnan, S.; Varkey, P.M.; Ravindran, M.; Ramachandran, L. Fuel regression rate enhancement studies in HTPB/GOX hybrid rocket motors. In Proceedings of the 34th AIAA/ASME/SAE/ASEE Joint Propulsion Conference and Exhibit, Cleveland, OH, USA, 13-15 July 1998. AIAA Paper No. 1998-3188.

59. Frederick, R.A.; Whitehead, J.J.; Knox, L.R.; Moser, M.D. Regression rates study of mixed hybrid propellants. J. Propul. Power 2007, 23, 175-180. [CrossRef]

60. Lengellé, G. Solid-Fuel Pyrolysis Phenomena and Regression Rate, Part 1: Mechanisms. In Fundamentals of Hybrid Rocket Combustion and Propulsion; Chiaverini, M.J., Kuo, K.K., Eds.; AIAA Progress in Astronautics and Aeronautics: Reston, VA, USA, 2007; Volume 218, pp. 127-166. 
61. Strand, L.D.; Ray, R.L.; Cohen, N.S. Hybrid rocket combustion study. In Proceedings of the 29th Joint Propulsion Conference and Exhibit (AIAA), Monterey, CA, USA, 28-30 July 1993. AIAA Paper No. 1993-2412.

62. Strand, L.D.; Jones, M.D.; Ray, R.L.; Cohen, N.S. Characterization of hybrid rocket internal heat flux and HTPB fuel pyrolysis. In Proceedings of the 30th Joint Propulsion Conference and Exhibit (AIAA), Indianapolis, IN, USA, 27-29 June 1994. AIAA Paper No. 1994-2876.

63. Paravan, C.; Manzoni, M.; Rambaldi, G.; DeLuca, L.T. Analysis of quasi-steady and transient burning of hybrid fuels in a laboratory-scale burner by an optical technique. Int. J. Energ. Mater. Chem. Propuls. 2013, 12, 385-410. [CrossRef]

64. Chen, S.; Tang, Y.; Zhang, W.; Shen, R.; Yu, H.; Ye, Y.; DeLuca, L.T. Innovative Methods to Enhance the Combustion Properties of Solid Fuels for Hybrid Rocket Propulsion. Aerospace 2019, 6, 47. [CrossRef]

65. Bisin, R.; Paravan, C.; Alberti, S.; Galfetti, L. An Innovative Strategy for Paraffin-based Fuels Reinforcement: Part I, Mechanical and Pre-Burning Characterization. In Proceedings of the 8th European Conference for Aeronautics and Space Sciences (EUCASS 2019), Madrid, Spain, 1-4 July 2019; pp. 1-14.

66. Bisin, R.; Paravan, C.; Verga, A.; Galfetti, L. An Innovative Strategy for Paraffin-based Fuels Reinforcement: Part II, Ballistic Characterization. In Proceedings of the 8th European Conference for Aeronautics and Space Sciences (EUCASS 2019), Madrid, Spain, 1-4 July 2019; pp. 1-14.

67. Grosse, M. Effect of a Diaphragm on Effect of a Diaphragm on Performance and Fuel Regression of a Laboratory Scale Hybrid Rocket Motor Using Nitrous Oxide and Paraffin. In Proceedings of the 45th AIAA/ASME/SAE/ASEE Joint Propulsion Conference \& Exhibit, Denver, CO, USA, 2-5 August 2009. AIAA Paper No. 2009-5113.

68. Yuasa, S.; Shiraishi, N.; Hirata, K. Controlling Parameters for Fuel Regression Rate of Swirling-oxidizer-flow-type Hybrid Rocket Engine. In Proceedings of the 48th AIAA/ASME/SAE/ASEE Joint Propulsion Conference \& Exhibit, Atlanta, GA, USA, 30 July-1 August 2012. AIAA Paper No. 2012-4106.

69. Yuasa, S.; Yamamoto, K.; Hachiya, H.; Kitagawa, K.; Oowada, Y. Development of a small sounding hybrid rocket with a swirling-oxidizer-type engine. In Proceedings of the 37th Joint Propulsion Conference and Exhibit (AIAA), Salt Lake City, UT, USA, 8-11 July 2001. AIAA Paper No. 2001-3537.

70. Ozawa, K.; Kitagawa, K.; Aso, S.; Shimada, T. Hybrid Rocket Firing Experiments at Various Axial-Tangential Oxidizer-Flow-Rate Ratios. J. Propul. Power 2019, 35, 94-108. [CrossRef]

71. Paravan, C.; Glowacki, J.; Carlotti, S.; Maggi, F.; Galfetti, L. Vortex Combustion in a Lab-scale Hybrid Rocket Motor. In Proceedings of the 52nd AIAA/SAE/ASEE Joint Propulsion Conference, Salt Lake City, UT, USA, 25-27 July 2016. AIAA Paper No. 2016-4562.

72. Connell, T.L., Jr.; Yetter, R.A.; Risha, G.A.; Huba, Z.J.; Epsheytn, A.; Fisher, B.T. Enhancement of Solid Fuel Combustion in a Hybrid Rocket Motor Using Amorphous Ti-Al-B Nanopowder Additives. J. Propul. Power 2019, 35, 662-664. [CrossRef]

73. Connell, T.L., Jr.; Risha, G.A.; Yetter, R.A.; Roberts, C.W.; Young, G. Boron and Polytetrafluoroethylene as a Fuel Composition for Hybrid Rocket Applications. J. Propul. Power 2015, 31, 373-385. [CrossRef]

74. Morothiya, G.; Ramakrishna, P.A. Utilization of Mechanical Activated Aluminum in Hybrid Rockets. J. Propul. Power 2018, 34, 1206-1213. [CrossRef]

75. Price, E.W.; Sigman, R.K. Combustion of Aluminized Solid Propellants. In Solid Propellant Chemistry, Combustion, and Motor Interior Ballistics; Yang, V., Brill, T.B., Ren, W.Z., Eds.; AIAA Progress in Astronautics and Aeronautics: Reston, VA, USA, 2000; Volume 185, pp. 66-687.

76. Babuk, V.A.; Vassiliev, V.A.; Sviridov, V.V. Formation of Condensed Combustion Products at the Burning Surface of Solid Rocket Propellant. In Solid Propellant Chemistry, Combustion, and Motor Interior Ballistics; Yang, V., Brill, T.B., Ren, W.Z., Eds.; AIAA Progress in Astronautics and Aeronautics: Reston, VA, USA, 2000; Volume 185, pp. 749-776.

77. DeLuca, L.T.; Galfetti, L.; Colombo, G.; Maggi, F.; Bandera, A.; Babuk, V.A.; Sindistskii, V.P. Microstructure Effects in Aluminized Solid Rocket Propellants. J. Propul. Power 2010, 26, 724-733. [CrossRef]

78. Gany, A. Micro and Nano Scale Phenomena of Aluminum Agglomeration during Solid Propellant Combustion. Eurasian Chem. Technol. J. 2016, 18, 161-170. [CrossRef]

79. Caveny, L.; Gany, A. Breakup of $\mathrm{Al} / \mathrm{Al}_{2} \mathrm{O}_{3}$ Agglomerates in Accelerating Flowfields. AIAA J. 1979, 17, 1368-1371. [CrossRef]

80. Rashkovskii, S.A. Statistical Simulation of Aluminum Agglomeration during Combustion of Heterogeneous Condensed Mixtures. Combust. Explos. Shock Waves 2005, 41, 174-184. [CrossRef] 
81. Jackson, T.; Najjar, F.; Buckmaster, J. New Aluminum Agglomeration Models and Their Use in Solid Propellant Rocket Simulations. J. Propul. Power 2005, 21, 925-936. [CrossRef]

82. Maggi, F.; DeLuca, L.T.; Bandera, A. A Pocket Model for Aluminum Agglomeration Based on Propellant Microstructure. AIAA J. 2015, 53, 3395-4303. [CrossRef]

83. Plaud, M.; Gallier, S. A Numerical Mesoscale Model for Aluminum Agglomeration in Solid Propellants. In Proceedings of the 7th European Conference for Aeronautics and Space Sciences (EUCASS 2017), Milan, Italy, 1-4 July 2017.

84. DeLuca, L.T.; Paravan, C.; Reina, A.; Marchesi, E.; Maggi, F.; Bandera, A.; Colombo, G.; Kosowski, B Aggregation and Incipient Agglomeration in Metallized Solid Propellants and Solid Fuels for Rocket Propulsion. In Proceedings of the 46th AIAA/ASME/SAE/ASEE Joint Propulsion Conference \& Exhibit, Nashville, TN, USA, 25-28 July 2010. AIAA Paper No. 2010-6752.

85. Purdue University. Heats of Formation and Chemical Compositions. Available online: https://engineering. purdue.edu/ \{\}propulsi/propulsion/comb/propellants.html (accessed on 19 April 2018).

86. Koch, E.C.; Hahma, A.; Klapotke, T.M.; Radies, H. Metal-Fluorocarbon Pyrolants: XI. Radiometric Performance of Pyrolants Based on Magnesium, Perfluorinated Tetrazolates, and Viton-A. Propellants Explos. Pyrotech. 2010, 35, 248-253. [CrossRef]

87. Koch, E.C. (Ed.) Metal-Fluorocarbon Based Energetic Materials; Wiley-VCH Verlag GmbH: Weinheim, Germany, 2012.

88. Biswas, S.W.; Vijaian, K. Friction and wear of PTFE-A review. Wear 1992, 158, 193-211. [CrossRef]

(C) 2019 by the author. Licensee MDPI, Basel, Switzerland. This article is an open access article distributed under the terms and conditions of the Creative Commons Attribution (CC BY) license (http://creativecommons.org/licenses/by/4.0/). 

Article

\title{
Nytrox as "Drop-in" Replacement for Gaseous Oxygen in SmallSat Hybrid Propulsion Systems
}

\author{
Stephen A. Whitmore \\ Department of Mechanical and Aerospace Engineering, Utah State University, Logan, UT 84322, USA; \\ stephen.whitmore@usu.edu; Tel.: +1-435-797-2951
}

Received: 13 February 2020; Accepted: 19 March 2020; Published: 12 April 2020

\begin{abstract}
A medical grade nitrous oxide $\left(\mathrm{N}_{2} \mathrm{O}\right)$ and gaseous oxygen (GOX) "Nytrox" blend is investigated as a volumetrically-efficient replacement for GOX in SmallSat-scale hybrid propulsion systems. Combined with 3-D printed acrylonitrile butadiene styrene (ABS), the propellants represent a significantly safer, but superior performing, alternative to environmentally-unsustainable spacecraft propellants like hydrazine. In a manner analogous to the creation of soda-water using dissolved carbon dioxide, Nytrox is created by bubbling GOX under pressure into $\mathrm{N}_{2} \mathrm{O}$ until the solution reaches saturation. Oxygen in the ullage dilutes $\mathrm{N}_{2} \mathrm{O}$ vapor and increases the required decomposition energy barrier by several orders of magnitude. Thus, risks associated with inadvertent thermal or catalytic $\mathrm{N}_{2} \mathrm{O}$ decomposition are virtually eliminated. Preliminary results of a test-and-evaluation campaign are reported. A small spacecraft thruster is first tested using gaseous oxygen and 3-D printed ABS as the baseline propellants. Tests were then repeated using Nytrox as a "drop-in" replacement for GOX. Parameters compared include ignition reliability, latency, initiation energy, thrust coefficient, characteristic velocity, specific impulse, combustion efficiency, and fuel regression rate. Tests demonstrate Nytrox as an effective replacement for GOX, exhibiting a slightly reduced specific impulse, but with significantly higher volumetric efficiency. Vacuum specific impulse exceeding $300 \mathrm{~s}$ is reported. Future research topics are recommended.
\end{abstract}

Keywords: hybrid rocket; "green" propellants; nitrous oxide decomposition; energy of activation; 3-D printing

\section{Introduction}

Over the past decade the Utah State University Propulsion Research Laboratory (USU-PRL) has developed and refined a novel High-Performance "Green" Hybrid Propulsion (HPGHP) system as an environmentally sustainable replacement for hydrazine and other highly-toxic propellants. In its most mature form, the HPGHP system uses gaseous oxygen (GOX) as the oxidizer with 3-D printed acrylonitrile-butadiene-styrene (ABS) as the fuel. The GOX/ABS propellants are highly mass efficient system, with a prototype light-weight $25 \mathrm{~N}$ thrust chamber achieving a vacuum specific impulse $\left(I_{\mathrm{sp}}\right)$ greater than $300 \mathrm{~s}$ [1]. Unfortunately, unless stored at very high pressures, GOX has a low specific gravity and is a volumetrically inefficient propellant. A higher density "green" alternative is highly desirable.

This paper will investigate the potential to use a blend of medical grade Nitrous Oxide $\left(\mathrm{N}_{2} \mathrm{O}\right)$ and gaseous oxygen (GOX), typically used for anesthesia applications, as a "drop-in" replacement for GOX in a legacy small spacecraft thruster system. Using Nytrox as the oxidizer in place of GOX allows a significant increase in overall volumetric efficiency, and with similar hazard levels. As will be described later in this paper, the "Nytrox" mixture is preferred to pure $\mathrm{N}_{2} \mathrm{O}$ which exhibits a significant potential decomposition hazard. Nytrox exhibits more than an order of magnitude increase in the activation energy for decomposition as compared to pure $\mathrm{N}_{2} \mathrm{O}$, allowing a significant increase in storage and handling safety results. Results of a testing campaign to demonstrate the compatibility of Nytrox with the current HPGHP thruster technology will be presented. 


\section{Background on Green Propellant Options}

Two studies performed by the European Space Agency Space Research and Technology Center (ESTEC) [2,3] have identified: (1) reduced production, operations, and transport costs resulting from propellant toxicity and explosion hazards and (2) reduced costs due to subsystems and overall systems interface complexity, as essential components for achieving low cost space access and operations. The ESTEC study showed also potential for significant cost savings by simplifying propellant ground handling procedures. Developing a "green" alternative for most commonly used toxic or potentially-hazardous propellants, especially hydrazine, was highly recommended.

\subsection{Ionic Liquid Propellants}

In response to ESTEC's report and other "green" propellant [4] recommendations, the US Air Force (USAF) and the Swedish Space Corporation Ecological Advanced Propulsion Systems (ECAPS) center have developed less hazardous alternatives to hydrazine. The two most mature propellants are based on aqueous solutions of the ionic liquids (ILs) ammonium dinitramide (ADN) $[5,6]$ and hydroxylamine nitrate (HAN) [7,8]. In August 2011, ECAPS published results from a year-long series of in-space tests of a 1-N thruster comparing their high performance green propellant, known by the brand name LMP-103S, to hydrazine on the Prisma spacecraft platform [9]. The report states that LMP-103S delivered equivalent-to-superior performance to hydrazine, with a vacuum specific impulse of $230 \mathrm{~s}$. NASA selected the USAF-developed HAN-based propellant, known by the product name AF-M315E, for its "Green" Propellant Infusion Mission (GPIM) [10]. The GPIM thruster system was successfully deployed by a Space-X Falcon Heavy Booster on June 25, 2019 [11]. Results from the GPIM spaceflight test are reported by McLean et al. [12]. In spite of being called "green," by their manufacturers, IL-based propellants are generally not environmentally-friendly. Both of the above-mentioned IL-propellants are toxic to organic tissue, for example AF-M315E contains Hydroxyethyl hydrazine (HEHN) as an ignition enhancer and burn stabilizer. In in high concentrations IL-based propellants are prone to energetic uncontrolled decomposition events. Thus, special handling precautions are required for processing and storage. The major advantage of both propellants is a low-vapor pressure at room temperature, allowing handling and servicing without the use of Self Contained Atmospheric Protective Ensemble (SCAPE) suits. Because of these properties, the USAF has recently begun to refer to such IL-formulations more properly as having "reduced toxicity" instead of being green.

In addition to potential toxicity and objective hazards, there exist several key developmental issues that make them IL-based propellants unsuitable for some small spacecraft applications. In order to reduce the decomposition hazard, both the LMP-103S and AFM-315E mono-propellants require a significant water content to keep the IL-salts in solution with up to 14\% for LMP-103S [13], and 16\% for AFM-315E [14]. The high water content makes IL-propellants notoriously hard to ignite. Also, undecomposed water vapor in the exhaust plume poses a significant contamination hazard, clouding or obscuring sensitive spacecraft optics or photo-voltaic cells [15].

Multiple catalyst systems have been developed to augment IL ignitability, but due to the high water content, room temperature ignition does not currently exist for either propellant. During ignition the catalyst beds must be preheated to greater than $340{ }^{\circ} \mathrm{C}(573 \mathrm{~K})$ for LMP-103S [16] and $370{ }^{\circ} \mathrm{C}(643 \mathrm{~K})$ for AFM-315E [17]. This preheat requirement places a large power burden on the spacecraft systems. Catalyst beds and associated heating systems add significantly to the inert mass of the spacecraft and the high-wattage preheat requirement presents a significant disadvantage for systems where power budgets are limited. For example, as stated by Pokrupa et al. [18], for the ECAPS 1-N LMP-103S thruster, "time required for $9.25 \mathrm{~W}$ of catbed preheating (to a minimum of $340^{\circ} \mathrm{C}$ ) has been shown in flight to be roughly 600-720 s." Thus, at a minimum the startup-cycle for this system requires between 5.6 and $6.7 \mathrm{~kJ}$ of energy input. For the Prisma flight demonstration of Reference [9], the pre-heat time was set to $30 \mathrm{~min}$, requiring $16.7 \mathrm{~kJ}$ of total energy input. The high pre-heat temperature also significantly limits the catbed system burn lifetime. 
Finally, due to the very slow reaction kinetics demonstrated by Hori and Katsumi [19] for HAN, and Whitmore and Burnside [20] for ADN at the moderate pressures levels required for SmallSat Thruster systems (1000-2000 kPa), ignition latencies can be significant, up to several seconds, and may limit the usefulness of IL-propellants for spacecraft maneuvering and control systems. Even under steady-state operating conditions, when compared to conventional solid, hybrid, or bi-propellant options, the performances of ionic liquid propellants are generally quite low-with achieved vacuum $I_{\mathrm{sp}}$ values at or less than $230 \mathrm{~s}$. The combination of these detrimental characteristics compared to their "green" advantages have led some in the industry to question whether ionic liquids as propellants have been "oversold [21]." Clearly, significant technological issues exist and must be overcome before the ionic-liquid propellants are used routinely as a hydrazine replacement.

\subsection{Hybrid Rockets as a Significantly Safer and More Efficient "Green" Propulsion Alternative}

The inherent safety and environmental friendliness of hybrid rocket systems have been known for several decades [22]. Propulsion systems using hybrid propellants offer the potential to replace the current generation of toxic or hazardous propellants with an environmentally-sustainable "green" alternative. Hybrids require only a single flow path, which significantly reduces the overall complexity when compared to bi-propellant systems. In fact, hybrids present a similar complexity to monopropellant systems, but with significantly higher performance and efficiency levels. Properly-optimized hybrid propulsion systems can achieve $I_{\text {sp }}$ and characteristic velocity $\left(c^{*}\right)$ values comparable to non-cryogenic bi-propellant systems, and in a highly storable form factor. The primary drawback to the wide-ranging deployment of hybrid systems is a low Technology Readiness Level (TRL). As the TRL matures, small hybrid systems have potential to fill an unmet and growing need for advanced propulsion both in-space and during launch stages for the emerging SmallSat market. Although requiring a small increase in overall dry mass compared to conventional solid-propellant systems, capabilities for on-demand throttle, shut-down, and relight, offset this mass-disadvantage. Such a "smart-stage" can potentially provide $\Delta V$ for orbit access, on-orbit maneuvering, and station keeping. Such a system could also provide extensive capabilities for endo-atmospheric maneuvering for a variety of defense applications.

\subsection{Hybrid Rocket Low-Power Arc-Ignition System}

Hybrid rockets are "safe" due to the relative stability of both the oxidizer and fuel components. This stability comes at a price, as traditionally hybrid rocket systems require a significant energy input for reliable ignition. For the vast majority of hybrid rocket-systems developed to date, pyrotechnic or "squib" charges are used to ignite a secondary solid-propellant motor whose high-enthalpy output provides sufficient heat to overcome the activation energy of the propellants. While effective, this pyrotechnic approach allows only a single-shot ignition, with no inherent restart capability. Thus, until very recently, the lack of a reliable, low-energy multiple-use ignition method has discouraged the use of hybrid rockets for in-space propulsion. This technology gap has limited the full potential for hybrid systems as restartable upper stages or in-space maneuvering systems.

The above-described hybrid restart issue has been overcome by leveraging the unique electrical breakdown properties of certain 3-D printed thermoplastics like ABS or polyamide. The authors discovered that when printed using a technique known as fused deposition modeling (FDM) [23], ABS possesses unique electrical breakdown properties that can be exploited to allow for rapid on-demand ignition [24]. Under normal conditions, ABS possesses a very high electrical resistivity and is not generally considered as an electrical conductor. However, as FDM-processed ABS is subjected to a moderate electrostatic potential between electrodes embedded in the material, the layered FDM structure concentrates electrical charges at points along the surface. Figure 1 shows a typical pyrolysis event, where the ablated hydrocarbon vapor results from the inductive arc carving a path across the fuel surface [25]. Because Joule heating pyrolizes a small mass of fuel material, when an oxidizing flow is introduced at oxygen partial pressures above two atmospheres (Reference [24]), combustion is initiated with the pyrolized vapor. 
Shortly after this discovery, the authors of this paper made several attempts to reproduce a similar phenomenon with other 3-D printed and extruded fuel materials [26]. Only a few printed material exhibited the required arcing-properties, with ABS being the best. None of the extruded materials exhibited the required arcing properties. It appears that 3-D printing is an essential feature for low-energy arc-ignition. As developed over the past five years, the HPGHP arc-ignition system is extremely power-efficient and can be started, stopped, and restarted with a high degree of reliability [27]. Once started, the system can be sequentially fired with no additional energy inputs required.

Multiple prototype ground-test units with thrust levels varying from 4.5 to $900 \mathrm{~N}$ have been developed and tested [28,29]. On 25 March, 2018 a flight experiment containing a 10-N prototype of this thruster system was launched aboard a two-stage Terrier-Improved Malemute sounding rocket from Wallops Flight Facility. The launch achieved apogee of $172 \mathrm{~km}$, allowing more than $6 \mathrm{~min}$ in hard-vacuum environment above the Von-Karman line. The thruster was successfully fired five times in a hard vacuum environment. Whitmore and Bulcher (2018) [30] report the details of this flight test experiment.

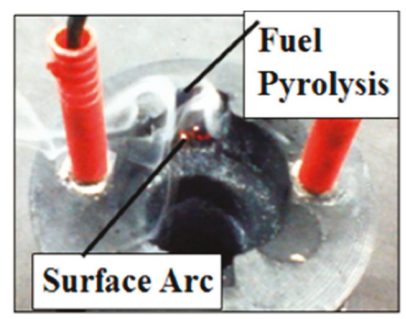

Figure 1. Inductive Charge Pyrolyzing acrylonitrile-butadiene-styrene (ABS) fuel. (Copyright Utah State University [1], reproduced with permission).

\subsection{Considering the Best Options for "Green" Oxidizers for Hybrid Space Propulsion}

Karabeyoglu (2014) [31] recently performed a comprehensive analytical study on oxidizer options for in-space hybrid propulsion systems. Key performance parameters or other beneficial characteristics identified by this study include non-toxicity, high characteristic velocity $\left(\mathrm{c}^{*}\right)$ and impulse density $\left(\rho \mathrm{I}_{\mathrm{sp}}\right)$, space storability, and safe performance under cold-soak conditions. This study identified only four options that can be reasonably considerable as "green," and still offer a vacuum specific impulse competitive with existing liquid bi-propellant and IL-monopropellants. These are: (1) Liquid Oxygen (LOX), (2) Gaseous Oxygen (GOX), (3) Hydrogen Peroxide $\left(\mathrm{H}_{2} \mathrm{O}_{2}\right)$, and (4) Nitrous Oxide. $\left(\mathrm{N}_{2} \mathrm{O}\right)$. Unfortunately, the oxidizer that offered the best $\rho \mathrm{I}_{\text {sp }}$ performance, $\mathrm{LOX}$, is not space-storable and must be eliminated from consideration for the proposed application.

Recent work by Whitmore et al. [32,33] and other organizations [34,35] have adapted hybrid rockets for use with medium-to-high grade $(90 \%) \mathrm{H}_{2} \mathrm{O}_{2}$. Hydrogen peroxide is a very efficient and dense propellant, and is an extremely strong oxidizer. Unfortunately, unless used in very high concentrations $(>98 \%)$, hydrogen peroxide is difficult to ignite in a hybrid rocket, and is marginally functional with the previously-described arc-ignition system. Most applications using hydrogen peroxide rely on catalytic ignition, which remains a significant development issue. None of the existing hybrid systems using peroxide are able to achieve a reliable cold-start. Significant ignition time latencies are also experienced.

Experience based on the results of References [32,33] have lead the authors to conclude that $90 \%$ peroxide is an unsuitable oxidizer for in-space applications, where cold-soak commonly occurs. It is likely that significantly higher oxidizer solutions with concentrations at $98 \%$ or greater could have avoided these pooling issues, however, at these concentrations peroxide becomes a class 4 oxidizer, and is no longer really a "green" propellant. As $98 \%$ peroxide is extremely hazardous to work with, it presents objective hazards that are on-par with hydrazine. It appears, that hybrid rockets using lower concentration ( $90 \%$ or lower) peroxide, are more suitable for launch vehicle stages where significant 
external power can be used to pre-heat all of the flow path components, and ignition latencies can be absorbed into the mission timeline.

Having eliminated LOX and $\mathrm{H}_{2} \mathrm{O}_{2}$ from consideration, only GOX and $\mathrm{N}_{2} \mathrm{O}$ remain as in-space hybrid oxidizers compatible with the HPGHP arc-ignition system. Gaseous oxygen is an excellent oxidizer that can be efficiently managed at pressures well below $14,000 \mathrm{kPa}$ (140 atmospheres) [36]. Unfortunately, GOX, even when stored at high pressures, has a low specific gravity and is a volumetrically inefficient propellant. For space missions requiring even moderate $\Delta V$ levels, GOX is an impractical propellant. Thus, by process of elimination $\mathrm{N}_{2} \mathrm{O}$ remains as the primary oxidizer option for this project. Nitrous oxide is by far the most commonly used oxidizer hybrid rocket "hobbyists". Nitrous oxide is an inexpensive, readily available and has the clear advantage of being non-toxic to human tissue.

\subsection{Hazards Associated with Using Nitrous Oxide as a Hybrid Rocket Propellant}

Nitrous Oxide exists as a saturated liquid below its critical temperature of $36.4^{\circ} \mathrm{C}$, and propulsion applications typically must deal with $\mathrm{N}_{2} \mathrm{O}$ in both liquid and vapor form. Studies performed by Rhodes at the Air Force Weapons Lab (AFWL) [37] show that it is nearly impossible to detonate liquid phase $\mathrm{N}_{2} \mathrm{O}$ in pure form. In fact, liquid-phase $\mathrm{N}_{2} \mathrm{O}$ is classified as non-explosive and non-flammable by the US Occupational Safety and Health Administration (OHSA) [38,39]. However, in vapor phase $\mathrm{N}_{2} \mathrm{O}$ can experience rapid thermal decomposition (Reference [37]), especially in the presence of contamination. The $\mathrm{N}_{2} \mathrm{O}$ decomposition reaction, as given by Equation (1), is strongly exothermic and releases heat up to $1864 \mathrm{~kJ} / \mathrm{kg}$ for full decomposition. Decomposition also rapidly produces large volumes of nitrogen and oxygen gas. Output gas temperatures can exceed $1900 \mathrm{~K}\left(1627^{\circ} \mathrm{C}\right)$. The combination of heat and high-temperature gas-volumes make the decomposition event potentially explosive.

$$
\mathrm{N}_{2} \mathrm{O} \rightarrow \mathrm{N}_{2(g)}+\frac{1}{2} \mathrm{O}_{2(g)}+\text { Heat }
$$

Generally, $\mathrm{N}_{2} \mathrm{O}$ has a large activation energy, $\mathrm{E}_{\mathrm{a}}$, for thermal decomposition, $5682 \mathrm{~kJ} / \mathrm{kg}$, and $\mathrm{N}_{2} \mathrm{O}$ vapor must be heated to temperatures near $1000{ }^{\circ} \mathrm{C}$ in order to induce thermal decomposition [40]. However, when $\mathrm{N}_{2} \mathrm{O}$ vapor is contaminated by a small amount of hydrocarbon material, the relative stability is lowered and $\mathrm{E}_{\mathrm{a}}$ drops significantly. Figure 2 illustrates the concept where "hydrocarbon-seeding" lowers the activation energy to a point where decomposition reactions can occur at near room-temperatures [41]. In effect, the addition of hydrocarbon material to nitrous oxide catalyzes the decomposition event. Because $\mathrm{N}_{2} \mathrm{O}$ is a highly polar molecule and an exceptionally good solvent, it readily picks up and dissolves hydrocarbons or other contaminating materials that may be exposed to the flow path. Since only a small amount of contamination is needed to catalyze decomposition, this physical property further exacerbates the potential safety hazards associated with $\mathrm{N}_{2} \mathrm{O}$ propulsion applications.

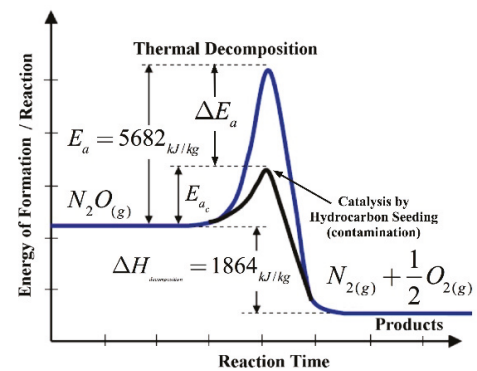

Figure 2. Hydrocarbon seeding reduces the $\mathrm{N}_{2} \mathrm{O}$ decomposition energy barrier. 
For flight applications, close-coupling of the oxidizer tank with the motor case creates a significant chance of introducing contamination into the thrust chamber. As the motor burns and nitrous oxide is depleted, adiabatic cooling forces a significant drop in the tank vapor pressure. This internal pressure drop provides the opportunity for backflow across the injector, allowing hot hydrocarbon gasses to enter the oxidizer feed lines and possibly the lower portion of the tank itself. The result is a significant potential for a run-away decomposition reaction. A notable number of accidents resulting from runaway $\mathrm{N}_{2} \mathrm{O}$ decomposition events have occurred. Karabeyoglu et al. (2008) [41] presented a summary of nine verified accidents related to inadvertent, uncontrolled nitrous oxide decomposition events.

\subsection{Mitigation of the $\mathrm{N}_{2} \mathrm{O}$ Decomposition Hazard}

Fortunately, it appears following procedures developed by the medical and dental anesthesia community offers a strong mitigation to this decomposition hazard [42]. In a manner directly analogous to creation of soda-water using dissolved carbon dioxide, an $\mathrm{N}_{2} \mathrm{O} / \mathrm{O}_{2}$ hybrid solution, referred to as Nytrox for the remainder of this paper, is created by bubbling gaseous $\mathrm{O}_{2}$ under pressure into liquid $\mathrm{N}_{2} \mathrm{O}$ until the solution reaches saturation. The oxygen in solution displaces much of the nitrous oxide vapor in the tank ullage, significantly reducing the potential for a decomposition reaction, and allows "laughing gas" to be safely worked with in a clinical environment.

Figure 3 plots the vapor/liquid isotherm diagram for a saturated "Nytrox" solution. Figure 3a plots the vapor and Figure $3 \mathrm{~b}$ plots the liquid phase mass concentrations of oxygen in solution as a function pressure and for isotherm curves for temperatures varying from $-30^{\circ} \mathrm{C}$ to $30^{\circ} \mathrm{C}$. The $0^{\circ} \mathrm{C}$ isotherm is highlighted as the solid blue line for both the liquid and vapor segments of the chart. The calculations of Figure 3 were performed using the Peng-Robinson [43] two-phase state-equation for binary solutions. The mixing rule used to combine the $\mathrm{O}_{2}$ and $\mathrm{N}_{2} \mathrm{O}$ binary components is based on the model of Zudkevitch and Joffe [44]. At phase equilibrium, the fugacity (for the purposes of this model the fugacity may be defined as the pressure of an ideal gas which has the same Gibbs free energy as the real gas at the same temperature. Fugacity-the "tendency to escape" from solution-is the measure of a mixture component's values for the liquid and vapor phases of each fluid component are equal. The implemented numerical algorithm that performs these calculations follows the procedure laid out by Karabeyoglu, Reference [31]. Reading the diagrams of Figure 3, at $0{ }^{\circ} \mathrm{C}$ and approximately 86 atmospheres $(8745 \mathrm{kPa})$, there exists a "sweet spot" where the concentration of oxygen in the vapor-phase is a maximum (note that the pressure required to hold the $\mathrm{O}_{2}$ in solution is significantly higher than the natural vapor pressure of $\mathrm{N}_{2} \mathrm{O}$, approximately 30 atmospheres at $0^{\circ} \mathrm{C}$ ) [45]. This optimal point, noted by the circular symbol on the graphs, shows that the vapor phase contains approximately $37 \% \mathrm{O}_{2}$ mass fraction, while the $\mathrm{O}_{2}$ mass fraction in the liquid phase lies at just below $13 \%$. This near-optimal point allows for the maximum proportion of vapor dilution while maintaining the highest possible density for the liquid phase.

a) Vapor, Dissolved $\mathrm{O}_{2}$ Fraction

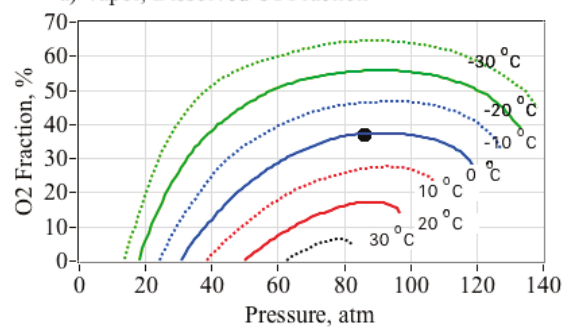

b) Liquid, Dissolved $\mathrm{O}_{2}$ Fraction

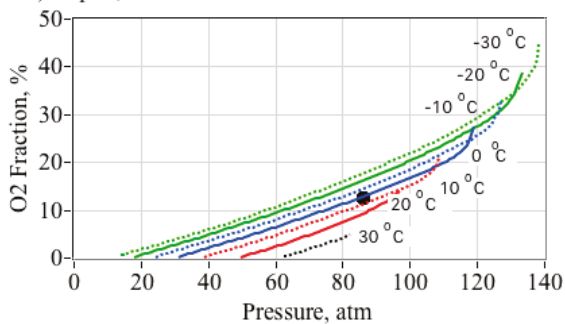

Figure 3. Nytrox vapor/liquid isotherm plots, mass fraction of $\mathrm{O}_{2}$ in solution. (Copyright Utah State University [46], reproduced with permission). 
Because $\mathrm{O}_{2}$ in solution dilutes the $\mathrm{N}_{2} \mathrm{O}$ vapor in the tank ullage, the required ignition energy $E_{\mathrm{i}}$, defined as the minimum energy required to initiate a deflagration wave, is significantly increased. Assuming a spherical flame kernel, when the input energy is smaller than $E_{\mathrm{i}}$, the resulting flame front decays rapidly because the released heat diffuses away from the surface faster that can be replaced by the decomposing material in the kernel volume. Dilution of the $\mathrm{N}_{2} \mathrm{O}$ vapor with $\mathrm{O}_{2}$ effectively increases the kernel volume, reducing the energy density. The diluent gas also acts to absorb heat, further quenching the reaction.

Figure 4 illustrates the effect of the $\mathrm{O}_{2}$ dilution in the Nytrox vapor phase upon $E_{\mathrm{i}}$. The data of Figure 4 are extrapolated from data presented by References [31] and [47]. For this calculation, Reference [31] assumes a spherical ignition kernel. Figure 4a plots the minimum ignition energy as a function of ullage $\mathrm{O}_{2}$ mass fraction for pressure levels varying from 40 to 100 atmospheres. Figure $4 \mathrm{~b}$ plots the ratio of the ignition energy at a given $\mathrm{O}_{2}$ mass concentration to the value for pure $\mathrm{N}_{2} \mathrm{O}$ vapor at the same pressure level. This ratio is referred to as the ignition energy amplification factor, $E_{\text {if }}$. Also plotted as solid symbols are the $E_{\mathrm{i}}$ values for Nytrox solutions at the $\mathrm{O}_{2}$ vapor mass fractions calculated (from Figure 3 ) at the given pressure levels and $0{ }^{\circ} \mathrm{C}$ storage temperature. Depending upon the shape of the flame kernel, the absolute energies of Figure 4a may vary, but the ratio plotted by Figure $4 \mathrm{~b}$ will remain generally constant.

Note that near the previously-described "sweet spot" pressure level at $86 \mathrm{~atm}$, the ignition energy has increased from less than 1 joule for pure $\mathrm{N}_{2} \mathrm{O}$, to a value greater than 2200 Joules for the Nytrox solution. This increase amounts to an amplification factor $\mathrm{E}_{\mathrm{if}}$ of greater than 10,000 or four orders of magnitude! This buffering effect significantly increases the handling safety for Nytrox, and it may be reasonably concluded that ignition cannot be achieved by any conceivable inadvertent ignition source. Only direct and purposeful action, such as the arc-ignition system or a pyrotechnic device can achieve the required ignition energy.

a) Minimum Ignition Energy

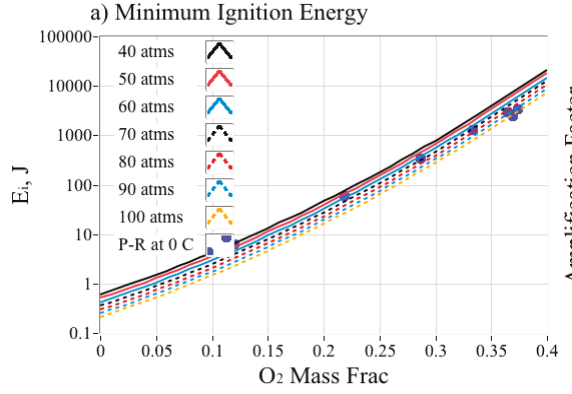

b) Minimum Ignition Energy Amplification Factor

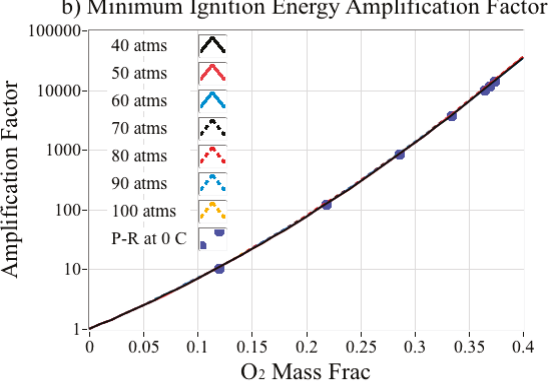

Figure 4. Minimum ignition energy $\mathrm{E}_{\mathrm{i}}$ for nytrox mixtures at 7 storage pressure levels. (A portion of this data is taken from Reference [31], data reproduced with permission).

\subsection{Effect of $\mathrm{O}_{2}$ in Solution Upon the Nytrox Mixture Density}

Figure 5 plots the densities of the Nytrox vapor and liquid phases, as calculated by the previously discussed Peng-Robinson model. Referring to Figures 3 and 5, near the "sweet spot" where the vapor $\mathrm{O}_{2}$ mass concentration peaks at $0{ }^{\circ} \mathrm{C}$ and 86 atmospheres, the liquid-phase density is approximately $780 \mathrm{~kg} / \mathrm{m}^{3}$. At this point the liquid-phase Nytrox density is slightly lower than for saturated nitrous saturated oxide at the same temperature, or approximately $900 \mathrm{~kg} / \mathrm{m}^{3}$. Thus, at the optimal point Nytrox is only $13 \%$ less dense than pure nitrous oxide. At a higher storage pressure of 120 atmospheres $(12,160 \mathrm{kPa})$ and $0{ }^{\circ} \mathrm{C}$, the percentage of $\mathrm{N}_{2} \mathrm{O}$ in the liquid-phase drops to around $70 \%$ with a corresponding density of only $600 \mathrm{~kg} / \mathrm{m}^{3}$. This difference amounts to a density drop of more than $40 \%$ compared to saturated $\mathrm{N}_{2} \mathrm{O}$ at the same temperature. This behavior occurs because the nitrous oxide and oxygen become mutually dissolved in each other, and as the oxygen content in solution rises, 
the density drops. Thus, maintaining the storage pressure near the best value of 80 atmospheres is essential to achieving volumetric efficiency with Nytrox.
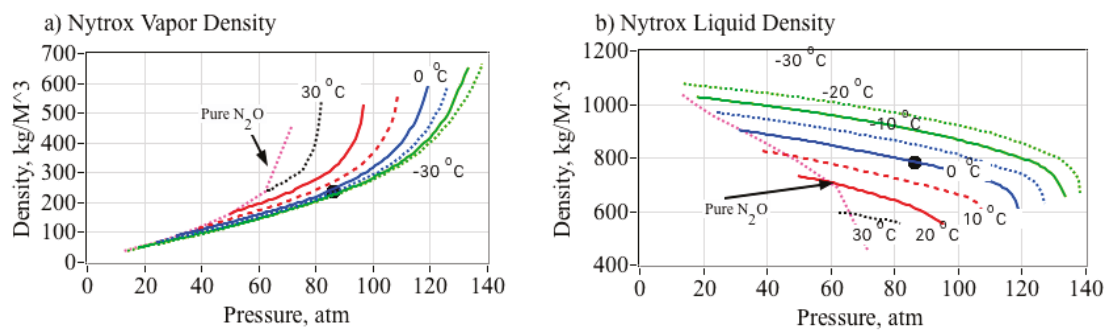

Figure 5. Density of nytrox vapor and liquid phases at vapor pressure for 7 different isotherms. (Copyright Utah State University [46], reproduced with permission).

\subsection{Predicted Propulsion Performance of the Nytrox/ABS Hybrid}

Although, when compared to pure $\mathrm{N}_{2} \mathrm{O}$ the liquid-phase density of Nytrox is slightly lower, the $\mathrm{O}_{2}$ in solution moderately increases the potential specific impulse $\left(I_{\mathrm{sp}}\right)$ and allows a significant reduction of the optimal oxidizer-to-fuel $(O / F)$ ratio. This performance-trade results in Nytrox being only slightly less volumetrically efficient than pure $\mathrm{N}_{2} \mathrm{O}$, and significantly more mass efficient. Figure 6 compares the performance of five different "green" $\mathrm{O}_{2}$ and $\mathrm{N}_{2} \mathrm{O}$ blend options when burned with ABS as the fuel source. In Figure 6, the plotted curves with differing colors compare pure GOX (black) and $\mathrm{N}_{2} \mathrm{O}$ (red), against Nytrox with three different liquid-phase $\mathrm{O}_{2}$ mass proportions, $90 \% \mathrm{~N}_{2} \mathrm{O} / 10 \%$ $\mathrm{O}_{2}$ (blue), $70 \% \mathrm{~N}_{2} \mathrm{O} / 30 \% \mathrm{O}_{2}$ (green), and 50\% $\mathrm{N}_{2} \mathrm{O} / 50 \% \mathrm{O}_{2}$ (violet). For notational simplicity, the Nytrox blends are referred by the mass-percentage of $\mathrm{N}_{2} \mathrm{O}$ in the liquid-phase of the solution: Nytrox 90, Nytrox 70, and Nytrox 50, respectively. The ABS fuel assumes a typical composition with 33\%, 33\%, and $34 \%$ acrylonitrile, butadiene, and styrene monomer mole-fractions. The associated density of this fuel blend is $1.04 \mathrm{~g} / \mathrm{cm}^{3}$.

The calculations of Figure 6 were performed using the industry-standard NASA Chemical Equilibrium Program (CEA) [48], and assuming six different chamber pressure levels varying from 690 to $3450 \mathrm{kPa}$ (100 to 500 psia) The propellants are assumed to be initially stored at $0{ }^{\circ} \mathrm{C}$. Parameters plotted on Figure 6 include: (a) characteristic velocity $c^{*}$, (b) vacuum $I_{\mathrm{sp}}$, (c) specific gravity, and (d) impulse density $\rho \mathrm{I}_{\mathrm{sp}}$ (impulse density is defined as the product of the specific impulse multiplied by the mean effective density of the propellants. Impulse density is typically expressed in units of N.s/L). For this analysis impulse density is defined as the product of the mean propellant density and the vacuum specific impulse, expressed in the unit of N.s/L. For each color grouping the pressure curves are represented by the different line styles, with the highest pressure levels exhibiting the highest $\mathrm{c}^{*}$ and $I_{\text {sp levels. }}$

The vacuum $\mathrm{I}_{\mathrm{sp}}$ calculations allow a 25:1 nozzle expansion-ratio, with the combustion products frozen at the nozzle throat. The specific gravity calculation for GOX (relative to the weight of water at $20^{\circ} \mathrm{C}$ ) assumes a storage pressure of $86 \mathrm{~atm}(8745 \mathrm{kPa})$ at $0^{\circ} \mathrm{C}$. The specific gravity calculation for pure $\mathrm{N}_{2} \mathrm{O}$ uses the saturation liquid density at $0{ }^{\circ} \mathrm{C}$ (from Reference [45]). The specific gravity for the Nytrox blends are calculated using the Peng-Robinson model, with the liquid-density values taken from Figure $3 b$ for the appropriate $\mathrm{N}_{2} \mathrm{O} / \mathrm{O}_{2}$ mass proportions.

For comparison purposes, $I_{\mathrm{sp}}$ and $\rho I_{\mathrm{sp}}$ of monopropellant-hydrazine are also plotted on Figure $6 \mathrm{~b}, \mathrm{c}$. Note that vacuum $I_{\mathrm{sp}}$ significantly exceeds that of hydrazine for all of the hybrid oxidizers. Even though hydrazine has a significantly higher specific gravity, $\rho I_{\mathrm{sp}}$ for the hybrid using either pure nitrous oxide or Nytrox 90 is still greater significantly greater than for hydrazine. The low GOX storage density results in the lowest impulse density for all of the hybrid oxidizers. Using pure $\mathrm{N}_{2} \mathrm{O}$ gives the best volumetric efficiency, but results in the lowest specific impulse and requires significantly more oxidizer 
at the optimal $O / F$ ratio. The curve corresponding to the Nytrox 90 mixture gives the best compromise with a distinct $\rho I_{\text {sp }}$ optimum occurring at an $O / F$ ratio of approximately 4.

Also of note, the data displayed in Figure 6 show that chamber pressure has only a second order effect upon the relative performances of the propellants, with the highest chamber pressure $(3450 \mathrm{kPa})$ exhibiting approximately $5 \%$ greater $\mathrm{c}^{*}$ and $I_{\mathrm{sp}}$ than the lowest chamber pressure $(690 \mathrm{kPa})$. Chamber pressure has essentially no effect upon the impulse density. Thus, even though the results to be presented later in this paper consider a very small thruster system operating at low chamber pressures, the results have a wider range of applicability.
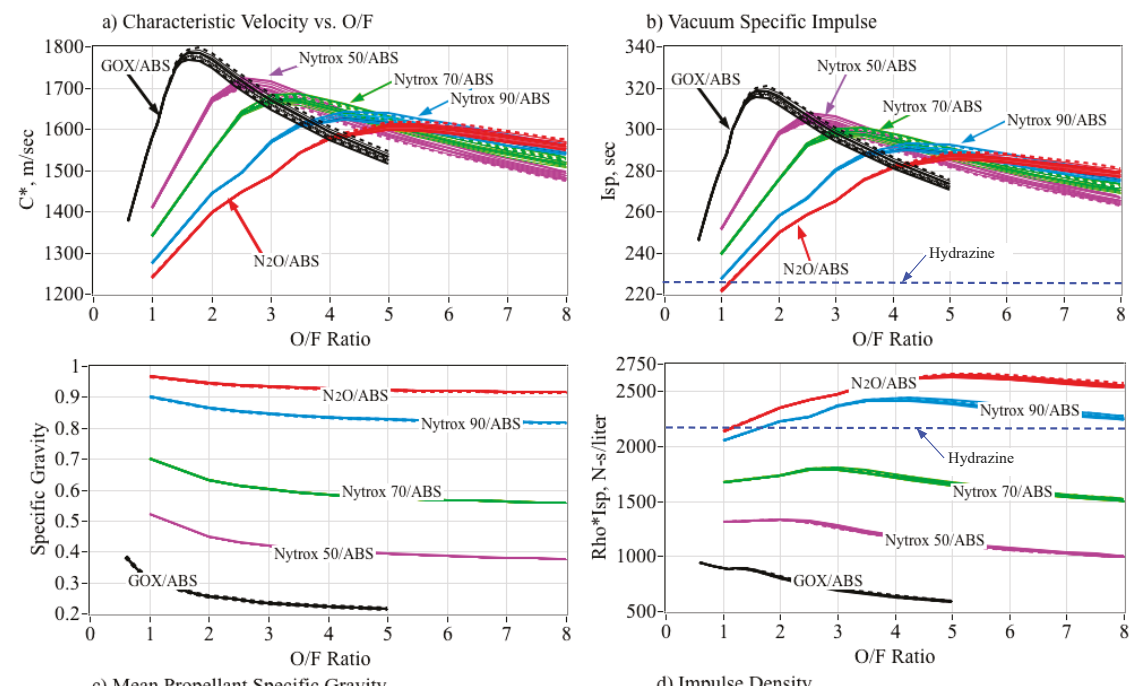

c) Mean Propellant Specific Gravity

d) Impulse Density

Figure 6. Performance of $3 \mathrm{~N}_{2} \mathrm{O} / \mathrm{O}_{2}$ concentrations compared against pure $\mathrm{N}_{2} \mathrm{O}$ and $\mathrm{GOX}$ as oxidizer. (Copyright Utah State University [46], reproduced with permission).

\subsection{Summary of Nytrox Properties and Safety Advantages Compared to Pure Nitrous Oxide}

The key advantages associated with using Nytrox mixtures to replace $\mathrm{N}_{2} \mathrm{O}$ as a hybrid oxidizer are:

(1) Nytrox is much safer than pure $\mathrm{N}_{2} \mathrm{O}$ because vapor phase has significant $\mathrm{O}_{2}$ concentration, thereby increasing the minimum ignition energy $E_{\mathrm{i}}$ by three or four orders of magnitude,

(2) The multiple order of magnitude increase in $E_{\mathrm{i}}$ using Nytrox allows for safe self-pressurization with high fluid densities.

(3) Self-pressurization greatly simplifies the system design and eliminates the need for a heavy, separate pressurant system using helium or nitrogen.

(4) Nytrox allows improved $I_{\text {sp }}$ performance compared to pure $\mathrm{N}_{2} \mathrm{O}$.

(5) From Figure $5 \mathrm{~b}$, at the optimal pressure level of 86 atmospheres, the Nytrox liquid density at $0{ }^{\circ} \mathrm{C}$ is higher than GOX by a factor approximately 6.4 , and allows for a significant improvement in the overall volumetric efficiency of the propellants.

(6) The optimal $\mathrm{O} / \mathrm{F}$ ratio is significantly reduced when using Nytrox, allowing a larger proportion of the total impulse to be delivered by the high-density fuel component, in this case, ABS.

(7) Maintaining the storage pressure near 80 atmospheres at $0{ }^{\circ} \mathrm{C}$ is essential to achieving best volumetric efficiency with Nytrox. 


\section{Experimental Apparatus, Instrumentation, and Test Procedures}

This section discusses the process for manufacture of the Nytrox solution, followed by a description of the test article, experimental apparatus, and hot-fire test procedures. The legacy hybrid thruster used for this evaluation was previously optimized for GOX as the oxidizer (Reference [1]) For this testing campaign the legacy hardware was deployed without change to demonstrate Nytrox as a "drop-in" replacement for GOX, and the results are not optimal for the Nytrox/ABS propellants. Considerable room for improvement and optimization of the Nytrox system exists.

\subsection{Nytrox Solution Processing}

For this study highly-purified grades [49] of $\mathrm{N}_{2} \mathrm{O}$ and GOX were used to ensure that the resulting Nytrox mixture was free from contaminants and any other possible catalytic agents. The objective of the developed procedure is to generate a Nytrox solution that lies near the previously-described "sweet spot," where the solution possesses a maximum concentration of oxygen in the vapor phase, with a minimum $\mathrm{O}_{2}$ concentration in the liquid phase. As described earlier, and depicted by Figure 3 , at $0{ }^{\circ} \mathrm{C}$ this optimum point concentration occurs at approximately 86 atmospheres. The resulting "Nytrox 87 " solution has a vapor phase $\mathrm{O}_{2}$ concentration of $37 \%$, and a liquid phase $\mathrm{O}_{2}$ concentration of approximately $13 \%$. For this condition the liquid-phase Nytrox 87 solution has a density of approximately $0.780 \mathrm{~g} / \mathrm{cm}^{3}$. Using the ideal gas law, GOX at the same temperature and pressure would have a density of $0.123 \mathrm{~g} / \mathrm{cm}^{3}$, or only $16 \%$ as dense.

Figure 7 shows the percolation apparatus block diagram. For this test campaign the procedure consists of filling the $4.5 \mathrm{~kg}$-capacity (10 lbs) Nytrox run tank half-full with nitrous oxide. During filling flow is passively initialed by placing the empty Nytrox run tank in an ice bath to lower the temperature to $0^{\circ} \mathrm{C}$, while the $\mathrm{N}_{2} \mathrm{O}$ service tank is kept at room temperature. The temperature difference lowers the vapor pressure of the run tank fluid, creating a pressure difference that motivates in fluid flow.

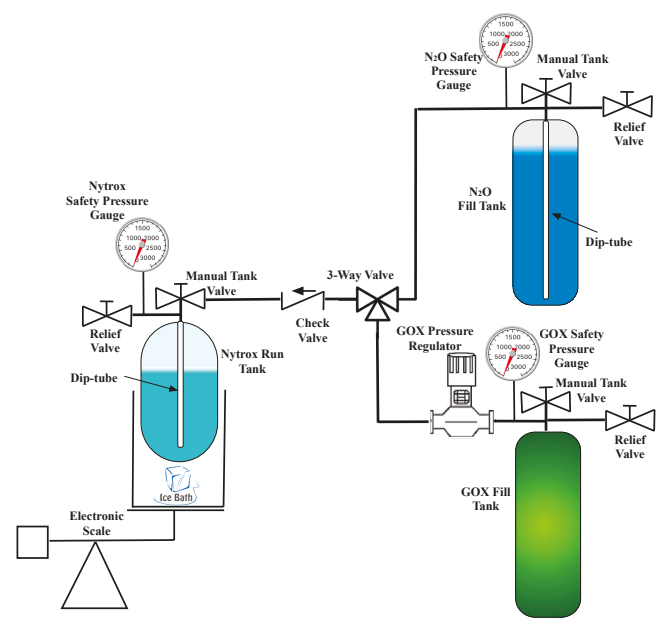

Figure 7. Nytrox percolation apparatus block diagram.

Once the Nytrox run tank is filled with the desired mass of nitrous oxide, the three-way valve is moved isolate the $\mathrm{N}_{2} \mathrm{O}$ fill tank, and to allow oxygen to begin flowing into the Nytrox run tank. The GOX regulator set pressure is maintained at 86 atmospheres throughout this process. A dip-tube in the run tank allows GOX to bubble up through the nitrous oxide. During passage through the liquid $\mathrm{N}_{2} \mathrm{O}$, oxygen dissolves into solution and also droplets of nitrous oxide are carried up into the gas phase.

The volume of liquid in the cylinder steadily diminishes until equilibrium conditions are reached for the fluid temperature. Equilibrium conditions are noted when the storage tank settles at a constant 
weight and internal pressure. With the fill-regulator set at $8720 \mathrm{kPa}$ (86 atm absolute pressure), the process takes about $2 \mathrm{~h}$ to reach equilibrium. An electronic scale was used to measure the nitrous oxide mass moved from the service tank to the run tank. After the Nytrox is blended at the desired density, the serviced Nytrox run tank is stored at $-15{ }^{\circ} \mathrm{C}$ temperatures in a portable freezer to keep vapor pressures low and ensure a minimal amount of $\mathrm{N}_{2} \mathrm{O}$ vapor in the tank ullage. By decreasing the temperature of the run tank, the activation energy barrier is raised even further and allows additional decomposition hazard mitigation.

\subsection{Hot-Fire Test Apparatus and Instrumentation}

This section summarizes the hardware, instrumentation, and test procedures used to perform the hot-fire evaluation tests. The hot-fire testing campaign was performed inside USU's on campus hazardous test facility. Whitmore and Bulcher [1], Whitmore and Stoddard [46], and Stoddard [50] describe the analytical methods, test apparatus, instrumentation, test procedures, and analysis methods used to derive the presented data in much fuller detail.

\subsubsection{Thrust Chamber}

The legacy GOX/ABS prototype of Reference [1] was reconfigured this testing campaign. Figure 8 presents the details of the thrust chamber assembly. Major system components are: (i) graphite nozzle, (ii) nozzle retention cap, (iii) motor case, (iv) 3D printed fuel grain with embedded electrodes, (v) insulating phenolic liner, (vi) chamber pressure fitting, and vii) single-port injector cap. The 38-mm diameter motor case, constructed from 6061-T6 aluminum, was procured commercially [51]. Table 1 summarizes the thrust chamber component geometry specifications, including the injector, fuel grain, motor case, and nozzle.

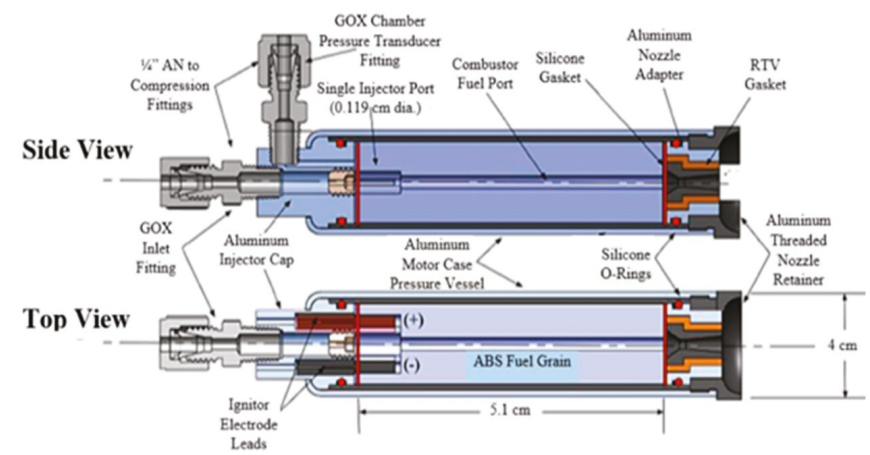

Figure 8. Test article thrust chamber assembly. (Copyright Utah State University [1], reproduced with permission).

Table 1. Motor component geometry specifications.

\begin{tabular}{|c|c|c|c|c|}
\hline \multicolumn{5}{|c|}{ Component } \\
\hline Single Port Injector & \multicolumn{4}{|c|}{ Diameter: $0.127 \mathrm{~cm}$} \\
\hline Fuel Grain (ABS) & Diameter: $3.168 \mathrm{~cm}$ & $\begin{array}{l}\text { Length: } \\
5.1 \mathrm{~cm}\end{array}$ & $\begin{array}{l}\text { Initial Weight: } \\
\quad 45.0 \mathrm{~g}^{1}\end{array}$ & $\begin{array}{c}\text { Initial Port } \\
\text { Diameter: } \\
0.53 \mathrm{~cm}\end{array}$ \\
\hline Motor Case & Diameter: $3.8 \mathrm{~cm}$ & $\begin{array}{l}\text { Length: } \\
7.92 \mathrm{~cm}\end{array}$ & $\begin{array}{r}\text { Wall T } \\
1.5\end{array}$ & ess: \\
\hline Nozzle (Graphite) & $\begin{array}{l}\text { Initial Throat Diameter: } \\
0.345 \mathrm{~cm}\end{array}$ & $\begin{array}{l}\text { Exit Diameter: } \\
0.483 \mathrm{~cm}\end{array}$ & $\begin{array}{l}\text { Expansion Ratio: } \\
2.07: 1, \text { Conical }\end{array}$ & $\begin{array}{c}\text { Exit Angle: } \\
5.0 \text { deg. }\end{array}$ \\
\hline
\end{tabular}

${ }^{1}$ The fuel grain print density was approximately, $1.04 \mathrm{~g} / \mathrm{cm}^{3}$. 


\subsubsection{Arc-Ignition System}

Figure 9a shows the motor head end layout with flow impingement shelves and embedded electrodes in the 3-D printed ABS fuel grain. Figure $9 \mathrm{~b}$ shows the ignition system electronics schematic. The ignition system power processing unit is based on the UltraVolt ${ }^{\circledR}$ line of high-voltage power supplies (HVPS) [52]. The HVPS provides a current-limited $(30 \mathrm{~mA})$ high voltage output of up to $1000 \mathrm{~V}$ or 30 Watts total output. Depending on the impedance on the arc path between the ignitor electrodes, the dissipated voltage typically varies between 100 and 400 volts. Ignition power to the thruster is initiated by sending a transistor-transistor logic (TTL)-level activate logic bit to the HVPS.

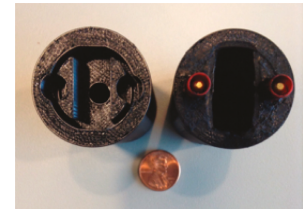

(a)

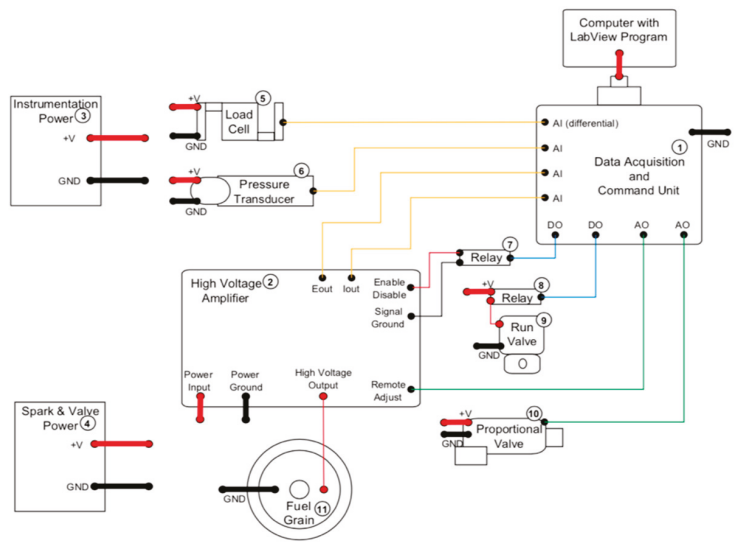

(b)

Figure 9. High-performance "green" hybrid propulsion (HPGHP) arc-igniton system: (a) motor head-end ignitor layout and (b) typical arc-ignition electrical system layout. (Copyright Utah State University [1], reproduced with permission).

\subsubsection{Test Stand Apparatus and Instrumentation}

Figure 10 shows the test motor assembled and mounted to the test stand. For this configuration support members allow bending along the direction of thrust to prevent them from interfering with the measured load. Figure 11 shows the piping and instrumentation diagram (P\&ID) of the test systems. The test stand measurements include Venturi-based oxidizer mass flow measurements, load-cell based thrust measurements, chamber pressure, and multiple temperature readings at various points along the flow path. The system was configured to allow rapid cycling between Nytrox and GOX using a 3-way valve. Directly aft of the thrust chamber lies the solenoid actuated oxidizer run-valve. Stoddard [50] presents a detailed summary of the test apparatus and procedures.

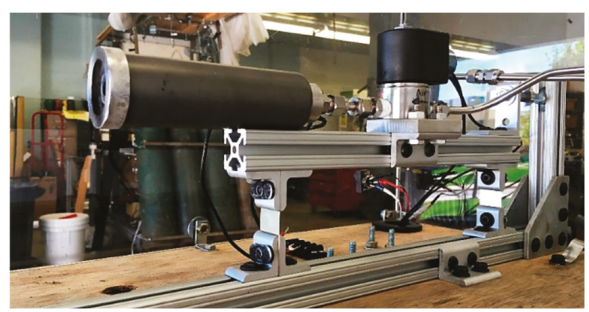

Figure 10. Thrust chamber mounted to load-balance test sled. (Copyright Utah State University [1], reproduced with permission). 


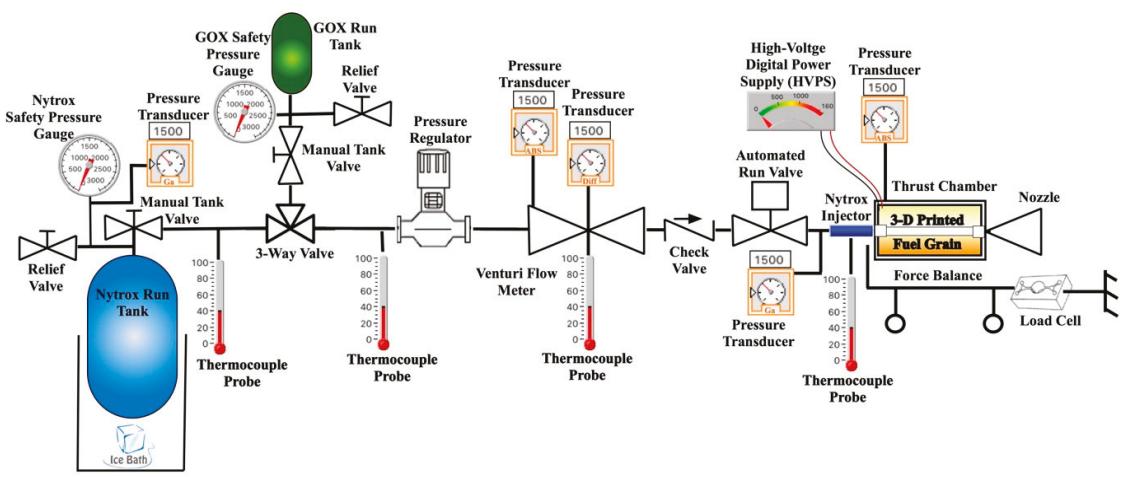

Figure 11. Piping and instrumentation diagram of the hot-fire test apparatus.

\subsection{Hot-Fire Test Procedures}

Initially, baseline tests were performed using gaseous oxygen as the oxidizer. This test series ensured that the "mothballed" system had been returned to the status that existed during the testing campaign of Reference [1]. Parameters measured include ignition power, thrust, chamber pressure, mass flow, fuel regression rate, specific impulse, and impulse density.

Following the baseline tests, the GOX tank was swapped for the run tank filled with the processed Nytrox. A commercial Holley Nitrous Oxide Systems (NOS ${ }^{\circledR}$ ) [53] storage tank was used as the nitrous oxide run tank. Other than the change in oxidizer and storage tank, the test assembly remained identical. Special attention was placed on establishing the required ignition power, and the resulting thrust, specific impulse and fuel regression rates. In order to simulate a true operational environment, during hot-fire testing the Nytrox is gradually allowed to warm to ambient conditions.

Test Procedures were nearly identical for both the GOX baseline and Nytrox tests. Pre-test measurements included fuel grain weight and port diameter, measured at both the top and bottom of the fuel port. The nozzle throat and exit plane diameters were also measured. Finally, the initial oxidizer run-tank tank weight, pressure, and temperature were measured. All data were logged on a spreadsheet for post-test analysis.

The upstream oxidizer flow regulator feed pressure was manually set to approximately 22 atmospheres $(2230 \mathrm{kPa})$ in order to choke the injector and ensure a constant oxidizer mass flow throughout the burn. For the two-phase Nytrox flow this pressure level also proved to be sufficiently high to quasi-choke the injector, and significantly reduced the risk of incurring injector-feed coupling instabilities during combustion.

In order to ensure Nytrox ignition reliability, the HVPS was activated sending power to the fuel grain electrodes one second before the oxidizer run valve was opened. Once the run valve opened, then HVPS power to the electrodes was continued to overlap for another $1 \mathrm{~s}$. Since the required power was so small, no attempt to shorten or optimize the "spark" length or overlap time was performed for this test series. For this test series, the motor run valve was programmed to open for a prescribed amount time that varied from 1 to $4 \mathrm{~s}$. The motor would snuff immediately after closure of the run valve. Allowing forma safety margin to prevent motor-case burn through, one fuel grain allows for $8 \mathrm{~s}$ of total burn time. Thus, on a single fuel grain a typical test series would prescribe four tests of $2 \mathrm{~s}$ each, or two tests of $4 \mathrm{~s}$ each. Following each burn, the weight and geometry measurements described in the previous paragraphs were repeated and logged for post-test analysis

\section{Data Analysis Methods}

This section details the analytical methods that were used to calculate key derived-parameters from the raw test data. These mass-flow based calculations include: (1) oxidizer mass flow, (2) mean 
fuel regression rate, (3) oxidizer-to-fuel ratio, (4) mean fuel port diameter, (5) oxidizer massflux, (6) total massflux, (7) equivalence ratio, and (8) specific gravity. Key performance parameters calculated from the raw data include: (1) combustion efficiency, (2) thrust coefficient, (3) specific impulse, (4) characteristic velocity, and (5) impulse density. The following section detail how these calculations were performed. The presented calculations for regression rate, fuel port diameter, and massflux are valid only for the longitudinal averages. There is no attempt to spatially resolve these parameters along the length of the fuel port.

\subsection{Calculating the Fuel Mass flow Rate.}

Although the inline Venturi measures the oxidizer mass flow in real-time, the test stand was not configured to directly measure the fuel mass flow. Instead, before and after each hot-firing the fuel grains were weighed to give the total fuel mass consumed during the test. As will be described later in this section, these mass measurements were used to anchor the "instantaneous" fuel mass flow rates, calculated as the difference between the nozzle exit and oxidizer mass flows:

$$
\dot{m}_{f u e l}(t)=\dot{m}_{\text {total }}(t)-\dot{m}_{o x}(t)
$$

Knowing the nozzle throat area $A^{*}$ and the plume exhaust gas properties, the nozzle exit (total) mass flow at each time point was calculated from the measured chamber pressure time history $P_{0}$, using the 1-dimensional choking mass flow equation, (Anderson [54], Chapter 4)

$$
\dot{m}_{\text {total }}(t)=A^{*} \cdot P_{0}(t) \cdot \sqrt{\frac{\gamma}{R_{g} \cdot T_{0}} \cdot\left(\frac{2}{\gamma+1}\right)^{\frac{\gamma+1}{\gamma-1}}}
$$

The calculation of Equation (2) assumes the flow composition is frozen at the nozzle entrance, (Anderson, [54], pp. 659-661) and nozzle erosion during the burn.

A table of thermodynamic and transport equilibrium properties of the GOX/ABS and Nytrox/ABS exhaust plumes were calculated using NASA's industry standard Chemical Equilibrium code (CEA) [48] with chamber pressure $P_{0}$ and mean $O / F$ ratio as independent look up variables for the tables. For each data point in the burn time history, the two-dimensional tables of thermodynamic and transport properties were interpolated using chamber pressure $P_{0}$ and mean $O / F$ ratio as lookup variables. Calculated parameters included the gas constant $R_{\mathrm{g}}$, ratio of specific heats $\gamma$, and flame temperature $T_{0}$. Defining the combustion efficiency as:

$$
\eta^{*}=\frac{c_{\text {actual }}^{*}}{c_{\text {ideal }}^{*}}=\sqrt{\frac{\left(\frac{\gamma+1}{2 \gamma}\right)^{\frac{\gamma+1}{\gamma-1}} R_{g} \cdot T_{0_{\text {actual }}}}{\left(\frac{\gamma+1}{2 \gamma}\right)^{\frac{\gamma+1}{\gamma-1}} R_{g} \cdot T_{0_{\text {ideal }}}}} \approx \sqrt{\frac{T_{0_{\text {actual }}}}{T_{0_{\text {ideal }}}}}
$$

The theoretical flame temperature was scaled by adjusting the combustion efficiency:

$$
T_{0_{\text {actual }}}=T_{0_{\text {ideal }}} \cdot\left(\eta^{*}\right)^{2}
$$

such that the calculated fuel mass consumption over the burn:

$$
\Delta M_{f u e l}\left(t_{\text {burn }}\right)=\int_{0}^{t_{\text {burn }}}\left(\dot{m}_{\text {total }}(t)-\dot{m}_{\text {ox }}(t)\right) \cdot d t
$$

matched the measured value from differences of the pre- and post-test weight measurements. As described earlier, the consumed fuel mass anchored the thermodynamic calculations. 
Adjusting input combustion efficiency upwards has the effect of increasing the calculated fuel mass consumption, and downwards decreases the calculated fuel mass consumption. The calculations of Equations (2)-(6) were iterated, adjusting $\eta^{*}$ after each iteration, until the calculated fuel mass matched the measured mass within a prescribed level of accuracy, in this case $0.5 \%$. For each iteration, the time-averaged oxidizer-to-fuel ratio was calculated as integrated oxidizer mass flow divided by the consumed fuel mass:

$$
O / F=\frac{\int_{0}^{t_{\text {burn }}} \dot{m}_{o x}(t) \cdot d t}{\Delta M_{\text {fuel }}\left(t_{\text {burn }}\right)}=\frac{\int_{0}^{t_{\text {burn }}} \dot{m}_{o x}(t) \cdot d t}{\int_{0}^{t_{\text {burn }}}\left(\dot{m}_{\text {total }}(t)-\dot{m}_{o x}(t)\right) \cdot d t}
$$

The thruster system to be tested exhibited very little $O / F$ ratio shift over the burn lifetime. Thus, the assumption of a constant $O / F$ ratio based on the total consumed masses had very little effect upon the presented results. Clearly, for propellants or thruster systems that exhibit a significant $O / F$ shift, the presented method must be modified with the $O / F$ being calculated as a function of time based upon the instantaneous mass flow calculations. The time-averaged $O / F$ method was used to reduce the complexity of the implemented model and also aid for numerical convergence.

\subsection{Calculating the Fuel Regression Rate}

Once the mass flow and consumed masses were calculated as described above, the instantaneous longitudinal mean of the regression rate was calculated from the rate of fuel mass depletion as:

$$
\dot{r}_{L}(t)=\frac{\dot{m}_{f u e l}(t)}{2 \pi \cdot \rho_{f u e l} \cdot r_{L}(t) \cdot L}
$$

In Equation (8) $\rho_{\text {fuel }}$ is the solid fuel density, $L$ is the fuel grain burn length, and $r_{L}$ is the longitudinal mean of the fuel port radius. Integrating Equation (8) from the initial condition to the current burn time solves for the instantaneous longitudinal mean of the port radius and cross-sectional area:

$$
r_{L}(t)=\sqrt{r_{0}^{2}+\frac{1}{\pi \cdot \rho_{f u e l} \cdot L} \int_{0}^{t} \dot{m}_{f u e l}(\tau) \cdot d \tau}
$$

and:

$$
A_{c}(t)=\pi \cdot r_{L}^{2}(t)
$$

In Equation (10) $r_{0}$ is the initial fuel port radius at the beginning of the burn. The terminal cross sectional area of the fuel port is:

$$
A_{c}\left(t_{\text {burn }}\right)=\pi \cdot r_{0}^{2}+\frac{\Delta M_{\text {fuel }}\left(t_{\text {burn }}\right)}{\rho_{\text {fuel }} \cdot L}
$$

The time-averaged fuel regression rate over the duration of the burn is calculated by:

$$
\overline{\dot{r}}=\frac{\Delta M_{\text {fuel }}\left(t_{\text {burn }}\right)}{2 \pi \cdot \rho_{\text {fuel }} \cdot L \cdot\left(\frac{r_{t_{\text {burn }}}+r_{0}}{2}\right) \cdot t_{\text {burn }}}
$$


The time-averaged- oxidizer mass flux, total mass flux, equivalence ratio, and effective specific gravity of the propellants are estimated by:

$$
\begin{array}{ll}
\bar{G}_{o x}=\frac{1}{t_{\text {burn }}}\left(\frac{\int_{0}^{t_{\text {burn }}} \dot{m}_{\text {ox }}(t) \cdot d t}{A_{c}(t)}\right), & \bar{G}_{\text {total }}=\frac{1}{t_{\text {burn }}}\left(\frac{\int_{0}^{t_{b u r n}} \dot{m}_{o x}(t) \cdot d t}{A_{c}(t)}+\frac{\Delta M_{\text {fuel }}}{A_{c}\left(t_{\text {burn }}\right)}\right) \\
\text { and } & s_{g}=\frac{s_{\text {gox }} \cdot s_{g_{\text {fuel }}} \cdot(O / F+1)}{\left(s_{g_{\text {fuel }}} \cdot O / F+s_{\text {gox }}\right)}
\end{array}
$$

For this discussion, the specific gravity is calculated based on the propellant density relative to the weight of water at $20^{\circ} \mathrm{C}$. For GOX flow, the specific gravity is based on mean tank storage density as calculated using the ideal gas law. For Nytrox flow the Peng-Robinson model was used to calculate the oxidizer specific gravity. As shown by Table 1, the ABS fuel print density was approximately $1.04 \mathrm{~g} / \mathrm{cm}^{3}$. The stoichiometric O/F ratio for each propellant combination was calculated using CEA [48].

\subsection{Calculating the Motor Performance Parameters}

The 1-dimensional de Laval flow equations (Anderson [54], Chapter 4) were used to calculate the thruster performance parameters. Thrust and thrust coefficient were calculated from chamber pressure as:

$$
\begin{gathered}
F_{\text {thrust }}=P_{0} A^{*} \cdot\left(\sqrt{\frac{2}{\gamma-1} \cdot\left(\frac{2}{\gamma+1}\right)^{\frac{\gamma+1}{\gamma-1}}}\left(1-\frac{p_{\text {exit }}}{P_{0}}\right)^{\frac{\gamma-1}{\gamma}}+\left(\frac{A_{\text {exit }}}{A^{*}}\right)\left(\frac{p_{\text {exit }}-p_{\infty}}{P_{0}}\right)\right) \\
C_{F}=\frac{F_{\text {thrust }}}{P_{0} A^{*}}=\gamma \sqrt{\frac{2}{\gamma-1} \cdot\left(\frac{2}{\gamma+1}\right)^{\frac{\gamma+1}{\gamma-1}}}\left(1-\frac{p_{\text {exit }}}{P_{0}}\right)^{\frac{\gamma-1}{\gamma}}+\left(\frac{A_{\text {exit }}}{A^{*}}\right)\left(\frac{p_{\text {exit }}-p_{\infty}}{P_{0}}\right)
\end{gathered}
$$

Specific Impulse, Characteristic Velocity, and Impulse density were calculated as:

$$
\begin{aligned}
I_{s p} & =\frac{F_{\text {thrust }}}{g_{0} \dot{m}_{\text {total }}} \\
c^{*} & =\frac{P_{0} A^{*}}{\dot{m}_{\text {total }}} \\
\rho I_{s p} & =s_{g} \cdot g_{0} \cdot I_{s p}
\end{aligned}
$$

In Equations (16) and (18) $g_{0}$ is normal acceleration of gravity at sea level, $9.8067 \mathrm{~m} / \mathrm{s}^{2}$. The thrust coefficient $C_{F}$ and specific impulse $I_{\mathrm{sp}}$ were also calculated directly from the thrust sensed by the test stand load cell. Values calculated from both sources will be presented later in order to support the verisimilitude of the collected test data.

\section{Results and Discussion}

This section presents results from the testing campaign. Nytrox-batch solution properties are presented first, followed by the Venturi flow meter calibration test results for Nytrox flow. Results of 32 successful hot-fires are presented, 13 with GOX as the oxidizer and 19 burns with Nytrox replacing GOX. Results from tests using GOX and Nytrox will first be presented individually and then compared. For both test series, the oxidizer regulator set-pressure was adjusted such that the nozzle exit pressure was near the optimal (ambient) condition. During preliminary testing, the optimal exit pressure was estimated from the measured chamber pressure, the nozzle expansion ratio, and combustion properties as determined from the earlier-described CEA analysis. The optimal exit pressure was desired for both test series in order to reduce or eliminate the effects nozzle efficiency as a factor in 
the performance comparisons. Thus, any performance differences would primarily result from the propellants. In order to achieve the optimal-nozzle exit pressure, the Nytrox/ABS thruster chamber pressure was approximately $6 \%$ higher than the corresponding chamber pressure for GOX/ABS.

\subsection{Making the Nytrox Mixture}

The procedures described by Section 3.1 were followed to generate the Nytrox batches used for this testing campaign. Table 2 summarizes these processing results.

Table 2. Nytrox mix batch specifications.

\begin{tabular}{|c|c|c|c|c|c|c|c|c|}
\hline Batch No. & 1 & 2 & 3 & 4 & 5 & 6 & Mean & Std. Dev \\
\hline Final Fill Pressure, kPa & 8704.4 & 8842.3 & 8739.4 & 8760.1 & 8725.6 & 8105.1 & 8745.4 & 269.3 \\
\hline Final Fill Temperature, ${ }^{\circ} \mathrm{C}$ & 0.1 & -0.2 & 0.0 & 0.0 & 0.2 & 0.2 & 0.05 & 0.15 \\
\hline Total Oxidizer Added, $\mathrm{kg}$ & 2.867 & 2.921 & 2.916 & 2.864 & 2.865 & 2.854 & 2.887 & 0.029 \\
\hline $\mathrm{N}_{2} \mathrm{O}$ Added, $\mathrm{kg}$ & 2.234 & 2.267 & 2.284 & 2.273 & 2.283 & 2.278 & 2.268 & 0.019 \\
\hline $\mathrm{O}_{2}$ Added to Tank, kg & 0.633 & 0.654 & 0.632 & 0.591 & 0.582 & 0.576 & 0.618 & 0.032 \\
\hline Mean Nytrox Density, $\mathrm{g} / \mathrm{cm}^{3}$ & 0.419 & 0.427 & 0.426 & 0.418 & 0.418 & 0.417 & 0.422 & 0.004 \\
\hline Liquid $\mathrm{O}_{2}$ Mass Fraction (\%) & 12.63 & 12.99 & 12.72 & 12.78 & 12.69 & 11.13 & 12.76 & 0.68 \\
\hline Vapor $\mathrm{O}_{2}$ Mass Fraction (\%) & 37.14 & 37.20 & 37.16 & 37.17 & 37.16 & 36.40 & 37.17 & 0.31 \\
\hline Vapor Fraction in Tank (\%) & 37.0 & 36.6 & 35.9 & 37.5 & 37.2 & 32.9 & 36.8 & 01.7 \\
\hline Nytrox Liquid Density, $\mathrm{g} / \mathrm{cm}^{3}$ & 0.774 & 0.770 & 0.773 & 0.773 & 0.774 & 0.790 & 0.773 & 0.007 \\
\hline Nytrox Vapor Density, $\mathrm{g} / \mathrm{cm}^{3}$ & 0.235 & 0.241 & 0.236 & 0.237 & 0.236 & 0.212 & 0.237 & 0.010 \\
\hline
\end{tabular}

At the time of this publication, six complete batches of Nytrox have been processed. The batch comparisons are remarkably similar. With the tank temperature closely regulated by submersion in an ice bath, the mean batch equilibrium pressure was $8745.4 \mathrm{kPa}(86.3 \mathrm{~atm})$ with a standard deviation of only $3 \%$. Also, the intensive fluid properties were remarkably similar. The mean effective fill density calculated using the known tank volume and pre- and post-fill weights was 0.422 , with a standard deviation of less than $1 \%$. The listed Nytrox vapor and liquid densities were calculated from the Peng-Robinson model based on the known tank fill pressure and temperature. Based on the Peng-Robinson model, the mean liquid $\mathrm{O}_{2}$ vapor mass fraction was $12.76 \%$, with a standard deviation of less than $0.68 \%$. The mean Nytrox liquid density was $0.773 \mathrm{~g} / \mathrm{cm}^{2}$ with a standard deviation of only $0.007 \%$. These values are remarkably close to the pre-planned "sweet spot" values as presented by Figures 3 and 5 .

\subsection{Oxidizer Venturi Calibration}

For the flow of gaseous oxygen, the associated Venturi mass flow calculations were developed using from material presented by Anderson [54]. The compressible mass flow equations are written in terms of the oxidizer stagnation downstream of the regulator $P_{0}$, the Venturi inlet pressure $P_{1}$, and the Venturi throat pressure $P_{2}$. The result is:

$$
\left.P_{0}=\left[\frac{\left(\frac{A_{1}}{A_{2}}\right)^{2} \cdot\left(P_{1}\right)^{\frac{\gamma+1}{\gamma}}-\left(P_{2}\right)^{\frac{\gamma+1}{\gamma}}}{\left(\frac{A_{1}}{A_{2}}\right)^{2} \cdot\left(P_{1}\right)^{\frac{2}{\gamma}}-\left(P_{2}\right)^{\frac{2}{\gamma}}}\right]^{\frac{\gamma}{\gamma-1}} \quad \dot{m}_{0 x}=C_{d} A_{2} P_{0} \sqrt{\frac{2 \gamma}{(\gamma-1)\left(R_{g} \cdot T\right)}\left[\left(\frac{P_{2}}{P_{0}}\right)^{\frac{2}{\gamma}}-\left(\frac{P_{2}}{P_{0}}\right)^{\frac{\gamma+1}{\gamma}}\right.}\right]
$$

Previously, References [1,30], performed extensive GOX cold flow tests at a multiple upstream set pressures varying from 15 to $30 \mathrm{~atm}$ were performed. Over this range the associated discharge coefficient was found to be essentially constant with a value of approximately $C_{d}=0.95$. The value was not found to vary over the range of mass flow tested in this experiment. Since the Venturi arrangement for GOX baseline tests did not change from the original tests series, the Venturi flow meter was not re-calibrated for GOX flow for this testing campaign.

In contrast to the GOX flow, the two-phase, binary nature of the Nytrox flow made mass flow measurements from the Venturi sensor rather more complicated. Multiple models have been previously developed for two phase nitrous oxide mass flows. These include models developed by Zilliac and 
Karabeyoglu [55], Dyer [56], Whitmore and Chandler [57], Zimmerman et al. [58], and Waxman et al. [59]. It is likely that these models, each developed for the flow of a single saturated liquid are applicable to the two phase binary fluid injector problem, but a solid theoretical foundation for Venturi flow has yet to be developed.

Thus, for this proof-of-concept testing campaign, the Nytrox mass flow was modeled using the compressible Venturi model of Equation (15), with discharge coefficient set specifically for Nytrox 87 flow. The associated ideal gas thermodynamic properties are calculated as the molar averages of the GOX and $\mathrm{N}_{2} \mathrm{O}$ fluid components, based on the Peng-Robinson model at the tank storage pressure and temperature.

Figure 12 plots the results of a series of cold-flow tests used to calibrate the Venturi for Nytrox flow. The actual thrust chamber and injector were used for these tests, but with the exit nozzle removed, and no fuel grain installed. For these tests regulator pressure was set at 22 atmospheres to match the set pressure to be used for the hot-fire tests. Run-time lengths of 1,2,3, and $7 \mathrm{~s}$ were performed, and the run tank mass was measured before and after each test. The oxidizer masses depleted from the run tank, measured by the differences in the before and after run tank weights, are plotted against the time integrals of the mass flows calculated by Equation (15), assuming $C_{d}=1.0$. The slope of the plotted curve, approximately $1.352 \mathrm{~g} / \mathrm{g}$, is equivalent to the true discharge coefficient at the upstream set pressure.

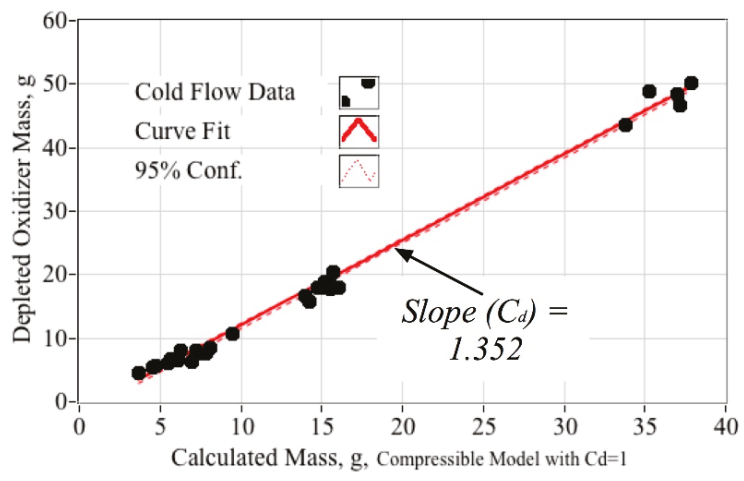

Figure 12. Venturi calibration for nytrox 90 flow at 22 atmospheres.

Clearly, since the calculated value $C_{d}=1.352$ is greater than unity, the model of Equation (15) is not a complete representation of the two-phase flow physics. It is likely that the ideal-gas compressible flow equations do not capture some of the interaction phenomena of the binary fluid components. If the tests were performed over a much wider mass flow range, it is possible that the slope curve plotted on Figure 12 would vary as a function of set pressure and mass flow. However, since the test data form a very tight curve for this series, the resulting $C_{d}$ value is considered to be accurate as long as the upstream regulator set pressure is maintained near 22 atmospheres.

This limited calibration was performed for time expediency to allow the testing campaign to be concluded within the available test window. With this method the molecular weight, gas constant, and ratio specific heats were calculated based on the mean tank vapor composition as shown by Table 2 . Pressing forward to the nytrox hot-fire testing campaign the following parameters were used for the Venturi nytrox flow calibration:

$$
M_{w}=37.67 \mathrm{~g} / \mathrm{mol}, R_{g}=220.72 \mathrm{~J} / \mathrm{kg}-\mathrm{K}, \gamma=1.40 \text {, and } C_{d}=1.352
$$




\subsection{Baseline $\mathrm{O}_{2}$ Hot-Fire Test Summary}

As stated previously, a series of hot-fire tests were performed using GOX as the oxidizer in order to establish a baseline for the small thruster system. Results from 13 hot-firings are reported here. Figure 13 plots the time histories from a typical GOX baseline test. Plotted are (a) thrust, (b) chamber pressure, (c) mass flow, and (d) consumed propellant mass.
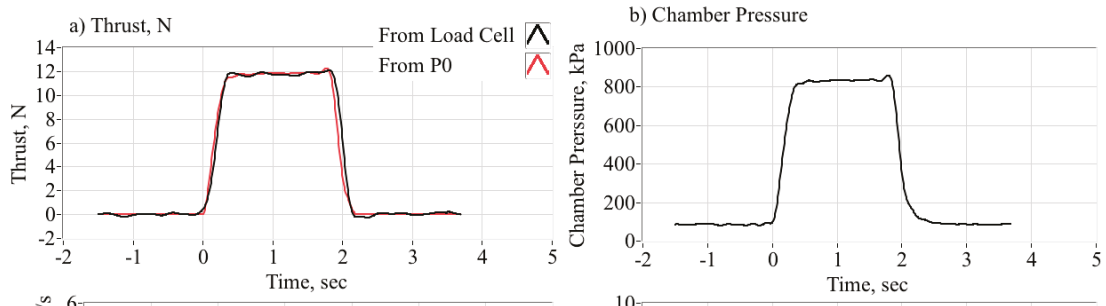

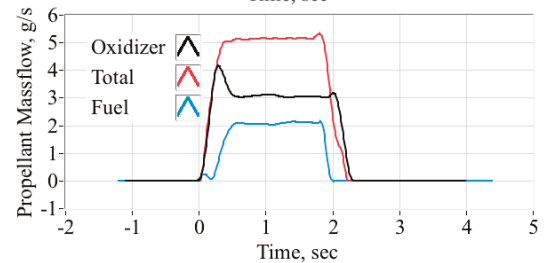

c) Massflow

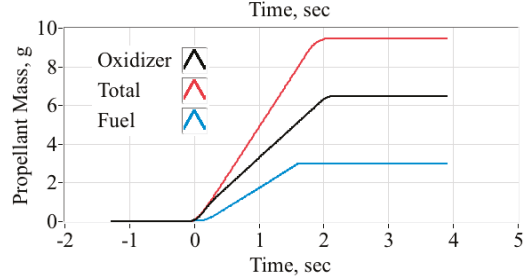

d) Consumed Propellant Mass (Integrated Massflow)

Figure 13. Time history of typical baseline GOX/ABS hot-firing.

The fuel mass flow and consumed mass time histories were calculated using the procedure previously laid out in Section 4.1. Note that although the oxidizer flow initiates at time zero, the steady chamber pressure is not reached until approximately $300 \mathrm{~ms}$ later. The initial GOX time history overshoot is likely due to an unchoked nozzle as gas streams into the thrust chamber. On Figure 13a thrust values as directly measured by the load call and as calculated from chamber pressure using Equation (12) show excellent agreement. This close comparison indicates that values for the nozzle-flow thermodynamic parameters, $T_{0}, \gamma, M_{w}$, and $R_{g}$, derived from the previously-described procedure of Section 4.1 are accurately calculated.

Figure 14 graphically summarizes the baseline tests, showing the results of 13 hot-firings. Plotted are $I_{\mathrm{sp}}, C_{F}, c^{*}$, and the mean ABS fuel regression rate. The mean fuel regression rate, calculated using Equations (10), is plotted as a function of both mean oxidizer $G_{o x}$ and total massflux $G_{\text {total }}$. The specific impulse and thrust coefficient curves plot values calculated using both the sensed thrust from the load cell, and the thrust calculated from chamber pressure. The plotted data of this section and the bar charts of the next section result from time history averages taken from the steady-state portions of each hot-fire test. For the purpose of this calculation the term "steady-state" means all data points that lie within $10 \%$ of the maximum observed thrust and chamber pressure levels.

The time-averaged $C_{F}$ values calculated using measured thrust and chamber pressure data were calculated as:

$$
\bar{C}_{F}=\frac{1}{t_{\text {burn }}} \int_{0}^{t_{\text {burn }}} \frac{F(t)}{P(t) \cdot A^{*}} \cdot d t
$$

are plotted as the black circular symbols. Time-averaged values calculated from the chamber pressure, calculated using 1-D De Laval flow model (Anderson, Reference [49]):

$$
\bar{C}_{F}=\frac{1}{t_{\text {burn }}} \int_{0}^{t_{\text {burn }}} \gamma \sqrt{\frac{2}{\gamma-1} \cdot\left(\frac{2}{\gamma+1}\right)^{\frac{\gamma+1}{\gamma-1}}}\left(1-\frac{p_{\text {exit }}}{P_{0}}\right)^{\frac{\gamma-1}{\gamma}}+\left(\frac{A_{\text {exit }}}{A^{*}}\right)\left(\frac{p_{\text {exit }}-p_{\infty}}{P_{0}}\right) \cdot d t
$$


are plotted as circular red symbols. In Equation (17), the exit pressure values were calculated from the chamber pressure based on nozzle expansion ratio using the previously-described values for $\gamma$ and $R_{g}$. For both the GOX and Nytrox testing campaign, pre- and post-test measurements indicated no discernable change in the nozzle throat area. For this calculation a constant throat area $A^{*}$ is used.

Dashed red lines overlaid on the $I_{\mathrm{sp}}, C_{F}$, and $c^{*}$ plots were values calculated from CEA [48]. assuming frozen flow at the nozzle throat. The plotted data generally support the theoretical calculations. Because the data of Figure 14 agree closely with results previously published by References [1,30], it is concluded that the reassembled test article and test stand were returned to the previous state of performance, for which there is an extensive data base.

a) Specific Impulse vs. $\mathrm{O} / \mathrm{F}$
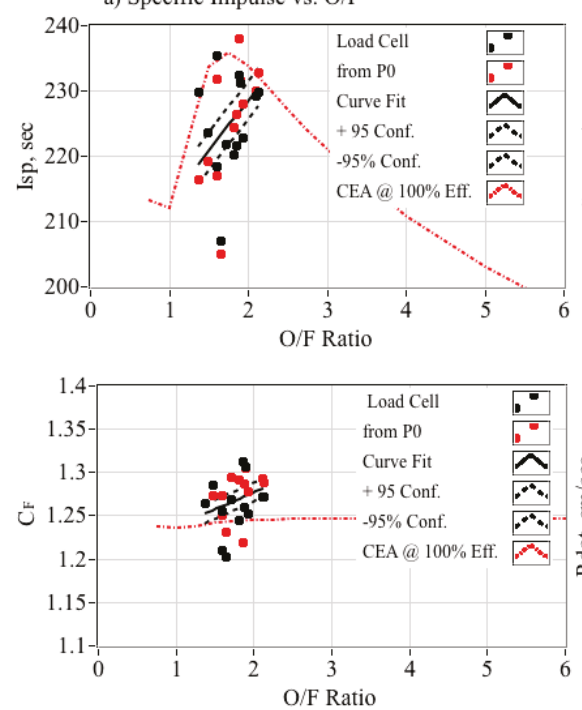

c) Thrust Coefficient b) Characteristic Velocity vs CEA
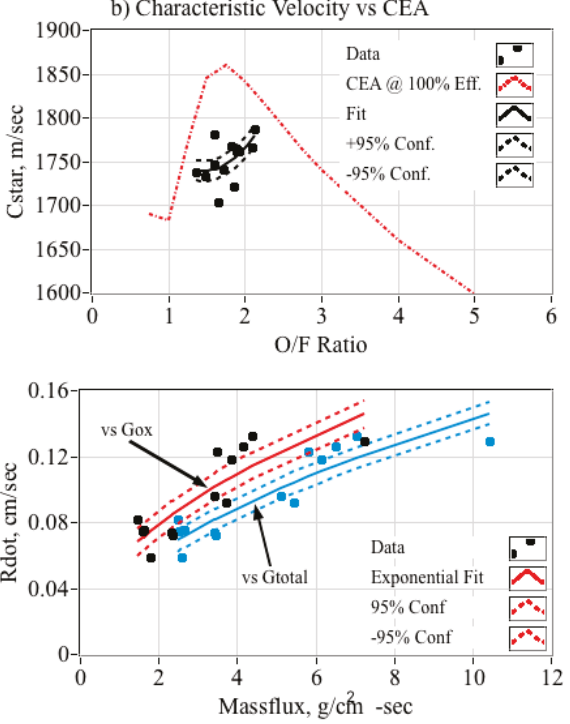

d) Regression Rate

Figure 14. Summary of the GOX/ABS baseline test results. (Copyright Utah State University [46], reproduced with permission).

\subsection{Nytrox 87 Hot-Fire Test Summary}

Data from a total of 19 Nytrox hot-firings are reported. Figure 15 plots the time histories from a typical hot-fire test. As with the previous plots of Figure 13, the plotted time histories are (a) thrust, (b) chamber Pressure, (c) mass flow, and (d) consumed propellant mass. The time scales have been adjusted so that the zero-time point corresponds with the motor-ignition, signaled by a sharp rise of the chamber pressure. As shown by the mass flow plot 15c, the Nytrox run valve was opened approximately $400 \mathrm{~ms}$ prior to this motor-ignition rise time. Prior to the motor ignition it appears that chilled, mostly liquid, Nytrox streamed into the combustion chamber. Because this pre-ignition flow stream was mostly-liquid, only a minor rise in thrust and chamber pressure occurs. This ignition latency was observed for the majority of the Nytrox tests, and will be described in detail later in this section. The Nytrox streaming into the thrust chamber prior to ignition is not included in the total Nytrox mass calculation of Figure 15b. Pre- and post-test measurements of the NOS ${ }^{\circledR}$ tank weights were compared against the calculation for the consumed oxidizer mass, e.g., from Figure 15d, and verified the accuracy of the nytrox Venturi discharge coefficient described in the previous section. 

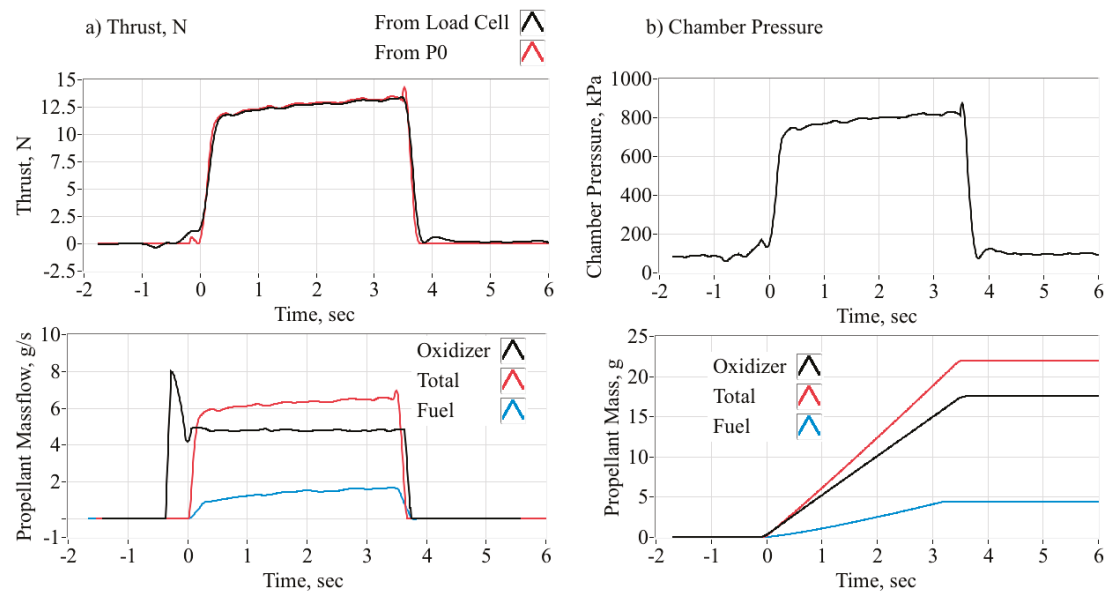

c) Massflow

d) Consumed Propellant Mass (Integrated Massflow)

Figure 15. Time history of typical Nytrox 87/ABS hot-firing.

Figure 16 graphically summarizes the nytrox test results. The nytrox mixtures used for these tests are described previously in Section 5.1, with the mixture properties listed by Table 2. As with the baseline data presentation, Figure 16 plots $I_{\mathrm{sp}}, C_{F}$, and $c^{*}$ as a function of $O / F$ ratio, and mean fuel regression rate as a function of $G_{0 x}$ and $G_{t o t a l}$. With regard to the regression rate plots, note that the $G_{0 x}$ and $G_{\text {total }}$ curves lie closer together as compared to the baseline data. This occurrence results from the significantly higher $\mathrm{O} / \mathrm{F}$ ratio for the nytrox flow, where the majority of total thruster mass flow is from the oxidizer and not ablated fuel.

a) Specific Impulse vs. $\mathrm{O} / \mathrm{F}$
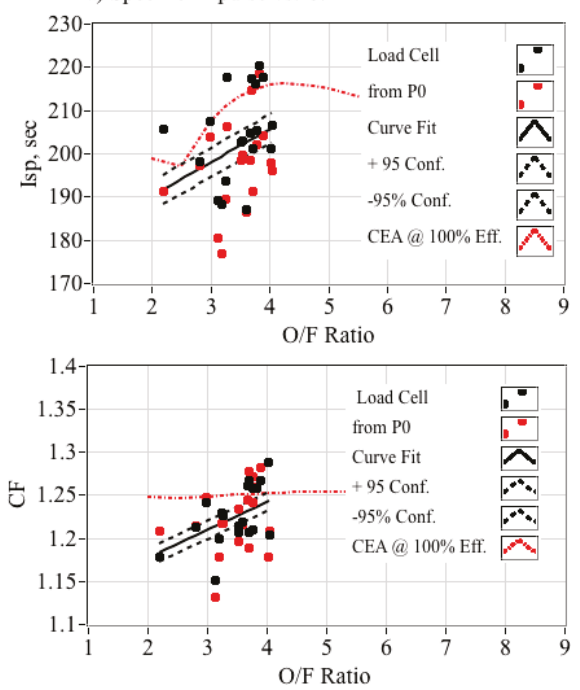

c) Thrust Coefficient b) Characteristic Velocity vs CEA
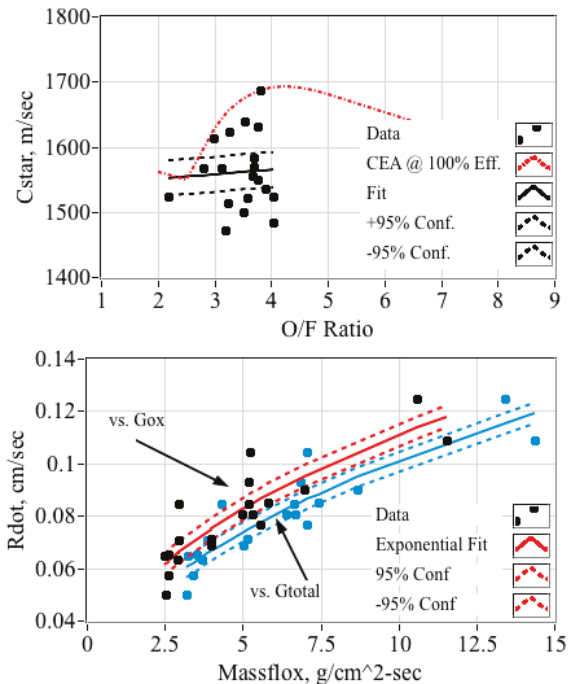

d) Regression Rate

Figure 16. Summary of the Nytrox 87/ABS hot-fire test results. (Copyright Utah State University [46], reproduced with permission). 
The corresponding CEA curves assuming a Nytrox $87\left(87 \% \mathrm{~N}_{2} \mathrm{O}\right)$ liquid composition are also plotted. Here there is significantly more scatter exhibited by the data, a likely result of the changing Nytrox fluid composition as the tank empties. The mean values for $I_{\mathrm{sp}}$ and $c^{*}$ are approximately $10 \%$ lower that theoretical CEA predictions. Comparing Figures 14 and 16 shows that when Nytrox is "dropped in" as a replacement for GOX, the motor tends to run slightly fuel-rich than the O/F required for optimal performance. Since the thruster had been previously optimized for best O/F ratio based on GOX as the oxidizer, this result was expected. The ensemble-mean Nytrox combustion efficiency, as calculated by Equation (3) for each burn was approximately $92.5 \%$.

\subsection{GOX/Nytrox Burn Data Comparisons}

This section compares the results of the GOX/ABS baseline against the Nytrox/ABS test results. Table 3 summarizes the GOX/ABS test statistics with data taken from Figure 14, and the Nytrox/ABS test statistics with data taken from Figure 16. The mean $\mu$, standard deviation $\sigma$ and $95 \% \mathrm{t}$-confidence intervals [56] for thrust, thrust coefficient, specific impulse, characteristic velocity, combustion efficiency, $\mathrm{O} / \mathrm{F}$ ratio, chamber pressure, and total mass flow are tabulated. Values for thrust, $\mathrm{C}_{\mathrm{F}}$, and $I_{\mathrm{sp}}$ are calculated using both the sensed load and thrust calculated from chamber pressure using Equations (12)-(14). Generally, the thrust comparisons are close, with the Nytrox/ABS thruster exhibiting a slightly higher thrust and chamber pressure that the GOX/ABS, a likely result from the higher Nytrox density. Conversely, the GOX/ABS thruster exhibits increased specific impulse $I_{\mathrm{sp}}$ and $c^{*}$ level, compared to Nytro/ABS. This result was predicted by Figure 6, and was expected. Also, note that the calculated values for $C_{F}$ for both test series are nearly identical, in fact they are statistically indistinguishable. This $C_{F}$ comparison indicates that the choice of propellants is the primary distinguishing actor with regard to the specific impulse performance differences.

Table 3. Summary of top-level parameter statistics for GOX/ABS baseline and Nytrox/ABS tests.

\begin{tabular}{|c|c|c|c|c|c|c|c|c|c|c|c|}
\hline \multicolumn{12}{|c|}{ Baseline GOX/ABS Test (13 Total Burns) } \\
\hline Statistic & \multicolumn{2}{|c|}{$\begin{array}{c}\text { Thrust, N } \\
\text { Load } P_{0}\end{array}$} & \multicolumn{2}{|c|}{$\begin{array}{c}C_{F} \\
\text { Load } P_{0}\end{array}$} & \multicolumn{2}{|c|}{$\begin{array}{c}I_{\mathrm{sp}} \\
\text { Load } P_{0}\end{array}$} & \multirow{2}{*}{$\begin{array}{c}\begin{array}{c}c^{*} \\
\mathrm{~m} / \mathrm{s}\end{array} \\
1751.4\end{array}$} & \multirow{2}{*}{$\begin{array}{c}\eta^{*} \\
0.919\end{array}$} & \multirow{2}{*}{$\begin{array}{c}O / F \\
1.772\end{array}$} & \multirow{2}{*}{$\begin{array}{c}P_{\mathbf{0}}, \\
\mathbf{k P a}\end{array}$} & \multirow{2}{*}{$\begin{array}{c}\begin{array}{c}\text { Total Mass } \\
\text { Flow, g/s }\end{array} \\
4.85\end{array}$} \\
\hline$\mu$ & 10.70 & 10.90 & 1.262 & 1.273 & 224.9 & 224.8 & & & & & \\
\hline$\sigma$ & 0.645 & 0.617 & 0.031 & 0.026 & 7.52 & 8.83 & 23.84 & 0.089 & 0.228 & 62.82 & 0.349 \\
\hline $95 \%$ t-conf. & 0.339 & 0.373 & 0.019 & 0.016 & 4.54 & 5.34 & 14.40 & 0.054 & 0.178 & 37.94 & 0.211 \\
\hline \multicolumn{12}{|c|}{ Nytrox/ABS Test (19 Total Burns) } \\
\hline$\mu$ & 11.75 & 11.70 & 1.227 & 1.222 & 204.4 & 198.4 & 560.84 & 0.927 & 3.464 & 809.7 & 6.14 \\
\hline$\sigma$ & 0.749 & 0.759 & 0.034 & 0.039 & 10.29 & 11.10 & 57.46 & 0.106 & 0.463 & 32.84 & 0.356 \\
\hline $95 \%$ t-conf. & 0.358 & 0.366 & 0.017 & 0.019 & 54.96 & 5.34 & 27.68 & 0.051 & 0.223 & 15.82 & 0.171 \\
\hline
\end{tabular}

\subsubsection{Top-Level Motor Performance Comparisons}

This section presents a series of bar charts that compare the mean properties of the thruster as derived from the 13 GOX-baseline and 19 Nytrox-evaluation hot-fire tests. In addition to the nominal performance parameters as listed by Table 3, mass-derived parameters like equivalence ratio, regression rate, and impulse-density are also compared. Effects of massflux on fuel regression rate will be assessed. Finally, the required ignition energy and ignition latencies will be assessed and compared.

Figure 17 bar charts compare the actual thrust, mean operating chamber pressure, oxidizer and total mass flows, and performance parameters of the thruster using the two propellant classes. In addition to the mean parameter levels, these plots also show the $95 \%$ confidence intervals based on the student $t$-distribution [60] for the appropriate degrees of freedom (based on the number of measurements). As shown by Figure 17a-c the Nytrox oxidizer, inserted as a higher-density "drop in" for GOX results in slightly higher absolute thrust, chamber pressure, and mass flow levels. These higher absolute levels are due to the higher density of Nytrox 87 oxidizer, as compared to GOX. 
a) Thrust
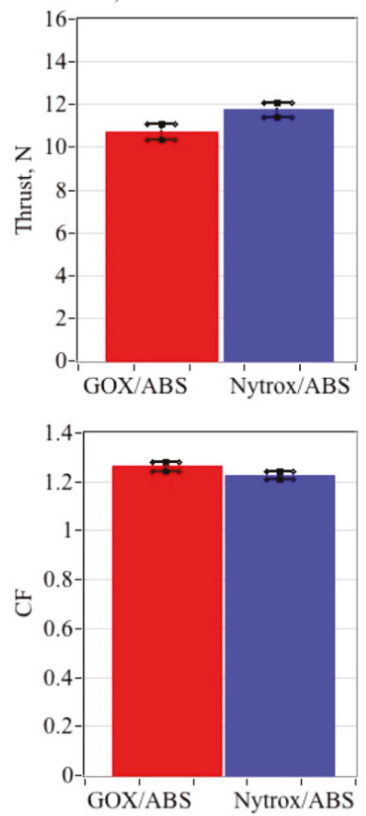

d) Thrust Coefficient b) Chamber Pressure
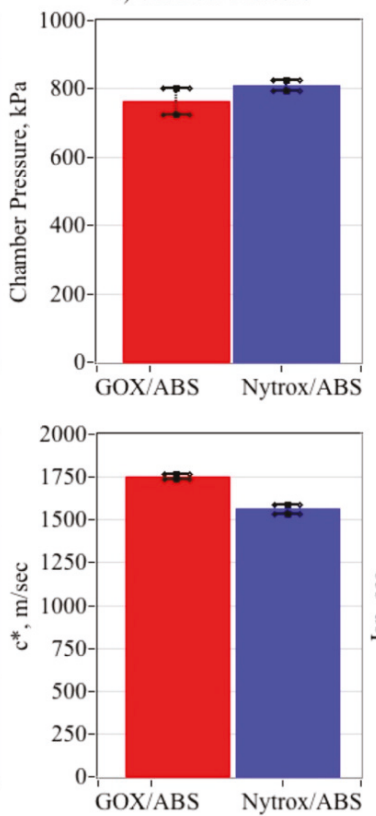

e) Characteristic Velocity c) Massflow
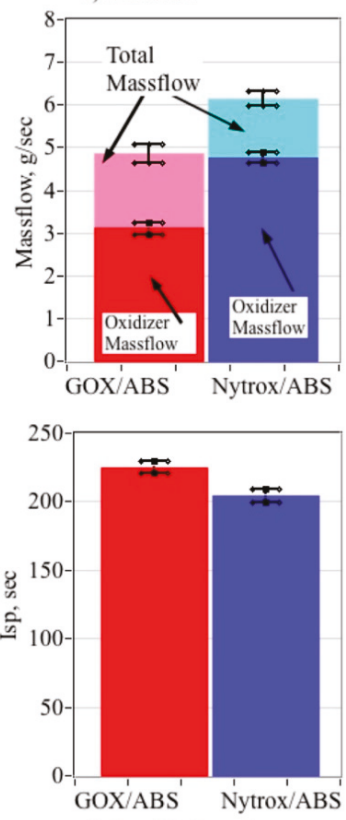

f) Specific Impulse

Figure 17. Comparing performance of test thruster using GOX/ABS and Nytrox 87/ABS.

Also, the mean values of the performance parameters $C_{F}, C^{*}$, and $I_{\mathrm{sp}}$, exhibit a slight-to-moderate reduction for Nytrox/ABS when compared to GOX/ABS. For example, as shown by Figure 17e, $\mathrm{f}$ the mean GOX/ABS $I_{\mathrm{sp}}$ and $c^{*}$ values are $224.8 \mathrm{~s}$ and $1751 \mathrm{~m} / \mathrm{s}$, respectively, as compared to $204.4 \mathrm{~s}$ and $1561 \mathrm{~m} / \mathrm{s}$ for Nytrox/ABS. This lowered performance results from two factors, the reduced optimal $c^{*}$ for Nytrox/ABS based on a lower flame temperature, and a less-than optimal O/F ratio for the Nytrox thruster. The first factor was previously predicted by the analytical comparisons of Figure 6, and was expected. The second factor can be corrected by slightly shortening the fuel grain length in order to better approach the optimal $O / F$ ratio.

\subsubsection{Comparing Combustion Properties}

Figure 18 compares the mean oxidizer to fuel ratio $O / F$, equivalence ratio $\Phi$ (Equivalence ratio $\Phi$ is defined as the stoichiometric $O / F$ divided by the actual $O / F$ achieved during the burn), combustion efficiency $\eta^{*}$, and mean regression rate $\overline{\dot{\gamma}}$ of the thruster burned with the two classes of propellants. Note, as shown by Figures $13 \mathrm{c}$ and $15 \mathrm{c}$, that the Nytrox mass flows were $30 \%$ higher than the corresponding GOX mass flows, and the resulting $O / F$ ratios are significantly higher for the Nytrox thruster. The higher $\mathrm{O} / \mathrm{F}$ ratio for Nytrox also results from the lower overall fuel regression rates shown by Figures 14d and $16 \mathrm{~d}$.

For both oxidizers the target equivalence ratio was between 1.5 and 2.0. Burning at these moderately fuel-rich equivalence ratios has the beneficial effect of reducing flame temperature, lowering nozzle throat erosion rate, and producing a plume with a lower molecular weight. The lowered molecular weight compensates for the reduced flame temperature, allowing an acceptable level of performance to be achieved. The fuel-rich equivalence ratios are a likely-reason why essentially no nozzle erosion was observed during this testing campaign.

Since, as shown by Figure 18b, both propellants burned at equivalence ratios between 1.5 and 2.0, the top-level design objectives were indeed achieved by these tests. The GOX/ABS thruster burned an 
equivalence ratio of approximately 1.65 , and as shown by Figure $14 a, b$, the resulting $O / F$ ratio tends to lie directly on top of the $\mathrm{O} / \mathrm{F}$ ratio for maximum $\mathrm{c}^{*}$.

When Nytrox 87 GOX was swapped-in for GOX, the ensemble mean value for $\Phi$ is approximately 1.95. As shown by Figure 16a,b, the mean Nytrox/ABS burn condition is slightly richer that would be required to achieve optimal performance. Because this test series was designed to evaluate Nytrox as a "drop-in" for GOX, the fuel port geometry was not optimized for best performance, thus, the non-optimal $c^{*}$ is not a concern here. A simple method for optimizing the system for Nytrox would be to slightly shorten the fuel port, thus, the resulting $\mathrm{O} / \mathrm{F}$ ratio towards leaner burning and back to near the optimal value.
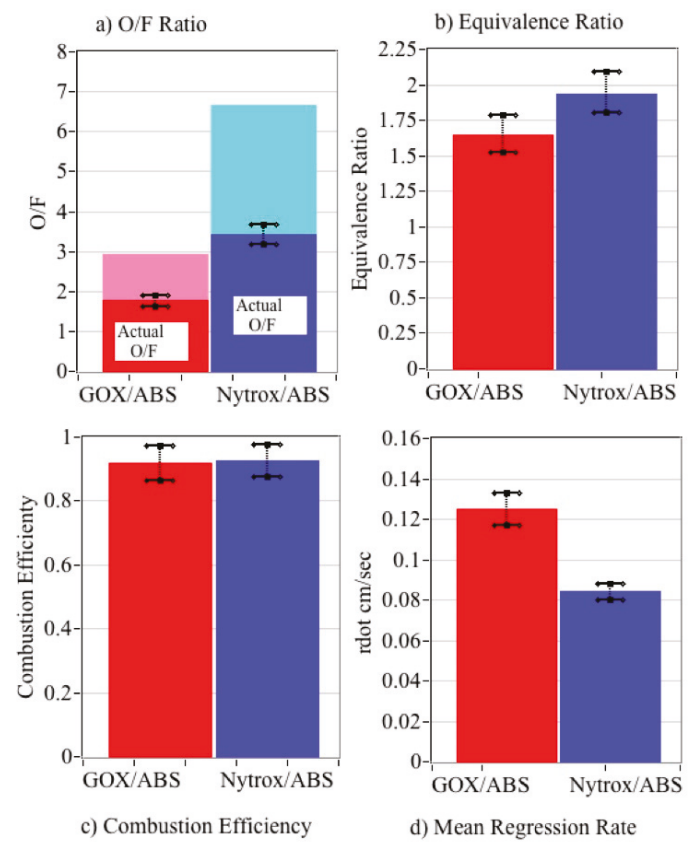

Figure 18. Comparing combustion properties of GOX/ABS and Nytrox 87/ABS motors.

\subsubsection{Detailed Fuel Regression Rate Comparisons}

Figure 19 presents the time-averaged, longitudinal-mean regression rates for GOX/ABS and Nytrox 87/ABS, overlaid with regression rate data for other propellant combinations. These data include previously collected by the Whitmore et al. (2013) [61] and Whitmore and Merkley [62] for pure $\mathrm{N}_{2} \mathrm{O}$ using HTPB (hydroxyl-terminated polybutadiene) and ABS as fuels. Power-law curve fits taken from Reference [63] for: (a) liquid oxygen (LOX)/paraffin, (b) LOX/HTPB, (c) $\mathrm{N}_{2} 0 /$ Escorez-HTPB, and (d) LOX/high-density-polyethylene (HDPE are also plotted.

The power-law curve fits take the form:

$$
\hat{\dot{r}}=a \cdot G_{o x}^{n}
$$

where $G_{o x}$ is the longitudinal mean of the oxidizer massflux, $\hat{\dot{\gamma}}$ is the curve-fit dependent variable, and $\{a, n\}$ are the burn parameters. The mean regression rate plotted on Figure $16 \mathrm{~d}$ calculated by integrating 
the power-law curve fits for GOX/BS and Nytrox 87/ABS over the operating massflux range, in this case, $0-20 \mathrm{~g} / \mathrm{cm}^{2} \cdot \mathrm{s}$ :

$$
\overline{\dot{r}}=\frac{a}{G_{o x_{\max }}} \int_{0}^{G_{o x_{\max }}} G_{o x}^{n} \cdot d G_{o x}=\frac{a}{n+1} G_{o x_{\max }}^{n}
$$

Table 4 summarizes the power-law fit parameters for the plotted curves on Figure 17. The Nytrox/ABS regression rates are moderately lower than result from GOX/ABS and LOX/Paraffin, a result that is primarily due to the reduced flame temperature and the associated heat transfer from the flame zone to the fuel surface. However, the resulting Nytrox87/ABS regression rates are clearly higher to $\mathrm{N}_{2} \mathrm{O}$ as a stand-alone oxidizer. The higher regression rate will lead to a reduced fuel grain length in order to achieve and equivalent fuel mass flow rate. This volumetric reduction offsets the loss in overall system density that derives from the switch to Nytrox from $\mathrm{N}_{2} \mathrm{O}$.

Also of note, for both propellant combinations GOX/ABS and Nytrox/ABS the burn exponents are close to a value of $n=0.5$. As noted by Zilliac and Karabeyoglu (Reference [56]), a hybrid-burn exponent of 0.5 corresponds to a propellant combination with essentially no O/F shift over the burn lifetime. This numerical calculation agrees well with the test observations, where very little $O / F$ shift was noted for this small thruster system. Ref. [62] explain this neutral burn profile as a balance between convective and radiative heat transfer in the combustion chamber. In small thruster systems of this size, radiation heating is no longer negligible and tends to compensate for the drop off in convective heat transfer as the fuel burns and the port opens up.

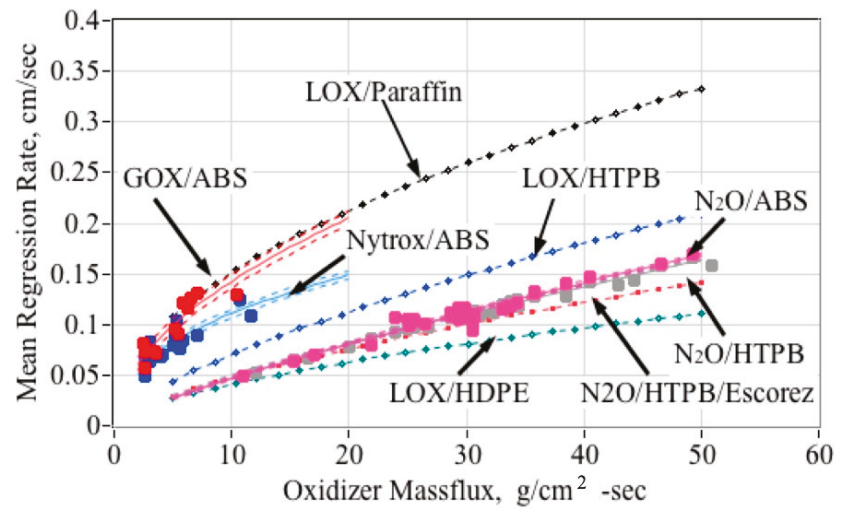

Figure 19. Comparing GOX/ABS and Nytrox 87/ABS fuel regression rates to previous tests of hybrid propellants.

Table 4. Power-law fit Parameters for GOX/ABS, Nytrox87/BS and other selected propellant regression rates. (A portion of this data is taken from References [61-63]. Data reproduced with permission).

\begin{tabular}{ccccccccc}
\hline \multirow{2}{*}{ Propellant } & $\begin{array}{c}\text { GOX/ } \\
\text { ABS }\end{array}$ & $\begin{array}{c}\text { Nytrox87/ } \\
\text { ABS }\end{array}$ & $\begin{array}{c}\mathbf{N}_{2} \mathbf{O} / \\
\text { ABS }^{58}\end{array}$ & $\begin{array}{c}\mathbf{N}_{2} \mathbf{O} / \\
\text { HTPB }^{58,59}\end{array}$ & $\begin{array}{c}\text { Paraffin/ } \\
\text { LOX }^{59}\end{array}$ & $\begin{array}{c}\text { LOX/ } \\
\text { HTPB }^{59}\end{array}$ & $\begin{array}{c}\text { Lox/ } \\
\text { HTPB-Escorez }^{59}\end{array}$ & $\begin{array}{c}\text { LOX/ } \\
\text { HDPE }^{59}\end{array}$ \\
\hline $\boldsymbol{a}$ coefficient & 0.0428 & 0.0354 & 0.00742 & 0.00795 & 0.0488 & 0.0146 & 0.0099 & 0.0098 \\
\hline$n$ exponent & 0.524 & 0.455 & 0.799 & 0.773 & 0.491 & 0.681 & 0.680 & 0.620 \\
\hline
\end{tabular}

\subsubsection{Volumetric Efficiency Comparisons}

In contrast to the mass-based $I_{\text {sp }}$ comparisons that favor the performance of the GOX/ABS propellants, the Nytrox/ABS propellants exhibit a significantly higher impulse density. The bar charts of Figure 20 show these comparisons. Plotted are effective specific gravity in Figure 20a and measured impulse density at ambient conditions in Figure 20b. Based on the Nytrox liquid storage density 
at 86 atmospheres (from Peng-Robinson model) and $0{ }^{\circ} \mathrm{C}$, as compared to the GOX storage density at 150 atmospheres (from ideal gas law), and the ABS Fuel density of $1.04 \mathrm{~g} / \mathrm{cm}^{3}$, the respective specific gravities are 0.842 and 0.512 , with ambient density impulses of 1710 and $1174 \mathrm{~N} \cdot \mathrm{s} / \mathrm{L}$. Thus, the Nytrox/ABS propellants exhibit at least a $45 \%$ increase in volumetric efficiency when compared to GOX/ABS.
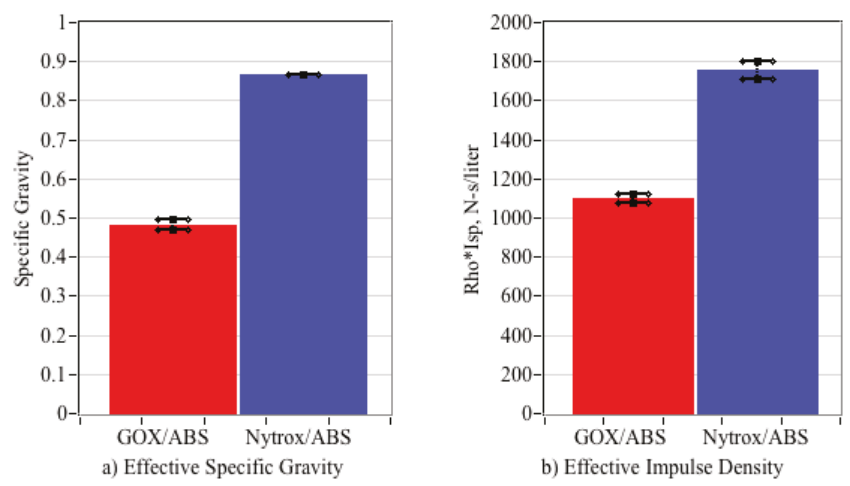

Figure 20. Volumetric Efficiency Comparisons.

\subsubsection{Extrapolating the Specific Impulse to Vacuum Conditions}

Recall that the specific impulse values plotted on Figure 14, Figure 16, and Figure 17 were derived from data collected under ambient test conditions at approximately $4700 \mathrm{ft}$. (1430 m) altitude, the elevation of the test facility in Logan Utah. The 2.07:1 nozzle expansion-ratio was designed to give near-optimal performance at this altitude, and the desired operating chamber pressure. Clearly, when matched with a high expansion-ratio nozzle, the vacuum performance will be significantly better. The ambient-test data can be extrapolated to altitude using the previously presented 1-D de Laval flow equations. (Anderson, Reference [49]) First, the thrust coefficient for the test condition is written in terms of the nozzle-exit-to-chamber pressure ratio, and the freestream-to-chamber pressure ratio:

$$
C_{\text {Ftest }}=\gamma \sqrt{\frac{2}{\gamma-1} \cdot\left(\frac{2}{\gamma+1}\right)^{\frac{\gamma+1}{\gamma-1}}}\left(1-\frac{p_{\text {exit }}}{P_{0}}\right)^{\frac{\gamma-1}{\gamma}}+\left(\frac{A_{\text {exit }}}{A^{*}}\right)\left(\frac{p_{\text {exit }}-p_{\infty}}{P_{0}}\right)
$$

For optimal operating conditions, the pressure thrust term of Equation (18) vanishes and the ratio of the optimal and test $C_{F}$ 's is used to scale specific impulse to the optimal condition:

$$
\frac{I_{\text {sp }} p_{\text {opt }}}{I_{\text {sptest }}}=\frac{\left(\frac{P_{0} A^{*}}{g_{0} \dot{m}}\right) C_{F_{\text {opt }}}}{\left(\frac{P_{0} A^{*}}{g_{0} \dot{m}}\right) C_{F_{\text {test }}}}=\frac{1}{1+\frac{\left(\frac{A_{\text {exit }}}{A^{*}}\right)\left(\frac{p_{\text {exit }}-p_{0}}{P_{0}}\right)}{\sqrt{\frac{2}{\gamma-1} \cdot\left(\frac{2}{\gamma+1}\right)^{\frac{\gamma+1}{\gamma-1}}\left(1-\frac{p_{\text {exit }}}{P_{0}}\right)^{\frac{\gamma-1}{\gamma}}}}}
$$

Using the motor geometry, thrust coefficient, mean chamber pressure, and the CEA-derived calculations for Nytrox 87/ABS, and assuming a 92.5\% combustion efficiency, from Figure 18c, Figure 21 plots the specific impulse extrapolations for GOX/ABS and Nytrox 87/ABS. For both propellant combinations, using the data of Figures 14 and 16, the plotted parameters are: (a) optimal expansion ratio as a function altitude, (b) optimal $\mathrm{C}_{\mathrm{F}}$ as a function of the optimal expansion ratio, (c) optimal $I_{\mathrm{sp}}$ as a function of expansion ratio, and (d) optimal impulse density as a function of altitude. Also plotted as the red and blue symbols are the actual experimental values for the GOX/ABS and Nytrox 87/ABS motors. The GOX/ABS data extrapolates to an $I_{\mathrm{sp}}$ of greater than $345 \mathrm{~s}$ under vacuum conditions, 
while the Nytrox/ABS data extrapolates to just over $300 \mathrm{~s}$. This $I_{\mathrm{sp}}$ value, although $8 \%$ lower than for GOX/ABS, is still nearly $25 \%-30 \%$ higher than can be achieved by any of the "green" ionic liquid propellants or by hydrazine. Using a similar process to scale the impulse density, the corresponding optimal vacuum $\rho I_{\text {sp }}$ values are 1,590 N.s/L for GOX/ABS, and $2510 \mathrm{~N} \cdot \mathrm{s} / \mathrm{L}$ for Nytrox 87/ABS. These values will be compared to Hydrazine and the ionic liquid propellants later in this report.

(a) Optimal Expansion Ratio at Altitude
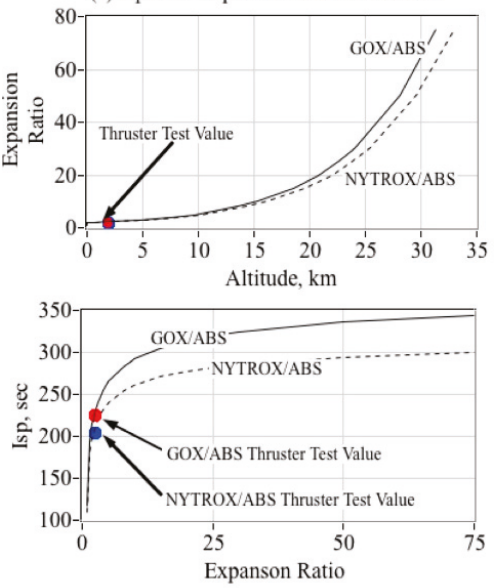

(c) Specific Impulse at Optimal Expansion Ratio (b) Thrust Coefficent at Optimal Expansion Ratio
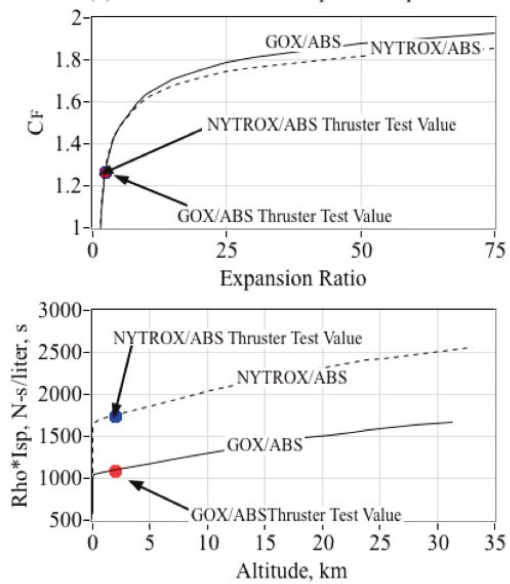

(d) Optimal Impulse Density vs Operating Altitude

Figure 21. Extrapolating ambient GOX/ABS and Nytrox 87/ABS test data to optimal vacuum conditions.

\subsubsection{Required Ignition Energy}

One of the key objectives of this research campaign was to demonstrate that Nytrox, can be "dropped in" as a replacement for GOX, but still function effectively with the previously-matured arc-ignition system. In that regard, the arc-ignition system was effective in igniting the Nytrox/ABS propellants, but did exhibit several minor issues that must still be overcome for an operation system. When a virgin-fuel grain is first burned with Nytrox, the observed ignition reliability was only about $50 \%$. "Setting" the arc path by first burning the grain using GOX, or by using a GOX pre-lead prior to initiating Nytrox flow overcame this problem. Once the first ignition is achieved, then the system reliably ignites using Nytrox, even with a dead-cold motor. The reason for this observed behavior appears to be that Nytrox expansion into the combustion chamber super-chills the ABS fuel, causing the surface impedance to increase to a point where the HVPS cannot provide sufficient power to pyrolize a conduction path through the material. Once a conduction path is set into the fuel material after the initial burn, then this issue goes away.

The data presented in Figure 22 supports this assertion. Here the required ignition energy is plotted as a function of the cumulative fuel grain burn lifetime. These data were taken from the burns plotted on Figures 14 and 16. Recall from the previous discussion of Section 2.3 that the HVPS is current limited at $30 \mathrm{~mA}$, and the output voltage is a function of the impedance path that the arc carves along the material surface. Total ignition energy is calculated as the integral of the output power. The HVPS output power is calculated as the product of the voltage and current-sense readings. Figure 22a plots the ignition energy for GOX/ABS propellants, and Figure 22b plots the ignition energy for Nytrox/ABS as a function of cumulative burn time for the fuel grain. The first two points on Figure 22b, near zero burn lifetime, were obtained from successful Nytrox tests of two previously unburned fuel grains without the GOX pre-lead. Both of these point show a considerably higher ignition energy requirement. The remaining lower ignition energy data points plotted on Figure $22 \mathrm{~b}$ were obtained from fuel grains 
that had been initially burned using a GOX pre-lead. For both GOX/ABS and Nytrox/ABS, the ignition energy correlates directly with burn time, indicating that the arc-path becomes better established, and the impedance drops following each ignition.
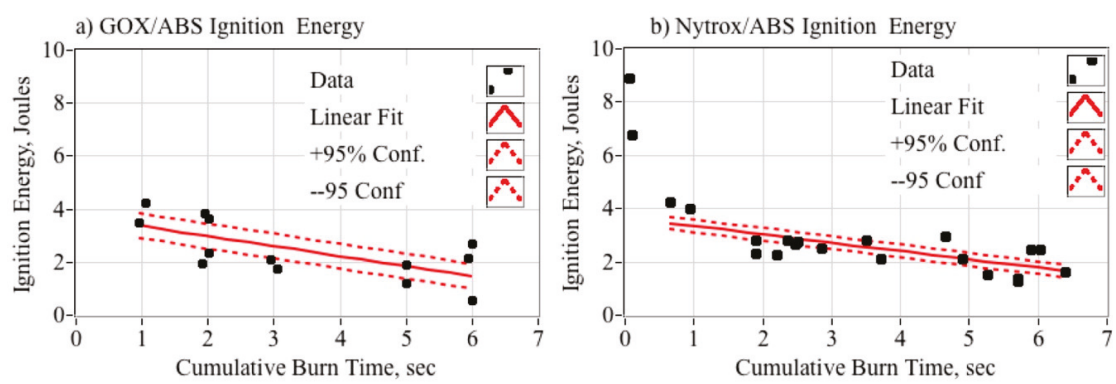

Figure 22. Effect of Fuel Grain Burn Lifetime on Required Ignition Energy.

Once the path is set, the required ignition energy is similar for both oxidizers. The bar chart of Figure 23 compares the mean ignition energies for GOX/ABS and Nytrox 87/ABS, calculated from the data presented in Figure 22, but with the virgin-grain Nytrox burns excluded. Both systems have a mean startup energy less than 2.5 joules, and to a $95 \%$ confidence level, neither require more than 4 joules for ignition (excluding the virgin-grain data). This energy level is contrasted to the ECAPS Prisma spacecraft [60] which used the ADN-based LMP-103s green propellant. For first ignition, this system required a 10 watt preheat for as long as $20 \mathrm{~min}$, consuming more than 12,000 joules of energy.

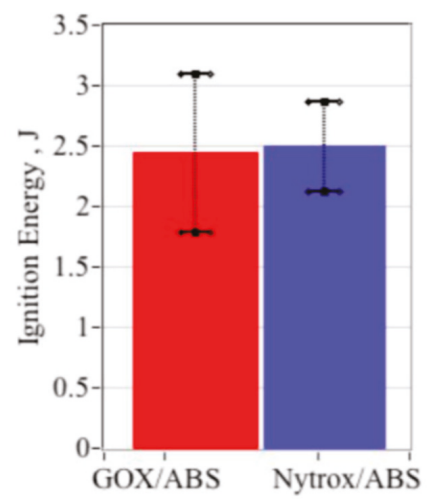

Figure 23. Mean Ignition Energy Levels for "Pre-Set" Fuel Grains.

\subsubsection{Ignition Latency}

Substituting Nytrox 87 for GOX in the 10-N thruster system, resulted in significant increase in ignition latency. Figure 24 illustrates this phenomenon. Plotted are time histories demonstrating the typical ignition behaviors for a 2-s pulse of the thruster system using first GOX/ABS, and then Nytrox 87/ABS. Plotted are: (a) thrust, (b) chamber pressure, (c) oxidizer mass flow, and (d) ignition power. Note that for both runs the ignition power profiles for both conditions are quite similar, but the chamber responses are significantly different. As shown by the mass flow data of Figure 24c, after opening the oxidizer valve flow into the combustion chamber is almost immediate. While the GOX/ABS motor lights and reaches within $63.2 \%$ of full operating pressure within about 150 milliseconds, the Nytrox/ABS Motor exhibits a more significant startup latency, in this example, an additional $250 \mathrm{~ms}$. 

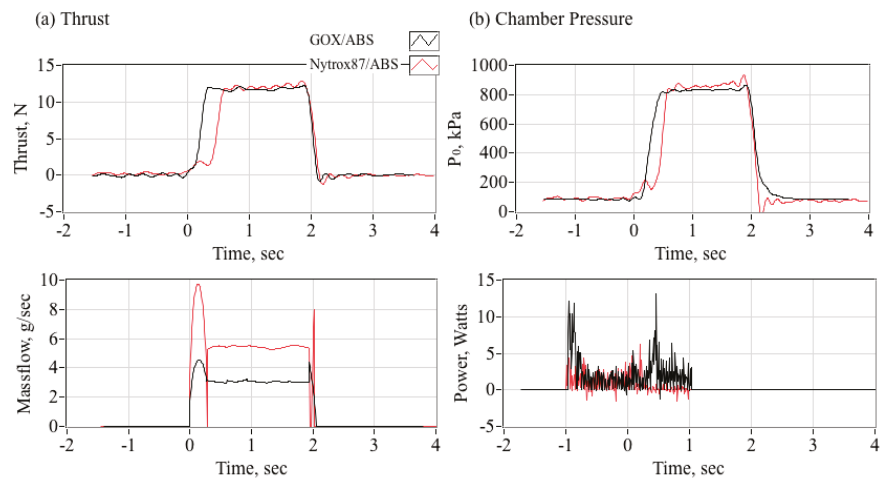

(c) Oxidizer Massflow

(d) Ignition Power

Figure 24. Typical ignition response time histories for GOX/ABS and Nytrox 87/ABS thrusters.

Over the burn lifetime of the fuel grains, the Nytrox/ABS propellants exhibit mean ignition latencies that are significantly larger than for GOX-ignition. It is hypothesized that the rapid expansion and subsequent phase change super-chills Nytrox fluid entering the combustion chamber. The very cold Nytrox reduces the pyrolysis rate of the fuel material and absorbs some of the spark energy. The result is a result in a slowed ignition process. The bar chart of Figure 25 shows this latency comparison.

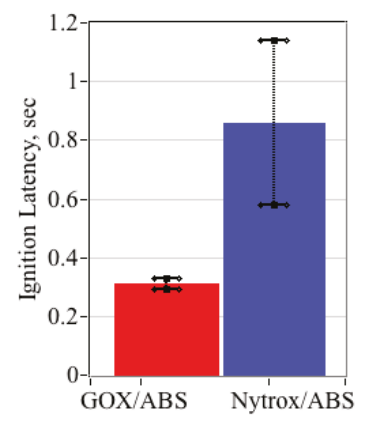

Figure 25. GOX/ABS, Nytrox/ABS mean ignition latency comparisons.

The additional latency exhibited by the Nitrox/ABS propellants occurred for all of the test runs, and appears to be endemic to the use of Nytrox as a hybrid oxidizer. Figure 26 compares the observed ignition latencies, calculated as the $63.2 \%$ first-order response rise time, for GOX/ABS and Nytrox 87/ABS. The GOX-ignition latency of Figure 24a correlates with the combustion chamber pressure, whereas the Nytrox-ignition latency of Figure 26b correlates strongly with the NOS ${ }^{\circledR}$ (Nytrox) run-tank pressure. The trend is very distinctive with the latency growing from only about $250 \mathrm{~ms}$ at the original tank pressure and temperature, to greater than $1.7 \mathrm{~s}$ at the highest internal tank pressures.

At first glance the behaviors exhibited by Figures 25 and 26 appear to be quite odd, but upon further examination, it becomes clear that the Nytrox latency trend results from the two-phase binary-mixture properties, and the concentration of oxygen in solution. Clearly for GOX-ignition the thruster chamber pressure is a key influence. With higher chamber pressures allowing nozzle to choke faster and build the internal oxygen partial pressure to greater than the observed critical value of 2 atmospheres (from Reference [24]) more rapidly.

In contrast, it appears that the observed latency trend of Figure $26 \mathrm{~b}$ results from the $\mathrm{O}_{2}$ mass concentration in the Nytrox solution. As described previously in Section 3.1, for a typical test the Nytrox run tank was chilled to $0{ }^{\circ} \mathrm{C}$ in an ice bath while filling. After the Nytrox is blended at the 
desired density manufactured, the filled run tank is stored at $-15^{\circ} \mathrm{C}$ to keep vapor pressures lower for safe storage. During testing the tank is removed from the freezer and gradually warms to the ambient temperature conditions.
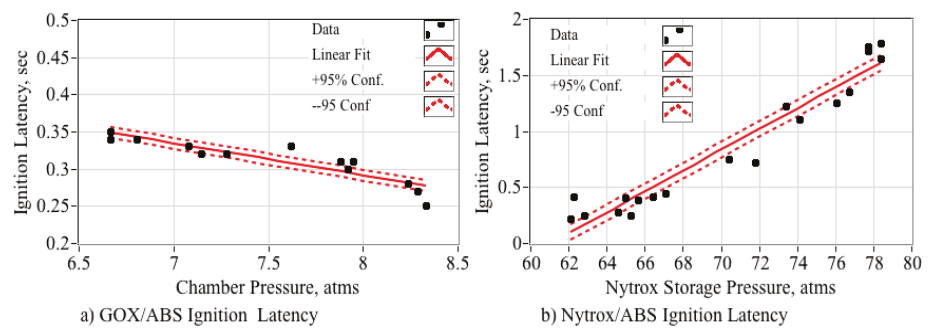

Figure 26. Comparing GOX/ABS and Nytrox/ABS ignition latencies.

The previously-described Peng-Robinson model [43] is used to analyze the quantitative effect of the rising tank temperature upon the Nytrox oxygen concentration. Assuming that the total molar volume remains constant, then as the tank warms from an initial temperature and pressure $\left\{P_{\text {init }}, T_{\text {init }}\right\}$ to a higher temperature $T_{\text {final }}$, then the corresponding pressure rise is calculated from the real gas equation of state:

$$
\frac{1}{v}=\frac{1}{v_{v}}+\frac{1}{v_{l}}=\left(\frac{1}{Z_{v}}+\frac{1}{Z_{l}}\right) \frac{P}{R_{u} \cdot T}
$$

where the parameters $v$ and $Z$ refer to the molar volume and compressibility factor, and the indices " 1 " and " $v$ " refer to the vapor and liquid phases, respectively. For the purposes of this discussion, the compressibility factor is a measure how much the fluid properties deviate from an ideal gas. Since the molar volume is constant, then from the initial-to-final state:

$$
\left(\frac{1}{Z_{v}}+\frac{1}{Z_{l}}\right)_{\text {init }} \frac{P_{\text {init }}}{T_{\text {init }}}=\left(\frac{1}{Z_{v}}+\frac{1}{Z_{l}}\right)_{\text {final }} \frac{P_{\text {final }}}{T_{\text {final }}}
$$

Solving for the final pressure level:

$$
P_{\text {final }}=\left(\frac{T_{\text {final }}}{T_{\text {init }}}\right) \cdot \frac{\left(\frac{1}{Z_{v}}+\frac{1}{Z_{l}}\right)_{\text {init }}}{\left(\frac{1}{Z_{v}}+\frac{1}{Z_{l}}\right)_{\text {final }}} \cdot P_{\text {init }}
$$

and the equilibrium solution at the final pressure is the state vector $\left\{P, T, Z_{v}, Z_{1}\right\}_{\text {final }}$ whose values are consistent with the Peng-Robinson model.

Figure 27 plots this numerical solution assuming a starting temperature and pressure of $-15^{\circ} \mathrm{C}$ (the Nytrox storage temperature) and $62 \mathrm{~atm}$. The solution then warms up to a peak temperature of $35{ }^{\circ} \mathrm{C}$. Plotted are: (a) the original latency data, (b) the Nytrox phase-equilibrium pressure as a function of temperature, (c) the $\mathrm{O}_{2}$ mass concentrations in the vapor and liquid phases as a function of solution temperature, and (d) the $\mathrm{O}_{2}$ mass concentrations in the vapor and liquid phases as a function Nytrox phase-equilibrium pressure. Note, as the tank warms, the pressure rises to 68 atmospheres at $0{ }^{\circ} \mathrm{C}$ (the optimal operating point) and then peaks at around 79 atmospheres at room temperature, or approximately $25^{\circ} \mathrm{C}$. This calculated maximum pressure agrees well with the maximum pressure observed in Figure 24a. Thus, the rising tank pressure correlates with a diminishing mass of oxygen in solution. 

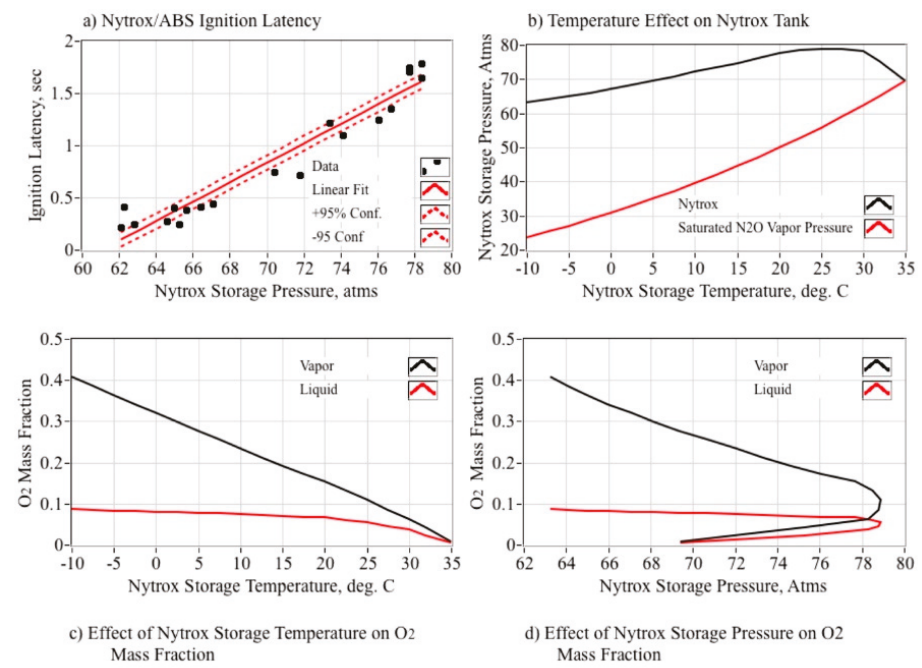

Figure 27. Nytrox ignition latency explained as diminishing $\mathrm{O}_{2}$ concentrations in Nytrox solution at higher tank temperatures.

As plotted in Figure $27 \mathrm{c}$, d, note that $\mathrm{O}_{2}$ mass concentrations drop rapidly as the solution warms. Eventually nearly all of the oxygen comes out of solution leading to a pure nitrous oxide liquid phase and stratified $\mathrm{O}_{2}$ and $\mathrm{N}_{2} \mathrm{O}$ vapor phases. Since oxygen is the lighter of the gasses, it would occupy the upper portion of the tank ullage. Thus, at the higher tank pressures of the warm tank, it appears that almost no $\mathrm{O}_{2}$ reaches the injector during the initial ignition stages. It appears that this phenomenon makes ignition increasingly more difficult, and it takes longer for the oxygen concentration to build to the critical level for ignition.

The results presented by Figures 26 and 27 were discovered only shortly before the publication of this paper, and have not been fully explored. Clearly, Nytrox response latencies have not been fully characterized at this point been, and are recommended as a point of emphasis for further study. A dedicated experiment with wider pressure and temperature ranges, and a range of initial saturation levels is proposed. When completely comprehended, it appears that super-chilling the Nytrox solution may be an effective engineering practice for reducing the observed ignition latencies using Nytrox.

\subsection{Comparing Nytrox to Existing Space Mono-Propellants}

With 19 successful hot-firings reported in this paper, Nytrox 87 has been demonstrated as a reliable on- "drop-in" replacement for GOX in the HPGHP thruster system. Table 5 compares the performance of the Nytrox/ABS system to the competing propellants: hydrazine, LMP-103S, and AF-M315-E. In Table 5 the data for Hydrazine and AF-M315E were extracted from Refs. [14] and [17]. Data for LMP-103S were extracted from Refs. [16] and [18]. Data for the Nytrox/ABS Hybrid System were extracted from Section 5 of this paper, and also from Ref. [1].

With the exception of impulse density, the Nytrox/ABS system outperforms the other propellants in every listed category. Even the lower $\rho I_{\text {sp }}$ value is a bit misleading. Because Nytrox had the ability to safely self-pressurize, there is no need for an additional volumetrically inefficient oxidizer pressurization system. The absence of this secondary system significantly decreases the overall system complexity, and leads to a clear advantage in volumetric efficiency. Due to the high pyrolysis energy of ABS fuel, 3.1 MJ $/ \mathrm{kg}$, the HPGHP motors are ablative and self-cooling, offering a final systems advantage. 
Table 5. Nytrox/ABS performance characteristics compared to existing space mono-propellants.

\begin{tabular}{|c|c|c|c|c|}
\hline Propellant & Hydrazine & LMP-103S & AF-M315E & $\begin{array}{l}\text { Nytrox/ABS } \\
\text { Hybrid }\end{array}$ \\
\hline Flame Temperature & $600-750^{\circ} \mathrm{C}$ & $1600^{\circ} \mathrm{C}$ & $1900^{\circ} \mathrm{C}$ & $3000^{\circ} \mathrm{C}$ \\
\hline Vacuum $I_{\mathrm{sp}}, \mathrm{s}$ & $220-225$ & $\begin{array}{c}252 \text { (theory) } \\
235 \text { (delivered) }\end{array}$ & $\begin{array}{c}266 \text { (theory) } \\
245 \text { (delivered) }\end{array}$ & $\begin{array}{c}320 \text { (theory) } \\
294 \text { (extrapolated) }\end{array}$ \\
\hline Specific Gravity & 1.01 & 1.24 & 1.465 & $0.650\left(87 \% \mathrm{~N}_{2} \mathrm{O}\right)$ \\
\hline Impulse density, N.s/L & 2270 & $\begin{array}{c}3125 \text { (theory) } \\
2915 \text { (delivered) }\end{array}$ & $\begin{array}{c}3900 \text { (theory) } \\
3650 \text { (delivered) }\end{array}$ & $\begin{array}{c}2510 \\
\text { (vacuum, extrapolated) } \\
1750 \\
\text { (ambient, delivered) }\end{array}$ \\
\hline Preheat Temperature & $\begin{array}{l}315^{\circ} \mathrm{C} \text {, cold-start } \\
\text { capable }\end{array}$ & $300^{\circ} \mathrm{C}$ & $370^{\circ} \mathrm{C}$ & $\begin{array}{c}\mathrm{N} / \mathrm{A} \\
\text { none-required }\end{array}$ \\
\hline $\begin{array}{l}\text { Required Ignition } \\
\text { Input Energy, Joules }\end{array}$ & N/A & $\begin{array}{c}12,000 \mathrm{~J} \\
(10 \text { Watts @ 1200 s) }\end{array}$ & 27,000 J (15 Watts @ $1800 \mathrm{~s}$ & $\begin{array}{c}2.5 \mathrm{~J} \\
(5 \text { Watts for } 500 \mathrm{~ms}) \\
\end{array}$ \\
\hline $\begin{array}{c}\text { Propellant Freezing } \\
\text { Temperature }\end{array}$ & $1-2^{\circ} \mathrm{C}$ & $-7^{\circ} \mathrm{C}$ & $\begin{array}{c}<0{ }^{\circ} \mathrm{C} \\
\text { (forms glass, no freezing point) }\end{array}$ & $-70^{\circ} \mathrm{C}$ \\
\hline Cost & $\$$ & $\$ \$ \$$ & $\$ \$ \$ \$$ & $\$$ \\
\hline Availability & Readily Available & Restricted Access & Limited Access & $\begin{array}{l}\text { Very Widely } \\
\text { Available }^{1}\end{array}$ \\
\hline $\begin{array}{l}\text { NFPA } 704 \text { Hazard } \\
\text { Class [64] }\end{array}$ & & & & \\
\hline
\end{tabular}

${ }^{1} 80 \%-90 \%$ Nytrox solutions easily manufactured, as per procedure in this paper. ${ }^{2}$ Based up the AF-M315E constituent components, Hydroxyl Ammonium Nitrate (HAN), and 2-Hydroxyethylhydrazine (HEHN) [65].

\section{Summary and Conclusions}

Over the past decade, a novel High-Performance "Green" Hybrid Propulsion (HPGHP) system has been developed as an environmentally sustainable replacement for hydrazine, and other highly-toxic spacecraft propellants. HPGHP is enabled by recent advances in 3-D printing and leverages unique electrical breakdown characteristics of printed plastics like ABS and polyamide. Additive manufacturing changes the electrical breakdown properties, and when printed materials are presented with a sufficiently high, low-current voltage, electrical-arcing along the layered surface pyrolizes material and seeds combustion when an oxidizing flow is introduced. The system has been engineered to a high level of reliability with the number of possible ignitions limited only by the amount of fuel. Typical startup sequences require less than 2 joules, and once started, the system can be sequentially fired with no additional energy inputs required.

In its most mature form, HPGHP uses gaseous oxygen as the oxidizer. Although gaseous oxygen is highly mass efficient, it is volumetrically inefficient due to its low specific gravity unless stored at very high pressures. In order to increase the HPGHP system volumetric efficiency, a two-phase blend of nitrous oxide and oxygen, "Nytrox," has been engineered as a higher-density "drop-in" replacement. Nytrox is similar to "laughing-gas" used for anesthesia applications and is blended by percolating oxygen under pressure into $\mathrm{N}_{2} \mathrm{O}$ until the solution reaches saturation.

With 19 successful hot-firings reported in this paper, results from the preliminary test-and-evaluation campaign have demonstrated Nytrox as an effective replacement for GOX in the HPGHP system, exhibiting a slightly reduced specific impulse, but with significantly higher volumetric efficiency. Vacuum specific impulse values exceeding $300 \mathrm{~s}$ are reported. This $I_{\mathrm{sp}}$ is significantly greater than can be achieved by hydrazine or the current generation of "green" propellants based on ionic-liquids, LMP-103S and AF-M315E. Because Nytrox had the ability to safely self-pressurize, there is no need for an additional volumetrically inefficient oxidizer pressurization system. This characteristic significantly decreases the overall system complexity.

The primary issue associated with using Nytrox a replacement for GOX as a hybrid oxidizer is a significant increase in the cold-start ignition latency. Presented data and supporting analysis 
shows that the higher Nytrox latency correlates strongly with the run tank storage temperature and pressure, and the mass fraction of oxygen in the Nytrox solution. Because of the reduced oxygen levels at warmer temperatures, it takes longer for the combustion chamber oxygen concentration to build to the critical level required for ignition. It appears that super-chilling the Nytrox solution may be an effective engineering practice for reducing the observed ignition latencies using Nytrox. Clearly, these Nytrox response latencies have not been fully characterized, and are recommended as a point of emphasis for further study.

\section{Patents}

Whitmore, Stephen A., "Restartable Ignition Devices, Systems, and Methods Thereof," USA Provisional Patent No. US 2015/A0322892 A1, 12 November, 2015.

Funding: This work was partially funded with a cooperative agreement with the NASA Marshall Spaceflight Center, Cooperative Agreement No. NNM16AA01A.

Acknowledgments: The author is especially grateful for the assistance of NASA Marshall Space Flight Center (MSFC) ER-23, by graciously provided access to the testing facilities used to collect vacuum chamber data for this project. I deeply appreciate MSFC employees Kevin Pedersen, Carlos Diaz, and Daniel Cavender for their time, technical support, and expert advice.

Conflicts of Interest: The author declares no conflict of interest.

\section{Nomenclature}

\section{Symbols}

$A_{\mathrm{C}}$

$A_{\text {exit }}$

$A^{*}$

$A_{1}$

$A_{2}$

$A_{\text {exit }} / A^{*}$

$C_{\mathrm{d}}$

$\mathrm{C}_{\mathrm{F}}$

$\bar{c}_{F}$

$c^{*}$

$E_{\mathrm{a}}$

$E_{\mathrm{a}_{\mathrm{c}}}$

$E_{\mathrm{a}}$

$E_{\mathrm{i}}$

$E_{\text {if }}$

F

$G_{\text {ox }}$

$G_{\text {tot }}$

$g_{0}$

H

$I_{\mathrm{sp}}$

$L_{\text {port }}$

$M_{\mathrm{W}}$

$M_{\text {fuel }}$

$M_{\text {oxl }}$

$m_{\text {flue }}$

$m_{\mathrm{Ox}}$

$m_{\text {total }}$

$\mathrm{O} / \mathrm{F}$

$\mathrm{O} / \mathrm{F}_{\text {actual }}$

$\mathrm{O} / \mathrm{F}_{\text {stoich }}$ abfuel port cross-sectional area, $\mathrm{cm}^{2}$

abnozzle exit area, $\mathrm{cm}^{2}$

abcross sectional area at which local flow chokes, $\mathrm{cm}^{2}$

abVenturi inlet area, $\mathrm{cm}^{2}$

abVenturi throat area, $\mathrm{cm}^{2}$

abnozzle expansion-ratio

abdischarge coefficient

abthrust coefficient

abtime averaged thrust coefficient

abcharacteristic velocity of propellants, $\mathrm{m} / \mathrm{s}$

abactivation energy for self-sustaining decomposition reaction, $\mathrm{kJ} / \mathrm{kg}$

abactivation energy for contaminated solution, $\mathrm{kJ} / \mathrm{kg}$

abreduction of energy of activation due to catalysis or hydrocarbon contamination, $\mathrm{kJ} / \mathrm{kg}$

abminimum energy required to initiate a deflagration wave in Nytrox mixture, J

abignition energy amplification factor

abthrust, $\mathrm{N}$

aboxidizer massflux, $\mathrm{g} / \mathrm{cm}^{2} \cdot \mathrm{s}$

abtotal massflux, $\mathrm{g} / \mathrm{cm}^{2} \cdot \mathrm{s}$

abnominal acceleration of gravity, $9.8067 \mathrm{~m} / \mathrm{s}$

abenergy of reaction, $\mathrm{kJ} / \mathrm{kg}$

abspecific impulse, $\mathrm{s}$

abfuel grain length, $\mathrm{cm}$

abmolecular weight, g/g-mol

abconsumed fuel mass, $g$

abconsumed oxidizer mass, $\mathrm{g}$

abfuel mass flow, g/s

aboxidizer mass flow, $\mathrm{g} / \mathrm{s}$

abtotal mass flow through the nozzle, g/s

aboxidizer/fuel ratio

abactual oxidizer-to-fuel ratio

abstoichiometric oxidizer-to-fuel ratio 
O/F abtime-averaged oxidizer-to-fuel ratio.

$P_{1} \quad$ abVenturi inlet pressure, psia

$P_{2} \quad$ abVenturi throat pressure, psia

$P_{0} \quad$ abchamber pressure or oxidizer flow stagnation pressure, psia

$p_{\text {exit }} \quad$ abexit plane static pressure, psia

$p_{\infty} \quad$ abambient pressure, psia

$R_{\mathrm{g}} \quad$ abgas constant, $\mathrm{J} / \mathrm{kg}-\mathrm{K}$

$R_{\mathrm{u}} \quad$ abuniversal gas constant, $8314.4612 \mathrm{~J} / \mathrm{kg}-\mathrm{mol}-\mathrm{K}$

$r_{\mathrm{L}} \quad$ ablongitudinal average of the fuel port radius, $\mathrm{cm}$

$r_{0} \quad$ abinitial fuel port radius, $\mathrm{cm}$

$\dot{r}_{L} \quad$ ablongitudinal mean of fuel regression rate, $\mathrm{cm} / \mathrm{s}$

$\overline{\dot{r}} \quad$ abmean regression rate over burn duration, $\mathrm{cm} / \mathrm{s}$

Acronyms

ABS abdependent variable for regression rate as fit by power-law curve, $\mathrm{cm} / \mathrm{s}$ abspecific gravity with respect to weight of water. abstagnation temperature, $\mathrm{K}$ abburn time, $\mathrm{s}$ abgeneric time symbol, $\mathrm{s}$ abcompressibility factor $a b$ mean value combustion efficiency $a b$ of specific heats abequivalence ratio absolid fuel density, $\mathrm{g} / \mathrm{cm}^{3}$ abimpulse density, N.s/L standard deviation

$\mathrm{ADN}$ abAcrylonitrile Butadiene Styrene

AF-M315E

AFWL

ECAPS

ESTEC

FDM

GOX

GPIM

HAN

HDPE

HPGHP

HTPB

HVPS

IL

LMP-103S

$\operatorname{NOS}^{\circledR}$

P\&ID abAmmonium Dinitramide abHAN Based Ionic Liquid Green Propellant abAir Force Weapons Lab abEcological Advanced Propulsion Systems abEuropean Space Agency Space Research and Technology Center abFused Deposition Modeling abGaseous Oxygen abGreen Propellant Infusion Mission abHydroxylamine Nitrate abHigh Density Polyethylene abHigh-Performance "Green" Hybrid Propulsion abHydroxyl-Terminated Polybutadiene abHigh Voltage Power Supply abIonic Liquid abADN-Based Ionic Liquid "Green" Propellant abNitrous Oxide Systems (Trademark)

TRL

TTL abPiping and Instrumentation

USU-PRL abTechnology Readiness Level abTransistor-Transistor Logic abUtah State University Propulsion Research Laboratory 


\section{References}

1. Whitmore, S.A.; Bulcher, A.M. Vacuum Test of a Novel Green-Propellant Thruster for Small Spacecraft, AIAA 2017-5044. In Proceedings of the 53rd AIAA/SAE/ASEE Joint Propulsion Conference, AIAA Propulsion and Energy Forum, Atlanta, GA, USA, 10-12 July 2017. [CrossRef]

2. Bombelli, V. Economic Benefits for the Use of Non-toxic Monopropellants for Spacecraft Applications, AIAA-2003-4783. In Proceedings of the 39th AIAA/ASME/SAE/ASEE Joint Propulsion Conference and Exhibit, Huntsville, AL, USA, 20-23 July 2003. [CrossRef]

3. Haeseler, D.; Bombelli, V.; Vuillermoz, P.; Lo, R.; Marée, T.; Caramelli, F. Green Propellant Propulsion Concepts for Space Transportation and Technology Development Needs. ESA SP-557. In Proceedings of the 2nd International Conference on Green Propellants for Space Propulsion, Cagliari, Sardinia, Italy, 7-8 June 2004; Available online: https://www.researchgate.net/publication/234265416_Green_Propellant_Propulsion_ Concepts_for_Space_Transportation_and_Technology_Development_Needs (accessed on 5 December 2019).

4. Goldstein, E. The Greening of Satellite Propulsion. Aerosp. Am. 2012, 26-28. Available online: https://uppsagd.files.wordpress.com/2012/05/aerospace-america-february-2012-the-greening-ofsatellite-propulsion-page-26-28.pdf (accessed on 5 December 2019).

5. Venkatachalam, S.; Santhosh, G.; Ninan, K.N. An Overview on the Synthetic Routes and Properties of Ammonium Dinitramide (ADN) and other Dinitramide Salts. Propellants Explos. Pyrotech. 2004, 29, 178-187. [CrossRef]

6. Nagamachi, M.Y.; Oliveira, J.I.; Kawamoto, A.M.; Dutra, R.D. ADN-The new oxidizer around the corner for an environmentally friendly smokeless propellant. J. Aerosp. Technol. Manag. 2009, 1, 153-160. Available online: http://www.redalyc.org/articulo.oa?id=309426551003 (accessed on 5 December 2019).

7. Rheingold, A.L.; Cronin, J.T.; Brill, T.B.; Ross, F.K. Structure of Hydroxylammonium Nitrate (HAN) and the Deuterium Homolog. Acta Crystallogr. 1987, 43, 402-404. Available online: https://scripts.iucr.org/cgi-bin/ paper?a26870 (accessed on 5 December 2019).

8. Hawkins, T.W.; Brand, A.J.; McKay, M.B.; Tinnirello, M. Reduced Toxicity, High Performance Monopropellant at the U.S. Air Force Research Laboratory. AFRL-RZ-ED-TP-2010-219. In Proceedings of the 4th International Association for the Advancement of Space Safety Conference, Huntsville, AL, USA, 19-21 May 2010; Available online: www.dtic.mil/dtic/tr/fulltext/u2/a522113.pdf (accessed on 5 December 2019).

9. Anflo, K.; Crowe, B. In-Space Demonstration of an ADN-based Propulsion System, AIAA-2011-5832. In Proceedings of the 47th AIAA/ASME/SAE/ASEE Joint Propulsion Conference \& Exhibit, San Diego, CA, USA, 31 July-03 August 2011. [CrossRef]

10. Spores, R.A.; Masse, R.; Kimbrel, S. GPIM AF-M315E Propulsion System, AIAA_2013-3849. In Proceedings of the 49th AIAA/ASME/SAE/ASEE Joint Propulsion Conference \& Exhibit, San Jose, CA, USA, 15-17 July 2013; Available online: https://ntrs.nasa.gov/archive/nasa/casi.ntrs.nasa.gov/20140012587.pdf (accessed on 5 December 2019).

11. Sempsrott, D. NASA's Green Propellant Infusion Mission Deploys. NASA Blog 2019. Available online: https://blogs.nasa.gov/spacex/2019/06/25/nasas-green-propellant-infusion-mission-deploys/ (accessed on 20 October 2019).

12. McLean, C.; Marotta, B.; Porter, B. Flight performance of the propulsion subsystem on the green propellant infusion mission. AAS 20-062. In Proceedings of the 30th AIAA/AAS Space Flight Mechanics Meeting, Orlando, FL, USA, 6-10 January 2020; Available online: http://www.univelt.com/AASPapers.html (accessed on 5 December 2019).

13. Wilhelm, M.; Negri, M.; Ciezki, H.; Schlechtriem, S. Preliminary tests on thermal ignition of ADN-based liquid monopropellants. Acta Astronaut. 2019, 158, 388-396. [CrossRef]

14. Anon, AF-M315E Safety Data Sheet. Available online: https://static1.squarespace.com/static/ 59de9c9c18b27ddf3bac610a/t/5e3af2d0fb8df43f9b2ae377/1580921553250/AF-M315E+SDS.pdf (accessed on 1 December 2019).

15. Button, K. Green Propellant. Aerospace America. March 2017. Available online: https://aerospaceamerica. aiaa.org/features/green-propellant/ (accessed on 1 December 2019).

16. Persson, M.; Anflo, K.; Dinardi, A. A Family of Thrusters for ADN-Based Monopropellant LMP-103S. AIAA-2012-3815. In Proceedings of the 48th AIAA/ASME/SAE/ASEE Joint Propulsion Conference \& Exhibit, Atlanta, GA, USA, 30 July-01 August 2012. [CrossRef] 
17. Brand, A. Reduced Toxicity, High Performance Monopropellant. AFRL-RZ-ED-VG-2011-326. Presentation at the Green Propellant Workshop, Sweden, 12-15 September 2011; p. 23. Available online: https://apps.dtic. mil/dtic/tr/fulltext/u2/a554667.pdf (accessed on 5 December 2019).

18. Pokrupa, N.; Anglo, K.; Svensson, O. Spacecraft System Level Design with Regards to Incorporation of a New Green Propulsion System. AIAA-2011-6129. In Proceedings of the 46th AIAA/ASME/SAE/ASEE Joint Propulsion Conference and Exhibit, San Diego, CA, USA, 31 July-3 August 2011. [CrossRef]

19. Katsumi, T.; Hori, K. Combustion Wave Structure of Hydroxylammonium Nitrate Aqueous Solutions. AIAA 2010-6900. In Proceedings of the 46th AIAA/ASME/SAE/ASEE Joint Propulsion Conference \& Exhibit, Nashville, TN, USA, 25-28 July 2010. [CrossRef]

20. Whitmore, S.A.; Burnside, C.G. Performance Analysis of a High Performance Green Propellant Thruster. NASA Marshall Space Flight Center Faculty Program, NASA TM-2015-218216. December 2015; pp. 125-151. Available online: https://ntrs.nasa.gov/search.jsp?R=20160000456 (accessed on 1 December 2019).

21. Lowe, D. The Overselling of Ionic Liquids. Science Magazine, In The Pipeline, 11 June 2013. Available online: http://blogs.sciencemag.org/pipeline/archives/2013/06/11/the_overselling_of_ionic_liquids (accessed on 28 March 2018).

22. Anon. Hazard Analysis of Commercial Space Transportation; Vol. 1: Operations, Vol. 2: Hazards, vol. 3: Risk Analysis, PB93-199040, Accession 1988; U.S. Dept. of Transportation: Washington, DC, USA, 1988. Available online: https://rosap.ntl.bts.gov/view/dot/35788 (accessed on 5 December 2019).

23. Palermo, E. Fused Deposition Modeling: Most Common 3D Printing Method. LIVESCIENCE. 19 September 2013. Available online: https://www.livescience.com/39810-fused-deposition-modeling.html (accessed on 2 December 2019).

24. Whitmore, S.A.; Inkley, N.R.; Merkley, D.P.; Judson, M.I. Development of a Power-Efficient, Restart-Capable Arc Ignitor for Hybrid Rockets. J. Propuls. Power 2015, 31, 1739-1749. [CrossRef]

25. Whitmore, S.A.; Mathias, S.D.; Harvey, R. High Voltage Breakdown and Arc-Tracking Mechanism of Thermoplastics with Applications to Hybrid Rocket Arc-Ignition. In Proceedings of the 53rd AIAA/SAE/ASEE Joint Propulsion Conference, Atlanta, GA, USA, 10-12 July 2017. AIAA 2017-4601. [CrossRef]

26. Whitmore, S.A.; Merkley, S.L.; Tonc, L.; Mathias, S.D. Survey of Selected Additively Manufactured Propellants for Arc Ignition of Hybrid Rockets. J. Propuls. Power 2016, 32, 1494-1504. [CrossRef]

27. Whitmore, S.A. Three-Dimensional Printing of "Green" Fuels for Low-Cost Small Spacecraft Propulsion Systems. J. Spacecr. Rocket. 2017, 54. [CrossRef]

28. Whitmore, S.A. Additive Manufacturing as an Enabling Technology for "Green" Hybrid Spacecraft Propulsion. In Proceedings of the RAST-1039, Conference on Recent Advances in Space Technology 2015, Istanbul, Turkey, 16-19 June 2015; Available online: https://ieeexplore.ieee.org/document/7208305 (accessed on 5 December 2019).

29. Whitmore, S.A.; Merkley, S.L.; Spurrier, Z.S.; Walker, S.D. Development of a Power Efficient, Restartable. Green" Propellant Thruster for Small Spacecraft and Satellites. SSC15-P-34. In Proceedings of the 29th AIAA/USU Conference on Small Satellites, Logan, UT, USA, 8-13 August 2015; Available online: https: //digitalcommons.usu.edu/smallsat/2015/all2015/90/ (accessed on 5 December 2019).

30. Whitmore, S.A.; Bulcher, A.M. A Green Hybrid Thruster Using Moderately Enriched Compressed Air as the Oxidizer. AIAA 2018-4841. In Proceedings of the 2018 Joint Propulsion Conference, AIAA Propulsion and Energy Forum, Cincinnati, OH, USA, 8-11 July 2018. [CrossRef]

31. Karabeyoglu, M.A. Nitrous Oxide and Oxygen Mixtures (Nytrox) as Oxidizers for Rocket Propulsion Applications. J. Propuls. Power 2014, 30, 696-706. [CrossRef]

32. Whitmore, S.A.; Armstrong, I.W.; Heiner, M.C.; Martinez, C.J. High-Performing Hydrogen Peroxide Hybrid Rocket with 3-D Printed and Extruded ABS Fuel. Aeronaut. Aerosp. Open Access J. 2018, 2. [CrossRef]

33. Whitmore, S.A.; Martinez, C.J.; Merkley, D.P. Catalyst Development for an Arc-Ignited Hydrogen Peroxide/ABS Hybrid Rocket System. Aeronaut. Aerosp. Open Access J. 2018, 2. [CrossRef]

34. Rommingen, J.E.; Husdal, J. Nammo Hybrid Rocket Propulsion TRL Improvement Program. AIAA $2012-4311$. In Proceedings of the 48th AIAA/ASME/SAE/ASEE Joint Propulsion Conference \& Exhibit, Atlanta, GA, USA, 30 July-1 August 2012. [CrossRef]

35. Anthoine, J.; Jean-Yves Lestrade, J.-Y.; Messineo, J.; Casu, S. Performances of a Multi-Pulsed Hybrid Rocket Engine Operating with Highly Concentrated Hydrogen Peroxide. AIAA 2017-4006. In Proceedings of the 53rd AIAA/SAE/ASEE Joint Propulsion Conference, AIAA Propulsion and Energy Forum, July 2017. [CrossRef] 
36. Anon. Safety Standard for Oxygen and Oxygen Systems; NSS 1740.15; NASA Office of Mission Assurance: Washington DC, USA, January 1996. Available online: https://ntrs.nasa.gov/search.jsp? $\mathrm{R}=19960021046$ (accessed on 5 December 2019).

37. Rhodes, G.W. Investigation of Decomposition Characteristics of Gaseous and Liquid Nitrous Oxide; Report AD-784 602; Air Force Weapons Laboratory: Kirtland AFB, NM, USA, July 1974; Available online: https: //www.freelists.org/archives/arocket/01-2014/pdfEE82jaPU9W.pdf (accessed on 5 December 2019).

38. Anon. Occupational Safety and Health Guideline for Nitrous Oxide; US Department of Labor, Occupational Health and Safety Administration. Available online: http://www.osha.gov/SLTC/healthguidelines/nitrousoxide/ recognition.html (accessed on 21 September 2018).

39. Whitmore, S.A. Additively Manufactured Acrylonitrile-Butadiene-Styrene-Nitrous-Oxide Hybrid Rocket Motor with Electrostatic Igniter. J. Propuls. Power 2015, 31, 1217-1220. [CrossRef]

40. Zakirov, V.; Sweeting, M.; Goeman, V.; Lawrence, T. Surrey Research on Nitrous Oxide Catalytic Decomposition for Space Applications. SSC00-XI-6. In Proceedings of the 14th Annual AIAA/USU Conference on Small Satellites, August 2000; Available online: https://digitalcommons.usu.edu/cgi/viewcontent.cgi? article $=2109 \&$ context $=$ smallsat (accessed on 5 December 2019).

41. Karabeyoglu, A.; Dyer, J.; Stevens, J.; Cantwell, B. Modeling of $\mathrm{N}_{2} \mathrm{O}$ Decomposition Events, AIAA 2008-4933. In Proceedings of the 44th AIAA/ASME/SAE/ASEE Joint Propulsion Conference and Exhibit, Hartford, CT, USA, 1-3 July 2008. [CrossRef]

42. Bracken, A.B.; Broughton, G.B.; Hill, W. Equilibria for Mixtures of Oxygen and Nitrous Oxide and Carbon Dioxide and Their Relevance to the Storage of N2O/O2 Cylinders for Use in Analgesia. J. Phys. D 1970, 3 , 1747-1758. [CrossRef]

43. Peng, D.-Y.; Robinson, D.B. A New Two Constant Equation of State. Ind. Eng. Chem. Fundam. 1976, 15, 59-64. [CrossRef]

44. Zudkevitch, D.; Joffe, J. Correlation and Prediction of Vapor- Liquid Equilibria with the Redlich-Kwong Equation of State. AIChE J. 1970, 16, 112-119. [CrossRef]

45. Linstrom, P.; Mallard, W. NIST Chemistry WebBook; NIST Standard Reference Database Number 69, National Inst. of Standards and Technology: Gaithersburg, Maryland, 2018. Available online: http://webbook.nist.gov/ (accessed on 28 June 2011).

46. Whitmore, S.A.; Stoddard, R.L. $\mathrm{N}_{2} \mathrm{O} / \mathrm{O}_{2}$ blends safe and volumetrically efficient oxidizers for small spacecraft hybrid propulsion. Aeronaut. Aerosp. Open Access J. 2019, 3. Available online: https://medcraveonline.com/ AAOAJ/AAOAJ-03-00097.pdf (accessed on 5 December 2019). [CrossRef]

47. Borisov, A.A.; Troshin, K.Y. Critical Conditions for Nitrous Oxide Ignition. Russ. J. Phys. Chem. 2009. [CrossRef]

48. Gordon, S.; McBride, B.J. Computer Program for Calculation of Complex Chemical Equilibrium Compositions and Applications. In NASA Technical Report RP-1311; 1994. Available online: https://ntrs.nasa.gov/archive/ nasa/casi.ntrs.nasa.gov/19950013764.pdf (accessed on 5 December 2019).

49. Anon. "Pure Gasses", Airgas, an Air Liquide Company. Pages 39, 40. Available online: http://airgassgcatalog. com/catalog/ (accessed on 2 July 2019).

50. Stoddard, R.L. Experimental Investigation of N2O/O2 Mixtures as Volumetrically Efficient Oxidizers for Small Spacecraft Hybrid Propulsion Systems. Master's Thesis, Utah State University, Logan, UT, USA, December 2019. Available on Digital Commons. Available online: https://digitalcommons.usu.edu/ (accessed on 5 December 2019).

51. Anon. Cesaroni Pro38 ${ }^{\circledR}$ Hardware. Available online: http://pro38.com/products/pro38/pro38.php (accessed on 5 January 2020).

52. Anon. ULTRAVOLT C Series High Voltage CAP-Charging Supplies. Advanced Energy, Inc. Available online: https://www.advancedenergy.com/globalassets/resources-root/data-sheets/ultravolt-c-series-datasheet.pdf (accessed on 9 December 2019).

53. Holley. Nitrous Oxide Systems. Available online: https://www.holley.com/brands/nos/ (accessed on 6 December 2019).

54. Anderson, J.D. Modern Compressible Flow, 3rd ed.; The McGraw Hill Companies, Inc.: New York, NY, USA, 2003; Chapter 4; pp. 127-187, ISBN-13: 978-0072424430; Available online: https://libcat.lib.usu.edu/search/ i0070016542 (accessed on 5 December 2019). 
55. Zilliac, G.; Karabeyoglu, M.A. Modeling of Propellant Tank Pressurization. AIAA 2005-3549. In Proceedings of the 41st AIAA/ASME/SAE/ASEE Joint Propulsion Conference \& Exhibit, Tucson, AZ, USA, 10-13 July 2005; pp. 1-25. [CrossRef]

56. Dyer, J.; Doran, E.; Dunn, Z.; Lohner, K. Modeling Feed System Flow Physics for Self-Pressuring Propellants. In Proceedings of the 43rd AIAA/ASME/ SAE/ASEE Joint Propulsion Conference \& Exhibit, Cincinnati, $\mathrm{OH}$, USA, 8-11 July 2007. AIAA Paper 2007-5702. [CrossRef]

57. Whitmore, S.A.; Chandler, S.N. Engineering Model for Self-Pressurizing Saturated-N2O-Propellant Feed Systems. J. Propuls. Power 2010, 26, 706-714. [CrossRef]

58. Zimmerman, J.E.; Waxman, B.S.; Cantwell, B.; Zilliac, G. Review and Evaluation of Models for Self-Pressurizing Propellant Tank Dynamics. AIAA 2013-4045. In Proceedings of the 49th AIAA/ASME/SAE/ASEE Joint Propulsion Conference \& Exhibit, San Jose, CA, USA, 14-17 July 2013. [CrossRef]

59. Waxman, B.S.; Zimmerman, J.E.; Cantwell, B.J.; Zilliac, G.G. Mass flow Rate Characterization of Injectors for Use with Self-Pressurizing Oxidizers in Hybrid Rockets. AIAA 2013-3636. In Proceedings of the 49th AIAA/ASME/SAE/ASEE Joint Propulsion Conference \& Exhibit, San Jose, CA, USA, 14-17 July 2013. [CrossRef]

60. Beckwith, T.G.; Marangoni, R.D.; Lienhard, J.H.V. Mechanical Measurements, 6th ed.; Prentice Hall: Upper Saddle River, NY, USA, 2006; pp. 43-73.

61. Whitmore, S.A.; Peterson, Z.W.; Eilers, S.D. Comparing Hydroxyl Terminated Polybutadiene and Acrylonitrile Butadiene Styrene as Hybrid Rocket Fuels. J. Propuls. Power 2013, 29, 582-592. [CrossRef]

62. Whitmore, S.A.; Merkley, S. Radiation Heating Effects on Oxidizer-to-Fuel Ratio of Additively Manufactured Hybrid Rocket Fuels. J. Propuls. Power 2019, 35, 863-878. [CrossRef]

63. Zilliac, G.; Karabeyoglu, M.A. Hybrid Rocket Fuel Regression Rate Data and Modeling. AIAA 2006-4504. In Proceedings of the 42nd AIAA/ASME/SAE/ASEE Joint Propulsion Conference \& Exhibit, Sacramento, CA, USA, 9-12 July 2006. [CrossRef]

64. Anon. NFPA 704, National Fire Protection Association Hazard Identification System. ACS Chemistry for Life. Available online: https://www.google.com/search?client=firefox-b-1-d\&q=NFPA+Hazard+Class (accessed on 10 December 2019).

65. Hussain, S.M.; Frazier, J.M. In vitro toxicity assessment of a new series of high energy compounds. Sci. Total Environ. 2001, 247, 151-160. Available online: https://www.ncbi.nlm.nih.gov/pubmed/11453292 (accessed on 25 March 2020). [CrossRef]

(C) 2020 by the author. Licensee MDPI, Basel, Switzerland. This article is an open access article distributed under the terms and conditions of the Creative Commons Attribution (CC BY) license (http://creativecommons.org/licenses/by/4.0/). 


\title{
Article \\ Design and Test of a Student Hybrid Rocket Engine with an External Carbon Fiber Composite Structure
}

\author{
Francesca Heeg ${ }^{*}{ }^{\dagger}$, Lukas Kilzer ${ }^{\dagger}$, Robin Seitz and Enrico Stoll \\ Institute of Space Systems (IRAS), Technische Universität Braunschweig (TUBS), 38108 Braunschweig, Germany; \\ 1.kilzer@tu-braunschweig.de (L.K.); r.seitz@tu-braunschweig.de (R.S.); e.stoll@tu-braunschweig.de (E.S.) \\ * Correspondence: f.heeg@tu-braunschweig.de; Tel.: +49-531-391-95951 \\ + These authors contributed equally to this work.
}

Received: 13 February 2020; Accepted: 9 May 2020; Published: 14 May 2020

\begin{abstract}
The development of hybrid rockets offers excellent opportunities for the practical education of students at universities due to the high safety and relatively low complexity of the rocket propulsion system. During the German educational program Studentische Experimental-Raketen (STERN), students of the Technische Universität Braunschweig obtain the possibility to design and launch a sounding rocket with a hybrid engine. The design of the engine HYDRA $4 X$ (HYbridDemonstrations-RaketenAntrieb) is presented, and the results of the first engine tests are discussed. The results for measured regression rates are compared to the results from the literature. Furthermore, the impact of the lightweight casing material carbon fiber-reinforced plastic (CFRP) on the hybrid engine mass and flight apogee altitude is examined for rockets with different total impulse classes (10 to $50 \mathrm{kNs})$. It is shown that the benefit of a lightweight casing material on engine mass decreases with an increasing total impulse. However, a higher gain on apogee altitude, especially for bigger rockets with a comparable high total impulse, is shown.
\end{abstract}

Keywords: hybrid rocket engine; sounding rocket; carbon fiber composite; engine test; total impulse; lightweight design

\section{Introduction}

Hybrid rocket propulsion systems offer several advantages compared to solid or liquid propulsion, such as operational safety, thrust throttling ability, and lower cost due to a simple system design, as stated in $[1,2]$. However, several drawbacks prevented the achievement of an advanced technical readiness level (TRL) in the past [3], which hindered the establishment of hybrid propulsion systems in the commercial space market [4]. One disadvantage of hybrid propulsion is a low fuel consumption rate, called the regression rate, which can lead to a low thrust density and sometimes complex fuel grain shapes [5]. Another challenging aspect is the inconstant oxidizer-to-fuel mass ratio during operation. It is caused by the fuel grain surface, which changes with continuing fuel consumption [6]. These disadvantages offer a wide range of research potential; hence, various aspects of hybrid rocket engines (HRE) are addressed in the respective research. One of the main parameters under research is the experimental characterization of the fuel regression rate as investigated in $[2,7,8]$ and how it is linked to heat transfer in the combustion chamber [9]. The regression rate also depends on other parameters like propellant choice $[10,11]$ and the oxidizer injecting system $[12,13]$. Other aspects like throttling, combustion modeling, or design optimization were investigated in [14-16], respectively. Due to the advantages of HRE, they are suitable for a variety of space applications such as space launch systems [1], upper stages [17], lunar and Mars ascent stages [5,18], and sounding rockets especially for university education. Hence, there are numerous activities of hybrid rocket engine development at universities around the globe. For example, Tsohas et al. [19] described the $4 \mathrm{kN}$ hybrid rocket development and launch at Perdue University, where the engine was propelled with hydrogen 
peroxide $\left(\mathrm{H}_{2} \mathrm{O}_{2}\right)$ and low density polyethylene (LDPE). At Tokai University, Japan, students developed engines with a thrust of up to $600 \mathrm{~N}$ that was achieved by using a propellant combination of nitrous oxide $\left(\mathrm{N}_{2} \mathrm{O}\right)$ and wax-based fuel [20]. Meanwhile, the Stratos hybrid rockets that were developed by team DARE (Delft Aerospace Rocket Engineering) of Technical University Delft used a fuel combination of sorbitol, paraffin, and aluminum powder. The engine DHX-200 Aurora of rocket Stratos II+ reached a thrust of $10 \mathrm{kN}$ and a total impulse of $100 \mathrm{kNs}$ [21]. In Germany, the hybrid rocket activities of student groups have been expedited by a national educational program called Studentische Experimental-Raketen (STERN) that was initiated by the German Aerospace Center (DLR) Space Administration in 2012 and is funded by the Federal Ministry of Economic Affairs and Energy (BMWi) [22]. This program allows students from German universities to develop and launch a supersonic sounding rocket [22]. The students are familiarized with the processes and reviews of a real aerospace project. The primary objectives of the rockets comprise a minimum altitude of $3 \mathrm{~km}$, a minimum velocity of Mach 1, and equipment with an onboard data transmission and a recovery system for a safe landing. During the project, the student teams were continuously supervised by experts in the field (Rocket propulsion (DLR Trauen, DLR Lampoldshausen) and DLR Mobile Raketenbasis (MORABA)) and the DLR Space Administration [23]. The participating student teams were able to launch their individual rockets from Esrange (European Space and Sounding Rocket Range) Space Center, Sweden [24]. Several of them used a hybrid propulsion system for their rockets, like the team of Hochschule Bremen. The rocket AQUASONIC used a $\mathrm{N}_{2} \mathrm{O}$ /polyethylene (PE) engine to reach an altitude of about $6.5 \mathrm{~km}$ [24]. The HyEnD (Hybrid Engine Development) team of Universität Stuttgart developed the rocket HEROS that also used $\mathrm{N}_{2} \mathrm{O}$ as the oxidizer, but in combination with paraffin as the fuel. This engine showed a design thrust of $10 \mathrm{kN}$ and a total impulse of over $100 \mathrm{kNs}$ [24]. Furthermore, the team of Universität Bremen contributed to the STERN program. They developed the rocket ZEpHyR (ZARM Experimental Hybrid Rocket), which applied an engine with liquid oxygen (LOX) and paraffin as propellants and achieved a total impulse of $54 \mathrm{kNs}$.

Another contribution to the program was made by the student association called Experimental

Raumfahrt-InteressenGemeinschaft e.V. (ERIG), on behalf of Technische Universität Braunschweig. The students were able to develop and launch successfully the sounding rocket Faust in 2015, which used a hybrid rocket engine [23]. Due to the success and the excellent opportunity for the practical education of university students, the STERN program was continued in a second phase since 2017 as STERN II. The rocket Faust II shall be developed as a successor of Faust, where the hybrid rocket engine shall be improved regarding performance and lightweight design. The design, main characteristics, and performance of the Faust engine HYDRA 3X is described in the following. Based on this, the design changes for the advanced engine HYDRA 4X, which will propel the Faust II rocket, are examined in detail, before the first engine test results are presented. Measured regression rates are compared to the results from the literature. Furthermore, a detailed analysis of the impact of two different engine casing materials is discussed. Finally, the conclusion and outlook on the effects of the new engine construction on lightweight design and rocket performance are presented.

\section{Materials and Methods}

\subsection{Overview of the Sounding Rocket Educational Project within the STERN Program}

The main objective of the STERN program is the provision of experience for students in overall system design. A complete rocket system is to be designed with different subsystems to achieve the project requirements. This includes ground segment design, system operation design, project planning, and documentation. An overview of the whole project content is presented in Figure 1. Various subsystems of the rocket are addressed within the flight segment. This is also shown in Figure 2, where the rocket subsystems are visualized. One main subsystem is the propulsion system, where the engine and valve and feeding systems are designed. The engine design and verification are emphasized in this article. 


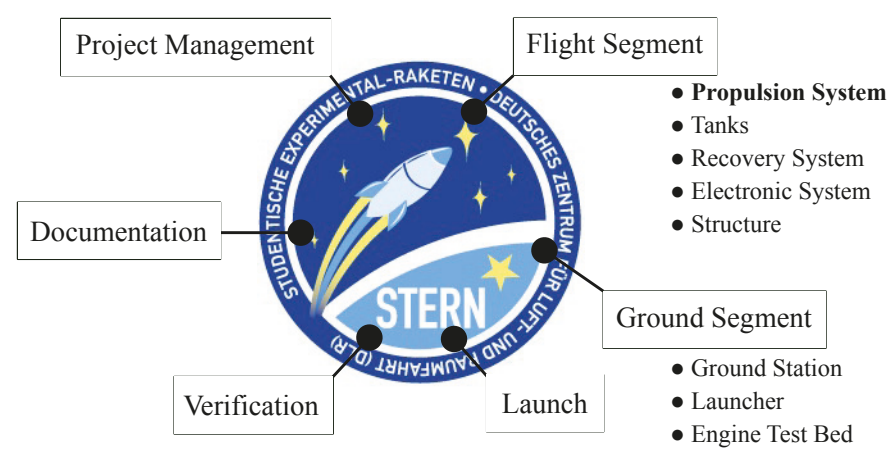

Figure 1. Overview of the STERN II project contents (STERN program logo [22]).

\subsection{Design of the Faust Rocket and HYDRA $3 X$ Engine}

Faust is a sounding rocket, which is propelled by a hybrid rocket engine. A schematic of the rocket is shown in Figure 2, and its main characteristics are listed in Table 1. Faust has a modular design of eight segments. The nose cone is part of the two-stage recovery system that is located on top of the electronics section. The complete propulsion system consists of a pressure vessel for pressurization of the oxidizer vessel, valves, feeding lines, and the hybrid engine. Fins for flight stability are connected to the body tube that accommodates the engine [25]. A successful launch with a maximum altitude of $5400 \mathrm{~m}$ was performed in 2015 .

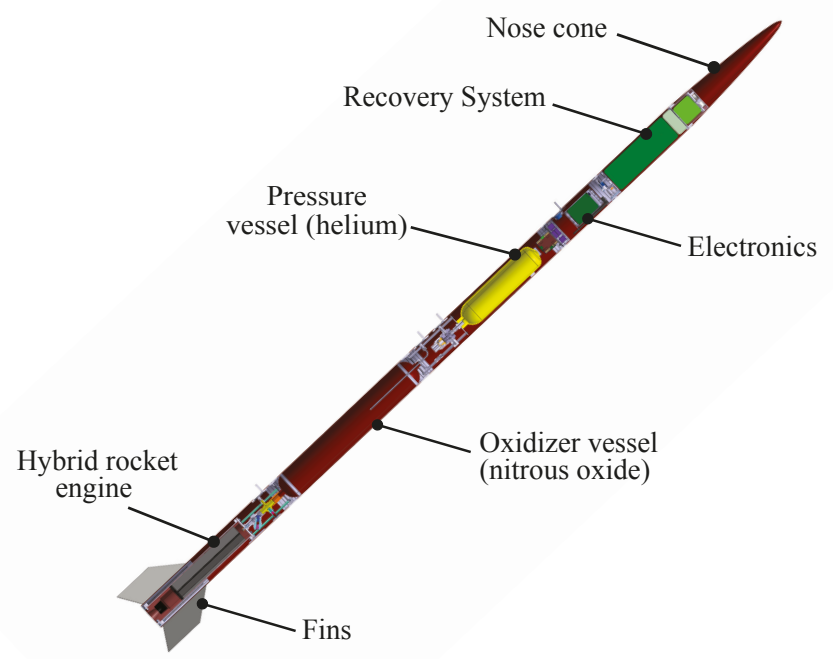

Figure 2. Design of the sounding rocket Faust.

Table 1. The Faust rocket's characteristics.

\begin{tabular}{lr}
\hline Parameter & Value \\
\hline Diameter & $0.12 \mathrm{~m}$ \\
Length & $3.2 \mathrm{~m}$ \\
Mass & $24.1 \mathrm{~kg}$ \\
Altitude & $5410.5 \mathrm{~m}$ \\
Engine & HYDRA 3X \\
\hline
\end{tabular}


A hybrid engine was chosen for the Faust rocket because of the advantages of the propulsion concept. The operational safety is suitable for a student project, and the propellants are storable and relatively safe to use. The hybrid engine HYDRA $3 X$ uses a propellant combination of liquid $\mathrm{N}_{2} \mathrm{O}$ as the oxidizer and a solid mixture of $70 \%$ hydroxyl-terminated polybutadiene (HTPB) and $30 \%$ aluminum powder as the fuel [26]. The aluminum was added to increase the fuel regression rate. Engine test runs with HYDRA 3X showed an increase in the regression rate of about $20 \%$ compared to tests without the addition of aluminum powder [26]. The engine design shows typical components of a hybrid engine, such as an injector for feeding and vaporizing oxidizer into the following combustion chamber [27]. It is based on a shower-head injector that additionally uses a cone for diverting the oxidizer to ensure a better distribution of the mass flow. The propellant fuel is cast directly into the combustion chamber, which uses a star-shaped mold for creating the required fuel geometry, which is presented in Figure 3. The fuel grain ends at the vortex disk, which generates a large vortex flow. This provides the required mixture of both propellants. Subsequently, a conical convergent-divergent nozzle is located at the end of the engine $[26,28,29]$.

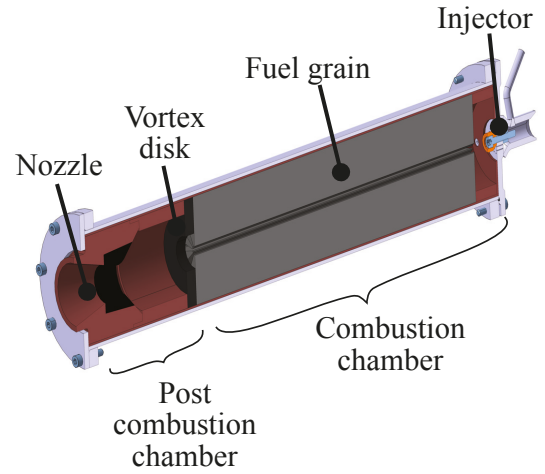

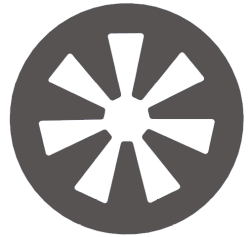

Fuel grain geometry

Figure 3. Design of the engine HYDRA 3X.

Most parts of the engine structure are manufactured from phenolic paper due to advantageous ablative cooling characteristics. The nozzle throat is additionally reinforced with graphite to withstand high thermal loads during combustion. The engine is encapsulated by a steel casing for resistance of combustion pressure loads. The engine outer diameter is $0.09 \mathrm{~m}$ and therefore smaller than the rocket diameter. Hence, an additional structure tube is necessary that is added to the engine casing mass. Structural and performance data of HYDRA $3 X$ are given in Table 2. A successful flight of the Faust rocket was performed with the HYDRA 3X engine. It burned for $10 \mathrm{~s}$ with a mean thrust of $1.25 \mathrm{kN}$. After a $37 \mathrm{~s}$ flight time, an apogee altitude of $5.4 \mathrm{~km}$ was reached [23]. The following objectives for the Faust II engine, HYDRA 4X, within the STERN II project, were chosen:

- $\quad$ Apogee altitude of $10 \mathrm{~km}$;

- Increased engine thrust and total impulse;

- Improved engine power-to-mass ratio;

- Integrated lightweight casing made from CFRP.

\subsection{Design of Hybrid Rocket Engine HYDRA $4 X$}

After the successful application of the HYDRA 3X engine, the HYDRA 4X engine for the Faust II propulsion system was planned. It uses pure HTPB as fuel, waiving additional aluminum powder. This propellant is cast into the engine at a liquid state. It cures by using hardener and therefore becomes solid. The grain geometry is created by using a casting mold that is removed after the curing process. The structure parts are made from phenolic paper, while the nozzle throat is additionally 
supported with graphite. The structure must be able to withstand the combustion pressure loads. Thus, the engine is equipped with a CFRP laminate coating that resists the operational pressure. The injector head is based on the design of HYDRA 3X. It is a shower-head injector with channel orifices of $0.8 \mathrm{~mm}$. A diverting cone is added, as shown in Figure $4 \mathrm{~b}$. The oxidizer flow impinges on this cone, which diverts the flow and directs it to the fuel grain. The current design of HYDRA 4X is presented in Figure 4a and key data in Table 2, respectively.

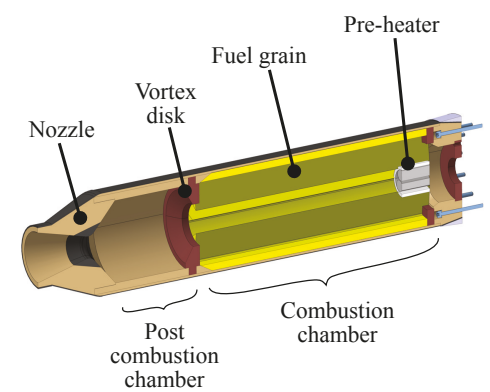

(a) HYDRA $4 X$ engine

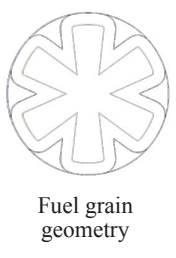

Injector channels

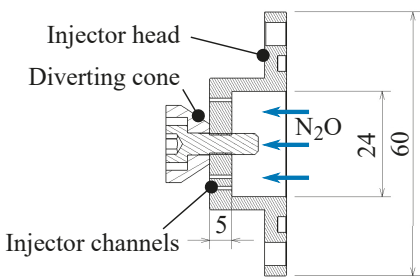

(b) Injector design

Figure 4. Design of the HYDRA 4X engine and injector with injector channels and the diverting cone.

Table 2. Structural and performance characteristics of the engines HYDRA $3 X$ and $4 X$.

\begin{tabular}{llrr}
\hline Parameter & Symbol & HYDRA 3X & HYDRA 4X \\
\hline Overall length & $l$ & $0.47 \mathrm{~m}$ & $0.62 \mathrm{~m}$ \\
Overall diameter & $d$ & $0.09 \mathrm{~m}$ & $0.14 \mathrm{~m}$ \\
Fuel grain length & $l_{f}$ & $0.31 \mathrm{~m}$ & $0.34 \mathrm{~m}$ \\
Post combustion chamber length & $l_{\text {post }}$ & $0.05 \mathrm{~m}$ & $0.10 \mathrm{~m}$ \\
Nozzle throat diameter & $d_{t}$ & $0.02 \mathrm{~m}$ & $0.03 \mathrm{~m}$ \\
Nozzle area ratio & $A_{e} / A_{t}$ & 5.06 & 4.97 \\
Mass & $m$ & $5.7 \mathrm{~kg}$ & $5.78 \mathrm{~kg}$ \\
Fuel mass & $m_{f}$ & $1.2 \mathrm{~kg}$ & $2.57 \mathrm{~kg}$ \\
Casing mass & $m_{\text {cas }}$ & $3.8 \mathrm{~kg}$ & $0.31 \mathrm{~kg}$ \\
Thrust & $F$ & $1.25 \mathrm{kN}$ & $2.25 \mathrm{kN}$ * \\
Total impulse & $I_{\text {tot }}$ & $12.5 \mathrm{kNs}$ & $35.55 \mathrm{kNs}$ * \\
Chamber pressure & $p_{c}$ & $3 \mathrm{MPa}$ & $2.5 \mathrm{MPa}$ * \\
\hline
\end{tabular}

${ }^{*}$ Design value.

\subsection{Performance Calculations}

The technical design of the HYDRA $4 \mathrm{X}$ is built upon a first evaluation of an ideal ratio of $\mathrm{N}_{2} \mathrm{O}$ and HTPB for a maximum specific impulse. NASAs CEA-Code (Chemical Equilibrium with Applications) suggests an oxidizer-to-fuel mass ratio of $\mathrm{O} / \mathrm{F}=7$, using input data from Table 3, with a frozen composition assumed after the nozzle throat. The $\mathrm{O} / \mathrm{F}$ is used to calculate combustion characteristics for the combustion chamber pressure and nozzle exit pressure. Since the ambient pressure of the rocket changes with altitude, a value for nozzle exit pressure needs to be defined. In this case, the nozzle is adapted to ambient pressure at half of the maximum flight altitude. Input data and relevant results of CEA are shown in Table 3.

Based on former flight tests' experience, a lift-off acceleration of around $5 \mathrm{~g}$ should be achieved to ensure a reliable launch. This is the boundary condition to determine the required thrust $F$ and, thus, mass flow $\dot{m}$. This results in a thrust of $2.25 \mathrm{kN}$ for the overall rocket mass of $46 \mathrm{~kg}$. From:

$$
\dot{m}=\frac{F}{\eta_{\text {engine }} \cdot I_{s p, C E A}}
$$


Ref. [1], and:

$$
\dot{m}_{f}=\frac{\dot{m}}{\mathrm{O} / \mathrm{F}+1}
$$

the required fuel mass flow $\dot{m}_{f}$ can be calculated. A global engine efficiency of $\eta_{\text {engine }}=91 \%$ was determined via engine tests during the first STERN program. This is a preliminary design assumption. Different star-shaped fuel grain geometries were iterated regarding the number of star spikes and shape. A constant regression, perpendicular to the fuel grain geometry over full burn time, was assumed. The regression rate for the preliminary design was approximated to $\dot{r}=0.6 \mathrm{~mm} / \mathrm{s}$ from previous in-house measurements. It was adapted with every hot fire test of HYDRA 4X for the design iteration. The mean regression rate of a hot fire test was obtained through weighing the engine before and after the test. The weight difference was converted into a geometric regression, by dividing the weight difference with burn time and fuel grain surface area prior to testing. An optimization was carried out for burn time $t_{b}$, thrust profile according to burn time, and initial mass flow levels, given by Equation (2). This led to a star-shaped fuel grain with the parameters shown in Section 4. Using fuel grain geometry and mass flow $\dot{m}_{f}$, the O/F-ratio determines the subsequent oxidizer mass flow $\dot{m}_{o x}$ :

$$
\dot{m}_{o x}=\mathrm{O} / \mathrm{F} \cdot \dot{m}_{f} .
$$

Table 3. Input data and results of CEA calculation.

\begin{tabular}{llr}
\hline Parameter & Symbol & Value \\
\hline Input parameter & & \\
Oxidizer to fuel mass ratio (-) & $\mathrm{O} / \mathrm{F}$ & 7 \\
Combustion chamber pressure (MPa) & $p_{c}$ & 2.5 \\
Nozzle exit pressure (MPa) & $p_{e}$ & 0.085 \\
\hline Output parameter & & \\
Temperature (chamber) (K) & $T_{c}$ & 3358.1 \\
Molar mass of reaction product (chamber) (g/mol) & $M$ & 26.534 \\
Ratio of specific heats (chamber) (-) & $\gamma_{c}$ & 1.1494 \\
Ratio of specific heats (throat) (-) & $\gamma_{t}$ & 1.1474 \\
Ratio of specific heats (exit) (-) & $\gamma_{e}$ & 1.2274 \\
Specific impulse (m/s) & $I_{s p, C E A}$ & 2391.9 \\
\hline
\end{tabular}

The propulsion system of Faust II was designed to inject liquid oxidizer into the engine. A pressure of $5 \mathrm{MPa}$ was chosen for pressurization of the oxidizer vessel. This pressure level ensured a liquid state of the nitrous oxide and thus operational temperatures up to $293 \mathrm{~K}$ [30]. This temperature agreed with ambient temperature requirements set by a planned launch from ESRANGE Space Center. With combustion chamber pressure $p_{c}$ and oxidizer vessel pressure $p_{o x}$, the theoretical injection speed $v_{t h}$ can be calculated using the oxidizer density $\rho_{o x}$, assuming incompressible, stationary, and isentropic Bernoulli flow (cf. [1]):

$$
v_{t h}=\sqrt{\frac{2}{\rho_{o x}}\left(p_{o x}-p_{c}\right)}
$$

A diameter of $0.8 \mathrm{~mm}$ and a length of $5 \mathrm{~mm}$ were chosen for a single injector orifice. The volume flow $\dot{Q}$ in a single circular orifice is decreased compared to its theoretical optimum due to stream tube contraction and pressure losses within the orifices [6]. This relation can be expressed by:

$$
\frac{\dot{Q}}{\dot{Q}_{t h}}=\frac{\dot{Q}}{v_{t h} \frac{\pi}{4} d^{2}}=C_{D}
$$

where the effects can be combined into a single factor $C_{D}$. This coefficient is measured in every test to iterate the number of injecting orifices. Equation (4) is only applicable to negligible changes of density along the injecting orifice length. Due to the strong temperature and pressure dependency, this is 
only partially applicable to nitrous oxide injection with a strong pressure gradient from the feeding line to the combustion chamber present. However, this approach was used in combination with the coefficient $C_{D}$ to approximate the real flow behavior of the nitrous oxide.

The total number of injecting orifices $n_{0 x}$ can be calculated by:

$$
n_{o x}=\frac{\dot{m}_{o x}}{\rho_{o x} \cdot \dot{Q}}
$$

The strong temperature and pressure dependency of the $\mathrm{N}_{2} \mathrm{O}$ density $\rho_{o x}$ and its effect on the number of injector orifices is to be handled with special consideration. A good control of the $\mathrm{N}_{2} \mathrm{O}$ parameters during fueling can circumvent off-design engine operation. This is also achieved by varying the number of injector orifices according to $\mathrm{N}_{2} \mathrm{O}$ parameters.

An auxiliary function $\Gamma$ is introduced in Equation (7) [7]. The mean of specific heat ratios $\gamma$ calculated by CEA in Table 3 is used as input. The characteristic velocity $C^{*}$ and the throat area $A_{t}$ are calculated by Equation (8) using molar mass $M$, the temperature $T_{c}$ of the reaction product in the chamber (cf. Table 3), and universal gas constant $R_{\infty}$ [7]. Subsequently, the nozzle throat diameter $d_{t}$ is computed in Equation (9).

$$
\begin{gathered}
\Gamma=\sqrt{\gamma} \cdot\left(\frac{2}{\gamma+1}\right)^{\frac{\gamma+1}{2 \cdot(\gamma-1)}} \\
C^{*}=\frac{1}{\Gamma_{c}} \sqrt{\frac{R_{\infty}}{M} \cdot T_{c}}=\frac{p_{c} \cdot A_{t}}{\dot{m}} \\
d_{t}=\sqrt{\frac{4}{\pi} \cdot A_{t}}
\end{gathered}
$$

The momentum thrust coefficient $C_{F}^{0}$ can be calculated according to Equation (10) by using the nozzle exit pressure $p_{e}$ [7]. Equations (7) and (10) are combined into the nozzle expansion ratio $A_{e} / A_{t}$ in Equation (11) [7]. A conical nozzle is chosen due to simplicity in manufacturing. The divergence cone half angle was set to $15^{\circ}$. The length of the divergent nozzle $l_{\text {nozzle }}$ is calculated through Equation (12).

$$
\begin{gathered}
C_{F}^{0}=\Gamma \cdot \sqrt{\frac{2 \cdot \gamma}{\gamma-1} \cdot\left[1-\left(\frac{p_{e}}{p_{c}}\right)^{\frac{\gamma-1}{\gamma}}\right]} \\
\frac{A_{e}}{A_{t}}=\frac{\Gamma^{2}}{C_{F}^{0} \cdot\left(\frac{p_{e}}{p_{c}}\right)^{\frac{1}{\gamma}}}=\frac{d_{e}^{2}}{d_{t}^{2}} \\
l_{\text {nozzle }}=\frac{d_{e}-d_{t}}{2} \cdot \frac{1}{\tan \left(15^{\circ}\right)}
\end{gathered}
$$

The thrust coefficient $C_{F}$ is computed by Equation (13), applying a correction factor to the momentum thrust coefficient $C_{F}^{0}$, correcting for the axial divergence of the exhaust of a conical nozzle [1]. The ambient pressure $p_{a}$ corresponds to the static pressure of the environment and is equal to $p_{e}$ at the design point. For following test data, the ambient pressure was measured at the test site. Note that this corresponds to an optimum $C_{F}$ achievable for conical nozzles.

$$
C_{F}=C_{F}^{0} \cdot \frac{1+\cos \left(15^{\circ}\right)}{2}+\frac{A_{e}}{A_{t}} \cdot \frac{p_{e}-p_{a}}{p_{c}}
$$

The combustion $\eta_{c}$ and nozzle expansion efficiency $\eta_{n o z z l e}$ of the engine are calculated by Equations (14) and (15) as ratios of experimentally measured parameters to ideal design values. This approach enables a comparison of actual engine performance with design performance. As the parameters might shift in the hot fire test, all experimental values are taken as mean over full burn 
time. According to [1], the overall engine efficiency is then calculated by Equation (16). All design data of the HYDRA 4X engine in its latest iteration after the fourth hot fire test are summarized in Table 4.

$$
\begin{aligned}
& \eta_{c}=\frac{C_{\text {exp }}^{*}}{C^{*}}=\frac{p_{c} \cdot A_{t}}{C^{*} \cdot\left(\dot{m}_{o x}+\dot{m}_{f}\right)} \\
& \eta_{\text {nozzle }}=\frac{C_{F, \exp }}{C_{F}}=\frac{F}{C_{F} \cdot p_{c} \cdot A_{t}} \\
& \eta_{\text {engine }}=\frac{I_{s p, \exp }}{I_{s p}}=\eta_{\text {nozzle }} \cdot \eta_{c}
\end{aligned}
$$

Table 4. Design data of HYDRA 4X.

\begin{tabular}{llr}
\hline Parameter & Symbol & Value \\
\hline Oxidizer pressure $(\mathrm{MPa})$ & $p_{o x}$ & 5 \\
Combustion chamber pressure $(\mathrm{MPa})$ & $p_{c}$ & 2.5 \\
Mean density $\mathrm{N}_{2} \mathrm{O}\left(\mathrm{kg} / \mathrm{m}^{3}\right)$ & $\rho_{o x}$ & 915.85 \\
Discharge coefficient $(-)$ & $C_{D}$ & 0.497 \\
Orifice diameter $(\mathrm{mm})$ & - & 0.8 \\
Orifice length $(\mathrm{mm})$ & - & 5 \\
Mass flow oxidizer $(\mathrm{kg} / \mathrm{s})$ & $\dot{m}_{o x}$ & 0.93 \\
Injector orifices $(-)$ & $n$ & 55 \\
\hline Density HTPB $\left(\mathrm{kg} / \mathrm{m}^{3}\right)$ & $\rho_{f}$ & 936.0 \\
Regression rate $(\mathrm{mm} / \mathrm{s})$ & $\dot{r}^{*}$ & 0.66 \\
Mass flow fuel $(\mathrm{kg} / \mathrm{s})$ & $\dot{m}_{f}$ & 0.13 \\
Outer diameter fuel grain $(\mathrm{m})$ & $d_{f}$ & 0.13 \\
Length fuel grain $(\mathrm{m})$ & $l_{f}$ & 0.34 \\
Burn time $(\mathrm{s})$ & $t_{b}$ & 15.8 \\
\hline Nozzle exit pressure $(\mathrm{MPa})$ & $p_{e}$ & 0.085 \\
Nozzle area ratio $(-)$ & $A_{e} / A_{t}$ & 4.97 \\
Momentum thrust coefficient $(-)$ & $C_{F}^{0}$ & 1.47 \\
Characteristic velocity $(\mathrm{m} / \mathrm{s})$ & $C^{*}$ & 1607 \\
Throat diameter $(\mathrm{m})$ & $d_{t}$ & 0.0295 \\
Divergent nozzle length $(\mathrm{m})$ & $l_{\text {nozzle }}$ & 0.07 \\
\hline
\end{tabular}

\section{Description of the Engine Test Bed}

All engine tests are performed at the test facility of DLR Trauen, where all safety requirements can be achieved. The engine test bed for HYDRA 4 X consists generally of two assemblies, the fluid supply system and the test bench. A schematic of the fluid supply system is presented in Figure 5a. The fluid supply system also includes the test bed electronics. An intermediate tank is used to store liquid $\mathrm{N}_{2} \mathrm{O}$ during the fueling process. Nitrous oxide is fed from cylinders directly into this tank where it can be pressurized to $6 \mathrm{MPa}$. In contrast to the rocket system, nitrogen is used for pressurizing the $\mathrm{N}_{2} \mathrm{O}$ tank on the test bed due to its better availability. Temperature and pressure within the intermediate tank are measured by three sensors. Strain gauges measure the tank weight during testing to control fueling and to determine oxidizer mass flow rates. After pressurization, the tank weight is set to zero. Thus, only the fueled nitrous oxide mass is measured. During the hot test, the intermediate tank is continuously pressurized by nitrogen. The change of tank weight during the hot test gives the oxidizer mass flow. The continuously added nitrogen mass flow for pressurization introduces a systematic error to the oxidizer mass flow measurement during the hot test. The additional weight of the nitrogen decreases the measured oxidizer mass flow. This systematic error is not corrected. Hence, all stated oxidizer mass flows are smaller than expected. 


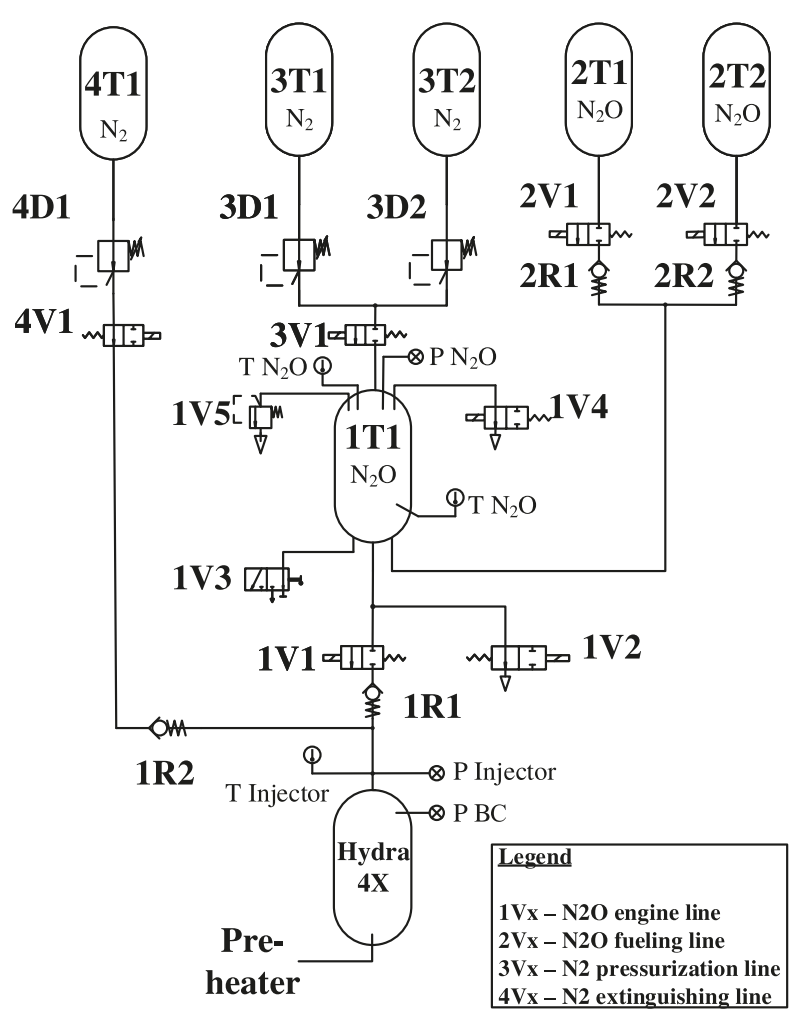

(a) PID

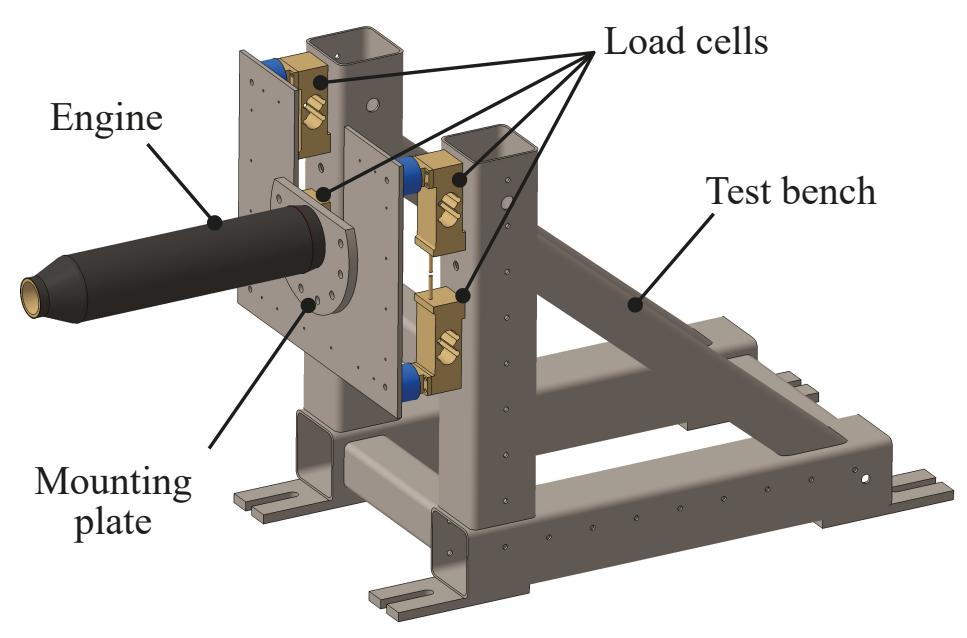

(b) Engine test bench

Figure 5. (a) Fluid plan of the fluid supply system; (b) Engine test bench with the engine HYDRA 4X. 
The oxidizer feeding line is mounted to the test bench and leads directly to the test injector. Liquid $\mathrm{N}_{2} \mathrm{O}$ is inserted into the engine through this component during the hot test. At this point, additional measurements are taken: temperature and pressure shortly in front of the injector are measured, as well as the combustion chamber pressure by a sensor connected to a small pipe that leads directly into the engine pre-chamber. Pressure is measured at a sampling rate of $1 \mathrm{kHz}$. The test bench is a robust steel construction that is modular and can be used for different engines, as is displayed in Figure $5 \mathrm{~b}$. The HYDRA $4 \mathrm{X}$ is mounted to the test bench using a mounting plate, which accommodates the injector and can easily be exchanged. Four load cells are integrated onto the test bench for thrust measurement. Data logging and valve controlling are realized by the National Instruments cRIO-9074 controller [29]. It is located within an electronic box near the fluid supply system and can be controlled remotely from the test control center.

\section{Results and Discussion}

\subsection{Hot Fire Tests and Iteration of Design}

The results for the first four hot fire tests of HYDRA 4 X engine are presented in the following. Design changes were made to increase engine performance between these tests. The changes of defined design parameters are summarized with the resulting measured values from corresponding hot fire tests in Table 5. The value $G_{0 x}$ was computed according to [7]:

$$
G_{o x}=\frac{\dot{m}_{o x}}{A_{p}}
$$

where $A_{p}$ is the free port area of the fuel grain. The mean port area over burn time was considered for the determination of the regression rate.

Table 5. Overview of the design and measured parameters of HYDRA 4X-01 to HYDRA 4X-04.

\begin{tabular}{|c|c|c|c|c|}
\hline Design parameter & H4X-01 & H4X-02 & H4X-03 & H4X-04 \\
\hline Fuel grain geometry & Geometry v1 & Geometry v1 & Geometry v2 & Geometry v2 \\
\hline Injector & 36 orifices & 40 orifices & 40 orifices & 55 orifices \\
\hline Post combustion chamber length & $0.06 \mathrm{~m}$ & $0.09 \mathrm{~m}$ & $0.1 \mathrm{~m}$ & $0.1 \mathrm{~m}$ \\
\hline \multicolumn{5}{|c|}{ Measured parameter as mean over burn time } \\
\hline$t_{b}(\mathrm{~s})$ & 8 & 15.7 & 12.2 & 12.3 \\
\hline$F(\mathrm{kN})$ & 1 & 1.2 & 1.6 & 2.3 \\
\hline$p_{c}(\mathrm{MPa})$ & 1.5 & 1.8 & 2 & 2.3 \\
\hline$\dot{m}_{f}(\mathrm{~kg} / \mathrm{s})$ & 0.08 & 0.1 & 0.13 & 0.12 \\
\hline$\dot{r}(\mathrm{~mm} / \mathrm{s})$ & 0.44 & 0.63 & 0.72 & 0.66 \\
\hline$p_{o x}(\mathrm{MPa})$ & 4.4 & 4.2 & 4.95 & 4.6 \\
\hline$\dot{m}_{o x}(\mathrm{~kg} / \mathrm{s})$ & 0.72 & 0.63 & 0.65 & 1.01 \\
\hline $\mathrm{G}_{O x}\left(\mathrm{~g} / \mathrm{cm}^{2} \mathrm{~s}\right)$ & 15.7 & 10 & 10.7 & 17.2 \\
\hline $\mathrm{O} / \mathrm{F}(-)$ & 8.75 & 6.3 & 5 & 8.4 \\
\hline
\end{tabular}

Pressure values of the intermediate tank, injector, and combustion chamber were measured during all engine tests. The measurements of the first four engine tests are presented in Figure 6 . Three general test phases are visible in all four graphs: The first phase describes the condition when the intermediate $\mathrm{N}_{2} \mathrm{O}$ tank was filled and pressurized to a pressure between 4.8 and 5.6 MPa. The combustion phase started with opening of the main oxidizer valve, and the tank pressure reduced slightly as the oxidizer flowed into the engine. A decrease of tank pressure over time could be noticed. This was a result of an insufficient pressurization of the fluid supply system used, which was originally designed for lower oxidizer mass flows. Therefore, the achieved oxidizer mass flow decreased over burn time in every test, i.e., initial and end values were in the range of a $10 \%$ deviation of mean oxidizer mass flow over burn time. Tank and injector pressure differed by a small value that complied with pressure loss in 
the feeding line. Injector and chamber pressure showed increased values after opening of the main valve. After closure of the main valve, thrust and combustion chamber pressure decreased fast to values prior to ignition, while the intermediate tank pressure increased again. The injector pressure did not drop to ambient pressure again, because the feeding line was still pressurized. For the first two tests, the feeding line extended with about a $2 \mathrm{~m}$ length between the main valve and engine; $\mathrm{cf}$. Figure $5 \mathrm{a}$. Due to this, the engine received oxidizer for a longer time than desired after main valve closure. To reduce this effect, the main valve was relocated directly in front of the engine for the third and fourth test.

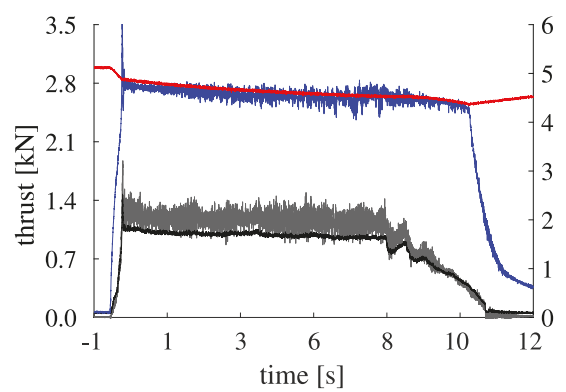

(a) HYDRA 4X-01

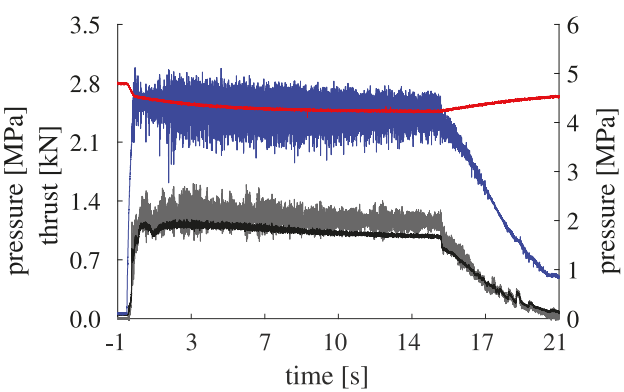

(b) HYDRA 4X-02

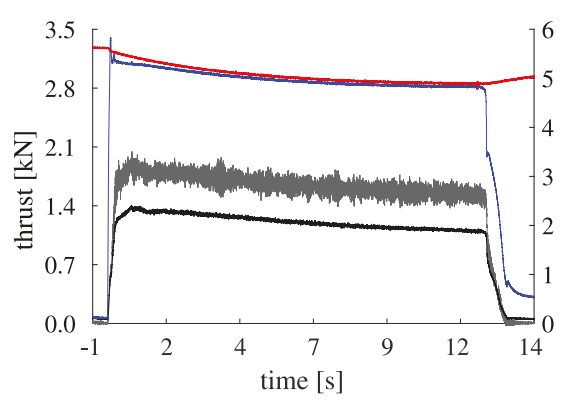

(c) HYDRA 4X-03

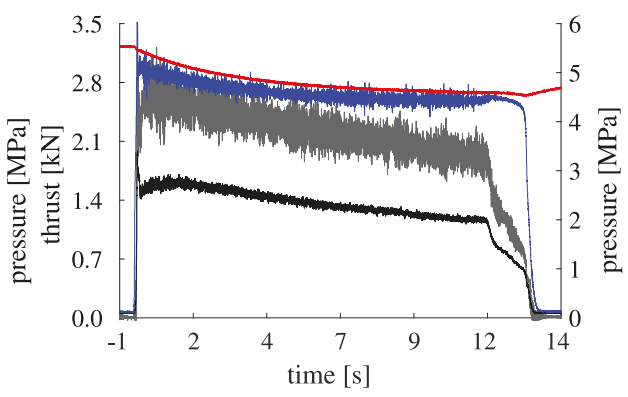

(d) HYDRA 4X-04

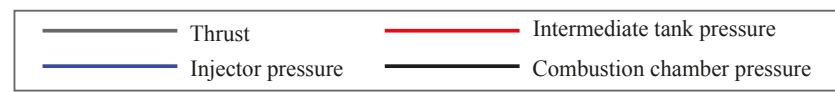

Figure 6. Engine test data for HYDRA 4X-01 (a), 4X-02 (b), 4X-03 (c), and 4X-04 (d).

The mean thrust of the first two engine tests was about $1.2 \mathrm{kN}$, which was apparently lower than the design thrust. Accordingly, the average combustion chamber pressure of $1.8 \mathrm{MPa}$ was low compared to the design value of $2.5 \mathrm{MPa}$. Analysis of test parameters yielded that the target value for oxidizer mass flow was not reached and the nozzle was manufactured inaccurately. Design changes between HYDRA 4X Versions 01 and 02 were made to increase engine performance: nozzle manufacturing was improved; post combustion chamber length was increased by $30 \%$; and injector design was changed to improve mass flow through an increase of orifice number and manufacturing quality and the combustion of both propellants by flattening the diverting cone angle. Since there were no remarkable changes between the performance results of HYDRA 4X-01 and $4 \mathrm{X}-02$, the consumption of the star-shaped solid propellant grain was examined for the second test in Figure $7 \mathrm{~b}$. Two main aspects could be determined. First of all, it was evident that HTPB consumption decreased with combustion chamber length. The mean consumption showed values between $30 \mathrm{~mm}$ at the fuel grain front and only $20 \mathrm{~mm}$ at the end considering the star peaks. This was also apparent 
for star indentations where consumption values varied from $7 \mathrm{~mm}$ to $0 \mathrm{~mm}$. The latter meant no consumption at all. Hence, the consumption of star indentations was significantly lower than that of star peaks. The authors assumed that although the target mean regression rate was reached, the uneven consumption over length and major differences between star peaks and indentations contributed to engine performance, as the overall fuel grain surface in the combustion process was decreased.

As a result, the fuel grain geometry was changed for the test of HYDRA 4 X-03 as presented in Figure $7 \mathrm{a}$ with parameter values summarized in Table 6 . The number of star peaks was decreased from eight to six, and the geometry was changed so that consumption of star indentations was augmented. The consumption measurements of HYDRA 4X-03 showed that the design changes enhanced the HTPB consumption, especially for star indentations, as can be seen in Figure 7c. Additionally, a more even consumption along engine length was demonstrated. Therefore, a higher mean thrust value of about $1.6 \mathrm{kN}$ at a chamber pressure of $2 \mathrm{MPa}$ could be reached, as presented in Figure $6 \mathrm{c}$. These values were still below the design parameters, which could be traced back to a low oxidizer mass flow. As a result, the injector was adapted once more between Tests 03 and 04 by increasing the number of injecting orifices.
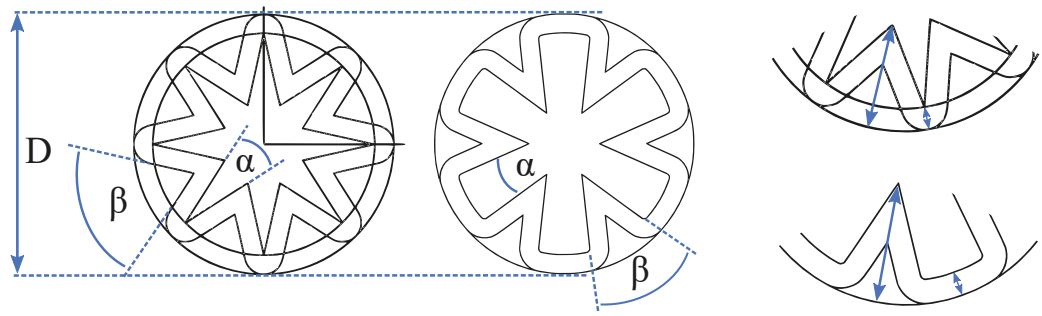

(a) Comparison of fuel grain geometries v1 (left) used in H4X-01 and H4X-02 and v2 (middle) used in $\mathrm{H} 4 \mathrm{X}-03$ and $\mathrm{H} 4 \mathrm{X}-04$. Measurement points for geometric consumption are indicated in the respective geometries on the right.

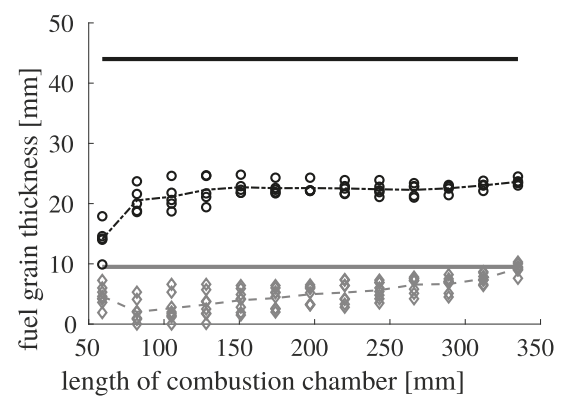

(b) HYDRA 4X-02

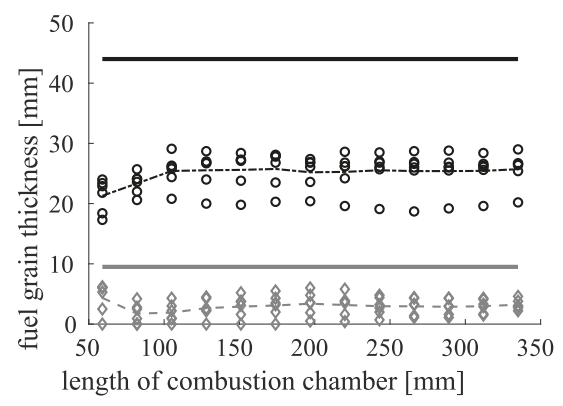

(c) HYDRA 4X-03

\begin{tabular}{lll}
\hline peak before test & o peak after test & ----- peak mean after test \\
indentation before test & $\diamond$ indentation after test & ----- indentation mean after test
\end{tabular}

Figure 7. (a) Comparison of fuel grain geometry iterations; (b,c) Thickness of the HTPB fuel grain at star peaks in black and indentations. Distances were measured originating from the chamber wall. Symbols represent measurement points as indicated in (a), and lines resemble the corresponding mean values. 
Table 6. Parameters of two fuel grain geometries of HYDRA 4X.

\begin{tabular}{ccc}
\hline & H4X-01, 02 & H4X-03, 04 \\
\hline $\mathrm{D}$ & \multicolumn{3}{c}{$130 \mathrm{~mm}$} \\
$\mathrm{n}$ & 8 & 6 \\
$\alpha$ & $25^{\circ}$ & $12^{\circ}$ \\
$\beta$ & $70^{\circ}$ & $48^{\circ}$ \\
\hline
\end{tabular}

With this adjustment, a mean thrust of approximately $2.3 \mathrm{kN}$ was reached at a chamber pressure of $2.3 \mathrm{MPa}$, an injector pressure of $4.6 \mathrm{MPa}$, and an oxidizer mass flow of $1.01 \mathrm{~kg} / \mathrm{s}$, as presented in Figure $6 \mathrm{~d}$. However, the mean oxidizer mass flow exceeded the design value of $0.931 \mathrm{~kg} / \mathrm{s}$. This resulted in a mean $\mathrm{O} / \mathrm{F}$ of 8.4 over the full test time, compared to a design $\mathrm{O} / \mathrm{F}$ of seven. The fuel mass flow of $0.123 \mathrm{~kg} / \mathrm{s}$ was in good accordance with the design value of $0.133 \mathrm{~kg} / \mathrm{s}$. To reach an appropriate oxidizer mass flow, the number of injecting orifices would be decreased, and the directional characteristic of the injector would be changed to supply oxidizer more directly at the star indentations. To achieve this directional behavior, the injector orifices would be aligned with the angular position of the star indentations. HYDRA 4 X-04 yielded $86 \%$ efficiency of the design $I_{s p}$. This global efficiency could be divided into $87 \%$ combustion efficiency and approximately $99 \%$ nozzle efficiency. Although the minimum design thrust was surpassed, it could only be achieved through an excess of injected oxidizer mass flow. As the flight fluid supply system would not be able to sustain this increased oxidizer mass flow for the complete burn time, further work would have to be carried out to achieve design performance at tolerable oxidizer mass flows.

Figure 8 shows the fuel grain regression rates obtained from the four tests compared against data from the literature [8,31-33]. The regression rate curves from these data follow the empirical power law [7]:

$$
\dot{r}=a \cdot G_{o x}^{n}
$$

in which the coefficients $a$ and $n$ were obtained through experimental data. These experiments were conducted for the same combination of propellant and oxidizer at similar combustion chamber pressures and comparable O/F. The main differences to HYDRA $4 \mathrm{X}$ were the fuel grain geometry and injector type. Only fuel grains with single circular port geometries were tested in the references presented. The determined regression rates from four HYDRA 4X tests were located between the fitting curves of $[8,31,32]$.

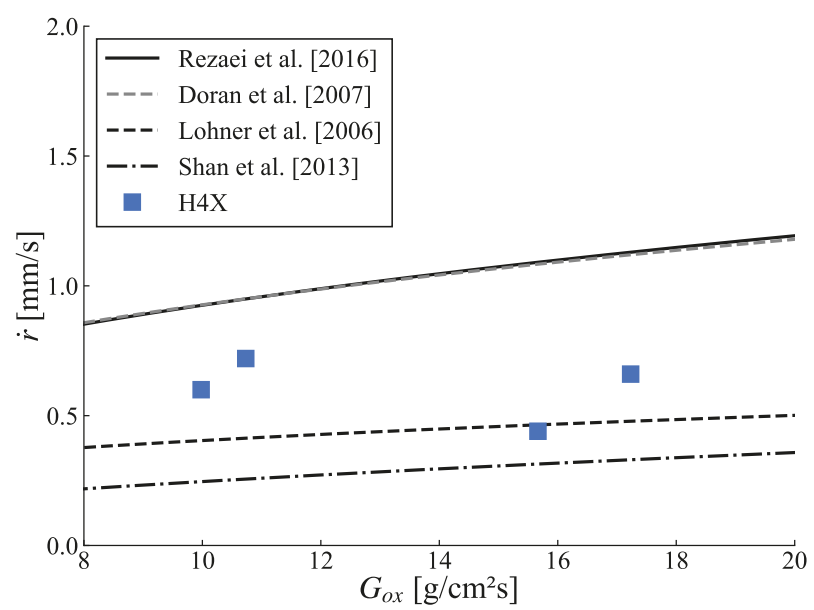

Figure 8. Comparison of fuel grain regression rates with literature data [8,31-33]. 


\subsection{Impact of Engine Casing Material on Flight Apogee}

The different designs of two HYDRA engines $3 X$ and $4 X$ were already discussed. Although there was a clear difference between engine performance data, structural changes were also made. A comparably heavy steel casing of HYDRA $3 X$ was replaced by a CFRP coating to reduce engine mass. This consequently resulted in a higher apogee. On the other hand, using CFRP also meant higher effort in design, calculation, manufacturing, and costs. Therefore, it was interesting to evaluate the impact of this change on engine mass, total hybrid rocket mass, and on flight apogee. Thus, a parametric analysis for fundamental hybrid rocket pre-design was conducted. This was done by a MATLAB tool that was established during the STERN II program. It uses geometric and mass relations for estimating the design of sounding rockets. The tool calculates the engine data, such as propellant mass, mass flow, and burn time, based on a requested mean thrust and total impulse. The regression rate was assumed to be constant over burn time. The fuel mass flow was calculated according to a mean fuel grain surface and a constant oxidizer mass flow. The selectable fuel grain geometry was either a monoport or a star shape, and a conical nozzle was used. Two pressure tanks made from CFRP for oxidizer and pressure gas were calculated based on engine data. Residual rocket components like electronics or parachutes were estimated by the user. Physical relations for hybrid engines and flight mechanics (described in $[1,7,34]$ ) were used to estimate flight performance, using the mean thrust as a constant thrust profile over burn time. The tool analyzes first estimations on rocket design factors like engine performance, geometric dimensions, and flight parameters, such as flight apogee. The analysis is suited for rockets in an impulse range of 10 to $50 \mathrm{kNs}$ and cell diameters of 100 to $180 \mathrm{~mm}$. A parametric study was carried out for a basic rocket design with a constant cell diameter of $150 \mathrm{~mm}$ while the total impulse was increased continuously from 10 to $50 \mathrm{kNs}$. The rocket engine became larger (as more fuel was required) and longer. The engine structure mass and casing mass rose with increasing total impulse. The total impulse of an engine could be calculated by thrust $F$ and engine burn time $t_{b}$ [1] according to Equation (19). This relation could be used here, since the design tool used a mean thrust value for calculations, even though the thrust profile was usually not constant for HRE.

$$
I_{t o t}=F \cdot t_{b}
$$

The launch acceleration of the rocket shall be about $5 \mathrm{~g}$ at a rail length of $9 \mathrm{~m}$. This results in a minimum thrust for an estimated rocket mass and a corresponding burn time. To characterize the relation between fuel and engine structure mass, two mass factors were introduced. Firstly, the relation factor $f_{c m}$ compares engine casing mass $m_{c a s}$ to fuel mass $m_{f}$ that can be accommodated by the engine. This factor shows how much casing mass is required for a defined fuel mass to reach a certain total impulse.

$$
f_{c m}=\frac{m_{c a s}+m_{f}}{m_{f}}
$$

A second relation factor $f_{e m}$ is defined, which represents a comparison between overall engine mass $m_{e}$ (including fuel mass) and fuel mass. This factor links fuel mass to engine structure mass $m_{s t r}$ that includes casing and other structural engine components.

$$
f_{e m}=\frac{m_{e}}{m_{f}}=\frac{m_{s t r}+m_{f}}{m_{f}}
$$

Using these engine parameters, a basic rocket design was developed and the results discussed. They were compared to the design parameters of the engine HYDRA 4X for the Faust II rocket that were presented. Figure 9 a shows the defined mass relation factors as a function of total impulse in a range from 10 to $50 \mathrm{kNs}$. 


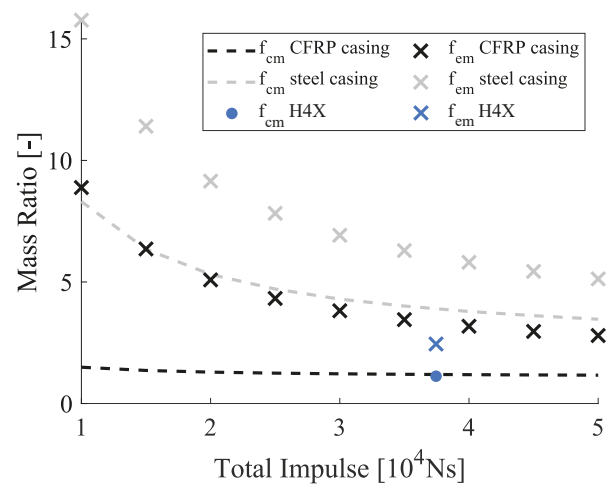

(a)

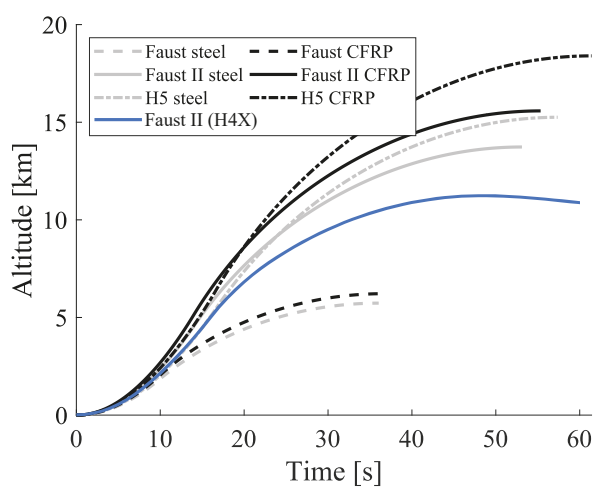

(b)

Figure 9. (a) Mass ratios $f_{c m}$ and $f_{e m}$ for steel and CFRP casing materials; (b) Flight trajectories until apogee altitude for three different rocket designs, again with steel or CFRP casing material.

Two main aspects can be derived from Figure 9a. A steel casing is heavier than a CFRP casing, but the relations between casing mass and fuel mass $\left(f_{c m}\right)$ change over the total impulse range. For smaller engines with a low total impulse of about $10 \mathrm{kNs}$, the weight of a steel casing is seven times higher than the respective fuel mass. This relation decreases with total impulse and shows a factor of about 3.5 for engines with a total impulse of $50 \mathrm{kNs}$. In comparison to that, $f_{c m}$ for a CFRP casing is nearly constant over the impulse range, where a mass factor of about 1.4 is reached. A second aspect addresses the total engine structure mass. It is derived from the casing (outer structure) and other structural components as presented. As Figure 9a shows for small engines $(10 \mathrm{kNs})$, the mass factor for overall engine mass $f_{e m}$ is at 15.7 for steel and 8.8 for CFRP casing material. This shows that the residual engine structure mass (disregarding the casing) cannot be neglected, although the mass factor $f_{e m}$ decreases with rising total impulse. The overall engine structure mass with a steel casing is about four times higher than the required fuel mass at an impulse of $50 \mathrm{kNs}$. In contrast, the structure mass with a CFRP casing is about twice as high as the accommodated fuel mass. Thus, the data showed that the engine structure mass was relatively high compared to the required fuel mass, especially, for a small impulse class. Less structure mass is needed relative to fuel mass for higher impulses. When using CFRP instead of a steel casing, the engine structure mass can be reduced by about $50 \%$ for the whole impulse range. The mass factor $f_{c m}$ for a steel casing shows a maximum for a small engine class, where the engine structure mass is very high compared to the fuel mass. Both mass factors decrease with increasing impulse. However, an engine with steel casing results in a mass twice as high as an engine with CFRP casing over the whole impulse range. The achieved values for the engine HYDRA $4 \mathrm{X}(35.5 \mathrm{kNs})$ are added in blue color. A $f_{c m}$ value of 1.12 was in good accordance with the results of the parametric analysis. The engine mass factor $f_{e m}$ of 2.45 was lower than the analysis result of 3.3, which meant that less structure mass was needed for this engine than the parametric tool assumed.

Figure $9 \mathrm{~b}$ shows the effect of casing material on flight apogee. Two existing $(\mathrm{H} 3 \mathrm{X}, \mathrm{H} 4 \mathrm{X})$ and one theoretical (H5) engine and subsequent rocket designs are presented in two versions: steel and CFRP, as presented in Table 7, where hybrid engine rockets for a range of impulses and rocket diameters are compared. For these designs, an approximated flight apogee was calculated to evaluate the casing mass impact. The flight trajectory is plotted until apogee, where the recovery system would be activated.

As Figure $9 \mathrm{~b}$ shows, an apogee of $5.7 \mathrm{~km}$ for Faust with a hybrid engine using a steel casing was reached. This was similar to Faust flown in 2015 with an apogee of roughly $5.4 \mathrm{~km}$. Therefore, the analysis provided reasonable results. An apogee difference for the two casing materials of about $500 \mathrm{~m}$ and a mass difference of about $2.2 \mathrm{~kg}$ were derived for Faust. A higher impact on apogee difference was observed for Faust II. Using a CFRP casing, Faust II was able to reach an altitude 
of $15.6 \mathrm{~km}$ (compared to $13.7 \mathrm{~km}$ when using a steel casing), which represented an apogee increase of about $14 \%$. The fully designed Faust II with a CFRP engine showed a higher mass than the pre-design $(+6.7 \mathrm{~kg})$, resulting in a lower apogee altitude of $11.2 \mathrm{~km}$. The trend for an increase of apogee rose for the larger engine HYDRA 5 (cf. Table 7). By using a CFRP engine casing, an increase of $3.1 \mathrm{~km}$ $(20.3 \%)$ was achieved compared to a steel casing. It was evident that a lighter CFRP engine casing was beneficial, especially for engines with higher total impulse and diameter values.

Table 7. Comparison of rocket design parameters for three different engines with two different casing materials. Results presented in parentheses correspond to real flight data for Faust and CAD data for Faust II.

\begin{tabular}{llll}
\hline Parameter & Faust & Faust II & H5 rocket \\
\hline Cell diameter $(\mathrm{m})$ & 0.123 & 0.143 & 0.17 \\
Engine & HYDRA 3X & HYDRA 4X & HYDRA 5 \\
Thrust $(\mathrm{kN})$ & 1.25 & 2.25 & 3 \\
Total impulse $(\mathrm{kNs})$ & 12.50 & 35.55 & 50 \\
Rocket mass $(\mathrm{kg})$ & Steel: $26.4(24.1)$ & Steel: 44.3 & Steel: 59.0 \\
& CFRP: 24.2 & CFRP: $39.6(46.3)$ & CFRP: 51.1 \\
Apogee altitude $(\mathrm{km})$ & Steel: $5.73(5.41)$ & Steel: 13.7 & Steel: 15.3 \\
& CFRP: 6.22 & CFRP: $15.6(11.2)$ & CFRP: 18.4 \\
\hline
\end{tabular}

\section{Conclusions and Outlook}

The previous and current development activities of hybrid rocket engines at Technische Universität Braunschweig were examined. The design of the rocket engine HYDRA 3X was described, which was launched at the Esrange Space Center in 2015. It was a hybrid engine using nitrous oxide as the oxidizer and HTPB as the fuel. Based on this, a new engine HYDRA 4X was developed, which was designed for the Faust II rocket. The main design changes compared to HYDRA 3X were a higher total impulse and thrust and the CFRP casing material. The test bed and first test results of the new HYDRA 4X engine were shown. The design changes to iterate a requested engine performance were explained. In addition, the obtained fuel grain regression rates were compared to literature data.

To quantify the impact of different casing materials on rocket mass parameters, a parametric study was performed. First, casing and engine structure masses were compared to the fuel mass. It was shown that the fraction of structure mass to overall engine mass decreased over increasing total impulse, although the impact of casing material on rocket apogee altitude increased. The analysis was suited best for rockets with diameters between 100 and $180 \mathrm{~mm}$ and impulses between 10 and $50 \mathrm{kNs}$. A CFRP engine casing material showed a clear advantage over a heavy metal casing when only flight altitude was considered. However, other design, manufacturing, and cost parameters have to be taken into account to quantify the benefit of the material choice. In a next step, the parametric analysis will be extended to a wider range of impulses, and the pre-design of other rocket components like the recovery and electronics system will be refined.

Author Contributions: Conceptualization, F.H. and R.S.; investigation, R.S., L.K. and F.H.; data curation, L.K and R.S.; writing, original draft preparation, F.H. and L.K.; writing, review and editing, L.K., R.S., and E.S.; visualization, L.K. and F.H.; supervision, F.H.; project administration, E.S. All authors have read and agreed to the published version of the manuscript.

Funding: We acknowledge support by the German Research Foundation and the Open Access Publication Funds of the Technische Universität Braunschweig. This work was supported by the Federal Ministry of Economics and Technology on the basis of a decision by the German Bundestag (FKZ: 50 RL1752).

Acknowledgments: The authors thank the DLR Administration for conducting the STERN II program and the whole review board for assistance and advice. Special thanks are given to DLR Trauen for enabling conducting the engine tests.

Conflicts of Interest: The authors declare no conflict of interest. 


\section{Abbreviations}

The following abbreviations are used in this manuscript:

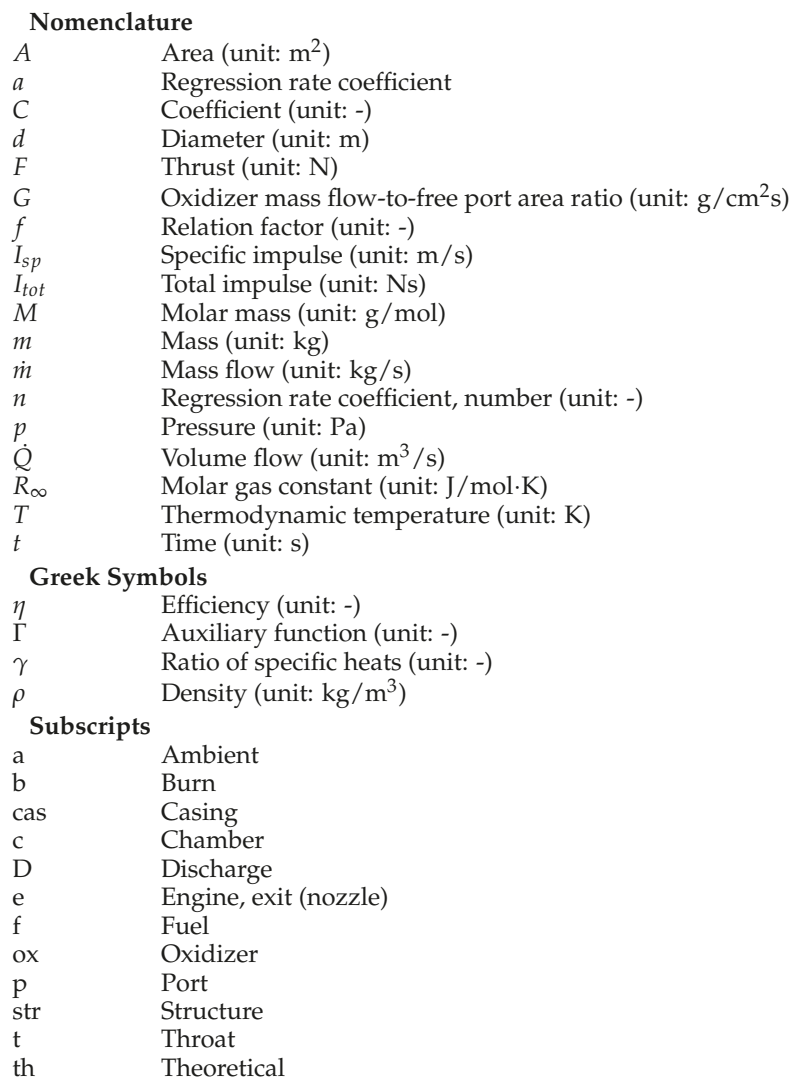

\section{Abbreviations}

TRL Technical readiness level

HRE Hybrid rocket engine

DLR German Aerospace Center

STERN Studentische Experimental-Raketen

MORABA Mobile Raketenbasis

IRAS Institute of Space Systems

ERIG ExperimentalRaumfahrt-InteressenGemeinschaft

BMWi Federal Ministry of Economic Affairs and Energy

HYDRA Hybrid Demonstrations-RaketenAntrieb

HTPB Hydroxyl-terminated polybutadiene

$\mathrm{N}_{2} \mathrm{O} \quad$ Nitrous oxide

$\mathrm{H}_{2} \mathrm{O}_{2} \quad$ Hydrogen peroxide

LDPE Low density polyethylene

PE polyethylene

LOX Liquid oxygen

CFRP Carbon fiber-reinforced plastic

$\mathrm{O} / \mathrm{F} \quad$ Oxidizer-to-fuel mass ratio

NASA National Aeronautics and Space Administration

CEA Chemical Equilibrium with Applications

PID Piping and instrumentation diagram 


\section{References}

1. Sutton, G.; Biblarz, O. Rocket Propulsion Elements, 7th ed.; John Wiley and Sons, Inc.: Hoboken, NJ, USA, 2001; pp. 27-38, 62-68, 77-82, 580.

2. Li, X.; Tian, H.; Yu, N.; Cai, G. Experimental investigation of fuel regression rate in a HTPB based lab-scale hybrid rocket motor. Acta Astronaut. 2014, 105, 95-100. [CrossRef]

3. Mazzetti, A.; Merotto, L.; Pinarello, G. Paraffin-based hybrid rocket engines applications: A review and a market perspective. Acta Astronaut. 2016, 126, 286-297. [CrossRef]

4. Schmierer, C.; Kobald, M.; Fischer, U.; Tomilin, K.; Petrarolo, A.; Hertel, F. Advancing Europe's Hybrid Rocket Engine Technology with Paraffin and LOX. In Proceedings of the 8th European Conference for Aeronautics and Aerospace Sciences (EUCASS), Madrid, Spain, 1-4 July 2019.

5. Schmierer, C. Analysis of a Hybrid Propulsion Lunar Sample Return Mission. Ph.D. Thesis, University of Stuttgart, Stuttgart, Germany, 2019.

6. Schmucker, R.H. Hybridraketenantriebe; Wilhelm Goldmann Verlag GmbH: Munich, Germany, 1972; pp. 22-24, 67-70.

7. Barrère, M.; Schneider, W.K.; Roy, M.; Von Kármán, T. Raketenantriebe; Elsevier Publishing Co.: New York, NY, USA, 1961; pp. 66-72, 98, 112-114.

8. Rezaei, H.; Soltani, M.R.; Mohammadi, A.R. Experimental Study of Fuel Regression Rate in a HTPB/ $\mathrm{N}_{2} \mathrm{O}$ Hybrid Rocket Motor. Sci. Iran. 2018, 25, 253-265. [CrossRef]

9. Chiaverini, M.J.; Kuo, K.K.; Peretz, A.; Harting, G. Regression-Rate and Heat-Transfer Correlations for Hybrid Rocket Combustion. J. Propuls. Power 2001, 17, 99-110. [CrossRef]

10. Carmicino, C.; Scaramuzzino, F.; Sorge, A.R. Trade-off between paraffin-based and aluminium-loaded HTPB fuels to improve performance of hybrid rocket fed with $\mathrm{N}_{2}$ O. Aerosp. Sci. Technol. 2014, 37, 81-92. [CrossRef]

11. Sun, X.; Tian, H.; Li, Y.; Yu, N.; Cai, G. Regression rate behaviors of HTPB-based propellant combinations for hybrid rocket motor. Acta Astronaut. 2016, 119, 137-146. [CrossRef]

12. Cheng, P.; Lia, Q.; Chen, H. Flow characteristics of a pintle injector element. Acta Astronaut. 2019, 154, 61-66. [CrossRef]

13. Di Martino, G.D.; Malgieri, P.; Carmicino, C.; Savino, R. A simplified computational fluid-dynamic approach to the oxidizer injector design in hybrid rockets. Acta Astronaut. 2019, 129, 8-21. [CrossRef]

14. Greatrix, D.R. Regression rate estimation for standard-flow hybrid rocket engines. Aerosp. Sci. Technol. 2009, 13, 358-363. [CrossRef]

15. Hui, T.; Yijie, L.; Peng, Z. Transient simulation of regression rate on thrust regulation process in hybrid rocket motor. Chin. J. Aeronaut. 2014, 27, 1343-1351.

16. Chiba, K.; Kanzaki, M.; Nakamiya, M.; Kitagawa, K.; Shimada, T. Conceptual Design of Single-Stage Launch Vehicle with Hybrid Rocket Engine for Scientific Observation Using Design Informatics. J. Space Eng. 2013, 6, 15-27. [CrossRef]

17. Bozic, O.; Poppe, G.; Porrmann, D. An advanced hybrid rocket engine for an alternative upper stage of the Brazilian VLM 1 LEO-launcher. In Proceedings of the 65th International Aeronautics Congress, Toronto, ON, Canada, 29 September-3 October 2014.

18. Karp, A.; Benito, J.; Nakazono, B.; Kim, H.; Shotwell, R.; Brandeau, E.; Vaughan, D.; Story, G. A Hybrid Mars Ascent Vehicle Design and FY 2016 Technology Development. In Proceedings of the IEEE Aerospace Conference, Big Sky, MT, USA, 4-11 March 2017.

19. Tsohas, J.; Appel, B.; Rettenmaier, A.; Walker, M.; Heister, S.D. Development and Launch of the Purdue Hybrid Rocket Technology Demonstrator. In Proceedings of the 45th AIAA/ASME/SAE/ASEE Joint Propulsion Conference, Denver, CO, USA, 2-5 August 2009.

20. Kawai, H.; Harada, J.; Oguro, J.; Banno, A.; Nakagawa, I. Launches of Hybrid Rockets with Wax Fuel. In Proceedings of the 49th AIAA/ASME/SAE/ASEE Joint Propulsion Conference, San Jose, CA, USA, 14-17 July 2013.

21. Werner, R.; Knop, T.; Wink, J.; Ehlen, J.; Huijsman, R.; Powell, S.; Florea, R.; Wieling, W.; Cervone, A.; Zandbergen, B. Development and Performance of the $10 \mathrm{kN}$ Hybrid Rocket Motor for the Stratos II Sounding Rocket. In Proceedings of the Space Propulsion 2016, Rome, Italy, 2-6 May 2016.

22. DLR-Raumfahrtmanagement. STERN: Studentische Experimental-Raketen. 2012. Available online: https:// www.dlr.de/rd/PortalData/28/Resources/dokumente/rl/STERN_Flyer.pdf (accessed on 13 January 2020). 
23. Becker, J.; Grefen, B.; Stoll, E. Results of the Leonis Project and Launch Campaign within the STERN Program. In Proceedings of the Deutscher Luft- und Raumfahrtkongress, Braunschweig, Germany, 13-15 September 2016.

24. Schuettauf, K.; Stamminger, A.; Lappoehn, K. The STERN Project-Hand On Rockets Science for University Student. In Proceedings of the 23rd ESA PAC Symposium, Visby, Sweden, 11-15 June 2017.

25. Rothe, D.; Hink, R. Faust Hybrid Rocket-Current Status. In Proceedings of the Deutscher Luft- und Raumfahrtkongress, Rostock, Germany, 22-24 September 2015.

26. Dullo, C.M.U.; Hink, R. Experimental Analyses of the HTPB-Aluminum Mixtures for Hybrid Rocket Engines. In Proceedings of the Deutscher Luft- und Raumfahrtkongress, Stuttgart, Germany, 10-12 September 2013.

27. Gamper, E.; Hink, R. Design and Test of Nitrous Oxide Injectors for a Hybrid Rocket Engine. In Proceedings of the Deutscher Luft- und Raumfahrtkongress, Rostock, Germany, 22-24 September 2015.

28. Baade, M.; Hink, R. Design of a Nozzle for a Hybrid Rocket Engine Using an Explicit Finite-Volume Based Simulation for Heat Transfer. In Proceedings of the Deutscher Luft- und Raumfahrtkongress, Stuttgart, Germany, 10-12 September 2013.

29. Hink, R. HYDRA 3 Hybrid Rocket Engine-Flight Version Test Results. In Proceedings of the Deutscher Luft- und Raumfahrtkongress, Rostock, Germany, 22-24 September 2015.

30. Lemmon, E.W.; McLinden, M.O.; Friend, D.G. NIST Chemistry WebBook, NIST Standard Reference Database Number 69; Chapter Thermophysical Properties of Fluid Systems; National Institute of Standards and Technology: Gaithersburg, MD, USA, 2018.

31. Lohner, K.; Dyer, J.; Doran, E.; Dunn, Z.; Zilliac, G. Fuel Regression Rate Characterization Using a Laboratory Scale Nitrous Oxide Hybrid Propulsion System. In Proceedings of the 42nd AIAA/ASME/SAE/ASEE Joint Propulsion Conference, Sacramento, CA, USA, 9-12 July 2006.

32. Doran, E.; Dyer, J.; Lohner, K.; Dunn, Z.; Cantwell, B.; Zilliac, G. Nitrous Oxide Hybrid Rocket Motor Fuel Regression Rate Characterization. In Proceedings of the 43rd AIAA/ASME/SAE/ASEE Joint Propulsion Conference, Cincinnati, OH, USA, 8-11 July 2007.

33. Shan, F.; Hou, L.; Piao, Y. Combustion performance and scale effect from $\mathrm{N}_{2} \mathrm{O} / \mathrm{HTPB}$ hybrid rocket motor simulations. Acta Astronaut. 2013, 85,1-11. doi:10.1016/j.actaastro.2012.12.013. [CrossRef]

34. Andrea, L. A Mathematical Perspective on Flight Dynamics and Control; Springer: Berlin, Germany, 2017.

(C) 2020 by the authors. Licensee MDPI, Basel, Switzerland. This article is an open access article distributed under the terms and conditions of the Creative Commons Attribution (CC BY) license (http:// creativecommons.org/licenses/by/4.0/). 



\title{
Technical Note \\ Oxygen-Methane Torch Ignition System for Aerospace Applications
}

\author{
Olexiy Shynkarenko * and Domenico Simone \\ Aerospace Engineering Department, Chemical Propulsion Laboratory, University of Brasília, \\ Brasília 70910-900, Brazil; domenico.simone@aerospace.unb.br \\ * Correspondence: olexiy@aerospace.unb.br
}

Received: 24 May 2020; Accepted: 8 July 2020; Published: 7 August 2020

\begin{abstract}
A new ignition system, based on a $\mathrm{CH}_{4} / \mathrm{O}_{2}$ torch has been developed by the Chemical Propulsion Laboratory of the University of Brasilia. Designed to ignite a hybrid rocket, this device has been improved to be used in testing of solid and liquid ramjet engines under development in our lab. The capability to provide multiple ignitions and to cool-down its combustion chamber walls by using a swirled injection of the oxidizer, along with a very low weight to power ratio, makes this device versatile. The igniter is controlled by a feedback system, developed by our group, which guarantees the possibility of operating in different design conditions enabling, therefore, complete integration with systems of different nature. The main characteristics of the igniter and the design solutions are presented including some considerations about the tests performed to evaluate the quality and performance of the ignition system.
\end{abstract}

Keywords: ignition system; gas torch; methane-oxygen combustion; vortex combustion chamber; hybrid rocket motor; ramjet motor

\section{Introduction}

A torch ignition system has been developed in the Chemical Propulsion Laboratory (CPL) of the University of Brasilia in the context of a project sponsored by the Brazilian Space Agency (AEB) promoting design and development of a scientific payload (the SARA capsule) [1] powered during the re-entry phase by a hybrid rocket.

Designed to ignite a hybrid rocket, this device has been improved to be installed and used to test other engines [2,3]. With a very low weight to power ratio, the ability to provide multiple ignitions and the possibility to cool its combustion chamber walls by using a swirled injection of the oxidizer this device is versatile. The igniter in fact is controlled by a feedback system developed in CPL which guarantees the possibility of operating in different design conditions enabling, therefore complete integration with systems of different nature.

The control system acts changing the $\mathrm{O} / \mathrm{F}$ ratio by increasing or decreasing the swirled mass flow rate of oxygen; a lean mixture corresponds to an increased cooling effect on the wall as result of the combined effect of an increased layer of oxygen protecting the walls and a reduced quantity of energy delivered inside the combustion chamber. The oxidizer-fuel combination selected is $\mathrm{O}_{2} / \mathrm{CH}_{4}$, because it is a green, well known and highly reacting mixture, able to deliver energy at various equivalence ratios with a relatively high density. Moreover this mixture is storable in space and nontoxic, reducing costs, and complexity of storage when compared to some other, more widely used, fuel-oxidizer combinations. Finally, when operating under lean conditions, combustion products are still rich in oxygen, thus favoring the ignition of the propellants in the main combustion chamber.

The aim of this work is to present the main characteristics required and the design solutions adopted for the igniter and to discuss the tests executed to evaluate quality and performance of the control system. 


\section{Igniter and Ignition System}

Different types of igniters for rocket motors are available in the literature: these include hypergolic reactants, resistive elements (low voltage), augmented high voltage spark (liquid bi-propellant torch), pyrotechnics, catalyzed monopropellants, gas-dynamics systems, and high power plasma arcs $[4,5]$. During the development of our hybrid propulsion system, we have constantly used a pyrotechnic ignition system, the main disadvantage of which is the impossibility of re-ignite the engine. Moreover, on a laboratory-scale it's preferable to have a controllable, reliable ignition system with a simple power regulation, which could be used in different aerospace applications, such as hybrid rocket motor or ramjet motor. Thus, a gas torch ignition system could be a good option for the motor initiation because of its relative simplicity and reliability $[6,7]$.

The experimental basis of the current work is a general flammability study [8] of various mixtures including the methane-oxygen with additives and impurities influencing the flame formation and ignition limits of gases. According to [8] a methane-oxygen pure mixture is flammable on a wide range of concentrations; however, a stable ignition could exist only when a methane concentration lies between $5 \%$ and $60 \%$ by volume. Another important parameter involved in the ignition process is the spark energy required for reliable flame initiation. From [8] it is known that the "ignitibility limit" for GOX/ $\mathrm{GCH}_{4}$ mixture is close to $1 \mathrm{~mJ}$.

The study of recent publications in the area of interest for this work has allowed to qualify and quantify the combustion properties of methane-oxygen flames. Analytical estimation of the flame structure in this paper is based on the work of Melvin and Moss [9] where the properties of methane-oxygen diffusion flames are given in a precise mathematical and physical model allowing a critical comparison between different reaction schemes and the elaboration of an analytical prediction of the mixture composition during the combustion process. Previously Bae et al. [10] have found the stability limits of the oxygen-methane diffusion flame using the jet and vortex injectors combination. A model of a compact size injector was elaborated showing a dependence of the visible flame length on Reynolds number and O/F ratio.

The work of Ellis [11] describes a background of a $\mathrm{LOX} / \mathrm{CH}_{4}$ swirled torch ignition system for a rocket engine and shows the flammability maps for various combinations of propellants. Pauly et al. [12] show conditions of the flame attachment to the injector when applied to coaxial jets, flame stabilization in time and its liftoff distance during the flame stabilization.

The ignition characteristics of a small thrust rocket engine working on methane-oxygen mixture were described by Jiaqi et al. [13]; the injectors' configuration was determined in order to achieve a stable motor ignition. It was confirmed that a spark-plug directly connected to the rocket engine's combustion chamber is not capable to supply enough energy for ignition. In this context, the design of a compact stand-alone torch igniter becomes an important key technology for reliable ignition of a rocket motor.

According to Li [14], swirling flows of pure methane-oxygen diffusion flame similar to that of our work are characterized by a stable tubular flame for $\Phi \in(0.52,1.05)$; outside of these limits, depending on the geometry of the combustion chamber and the injector, the flame could oscillate or be unstable.

The development of the torch ignition system for the Vinci upper stage engine was described by Frenken and Vermeulen [15]. The components of the system were described and the main parameters of the system operation were discussed. It was shown that a compact gas torch igniter of $440 \mathrm{~kW}$ of thermal power could show a reliable operation when its design and flow conditions are optimized.

Kinetic mechanisms for modern CFD tools of methane-oxygen mixture combustion were studied by Haidn et al. [16] in order to estimate a design approach for effective injectors geometry and flow conditions for startup combustion sequence and key requirements for the feasible ignition system.

Moon et al. [17] carried out an experimental study on a methodology to estimate the reliability of a pyrotechnic ignition system for a hybrid rocket motor, which could be extended to the gas torch ignition system discussed in this work. 
Vortex combustion chambers are thus known in the literature for their capability to improve fuel mixing and combustion. For example, Yi et al. [18] shows an igniter, based on a pre-mixed flame, in which it is possible to obtain an excellent level of mixing in the combustion chamber by using crossed flow injection. In this work another concept has been developed: the swirled flow, in fact, surrounds the methane jet so as to keep the flame away from the walls of the chamber up to its outlet section. This allows small size and weight of the igniter with undoubted advantages. Furthermore, on [18] the effects of a flame not parallel to the axis on the grain combustion is not described. In our tests we found that a pilot flame parallel to the engine axis guarantees uniform combustion in the radial direction. For these reasons the preliminary design of our igniter was challenging: before manufacturing the prototype, many configurations were designed and tested, with the help of numerical fluid dynamics, varying combustion chamber length, fuel injector and nozzle geometries. Figure 1 shows the baseline geometry selected with the expected flow lines distribution colored by temperature.

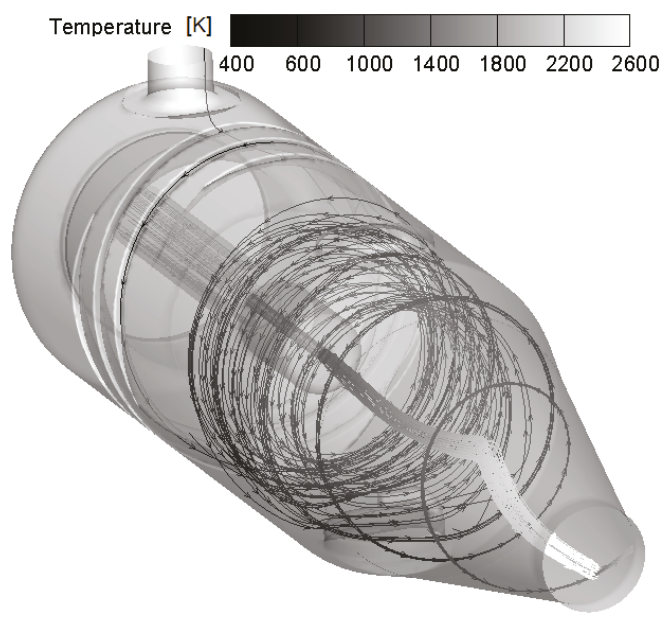

Figure 1. Streamlines distribution colored by temperature (cylindrical injector, $\Phi=0.4$ ).

During this phase, many solutions for the $\mathrm{CH}_{4}$ injector geometry were tested; as a result, the conical injector configuration was selected and manufactured because of its ability to reduce the surfaces exposed to the aggressive attack of radicals combined with intense heat fluxes. The igniter assembly with the rocket motor shown on Figure 2 consists of the following components: 1 -fuel inlet, 2-oxidizer injector, 3-fuel injector, 4-sensor interface, 5-fuel inlet fastener, 6-oxidizer inlet, 7-igniter casing, 8-combustion chamber, 9-spark plug interface, 10-oxidizer closure, 11-injector plate, 12-injector interface, 13-igniter sealing.

As shown in the schematic above, while methane is injected by means of a jet parallel to the axis (1), oxygen enters the combustion chamber (6) passing through helical ducts (2) which impart the desired circumferential motion. The swirled flow thus, surrounding the fuel jet, controls the extension of the reacting region and its temperature; by increasing or decreasing the oxygen mass flow rate it is thus possible to protect the walls until the exit section. Downstream of the $\mathrm{CH}_{4}$ injector are located the spark plug (9) and thermocouple (4) interfaces; flow temperatures have been measured varying the position of the thermocouple along the $24 \mathrm{~mm}$ long diameter $\mathrm{AB}$.

To ensure a high resistance to thermal fluxes and corrosion all the components shown in Figure 2 are made of stainless steel 310 . 


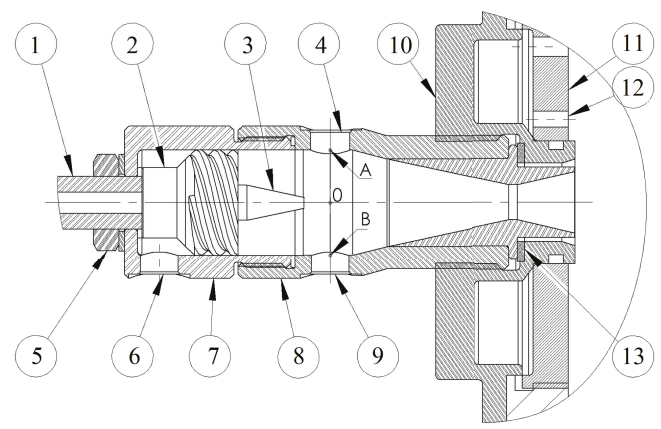

Figure 2. Igniter assembled into the hybrid motor.

Table 1 completes the description of the igniter summarizing the required performance in case of use as hybrid rocket igniter and also as solid or liquid fueled ramjet pre-heater (simulating the same conditions expected, for different Mach numbers, downstream of their inlet).

Table 1. Design criteria for the ignition system.

\begin{tabular}{cc}
\hline Parameter & Value \\
\hline Minimal power (hybrid), $\mathrm{kW}$ & 3 \\
Minimal power (ramjet), $\mathrm{kW}$ & 40 \\
Minimal number of ignitions & $16 \times 3 \mathrm{~s}$ \\
Minimal system operation, s & 48 \\
Ignition probability in a & $99.7 \%$ \\
sequence of 3 ignitions & \\
Electric power, $\mathrm{W}$ & 20 \\
\hline
\end{tabular}

As the table shows, attention has been paid especially to the reliability of the ignition requiring a very high success rate within three consecutive ignitions. Indeed the control system is able to manage up to 16 ignition attempts, each $3 \mathrm{~s}$ long, thus ensuring a high probability of ignition.

The ignition system, shown in Figure 3, is composed by a feeding system and a control system.

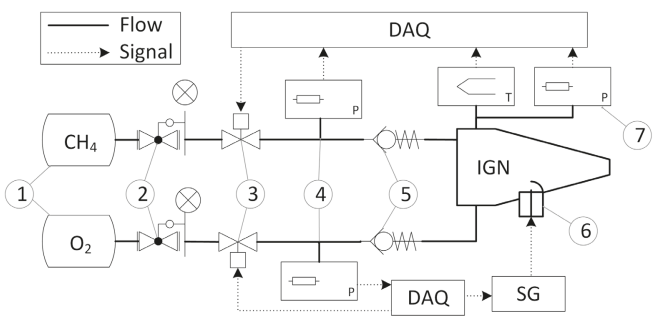

Figure 3. Ignition system schematics.

The feeding system is composed by two high-pressure tanks filled by oxygen and methane. Each flow, passing through a pressure regulator valve (2), reduces its pressure; then, downstream of a flow valve (3) controlled by the ignition algorithm, a non-return spring loaded check valve (5) is preventing backflow phenomena. Finally both flows are injected into the combustion chamber through the injectors and ignited by a spark plug (6) through the spark generator (SG).

The pressure sensors (4) along the feeding lines and inside the combustion chamber are used to determine the mass flow rate of the propellants; moreover a thermocouple installed inside the combustion chamber reads the temperature. These data, sent to the 14 bit A/D converter of DAQ are 
processed by using a control algorithm developed in the CPL and written using the G-Language of the LabVIEW environment.

The pressure transducers have been installed using T-adapters of the same diameter of pipes to avoid losses allowing thus a more precise measure of the static pressure in the system. They can be positioned, if required, upstream of the flow valves (3) to estimate the pressure drop and flow coefficients inside the valve. Finally, the thermocouple installed inside the combustion chamber is connected to an adjustable adapter, allowing measurements along the chamber diameter; the adapter connection is also compatible with the pressure transducer (7).

\section{Qualification Tests}

More than 400 tests were performed, verifying mainly resistance and reliability of the components. Particular attention has been paid to measuring temperature and pressure in the feed lines and inside the combustion chamber. These data have been used as feedback informations to capture the ignition moment and to control the mass flow rate and energy output of the igniter. Performance in terms of ignition limits, repeatability and delay have been verified; finally, flame stability at low and high-pressure and power released have been investigated. All these tests have been performed using the system to ignite a hybrid rocket.

A series of measurements has been performed, for pressure and temperature, varying the mass flow rates and thus the $\mathrm{O} / \mathrm{F}$ ratio of the mixture; every measure was repeated at least 25 times for all points of interest in order to collect statistical data assessing the repeatability of the ignition process.

The mass flow rate of oxidizer and fuel has been calculated carefully by using the water displacement method, a procedure allowing the calculation with a very low margin of error. To perform this measurement, the ignition system was assembled downstream of a large volume water tank. Thus the valves have been opened for a period of time sufficient to ignore inertia effects; the amount of water displaced during this time was measured and the gas compressibility correction was recalculated.

Test have also been performed to evaluate the "energy absorbed", that is the quantity of the heat efficiently transferred to the solid grain of paraffin. This is a fundamental parameter for solid fueled hybrid rocket and ramjet because it is connected with the energy required to liquefy and or vaporize the fuel allowing homogeneous reactions in the main combustion chamber. The ignition system has been assembled with our hybrid engine; thus, many tests were executed, all with constant motor configuration and same initial grain mass and geometry. Varying the mass flow rate of $\mathrm{CH}_{4}$ and $\mathrm{O}_{2}$ and thus their $\mathrm{O} / \mathrm{F}$ ratio, the grain consumption due to the paraffin melting and evaporation processes were measured; finally, the power absorbed by the fuel grain has been evaluated by calculating the variation of its mass:

$$
W=\Delta m\left(c_{\text {sol }} \Delta T_{\text {sol }}+c_{\text {fusion }}+c_{\text {liq }} \Delta T_{\text {liq }}\right) / \Delta t
$$

where $\Delta m$ is the mass of melted paraffin, $c_{s o l}$ and $c_{l i q}$ are the averaged specific heat capacity of the paraffin in solid and liquid state, respectively, $\Delta T_{\text {sol }}$ is a difference between paraffin fusion point and ambient temperature, $\Delta T_{l i q}$ is a difference between liquid paraffin temperature at the end of the experiment and the fusion temperature, $c_{\text {fusion }}$ is a specific heat of paraffin fusion, $\Delta t$ is the duration of the experiment. The quantity of evaporated paraffin was considered negligibly small and was not considered in the Equation (1).

Table 2 finally shows the maximum error allowed in the measurement of the quantities of interest, evaluated considering all the possible sources of error in the system.

All the experimental tests have been repeated at least 2 times to minimize the possibility of errors during the execution. 
Table 2. Measurement errors.

\begin{tabular}{ccc}
\hline Parameter & Units & Value \\
\hline Temperature & $\mathrm{K}$ & 5 \\
Thermocouple position & $\mathrm{mm}$ & 0.05 \\
Pressure & $\%$ & 0.05 \\
Mass flow & $\%$ & 0.5 \\
Absorbed energy & $\%$ & 3.4 \\
\hline
\end{tabular}

\section{Results and Discussions}

Opening the valves in angles ranging from $0^{\circ}$ (fully closed position) to $90^{\circ}$ (fully open position), the pressure difference registered between the sensors (4) and (7) increased from 1 to 5 bar inside the fuel line and 1.5 to 5 bar inside the oxidizer line. Measurement of the pressure difference between sensors (4) and (7) takes into account the check valves and other local pressure losses.

Figure 4 shows the results of all the experiments for both propellants, where the measured mass flow rate has been normalized by using a reference values of $4 \mathrm{~g} / \mathrm{s}$ for the oxidizer and $0.7 \mathrm{~g} / \mathrm{s}$ for the fuel.

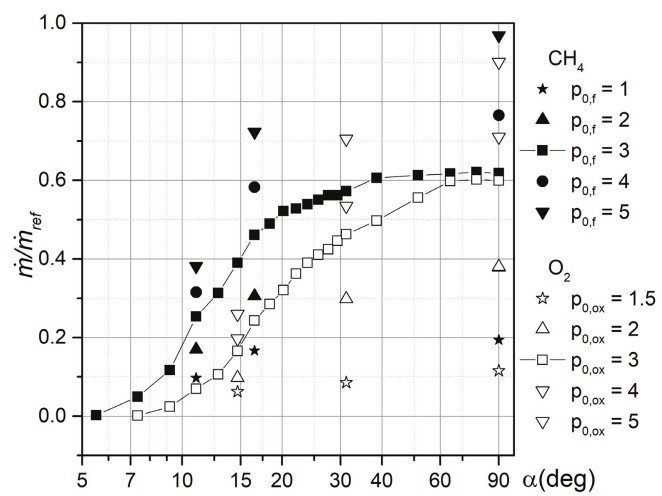

Figure 4. Relative mass flow rate as a function of the valves opening angle $\alpha$ and line total pressure $p_{0}$.

Here we can observe a strong correlation between the inlet total pressure and mass flow rate, as well as the influence of the valves opening angles. With these data it is possible to easily calculate the $\mathrm{O} / \mathrm{F}$ ratio (and total mass flow rate) by using the following correlation:

$$
O / F=\frac{\dot{m}_{o x}}{\dot{m}_{f}}=\frac{f_{1}\left(p_{0, o x}, \alpha_{o x}\right)}{f_{2}\left(p_{0, f}, \alpha_{f}\right)}
$$

The minimal amount of gas required for 16 ignitions of the hybrid rocket motor [1] are $m_{o x}=$ $105 \mathrm{~g}$, and $m_{f}=20.56 \mathrm{~g}$, calculated taking into account the average ignition delay of $1.16 \pm 0.48 \mathrm{~s}$ measured experimentally.

Pressure levels after the check valves and in the combustion chamber have been measured during the ignition process and are shown on Table 3, as an average of 50 tests. Here, two opening levels have been used: partially open, used for the flame initiation process, and fully open required during the fully developed combustion process. The pressure regulator valves (2), has been set to a pressure $p_{2}=3$ bar for both the fuel and oxidizer lines. After a group of 50 tests mean pressure values and standard deviation have been estimated in order to provide the flame temperature and energy output variation in a group of tests. 
Table 3. Pressure variation during the ignition process.

\begin{tabular}{cccc}
\hline Valve Opening & Location & $p$, mbar & $\sigma$, mbar \\
\hline \multirow{3}{*}{ Partial } & Oxidizer & 32.1 & 1.1 \\
& Fuel & 771 & 61.9 \\
& Chamber & 0.4 & 0.14 \\
\hline \multirow{3}{*}{ Full } & Oxidizer & 567.4 & 4.5 \\
& Fuel & 2194 & 47.6 \\
& Chamber & 95 & 11 \\
\hline
\end{tabular}

These values of the standard deviation show the required repeatability of the flow properties. A sequence of 100 attempts was performed to test the ignition probability, each attempt with duration of $1 \mathrm{~s}$. Not a single failure of the ignition system was found during this test and the ignition probability has been shown to be $P \geq 99 \%$ for the single attempt, and $P \geq 99.99 \%$ for three attempts in a sequence. These values can be considered strictly speaking valid just for the flame initiation process because other possible failure probabilities such as malfunction of sub-components like spark plug, valves, sensors, control system or others have not been included. However, after more than 400 tests not a single sub-system component failure has found, suggesting a very high level of reliability for the whole system.

Figure 5 shows the results obtained during the tests done to evaluate the power absorbed by the grain; data are reported as total propellant mass flow rate vs. O/F ratio with the absorbed power as parameter. A strong relation between the mass flow rate and the absorbed energy has been observed; among all the possible combinations, the couple $\dot{m}_{\Sigma}=2.38 \mathrm{~g} / \mathrm{s}$ and $\mathrm{O} / \mathrm{F}=5.78$ has been considered optimal because it provides sufficient power for the rocket or ramjet motor ignition (no ignition failure was detected among hundreds of tests) and allows an efficient cooling process.

There were 14 experimental points in the O/F ratio and mass-flow-rate domain used to draw the contours of Figure 5 .

The left-upper corner of the Figure 5 corresponds to high energy output at near stoichiometric values; the low $\mathrm{O} / \mathrm{F}$ ratio of this region makes it useless because decreasing the mass flow rate of the swirling flow we are decreasing the efficiency of the cooling layer protecting the wall against overeating.

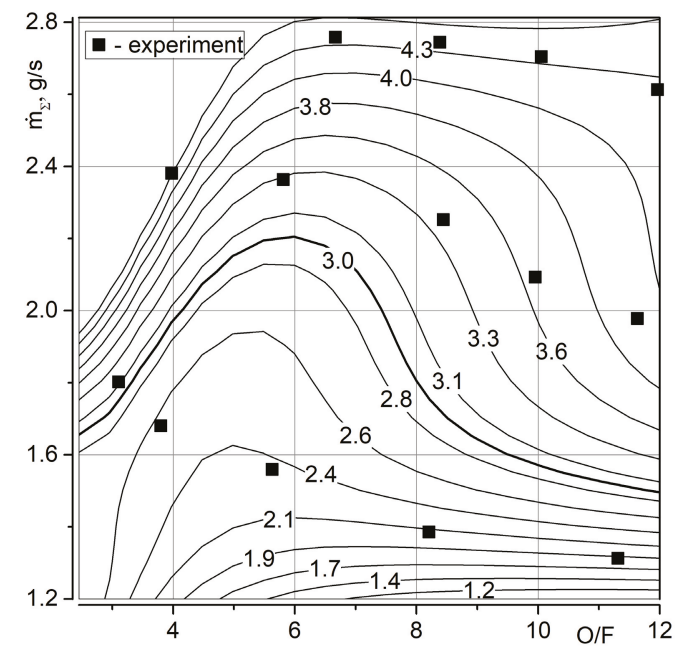

Figure 5. Average power in $\mathrm{kW}$ absorbed by the fuel grain: contour lines are approximations of the experimental data. 
Figure 6 shows the temperature values measured along the vertical line AB, defined earlier (Figure 2). The flow conditions are the same giving the optimal solution identified with the previous tests.

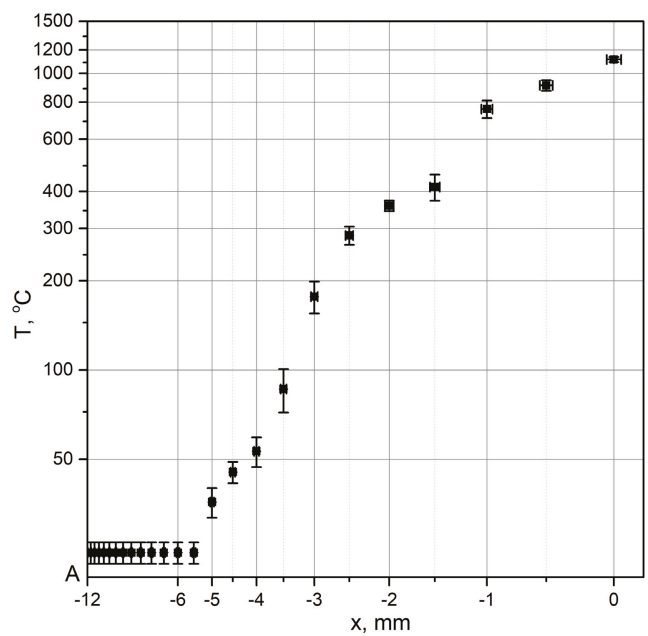

Figure 6. Temperature measured on line AB.

First of all, the effect of the thermocouple on the flow was tested: it partially brakes the cooling vortex and increases the wall heating. This effect is dependent on the thermocouple position. After various tests, the optimal thermocouple position was found to be at $y=-3.0 \mathrm{~mm}$ from the axis, being $\mathrm{y}$ the vertical coordinate along the $\mathrm{AB}$ segment with zero coincident with the injector axis. This position allows a detection of the temperature rise during the ignition process reducing the interference with the cooling vortex. To confirm the efficient effect of the swirling flow in cooling the wall, the maximum temperature registered during $20 \mathrm{~s}$ of combustion has been $\mathrm{T}=95 \pm 2{ }^{\circ} \mathrm{C}$ which is significantly lower than $1150^{\circ} \mathrm{C}$ allowed for the alloy AISI $310 S$ in continuous service [19]. The efficiency of the cooling effect has been also qualitatively observed during a long test sequence of $180 \mathrm{~s}$. The thermocouple was removed from the combustion chamber in order to reduce the disturbances in a swirling flow. Ignition was detected by the change of the exhaust temperature. No significant heat loads, capable of damaging the walls of the combustion chamber or its components, including the nitrile o-rings, have been experienced; the igniter has been disassembled without using heat protection.

It is important to highlight that the total measurement error reported in Figure 6 includes the error due to the thermocouple positioning, estimated to be of order of $\pm 0.05 \mathrm{~mm}$.

\section{Conclusions}

All the performed tests show a stable, reliable and predictable behavior of the whole ignition system. The system overheating has been efficiently prevented by burning oxidizer-reach mixtures with $\mathrm{O} / \mathrm{F}$ ranging between 5 and 8 , being $\mathrm{O} / \mathrm{F}=5.78$ the value allowing the best compromise between energy released and cooling effect on the walls. Moreover, optimal pressure limits for the energy delivery have been found equal to $p_{0}=2.5-3$ bar for both oxidizer and fuel feeding lines. With the use of the displacement method it has been possible to find and test the relation between pressure and mass flow rates, to show their relationship with the released power and also to calculate the minimal amount of propellants necessary for the multiple re-ignition of the hybrid rocket motor. The large number of tests performed shows the high repeatability of the ignitions with a probability higher than $99 \%$. 
With these characteristics, the igniter, and in general the ignition system responded exactly to the requirements imposed during the design phase: future works will focus on test and improvement of the integration of the system in hybrid and ramjet motors to investigate its behavior as a part of the complete propulsion system.

Although based on a preliminary analytical and numerical investigation, this work is purely experimental. A theoretical-numerical study is in progress; based on CFD, it will describe quantitatively heat fluxes and the influence of mass flow rate for different internal geometries focusing on future improvement of this igniter design in terms of total pressure losses, materials and energy released into the main combustion chamber.

Author Contributions: Conceptualization, O.S.; formal analysis, O.S. and D.S.; investigation, O.S. and D.S; writing-original draft preparation, O.S.; writing-review and editing, D.S.; visualization, O.S and D.S.; project administration, O.S.; funding acquisition, O.S. All authors have read and agreed to the published version of the manuscript.

Funding: This research was funded by the Foundation for the Scientific Research Support of the Federal District (FAPDF), Brazil, grant No.0193.001001/2015.

Acknowledgments: The authors are grateful for the technical support of collaborators of the Chemical Propulsion Laboratory of the University of Brasilia.

Conflicts of Interest: The authors declare no conflict of interest.

\section{Abbreviations}

The following abbreviations are used in this manuscript:

AEB Brazilian Space Agency

UnB University of Brasilia

CPL Chemical Propulsion Laboratory

SG Spark Generator

DAQ Data Acquisition

\section{Nomenclature}

$\begin{array}{ll}c & \text { specific heat capacity } \\ m & \text { mass } \\ \dot{m} & \text { mass flow rate, } \mathrm{g} / \mathrm{s} \\ \mathrm{O} / \mathrm{F} & \text { oxidizer-to-fuel ratio } \\ p & \text { pressure, bar } \\ P & \text { probability } \\ Q & \text { energy, } \mathrm{J} \\ t & \text { time } \\ T & \text { temperature, }{ }^{\circ} \mathrm{C} \\ W & \text { power, } \mathrm{W} \\ \alpha & \text { angle, degrees } \\ \Phi & \text { equivalence ratio } \\ \text { Subscripts } \\ 0 & \text { stagnation property } \\ f & \text { fuel } \\ \text { liq } & \text { liquid property } \\ \text { ox } & \text { oxidizer } \\ r e f & \text { reference parameter } \\ \text { sol } & \text { solid property }\end{array}$




\section{References}

1. Andrianov, A.; Shynkarenko, O.; de Morais Bertoldi, A.E.; Barcelos Junior, M.N.D.; Veras, C.A.G. Concept and design of the hybrid test-motor for development of a propulsive decelerator of SARA reentry capsule. In Proceedings of the 51st AIAA/SAE/ASEE Joint Propulsion Conference, Orlando, FL, USA, 27-29 July 2015.

2. Azevedo, V.A.; Alves, I.P.M.; Shynkarenko, O.; Veras, C.A.G. Experimental Investigation of High Regression Rate Paraffin for Solid Fuel Ramjet Propulsion. In Proceedings of the AIAA Propulsion and Energy 2019 Forum, Indianapolis, IN, USA, 19-22 August 2019.

3. CPL. Design and Development of a High-Maneuverability Supersonic Missile with Ramjet Engine. 2019. Available online: http:/ / fga.unb.br/cpl/project-fapdf-ramjet (accessed on 1 July 2020).

4. Jain, S. Self-igniting fuel-oxidizer systems and hybrid rockets. J. Sci. Ind. Res. 2003, 62, 293-310.

5. Whitmore, S.A.; Inkley, N.R.; Merkley, D.P.; Judson, M.I. Development of a Power-Efficient, Restart-Capable Arc Ignitor for Hybrid Rockets. J. Propul. Power 2015, 31, 1739-1749. [CrossRef]

6. Souza, K.; Shynkarenko, O. Development of a measurement system of temperature and pressure in the combustion chamber of a torch ignition system. In Proceedings of the 24th International Congress of Mechanical Engineering, Curitiba, Brazil, 3-8 December 2017.

7. Souza, K. Analysis of the Transient Combustion Process in the Igniter Type Torch. Bachelor's Thesis, University of Brasilia, Brasília, Brazil, 2017.

8. Zabetakis, M. Flammability Characteristics of Combustible Gases and Vapors; U.S. Department of the Interior, Bureau of Mines: Washington, DC, USA, 1965; pp. 9-47.

9. Melvin, A.; Moss, J. Structure in methane-oxygen diffusion flames. Symp. (Int.) Combust. 1975, 15, $625-636$. [CrossRef]

10. Seong, B.; Joon, H.; Dae, B.; Jeong, K. Combustion Characteristics of the Methane-oxygen Diffusion Flame Using Swirl-coaxial Injector in a Model Combustor. In Proceedings of the 53rd AIAA/SAE/ASEE Joint Propulsion Conference, Atlanta, GA, USA, 10-12 July 2017. [CrossRef]

11. Ellis, R.J. Evaluation of a Torch Ignition System for Propulsion. Master's Thesis, The University of Texas at El Paso, El Paso, TX, USA, 2014.

12. Pauly, C.; Sender, J.; Oschwald, M. Ignition of a Gaseous Methane/Oxygen Coaxial Jet. Prog. Propuls. Phys. 2009, 1, 154-170. [CrossRef]

13. Jiaq, Z.; Qing-Lian, L.; Chi-bing, S. Ignition characteristics and combustion performances of a LO2/GCH4 small thrust rocket engine. J. Cent. South Univ. 2018, 25, 646-652. [CrossRef]

14. Li, B.; Shi, B.; Zhao, X.; Ma, K.; Dingjiang, X.; Zhao, D.; Li, J. Oxy-fuel combustion of methane in a swirl tubular flame burner under various oxygen contents: Operation limits and combustion instability. Exp. Therm. Fluid Sci. 2017, 90. [CrossRef]

15. Frenken, G.; Vermeulen, E.; Bouquet, F.; Sanders, B. Development Status of the Ignition System for Vinci. In Proceedings of the 38th AIAA/ASME/SAE/ ASEE Joint Propulsion Conference \& Exhibit, Indianapolis, IN, USA, 7-10 July 2002. [CrossRef]

16. Haidn, O.; Celano, M.P.; Meng, L.; Christof, R.; Silvestri, S.; Slavinskaya, N. On Methane/Oxygen Combustion for Rocket Applications. In Proceedings of the International Symposium on Innovation and Prospects of Liquid Propulsion Technology, Xi'an, China, 4-6 September 2016.

17. Moon, K.H.; Moon, H.; Joo-Ho, C.; Jin-Kon, K. Reliability Prediction of Hybrid Rocket Ignition System. J. Korean Soc. Aviat. Aeronaut. 2016, 24, 26-34. [CrossRef]

18. Yi, W.; Zhang, Z.; Fuwen, L.; Wang, N. Combustion characterization of a $\mathrm{CH}_{4} / \mathrm{O}_{2}$ rapid mixed swirl torch igniter for hybrid rocket motor. Aerosp. Sci. Technol. 2019, 98. [CrossRef]

19. Davis, J. (Ed.) ASM Specialty Handbook: Heat-Resistant Materials; ASM International: Geauga County, OH, USA, 1997; pp. 89-122.

(C) 2020 by the authors. Licensee MDPI, Basel, Switzerland. This article is an open access article distributed under the terms and conditions of the Creative Commons Attribution (CC BY) license (http:/ / creativecommons.org/licenses/by/4.0/). 
MDPI

St. Alban-Anlage 66

4052 Basel

Switzerland

Tel. +41616837734

Fax +41 613028918

www.mdpi.com

Aerospace Editorial Office

E-mail: aerospace@mdpi.com

www.mdpi.com/journal/aerospace

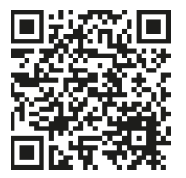



MDPI

St. Alban-Anlage 66

4052 Basel

Switzerland

Tel: +41 616837734

Fax: +41 613028918

www.mdpi.com 